\title{
Solution approaches for facility layout problems
}

\author{
Dissertation \\ zur Erlangung des mathematisch-naturwissenschaftlichen Doktorgrades \\ „Doctor rerum naturalium“" \\ der Georg-August-Universität Göttingen \\ im Promotionsstudiengang Mathematical Sciences \\ der Georg-August University School of Science (GAUSS)
}

vorgelegt von

Mirko Dahlbeck

aus Herne

Göttingen, 2020 


\section{Betreuungsausschuss:}

JProf. Dr. Anja Fischer

Juniorprofessur Management Science

Technische Universität Dortmund

Prof. Dr. Anita Schöbel

Fachbereich Mathematik

Technische Universität Kaiserslautern

Mitglieder der Prüfungskommission:

Referentin:

JProf. Dr. Anja Fischer

Juniorprofessur Management Science

Technische Universität Dortmund

Korreferent:

Prof. Dr. Christoph Lehrenfeld

Institut für Numerische und Angewandte Mathematik

Georg-August-Universität Göttingen

2. Korreferent:

Univ.-Prof. Dipl.-Ing. Dr. Franz Rendl

Institut für Mathematik

Alpen-Adria-Universität Klagenfurt

Weitere Mitglieder der Prüfungskommission:

Prof. Dr. Gerlind Plonka-Hoch

Institut für Numerische und Angewandte Mathematik

Georg-August-Universität Göttingen

Prof. Dr. Thorsten Hohage

Institut für Numerische und Angewandte Mathematik

Georg-August-Universität Göttingen

Prof. Dr. Stephan Huckemann

Institut für Mathematische Stochastik

Georg-August-Universität Göttingen

Tag der mündlichen Prüfung: 20.01.2021 


\section{Contents}

1. Introduction 5

2. Literature review 9

2.1. The single-row and the directed circular facility layout problem . . . . . . . . . . 9

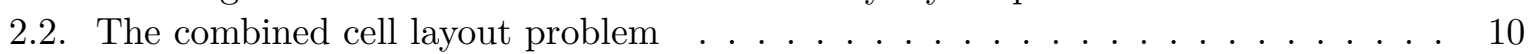

2.3. The multi-row facility layout problem . . . . . . . . . . . . . . 12

3. Summary of the publications 14

3.1. A mixed-integer linear programming approach for the T-row and the multi-bay

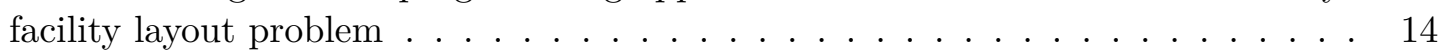

3.1.1. Our MILP approach . . . . . . . . . . . . . . . . . . . . . . . 14

$3.1 .2 . \quad$ Cutting planes $\ldots \ldots \ldots \ldots \ldots \ldots$

3.1.3. The multi-bay facility layout problem with three rows . . . . . . . . . 18

3.1.4. Computational experiments . . . . . . . . . . . . . . . . 19

3.2. New exact approaches for the combined cell layout problem and extensions of the

multi-bay facility layout problem . . . . . . . . . . . . . . . 22

3.2.1. The combined cell layout problem . . . . . . . . . . . . . . 22

3.2.2. Extensions of the multi-bay facility layout problem . . . . . . . . . . 25

$3.2 .3 . \quad$ Computational results $\ldots \ldots \ldots \ldots \ldots \ldots \ldots$

3.3. Combining simulation and optimization for extended double row facility layout

problems in factory planning . . . . . . . . . . . . . . . 31

3.3.1. Extensions of a DRFLP model . . . . . . . . . . . . . . . . 31

3.3.2. Iterative combination of optimization and simulation . . . . . . . . . . . 34

$3.3 .3 . \quad$ Computational results $\ldots \ldots \ldots \ldots \ldots \ldots \ldots$

3.3.4. Manufacturing different products . . . . . . . . . . . . . . . 36

3.4. Decorous combinatorial lower bounds for row layout problems . . . . . . . . . . . 38

3.4.1. Combinatorial lower bounds . . . . . . . . . . . . . . 38

3.4.2. A lower bounding model . . . . . . . . . . . . . . . . . . . . . 41

3.4.3. Computational results . . . . . . . . . . . . . . . . . . . 43

3.5. A study of the relation between the single-row and the double-row facility layout

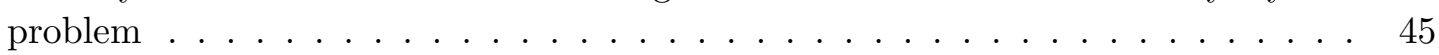

3.5.1. The equidistant case . . . . . . . . . . . . . . . . . . 45

3.5.2. The double-row facility layout problem. . . . . . . . . . . . . . . . . . 46

$3.5 .3 . \quad$ Heuristic approaches for the DRFLP . . . . . . . . . . . . . . . . . . . . . 47

3.5.4. Computational results $\ldots \ldots \ldots \ldots \ldots$. . . . . . . . . . . . 48

\begin{tabular}{ll}
\hline 4. Discussion & 51
\end{tabular}

5. Conclusion and future work

\begin{tabular}{ll}
\hline References & 62
\end{tabular}

\begin{tabular}{ll}
\hline Appendix & 63
\end{tabular}

A. A mixed-integer linear programming approach for the T-row and the multi-bay

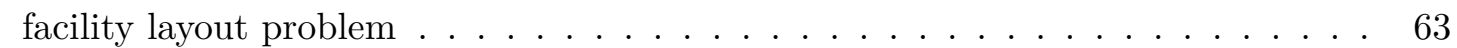

B. New exact approaches for the combined cell layout problem and extensions of the multi-bay facility layout problem . . . . . . . . . . . . . . . . . . 101

C. Combining simulation and optimization for extended double row facility layout problems in factory planning . . . . . . . . . . . . . . . 132

D. Decorous combinatorial lower bounds for row layout problems . . . . . . . . . . . 154 
E. A study of the relation between the single-row and the double-row facility layout

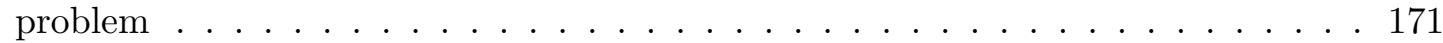




\section{Introduction}

The chief executive officers of real-world factories have to deal with several challenges such as globalization, the growing dynamics of the markets, the increase in customized products, decreasing product life cycles and technological innovations. Therefore, they aim to conduct a cost efficient production in order to remain competitive. The layout of the operating equipment (in the following denoted as departments) is one of the main influencing factors and provides a basis to uphold the long-term productivity and competitiveness [49, 96, 104]. This leads to facility layout problems. One looks for a non-overlapping arrangement of the departments within a given factory or within a factory with varying areas. The goal is to minimize the weighted distances between the departments where the transport weights are based on aggregated information about the transports between the departments 20 . The distance calculation between the departments depends on the structure of the layout and the material handling-systems. Without restrictions on the layout, even small instances are well-known to be rather challenging and deriving optimal solutions for small instances is very time-consuming, see, e.g., [87, 103]. For this reason, one often concentrates on special cases where the structure of the layout and the paths are restricted. These facility layout problems can often be divided into smaller subproblems and these subproblems can be solved independently, see Section B. Therefore, we mainly contribute to the Multi-Bay Facility Layout Problem and its extensions as well as the Multi-Row Facility Layout Problem.

We start our description with the Multi-Bay Facility Layout Problem (MBFLP). We are given a set of departments $[n]:=\{1, \ldots, n\}, n \in \mathbb{N}$, with lengths $\ell_{i}, i \in[n]$, and pairwise weights $w_{i j}, i, j \in[n], i \neq j$, and $m \in \mathbb{N}$ non-overlapping parallel rows $\mathcal{R}:=[m]$. The MBFLP looks for an assignment $r_{i} \in \mathcal{R}, i \in[n]$, of the departments to the rows $\mathcal{R}$ minimizing the weighted sum of the center-to-center distances between the departments such that departments in the same row do not overlap, see, e. g., [27, 28, 86]. The distance between departments in the same row equals their horizontal distance and the distance between departments in distinct rows equals the sum of their horizontal distance to a fixed left border plus the vertical distance $\left|r_{i}-r_{j}\right| w_{\text {path }}^{B}$ for departments $i, j \in[n], i<j$, where $w_{\text {path }}^{B} \in \mathbb{R}_{\geq 0}$ denotes the width of a path between two rows. For an illustration we refer to Figure 1.0.1b. The distance calculation can be interpreted in the following way. There are inner-row and inter-row material handling-systems, whereby the inter-row material handling-system, e. g., an overhead bridge crane [26, 86], is fixed at the left border. The rows might be separated by equipment, some free space for maintenance or passageways [28. The inter-row transport is more costly than the inner-row transport, because two separate material handling systems have to be coordinated, the transfer mechanism is costly (typically a larger capacity crane is used) and at this point the most delay and damages occur, see [28, 86]. High inter-row transport costs can be included by enlarging $w_{\text {path }}^{B}$. The departments are given as one-dimensional objects, so we assume implicitly that the height of the departments equals one. Thus, one can include the height of the departments in the distance calculation by enlarging $w_{\text {path }}^{B}$, for an illustration we refer to Figure $1.0 .1 \mathrm{~b}$. If $w_{\text {path }}^{B}=1$, the height of the departments is included and if $w_{\text {path }}^{B}>1$, then the width of the path is additionally taken into account, and otherwise the width of the path is neglected.

We use distance variables $d_{i j}=d_{j i}, i, j \in[n], i<j$, to measure the center-to-center distances between $i$ and $j$ as described above. We look for a vector $r \in \mathcal{R}^{n}$ of the assignment of the departments to the rows and for a vector $p \in \mathbb{R}^{n}$ where $p_{i}, i \in[n]$, denotes the position of the center of $i$, measured from a fixed left border such that all departments are to the right of this border. Then, we aim to solve the following optimization problem to optimality

$$
\begin{array}{ll}
\min _{r \in \mathcal{R}^{n}, p \in \mathbb{R}^{n}} & \sum_{\substack{i, j \in[n] \\
i<j}}\left(w_{i j}+w_{j i}\right) d_{i j} \\
\text { s.t. } & \left|p_{i}-p_{j}\right| \geq \frac{\ell_{i}+\ell_{j}}{2},
\end{array} \quad i, j \in[n], i<j, r_{i}=r_{j},
$$




$$
\begin{array}{rlrl}
d_{i j} & =\left|p_{i}-p_{j}\right|, & & i, j \in[n], i<j, r_{i}=r_{j}, \\
d_{i j} & =p_{i}+p_{j}+\left|r_{i}-r_{j}\right| w_{\text {path }}^{B}, & i, j \in[n], i<j, r_{i} \neq r_{j} .
\end{array}
$$

Furthermore, we introduce an extension of the MBFLP with $m=3$ rows denoted by (3-BFLP), the so-called T-Row Facility Layout Problem (TRFLP). We are given two orthogonal rows $\mathcal{R}:=\{1,2\}$ which form a $\mathrm{T}$ such that departments assigned to distinct rows cannot overlap. The distances between the departments are measured in rectilinear directions and measuring the distances between departments in distinct rows, one has to take the width of the path $w_{\text {path }}^{T} \in \mathbb{R}_{\geq 0}$ into account. The horizontal row is denoted by row 1 and the vertical row by row 2 . Let $p_{M} \in \mathbb{R}$ denote the position in row 1 measured from a fixed left border which is directly opposite row 2 . If we interpret the rows as line segments and if $w_{p a t h}^{T}=0$, then $p_{M}$ is the point of intersection of row 1 and row 2. Then, the distance calculation in (1.0.1) is adapted such that

$$
d_{i j}=\left|p_{i}-p_{M}\right|+p_{j}+w_{\text {path }}^{T}, \quad i, j \in[n], r_{i}=1, r_{j}=2,
$$

where $p_{i} \in \mathbb{R}$ denotes the center position of $i$ measured from a fixed left (upper) border if $r_{i}=1$ $\left(r_{i}=2\right), i \in[n]$. Note that in the model for the 3-BFLP and the TRFLP one might obtain $p_{i} \leq 0$ for some $i \in[n]$.

The MBFLP is of special interest in practice because these layouts are commonly used layouts in industry such as heavy manufacturing, e.g., steel production and bridge crane manufacturing, and semiconductor fabrication [76, 86, 107]. Further, many real-world factory layouts implicitly use these layout structures, see, e.g., [26, 76]. The TRFLP is a new facility layout problem and an extension of the 3-BFLP as we will show in Section A, and hence the TRFLP arises in the same areas of applications. The more complex path structure of the TRFLP in comparison to the 3-BFLP can be used to divide large facility layout problems into smaller subproblems which can be solved independently, see Section $B$. One advantage of the TRFLP in comparison to the 3-BFLP is that the inter-row material handling-system is not fixed at the left border, and hence the weighted transport distances can be significantly smaller, which can be seen in Section A.

The special case of the MBFLP with $m=1$ is called Single-Row Facility Layout Problem (SRFLP) and is well-known to be $\mathcal{N} \mathcal{P}$-hard [5, 43, 94]. Thus, the MBFLP and the TRFLP are $\mathcal{N} \mathcal{P}$-hard as well. Besides its application in factory planning the SRFLP arises in the arrangement of rooms in hospitals [25, 40, 48] and the arrangement of books on a shelf [8]. The SRFLP is widely studied [3, 4, 58, 64.

We illustrate the distance calculation of the SRFLP, the 3-BFLP and the TRFLP in the following example (this example is presented in Section A). We set $w_{\text {path }}^{T}+1=w_{\text {path }}^{B}$, and thus the heights of the departments in the 3-BFLP are taken into account.

Example 1.0.1. We consider an instance with $n=5$ departments with lengths $\ell_{1}=\ell_{3}=\ell_{4}=$ $4, \ell_{2}=5, \ell_{5}=2$, and non-zero weights $w_{12}=w_{23}=w_{24}=w_{34}=w_{45}=1, w_{14}=3$ with $w_{\text {path }}^{T}=0$ and $w_{\text {path }}^{B}=1$. In Figure 1.0.1 optimal layouts of the SRFLP, the 3-BFLP and the TRFLP are illustrated:

a) An optimal single-row layout is illustrated in Figure $1.0 .1 \mathrm{a}$ with an objective value of

$$
1 \cdot 4.5+3 \cdot 4+1 \cdot 4.5+1 \cdot 8.5+1 \cdot 13+1 \cdot 3=45.5 ;
$$

b) An optimal 3-Bay layout is depicted in Figure 1.0.1b with an objective value of

$$
1 \cdot 6.5+3 \cdot 5+1 \cdot 4.5+1 \cdot 5.5+1 \cdot 10+1 \cdot 3=44.5 ;
$$

c) An optimal T-row layout is shown in Figure 1.0.1c with an objective value of

$$
1 \cdot 6.5+3 \cdot 2+1 \cdot 4.5+1 \cdot 4.5+1 \cdot 9+1 \cdot 3=33.5 \text {. }
$$




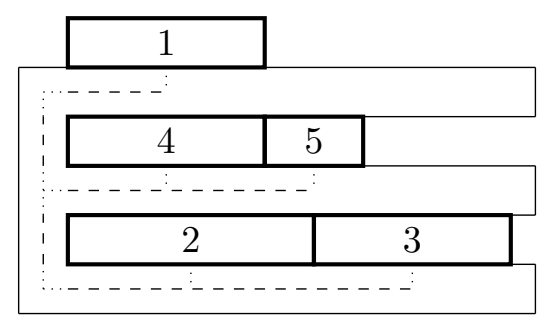

(b) An optimal 3-Bay layout with objective value 44.5 . (a) An optimal single-row layout with objective value 45.5.

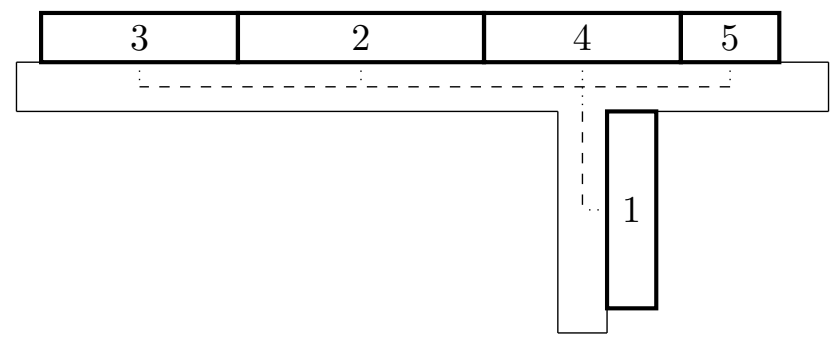

(c) An optimal T-row layout with objective value 33.5 where the center of 4 is on position $p_{M}$ in row 1 .

Figure 1.0.1: We are given an instance with $n=5$ departments with lengths $\ell_{1}=\ell_{3}=\ell_{4}=$ $4, \ell_{2}=5, \ell_{5}=2$, and non-zero weights $w_{12}=w_{23}=w_{24}=w_{34}=w_{45}=1, w_{14}=3$ with $w_{\text {path }}^{T}=0, w_{\text {path }}^{B}=1$. We illustrate optimal layouts for the SRFLP, the 3-BFLP and the TRFLP where the center of 4 is on position $p_{M}$ in row 1 in the illustrated T-row layout.

Highly related to the MBFLP is the Multi-Row Facility Layout Problem (MRFLP), see, e.g., $[19$, 20] with $m \in \mathbb{N}$ rows where the center-to-center distances between the departments are measured in horizontal directions. For $m=2$ this problem is called Double-Row Facility Layout Problem (DRFLP), see, e. g., [12, 89, 101]. As all considered facility layout problems, the MRFLP has application in factory planning and the MRFLP is $\mathcal{N} \mathcal{P}$-hard [12, 101. Additionally, one can use the DRFLP and the MRFLP to determine an arrangement of rooms in hospitals or schools [7, 25, 40, 48,

The main difference between the MBFLP and the MRFLP concerns the distance calculation between departments in distinct rows. In practice, this can be due to restrictions on the layout, e. g., the rows might be separated by equipment, some free space for maintenance or passageways [28]. Additionally, the available material handling systems determine the distance calculation. As a result, in the MRFLP and in the DRFLP one might obtain free-spaces between neighboring departments in the same rows. We illustrate the distance calculation of the DRFLP in the following example (this example is presented in Section E).

Example 1.0.2. We are given an instance with $n=5$ departments with lengths $\ell_{1}=\ell_{3}=\ell_{4}=2$, $\ell_{2}=\ell_{5}=1$, and non-zero weights $w_{12}=w_{45}=3, w_{23}=w_{34}=1$.

a) An optimal single-row layout is depicted in Figure 1.0.2a with objective value

$$
3 \cdot 1.5+1 \cdot 1.5+1 \cdot 2+3 \cdot 1.5=12.5 \text {. }
$$

b) An optimal double-row layout is illustrated in Figure 1.0.2b with objective value

$$
3 \cdot 0+1 \cdot 1.5+1 \cdot 1.5+3 \cdot 0=3 .
$$

In this thesis we mainly contribute to the MBFLP and its extensions and the MRFLP. Several heuristics derive solutions for these problems rather fast but without any knowledge about the 


\begin{tabular}{|l|l|l|l|l|}
\hline 1 & 2 & 3 & 4 & 5 \\
\hline
\end{tabular}

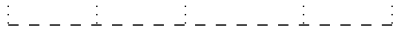

(a) Illustration of an optimal single-row layout with objective value 12.5 .

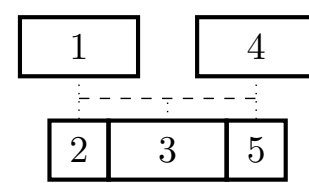

(b) Illustration of an optimal double-row layout with objective value 3 .

Figure 1.0.2: We consider an instance with $n=5$ departments with lengths $\ell_{1}=\ell_{3}=\ell_{4}=2$, $\ell_{2}=\ell_{5}=1$ and non-zero weights $w_{12}=w_{45}=3, w_{23}=w_{34}=1$. We illustrate optimal layouts for the SRFLP and the DRFLP and in the double-row layout there arises free-space between department 1 and department 4 in row 1 .

quality of the solution [12, 27, 33, 86]. Furthermore, exact approaches for the MBFLP are rare in the literature and only small instances can be solved to optimality in reasonable time [42, 66. In contrast to the approaches in the literature, we focus on exact approaches for the MBFLP and we present realistic extensions of the MBFLP in order to include aspects which are relevant in practice. Since the size of the factory, and thus the costs of the initial investment increases with a large number of rows, we focus on the 3-BFLP and the MBFLP with $m=4$ rows (4-BFLP). For instance, in a hospital each floor corresponds to a row and the initial investment costs for building a hospital increases with the number of floors. In Section $\mathrm{A}$ we present an mixed-integer linear programming (MILP) approach for the TRFLP based on extension of the well-known betweenness variables. We show that the TRFLP is a generalization of the 3-BFLP and we adapt our approach to the 3-BFLP. In Section B we present a new exact approach for the 3-BFLP and the 4-BFLP and several further layout problems including extensions of the MBFLP. By comparing the optimal solution value of several facility layout problems we support the chief executive officer to choose a layout for a factory when a factory is built up from the ground.

Usually, in mathematical models only the transport loads are taken into account and for a successful production further key performance indicators play an important role. Hence, in Section C we present a combined optimization-simulation approach for the DRFLP. We extend the current fastest model for the DRFLP [42] in various directions and the simulation is used to detect possible bottlenecks in the production. In order to evaluate the quality of heuristically determined double-row layouts, see, e.g., [12, 33, 89, we present in Section D the first known non-trivial lower bounds for the DRFLP by interpreting a subproblem of the DRFLP as a parallel identical machine scheduling problem. We further improve these lower bounds by an MILP model, which is indeed not a formulation for the DRFLP. In Section E we study the relation between the optimal value of the SRFLP and the MRFLP in the equidistant case where all departments have the same length. In the equidistant case, we prove that the optimal value of the MRFLP is less than or equal to the optimal value of the SRFLP divided by the number of rows. Considering the DRFLP with arbitrary department lengths, we present a formula which states that the optimal value of the SRFLP and the DRFLP might be close and we present an example which shows that this formula is tight. We present heuristics for the DRFLP in the equidistant case and for the general DRFLP and for both problems we improve some of the best known upper bounds for large instances.

This thesis is structured as follows. In Section 2 we present a literature review on facility layout problems. In Section 3 we summarize the five publications contributing to this thesis and we outline the author's contribution to the individual publications. In Section 4 the contributions of the five publications and the connections between the individual publications are discussed. A conclusion and an outlook for future work is given in Section 5 . Finally, all five publications are provided in the appendix. 


\section{Literature review}

Several classes of facility layout problems are studied in the literature, we refer to the surveys [20, 38, 51] for an overview. This thesis contributes to mathematical optimization approaches for facility layout problems and we mainly contribute to the Combined Cell Layout Problem and extensions of the MBFLP, see Section 2.2, as well as the MRFLP, see Section 2.3. So in the literature review we mainly focus on these problems as well. In Section 2.1 we mainly consider special well-studied cases of the Combined Cell Layout Problem. In the following, let a set of departments with lengths and weights be given as described in Section 1 .

\subsection{The single-row and the directed circular facility layout problem}

We start our literature review with the SRFLP. In [94] a dynamic programming algorithm for solving the SRFLP exactly is presented. However, in the last 20 years almost all exact approaches for the SRFLP are either based on MILP formulations, see, e.g., [3, 4, [5, 13], or semidefinite programming (SDP), see [17, 18, 21, 58, 59. At first, we focus on the MILP approaches. The MILP model of [3] and the integer linear programming (ILP) model [4] make use of ordering variables. In 3 instances with up to 15 departments are solved to optimality in at most 7 hours and in [4] instances with up to 18 departments are solved to optimality in less than 6 hours. Several further classes of valid inequalities are presented in [13] and lower bounds for the SRFLP are obtained with gaps less than $8 \%$ to the best known upper bounds within a time limit of 1 day for instances with up to 100 departments. The most promising ILP approach [5] for calculating optimal single-row layouts is based on betweenness variables and instances with up to 35 departments are solved to optimality in at most 6 hours. A polyhedral study concerning this approach can be found in [99. The SDP approaches use products of ordering variables and the SDP approach of $[18$ is able to solve instances with up to 30 departments to optimality within 32 hours. This approach is extended by [21] such that lower bounds with gaps of less than $5 \%$ for instances with up to 100 departments can be calculated in 256 hours. Then, [58, 59] suggested a strengthening of the semidefinite relaxation via inequalities based on 3-cycle-inequalities, see [81]. This leads to the current fastest approach for the SRFLP [58, 59] which is able to solve instances with 36 departments in at most 20 minutes and one instance with 42 departments in less than 2 hours. Furthermore, for instances with up to 100 departments lower and upper bounds are provided with gaps less than $2 \%$ in around 200 hours.

In the last years, several heuristic approaches are provided for the SRFLP using tabu search [71, 98, scatter search [73], a genetic algorithm [36, 72], Lin-Kernighan [70] and variable neighborhood search [46, 91]. A multi-start simulated annealing heuristic is presented in [93] for the SRFLP which obtains the best known solutions or small gaps for instances from the literature with $60 \leq n \leq 80$ departments and even for instances with $n=1000$ departments the running time of this heuristic is at most one hour. Recently, a greedy randomized adaptive search procedure is presented in [34] which could improve several results of 93 for instances with $200 \leq n \leq 500$ departments and obtained solutions with objective value close to the best known values presented in [93. Besides that, there are heuristic approaches concerning extensions of the SRFLP. In [79] a heuristic approach using a firework algorithm is presented for the SRFLP where the positions of some departments are fixed or the ordering of some departments is known in advance. The SRFLP with asymmetric and sequence-dependent clearance conditions between the departments is considered in [84] and an artificial bee colony algorithm is presented. Further, a construction heuristic based on three heuristic rules (greedy, random, linear program (LP) based) is presented in [63] for the SRFLP with so called machine-spanning clearances, i.e., clearance conditions which additionally have to be taken into account between departments which are not neighboring. We refer to [64, 69] for two surveys on the SRFLP.

Given the SRFLP where all departments have the same length, we obtain the Single-Row Equidistant Facility Layout Problem (SREFLP). The SREFLP is a special case of the Koopmans- 
Beckmann version of the Quadratic Assignment Problem (KBQAP), see, e.g., [67, 177. In the KBQAP, we are given $n$ departments and $n$ locations, pairwise distances between the locations and pairwise weights between the departments. Then, one looks for an assignment of the departments to the locations such that the weighted distances between the departments are minimized. In 53 it is shown that the best method for the SRFLP is better than methods especially designed for the SREFLP, see, e.g., [90, 92].

In the Directed Circular Facility Layout Problem (DCFLP) the task is to find an arrangement of the departments along a circle such that the weighted sum of the center-to-center distances measured in clockwise direction is minimized. The DCFLP can be modeled as a Linear Ordering Problem (LOP) [57, 60] where a set of nodes $V=[n]$ and weights $w_{i j}$ and $w_{j i}, i, j \in V, i<j$, are given. Then, the LOP looks for a permutation of the nodes maximizing the sum of the weights where the weight $w_{i j}, i, j \in[n], i \neq j$, is only taken into account if $i$ is left to $j$, see, e. g., [44, 45]. Hence, in practice the DCFLP can be solved faster than the SRFLP, see [57, 60], which was so far considered as the simplest available layout type. An SDP and an ILP approach is given in [60] as well as heuristic approaches such that tight lower and upper bounds for instances with up to 100 departments are provided. According to [1, 74], the DCFLP has several practical advantages over the SRFLP, e.g., relative low initial investment costs because of their space-saving design and high material handling flexibility. The DCFLP arises by, e. g., determining a space-free alignment around a cyclic conveyor system or the cyclic motion path of an industrial robot. We refer to [60] for an overview of further circular layout problems.

\subsection{The combined cell layout problem}

Now, we consider a generalization of the SRFLP and the DCFLP, the so-called Combined Cell Layout Problem (CCLP). We are given a set of cells $\mathcal{C}:=\{1, \ldots, m\}, m \in[n], m \geq 2$, each with an (un-) loading station whose position is denoted by $p_{E_{k}}, k \in \mathcal{C}$. The function $t: \mathcal{C} \rightarrow\{$ SRFLP, DCFLP $\}$ specifies the associated layout type of each cell. For $t(k)=\operatorname{SRFLP}, k \in \mathcal{C}$, the position $p_{E_{k}}$ of the loading station is fixed at the left or right border of cell $k$ and for $t(k)=$ DCFLP, $k \in \mathcal{C}$, the loading station can be placed on an arbitrary position along the circle. The inner-cell distances depend on the type of the cell. The inter-cell distance between cell $k \in \mathcal{C}$ and cell $o \in \mathcal{C}, k<o$, is denoted by $u_{k o}$ and the distance between departments in different cells equals the sum of the distances of the departments to the respective loading station in the same cell plus the corresponding inter-cell distance $u_{k o}$. We also write CCLP $\left(m_{1}, m_{2}\right), m=m_{1}+m_{2}$, where $m_{1} \in \mathbb{Z}_{\geq 0}$ denotes the number of cells of type SRFLP and $m_{2} \in \mathbb{Z}_{\geq 0}$ denotes the number of cells of type DCFLP.

Several special cases of the CCLP have been studied in the literature. If the assignment of the departments to the cells is fixed, the problem is called Fixed-Cell Combined Cell Layout Problem (FC-CCLP). For the FC-CCLP where additionally in each cell one department is fixed as the loading station, the ILP model of [16] outperformed the SDP approach of [55]. To the best of our knowledge, the approach of the publication in Section $B$ is the first that considers the CCLP without fixing one department as loading station and without pre-assigning departments to given cells.

Considering the CCLP with $t(k)=\operatorname{SRFLP}$ for all $k \in \mathcal{C}$ and $u_{k o}=|k-o| w_{\text {path }}^{B}, k, o \in \mathcal{C}, k<o$, we obtain the MBFLP where the cells are interpreted as parallel non-overlapping rows. Thus, the CCLP is a generalization of the MBFLP. Often, two-stages procedures are used for the MBFLP and its extensions where in the first step the assignment of the departments to the rows is determined heuristically, and then a layout of the departments in the same row is calculated heuristically, see, e. g., [27, 28, 86]. These layouts are usually calculated fast but without any knowledge about the quality of the obtained solutions. In [86], the MBFLP is considered where additionally the size of the rows is restricted, i. e., the sum of the lengths of the departments assigned to row $k$ is at most $F_{k}, k \in[m]$. However, in the computational experiments $F_{k}=F \in \mathbb{R}_{\geq 0}, k \in[m]$, is chosen. Then, in the first step the assignment of the departments to the rows is determined by solving an MILP model neglecting distances between departments in the same row and minimizing the 
sum of the weighted inter-row distances between the departments in distinct rows such that the sum of the lengths of the departments in row $k$ does not exceed $F_{k}, k \in[m]$. In the second step a dummy department $n+1$ with length $\ell_{n+1}=0$ and weights $w_{i(n+1)}=w_{(n+1) i}, i \in[n]$, which are equal to the sum of the weights of $i$ to departments $[n] \backslash\{i\}$ which lie in a different row than $i$, is added. One determines a layout of the departments in the same row respecting the dummy department $n+1$ which is fixed at the border of the layout and the lengths of the departments are set to one. Then, the dynamic programming algorithm of [94] is used to solve $m$ single-row instances independently.

Exact approaches for the MBFLP are rarely studied in the literature. In [42] an ILP model is presented for the MBFLP where the assignment of the departments to the rows is fixed, and instances with up to 25 departments and up to 5 rows are solved in less than one second. In order to compute an optimal solution for the MBFLP one has to enumerate over all distinguishable assignments of the departments to the rows. These results can be found in the computational experiments in Section A. A survey on the MBFLP is given in [37].

The Flexible Multi-Bay Facility Layout Problem (FBFLP) is an extension of the MBFLP, see, e. g., [28, 66, [76], where the departments are given as two-dimensional objects such that every department has a length and a height. Then, the height of a row equals the height of a department with the greatest height in that row and the vertical distance between two rows equals half of the heights of these two rows plus the sum of the heights of the rows between them. The motivation is that the rows are parallel and by calculating distances between departments in distinct rows, the heights of the intermediate rows have to be taken into account. The departments have to be arranged space-free within the bays. In contrast to the MBFLP, the number of rows is not restricted. The MILP approach of [66] can solve one instance with 12 departments in less than two hours but is not able to solve an instance with 11 departments within a given time limit of 24 hours. Besides the exact approach, the two-stage procedure of [86] is adapted to this case by [28] and several further heuristics are presented using ant colony optimization algorithms [30, 31, 76, 106]. A particle swarm optimization algorithm is presented in [75] for the FBFLP where free-spaces between departments in horizontal and vertical directions are allowed within the bays. Another extension of the FBFLP has been considered in [85] where several periods are considered and the pairwise weights of the departments might change after a period. The departments can be rearranged between the periods and the rearrangement leads to extra costs. For this problem, a genetic algorithm is provided in [85. In conclusion, exact approaches for the MBFLP and the FBFLP are rare in the literature. A survey enclosing the FBFLP is given in [20].

The layout problem similar to the MBFLP but with $u_{k o}=w_{\text {path }}^{B}, k, o \in \mathcal{C}, k<o$, for some $w_{\text {path }}^{B} \in \mathbb{R}_{\geq 0}$ is denoted by Pier-Type Material Flow Pattern (PMFP). In [32] an MILP model for the PMFP is presented, and an instance with 12 departments and up to five rows is considered where a gap of around $95 \%$ is obtained after a time limit of 2 hours. The authors conclude with the research question to develop a more efficient approach for the PMFP. The PMFP has application in designing the layout of cross docking warehouses, e. g., there are some departments for receiving incoming materials and the other departments are used for direct shipping of shipping supplies [32]. We present a well-performing exact approach for the PMFP with $m=3$ (3-PMFP) and $m=4$ (4-PMFP) rows in Section B.

Furthermore, we present an exact approach for the 4-BFLP in Section B and we introduce an extension of the 4-BFLP, the so-called X-Row Facility Layout Problem (XRFLP). The departments are given as one-dimensional objects, so we assume that the height of the departments equals one. Given four non-overlapping cells of type SRFLP which form an X and let $d_{i p_{E_{k}}}$ denote the distance of the center of $i \in[n]$ to $p_{E_{k}}$ if $i$ is assigned to cell $k \in[4]$. Let $C_{2}\left(C_{3}\right)$ denote the set of departments assigned to cell 2 (cell 3), then one has to ensure that the departments in cell 2 and in cell 3 do not overlap, i. e., either $d_{i p_{E_{2}}} \geq 1+\frac{\ell_{i}}{2}, i \in C_{2}$, or $d_{i p_{E_{3}}} \geq 1+\frac{\ell_{i}}{2}, i \in C_{3}$, has to be satisfied. For an illustration we refer to Figure 2.2.1 (the Figure is presented in Section B). The XRFLP is an extension of the 4-BFLP because one additionally has to ensure that departments in 

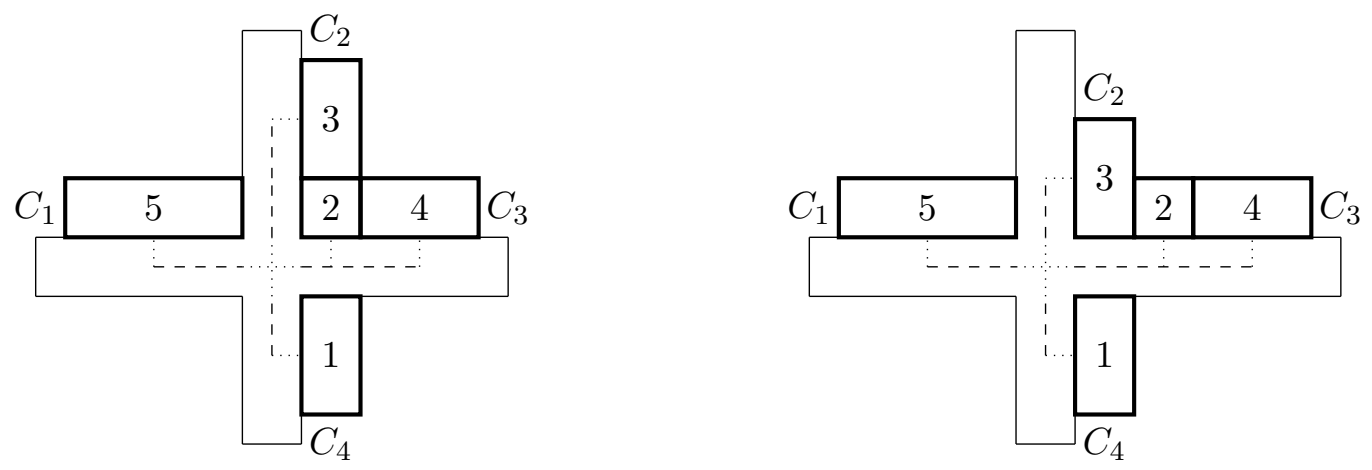

Figure 2.2.1: Illustration of two feasible X-row layouts with $n=5$ departments. Note that one has to ensure that departments in cell 2 and cell 3 do not overlap.

cell 2 and cell 3 do not overlap. In factory planning it is realistic to take the width of the path between cells into account because the products have to be transported between the departments by a forklift or an automatic guided vehicle and the transportation systems usually travel in a rectangular manner. Considering the XRFLP, let $w_{\text {path }}^{1}\left(w_{\text {path }}^{2}\right)$ denote the width of the path between cell 1 and cell 3 (cell 2 and cell 4). Measuring the distances between cell 2 and cell 3 we do not cross a path, and hence we set the inter-cell distance to zero. In contrast, going from cell 1 to cell 4 we cross both paths, i. e., $u_{14}=w_{\text {path }}^{1}+w_{\text {path }}^{2}$. For the remaining inter-cell distances we obtain $u_{12}=u_{13}=w_{\text {path }}^{1}$ and $u_{24}=u_{34}=w_{\text {path }}^{2}$.

We refer to Section $B$ for a theoretical and computational study of the relation of the optimal solution values of the SRFLP, DCFLP, CCLP $(2,0)$, CCLP $(1,1)$, CCLP $(0,2)$, TRFLP, 3-BFLP, 4-BFLP, 3-PMFP, 4-PMFP, and the XRFLP.

\subsection{The multi-row facility layout problem}

Several MILP approaches are available for the DRFLP [8, 10, 11, 29, 33, 102] (see [110] for a correction of [33]). The ILP-based approach in [33] (see also the corresponding corrections by [110]) can handle instances with up to 10 departments whereas the exact ILP approach of [8] can solve instances with up to 12 departments to optimality. The latter model was improved in [101 such that one is able to solve a DRFLP instance with 15 departments in at most 11 hours. However, the approach of [42] is the current fastest exact approach for the DRFLP as well as the MRFLP and can solve DRFLP instances with up to 16 departments in less than 12 hours and multi-row instances with up to 5 rows and 13 departments in less than 7 hours. From a practical point of view the DRFLP seems much harder than the SRFLP. However, for both problems MILP approaches based on betweenness variables outperform MILP approaches based on ordering variables [4, 5, 8, 101].

To the best of our knowledge there has not been research done on computing non-trivial lower bounds for the DRFLP. The approach of [42] enumerates over all distinguishable assignments of the departments to the rows. Thus, one cannot use this approach for deriving lower bounds for larger instances. The MILP models, see, e.g., [8, 101], make us of big- $M$-type constraints to ensure the correct relation of position variables and ordering variables, and hence their linear relaxations are rather weak. So using them in a branch-and-cut approach leads to weak lower bounds and so to large gaps for medium-sized and large DRFLP instances, even after a longer time limit because the root node gaps are hardly improved. For detailed computational results we refer to Section D.

We are aware of only a few problem specific heuristic approaches for the DRFLP. In [33], two departments with the pairwise greatest product of their weights and the sum of their lengths are fixed directly opposite, and then according to five different rules the remaining departments are 
added to the layout, where in each step one department is assigned via a 1-opt algorithm. Note that, given the assignment of the departments to the rows and the ordering of the departments in the same row, one can determine the exact positions of the departments by solving an LP. A related heuristic approach is given in [89. The heuristic of [12] seems to calculate good double-row layouts, but one drawback is the high running time. In [112] a multi-objective tabu search is used for the DRFLP with the additional goal to minimize the area of the layout. Another heuristic for an extended version of the DRFLP is given in [113] where the departments are given as two-dimensional objects and vertical distances as well as certain clearance conditions are taken into account. The DRFLP with clearance conditions is considered in [47] and a decomposed-based heuristic is applied. Further heuristics for the DRFLP include dynamic aspects [22, 105]. A genetic algorithm for an extended version of the MRFLP is presented in [97] and a three-stage heuristic is provided in [111]. Recently, a two-stage heuristic has been presented in [19] for the MRFLP which derives solutions quickly for $m \geq 3$. However, without knowledge of good lower bounds it is difficult to evaluate the quality of these heuristics.

Several special cases of the MRFLP and the DRFLP are studied in the literature which often concern the arrangement or the lengths of the departments. In the Space-Free MRFLP and Space-Free DRFLP (SF-MRFLP and SF-DRFLP) one restricts to a common left border of the rows and spaces between neighboring departments in the same row are not allowed. Note that for the CCLP and its special cases there always exists an optimal layout without spaces between neighboring departments. Exact approaches for the SF-DRFLP were presented in [7, 41, 42, 54] and heuristic approaches in 2, 68. The approach of 42$]$ is the current fastest approach for the SF-DRFLP and, similar to the general DRFLP, instances with up to 16 departments are solved to optimality in less than 12 hours. If additionally the row assignment of the departments is fixed in the SF-MRFLP, we obtain the $m$-Parallel Row Ordering Problem (mPROP) and for $m=2$ we obtain the Parallel Row Ordering Problem (PROP) [9, 52, 82, 108. The current fastest approach for these problems is able to solve instances with up to 25 departments to optimality [42]. For larger instances one can derive lower bounds via the SDP approach in [52].

Now we consider the MRFLP and the DRFLP where all departments have the same length, then we obtain the Multi-Row Equidistant Facility Layout Problem (MREFLP) and the Double-Row Equidistant Facility Layout Problem (DREFLP) [6, 56]. We assume in the following that the lengths of the departments are equal to one in the MREFLP and in the DREFLP. In the MREFLP the departments can be arranged on an integer grid and an ILP and an SDP model are presented [14, 15]. Equidistant double-row and equidistant multi-row instances with up to 25 departments are solved to optimality for $2 \leq m \leq 5$. Moreover, gaps with less than $4 \%$ are obtained for instances with up to 50 departments and $2 \leq m \leq 5$ [14, 15]. Due to the grid structure of optimal solutions the MREFLP can be seen as a special case of the KBQAP, see, e. g., [80]. 


\section{Summary of the publications}

In this chapter we summarize each of the five research publications which are the core of this cumulative thesis. At the end of each section, the author's contribution is summarized. The first publication concerns an MILP approach for the TRFLP and the 3-BFLP and in the second publication new exact approaches for the CCLP and extensions of the MBFLP are presented. In the third, fourth and fifth publication we focus on the DRFLP presenting a combined simulation and optimization approach, combinatorial lower bounds and a study of the relation between the SRFLP and the DRFLP. Additionally, in the fifth publication the MREFLP is considered.

In order to secure a consistent terminology in this thesis, the notation in this Section might differ from the notation in the publications.

\subsection{A mixed-integer linear programming approach for the T-row and the multi-bay facility layout problem}

In this section we summarize the publication provided in Section $\mathrm{A}$ and Mirko Dahlbeck is the sole author of this work.

In this work we introduce the TRFLP and we show that the TRFLP is a generalization of the 3-BFLP. Further, if $w_{\text {path }}^{T} \leq w_{\text {path }}^{B}$, the optimal value of the TRFLP is less than or equal to the optimal value of the 3-BFLP. We present an MILP approach for the TRFLP and the 3-BFLP based on an extension of the well-known betweenness variables, see, e.g., [5, 42, which now can be equal to one if the corresponding departments lie in different rows. We use transitivity constraints to combine the extended betweenness variables with the remaining variables and we provide cutting planes exploiting the crossroad structure. Hence, T-row (3-Bay) instances with up to 18 (17) departments are solved to optimality in less than 7 hours and we outperform the current best exact approach for the 3-BFLP.

\subsubsection{Our MILP approach}

We start this section with the following proposition, which is essential for our algorithm:

Proposition 3.1.1. Given a T-row instance, there exists an optimal T-row layout where the center of one department lies on position $p_{M}$ in row 1.

Therefore, we fix the center of $s_{M} \in[n]$ on position $p_{M}$ in row 1 and we denote the resulting problem by $\left(s_{M}\right.$-TRFLP). The $s_{M}$-TRFLP is $\mathcal{N} \mathcal{P}$-hard, but one can calculate the rectilinear centerto-center distances between departments in distinct rows easier than in the TRFLP. So we set up a MILP model for the $s_{M}$-TRFLP which we solve with a branch-and-cut algorithm.

Then, our algorithm for the TRFLP works in the following way. For each $s_{M} \in[n]$ we heuristically determine an $s_{M}$-T-row layout, and we sort the departments by increasing order of the objective values of the $s_{M}$-T-row layouts. Let $s_{M} \in[n]$ be the first not yet considered department in this sorting. Then, we solve the $s_{M}$-TRFLP with our branch-and-cut algorithm where the current best objective value is set as an upper bound, i. e., the branch-and-cut algorithm is interrupted if the best lower bound exceeds the upper bound. We repeat this until the $s_{M}$-TRFLP with the described upper bound is solved for each $s_{M} \in[n]$.

We present a short summary of our MILP model for the $s_{M}$-TRFLP. So, in the following, let $s_{M} \in[n]$ be fixed. If $i \in[n]$ and $j \in[n] \backslash\{i\}$ lie in row 2 and $i$ is above (below) $j$, we say that $i$ is left (right) to $j$. Then, we use ordering and assignment variables

$$
z_{i j}=\left\{\begin{array}{ll}
1, & i \text { is left to } j \text { and } i \text { and } j \text { are in the same row, } \\
0, & \text { otherwise, }
\end{array} \quad i, j \in[n], i \neq j,\right.
$$




$$
y_{i}= \begin{cases}1, & \text { if } i \text { lies in row } 1 \\ 0, & \text { otherwise }\end{cases}
$$

$i \in[n]$.

At first, we fix $s_{M}$ to row 1 , see equation (3.1.1), and we ensure that $i \in[n] \backslash\left\{s_{M}\right\}$ is left or right to $s_{M}$ if and only if $i$ is in row 1 , see equations 3.1 .2

$$
\begin{aligned}
& y_{s_{M}}=1 \text {, } \\
& z_{i s_{M}}+z_{s_{M} i}-y_{i}=0, \quad i \in[n] \backslash\left\{s_{M}\right\} .
\end{aligned}
$$

We add two dummy departments $n+1$ and $n+2$ with lengths $\ell_{n+1}=\ell_{n+2}=0$ and weights $w_{i j}=w_{j i}=0, i, j \in[n+2], i<j,|\{i, j\} \cap\{n+1, n+2\}| \geq 1$, to our model, and we fix $n+1$ at the left border of row 1 and $n+2$ at the left (upper) border of row 2 . Then, we define betweenness variables where at least one of the three departments is a dummy department or $s_{M}$ in the following way

$$
x_{i k j}=x_{j k i}= \begin{cases}1, & \text { if } k \text { lies between } i \text { and } j \text { in the same row } \\ 0, & \text { otherwise }\end{cases}
$$

$i, j, k \in[n+2],\left|\{i, j, k\} \cap\left\{s_{M}, n+1, n+2\right\}\right| \geq 1, i<j,|\{i, j, k\}|=3$. Remark that, given three departments and one of them is equal to $s_{M}, n+1$ or $n+2$, the corresponding betweenness variables are only equal to one if all three departments lie in the same row. We show some inequalities to combine these betweenness variables with the ordering and assignment variables

$$
\begin{array}{ll}
x_{i s j}+x_{s i j}+x_{s j i}-y_{i}-y_{j} \geq-1, & i, j \in[n] \backslash\left\{s_{M}\right\}, i<j, s \in\left\{s_{M}, n+1\right\}, \\
x_{i s j}+x_{s i j}+x_{s j i}-y_{i} \leq 0, & i, j \in[n] \backslash\left\{s_{M}\right\}, i \neq j, s \in\left\{s_{M}, n+1\right\}, \\
x_{i s_{M} j}-z_{i s_{M}}-z_{s_{M} j} \geq-1, & i, j \in[n] \backslash\left\{s_{M}\right\}, i \neq j, \\
x_{(n+1) i j}+x_{(n+2) i j}-z_{i j}=0, & i, j \in[n] \backslash\left\{s_{M}\right\}, i \neq j .
\end{array}
$$

If $i \in[n] \backslash\left\{s_{M}\right\}$ and $j \in[n] \backslash\left\{s_{M}\right\}, i<j$, are assigned to row 1 and $s \in\left\{s_{M}, n+1\right\}$, then the sum of the corresponding three betweenness variable equals one, see inequalities (3.1.3). Let $i, j \in[n] \backslash\left\{s_{M}\right\}, i \neq j$. Inequalities 3.1 .4 ensure that betweenness variables containing $i, j$ and $s_{M}$ or $n+1$ are equal to zero if $i$ or $j$ (or both) are assigned to row 2. Inequalities 3.1.5) are related to the standard linearization, so if $i$ is left to $s_{M}$ and $j$ is right to $s_{M}$ in the same row, then $s_{M}$ lies between $i$ and $j$ in the same row. Equations 3.1.6 ensure that $i$ and $j$ are in the same row and $i$ is left to $j$ if and only if $i$ is left to $j$ in row 1 or row 2 . So we are able to combine $x_{(n+2) i j}, i, j \in[n] \backslash\left\{s_{M}\right\}, i \neq j$, with the ordering variables without using standard linearization. Further inequalities are shown in Section A.

We want to give some insights about our branch-and-cut algorithm. At first, the LP consisting of the inequalities included in the beginning is solved with $x_{i k j} \in[0,1], i, j, k \in[n+2],|\{i, j, k\}|=$ $3, i<j, z_{i j} \in[0,1], i, j \in[n] \backslash\left\{s_{M}\right\}, i \neq j, z_{i s_{M}} \in[0,1], i \in[n] \backslash\left\{s_{M}\right\}, y_{i} \in[0,1], i \in[n]$, where we define the betweenness variables $x_{i k j}, i, j, k \in[n] \backslash\left\{s_{M}\right\},|\{i, j, k\}|=3, i<j$, below. Then, violated cutting planes are added according to our separation strategy, we refer to Section $\mathrm{A}$ for details. This LP is solved again until we obtain a binary solution, i.e., the $y, z$ and $x$ variables are binary, or until no violated cutting plane can be found. The objective value of this (fractional) solution is defined as the value of the root relaxation.

The distance between $i \in[n]$ and $j \in[n] \backslash\{i\}$ in the $s_{M}$-TRFLP can be expressed by $\left|p_{i}-p_{M}\right|+$ $p_{j}+w_{\text {path }}^{T}$ if $i$ lies in row 1 and $j$ in row 2 , see equations 1.0 .2 . So one can use these equations with big- $M$-constraints to combine the distance variables with the assignment variables to take the row assignment of $i$ and $j$ into account. However, this leads to a rather bad root relaxation, because these inequalities are often redundant for fractional values of $y$, e. g., if $y_{i}=y_{j}=\frac{1}{2}$ these inequalities are redundant. In further steps in the branch-and-cut algorithm, these lower bounds are hardly improved for fractional values of $y$, so this leads to a poorly performing algorithm as we will see in Section 3.1.4. 
Therefore, we aim to calculate the rectilinear center-to-center distances between the departments without big- $M$-constraints. Our idea is to extend the definition of the betweenness variables. Given an $s_{M}$-T-row layout, we define $B_{1}\left(B_{2}\right)$ as the set of departments left (right) to $s_{M}$ and $B_{3}$ as the set of departments in row 2 . Let $i^{\prime}\left(j^{\prime}\right)$ be the department in $B_{a}\left(B_{b}\right)$ closest to $p_{M}, a, b \in\{1,2,3\}, a \neq b$. We arrange the departments $B_{a} \cup B_{b}$ on a straight line such that $i^{\prime}$ and $j^{\prime}$ are neighboring as well as neighboring departments in the $s_{M}$-T-row layout are neighboring. Let $i, j, k \in[n] \backslash\left\{s_{M}\right\},|\{i, j, k\}|=3, i<j$. We say that $k \in[n] \backslash\left\{s_{M}\right\}$ lies between $i$ and $j$ if there exists $a, b \in\{1,2,3\}$ such that $k$ lies between $i$ and $j$ on the with $B_{a} \cup B_{b}$ associated straight line. If $a=b$, then the departments in the set $B_{a}$ are already arranged on a straight line. In the $s_{M}$-TRFLP an automated guided vehicle has to traverse every department which lies between $i$ and $j$. This motivates the following extension of the betweenness variables

$$
x_{i k j}=x_{j k i}= \begin{cases}1, & \text { if } k \text { lies between } i \text { and } j, \\ 0, & \text { otherwise }\end{cases}
$$

$i, j, k \in[n] \backslash\left\{s_{M}\right\},|\{i, j, k\}|=3, i<j$. So our new betweenness variables $x_{i k j}$ might also be equal to one if $i \in[n] \backslash\left\{s_{M}\right\}$ and $j \in[n] \backslash\left\{s_{M}\right\}, i<j$, lie in distinct rows, $k \in[n] \backslash\left\{s_{M}, i, j\right\}$. Since this version of betweenness variables seems to be a novelty in the layout planning literature, we illustrate their usage by an example:

Example 3.1.2. We are given a T-row instance with $n=6$ departments with lengths $\ell_{1}=\ell_{5}=$ $3, \ell_{2}=4, \ell_{3}=1, \ell_{4}=\ell_{6}=2$. An $s_{M}$-T-row layout with $s_{M}=2$ is illustrated in Figure 3.1.1 including the dummy departments 7 and 8. We summarize all betweenness variables which are equal to one. We start with $x_{i k j}, i, j, k \in[n], i<j,|\{i, k, j\}|=3$, where $i$ and $j$ lie in distinct rows: $x_{156}=x_{356}=x_{435}=x_{436}=x_{456}=1$. For betweenness variables containing $s_{M}$ or at least one of the dummy departments, we get: $x_{123}=x_{124}=x_{234}=x_{217}=x_{317}=x_{327}=x_{417}=$ $x_{427}=x_{437}=x_{658}=1$. Besides that we get $x_{134}=1$ and the remaining betweenness variables are equal to zero.

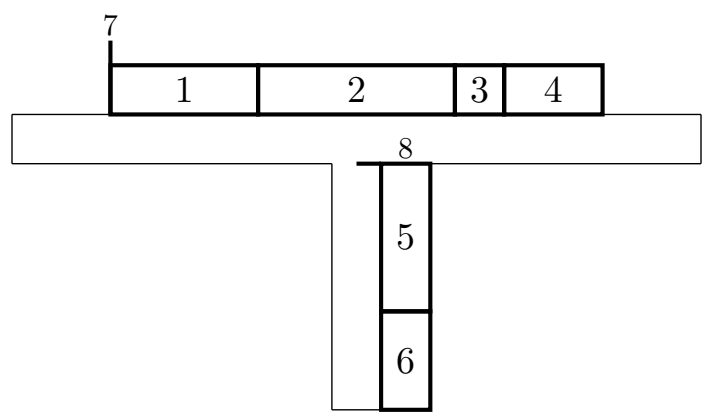

Figure 3.1.1: Illustration of a $s_{M}$-TRFLP layout with $s_{M}=2$ where the dummy department 7 (8) is at the left (upper) border of row 1 (row 2). We summarize all extended betweenness variables, i. e., betweenness variables which do not contain a dummy department or $s_{M}$, which are equal to one: $x_{156}=x_{356}=x_{435}=x_{436}=x_{456}=1$.

We present the following inequalities to obtain the correct relation between our new extended betweenness variables and betweenness variables containing $s_{M}$ or $n+1$

$$
\begin{array}{ll}
x_{i k j}-x_{s_{M} k i}+x_{s_{M} k j}-x_{(n+2) k i}+x_{(n+2) k j} \geq 0, & i, j, k \in[n] \backslash\left\{s_{M}\right\},|\{i, j, k\}|=3, \\
x_{i k j}-x_{s_{M} k i}-x_{s_{M} k j}-x_{(n+2) k i}-x_{(n+2) k j} \leq 0, & i, j, k \in[n] \backslash\left\{s_{M}\right\},|\{i, j, k\}|=3, i<j, \\
& i, j, k \in[n] \backslash\left\{s_{M}\right\}, i<j<k .
\end{array}
$$




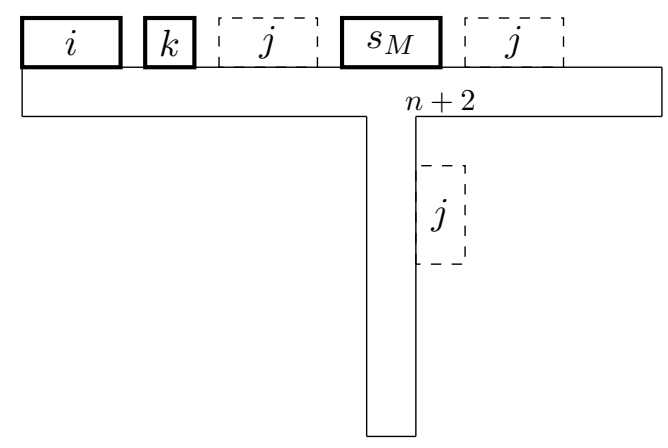

(a) Let $x_{i k s_{M}}=1$. Then, $k$ lies between $i$ and $j$ if and only if $k$ does not lie between $s_{M}$ and $j$.

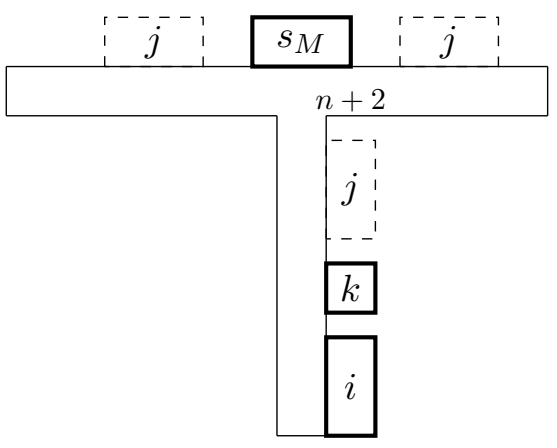

(b) Let $x_{(n+2) k i}=1$. Then, $k$ lies between $i$ and $j$ if and only if $k$ is not left to $j$ in row 2 , i. e., $x_{(n+2) k j}=0$.

Figure 3.1.2: Let $i, j, k \in[n] \backslash\left\{s_{M}\right\},|\{i, j, k\}|=3$. Visualization of inequalities (3.1.7) with $x_{s_{M} k i}=1$ in Figure $3.1 .2 \mathrm{a}$ and $x_{(n+2) k i}=1$ in Figure $3.1 .2 \mathrm{~b}$. We illustrate possible positions for $j$ in dashed rectangles such that $x_{i k j}=1$. The dummy department $n+1$ is not illustrated here.

Considering three departments, at most one of them lies in the middle, see inequalities (3.1.9). Let $i, j, k \in[n] \backslash\left\{s_{M}\right\},|\{i, j, k\}|=3$, be fixed, and we consider inequalities (3.1.7). Let $k$ lie between $i$ and $s_{M}(n+2)$ and let $k$ not lie between $s_{M}(n+2)$ and $j$. Then, $k$ lies between $i$ and $j$, for an illustration we refer to Figure 3.1.2a $(3.1 .2 \mathrm{~b})$. Otherwise, inequalities (3.1.7) are redundant. By inequalities (3.1.8) we obtain upper bounds for $x_{i k j}, i, j, k \in[n] \backslash\left\{s_{M}\right\},|\{i, j, k\}|=3, i<j$, we refer to Section $\mathrm{A}$ for details.

It remains to calculate the rectilinear center-to-center distances between the departments. For the $s_{M}$-TRFLP there always exists an optimal space-free layout, i. e., an optimal layout without free-spaces between neighboring departments in the same row. So we set

$$
\begin{aligned}
& d_{i s_{M}}=\frac{\ell_{i}}{2}+\frac{\ell_{s_{M}}}{2} y_{i}+w_{\text {path }}^{T}\left(1-y_{i}\right)+\sum_{k \in \backslash\left\{s_{M}, i\right\}} \ell_{k}\left(x_{i k s_{M}}+x_{(n+2) k i}\right), \quad i \in[n] \backslash\left\{s_{M}\right\}, \quad \\
& d_{i j}=\frac{\ell_{i}+\ell_{j}}{2}+\left(1-z_{i j}-z_{j i}\right)\left(w_{\text {path }}^{T}+\frac{\ell_{s_{M}}}{2}\right)+\sum_{k \in[n] \backslash\{i, j\}} \ell_{k} x_{i k j}, \quad i, j \in[n] \backslash\left\{s_{M}\right\}, i<j .
\end{aligned}
$$

By equations 3.1.10 we calculate the rectilinear center-to-center distance between $s_{M}$ and $i \in[n] \backslash\left\{s_{M}\right\}$ by summing up the lengths of all departments which are between $i$ and $s_{M}$ and $i$ and $n+2$. Additionally, we add $\frac{\ell_{s_{M}}}{2}$ if $i$ lies in row 1 and $w_{\text {path }}^{T}$ if $i$ is in row 2. By equations (3.1.11) we measure the rectilinear center-to-center distance between $i \in[n] \backslash\left\{s_{M}\right\}$ and $j \in[n] \backslash\left\{s_{M}\right\}, i<j$. We add $\frac{\ell_{s_{M}}}{2}+w_{\text {path }}^{T}$ if $i$ and $j$ lie in distinct rows and we add the sum of the lengths of the departments between $i$ and $j$. In Section A we exclude some constants and we show how the distance variables can be neglected.

Additionally, we aim to eliminate symmetrical layouts. Let $S=\left(\pi_{1}, \ldots, \pi_{n-1}\right)$ denote an arbitrary sorting of the departments $[n] \backslash\left\{s_{M}\right\}$. If $\pi_{1}$ is assigned to row 1 , we fix $\pi_{1}$ to the left of $s_{M}$, see equation (3.1.12). However, if $\pi_{1}$ is assigned to row 2, we fix another department in row 1 to the left of $s_{M}$. We continue in this manner, see inequalities (3.1.13). These inequalities are related to symmetry breaking constraints for the graph coloring problem, see, [83, 88. We set

$$
\begin{aligned}
& z_{\pi_{1} s_{M}}-y_{\pi_{1}}=0 \\
& z_{\pi_{j} s_{M}}-y_{\pi_{j}}+\sum_{k=1}^{j-1} y_{\pi_{k}} \geq 0, \quad j=2, \ldots, n-1 .
\end{aligned}
$$




\subsubsection{Cutting planes}

In the following, let $s_{M} \in[n]$ be fixed. In this section we describe further inequalities to strengthen our formulation for the $s_{M}$-TRFLP. Let $i, j, k \in[n] \backslash\left\{s_{M}\right\}, i<j<k$, be given. In the SRFLP the sum of the corresponding three betweenness variables, i. e., $x_{i k j}+x_{j i k}+x_{i j k}$, equals one but this sum might be equal to zero in the $s_{M}$-TRFLP. This is a significant difference because in the root relaxation of our branch-and-cut algorithm the value of the betweenness variables might be close to zero. This would lead to a rather bad objective value at the root relaxation. Therefore, we present various lower bounds for the sum of the betweenness variables.

Proposition 3.1.3. Let $s_{M} \in[n]$. Then, the following inequalities are valid for the $s_{M}-T R F L P$

$$
\begin{array}{ll}
x_{i k j}+x_{j i k}+x_{i j k}+x_{i s_{M} j}+x_{i s_{M} k}+x_{j_{M} k} \geq 1, & i, j, k \in[n] \backslash\left\{s_{M}\right\}, i<j<k, \\
x_{i k j}+x_{j i k}+x_{i j k}+x_{i_{s_{M}}}+x_{j_{s_{M}}}-y_{k} \geq 0, & i, j, k \in[n] \backslash\left\{s_{M}\right\},|\{i, j, k\}|=3, i<j, \\
& i, j, k \in[n] \backslash\left\{s_{M}\right\}, i<j<k .
\end{array}
$$

Proof. Let $S:=\{i, j, k\} \subseteq[n] \backslash\left\{s_{M}\right\},|\{i, j, k\}|=3, i<j$, be given. Note first that inequalities 3.1.14-3.1.16 are satisfied if $x_{i k j}+x_{i j k}+x_{j i k}$ equals one. It turns out that $x_{i k j}+x_{i j k}+x_{j i k}$ equals zero if and only if $s_{M}$ lies between two departments of the set $S$ and the remaining department in $S$ lies in row 2. Otherwise, two or three departments are left or right to $s_{M}$ $(n+2)$ and then by inequalities (3.1.7) one of the betweenness variables equals one. Thus, inequalities (3.1.14) are valid. Consider inequalities (3.1.15) with $y_{k}=1$ and let the sum of the corresponding betweenness variables be equal to zero, then $s_{M}$ lies between $i$ and $k$ or between $j$ and $k$. Inequalities (3.1.15) are satisfied if $y_{k}=0$. Given three departments which are assigned to row 1 , then the sum of the corresponding betweenness variables equals one, see inequalities (3.1.16).

We want to give two comments regarding this proposition. At first, inequalities (3.1.14)-3.1.15) are not valid for betweenness variables known in the literature [5, 42] where the betweenness variables are only equal to one if the corresponding three departments lie in the same row. To see this, we consider an $s_{M}$-T-row layout with $k$ in row 1 and $i$ and $j$ in row $2, i, j, k \in$ $[n] \backslash\left\{s_{M}\right\},|\{i, j, k\}|=3$. Then, one would obtain $x_{i k j}+x_{j i k}+x_{i j k}+x_{i s_{M} j}+x_{i s_{M} k}+x_{j s_{M} k}=0$ and $y_{k}=1$, so inequalities $(3.1 .14)-(3.1 .15)$ are not satisfied.

At second, we consider the following inequalities, which are related to inequalities 3.1.16)

$$
x_{i k j}+x_{i j k}+x_{j i k}+y_{i}+y_{j}+y_{k} \geq 1, \quad i, j, k \in[n] \backslash\left\{s_{M}\right\}, i<j<k .
$$

These inequalities are implied by inequalities (3.1.14) and inequalities (3.1.4).

We conclude our description of our MILP model for the $s_{M}$-TRFLP by summarizing where we use explicitly that the center of $s_{M}$ is fixed on position $p_{M}$ in row 1. At first, we use this by the rectilinear center-to-center distance calculation between the departments. At second, we combine our new extended betweenness variables with betweenness variables containing $s_{M}$ or $n+2$, see inequalities (3.1.7)-(3.1.8). In addition, in the symmetry breaking constraints $(3.1 .12)-(3.1 .13)$ we use this as well. An alternative could be to set up an MILP model for the TRFLP and to determine via binary variables the department whose center is on position $p_{M}$ in row 1 . Then, these new binary variables have to be added to the above mentioned inequalities. Recall that during the branch-and-cut algorithm one most often obtains fractional solutions, so in this case the above mentioned inequalities are significantly weaker or even redundant.

\subsubsection{The multi-bay facility layout problem with three rows}

Given a T-row instance, we add an additional dummy department $n+3$ with length $\ell_{n+3}=2 w_{\text {path }}^{B}$ and weights $w_{i(n+3)}=w_{(n+3) i}=0, i \in[n+2]$, to our model and we fix the center of the 
dummy department $n+3$ on position $p_{M}$ in row 1 . Then, the obtained problem is denoted by $((n+3)$-TRFLP $)$. So let an optimal $(n+3)$-T-row layout be given with $w_{\text {path }}^{T}=0$. Then, we assign the departments in the $(n+3)$-T-row layout to the left (right) of $n+3$ to row 1 (row 3 ) in the 3-Bay layout in reversed order (without changing the order of the departments). The departments in row 2 in the $(n+3)$-T-row layout are assigned to row 2 in the 3-Bay layout without changing the order of the departments in the same row. Hence, we obtain an optimal 3-Bay layout and vice versa. An immediate consequence is the following:

Proposition 3.1.4. Given a 3-BFLP instance with $w_{\text {path }}^{B} \in \mathbb{R}_{\geq 0}$ and a dummy department with length $\ell_{n+3}=2 w_{\text {path }}^{B}$ and weights $w_{i(n+3)}=w_{(n+3) i}=0, i \in[n+2]$. Then, the 3-BFLP is equivalent to the $(n+3)-T R F L P$ with $w_{\text {path }}^{T}=0$.

As a consequence, we can use our MILP model for the $s_{M}$-TRFLP with $s_{M}=n+3$ for the 3-BFLP. In this case further improvements are presented in Section A. Note that one main difference between solving the TRFLP and the 3-BFLP with our approach is that for the 3-BFLP only one MILP model has to be solved. In Section A we discuss the relationship of the optimal values of the TRFLP and the 3-BFLP and it turns out that, if $w_{\text {path }}^{T} \leq w_{\text {path }}^{B}$, the optimal value of the TRFLP is less than or equal to the optimal value of the 3-BFLP.

\subsubsection{Computational experiments}

\begin{tabular}{|c|c|c|c|c|c|c|}
\hline Instances & Source & SRFLP & 3-BFLP & TRFLP & $\mathrm{H}_{3-\mathrm{BFLP}}$ & $\mathrm{H}_{\text {TRFLP }}$ \\
\hline$A m 11 a$ & 11 & 10630.5 & 8795.5 & 8407.0 & 8814.5 & 8411.5 \\
\hline$A m 11 b$ & 11 & 7375.5 & 6021.5 & 5665.0 & 6021.5 & 5667.5 \\
\hline Am12a & (7) 8 & 2901.0 & 2508.0 & 2354.5 & 2515.0 & 2354.5 \\
\hline$A m 12 b$ & 7. 8 & 3280.5 & 2691.5 & 2539.5 & 2697.5 & 2548.5 \\
\hline$A m 13 a$ & 7 & 4902.5 & 4021.5 & 3836.0 & 4204.5 & 3836.0 \\
\hline$A m 13 b$ & [7] & 5698.0 & 4529.0 & 4362.5 & 4529.0 & 4362.5 \\
\hline Am14_1 & 42 & 5481.5 & 4560.5 & 4350.5 & 4565.5 & 4358.0 \\
\hline$A m 14 \bar{a}$ & 102 & 5673.0 & 4687.0 & 4446.5 & 4734.0 & 4448.0 \\
\hline$A m 14 b$ & 102 & 5595.0 & 4665.0 & 4430.5 & 4683.0 & 4433.0 \\
\hline$A m 15$ & 3 & 6305.0 & 5291.0 & 5071.0 & 5294.0 & 5079.0 \\
\hline$H K 15$ & 50 & 33220.0 & 26494.0 & 26124.0 & 26495.0 & 26125.0 \\
\hline$P 16 a$ & 9 & 14829.0 & 12287.5 & 11943.0 & 12326.0 & 11943.0 \\
\hline$P 16 b$ & 9 & 11878.5 & 9781.0 & 9469.5 & 9784.5 & 9469.5 \\
\hline$P 17 a$ & new & 14436.5 & 11852.0 & 11524.5 & 11888.5 & 11528.0 \\
\hline$P 17 b$ & new & 15682.0 & 12691.5 & 12317.0 & 12874.0 & 12389.0 \\
\hline$A m 17$ & 4 & 9254.0 & 7647.0 & 7315.0 & 7690.0 & 7318.0 \\
\hline$P 18 a$ & new & 16118.5 & 12022.0' & TL & 12863.5 & 12516.0 \\
\hline$P 18 b$ & new & 17716.5 & $12972.5^{\prime}$ & $\mathrm{TL}$ & 14616.5 & 14072.0 \\
\hline$A m 18$ & 4 & 10650.5 & 7990.5 & 8413.5 & 8835.5 & 8413.5 \\
\hline
\end{tabular}

Table 3.1.1: Optimal values of the SRFLP, the 3-BFLP and the TRFLP for instances from the literature with $w_{\text {path }}^{T}=0, w_{\text {path }}^{B}=1$. We write "TL" if the time limit of 8 hours is exceeded. Lower bounds for the 3-BFLP are marked with " " " if the time limit is exceeded or if we run out of memory storage. The optimal value of the TRFLP is up to $6.1 \%$ smaller than the optimal value of the 3-BFLP and up to $23.4 \%$ than the optimal value of the SRFLP.

In this section we summarize our computational results and we set $w_{\text {path }}^{T}=0$ and $w_{\text {path }}^{B}=1$, so the heights of the departments in the 3-BFLP are taken into account. Further computational experiments for greater values of $w_{\text {path }}^{T}$ and $w_{\text {path }}^{B}$ can be found in Section A The optimal solution values of the SRFLP, the 3-BFLP and the TRFLP are compared in columns three, four and five of Table 3.1.1, and we write "TL" if the time limit of 8 hours is exceeded. If the 3-BFLP is 
not solved to optimality, the obtained lower bound is displayed and marked with " " ". Our heuristically determined upper bounds for the 3-BFLP (TRFLP) are given in the sixth (seventh) column denoted by "H $\mathrm{H}_{3-B F L P}$ " ("H $\mathrm{H}_{\text {TRFLP }}$ ").

The optimal value of the TRFLP (3-BFLP) is between $18.8 \%$ and $23.4 \%$ (13.5\% and $20.5 \%$ ) smaller than the optimal value of the SRFLP, so the optimal values of the TRFLP and the 3-BFLP are significantly smaller than the optimal value of the SRFLP. Further, the optimal value of the TRFLP is between $1.4 \%$ and $6.1 \%$ smaller than the optimal value of the 3-BFLP, see Table 3.1.1. These reductions on the sum of the weighted transport distances are remarkable since factories are built for a long period, and the rearrangement of the departments is expensive. Hence, for these instances the TRFLP is preferable to the 3-BFLP and the SRFLP. The 3-Bay instances with 18 departments are not solved to optimality, but we obtain lower bounds with gaps between $4.0 \%$ and $7.9 \%$ to heuristically determined upper bounds.

\begin{tabular}{|c|c|c|c|c|c|c|c|c|}
\hline Instances & $\mathrm{Enu}_{3-\mathrm{BFLP}}$ & MILP $_{3-B F L P}$ & $E_{\text {TRFLP }}$ & MILP $_{1}$ & $\mathrm{MILP}_{2}$ & MILP $_{3}$ & $\mathrm{MILP}_{4}$ & MILP $_{\text {same-row }}$ \\
\hline$A m 11 a$ & $3: 26$ & 48 & $9: 31$ & 33 & 54 & 34 & $1: 04$ & $1: 37$ \\
\hline$A m 11 b$ & 3:35 & $1: 19$ & $10: 11$ & 20 & 41 & 16 & 41 & $1: 27$ \\
\hline$A m 12 a$ & $13: 26$ & $1: 53$ & $38: 29$ & 51 & $1: 29$ & $1: 20$ & $2: 44$ & $6: 18$ \\
\hline$A m 12 b$ & $13: 30$ & 1:01 & $40: 19$ & 42 & $1: 15$ & 50 & $2: 35$ & $6: 40$ \\
\hline$A m 13 a$ & $53: 46$ & $4: 16$ & $2: 58: 52$ & $2: 17$ & $3: 05$ & $3: 56$ & $14: 03$ & $24: 18$ \\
\hline$A m 13 b$ & $53: 34$ & $9: 31$ & $3: 01: 51$ & $2: 17$ & $2: 58$ & 5:02 & $10: 16$ & $18: 33$ \\
\hline Am14_1 & $3: 45: 33$ & $1: 28: 24$ & TL & $9: 11$ & $16: 27$ & $24: 47$ & $1: 16: 10$ & $1: 17: 49$ \\
\hline$A m 14 \bar{a}$ & $3: 28: 30$ & $13: 23$ & $\mathrm{TL}$ & $6: 00$ & $15: 32$ & $12: 13$ & $52: 54$ & $58: 26$ \\
\hline$A m 14 b$ & $3: 28: 47$ & $13: 37$ & TL & $6: 51$ & $15: 59$ & $17: 34$ & $1: 22: 07$ & $1: 17: 58$ \\
\hline$A m 15$ & TL & $25: 24$ & TL & $15: 31$ & $33: 45$ & $1: 49: 33$ & $3: 20: 07$ & $3: 35: 25$ \\
\hline$H K 15$ & TL & $17: 51$ & TL & $13: 48$ & $20: 05$ & $58: 54$ & $3: 54: 52$ & $2: 56: 30$ \\
\hline$P 16 a$ & TL & 44:00 & TL & $1: 12: 31$ & $3: 27: 04$ & TL & TL & TL \\
\hline$P 16 b$ & TL & 1:21:35 & TL & 1:09:58 & $2: 42: 40$ & $6: 04: 38$ & TL & TL \\
\hline$P 17 a$ & TL & $3: 51: 48$ & $\mathrm{TL}$ & $3: 32: 27$ & TL & TL & TL & TL \\
\hline$P 17 b$ & TL & 6:31:46 & TL & $3: 37: 59$ & TL & TL & TL & TL \\
\hline Am 17 & TL & 4:58:37 & TL & $2: 19: 20$ & $5: 53: 06$ & TL & TL & TL \\
\hline$P 18 a$ & TL & TL & TL & TL & TL & TL & TL & TL \\
\hline$P 18 b$ & TL & $5: 23: 22^{\dagger}$ & TL & TL & TL & TL & TL & TL \\
\hline$A m 18$ & TL & TL & TL & 6:01:58 & TL & TL & TL & TL \\
\hline
\end{tabular}

Table 3.1.2: Running times are given in sec, min:sec or in h:min:sec for instances from the literature with $w_{\text {path }}^{T}=0, w_{\text {path }}^{B}=1$. We write "TL" if the time limit of 8 hours is exceeded and the running time is marked with " $\dagger$ " if we run out of memory storage. For the TRFLP, variant 1 delivers the fastest approach. We clearly outperform the enumerative approach of [42] for the TRFLP and the 3-BFLP.

We are able to solve T-row instances with up to 18 departments and 3-Bay instances with up to 17 departments to optimality, see Table 3.1.2. Our approach for the 3-BFLP (column three) clearly outperforms the current best exact approach for the 3-BFLP 42 (column two). We adapted the approach [42] to the TRFLP (column four) and our approach for the TRFLP (column five) outperforms this approach as well. Neglecting inequalities (3.1.14)-(3.1.15) in our approach (see column six), the running time is for almost all instances with at least 14 departments more than twice as high as the running time of our best approach, so these inequalities significantly improve the performance of our branch-and-cut algorithm. Several further inequalities are added to our approach for the $s_{M}$-TRFLP (column seven), and thus the objective value at the root node is greater. However, this increases the running time in the further branching steps, and this variant is slower than our best approach. Using this approach we are able to calculate lower bounds for larger instances, we refer to Section A for the corresponding computational results. We use standard linearization instead of the transitivity inequalities (3.1.7)- 3.1 .8 (column eight) to combine the extended betweenness variables with betweenness variables containing $s_{M}$ or $n+2$ 
and the ordering and assignment variables, we refer to Section A for details. The running time is significantly increased and this shows that the transitivity inequalities (3.1.7)-(3.1.8) significantly improve the performance of our approach in comparison to inequalities based on the standard linearization. Additionally, we clearly outperform an approach based on an MILP model for the $s_{M}$-TRFLP which consists of betweenness variables which are only equal to one if all departments are in the same row (in this interpretation, betweenness variables are known in literature [5, 42]), see column nine. In this approach big- $M$-constraints are used to combine distance variables and assignments variables and the drawbacks of these inequalities in branch-and-cut algorithms are discussed in Section 3.1.1.

\section{The author's contribution:}

Mirko Dahlbeck is the sole author of this work, so the ideas, the proofs, the write-up and the implementation are the author's work. 


\subsection{New exact approaches for the combined cell layout problem and extensions of the multi-bay facility layout problem}

In this section we summarize the publication given in Section B. This publication is joint work with Anja Fischer, Kerstin Maier and Philipp Hungerländer.

In this summary we use the term "cell" instead of "row" in order to get a consistent terminology.

In this work we present a new exact approach for the CCLP where we enumerate over all assignments of the departments to the cells and then solve several FC-CCLP. Then, we solve the FC-CCLP by solving $m$ appropriate subproblems independently. Considering the CCLP where all cells of type SRFLP have the same inter-cell distances, we merge two cells of type SRFLP. Thus, if the number of cells of type SRFLP is even, we halve the number of cells of type SRFLP, and hence we significantly reduce the number of cell assignments that have to be considered. Additionally, we describe the adaption of this approach to the 3-BFLP, the 4-BFLP and the XRFLP. In a computational study we compare the computation times and the optimal values of these facility layout problems in order to support the chief executive officer to choose a layout if a factory is built up from the ground.

\subsubsection{The combined cell layout problem}

We consider the FC-CCLP and our goal is to solve the optimization problems in each cell independently as done in [86] for an extension of the MBFLP. Considering cells of type SRFLP, one can add a dummy department to each cell and fix it at the left border with length zero and the weights between the dummy department and a department in the same cell equals the sum of the weights of this department to departments in distinct cells. The weights between the dummy department and departments in distinct rows are set to zero. For cells of type DCFLP one can use a similar idea, but one does not necessarily need to add a dummy department. At the end of this section we describe how these problems can be solved to optimality. We use the following definition:

Definition 3.2.1. Given an FC-CCLP instance and let $\mathcal{D} \subseteq\left[n+m^{\prime}\right], m^{\prime} \in \mathbb{N}_{\geq 0}$, be a set of departments where $n+1, n+2, \ldots, n+m^{\prime}$ are dummy departments with lengths $\ell_{n+1}, \ell_{n+2}, \ldots, \ell_{n+m^{\prime}} \in$ $\mathbb{R}_{\geq 0}$ and weights $w_{i z}=w_{z i}=0, i \in\left[n+m^{\prime}\right], z \in\left\{n+1, \ldots, n+m^{\prime}\right\}, i \neq z$. For the department $s_{M} \in \mathcal{D}$ we set

$$
\begin{aligned}
w_{i s_{M}} \leftarrow w_{i s_{M}}+\sum_{j \in[n] \backslash \mathcal{D}} w_{i j}, & i \in \mathcal{D} \backslash\left\{s_{M}\right\}, \\
w_{s_{M} i} \leftarrow w_{s_{M} i}+\sum_{j \in[n] \backslash \mathcal{D}} w_{j i}, & i \in \mathcal{D} \backslash\left\{s_{M}\right\},
\end{aligned}
$$

all other weights remain the same. Then, the aim of the optimization problem $W_{\left(s_{M}, p_{z}\right)}^{A}(\mathcal{D})$ for the updated weights is to find an optimal layout of the departments $\mathcal{D}$ respective the structure of the cell $A \in\{S R F L P, D C F L P\}$. Additionally, $p_{z}, z \in\{a, b\}$, specifies the position of $s_{M}$, where $p_{a}$ denotes that the position of $s_{M}$ can be chosen arbitrarily and $p_{b}$ expresses that $s_{M}$ has to lie at the leftmost position of the layout. Let $A=D C F L P$ and $s_{M} \in\left\{n+1, \ldots, n+m^{\prime}\right\}$, then one department of the set $\mathcal{D} \backslash\left\{s_{M}\right\}$ may overlap with $s_{M}$.

Note that in cells of type DCFLP one department may overlap with the dummy department in the same cell because one department may overlap with the (un-) loading station as well. Given an FC-CCLP instance, we add a dummy department with length zero and weights calculated via (3.2.1) - 3.2.2) to each cell. Then, the weighted inter-cell distances are taken into account by the dummy departments and hence, we are able to solve the FC-CCLP by solving the optimization problems in each cell independently. 
Lemma 3.2.2. Given a fixed-cell combined cell layout instance where $C_{k}, k \in \mathcal{C}$, denotes the set of departments assigned to cell $k$ and let the dummy department $n+k$ be added to cell $k$ with length $\ell_{n+k}=0$ and adapted weights for dummy department $n+k$ as described in (3.2.1)-(3.2.2) for $\mathcal{D}=C_{k} \cup\{n+k\}$ and $s_{M}=n+k$. Then, the FC-CCLP is equivalent to solving the problems

$$
\begin{array}{ll}
W_{\left(n+k, p_{b}\right)}^{S}\left(C_{k} \cup\{n+k\}\right), & k \in \mathcal{C} \text { with } t(k)=S R F L P, \\
W_{\left(n+k, p_{a}\right)}^{D}\left(C_{k} \cup\{n+k\}\right), & k \in \mathcal{C} \text { with } t(k)=D C F L P,
\end{array}
$$

and the sum of the optimal values (plus constant inter-cell weights) is equal to the optimal value of the FC-CCLP.

Hence, the FC-CCLP can be divided into $m$ sub-problems. In Section B we concentrate on cells of type SRFLP and DCFLP, but note that the result of Lemma 3.2.2 is independent of the layout type of the cells, and thus our approach can be extended to cells of other types such as the DRFLP.

Now we consider an instance where all cells of type SRFLP have the same inter-cell distance, i. e., $u_{k o}=c$ for some constant $c \in \mathbb{R}_{\geq 0}$ for all $k, o \in \mathcal{C}, k<o$, with $\{t(k), t(o)\} \cap\{\operatorname{SRFLP}\} \neq \emptyset$. We briefly describe our main idea. We merge two cells of type SRFLP and we add one dummy department with weights as described in (3.2.1)- 3.2 .2 to this merged cell. The length of this dummy department is chosen appropriate and now, the dummy department is not fixed at the border. We solve this optimization problem, and then we assign the departments left (right) of this dummy department to an original cell of type SRFLP in reversed order (without changing the order of these departments). Thus, we halve the number of cells of type SRFLP if the number of cells of type SRFLP is even.

Theorem 3.2.3. The CCLP $\left(m_{1}, m_{2}\right)$ with $u_{k o}=c \in \mathbb{R}_{\geq 0}$ for all $k, o \in \mathcal{C}, k<o$, with $\{t(k), t(o)\} \cap$ $\{S R F L P\} \neq \emptyset$ is equivalent to enumerate over $\frac{\left(\left\lceil\frac{m_{1}}{2}\right\rceil+m_{2}\right)^{n}}{\left[\frac{m_{1}}{2}\right] ! m_{2} !}$ cell assignments and solve the following optimization problems for a fixed cell assignment exactly

$$
\begin{aligned}
& W_{\left(n+k, p_{a}\right)}^{S}\left(C_{k} \cup C_{k+1} \cup\{n+k\}\right), \\
& W_{\left(n+m_{1}, p_{b}\right)}^{S}\left(C_{m_{1}} \cup\left\{n+m_{1}\right\}\right), \\
& W_{\left(n+k, p_{a}\right)}^{D}\left(C_{k} \cup\{n+k\}\right),
\end{aligned}
$$

$$
\begin{aligned}
& k=1,3, \ldots, h, \\
& \text { if } m_{1} \text { is odd, } \\
& k=m_{1}+1, \ldots, m,
\end{aligned}
$$

with $h=m_{1}-1$ if $m_{1}$ is even and $h=m_{1}-2$ if $m_{1}$ is odd and the departments $C_{k}$ are assigned to cell $k \in[m]$. Apart from this the dummy department $n+k$ is added to cell $k$ for $k=1,3, \ldots, h$, $k=m_{1}$ if $m_{1}$ odd and $k=m_{1}+1, \ldots, m$. Additionally, we have to compute some constants such that inter-cell distances are calculated correctly.

Further, the SRFLP is equivalent to the CCLP (2,0).

In our algorithm we enumerate over at most $\frac{\left(\left\lceil\frac{m_{1}}{2}\right\rceil+m_{2}\right)^{n}}{\left[\frac{m_{1}}{2}\right] ! m_{2} !}$ cell assignments because we only need to consider distinguishable cell assignments. The current best objective value is set as an upper bound, and if the upper bound is exceeded, we neglect the current cell assignment and go to the next one. We solve cells of type DCFLP first since in practice the DCFLP is easier to solve than the SRFLP, see [57, 60], and the results of [16, 55] indicate that in general the optimal values of cells of type DCFLP are higher than the optimal values of cells of type SRFLP. Thus, we hope to exceed the upper bound earlier such that we can neglect the current cell assignment.

Considering cells of type SRFLP, the $W_{\left(n+k, p_{a}\right)}^{S}\left(C_{k} \cup C_{k+1} \cup\{n+k\}\right)$ can be solved by solving a single-row instance with departments $C_{k} \cup C_{k+1} \cup\{n+k\}$. For solving $W_{\left(n+\tilde{m}_{1}, p_{b}\right)}^{S}\left(C_{\tilde{m}_{1}} \cup\left\{n+\tilde{m}_{1}\right\}\right)$ if $m_{1}$ is odd with $\tilde{m}_{1}=\left\lceil\frac{m_{1}}{2}\right\rceil$, we can simply fix the dummy department $n+\tilde{m}_{1}$ at the border, i. e.,

$$
x_{i\left(n+\tilde{m}_{1}\right) j}=0, \quad i, j \in C_{\tilde{m}_{1}}, i<j,
$$


and then solve a single-row instance with departments $C_{\tilde{m}_{1}} \cup\left\{n+\tilde{m}_{1}\right\}$ and these additional equations.

Next, we focus on the subproblems of the FC-CCLP concerning cells of type DCFLP. Let $C_{k}$ denote the set of departments assigned to some cell $k$ with $t(k)=$ DCFLP. By the following proposition we can use techniques from the directed-circular literature [16, 157, 60] for solving the $W_{\left(n+k, p_{a}\right)}^{D}\left(C_{k} \cup\{n+k\}\right)$ to optimality.

Proposition 3.2.4. Let an $F C-C C L P$ instance be given where $C_{k}, k \in \mathcal{C}$, denotes the set of departments assigned to the cell $k$ with $t(k)=D C F L P$. Let dummy department $n+k$ be assigned to cell $k$ with length $\ell_{n+k}=0$ and weights $w_{i(n+k)}=\sum_{j \in[n] \backslash C_{k}} w_{i j}, w_{(n+k) i}=\sum_{j \in[n] \backslash C_{k}} w_{j i}$ (see (3.2.1), 3.2.2) and let $V_{k}:=\left\{i \in C_{k}: w_{i(n+k)}+w_{(n+k) i}>0\right\} \neq \emptyset$. Further, let $v_{n+k}^{*}$ denote the optimal value of the $W_{\left(n+k, p_{a}\right)}^{D}\left(C_{k} \cup\{n+k\}\right)$ and let $v_{s_{M}}^{*}$ denote the optimal value of the $W_{\left(s_{M}, p_{a}\right)}^{D}\left(C_{k}\right), s_{M} \in V_{k}$, where in $W_{\left(s_{M}, p_{a}\right)}^{D}\left(C_{k}\right)$ the weights of $s_{M}$ are adjusted according to (3.2.1), (3.2.2. Then

$$
v_{n+k}^{*}=\min \left\{v_{s_{M}}^{*}: s_{M} \in V_{k}\right\} .
$$

If $V_{k}=\emptyset$ for some $k \in \mathcal{C}$ with $t(k)=$ DCFLP, we simply neglect the dummy department $n+k$, and then $W_{\left(n+k, p_{a}\right)}^{D}\left(C_{k} \cup\{n+k\}\right)$ is equivalent to the DCFLP with departments $C_{k}$. If $V_{k} \neq \emptyset$, one can fix one department $i \in V_{k}$ opposite $n+k$ and enumerate over each department in $C_{k}$ fixed opposite $n+k$, see Proposition 3.2.4. In summary, we obtain an optimal layout of the $W_{\left(n+k, p_{a}\right)}^{D}\left(C_{k} \cup\{n+k\}\right)$ by solving $\max \left\{1,\left|V_{k}\right|\right\}$ directed-circular instances.

To reduce the number of directed-circular instances that have to be solved, we set up an MILP model to solve the $W_{\left(n+k, p_{a}\right)}^{D}\left(C_{k} \cup\{n+k\}\right), C_{k} \subseteq[n], k \in \mathcal{C}, V_{k} \neq \emptyset$. One advantage of an MILP model in comparison to an enumerative approach is that lower bounds are obtained quickly, and hence to exclude unbalanced cell assignments earlier in our algorithm. Therefore, we use the following binary variables

$$
y_{i}= \begin{cases}1, & \text { if } i \text { lies opposite } n+k, \\ 0, & \text { otherwise }\end{cases}
$$

for $i \in C_{k}$ and we use ordering variables

$$
z_{i j}= \begin{cases}1, & \text { if } i \text { lies to the left of } j \\ 0, & \text { otherwise }\end{cases}
$$

$i, j \in C_{k}, i<j$. Note that $n+k$ is the leftmost department, and hence we neglect ordering variables containing $n+k$, see, e.g., [57, 60].

Let $D_{i j}, i, j \in C_{k}, i<j$, denote the sum of the lengths of the departments $k \in C_{k} \backslash\{i\}$ which are left of $j$ minus the sum of the lengths of the departments $k \in C_{k} \backslash\{j\}$ which are left of $i$. Note that $D_{i j}, i, j \in C_{k}, i<j$, is negative if $j$ is left to $i$. Further, let $L=\sum_{i \in C_{k}} \ell_{i}$ and $\hat{w}_{i j}=w_{i j}-\min \left\{w_{i j}, w_{j i}\right\}, i, j \in C_{k}, i \neq j, \hat{w}_{i(n+k)}=w_{i(n+k)}, \hat{w}_{(n+k) i}=w_{(n+k) i}, i \in C_{k}$. We exclude the constant

$$
W_{k}^{D}=\sum_{\substack{i, j \in C_{k} \cup\{n+k\} \\ i \neq j}} \hat{w}_{i j} \frac{\ell_{i}+\ell_{j}}{2}+\sum_{\substack{i, j \in C_{k} \\ i<j}} \min \left\{w_{i j}, w_{j i}\right\} L .
$$

Let $d_{i j}$ combined with the excluded constant measure the center-to-center distances between $i \in C_{k}$ and $j \in C_{k}, i \neq j$, measured in clockwise directions. We define $L_{i}=\sum_{j \in C_{k} \backslash\{i\}} \ell_{j}, i \in C_{k}$, and $L_{i j}=L-\ell_{i}-\ell_{j}, i, j \in C_{k}, i<j$. Then, our MILP model with $V_{k} \neq \emptyset$ reads as follows

$$
\min \sum_{\substack{i, j \in C_{k} \cup\{n+k\} \\ i \neq j}} \hat{w}_{i j} d_{i j}
$$




$$
\begin{array}{ll}
\text { s.t. } 0 \leq z_{i j}+z_{j k}-z_{i k} \leq 1, & i, j, k \in C_{k}, i<j<k, \\
& D_{i j}-\sum_{\substack{k \in C_{k} \backslash\{i\} \\
k<j}} \ell_{k} z_{k j}+\sum_{\substack{k \in C_{k} \backslash\{i\} \\
k>j}} \ell_{k} z_{j k} \\
\quad+\sum_{\substack{k \in C_{k} \backslash\{j\} \\
k<i}} \ell_{k} z_{k i}-\sum_{\substack{k \in C_{k} \backslash\{j\} \\
k>i}} \ell_{k} z_{i k}=-\sum_{\substack{k \in C_{k} \\
i<k<j}} \ell_{k}, & i, j \in C_{k}, i<j, \\
d_{i j}+L_{i j} z_{i j}-D_{i j}=L_{i j}, & \\
d_{j i}-L_{i j} z_{i j}+D_{i j}=0, & i, j \in C_{k}, i<j, \\
\sum_{i \in C_{k}} y_{i}=1, & i, j \in C_{k}, i<j, \\
z_{i j}-y_{i} \geq 0, & \\
z_{i j}+y_{j} \leq 1, & i, j \in C_{k}, i<j, \\
y_{i}=0, \quad & i, j \in C_{k}, i<j, \\
d_{(n+k) i}-\sum_{j \in C_{k}} \ell_{j} z_{j i}+\sum_{j \in C_{k}} \ell_{j} z_{i j}+\sum_{j \in C_{k}} \frac{\ell_{j}}{2} y_{j}=\sum_{j \in C_{k}} \ell_{j}, & i \in C_{k}, \\
j>i & \\
d_{i j} \geq 0, & i, j \in C_{k}, i \neq j, \\
d_{i(n+k)}+d_{(n+k) i}+\left(L_{i}+\ell_{i}\right) y_{i}=L_{i}, & i \in C_{k}, \\
d_{i j} \geq-\frac{\ell_{i}}{2}, & i, j \in C_{k} \cup\{n+k\}, i \neq j, \\
y_{i} \in\{0,1\}, & |\{i, j\} \cap\{n+k\}|=1, \\
& i \in C_{k} .
\end{array}
$$

Let $k \in \mathcal{C}$. Inequalities $(3.2 .4)$ are the well-known 3-cycle-inequalities, which ensure transitivity and suffice to induce a feasible ordering of the departments, see, e. g., [16, 57, 60]. Equations (3.2.5) compute for each pair of departments $i$ and $j$ with $i, j \in C_{k}, i<j$, the sum of the lengths of the departments $k \in C_{k} \backslash\{i\}$ which are left of $j$ minus the sum of the lengths of the departments $k \in C_{k} \backslash\{j\}$ which are left of $i$. By equations 3.2.6 $-(3.2 .7)$ we obtain the correct distance between $i$ and $j$ with $i, j \in C_{k}, i<j$, i. e., if $i$ lies left to $j$ we get $d_{i j}=D_{i j}$ and $d_{j i}=L_{i j}-D_{i j}$ and otherwise $d_{i j}=L_{i j}+D_{i j}$ and $d_{j i}=-D_{i j}$ with negative values of $D_{i j}$ in the latter case. Inequalities (3.2.4)-(3.2.7) are taken from [16, 60.

Equations (3.2.8) ensure that exactly one department lies opposite $n+k$, see Proposition 3.2.4. According to the $z$ variables we obtain an ordering of the departments and we ensure via inequalities (3.2.9)-(3.2.10) that the department $i^{\prime} \in C_{k}$ with $y_{i^{\prime}}=1$ is the leftmost department of the set $C_{k}$. By Proposition 3.2.4 and our assumption $V_{k} \neq \emptyset$, we set $y_{i}, i \in C_{k} \backslash\left\{V_{k}\right\}$, equal to zero, see equations (3.2.11). The distance calculation in equations $(3.2 .12)$ is similar to the distance calculation in [16, 57, 60] where we additionally subtract half the length of the department that is fixed opposite $n+k$ because we excluded the constant $W_{k}^{D}$. By equations (3.2.14) and inequalities 3.2.15) we obtain $d_{i(n+k)}=d_{(n+k) i}=-\frac{\ell_{i}}{2}, i \in C_{k}$, if $i$ lies opposite $n+k$. In this case the distances $d_{i(n+k)}$ and $d_{(n+k) i}, i \in C_{k}$, are negative since we excluded the constant $\sum_{i \in C_{k}}\left(w_{i(n+k)}+w_{(n+k) i}\right) \frac{\ell_{i}}{2}$. If $i \in C_{k}$ does not lie opposite $n+k$, we obtain $d_{i(n+k)}+d_{(n+k) i}=L_{i}$, see equations (3.2.14).

\subsubsection{Extensions of the multi-bay facility layout problem}

If $w_{\text {path }}^{B}=0$, one can immediately apply the results of Theorem 3.2 .3 to the MBFLP. In this section we investigate the case $w_{\text {path }}^{B}>0$ for the 3-BFLP and the 4-BFLP and we describe how our approach can be extended to the XRFLP.

At first, we consider the 3-BFLP. We merge cell 1 and cell 2 and interpret cell 3 as new 
cell 2. Let $C_{1}\left(C_{2}\right)$ denote the set of departments assigned to the merged cell 1 (cell 2), $C_{1}, C_{2} \subseteq[n], C_{1} \cap C_{2}=\emptyset$, such that $C_{1} \dot{\cup} C_{2}=[n]$. The dummy department $n+1(n+2)$ is added to the merged cell 1 (cell 2) with $\ell_{n+1}=0\left(\ell_{n+2}=0\right)$ and weights $w_{i(n+1)}=\sum_{j \in[n] \backslash C_{1}} w_{i j}$, $w_{(n+1) i}=\sum_{j \in[n] \backslash C_{1}} w_{j i}\left(w_{i(n+2)}=\sum_{j \in[n] \backslash C_{2}} w_{i j}, w_{(n+2) i}=\sum_{j \in[n] \backslash C_{2}} w_{j i}\right)$ (see $\left.(3.2 .1)-3.2 .2\right)$. We exclude the constant $\sum_{i \in C_{1}, j \in C_{2}}\left(w_{i j}+w_{j i}\right) \cdot w_{p a t h}^{B}$ and we fix an additional dummy department $n+3$ to the merged cell 1 neighboring $n+1$ with lengths $\ell_{n+3}=w_{\text {path }}^{B}$ and weights $w_{i(n+3)}=$ $w_{(n+3) i}=0, i \in C_{1} \cup\{n+1\}$. Then, we obtain an optimal solution for the 3-BFLP by enumerating over $C_{1}, C_{2} \subseteq[n], C_{1} \cap C_{2}=\emptyset, C_{1} \cup C_{2}=[n]$ and solving the $W_{\left(n+1, p_{a}\right)}^{S}\left(C_{1} \cup\{n+1, n+3\}\right)$ and the $W_{\left(n+2, p_{b}\right)}^{S}\left(C_{2} \cup\{n+2\}\right)$.

If $w_{\text {path }}^{B}=0$, we can fix two departments to $C_{1}$ and one has to consider $2^{n-2}$ generalized cell assignments. If $w_{\text {path }}^{B}>0$, one department can be fixed to $C_{1}$, and hence we have to consider $2^{n-1}$ generalized cell assignments. Hence, we can solve the 3-BFLP similar to the approach summarized in the previous section. This approach differs from the approach of Section $\mathrm{A}$ where a dummy department $n+1$ with length $\ell_{n+1}=2 w_{\text {path }}^{B}$ is added to cell 1 and its center position is fixed on position $p_{M}$. Then, we could add a dummy department $n+2$ to cell 2 as described above, however, we cannot fix one department to cell 1 and hence, we cannot reduce the number of cell assignments that have to be considered.

Next we consider layout problems with four original cells in detail. Recall that we assume that the height of each department equals one. The following proposition is essential to solve the cells of the XRFLP independently, and thus we derive a result similar to Theorem 3.2.3.

Proposition 3.2.5. Given an XRFLP instance. There always exists an optimal X-row layout where some $i \in[n]$ is contained in cell 3 and $d_{i p_{E_{3}}}=\frac{\ell_{i}}{2}$.

Given an X-row instance, we want to ensure that departments in cell 2 and cell 3 do not overlap. Therefore, we fix an additional dummy department $n+3$ at the border of cell 2 with lengths $\ell_{n+3}=1$ and weights $w_{i(n+3)}=w_{(n+3) i}=0, i \in[n+2]$, see Proposition 3.2.5.

At first, we consider the case where inter-cell distances are equal to zero, i. e., $w_{\text {path }}^{1}=w_{\text {path }}^{2}=0$. Then, one can solve the cells independently, see Lemma 3.2.2 and hence we can apply Theorem 3.2 .3 with $u_{k o}=0, k, o \in[4], k<o$, and we merge cell 1 and cell 3 as well as cell 2 and cell 4. The dummy department $n+1(n+2)$ is added to the merged cell 1 (cell 2) with $\ell_{n+1}=0$ $\left(\ell_{n+2}=0\right)$ and weights as described in (3.2.1)-(3.2.2) and the merged cell 2 additionally contains the dummy department $n+3$ which is neighboring $n+2$.

We include inter-cell distances in our approach for the XRFLP in the following way. For an illustration we refer to Figure 3.2 .1 .

Corollary 3.2.6. Given an $X$-row instance with $w_{\text {path }}^{1}, w_{\text {path }}^{2} \geq 1$, and we set $\ell_{n+1}=0, \ell_{n+2}=$ $2 \cdot \min \left\{w_{\text {path }}^{2}, 1\right\}, \ell_{n+3}=\left|w_{\text {path }}^{2}-1\right|, \ell_{n+4}=w_{\text {path }}^{1}$, and $n+1(n+2)$ is neighboring $n+4(n+3)$. We obtain an optimal $X$-row layout by enumerating over all assignments of the departments to the cells $\mathcal{C}=\{1,2\}$ and solving the $W_{\left(n+1, p_{a}\right)}^{S}\left(C_{1} \cup\{n+1, n+4\}\right)$ and the $W_{\left(n+2, p_{a}\right)}^{S}\left(C_{2} \cup\{n+2, n+3\}\right)$ where $C_{k}, k \in[2]$, denotes the set of departments assigned to (merged) cell $k$ and the weights of the additional dummy departments are set to $w_{i(n+3)}=w_{(n+3) i}=w_{i(n+4)}=w_{(n+4) i}=0, i \in[n+2]$.

In our algorithm we solve the $W_{\left(n+2, p_{a}\right)}^{S}\left(C_{2} \cup\{n+2, n+3\}\right)$ first if $\left|C_{2}\right| \leq\left|C_{1}\right|+1$ and otherwise we solve the $W_{\left(n+1, p_{a}\right)}^{S}\left(C_{1} \cup\{n+1, n+4\}\right)$ first with the idea to exclude unbalanced cell assignments earlier.

Next we describe how to include inter-cell distances to the 4-BFLP in our approach. At first, we merge cell 1 and cell 2 as well as cell 3 and cell 4 . Hence, let $C_{1}\left(C_{2}\right)$ denote the set of departments assigned to the merged cell 1 (cell 2). We exclude the constant $\sum_{i \in C_{1}, j \in C_{2}}\left(w_{i j}+w_{j i}\right) \cdot w_{p a t h}^{B}$ and solve the $W_{\left(n+k, p_{a}\right)}^{S}\left(C_{k} \cup\{n+k, n+k+2\}\right), k \in\{1,2\}$, with an additional dummy department $n+k+2$ with length $\ell_{n+k+2}=w_{\text {path }}^{B}$ and weights $w_{i(n+k+2)}=w_{(n+k+2) i}=0, i \in C_{k} \cup\{n+k\}$, where $n+k+2$ is a neighboring department of $n+k$. By Corollary 3.2.6 we obtain an optimal 

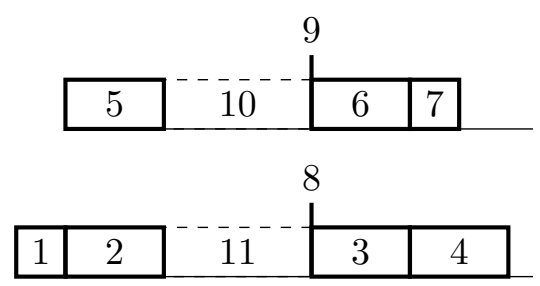

(a) Feasible layouts for the $W_{\left(8, p_{a}\right)}^{S}(\{1,2,3,4\} \cup$ $\{8,11\})$ and the $W_{\left(9, p_{a}\right)}^{S}(\{5,6,7\} \cup\{9,10\})$ with $\ell_{10}=\ell_{11}=w_{\text {path }}^{B}=3$.

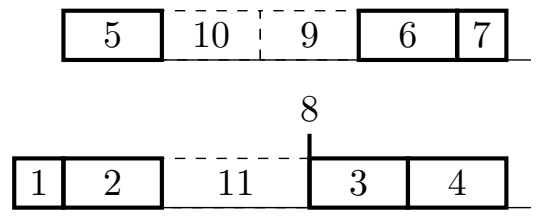

(c) Feasible layouts for the $W_{\left(8, p_{a}\right)}^{S}(\{1,2,3,4\} \cup$ $\{8,11\})$ and the $W_{\left(9, p_{a}\right)}^{S}(\{5,6,7\} \cup\{9,10\})$ with $w_{\text {path }}^{1}=w_{\text {path }}^{2}=3$ and $\ell_{9}=\ell_{10}=2, \ell_{11}=3$.

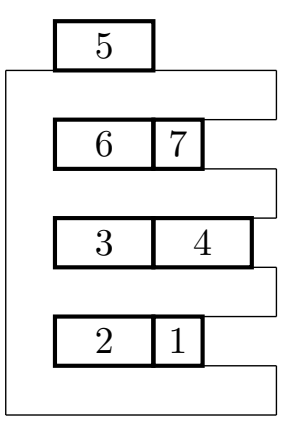

(b) 4-Bay layout with $\ell_{10}=\ell_{11}=w_{\text {path }}^{B}$ deduced from the single-row layouts illustrated in Figure $3.2 .1 \mathrm{a}$

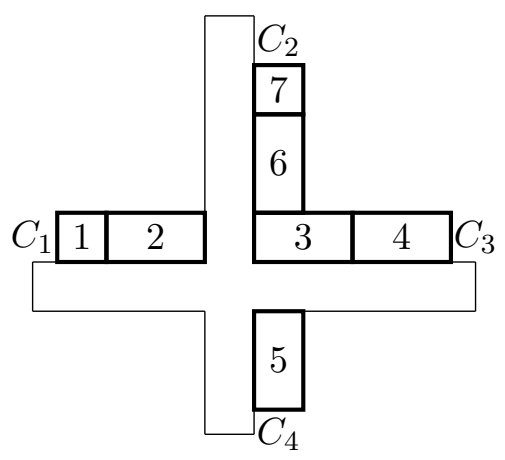

(d) X-row layout with $\ell_{9}=\ell_{10}=2, \ell_{11}=3$, deduced from the single-row layouts illustrated in Figure $3.2 .1 \mathrm{c}$

Figure 3.2.1: Visualization of obtained 4-Bay and X-row layouts with positive inter-cell distances by solving appropriate single-row instances. In order to construct a 4-Bay layout the departments left (right) to the dummy department 8 are assigned to cell 1 (cell 2 ) in reversed (the same) order and the departments left (right) to the dummy department 9 are assigned to cell 4 (cell 3) in reversed (the same) order.

solution for the 4-BFLP by this method. For an illustration we refer to Figure 3.2.1. In our algorithm we solve the $W_{\left(n+2, p_{a}\right)}^{S}\left(C_{2} \cup\{n+2, n+3\}\right)$ first if $\left|C_{2}\right| \leq\left|C_{1}\right|$ and otherwise we solve the $\left.W_{\left(n+1, p_{a}\right)}^{S}\left(C_{1} \cup\{n+1, n+4\}\right)\right)$ first.

Considering the 4-BFLP with $w_{\text {path }}^{B}=0$, we can fix two departments to cell 1 , and hence it is sufficient to consider $2^{n-2}$ cell assignments. If $w_{\text {path }}^{B}>0$, one can fix one department to cell 1 , and hence it is sufficient to consider $2^{n-1}$ cell assignments. In the proof of Proposition 3.2.5 symmetry breaking is used, see Section B, so in order to solve the XRFLP to optimality with our approach one has to consider $2^{n}$ cell assignments.

\subsubsection{Computational results}

In Table 3.2.1 we compare the optimal values of several facility layout problems where the intercell distances are set to zero. For computational experiments with positive inter-cell distances we refer to Section B. In the column "Source" we display the source of the symmetric instances. We observe that the optimal value of the TRFLP is smaller than the optimal value of the 3-BFLP. The optimal value of the 4-BFLP is slightly smaller than the optimal value of the XRFLP. For all instances the optimal value of the XRFLP is smaller than the optimal value of the TRFLP and the 4-BFLP has the smallest optimal value. Recall that the SRFLP is equivalent to the CCLP $(2,0)$, see Theorem 3.2 .3 . The optimal value of the SRFLP is smaller than the optimal value of the CCLP 


\begin{tabular}{|c|c|c|c|c|c|c|c|c|}
\hline Instances & Source & SRFLP & $\operatorname{CCLP}(1,1)$ & $\operatorname{CCLP}(0,2)$ & 3-BFLP & TRFLP & 4-BFLP & XRFLP \\
\hline$A m 11 a$ & 11 & 10630.5 & 9840.0 & 11178.5 & 8466.5 & 8407.0 & 6899.5 & 7038.5 \\
\hline$A m 11 b$ & 11 & 7375.5 & 6802.5 & 7262.0 & 5694.5 & 5665.0 & 4864.5 & 4990.5 \\
\hline$A m 12 a$ & 7. 8 & 2901.0 & 2702.5 & 3266.5 & 2382.0 & 2354.5 & 1994.0 & 2047.0 \\
\hline$A m 12 b$ & 77, 8 & 3280.5 & 3042.5 & 3389.5 & 2557.5 & 2539.5 & 2172.5 & 2234.5 \\
\hline$A m 13 a$ & 7 & 4902.5 & 4404.5 & 5283.5 & 3863.5 & 3836.0 & 3258.5 & 3327.5 \\
\hline$A m 13 b$ & [7] & 5698.0 & 5046.0 & 6029.0 & 4376.0 & 4362.5 & 3642.0 & 3702.0 \\
\hline Am14_1 & 42 & 5481.5 & 5132.0 & 5766.5 & 4370.5 & 4350.5 & 3557.5 & 3634.5 \\
\hline$A m 14 \bar{a}$ & 102 & 5673.0 & 5263.0 & 6619.0 & 4475.0 & 4446.5 & 3773.0 & 3872.0 \\
\hline$A m 14 b$ & 102 & 5595.0 & 5166.0 & 5725.0 & 4451.0 & 4430.5 & 3749.0 & 3838.0 \\
\hline$A m 15$ & 3 & 6305.0 & 5961.5 & 6899.0 & 5093.0 & 5071.0 & 4237.0 & 4319.0 \\
\hline$H K 15$ & 50 & 33220.0 & 30880.0 & 37440.0 & 26290.0 & 26125.0 & 21810.0 & 21891.0 \\
\hline$P 16 a$ & 9 & 14829.0 & 14087.0 & 15125.0 & 11999.0 & 11943.0 & 10076.0 & 10194.0 \\
\hline$P 16 b$ & [9] & 11878.5 & 11360.0 & 12768.5 & 9499.5 & 9469.5 & 7805.5 & 7921.5 \\
\hline$P 17 a$ & Section $\mathrm{A}$ & 14436.5 & 14066.0 & 15930.0 & 11551.5 & 11524.5 & 9574.5 & - \\
\hline$P 17 b$ & Section $\bar{A}$ & 15682.0 & 14870.5 & 16034.0 & 12389.0 & 12317.0 & 10174.0 & - \\
\hline$A m 17$ & 4 & 9254.0 & 8604.0 & 10896.0 & 7345.0 & 7315.0 & 6044.0 & - \\
\hline$P 18 a$ & Section A & 16118.5 & 15043.5 & 17904.0 & 12528.5 & - & 10266.5 & - \\
\hline$P 18 b$ & Section $\bar{A}$ & 17716.5 & 16733.0 & 18022.5 & 14138.5 & - & - & - \\
\hline$A m 18$ & [4] & 10650.5 & 10050.5 & 12274.5 & 8446.5 & 8413.5 & 6914.5 & - \\
\hline
\end{tabular}

Table 3.2.1: We illustrated the optimal values of several facility layout problems for instances from the literature where the inter-cell distances are set to zero. Instances marked with "-" could not be solved to optimality within the time limit of 8 hours.

$(0,2)$ here but the CCLP $(1,1)$ has the smallest optimal value of these three problems in our tests. The optimal values of the CCLP are greater than the ones of the 3-BFLP.

Our approach for the 3-BFLP as well as our enumerative approach for the TRFLP, see Section B for details, can be found in column "our" in Table 3.2.2. The CCLP $(0,2)$ with up to 18 departments could be solved in less than 30 minutes, see Table 3.2 .2 by using our MILP approach (column "MILP"). For these instances our MILP approach clearly outperforms the enumerative approach (column "enu") which can be derived immediately by the results of Lemma 3.2 .2 and Proposition 3.2.4. Therefore, our approach outperforms the approach of [16, 55] as well because their models do not contain dummy departments, and hence one would have to use the enumerative approach. In order to compare our approach for the TRFLP and the 3-BFLP with the approach of Section A, we make use of the heuristically determined upper bounds in Section A. Considering the 3-BFLP we outperform the approach of Section A, see Table 3.2.2. With our approach the TRFLP (the 4-BFLP) could be solved with up to 17 (18) departments within a time limit of 8 hours and the XRFLP with up to 16 departments. Our MILP model for the optimization problem in cell 1 in the TRFLP, see Section $B$, leads to a smaller running time than the corresponding enumerative approach, see Table 3.2.2 However, the approach of Section A can even solve one T-row instance with 18 departments and is for most T-row instances faster than our approach. Additionally, computational tests for the 3-PMFP and the 4-PMFP can be found in Section B,

\section{The author's contribution:}

The main ideas of this publication which concern merging cells of type SRFLP and adapting the approach to the 3-BFLP, 4-BFLP are the author's work. Large parts of the ideas of adapting this approach to the XRFLP are the author's work as well. He contributed to ideas for the MILP model in Section 4.2. Section 3 is joint work with Anja Fischer. The proof of Lemma 4 is joint work with Anja Fischer and Kerstin Maier. The main result, Theorem 5, was proven by the author under the revision of Anja Fischer. The proof of Proposition 8 is joint work with Kerstin Maier and the proof of Proposition 7 and Proposition 10 were done by the author in 


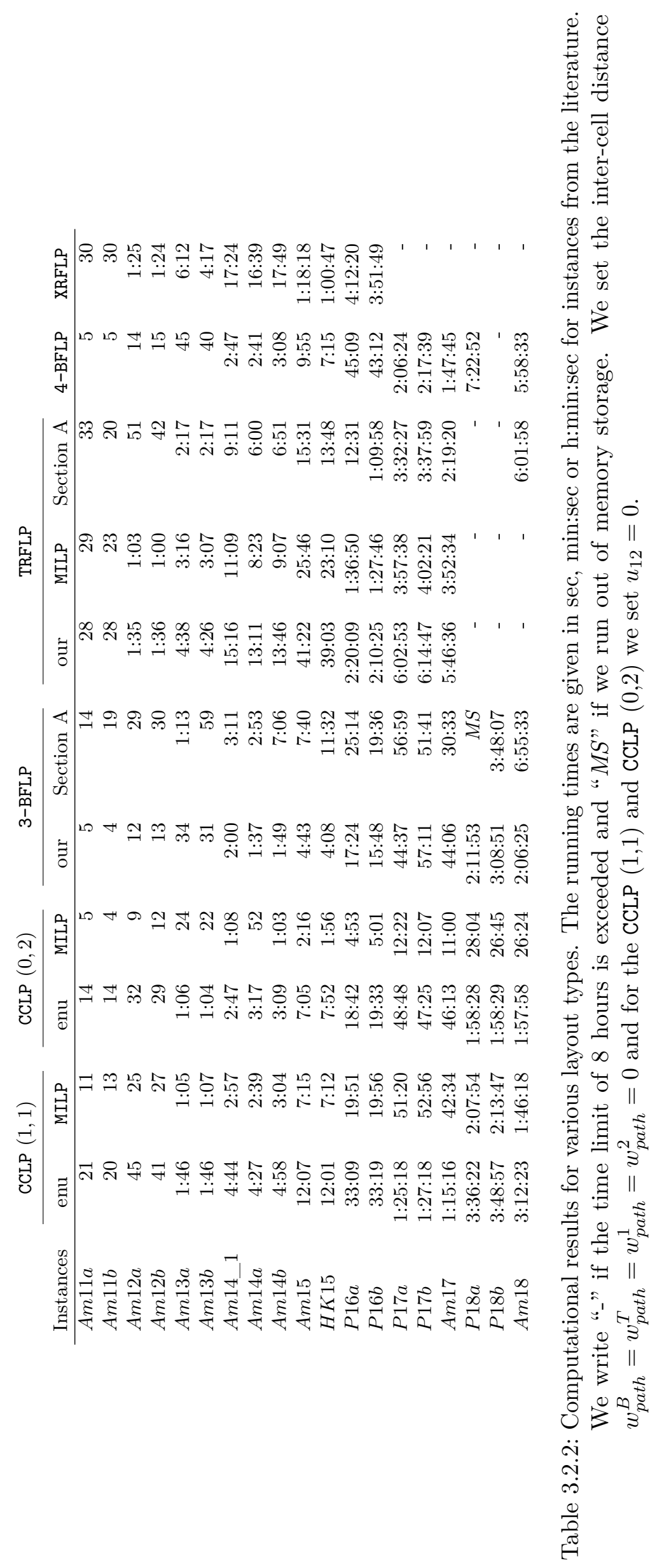


advice of the coauthors. The write-up of Section 1 is joint work with Kerstin Maier and the author is responsible for large parts of the write-up of Section 4 and Section 5. Additionally, the implementation and the computational experiments are the author's work. 


\subsection{Combining simulation and optimization for extended double row facility layout problems in factory planning}

In this section we summarize the publication provided in Section C which is joint work with Uwe Bracht, Anja Fischer and Thomas Krüger.

In this work we present a combined optimization-simulation approach for the DRFLP. In order to handle many of the requirements posed on the layout in real-world production, the existing mathematical optimization models have to be extended. For this we extend the mathematical approach of [42] and we are able to solve a realistic double-row instance with 21 departments. The start solution obtained via mathematical optimization is then the basis for the following steps of the factory planners on a much finer level of detail.

\subsubsection{Extensions of a DRFLP model}

In the classic models for the DRFLP, see, e.g., [8, 33, 42], the following three assumptions are made

1. the total size of the area needed for the arrangement is not limited,

2. vertical distances between the departments are neglected,

3. each department can be assigned to any of the two rows.

The approach of 42 enumerates over all distinguishable row assignments of the departments. Then, the dummy department $n+1(n+2)$ is arranged at the left (right) border with $\ell_{n+1}=$ $\ell_{n+2}=0, w_{i j}=w_{j i}=0, i \in[n+2], j \in\{n+1, n+2\}, i<j$, and an MILP model is used to solve several DRFLP with fixed-row assignment (FR-DRFLP). In order to consider a fixed row assignments, let $R_{1} \subseteq[n]\left(R_{2} \subseteq[n]\right)$ denote the set of departments assigned to row 1 (row 2 ). The dummy departments $n+1$ and $n+2$ are assigned to both rows. The MILP model of [42] makes use of betweenness variables

$$
x_{i k j}=x_{j k i}= \begin{cases}1, & k \text { lies between } i \text { and } j \text { in the same row } \\ 0, & \text { otherwise }\end{cases}
$$

for $l \in\{1,2\}, i, j, k \in R_{l} \cup\{n+1, n+2\},|\{i, j, k\}|=3, i<j$. Further, let $d_{i j}=d_{j i}, i, j \in$ $[n+2], i<j$, measure the horizontal center-to-center distance between $i$ and $j$. The betweenness variables containing a dummy department $n+1$ or $n+2$ are combined with the distance variables via big- $M$-inequalities. We refer to [42] for a description of the MILP model and to Section C for a summary of the MILP model.

Usually, in factory planning the incoming warehouse and the shipping warehouse of a factory are arranged at the left and at the right border, respectively. If this is the case, the dummy departments $n+1$ and $n+2$ can be interpreted as these warehouses, see Figure 3.3.1. Of course, we might obtain a better overall solution value if we drop the restriction on the position of both warehouses. In this case they are treated as ordinary departments that have transport weights to other departments and need a certain space.

In this section we extend the approach of [42] in order to overcome these three assumptions. Let the departments be given as two-dimensional objects such that each department has a length and a height. Then, the area of a layout is defined in the following way:

Definition 3.3.1. The area of a given layout is defined as the area of the minimum boundary rectangle containing all departments.

By definition the area of a layout is equal to $d_{(n+1)(n+2)} \cdot h$, where $h$ is the height of the layout. Let $h_{i}$ denote the height of department $i \in[n]$. In the FR-DRFLP we compute the height of the 


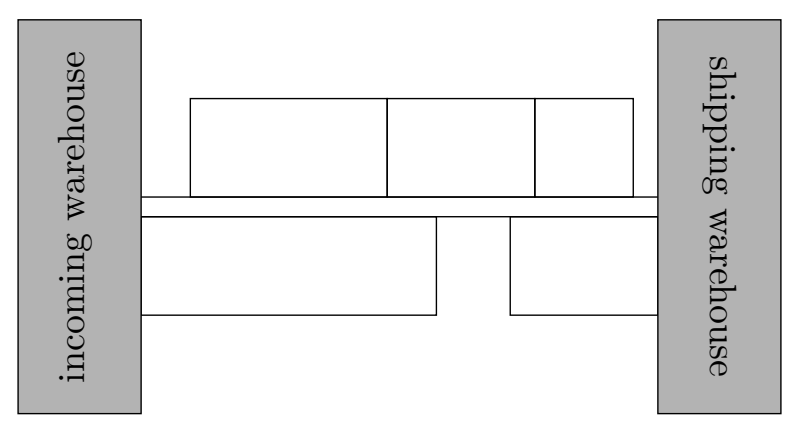

Figure 3.3.1: Visualization of an extended DRFLP layout where we fixed the incoming and the shipping warehouse to the border of the layout. One motivation for this arrangement is that one hopes to receive rather linear transport flows between the departments.

layout by summing up the height of a department with the largest height in each row plus the width of the path $w_{\text {path }}^{D}$ between the two rows, i. e., $h=\max _{i \in R_{1}} h_{i}+\max _{i \in R_{2}} h_{i}+w_{\text {path }}^{D}$. So for a fixed row assignment the height of a layout is constant. Assume that the area of the layout may be at most $F \in \mathbb{R}_{\geq 0}$. Then, the linear inequality $d_{(n+1)(n+2)} \leq \frac{F}{h}$ ensures that the area of the layout is bounded by $F$. Further, we can neglect all row assignments where the sum of the lengths of the departments in the same row exceeds $\frac{F}{h}$.

Apart from a restriction of the used area, there might appear so called blocked areas in real-world factory planning problems. It is not allowed to place departments in these areas. This might be due to already existing departments or due to safety restrictions. Let $B_{1}=\left\{\left[b_{1}, b_{1}+g_{1}\right], \ldots,\left[b_{u}, b_{u}+\right.\right.$ $\left.\left.g_{u}\right]\right\}$ be the blocked areas in row 1 and $B_{2}=\left\{\left[b_{u+1}, b_{u+1}+g_{u+1}\right], \ldots,\left[b_{v}, b_{v}+g_{v}\right]\right\}$ be the blocked areas in row 2 for given $b_{k}, g_{k} \geq 0, k \in[v]$. For each blocked area we introduce a new dummy department, which we will call blocked department, with length equal to the length of the blocked area. We place the center of the blocked department in the middle of the blocked area. So we get the blocked departments $n+3, n+4, \ldots,\left(n+2+\left|B_{1}\right|+\left|B_{2}\right|\right)$ with length $\ell_{n+2+k}=g_{k}$ for $k \in[v]$. The row assignment of the blocked departments is fixed, namely $R^{1}=\left\{(n+3), \ldots,\left(n+2+\left|B_{1}\right|\right)\right\}$ are assigned to row 1 and $R^{2}=\left\{\left(n+2+\left|B_{1}\right|+1\right), \ldots,\left(n+2+\left|B_{1}\right|+\left|B_{2}\right|\right)\right\}$ to row 2. To ensure that the blocked department $n+2+k$ lies exactly on the interval $\left[b_{k}, b_{k}+g_{k}\right]$, we set the distance variable to

$$
d_{(n+1)(n+2+k)}=b_{k}+\frac{g_{k}}{2}, k \in[v] .
$$

Next we include vertical distances between the departments in order to overcome the second assumption stated in the beginning of this section. The departments are given as 2-dimensional objects. The vertical center-to-center distance between two departments in the same row equals the sum of the heights of these two departments. If two departments are in distinct rows, one additionally adds $w_{\text {path }}^{D}$. An example is illustrated in Figure 3.3.2. In conclusion, we only need to add the following constant value to the objective value of some FR-DRFLP to include vertical distances

$$
\sum_{\substack{i, j \in[n] \\ i<j}} \frac{h_{i}+h_{j}}{2}\left(w_{i j}+w_{j i}\right)+\sum_{\substack{i \in R_{1} \\ j \in R_{2}}} w_{\text {path }}^{D}\left(w_{i j}+w_{j i}\right) .
$$

Naturally, the third assumption mentioned at the beginning of this section can easily be dropped. If the row assignment of some departments is fixed in advance, this only helps us because the number of possible row assignments decreases.

In this exact approach, we enumerate over all row assignments of the departments and solve some MILP model in each step. One can fix one department to row 1, so there are in general $\frac{1}{2} \cdot 2^{n}$ distinguishable row assignments that have to be considered. In realistic instances there 


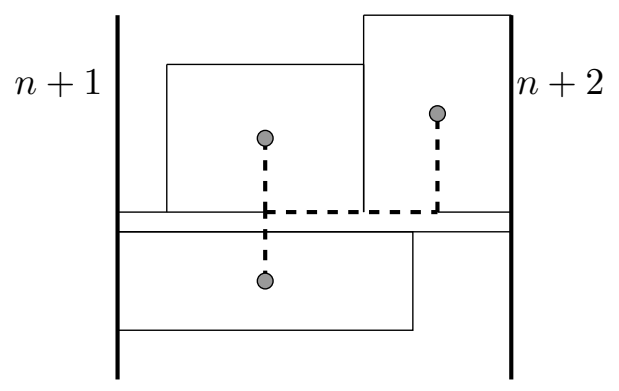

Figure 3.3.2: Visualization of the vertical and horizontal center-to-center distances between three departments.

appear departments of the same type, i. e., departments that have the same length and the same transport weight $w$ to all other departments. In our test case in Section 3.3 .3 we consider such a realistic instance. We use this additional information to reduce the number of distinguishable row assignments significantly.

Theorem 3.3.2. Let $k$ denote the number of different department types and let $a_{i}$ be the number of departments of type $i \in[k]$. Then there are at most

$$
\left\lceil\frac{1}{2} \prod_{i \in[k]}\left(a_{i}+1\right)\right\rceil
$$

distinguishable row assignments.

This formula is also correct if all departments have different types, because then $a_{i}=1$ for all $i \in[k]$ and $k=n$. We illustrate the advantages of Theorem 3.3.2 by a realistic example, see [95] and Section 3.3.3.

Example 3.3.3. We are given $n=21$ departments, where two departments appear four times, three departments twice and seven departments just once. Without reduction, we have to test $2^{20}=1048576$ row assignments. By Theorem 3.3.2 we obtain at most $\frac{1}{2} \cdot 5 \cdot 5 \cdot 3 \cdot 3 \cdot 3 \cdot 2^{7}=43200$ distinguishable row assignments.

Considering departments of the same type, we can strengthen our MILP model. We break symmetries by fixing the ordering of departments of the same type in the same row. This symmetry breaking is done in such a way that at least one optimal solution is preserved. Let $a_{i_{1}}$ departments of the same type $i, i \in[k]$, be in row 1 . We denote these departments, w.l.o.g., by $1, \ldots, a_{i_{1}}$. Then, we fix the ordering of these departments by additional constraints, w.l.o.g., we use an ascending order. Since these departments are of the same type, they have the same length and we can add

$$
d_{(n+1) 1} \leq d_{(n+1) 2}+\ell_{1} \leq \ldots \leq d_{(n+1)\left(a_{i_{1}}\right)}+\left(a_{i_{1}}-1\right) \cdot \ell_{1}
$$

to our model. It follows immediately that we can set the betweenness variables which contain dummy departments to

$$
x_{(n+1) k l}= \begin{cases}1, & k, l \in\left[a_{i_{1}}\right], k<l, \\ 0, & k, l \in\left[a_{i_{1}}\right], k>l .\end{cases}
$$

Similar equations can be added for department $n+2$. Furthermore, we fix the associated betweenness variables

$$
x_{k u v}= \begin{cases}1, & k, u, v \in\left[a_{i_{1}}\right], k<u<v, \\ 0, & k, u, v \in\left[a_{i_{1}}\right], k<v \text { and }(u<k \text { or } u>v) .\end{cases}
$$




\subsubsection{Iterative combination of optimization and simulation}

In mathematical models usually only the transport loads are taken into account. For a successful production system, which highly depends on the decisions made during the factory planning process, several further key performance indicators play a significant role. Therefore, we use simulation. Given a layout of the departments, we generate dynamic and realistic information about the transport processes by running a simulation. The simulation model allows

- the consideration of different distribution strategies for the material flow,

- adding different products,

- adjusting the processing sequences, i. e., in which order the products have to be processed,

- adjusting a production program which includes a production schedule and the number of products.

A benefit of the simulation is that we can consider additional key performance indicators of the production systems, among them output, throughput times, inventory, capacities, utilization of resources. Further, the visualization of processes simplifies the understanding of complex relations [24].

An analysis of the results including the key performance indicators is then the basis for further improvements. If the current layout has to be improved, the DRFLP models are customized by extending or adapting the mathematical models and the interplay between optimization and simulation continues. One big advantage of our iterative layout creation is that afterwards we can nicely compare the found solutions with respect to several indicators. So, the effect of certain decisions becomes clear. The simulation might show, for instance, that the distances between certain departments are too large for a smooth production. Then, we can restrict these distances in the model, see Section 3.3.4.

Many simulation models are generated in 2D. This kind of department representation is quite abstract and impedes the intuitive understanding of the layout and the production process. Especially for layouts with an increased number of departments, the transparency of a DRFLP solution with the $2 \mathrm{D}$ simulation model is limited. The integration of $3 \mathrm{D}$ models provides a better overview for the planner as illustrated in Figure 3.3 .3 . Especially the product flow can be demonstrated very quickly. Using a 3D simulation model on basis of the optimized layout can simplify the virtual validation of the planned production area and the detection of bottlenecks. All in all, simulation allows to control whether it will be possible to achieve the desired output of the production system afterwards in real production.

\subsubsection{Computational results}

For testing our new approach for the extended DRFLP models in the simulation we use a well-known application example [95. It represents a real gearbox production and includes 21 departments (with 12 types) and eight different products which are combined in an assembly department to an end product. This example provides a solid data basis for the layout planning problem and the simulation model. A 3D illustration of some layout for this instance, where the incoming and the shipping warehouse are arranged at the borders, is given in Figure 3.3.3. This instance has already been taken into account in Example 3.3.3. In our computational tests we used Theorem 3.3.2 and the additional symmetry breaking constraints for departments of the same type in the same row. In Figure 3.3 .4 we show the development of the running times in a logarithmic scale of our optimization approach when we enlarge the number of the departments. In the tests we start with 12 departments of different types and successively add one department in each step. Hence, department type 3 appears twice when we consider 13 departments and three times when we consider 14 departments and so on. Figure 3.3.4 shows that the original instance with all 


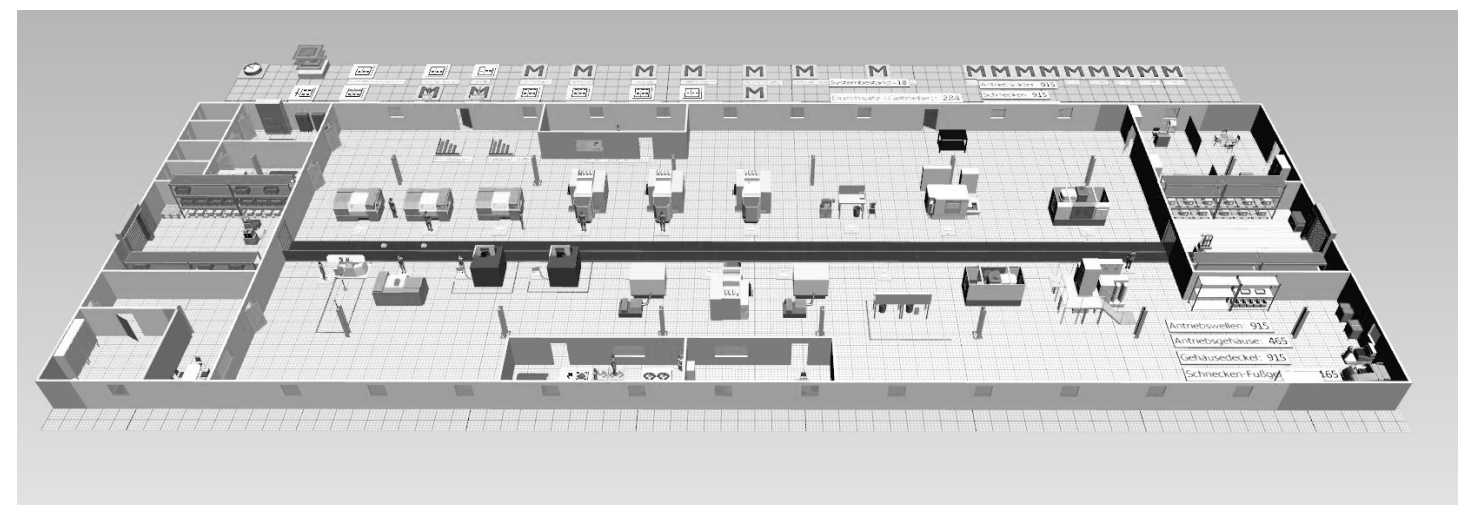

Figure 3.3.3: 3D illustration of a DRFLP layout with 21 departments [95. This simulation was derived using mathematical optimization. The incoming and the shipping warehouse are arranged at the left and the right border, respectively.

departments can be solved in less than 14 hours, although it contains 21 departments and so five departments more than the largest DRFLP instance solved to optimality in the literature before.

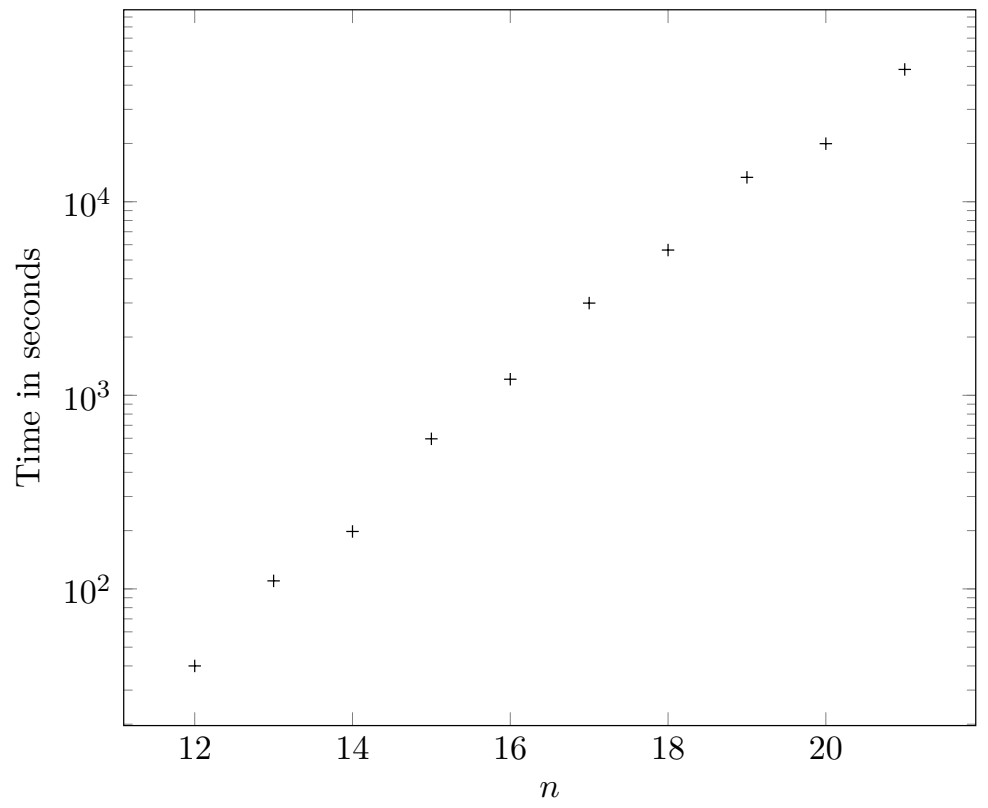

Figure 3.3.4: Running times in seconds for variations of some realistic instance from [95. We start with 12 different department types and 12 departments and we add departments successively according to the following order of the number of departments of each of the 12 types: 114122412111 . The largest instance contains 21 departments.

In the simulation we tested the following five different solutions where in the first four variants the incoming and shipping warehouse were arranged at the border:

1. A solution determined according to criteria usually used in factory planning where apart from the transport weights one had a special look at the linearity of the flows. The heuristic of Schmigalla [65, 100] was applied and afterwards the solution was improved by hand.

2. A solution determined according to criteria usually used in factory planning where all departments of a type were interpreted as one big block and then these blocks were arranged. The number of blocks that had to be arranged is smaller than the total number of departments. So it was easier to build this layout by hand. 
3. We used our mathematical DRFLP model for deriving a solution. A 3D visualization of the layout can be found in Figure 3.3.3.

4. We used our mathematical DRFLP model for deriving a solution. Additionally, all departments of the same type were interpreted as one big block and then these 12 blocks were arranged.

5. Solution derived using our mathematical DRFLP model with arbitrary position of all departments as well as of the incoming and the shipping warehouse (in our model these are departments, too). This approach was also used for deriving the results in Figure 3.3.4.

In all five simulations we manufactured 36000 end products and determined afterwards the average distance of each single product and the total distance traveled. The results can be found in Table 3.3.1. The end product, which is obtained by combining all eight products in an assembly department, is denoted as product 9. The second column in Table 3.3.1 shows the number of transports ("Trans") needed for each product $1, \ldots, 9$, and the next ten columns show the distances for the five simulation variants where the left column ("single") for each type contains the information on the average transport distance of each single product and the right column the total distance ("total") traveled for all products of the same type.

The results show that our optimization model improves the solution significantly in comparison to the solution determined by hand, especially if we do not restrict the positions of the warehouses (Layout 5). Comparing Layout 1 and Layout 3, the solution obtained using optimization is better than the solution obtained by applying the heuristic of Schmigalla followed by some improvement steps by the factory planners. But even the optimized solution with blocks and fixed border, illustrated in Layout 4, is better than the solutions determined by hand, illustrated in Layout 2 .

\begin{tabular}{|c|c|c|c|c|c|c|c|c|c|c|c|}
\hline \multirow[b]{2}{*}{$\mathrm{P}$} & \multirow[b]{2}{*}{ Trans } & \multicolumn{2}{|c|}{ Layout 1} & \multicolumn{2}{|c|}{ Layout 2} & \multicolumn{2}{|c|}{ Layout 3} & \multicolumn{2}{|c|}{ Layout 4} & \multicolumn{2}{|c|}{ Layout 5} \\
\hline & & single & total & single & total & single & total & single & total & single & total \\
\hline 1 & 480 & 37.90 & 18192.0 & 38.70 & 18576.0 & 28.70 & 13776.00 & 29.15 & 13992.00 & 16.31 & 7828.8 \\
\hline 2 & 90 & 37.90 & 3411.0 & 38.70 & 3483.0 & 28.70 & 2583.00 & 29.15 & 2623.50 & 16.31 & 1467.9 \\
\hline 3 & 144 & 38.50 & 5544.0 & 44.85 & 6458.4 & 29.32 & 4222.08 & 35.91 & 5171.04 & 30.75 & 4428.0 \\
\hline 4 & 60 & 37.90 & 2274.0 & 37.60 & 2256.0 & 28.70 & 1722 & 29.15 & 174 & 39.60 & 2376.0 \\
\hline 5 & 30 & 65.60 & 1968.0 & 71.90 & 2157.0 & 69.20 & 2076.00 & 70.40 & 2112.00 & 66.51 & 1995.3 \\
\hline 6 & 60 & 55.96 & 3357.6 & 49.03 & 2941.8 & 55.66 & 3339.60 & 54.18 & 3250.80 & 54.27 & 3256.2 \\
\hline 7 & 30 & 66.12 & 1983.6 & 71.89 & 2156.7 & 66.42 & 1992.60 & 70.90 & 2127.00 & 66.51 & 1995.3 \\
\hline 8 & 120 & 37.90 & 4548.0 & 37.60 & 4512.0 & 28.70 & 3444.00 & 29.15 & 3498.00 & 39.00 & 4680.0 \\
\hline 9 & 720 & 2.00 & 1440.0 & 2.00 & 1440.0 & 10.80 & 7776.00 & 11.20 & 8064.00 & 0.00 & 0.0 \\
\hline Total & & & 42718.2 & & 43980.9 & & 40931.28 & & 42587.34 & & 28027.5 \\
\hline
\end{tabular}

Table 3.3.1: Visualization of the results of the five simulations for our test case. In this production all eight products are combined to an end product, denoted as product 9 , see column "P". The entry "Total" in the first column refers to the total distance traveled in each of the five layouts. Note that using our approach from mathematical optimization with arbitrary department positions reduces the total distances significantly in comparison with the other four variants.

\subsubsection{Manufacturing different products}

The simulation showed that transport distances might be rather high in an optimal layout if the number of products of this type is small in comparison to the others. However, high transport distances can increase the cycle time. For a smooth production we want to bound the transport distances associated to single products. Let $P$ denote the set of products and $z_{p}$ be the desired number of product $p \in P$. Every product has its own transport matrix $W^{p}$. The $i j$-th entry of the matrix $W^{p}$ denotes the transport weights between department $i$ and $j$ for producing product 
$p$. The transport weight matrix $W$ that we used before is built on the sum of the transport matrices times the associated desired number of products, i. e., $W=\sum_{p \in P} z_{p} \cdot W^{p}$. Our aim is now to investigate the influence of single products to the whole production.

Definition 3.3.4. For a product $p \in P$ we define the transport distance as

$$
\sum_{\substack{i, j \in[n] \\ i<j}}\left(w_{i j}^{p}+w_{j i}^{p}\right) d_{i j},
$$

where $w_{i j}^{p}$ is the ij-th entry of the matrix $W^{p}$.

We set up a desired distance $d_{p} \in \mathbb{R}_{+}$for the transport distance for each single product $p \in P$. Of course, the desired transport distance depends on the amount of products $z_{p}$ for $p \in P$. If this value is exceeded, we want to penalize this with a quadratic function $\tilde{f}$ which is later approximated by some piecewise linear function. Let $a \geq d_{p}$ be the highest possible transport distance of product $p \in P$. Then we set $\tilde{f}:[0, a] \rightarrow[0, \tilde{f}(a)]$ such that

$$
\tilde{f}(x)= \begin{cases}d_{p}-x, & x<d_{p}, \\ 0, & x=d_{p}, \\ \left(x-d_{p}\right)^{2}, & x>d_{p} .\end{cases}
$$

To avoid a non-linear objective function, we approximate $\tilde{f}$ on the interval $\left[d_{p}, a\right]$ with a piecewise linear, continuous, convex function $f$. Therefore, we use linear interpolation [23].

Let a product $p \in P$ and points $u_{1}, \ldots, u_{o}$ be given with $o \geq 1$ and $u_{i} \geq d_{p}$ sorted in ascending order for $i \in[o]$. Then, we compute a linear approximation of $\tilde{f}$ between the points $\left(u_{i},\left(u_{i}-d_{p}\right)^{2}\right)$ and $\left(u_{i+1},\left(u_{i+1}-d_{p}\right)^{2}\right)$ for $i=1, \ldots, o-1$. The resulting function $f$ is piecewise linear and can be written as $f(x)=\max _{i=1, \ldots, o}\left(a_{i}\right)^{T} x+b_{i}$ for $a_{i}, b_{i} \in \mathbb{R}$ and $i \in[m]$. We add the following term to the objective function

$$
z_{p} \cdot f\left(\sum_{\substack{i, j \in[n] \\ i<j}}\left(w_{i j}^{p}+w_{j i}^{p}\right) d_{i j}\right) .
$$

This term can be linearized by replacing (3.3.1) with

$$
z_{p} \cdot t
$$

in the objective function and adding the constraints

$$
a_{i}^{T} x+b_{i} \leq t, \quad i \in[o] .
$$

We may set up such a penalty function for every product $p \in P$.

\section{The author's contribution:}

The ideas of the extensions of the basic DRFLP model as well as the result of Theorem 2 including the proof are joint work with Anja Fischer. The write-up of Section 1 and Section 2 are joint work with Anja Fischer as well. The author contributed to the write-up of Section 4 and he is responsible for large parts of the write-up of Section 3 and Section 5.2. 


\subsection{Decorous combinatorial lower bounds for row layout problems}

In this section we summarize the publication provided in Section $\mathrm{D}$ which is joint work with Anja Fischer and Frank Fischer.

The current best approach [42] for the DRFLP can solve only small double-row instances to optimality in reasonable time and this enumerative approach cannot be used to derive lower bounds for large-sized double-row instances. Further, lower bounds received via some branchand-cut algorithm within a given time limit of one hour for some DRFLP formulation from the literature are rather weak as we will see in Table 3.4.1. In Section 3.4.1 we present two different combinatorial lower bounds for the DRFLP based on a relation between some special DRFLP and some parallel identical machine scheduling problem. In addition, these lower bounds are combined with a new MILP model, see Section 3.4.2, which is indeed not a formulation for the DRFLP, to further strengthen the lower bounds. The combinatorial lower bounds as well as the lower bounding model can further be improved in the equidistant case.

\subsubsection{Combinatorial lower bounds}

Our approach for calculating lower bounds for the DRFLP is related to the following problem:

Definition 3.4.1. Given a set of jobs $J$ with processing times $q_{k} \in \mathbb{R}_{+}$and weights $w_{k} \in \mathbb{R}_{+}, k \in$ $J$, one looks for an assignment of start times $t_{k} \in \mathbb{R}_{+}$of the jobs $J$ to $u \in \mathbb{N}$ parallel identical machines such that no two jobs overlap on one machine and such that the sum of the weighted completion times $\sum_{k \in J} w_{k} C_{k}$ with $C_{k}=t_{k}+q_{k}$ is minimized. For constant $u$ we denote this problem by $P_{u} \| \sum w_{k} C_{k}$.

The scheduling problem $P_{u} \| \sum w_{k} C_{k}$ is weakly $\mathcal{N} \mathcal{P}$-hard, see, e.g., [78]. It is well known that the unweighted case, i. e., $P_{u} \| \sum C_{k}$ with $w_{k}=1$ for $k \in J$, can be solved to optimality in polynomial time by the Shortest Processing Time rule (SPT), where one processes the jobs in increasing order of their processing time.

Our aim is to compute lower bounds for the weighted distances of department $i \in[n]$ to departments $S \subseteq[n] \backslash\{i\}$. Let $p \in \mathbb{R}^{n}$ denote the center positions of the departments and let $r \in\{1,2\}^{n}$ denote the assignment of the departments to the rows. Further, let $\widehat{W}_{i}(S)$ denote the objective value of a feasible double-row layout with departments $\{i\} \cup S, S \subseteq[n] \backslash\{i\}$, which minimizes $\sum_{j \in S}\left(w_{i j}+w_{j i}\right)\left|p_{i}-p_{j}\right|$. Adding the additional constraint $p_{i}=p_{j}$ for some $j \in S$, i. e., $i$ lies directly opposite to $j$, then the corresponding optimization problem is denoted by $\widehat{W}_{(i, j)}(S)$. It turns out that:

Proposition 3.4.2. Let $(n, w, \ell)$ be a DRFLP instance and let $i \in[n], S \subseteq[n] \backslash\{i\}$. Then $\widehat{W}_{i}(S)=\min _{j \in S} \widehat{W}_{(i, j)}(S)$.

In the following, we determine two different lower bounds for $\widehat{W}_{(i, j)}(S)$ given some DRFLP instance. In both variants we interpret the optimization problem for computing $\widehat{W}_{(i, j)}(S)$ as a scheduling problem $P_{4} \| \sum w_{k} C_{k}$ with weights $w_{k}=w_{i k}+w_{k i}, k \in S \backslash\{j\}$. The departments correspond to the jobs in the $P_{4} \| \sum w_{k} C_{k}$ and the lengths of the departments to the processing times, i. e., $q_{k}=\ell_{k}, k \in S \backslash\{j\}$. Given a feasible solution of $\widehat{W}_{(i, j)}(S)$, then, as illustrated in Figure 3.4.1, machine 1 and machine 2 of the scheduling problem correspond to row 1 in this solution and machine 3 and machine 4 to row 2. Additionally, we have to take into account that in the scheduling problem the completion times of the jobs are considered while in the DRFLP one measures center-to-center distances between the departments.

Thus we are able to use methods from the scheduling literature to compute lower bounds for the DRFLP. All lower bound calculations have in common that we sort the jobs in $S \backslash\{j\}$ by some given order. Respecting some machine-dependent non-availability times from zero to $a=\left(a_{1}, \ldots, a_{4}\right) \in \mathbb{R}_{+}^{4} \cup\{\infty\}$ (i. e., no job on machine $k$ may start before $a_{k}, k=1, \ldots, 4$ ), the 


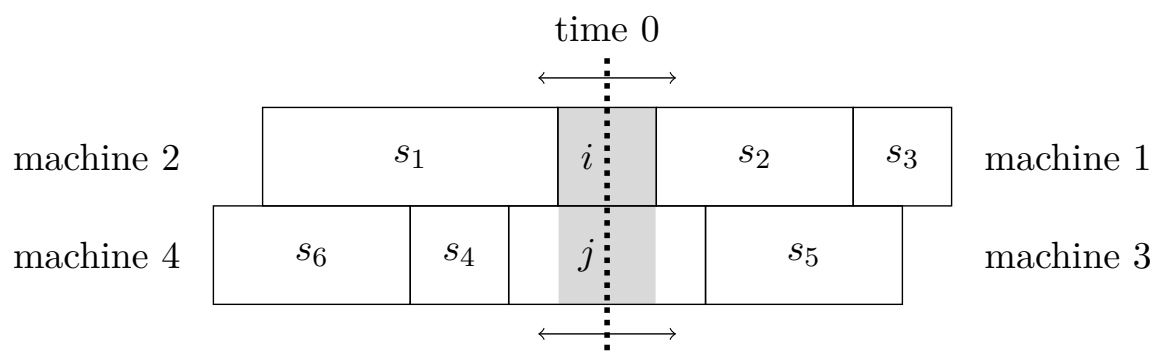

Figure 3.4.1: Visualization of the connection of the DRFLP and parallel machine scheduling on four machines. Here departments $i$ and $j$ lie opposite and we have to arrange departments $\left\{s_{1}, \ldots, s_{6}\right\}$. In the lower bound calculations we will partially adjust the start of the jobs (departments) at a machine by half the length of $i$ (see gray area) or half the length of $j$. In the scheduling problem one considers the completion times of the jobs while in the DRFLP one measures the center-to-center distances between the departments.

jobs are assigned in a greedy manner. Whenever a machine becomes idle and is available one assigns the next unscheduled job in the list non-preemptively. Our basic algorithm is summarized in Algorithm 3.4.1.

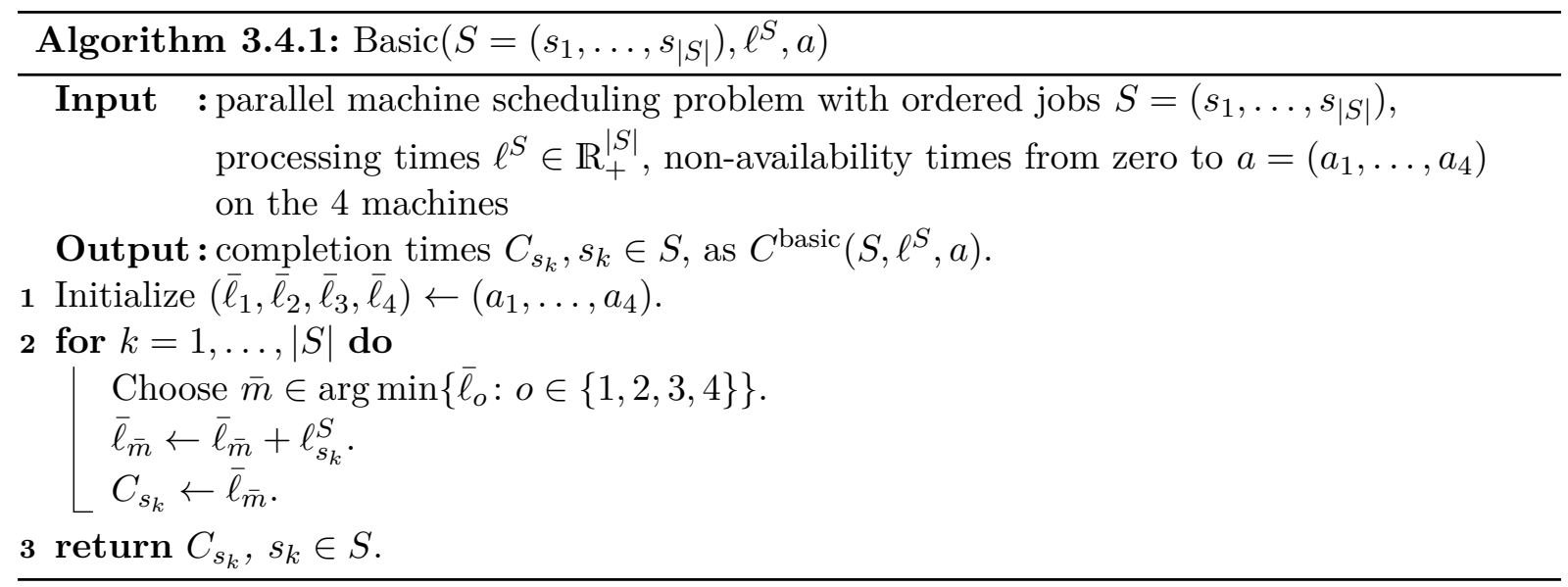

In our first combinatorial lower bound for $\widehat{W}_{(i, j)}(S), i \in[n], S \subseteq[n] \backslash\{i\}$, we fix $i$ opposite $j \in S$ and we make use of the SPT rule, so we sort the departments $S \backslash\{j\}$ by increasing length. The departments are assigned as described in Algorithm 3.4.1. Recall that the SPT rule determines an optimal solution for the $P_{4} \| \sum C_{k}$ (with $w_{k}=1, k \in J$ ). Then, we assign the highest weights to departments closest to $i$ and obtain a lower bound for $\widehat{W}_{(i, j)}(S)$. As illustrated in Figure 3.4.1. machine 1 and machine 2 are not available from 0 to $\frac{\ell_{i}}{2}$ and machine 3 and machine 4 from 0 to $\frac{\ell_{j}}{2}$.

Definition 3.4.3. Let $(n, w, \ell)$ be a DRFLP instance. Let $i \in[n], S \subseteq[n] \backslash\{i\}, j \in S$ with $S_{j}^{s p t}=\left(s_{1}, \ldots, s_{|S|-1}\right)$ a sequence of departments in $S \backslash\{j\}$ with length $\ell_{j}^{S_{j}^{s p t}}=\left(\ell_{s_{1}}, \ldots, \ell_{s_{|S|-1}}\right)$ ordered by increasing lengths and let

$$
C^{s p t,(i, j)}(S, \ell):=C^{\text {basic }}\left(S_{j}^{s p t}, \ell^{S_{j}^{s p t}},\left(\frac{\ell_{i}}{2}, \frac{\ell_{i}}{2}, \frac{\ell_{j}}{2}, \frac{\ell_{j}}{2}\right)\right)
$$

denote the completion times returned by Algorithm 3.4.1. Furthermore, let $w_{i \bullet}^{\prime}=\left(w_{i 1}^{\prime}+\right.$ $\left.w_{1 i}^{\prime}, \ldots, w_{i(|S|-1)}^{\prime}+w_{(|S|-1) i}^{\prime}\right)$ be the weights $w_{i k}+w_{k i}$ of $k \in S \backslash\{j\}$, ordered decreasingly. 
Then the SPT-lower-bound is

$$
W_{(i, j)}^{s p t}(S):=\sum_{k=1}^{|S|-1} w_{i k}^{\prime}\left(C_{s k}^{s p t,(i, j)}(S, \ell)-\frac{\ell_{s_{k}}}{2}\right) .
$$

In the special case of all weights being equal to one the SPT-distance-bound is

$$
W_{(i, j)}^{d s t}(S):=\sum_{k=1}^{|S|-1}\left(C_{s_{k}}^{s p t,(i, j)}(S, \ell)-\frac{\ell_{s_{k}}}{2}\right) .
$$

The SPT-distance-bound cannot be used to derive bounds for the optimal value of the DRFLP. However, it can be used to derive lower bounds for the (geometric) distances between the departments themselves without regarding the amount of transports.

Now we consider a second variant for computing a lower bound for the $\widehat{W}_{(i, j)}(S)$ based on the Smith rule which has been extended to $P \| \sum w_{k} C_{k}$ in the following way: The jobs are ordered non-increasingly by their relative weights $\frac{w_{k}}{q_{k}}$ for $k \in J$ and we assign each of the jobs using this order to the next machine that gets idle. It is proven in [62 that the Smith rule for sorting the jobs leads to a $\frac{1+\sqrt{2}}{2}$-approximation algorithm for the $P \| \sum w_{k} C_{k}$. We set $\alpha^{K K}:=\frac{1+\sqrt{2}}{2}$.

Definition 3.4.4. Let $(n, w, \ell)$ be a DRFLP instance, and let $i \in[n], S \subseteq[n] \backslash\{i\}$ and $j \in S$. We denote by $S_{j}^{s c}=\left(s_{1}, \ldots, s_{|S|-1}\right)$ a sequence of departments $S \backslash\{j\}$ with length vector $\ell^{S_{j}^{s c}}$ ordered non-increasingly by $\frac{w_{i k}+w_{k i}}{\ell_{k}}, k \in S \backslash\{j\}$. Denote by

$$
C^{s c,(i, j)}(S, \ell):=C^{b a s i c}\left(S_{j}^{s c}, \ell^{S_{j}^{s c}},(0,0,0,0)\right)
$$

the completion times returned by Algorithm 3.4.1 for this ordering. Then the SCHED1-lower-bound is

$$
W_{(i, j)}^{s c}(S):=\frac{1}{\alpha^{K K}} \sum_{k=1}^{|S|-1}\left(w_{i s_{k}}+w_{s_{k} i}\right) \cdot C_{s_{k}}^{s c,(i, j)}(S, \ell)+\sum_{k=1}^{|S|-1}\left(w_{i s_{k}}+w_{s_{k} i}\right) \cdot\left(\frac{1}{2} \min \left\{\ell_{i}, \ell_{j}\right\}-\frac{\ell_{s_{k}}}{2}\right) .
$$

In Section $\mathrm{D}$ we prove that $W_{(i, j)}^{\mathrm{spt}}(S)$ and $W_{(i, j)}^{\mathrm{sc}}(S)$ are indeed lower bounds for $\widehat{W}_{(i, j)}(S), i \in$ $[n], j \in S \subseteq[n] \backslash\{i\}$. Combining these results leads to a first combinatorial lower bound for the optimal value of the DRFLP.

Theorem 3.4.5. Let $V_{i}=\left\{k \in[n]: w_{i k}+w_{k i}>0\right\}$. The value

$$
\frac{1}{2} \sum_{i \in[n]} \min _{j \in V_{i}} \max \left\{W_{(i, j)}^{s p t}\left(V_{i}\right), W_{(i, j)}^{s c}\left(V_{i}\right)\right\}
$$

is a lower bound on the optimal value of the DRFLP and can be computed in $\mathcal{O}\left(n^{3} \cdot \log (n)\right)$.

In Section $\mathrm{D}$ a third combinatorial lower bound for $\widehat{W}_{(i, j)}(S)$ is presented and used in the calculation of (3.4.2). Additionally, in Section $\mathrm{D}$ it is shown that the combinatorial lower bounds can be extended to the MRFLP.

In the equidistant case, we can simplify the calculation of the SPT-lower-bound since an optimal solution for the $P_{4} \| \sum w_{k} C_{k}$ can be determined by assigning the departments with the highest weights first. So for $i \in[n]$ we sort the departments in $S \subseteq[n] \backslash\{i\}$ by decreasing weights $w_{i k}+w_{k i}, k \in S$, and assign the departments in that order as close as possible to $i$, i. e., a department with highest weight $w_{i k}+w_{k i}, k \in S$, lies directly opposite $i$. We denote this lower bound by $W_{i}^{\text {sort }}(S)$. 
If we know that two departments $i, j \in[n], i<j$, overlap and so lie exactly opposite due to the grid structure [15], we can determine a lower bound for the weighted distances of $i$ and $j$ to the departments $S \subseteq[n] \backslash\{i, j\}$. For this we order the departments in $S$ by decreasing weight $w_{i k}+w_{k i}+w_{j k}+w_{k j}, k \in S$, and get a sequence $S_{i, j}^{\mathrm{E}-\mathrm{spt}}=\left(s_{1}, \ldots, s_{|S|}\right)$. With

$$
C^{\mathrm{E}-\mathrm{spt},(i, j)}(S)=C^{\mathrm{basic}}\left(S_{i, j}^{\mathrm{E}-\mathrm{spt}},(1, \ldots, 1),(0,0,0,0)\right)
$$

we denote the completion times returned by Algorithm 3.4.1 and we set

$$
W_{(i, j)}^{\mathrm{E}-\mathrm{spt}}(S):=\sum_{k=1}^{|S|}\left(w_{i s_{k}}+w_{s_{k} i}+w_{j s_{k}}+w_{s_{k} j}\right)\left(C_{s_{k}}^{\mathrm{E}-\mathrm{spt},(i, j)}(S)\right) .
$$

Proposition 3.4.6. Let $(n, w, \mathbf{1})$ be a DREFLP instance. Let $i, j \in[n], i<j$, and $S \subseteq[n] \backslash\{i, j\}$, then for all equidistant double-row layouts with $p_{i}=p_{j}$ we have

$$
W_{(i, j)}^{E-s p t}(S) \leq \sum_{k \in S}\left(w_{i k}+w_{k i}+w_{j k}+w_{k j}\right)\left|p_{i}-p_{k}\right| .
$$

\subsubsection{A lower bounding model}

We aim to further improve the combinatorial lower bounds via an MILP model, which is indeed not a formulation for the DRFLP. We use distance variables $d_{i j}=d_{j i} \geq 0, i, j \in[n], i<j$, measuring the horizontal center-to-center distances between $i$ and $j$. In contrast to the literature, see, e. g., [42, 101], where left-right ordering variables were used, we use binary overlap variables $x_{i j}=x_{j i} \in\{0,1\}, i, j \in[n], i<j$. Two departments $i$ and $j$ overlap if their positions satisfy $\left|p_{i}-p_{j}\right|<\frac{\ell_{i}+\ell_{j}}{2}$. The associated variables have the following interpretation

$$
x_{i j}= \begin{cases}1, & \text { departments } i \text { and } j \text { lie in different rows and overlap, } \\ 0, & \text { otherwise. }\end{cases}
$$

We define a parameter $\iota \in\{0,1\}$ which is one if and only if all department lengths are integral. This is the case in almost all test instances in the literature. A short summary of our lower bounding model for the DRFLP reads as follows.

$$
\begin{aligned}
& \min \sum_{\substack{i, j \in[n] \\
i<j}}\left(w_{i j}+w_{j i}\right) d_{i j} \\
& \sum_{\substack{i, j \in S \\
i<j}} x_{i j} \leq|S|-1, \\
& S \subseteq[n],|S| \geq 2, \\
& \sum_{j \in S} d_{i j} \geq \min _{j \in S} W_{(i, j)}^{\mathrm{dst}}(S) \\
& i \in[n], S \subseteq[n] \backslash\{i\} \\
& \sum_{j \in S}\left(w_{i j}+w_{j i}\right) d_{i j} \geq \min _{j \in S} \max \left\{\begin{array}{c}
W_{(i, j)}^{\mathrm{spt}}(S), \\
W_{(i, j)}^{\mathrm{sc}}(S)
\end{array}\right\}, \quad i \in[n], S \subseteq V_{i}, \\
& d_{i j}+\left(\frac{\ell_{i}+\ell_{j}}{2}\right) x_{i j} \geq \frac{\ell_{i}+\ell_{j}}{2}, \quad i, j \in[n], i<j, \\
& d_{i j}+\left(M-\ell_{i}-\ell_{j}+\frac{1}{2} \iota\right) x_{i j} \leq M-\frac{\ell_{i}+\ell_{j}}{2}, \quad i, j \in[n], i<j, \\
& d_{i j}+d_{j k}-d_{i k} \geq 0 \text {, } \\
& i, j, k \in[n],|\{i, j, k\}|=3, i<k, \\
& x_{i j}=x_{j i} \in\{0,1\} \text {, } \\
& i, j \in[n], i<j, \\
& d_{i j}=d_{j i} \geq 0 \text {, } \\
& i, j \in[n], i<j \text {. }
\end{aligned}
$$

The overlap variables in a double-row layout have to build a forest if we interpret them as edges in a graph where each department represents a single node of the associated graph and two 


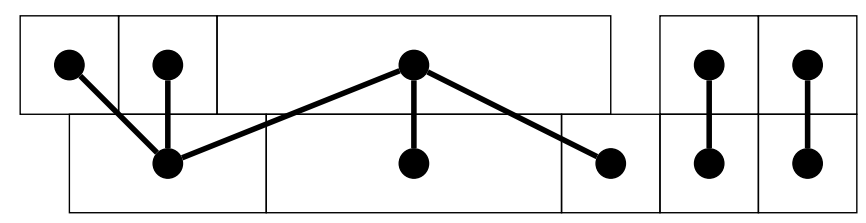

Figure 3.4.2: Visualization of the forest associated to the overlap variables of a double-row layout. Each node represents a department and there exists an edge between two different departments if and only if both overlap.

nodes (departments) are connected by an edge if both overlap in the layout, see Figure 3.4.2. We ensure this by the well-known subtour elimination constraints (3.4.4), see, e. g., [35, 39], for the complete description of the forest polytope.

We use our combinatorial bounds to bound the sum of the (weighted) distances between all departments of some set $S \subset[n]$, see (3.4.5)-3.4.6). The distance of two departments that do not overlap is at least the sum of half the lengths of these two departments, see inequalities (3.4.7). On the other hand the distance of two departments that overlap cannot be larger than the sum of half the lengths of both departments, see inequalities (3.4.8). Inequalities (3.4.8) are redundant if two departments do not overlap, we refer to Section $\mathrm{D}$ for the calculation of the big- $M$-value. Assuming integral department length we can even enforce that this value is $\frac{1}{2}$ less because the overlap is then at least one half (the departments are arranged on the half grid according to [56]). As used in previous layout models, see, e. g., [13], the distance variables have to satisfy the triangle inequalities (3.4.9).

We can further improve this lower bounding MILP model in the equidistant case. For the DREFLP there always exists an optimal solution on the grid [15]. Therefore, we can restrict to solutions where two departments overlap if and only if they lie directly opposite each other. So the interpretation of our overlap variables changes to

$$
x_{i j}^{e}=x_{j i}^{e}= \begin{cases}1, & \text { if } i \text { and } j \text { lie directly opposite each other } \\ 0, & \text { otherwise }\end{cases}
$$

$i, j \in[n], i<j$. A short summary of our model specialized to the DREFLP reads as follows.

$$
\begin{aligned}
& \min \sum_{\substack{i, j \in[n] \\
i<j}}\left(w_{i j}+w_{j i}\right) d_{i j} \\
& \sum_{\substack{j \in[n] \\
j \neq i}} x_{i j}^{e} \leq 1, \quad i \in[n], \\
& \sum_{\substack{i, j \in[n] \\
i<j}} x_{i j}^{e} \geq n-\left\lceil\frac{2 n}{3}\right\rceil+1, \quad n \geq 9, \\
& \begin{array}{ll}
d_{i j}+x_{i j}^{e} \geq 1, & i, j \in[n], i<j, \\
d_{i j}+M x_{i j}^{e} \leq M, & i, j \in[n], i<j,
\end{array} \\
& \sum_{\substack{i, j \in[n] \\
i<j}} d_{i j} \geq \begin{cases}\frac{(n+1) n(n-1)}{12}, & n \text { odd }, \\
\frac{(n+2) n(n-2)}{12}, & n \text { even, }\end{cases} \\
& \sum_{j \in S}\left(w_{i j}+w_{j i}\right) d_{i j} \geq W_{i}^{\mathrm{sort}}(S), \quad i \in[n], S \subseteq V_{i}, \\
& \sum_{k \in S}\left(\left(w_{i k}+w_{k i}\right) d_{i k}+\left(w_{j k}+w_{k j}\right) d_{j k}\right)
\end{aligned}
$$




$$
\begin{array}{rlrl} 
& -x_{i j}^{e}\left(W_{(i, j)}^{\mathrm{E}-\mathrm{spt}}(S)\right) \geq 0, & & i, j \in[n], i<j, S \subseteq[n] \backslash\{i, j\}, \\
x_{i j}^{e}=x_{j i}^{e} \in\{0,1\}, & & i, j \in[n], i<j .
\end{array}
$$

There always exists an optimal solution to the DREFLP on the grid [15]. So each department may overlap with at most one department, see (3.4.10). For $n \geq 9$ there always exists an optimal equidistant double-row layout which uses at most $\left\lceil\frac{2 n}{3}\right\rceil-1$ columns of the grid [15]. It follows that at least $n-\left\lceil\frac{2 n}{3}\right\rceil+1$ columns contain two departments, see 3.4.11). If two departments overlap, their distance is zero, see (3.4.13), and otherwise at least one, see (3.4.12). In the unweighted case of the DREFLP, i. e., if all weights are equal to one, an optimal solution can be determined directly, see Section $\mathrm{E}$ and this value is a lower bound for the sum of the distances in the DREFLP, see (3.4.14). Note that we are not aware of a similar result for the DRFLP, so we take advantage of the DREFLP structure here. Apart from this we can bound the sum of the weighted distances of some $i \in[n]$ to all departments $S \subseteq V_{i}$ from below using our combinatorial bounds, see 3.4.15. If two departments $i, j \in[n], i<j$, overlap, we can use $W_{(i, j)}^{\mathrm{E}-\text { spt }}(S)$ defined in 3.4.3 as a lower bound for the weighted distances of $i$ and $j$ to the departments $S \subseteq[n] \backslash\{i, j\}$, see (3.4.16). If $i$ and $j$ do not overlap, inequality (3.4.16) is redundant.

Additionally, in Section D the combinatorial lower bounds as well as the lower bounding MILP model are adapted to the PROP.

\subsubsection{Computational results}

Since only smaller instances were solved to optimality in the literature, we generate random instances with $n \in\{20,30,40,50\}$. To obtain a wide set of random instances we set the transport density to $10 \%, 50 \%$ and $100 \%$ and we choose integer transport weights randomly between 1 and 10. The integral lengths of the departments are chosen randomly between 1 and 15 (see Table 3.4.1). For each type we created ten instances. We denote these instances by $n_{k}$ where $n$ is the number of departments and $k$ is the transport density. The first column "Instance" of the tables displays the instances name.

The lower bound value obtained by applying Cplex with a given time limit of one hour for the DRFLP formulation in [8] is given in column "Amaral". Apart from this the tables contain our combinatorial bound (3.4.2) as well as the lower bounds derived using branch-and-cut for our lower bounding model within a given time limit of three or ten minutes, see columns "MILP 3 min" and "MILP ${ }_{10 \mathrm{~min}}$ ", respectively. In order to show that our combinatorial lower bounds significantly strengthen our lower bounding model we tested our MILP without using the combinatorial results from Section 3.4.1. These results can be found in column MILP 3 min . We applied the heuristic of [33] and afterwards a 1-opt and 2-opt exchange algorithm is applied. The resulting value is given in column "heuristic" and the time spent for the heuristic including the exchange algorithms in seconds is given in column "time heur." "Gap Ama." refers to the average gaps using Cplex for the model in [8] after a time limit of one hour and "Gap [3.4.2)", "Gap MILPpure" and "Gap $_{\text {MiLP " to }}$ the average gaps of our combinatorial lower bound and of our lower bounding model without and with the use of the combinatorial lower bounds, respectively.

Table 3.4.1 shows that our combinatorial lower bounds, which were computed in less than one second, clearly outperform the lower bounds obtained via using Cplex within a time limit of one hour for the DRFLP formulation in [8] on the randomly generated instances. These lower bounds are rather weak because of big-M type constraints and a quadratic number of binary decision variables and so the gaps are close to $100 \%$ for large $n$. Using branch-and-cut to improve our bounds allows a significant strengthening to final gaps between $14 \%$ and $55 \%$. For the MILP variant that does not use the combinatorial bounds the gaps are much higher and grow by increasing the number of departments. For instances with at least 40 departments the average gaps are higher than $70 \%$. Regarding (3.4.2) and our MILP the gaps are smaller for dense instances. Enlarging the time limit for our MILP approach from 3 to 10 minutes usually has only 


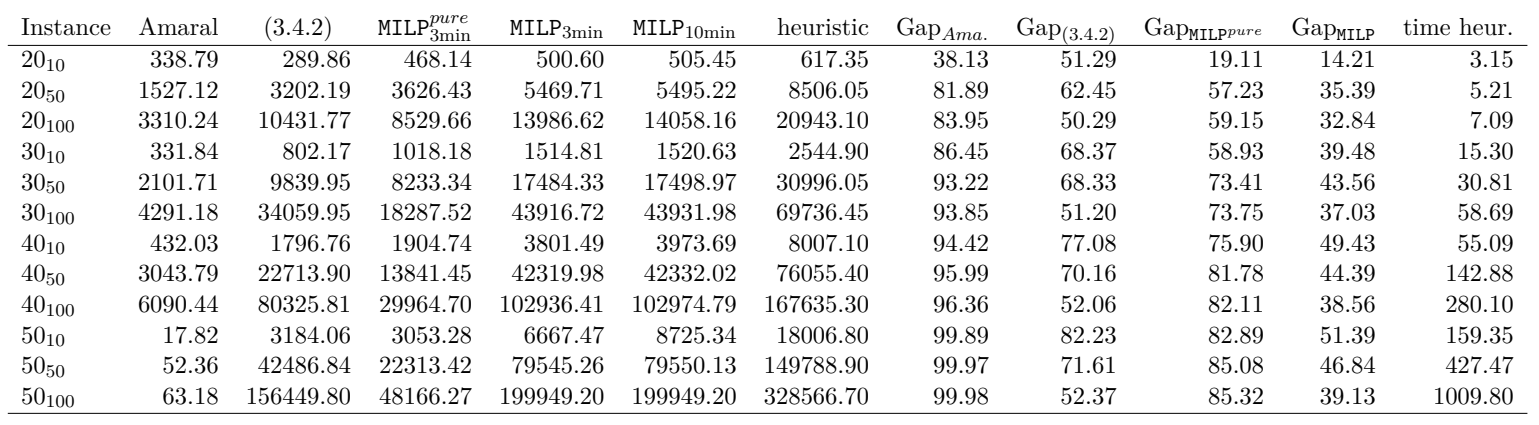

Table 3.4.1: Results for randomly generated double-row instances with integral department lengths between 1 and 15. We display the average values over ten instances each. The average gaps are given in percent. Note that for six instances with $n=50$ and density $10 \%$ we had to enlarge the time limit to five minutes for MILP ${ }^{\text {pure }}$.

\begin{tabular}{|c|c|c|c|c|c|c|c|}
\hline Instances & 3.4.2 for DREFLP & MILP $_{3 \min }$ & best ub & $\operatorname{Gap}_{3.4 .2}$ & $\mathrm{Gap}_{\text {MILP }}$ & Gap $_{\text {Anjos ILP } 3 \mathrm{~h}}$ & $\mathrm{Gap}_{\mathrm{SDP} 3 \mathrm{~h}}$ \\
\hline $\bar{Y}$ & 4301 & 5821 & 6046 & 28.86 & 3.72 & 0.00 & 0.00 \\
\hline$Y_{25}$ & 7032 & 9887 & 10170 & 30.86 & 2.78 & 1.22 & 0.36 \\
\hline$Y_{30}$ & 9237 & 13315 & 13790 & 33.02 & 3.44 & 2.78 & 0.14 \\
\hline$Y_{35}$ & 12607 & 18595 & 19087 & 33.95 & 2.58 & 21.27 & 0.26 \\
\hline$Y_{40}$ & 15332 & 22809 & 23739 & 35.41 & 3.92 & 23.88 & 0.37 \\
\hline$Y_{45}$ & 19952 & 29639 & 31442 & 36.54 & 5.73 & 26.35 & 0.65 \\
\hline$Y_{50}$ & 25839 & 39450 & 41517 & 37.76 & 4.98 & 28.35 & 0.62 \\
\hline
\end{tabular}

Table 3.4.2: Results for equidistant instances from the literature [15, 109]. The upper bounds "best ub" are taken from [15]. We compared our lower bounding model with the ILP and the SDP from [15] with a given time limit of three hours. The value of (3.4.2) and MILP 3 min are rounded to integers.

a very small effect on the bound. So three minutes seem to be a good value. Furthermore, the tables show that the quality of our lower bounds hardly deteriorates if one increases the number of departments.

In Table 3.4.2 we compare our lower bounding model specialized to the DREFLP with a time limit of three minutes with an ILP model for the DREFLP (denoted by "Gap Anjos ILP 3h") and an SDP approach for the DREFLP [15] (denoted by "Gap SDP $3 \mathrm{~h}$ ") with a time limit of three hours. The upper bounds ("best ub") in Table 3.4.2 are taken from [15]. For benchmark instances from the literature, see, e.g., [53, 109, with 20 to 50 departments the gaps of our combinatorial bounds are around $35 \%$ and the gaps of our lower bounding model are between $2.58 \%$ and $5.73 \%$. While our lower bounding model outperforms the ILP approach of [15] for $n \geq 35$, the SDP approach of [15] provides the best lower bounds. One advantage of our approach is that the running time is significantly smaller. For additional computational tests including lower bounds for the PROP we refer to Section D.

\section{The author's contribution:}

The author's ideas concern the improvement of the combinatorial lower bounds and the MILP approaches to the DREFLP and the PROP. The proofs for the correctness of the combinatorial lower bounds are joint work by the author, Anja Fischer and Frank Fischer. A large part of the write-up was done by the author and revised and further improved by Anja Fischer and Frank Fischer. The author is responsible for a considerable part of the implementation and fully responsible for the computational experiments. 


\subsection{A study of the relation between the single-row and the double-row facility layout problem}

In this section we summarize the publication given in Section E which is joint work with Anja Fischer and Philipp Hungerländer.

Only small instances can be solved to optimality in reasonable time for the DRFLP and the DREFLP while good or optimal solutions for the SRFLP can be derived very fast. In the first part, we focus on the equidistant case and we show that the optimal value of the MREFLP is less than or equal to the optimal value of the SRFLP divided by the number of rows of the MREFLP. We derive equidistant double-row layouts satisfying this property in a very short time and we improve some of the best known upper bounds for the DREFLP. In the second part, we provide a formula for the relation of the optimal value of the DRFLP and the SRFLP which shows that the optimal value of the DRFLP and the SRFLP might be close. Additionally, we provide an example which shows that this formula is tight. Furthermore, we present heuristic approaches for the DRFLP based on good or optimal single-row layouts which can be calculated very fast and we improve the best known upper bounds for large-sized instances. In the following, we denote the optimal value of the MRFLP with $m$ rows by $v_{m}^{*}$.

\subsubsection{The equidistant case}

We are given an MREFLP instance. In [56] it is proven that there always exists an optimal multi-row layout where the departments are arranged on the grid. We say that $i \in[n]$ lies in column $j \in[n]$ if the center of $i$ is located at the $j^{\text {th }}$ grid point. Then, we construct equidistant multi-row layouts on the grid in the following way:

Definition 3.5.1. We are given an equidistant multi-row instance and an equidistant single-row layout $\pi$. Then, the equidistant multi-row layout $L_{k}(\pi), k \in[m]$, is constructed by assigning the first $k$ departments in the order of $\pi$ to the first column and totally filling up all other columns with the remaining departments in the order of $\pi$.

Let $\pi$ be an equidistant single-row layout. The layouts $L_{k}(\pi), k \in[m]$, contain possible spaces only in the first and last column, we refer to Figure 3.5.1 for an illustration of the special case $m=2$. In the following theorem we provide a relation between the objective value of an equidistant single-row layout $\pi$ and the objective value of the associated multi-row layouts $L_{1}(\pi), \ldots, L_{m}(\pi)$.
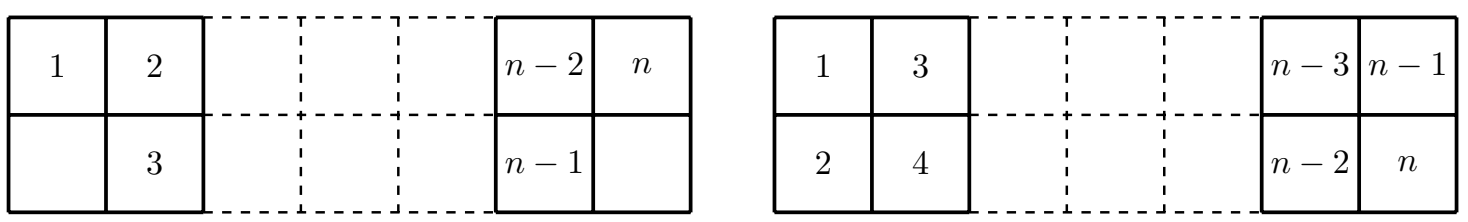

Figure 3.5.1: Illustration of the equidistant double-row layouts $L_{1}(\pi)$ on the left-hand side and $L_{2}(\pi)$ on the right-hand side deduced from the single-row layout $\pi=(1, \ldots, n)$. Note that in this drawing we assume, w.l.o.g., that $n$ is even.

Theorem 3.5.2. Given an equidistant multi-row instance and an associated equidistant single-row layout $\pi$ with objective value $v_{1}$ and let $v_{L_{k}(\pi)}$ denote the objective value of layout $L_{k}(\pi), k \in[m]$. Then

$$
\sum_{k \in[m]} v_{L_{k}(\pi)}=v_{1}
$$


Note that an equidistant instance with $n$ departments where $n$ is odd with weights $w_{i j}+w_{j i}=$ $1, i, j \in[n], i<j$, satisfies the equation $v_{m}^{*}=\frac{v_{1}^{*}}{m}$. Thus, in view of Theorem 3.5.2 we present the relation between $v_{1}^{*}$ and $v_{m}^{*}$ in the equidistant case.

Corollary 3.5.3. Given an equidistant multi-row instance and let $v_{m}^{*}\left(v_{1}^{*}\right)$ denote the optimal value of the MREFLP (SRFLP). Then

$$
v_{m}^{*} \leq \frac{v_{1}^{*}}{m} .
$$

Given an equidistant multi-row instance and a single-row layout $\pi$ with objective value $v_{1}$. Recall that several approaches are able to calculate good or optimal single-row layouts, see, e. g., [34, 58, 59, 93. Then, Theorem 3.5.2 and Corollary 3.5.3 provide an easy way to construct an equidistant multi-row layout with objective value $v_{m}$ based on $\pi$ that satisfies $v_{m} \leq \frac{v_{1}}{m}$ by computing the layouts $L_{1}(\pi), \ldots, L_{m}(\pi)$ and choosing one layout with minimal objective value. To further improve the layouts $L_{i}(\pi), i \in[m]$, in Section E an ILP model is set up which simplifies to some LP to include free-space not only in the first and in the last column. Afterwards, exchange algorithms are applied to further improve the resulting layout, we refer to Section E for details.

\subsubsection{The double-row facility layout problem}

Now we focus on the DRFLP with arbitrary department lengths. Since every single-row layout is also a valid double-row layout, the optimal value of the DRFLP is less than or equal to the optimal value of the SRFLP. We provide the following result:

Proposition 3.5.4. Let $v_{1}^{*}\left(v_{2}^{*}\right)$ denote the optimal value of the SRFLP (DRFLP). Then we get

$$
(n-1) v_{2}^{*} \leq(n-2) v_{1}^{*} .
$$

We present the following instance to show that inequality $(3.5 .2)$ is tight. So for this instance the optimal value of the SRFLP and the optimal value of the DRFLP are very close.

Instance 1. We are given an instance with $n$ departments and we choose $0<\varepsilon \leq \frac{1}{10}$. The lengths are set to $\ell_{1}=\ell_{2}=\ell_{3}=\varepsilon$ and $\ell_{k}=\frac{2}{\varepsilon^{k-4}}-\ell_{k-1}, k \in[n], k \geq 4$, and non-zero weights are set to $w_{12}=w_{23}=1$ and $w_{(k-1) k}=\varepsilon^{k-3}, k \in[n], k \geq 3$.

In the following example we consider optimal single-row and double-row layouts of Instance 1 with $n=3,4,5$ :

Example 3.5.5. a) We are given $n=3$ : Then, we get $v_{1}^{*}=2 \varepsilon$ and $v_{2}^{*}=\varepsilon$ and hence inequality $\frac{v_{2}^{*}}{v_{1}^{*}}>\frac{1}{2}-\delta$ is satisfied for $\delta>0$.

b) Now we consider $n=4$ : Clearly, $v_{1}^{*}=3 \varepsilon$ and an optimal double-row layout is depicted in Figure 3.5.2 and has objective value $v_{2}^{*}(\varepsilon)=\varepsilon+(1-\varepsilon) \varepsilon$. Hence we have $\frac{v_{2}^{*}}{v_{1}^{*}}=\frac{\varepsilon(2-\varepsilon)}{3 \varepsilon}=$ $\frac{2-\varepsilon}{3}>\frac{2}{3}-\delta$ if $\varepsilon<3 \delta$.

c) Next we are given $n=5$ : Then, $v_{1}^{*}=4 \varepsilon$. An optimal double-row layout can be obtained by arranging the first 4 departments as good as possible as illustrated in Figure 3.5.2, and then additionally arrange department 5 such that the centers of departments 4 and 5 are as close as possible, see Figure 3.5.2. Then, $d_{45} w_{45}=\left(p_{5}-p_{4}\right) w_{45}=\left(\frac{\ell_{5}}{2}+\ell_{3}-\frac{\ell_{4}}{2}\right) \varepsilon^{2}=\varepsilon-2 \varepsilon^{2}+2 \varepsilon^{3}$ and thus $v_{2}^{*}=\varepsilon+(1-\varepsilon) \varepsilon+\varepsilon\left(1-2 \varepsilon+2 \varepsilon^{2}\right)$ and the desired inequality $\frac{v_{2}^{*}}{v_{1}^{*}}>\frac{3}{4}-\delta$ is satisfied for $\delta>\frac{3 \varepsilon-2 \varepsilon^{2}}{4}$.

In the following lemma we show how Instance 1 can be solved to optimality and an immediate consequence is that the double-row layouts considered in Example 3.5.5 are optimal: 


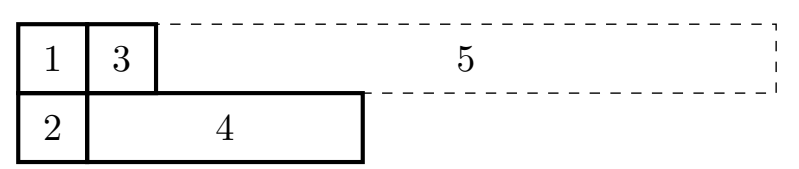

Figure 3.5.2: We are given Instance 1 with $n=4(n=5)$ with lengths $\ell_{1}=\ell_{2}=\ell_{3}=$ $\varepsilon>0, \ell_{4}=2-\varepsilon,\left(\ell_{5}=\frac{2}{\varepsilon}-\ell_{4}\right)$ and non-zero weights $w_{12}=w_{23}=1, w_{34}=\varepsilon$ $\left(w_{45}=\varepsilon^{2}\right)$. An optimal single-row layout is $\pi^{*}=(1,2,3,4)\left(\pi^{*}=(1,2,3,4,5)\right)$ and has objective value $v_{1}^{*}=3 \varepsilon\left(v_{1}^{*}=4 \varepsilon\right)$. The structure of an optimal doublerow layout for $0<\varepsilon \leq \frac{1}{10}$ is illustrated above for $n=4$ and $n=5$ where 5 is illustrated as a dashed rectangle. We obtain $v_{2}^{*}=\varepsilon+(1-\varepsilon) \varepsilon$ for $n=4$ and $v_{2}^{*}=\varepsilon+(1-\varepsilon) \varepsilon+\varepsilon\left(1-2 \varepsilon+2 \varepsilon^{2}\right)$ for $n=5$.

Lemma 3.5.6. Considering Instance 1 with $n$ departments and $0<\varepsilon \leq \frac{1}{10}$. Then, an optimal double-row layout is obtained by arranging the departments in the order $1, \ldots, n$ in an alternating manner to the rows. In each step, one department is assigned at the rightmost possible position such that the layout is space-free and such that 1 lies directly opposite 2.

We conclude that inequality 3.5 .2 is tight for Instance 1 with $n$ departments and $0<\varepsilon \leq \frac{1}{10}$.

Theorem 3.5.7. Let $n \geq 3$. Then, there exists a constant $C>0$ such that for every $0<\varepsilon \leq \frac{1}{10}$ there exists a $\delta$ with $0<\delta \leq C \varepsilon$ such that

$$
\frac{v_{2}^{*}}{v_{1}^{*}}>\frac{n-2}{n-1}-\delta
$$

where $v_{1}^{*}\left(v_{2}^{*}\right)$ denotes the optimal value of the SRFLP (DRFLP) with $n$ departments of Instance 1 .

\subsubsection{Heuristic approaches for the DRFLP}

There are three reasons for constructing double-row layouts based on single-row layouts. At first, good or optimal single-row layouts can be obtained very fast, see, e. g., [34, 58, 91, 93. At second, our computational results, see Section 3.5.4, indicate that by going from single-row layouts to double-row layouts the objective value is approximately halved. And the third reason is that these double-row layouts can be calculated very fast, in particular, for heuristically determined single-row layouts.

We present a heuristic based on a single-row layout $\pi$ and we assume, w. l. o.g., $\pi=(1, \ldots, n)$. The idea of our heuristic is that the ordering of departments in the same row is given via $\pi$, so it remains to determine the row assignment of the departments as well as their exact positions. We are given $n_{\min }, n_{\max } \in \mathbb{N}, n_{\min } \leq n_{\max }$, and in each step we add a set $S$ of departments which contains, if possible, at least $n_{\min }$ departments and at most $n_{\max }$ departments.

Let the set of departments $[h], 0 \leq h \leq n, h \in \mathbb{N}_{0}$, be already added to the double-row layout (we start with $h=0$ and we stop if $h=n$ ). If $h+n_{\max } \geq n$, all remaining departments are added and we set $S=\{h+1, \ldots, n\}$. Otherwise, we interpret the departments $H:=\{h+1, \ldots, n\}$ as nodes in a complete graph with weights $w_{i j}+w_{j i}, i, j \in H, i<j$. Our goal is to determine some $k^{\prime}$ and an associated set $S:=\left\{h+1, h+2, \ldots, k^{\prime}\right\}$ such that the sum of the total transport weights between $S$ and $[n] \backslash\left[k^{\prime}\right]$ is small. So we detect which departments should be considered together in the next step. We set

$$
k^{\prime}:= \begin{cases}\arg \min _{h+n_{\min } \leq k \leq h+n_{\max }} \sum_{\substack{i=h+1, \ldots, k \\ j=k+1, \ldots, n}} w_{i j}+w_{j i}, & h+n_{\max }<n, \\ n, & h+n_{\text {max }} \geq n\end{cases}
$$

So $|S| \leq n_{\max }$, and, if $h+n_{\min } \leq n$, then $|S| \geq n_{\min }$. The calculation of $k^{\prime}$ is related to the calculation of a constrained minimum cut in the graph described above. 
Then, we add the dummy department $n+1(n+2)$ to row 1 (row 2) with length $\ell_{n+1}=\ell_{n+2}=0$ and weights $w_{i(n+1)}=w_{(n+1) i}=w_{i(n+2)}=w_{(n+2) i}=\frac{1}{2} \sum_{\substack{i=h+1, \ldots, k^{\prime} \\ j=k^{\prime}+1, \ldots, n}} w_{i j}+w_{j i}$ such that $n+1$ $(n+2)$ is the rightmost department in row 1 (row 2). Knowing $k^{\prime}$ and so $S$, our goal is to determine a row assignment of the departments $S$ such that departments in the same row are sorted according to $\pi$ and such that sum $\sum_{i, j \in\left[k^{\prime}\right] \cup\{n+1, n+2\}}\left(w_{i j}+w_{j i}\right) d_{i j}$ is minimized, i. e., we have to solve a (small) double-row instance where the order of the departments in the same row is known. For solving this problem we apply the approach of [42] and enumerate over all distinguishable assignments of the departments $S$ to the rows. Knowing the order of the departments in the rows, each subproblem reduces to some LP with $k^{\prime}+2$ departments. We choose one of the row assignments for $S$ where the layout has minimal objective value. In the last step, when $n \in S$, the solution of the LP corresponds to a double-row layout including possible free-spaces. The algorithm stops after returning this layout. We denote this heuristic by $\operatorname{mc}\left(n_{\min }, n_{\max }\right)$. To further improve this layout, we set up an MILP model for the 1-opt algorithm. Then, we apply the 1-opt (2-opt) approach until the solution cannot be improved by a 1-opt (2-opt) step.

\subsubsection{Computational results}

We apply our heuristics based on best known single-row layouts, see, e. g., [70, 71, 73], and we use a heuristic for the SRFLP with a short running time and which is easy to implement, i. e., we start with a random single-row layout and apply a 1-opt algorithm and a 2-opt algorithm until the single-row layout cannot be improved by a 1-opt or 2-opt step, respectively.

We start our computational study with the equidistant case. In Table 3.5.1 we display in column two (column three) the objective value of a best known (heuristically determined) singlerow layout denoted by "Best known" ("Heuristic"). The objective value of the start layout and of the final layout after applying our exchange algorithm is denoted by $H_{\text {Best }}\left(H_{\text {Heur }}\right)$ and is based on a best known single-row layout (heuristically determined single-row layout). The current best upper bounds for these instances are derived by the SDP approach of [15] and the time limit is set to 3 hours.

We observe that for all instances in Table 3.5.1 our heuristic based on best known single-row layouts is better than the one based on heuristically determined single-row layouts. Note that the obtained gaps of our single-row heuristic are rather small and the running time is at most one minute, even for $n=100$. For all large sko-instances with $n \geq 49$ and given some best known single-row layout, we improve the previously known best upper bounds in [15] with a significantly smaller running time. Using our approach based on a heuristically determined single-row layout, we obtain small gaps to the approach of [15], however, these layouts can be calculated in a few minutes, including the corresponding single-row layout. Comparing the best solution values of the SREFLP and the DREFLP one can see that the value of the DREFLP is strictly less than halve the value of the SREFLP, but rather close to this value in our tests.

In Table 3.5.2 we consider the DRFLP and the notation is similar to Table 3.5.1. We compare our results with the heuristic approach of [33]. We focus on sko-instances where good heuristically determined single-row layouts are available at https://www.philipphungerlaender.com/ benchmark-libraries/layout-lib/row-layout-instances/. Looking at the results for the sko-instances in Table 3.5 .2 all solutions derived using the mc heuristic based on best known single-row layouts are better than the results of [33]. If we use the mc heuristic in combination with our simple single-row heuristic, we could improve 5 out of 9 upper bounds in comparison to the approach in [33]. The running time of the mc heuristic is slightly reduced by using a best known single-row layout instead of a heuristically determined single-row layout and the heuristic of [33] is a bit faster than the mc heuristic. For the mc heuristic based on some known single-row layout, the exchange algorithm only slightly improves the start layout, so the running time could be improved, by neglecting the exchange algorithms. Note that the layouts of [33] are 


\begin{tabular}{|c|c|c|c|c|c|c|c|c|c|}
\hline \multirow[b]{2}{*}{ Instance } & \multicolumn{2}{|c|}{ SRFLP } & \multicolumn{2}{|c|}{ Start layout } & \multicolumn{2}{|c|}{ Exchange } & \multirow[b]{2}{*}{15} & \multicolumn{2}{|c|}{ Time } \\
\hline & Best known & Heuristic & $H_{\text {Best }}$ & $H_{\text {Heur }}$ & $H_{\text {Best }}$ & $H_{\text {Heur }}$ & & $H_{\text {Best }}$ & $H_{\text {Heur }}$ \\
\hline sko42-1 & 25525 & 25525 & 12749 & 12749 & 12743 & 12743 & 12731 & 2 & 2 \\
\hline sko49-1 & 40967 & 42469 & 20477 & 21226 & 20470 & 21224 & 20512 & 6 & 6 \\
\hline sko56-1 & 64024 & 66083 & 31975 & 33011 & 31972 & 32932 & 31988 & 11 & 24 \\
\hline sko64-1 & 96883 & 98122 & 48418 & 49052 & 48409 & 49004 & 48574 & 23 & 45 \\
\hline sko72-1 & 139150 & 143317 & 69535 & 71607 & 69531 & 71603 & 69621 & 41 & 42 \\
\hline sko81-1 & 205106 & 208554 & 102549 & 104263 & 102549 & 104067 & 102793 & $1: 10$ & $4: 36$ \\
\hline sko100-1 & 378234 & 384049 & 189062 & 191982 & 189056 & 191964 & - & $3: 31$ & $3: 56$ \\
\hline
\end{tabular}

Table 3.5.1: Heuristically determined upper bounds for equidistant double-row instances from the literature [15. Our heuristics are based on best known single-row layouts as well as heuristically determined single-row layouts.

significantly improved by the exchange algorithms.

\section{The author's contribution:}

The proofs of Theorem 5, Proposition 9 and Theorem 12 were done by the author in consultation with Anja Fischer. The proof of Lemma 11, which is by far the largest proof in this publication, was mainly done by the author and revised multiple times by Anja Fischer. The author's ideas concern the mincut heuristic and the MILP model for the 1-opt algorithm. He is responsible for a large part of the implementation and fully responsible for the computational experiments. 


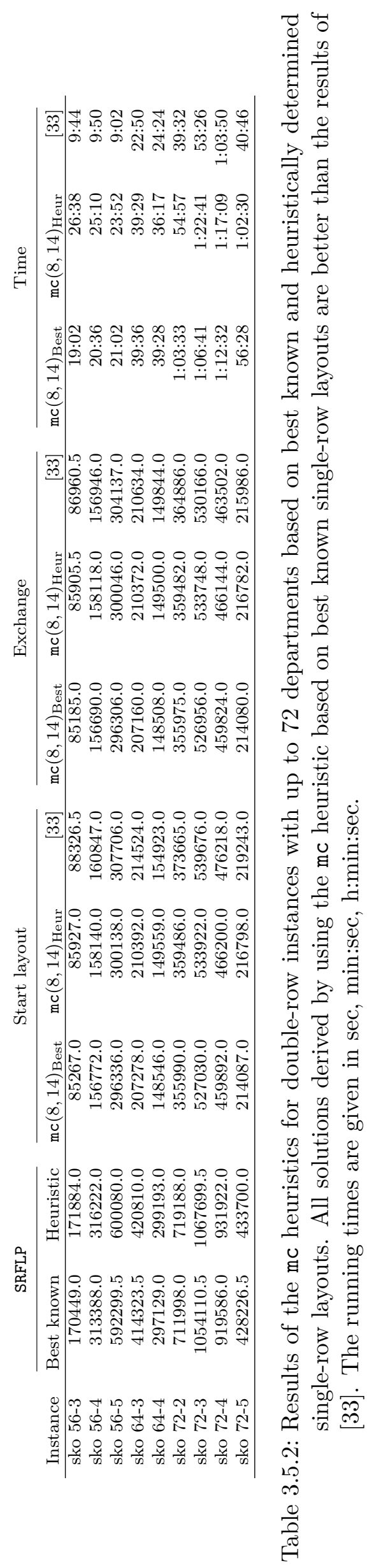




\section{Discussion}

In this section we first discuss the novelties of the five publications and afterwards we outline connecting factors between them.

In Section $\mathrm{A}$ we show that there always exists an optimal T-row layout where one department has its center position on position $p_{M}$ in row 1 . Then, we enumerate over each department with its center fixed on position $p_{M}$ in row 1 . We present an MILP model for this subproblem of the TRFLP based on an extension of the betweenness variables which can be in contrast to the literature equal to one if the corresponding departments lie in different rows. This extension seems to be a novelty in the layout planning literature and has two advantages. At first, the distances between departments in different rows can be calculated without big- $M$-constraints. At second, we are able to use transitivity constraints to ensure the correct relation of the extended betweenness variables and the remaining variables. Thus, we avoid the usage of standard linearization to couple the extended betweenness variables with the remaining variables. We provide cutting planes exploiting the crossroad structure of the TRFLP. We prove that the TRFLP is a generalization of the 3-BFLP, and hence we can adapt our MILP approach to the 3-BFLP. In order to evaluate the performance of our approach, we set up an MILP approach based on betweenness variables which can only be equal to one if the corresponding three departments lie in the same row (as known in the literature). Further, we use a variant which uses standard linearization instead of the transitivity inequalities and in another variant, we neglect some of the cutting planes. In our computational study we show that we clearly outperform these approaches as well as the current fastest exact approach for the 3-BFLP. Additionally, we consider star instances where one department has a high length and high weights to the remaining departments. The lengths and the weights of the remaining departments are smaller and the transport density of the remaining departments is set to $20 \%$, see Section A. It turns out that our approach works even better on the considered star instances and T-row and 3-Bay instances with up to 20 departments are solved within 4 hours. Besides the exact approaches, we obtain tight lower bounds for even larger T-row and 3-Bay instances, and thus we can evaluate the quality of heuristically determined layouts.

In Section $\mathrm{B}$ we present a new exact approach for the CCLP. Given a CCLP instance where all cells of type SRFLP have the same inter-cell distances, we merge two cells of type SRFLP. If the number of cells of type SRFLP is even, this result allows us to halve the number of cells of type SRFLP. Then, we enumerate over all assignments of the departments to the (merged) cells and solve several FC-CCLP. Thus, merging cells of type SRFLP reduces the number of cell assignments that have to be considered significantly. We add a dummy department with appropriate length and weights to each (merged) cell, and then the optimization problem in each (merged) cell can be solved independently. Considering cells of type DCFLP, one department may overlap with the dummy department and for the arising optimization problem we present a new MILP model. In our computational results we show that the MILP approach outperforms an associated enumerative approach. Furthermore, we introduce the XRFLP, which is a realistic extension of the 4-BFLP with a more complex path structure. We extend our main result to the 3-BFLP, the TRFLP, the 4-BFLP with positive inter-row distances and to the XRFLP with positive inter-cell distances. A computational study is presented to compare the optimal solution values of several facility layout problems to support the chief executive officer to choose a layout if a factory is built from the ground up.

In Section C a combined optimization-simulation approach for the DRFLP is presented. We extend the current fastest exact approach for the DRFLP in order to cover several aspects which are important in practice. The simulation can detect possible bottlenecks in the production, and then one can add further constraints to the DRFLP model. Considering departments of the same type, we reduce the number of distinguishable row assignments that have to be considered such that for the first time a double-row instance with 21 departments containing departments of the same type is solved to optimality. We compare our mathematical model with classic methods 
from factory planning and we reduce the sum of the weighted transport distances significantly, especially by using arbitrary positions for the warehouses.

In Section $D$ we develop the first combinatorial lower bounds for the optimal solution value of the DRFLP. Therefore, we interpret a subproblem of the DRFLP as a parallel identical machine scheduling problem. Furthermore, we combine these bounds with a new MILP model, which is indeed not a formulation for the DRFLP, to obtain even better lower bounds. We further improve the combinatorial lower bounds and the lower bounding MILP model for the DREFLP and the PROP. Even our combinatorial lower bounds are better than lower bounds received via some branch-and-cut algorithm within a given time limit of one hour for some DRFLP formulation from the literature. Additionally, our lower bounds can be calculated very fast and the combinatorial lower bounds can be adapted to the MRFLP.

In Section E we study the relationship of the MREFLP and the SREFLP and we prove that $v_{m}^{*} \leq \frac{v_{1}^{*}}{m}$ where $v_{m}^{*}$ denotes the optimal value of the MREFLP with $m \in \mathbb{N}$ rows. Given an equidistant single-row layout with objective value $v_{1}$, we derive a multi-row layout with objective value less than or equal to $\frac{v_{1}}{m}$. We can further improve such a layout by the inclusion of free spaces via a new ILP model which simplifies to some LP model. For the DREFLP we improve some of the best known upper bounds, in particular, for large instances and these layouts can be calculated in a few minutes. In comparison, the SDP approach of [15] has a time limit of three hours and includes a construction heuristic. Furthermore, we show that the optimal solution value of the SRFLP and the DRFLP for the same instance might be close. In particular, we prove that the following inequality holds $(n-1) v_{2}^{*} \leq(n-2) v_{1}^{*}$, where $v_{1}^{*}$, $v_{2}^{*}$ denote the optimal solution values of the two problems. Additionally, we present an example where this bound is tight. However, good or optimal single-row layouts can be a good starting point for deriving good double-row layouts. We present two heuristics for the DRFLP, which rely on the ideas used for the MREFLP and which can be calculated very fast. We obtain very small gaps to the best known upper bounds for instances with 30 and 40 departments, but derive these solutions much faster and for larger instances we outperform the heuristic of 33 .

All five publications concern solution approaches for facility layout problems and these approaches bear many similarities. The considered facility layout problems are very challenging, and thus we most often divide these problems into smaller subproblems. In Section A we set up an MILP model for the $s_{M}$-TRFLP instead of the TRFLP, $s_{M} \in[n]$, because this problem can be solved easier in practice and this allows us to exploit the crossroad structure nicely. In Section B (Section C) we enumerate over all distinguishable cell (row) assignments of the departments, and thus we consider layout problems where the assignment of the departments to the cells (rows) is known. Thus, in Section B we solve the optimization problem in each (merged) cell independently. In Section C this enables us to include several realistic extensions and to improve the approach for realistic instances with departments of the same type. In Section $\mathrm{D}$ we fix $i \in[n]$ opposite $j \in[n] \backslash\{i\}$, and then we are able to use a relation to a scheduling problem to calculate combinatorial lower bounds for the weighted distances of $i$ to departments $S \subseteq[n] \backslash\{i\}$. In our heuristic approaches in Section E we assume that the order of the departments in the same row is given by a single-row layout $\pi$, and hence it remains to determine the row assignment of the departments and their exact positions.

The publications in Sections $\mathrm{A} B$ are highly related since the same facility layout problems are considered. Considering instances known in the literature, the approach of Section $\mathrm{B}$ is able to solve larger 3-Bay instances than the approach of Section A. However, the approach of Section B is not applied on star instances while the approach of Section A for the TRFLP and the 3-BFLP performs significantly better on star instances. Additionally, the approach of Section $\mathrm{A}$ is able to calculate tight lower bounds for the TRFLP and the 3-BFLP. For the TRFLP the approach of Section $\mathrm{A}$ is for almost all instances from the literature faster than the approach of Section B. One advantage of the approach of Section $B$ is that several further facility layout problems are considered. It is interesting to extend the MILP approach of Section B to the 4-BFLP and the 
XRFLP as well.

One can combine the results of Sections $\mathrm{D} E$ such that one obtains lower and upper bounds for the DRFLP. Thus, the lower bounds allow us to evaluate the quality of heuristically determined layouts. Both publications have in common that we nicely exploit the grid structure of equidistant double-row layouts, and thus in both publications the results in the equidistant case are significantly better than in the general case.

Furthermore, it is possible to adapt the combinatorial lower bounds to facility layout problems considered in Sections $\mathrm{A} B$ and these combinatorial lower bounds can then be improved by a lower bounding MILP model. We expect that these lower bounds can be calculated faster than the lower bounds in Section A for the TRFLP and the 3-BFLP, and thus one can calculate lower bounds for even larger instances. However, one might obtain larger gaps between heuristically determined upper bounds and the derived lower bounds than in Section A. Additionally, one can adapt the heuristic approach of Section E to the TRFLP, see Section A.

Furthermore, the combined optimization-simulation approach of Section C can be used to evaluate the determined layouts in Sections $\mathrm{A} B$ and Section $\mathrm{E}$ in practice. Further key indicators can be taken into account which are not included in the mathematical models to guarantee a smooth production.

Our approaches in Sections $\mathrm{A}$ C concern realistic extensions of facility layout problems. In Sections $\mathrm{A} B$ we introduce the TRFLP and the XRFLP, and therefore we consider generalizations of the 3-BFLP and the 4-BFLP with a more complex crossroad structure, and thus a more complex path structure. So we continue the line of research of Section $\mathrm{C}$ to extend facility layout problems in order to include realistic aspects. Hence, these publications are closely related. In view of the results of Section B facility layout problems can be decomposed in a combination of the CCLP, the TRFLP, the XRFLP and the MRFLP. Thus, by solving the TRFLP and the XRFLP one can solve real-world facility layout problems with more complex crossroad structures. One of our goals is to support the chief executive officer to choose a layout when a factory is built from the ground up. Besides the optimal solution values the available area as well as the available material handling-systems play an enormous role to determine the layout. These two factors can determine, for instance, whether the layout has a multi-row or multi-bay structure (or both). Therefore, in this thesis several facility layout problems are studied and the corresponding optimal solution values are compared. 


\section{Conclusion and future work}

Facility layout problems have wide applications in factory planning and the layout of departments is one of the main influencing factors for the costs of the production within a factory [49, 104]. Without any restrictions on the layout, these problems are very challenging [87, 103]. For this reason we concentrated on special cases where the structure of the layout and the paths are restricted. In this thesis we mainly focused on the Combined Cell Layout Problem (CCLP) and its special case the Multi-Bay Facility Layout Problem (MBFLP) and the Multi-Row Facility Layout Problem (MRFLP). Further, we introduced the T-Row Facility Layout Problem (TRFLP) and the X-Row Facility Layout Problem (XRFLP) which are extensions of the MBFLP with three (3-BFLP) and four (4-BFLP) rows, respectively, with a more complex path structure.

Exact approaches for the CCLP and the MBFLP are rare in the literature, so we set up an mixed-integer linear programming (MILP) approach for the TRFLP and the 3-BFLP based on an extension of the betweenness variables which can now be equal to one even if the corresponding departments lie in distinct rows. Several inequalities were provided exploiting the crossroad structure of the layouts.

Further, we presented a new exact approach for the CCLP where we enumerated over all assignments of the departments to the cells. If all cells of type Single-Row Facility Layout Problem (SRFLP) have the same inter-cell distances, we merged two cells of type SRFLP, and thus we halved the number of cells of type SRFLP if the number of cells of type SRFLP is even. We extended this approach to the 3-BFLP, the 4-BFLP and the TRFLP with positive inter-row distances and to the XRFLP with positive inter-cell distances. A theoretical and computational study was presented to compare the optimal solution values of several facility layout problems to support the chief executive officer to choose a layout if a factory is built from the ground up.

We extended the current fastest approach for the Double-Row Facility Layout Problem (DRFLP) in order to include realistic aspects and we used a combined optimization-simulation approach for the DRFLP. The simulation was used to detect possible bottlenecks in the production, and then the model for the DRFLP could be further extended. We reduced the number of row assignments of the departments that have to be considered if there are departments of the same type and we were able for the first time to solve a double-row instance with 21 departments containing departments of the same type.

Currently, heuristics are the only way to determine solutions for large DRFLP instances. In order to evaluate the quality of heuristically determined layouts, we provided the first known non-trivial lower bounds on the optimal solution value of the DRFLP. Interpreting some subproblem of the DRFLP as a parallel identical machine scheduling problem, we computed combinatorial lower bounds for the DRFLP. We set up an MILP model, which is not a formulation for the DRFLP, to further strengthen our lower bounds. Additionally, we considered the Double-Row Equidistant Facility Layout Problem (DREFLP) and the Parallel Row Ordering Problem (PROP) and we adapted the combinatorial lower bounds and the MILP model to these cases.

We studied the relation between the optimal solution value of the Multi-Row Equidistant Facility Layout Problem (MREFLP) and the Single-Row Equidistant Facility Layout Problem (SREFLP) and proved that the optimal value of the MREFLP is less than or equal to the optimal value of the SREFLP divided by the number of rows in the MREFLP. We constructed equidistant double-row layouts satisfying this property and improved some of the best known upper bounds for the DREFLP in combination with a short running time. For the general SRFLP and the general DRFLP we provided a formula which shows that the corresponding optimal solution values might be close. Further, we presented an example where this formula is tight. However, we constructed two heuristics which derive good double-row layouts based on good or optimal single-row layouts in a very short time.

It remains for future work to consider a facility layout problem in the shape of an $U$ and to extend the Directed Circular Facility Layout Problem (DCFLP) such that the departments are arranged on a layout in the shape of a square. One can set up an MILP model for these 
two problems based on our extension of the betweenness variables in Section A. Further, it is interesting to set up an MILP model for the XRFLP and the MBFLP with at least four rows. Therefore, one can use our extension of the betweenness variables and one can adapt our MILP approach as well as our cutting planes, see Section A.

In our approach in Section B we considered cells of type SRFLP and DCFLP. A realistic extension is to consider cells of other types, e. g., cells of type DRFLP. One can extend the TRFLP such that it is allowed to arrange departments on both sides of the path. For this new problem, one can adapt the MILP approach of Section $\mathrm{A}$ or one can use the approach of Section $\mathrm{B}$ and decompose this problem into two cells of type DRFLP with additional constraints to ensure that the departments at the crossroad do not overlap. A similar extension for the XRFLP is of practical interest as well. Furthermore, a realistic extension is to consider two-dimensional departments with varying height. Then, it might be harder to ensure in the XRFLP that departments in cell 2 and cell 3 do not overlap.

An interesting extension of the MBFLP is the case where inter-row material handling-systems are fixed at the left and at the right border of the layout. In this case, one can add two dummy departments to each row and the idea of merging cells of type SRFLP, see Section B, might be useful. Additionally, the CCLP can be extended such that in cells of type DCFLP the loading and unloading stations might be on distinct positions.

From a practical point of view, it is interesting to extend the betweenness model for the SRFLP such that it is capable of more realistic extensions such as individual in- and output positions of the departments and to take certain clearance conditions into account.

The rearrangement of the departments in a factory is expensive, so the departments are arranged for several years. Thus, the inclusion of robustness aspect is important because the production program and hence the transport weights between the departments might change, and hence it seems to be fruitful to continue the line of research of [61.

\section{References}

[1] Panos Afentakis. A loop layout design problem for flexible manufacturing systems. International Journal of Flexible Manufacturing Systems, 1(2):175-196, 1989.

[2] Hannu Ahonen, Arlindo Gomes de Alvarenga, and André R. S. Amaral. Simulated annealing and tabu search approaches for the corridor allocation problem. European Journal of Operational Research, 232(1):221-233, 2014.

[3] André R. S. Amaral. On the exact solution of a facility layout problem. European Journal of Operational Research, 173(2):508-518, 2006.

[4] André R. S. Amaral. An exact approach to the one-dimensional facility layout problem. Operations Research, 56(4):1026-1033, 2008.

[5] André R. S. Amaral. A new lower bound for the single row facility layout problem. Discrete Applied Mathematics, 157(1):183-190, 2009.

[6] André R. S. Amaral. On duplex arrangement of vertices. Technical report, Departamento de Informática, Universidade Federal do Espírito Santo (UFES), Brazil, 2011.

[7] André R. S. Amaral. The corridor allocation problem. Computers $\&$ Operations Research, 39(12):3325-3330, 2012.

[8] André R. S. Amaral. Optimal solutions for the double row layout problem. Optimization Letters, 7(2):407-413, 2013.

[9] André R. S. Amaral. A parallel ordering problem in facilities layout. Computers $\&$ Operations Research, 40(12):2930-2939, 2013. 
[10] André R. S. Amaral. A mixed-integer programming formulation of the double row layout problem based on a linear extension of a partial order. Technical report, Graduate School of Computer Science (PPGI), Federal University of Espírito Santo (UFES), Brazil, 2018. Available at http://www.optimization-online.org/DB_HTML/2020/04/7739.html.

[11] André R. S. Amaral. A mixed-integer programming formulation for the double row layout of machines in manufacturing systems. International Journal of Production Research, 57(1):34-47, 2019.

[12] André R. S. Amaral. A heuristic approach for the double row layout problem. Annals of Operations Research, pages 1-36, 2020.

[13] André R. S. Amaral and Adam N. Letchford. A polyhedral approach to the single row facility layout problem. Mathematical Programming, 141(1-2):453-477, 2013.

[14] Miguel F. Anjos, Anja Fischer, and Philipp Hungerländer. Solution approaches for the double-row equidistant facility layout problem. In Marco Lübbecke, Arie Koster, Peter Letmathe, Reinhard Madlener, Britta Peis, and Grit Walther, editors, Operations Research Proceedings 2014, pages 17-23, Cham, 2016. Springer International Publishing.

[15] Miguel F. Anjos, Anja Fischer, and Philipp Hungerländer. Improved exact approaches for row layout problems with departments of equal length. European Journal of Operational Research, 270(2):514-529, 2018.

[16] Miguel F. Anjos, Philipp Hungerländer, and Kerstin Maier. An integer linear programming approach for the combined cell layout problem. In 2018 IEEE International Conference on Industrial Engineering and Engineering Management (IEEM), pages 705-709. IEEE, 2018.

[17] Miguel F. Anjos, Andrew Kennings, and Anthony Vannelli. A semidefinite optimization approach for the single-row layout problem with unequal dimensions. Discrete Optimization, 2(2):113-122, 2005.

[18] Miguel F. Anjos and Anthony Vannelli. Computing globally optimal solutions for single-row layout problems using semidefinite programming and cutting planes. INFORMS Journal On Computing, 20(4):611-617, 2008.

[19] Miguel F. Anjos and Manuel V. C. Vieira. Mathematical optimization approach for facility layout on several rows. Optimization Letters, pages 1-15, 2020.

[20] Miguel F. Anjos and Manuel V.C. Vieira. Mathematical optimization approaches for facility layout problems: The state-of-the-art and future research directions. European Journal of Operational Research, 261(1):1-16, 2017.

[21] Miguel F. Anjos and Ginger Yen. Provably near-optimal solutions for very large single-row facility layout problems. Optimization Methods and Software, 24(4):805-817, 2009.

[22] Maria M. Azevedo, José A. Crispim, and Jorge P. de Sousa. A dynamic multi-objective approach for the reconfigurable multi-facility layout problem. Journal of manufacturing systems, 42:140-152, 2017.

[23] Thierry Blu, Philippe Thévenaz, and Michael Unser. Linear interpolation revitalized. IEEE Transactions on Image Processing, 13(5):710-719, 2004.

[24] Uwe Bracht, Dieter Geckler, and Sigrid Wenzel. Digitale Fabrik: Methoden und Praxisbeispiele. Springer-Verlag, 2011. 
[25] Timothy W. Butler, Kirk R. Karwan, James R. Sweigart, and Gary R. Reeves. An integrative model-based approach to hospital layout. IIE transactions, 24(2):144-152, 1992.

[26] Gino Cardarelli and Pacifico M. Pelagagge. Simulation tool for design and management optimization of automated interbay material handling and storage systems for large wafer fab. IEEE Transactions on Semiconductor Manufacturing, 8(1):44-49, 1995.

[27] Ignacio Castillo and Brett A. Peters. Integrating design and production planning considerations in multi-bay manufacturing facility layout. European Journal of Operational Research, 157(3):671-687, 2004.

[28] Junjae Chae and Brett A. Peters. Layout design of multi-bay facilities with limited bay flexibility. Journal of manufacturing systems, 25(1):1-11, 2006.

[29] Junjae Chae and Amelia C. Regan. A mixed integer programming model for a double row layout problem. Computers E Industrial Engineering, 140:106244, 2020.

[30] Mei-Shiang Chang and Hsin-Yi Lin. A flexible bay structure representation and ant colony system for unequal area facility layout problems. In Proceedings of the World Congress on Engineering, volume 3, pages 1-6, 2012.

[31] Mei-Shiang Chang and Hsin-Yi Lin. An immunized ant colony system algorithm to solve unequal area facility layout problems using flexible bay structure. In Proceedings of the Institute of Industrial Engineers Asian Conference 2013, pages 9-17. Springer, 2013.

[32] Jaewoo Chung and Yunjung Suh. Analysis on the pier-type material flow pattern for facility layout applications. Journal of Applied Sciences, 14(3):237-244, 2014.

[33] Jaewoo Chung and J.M.A. Tanchoco. The double row layout problem. International Journal of Production Research, 48(3):709-727, 2010.

[34] Gildásio L. Cravo and André R. S. Amaral. A grasp algorithm for solving large-scale single row facility layout problems. Computers \& Operations Research, 106:49-61, 2019.

[35] George B. Dantzig, Delbert R. Fulkerson, and Selmer Johnson. Solution of a large scale traveling salesman problem. Journal of the Operations Research Society of America, 2(4):393-410, 1954.

[36] Dilip Datta, André R. S. Amaral, and José Rui Figueira. Single row facility layout problem using a permutation-based genetic algorithm. European Journal of Operational Research, 213(2):388-394, 2011.

[37] Hamid Davoudpour, Amir A. Jaafari, and Leila N. Farahani. Facility layout problems using bays: A survey. AIP Conference Proceedings, 1247(1):485-491, 2010.

[38] Amine Drira, Henri Pierrval, and Sonia Hajri-Gabouj. Facility layout problems: A survey. Annual Reviews in Control, 31(2):255-267, 2007.

[39] Jack Edmonds. Matroids and the greedy algorithm. Mathematical Programming, 1(1):127$136,1971$.

[40] Alwalid N. Elshafei. Hospital layout as a quadratic assignment problem. Journal of the Operational Research Society, 28(1):167-179, 1977.

[41] Anja Fischer, Frank Fischer, and Philipp Hungerländer. A new exact approach to the spacefree double row layout problem. In Operations Research Proceedings 2015, Selected Papers of the International Conference of the German, Austrian and Swiss Operations Research Societies (GOR, ÖGOR, SVOR/ASRO), University of Vienna, Austria, September 1-4, 2015., pages 125-130, 2015. 
[42] Anja Fischer, Frank Fischer, and Philipp Hungerländer. New exact approaches to row layout problems. Mathematical Programming Computation, 11(4):703-754, 2019.

[43] Michael Randolph Garey, David S. Johnson, and Larry J. Stockmeyer. Some simplified NP-complete problems. In STOC '74: Proceedings of the sixth annual ACM symposium on Theory of computing, pages 47-63. ACM, 1974.

[44] Martin Grötschel, Michael Jünger, and Gerhard Reinelt. A cutting plane algorithm for the linear ordering problem. Operations Reasearch, 32(6):1195-1220, 1984.

[45] Martin Grötschel, Michael Jünger, and Gerhard Reinelt. Facets of the linear ordering polytope. Mathematical Programming, 33(1):43-60, 1985.

[46] Jian Guan and Geng Lin. Hybridizing variable neighborhood search with ant colony optimization for solving the single row facility layout problem. European Journal of Operational Research, 248(3):899-909, 2016.

[47] Jian Guan, Geng Lin, Hui-Bin Feng, and Zhi-Qiang Ruan. A decomposition-based algorithm for the double row layout problem. Applied Mathematical Modelling, 77:963-979, 2020.

[48] Peter M. Hahn and Jakob Krarup. A hospital facility layout problem finally solved. Journal of Intelligent Manufacturing, 12(5-6):487-496, 2001.

[49] Mohsen M. D. Hassan. Machine layout problem in modern manufacturing facilities. International Journal of Production Research, 32(11):2559-2584, 1994.

[50] Sunderesh S. Heragu and Andrew Kusiak. Machine Layout Problem in Flexible Manufacturing Systems. Operations Research, 36(2):258-268, 1988.

[51] Hasan Hosseini-Nasab, Sepideh Fereidouni, Seyyed M. T. F. Ghomi, and Mohammad B. Fakhrzad. Classification of facility layout problems: a review study. The International Journal of Advanced Manufacturing Technology, 94(1-4):957-977, 2018.

[52] Philipp Hungerländer. A semidefinite optimization approach to the parallel row ordering problem. Technical report, Alpen-Adria Universität Klagenfurt, Mathematics, Optimization Group, TR-ARUK-M-O-14-05, 2014.

[53] Philipp Hungerländer. Single-row equidistant facility layout as a special case of single-row facility layout. International Journal of Production Research, 52(5):1257-1268, 2014.

[54] Philipp Hungerländer and Miguel F. Anjos. A semidefinite optimization approach to space-free multi-row facility layout. Cahiers du GERAD G-2012-03, GERAD, Montreal, QC, Canada, 2012.

[55] Philipp Hungerländer and Miguel F. Anjos. An exact approach for the combined cell layout problem. In Stefan Helber, Michael Breitner, Daniel Rösch, Cornelia Schön, JohannMatthias Graf von der Schulenburg, Philipp Sibbertsen, Marc Steinbach, Stefan Weber, and Anja Wolter, editors, Operations Research Proceedings 2012, pages 275-281, Cham, 2014. Springer International Publishing.

[56] Philipp Hungerländer and Miguel F. Anjos. A semidefinite optimization-based approach for global optimization of multi-row facility layout. European Journal of Operational Research, 245(1):46-61, 2015.

[57] Philipp Hungerländer, Kerstin Maier, Jörg Pöcher, and Christian Truden. On a new modelling approach for circular layouts and its practical advantages. In 2017 IEEE International Conference on Industrial Engineering and Engineering Management (IEEM), pages 1581-1585, Dec 2017. 
[58] Philipp Hungerländer and Franz Rendl. A computational study and survey of methods for the single-row facility layout problem. Computational Optimization and Applications, $55(1): 1-20,2013$.

[59] Philipp Hungerländer and Franz Rendl. Semidefinite relaxations of ordering problems. Mathematical Programming, 140(1):77-97, 2013.

[60] Philipp Hungerländer, Kerstin Maier, Veronika Pachatz, and Christian Truden. Exact and heuristic approaches for a new circular layout problem. SN Applied Sciences, 2(6):1035, May 2020.

[61] Niloufar Izadinia, Kourosh Eshghi, and Mohammad Hassan Salmani. A robust model for multi-floor layout problem. Computers \& Industrial Engineering, 78:127-134, 2014.

[62] Tsuyoshi Kawaguchi and Seiki Kyan. Worst case bound of an lrf schedule for the mean weighted flow-time problem. SIAM Journal on Computing, 15(4):1119-1129, 1986.

[63] Birgit Keller. Construction heuristics for the single row layout problem with machinespanning clearances. INFOR: Information Systems and Operational Research, 57(1):32-55, 2019.

[64] Birgit Keller and Udo Buscher. Single row layout models. European Journal of Operational Research, 245(3):629-644, 2015.

[65] Hans Kettner, Jürgen Schmidt, and Hans-Robert Greim. Leitfaden der systematischen Fabrikplanung. Hanser München, 1984.

[66] Abdullah Konak, Sadan Kulturel-Konak, Bryan A. Norman, and Alice E. Smith. A new mixed integer programming formulation for facility layout design using flexible bays. Operations Research Letters, 34(6):660-672, 2006.

[67] Tjalling C. Koopmans and Martin Beckmann. Assignment problems and the location of economic activities. Econometrica: journal of the Econometric Society, 25(1):53-76, 1957.

[68] Ravi Kothari and Diptesh Ghosh. Population heuristics for the corridor allocation problem. Technical report, Working paper / Indian Institute of Management Ahmedabad, 2012.

[69] Ravi Kothari and Diptesh Ghosh. The single row facility layout problem: state of the art. OPSEARCH, 49(4):442-462, 2012.

[70] Ravi Kothari and Diptesh Ghosh. Insertion based Lin-Kernighan heuristic for single row facility layout. Computers \& Operations Research, 40(1):129 - 136, 2013.

[71] Ravi Kothari and Diptesh Ghosh. Tabu search for the single row facility layout problem using exhaustive 2-opt and insertion neighborhoods. European Journal of Operational Research, 224(1):93-100, 2013.

[72] Ravi Kothari and Diptesh Ghosh. An efficient genetic algorithm for single row facility layout. Optimization Letters, 8(2):679-690, 2014.

[73] Ravi Kothari and Diptesh Ghosh. A scatter search algorithm for the single row facility layout problem. Journal of Heuristics, 20(2):125-142, 2014.

[74] Panagiotis Kouvelis, Wen-Chyuan Chiang, and Ali S. Kiran. A survey of layout issues in flexible manufacturing systems. Omega, 20(3):375-390, 1992. 
[75] Sadan Kulturel-Konak and Abdullah Konak. A new relaxed flexible bay structure representation and particle swarm optimization for the unequal area facility layout problem. Engineering Optimization, 43(12):1263-1287, 2011.

[76] Sadan Kulturel-Konak and Abdullah Konak. Unequal area flexible bay facility layout using ant colony optimisation. International Journal of Production Research, 49(7):1877-1902, 2011.

[77] Eugene L. Lawler. The quadratic assignment problem. Management science, 9(4):586-599, 1963.

[78] Chung-Yee Lee and Reha Uzsoy. A new dynamic programming algorithm for the parallel machines total weighted completion time problem. Operations research letters, 11(2):73-75, 1992.

[79] Silu Liu, Zeqiang Zhang, Chao Guan, Lixia Zhu, Min Zhang, and Peng Guo. An improved fireworks algorithm for the constrained single-row facility layout problem. International Journal of Production Research, pages 1-19, 2020.

[80] Eliane Maria Loiola, Nair Maria Maia de Abreu, Paulo Oswaldo Boaventura-Netto, Peter Hahn, and Tania Querido. A survey for the quadratic assignment problem. European Journal of Operational Research, 176(2):657-690, 2007.

[81] László Lovász and Alexander Schrijver. Cones of matrices and set-functions and 0-1 optimization. SIAM Journal on Optimization, 1:166-190, 1991.

[82] Mansoureh Maadi, Mohammad Javidnia, and Rasoul Jamshidi. Two strategies based on meta-heuristic algorithms for parallel row ordering problem (PROP). Iranian Journal of Management Studies, 10(2):467-498, 2017.

[83] Enrico Malaguti and Paolo Toth. A survey on vertex coloring problems. International transactions in operational research, 17(1):1-34, 2010.

[84] Laura Manzke, Birgit Keller, and Udo Buscher. An artificial bee colony algorithm to solve the single row layout problem with clearances. In International Conference on Information Systems Architecture and Technology, pages 285-294. Springer, 2017.

[85] Mostafa Mazinani, Mostafa Abedzadeh, and Navid Mohebali. Dynamic facility layout problem based on flexible bay structure and solving by genetic algorithm. The International Journal of Advanced Manufacturing Technology, 65(5-8):929-943, 2013.

[86] Russell D. Meller. The multi-bay manufacturing facility layout problem. International Journal of Production Research, 35(5):1229-1237, 1997.

[87] Russell D. Meller, Weiping Chen, and Hanif D. Sherali. Applying the sequence-pair representation to optimal facility layout designs. Operations Research Letters, 35(5):651$659,2007$.

[88] Isabel Méndez-Díaz and Paula Zabala. A cutting plane algorithm for graph coloring. Discrete Applied Mathematics, 156(2):159-179, 2008.

[89] Chase C. Murray, Alice E. Smith, and Zeqiang Zhang. An efficient local search heuristic for the double row layout problem with asymmetric material flow. International Journal of Production Research, 51(20):6129-6139, 2013.

[90] Gintaras Palubeckis. A branch-and-bound algorithm for the single-row equidistant facility layout problem. OR Spectrum, 34(1):1-21, 2012. 
[91] Gintaras Palubeckis. Fast local search for single row facility layout. European Journal of Operational Research, 264(3):800-814, 2015.

[92] Gintaras Palubeckis. Fast simulated annealing for single-row equidistant facility layout. Applied Mathematics and Computation, 263:287-301, 2015.

[93] Gintaras Palubeckis. Single row facility layout using multi-start simulated annealing. Computers $\&$ Industrial Engineering, 103:1-16, 2017.

[94] Jean-Claude Picard and Maurice Queyranne. On the one-dimensional space allocation problem. Operations Research, 29(2):371-391, 1981.

[95] Uwe Prêt. Komplexes fallbeispiel teilefertigungs- und montageprojekt "schneckengetriebeproduktion". Available at (last download on 20.11.2020): http://www.uwe-pret.de/ getriebe.pdf, 2017.

[96] Tobias Rooks. Rechnergestützte Simulationsmodellgenerierung zur dynamischen Absicherung der Montagelogistikplanung bei der Fahrzeugneutypplanung im Rahmen der digitalen Fabrik. Shaker, 2010.

[97] Soroush Safarzadeh and Hamidreza Koosha. Solving an extended multi-row facility layout problem with fuzzy clearances using ga. Applied Soft Computing, 61:819-831, 2017.

[98] Hamed Samarghandi and Kourosh Eshghi. An efficient tabu algorithm for the single row facility layout problem. European Journal of Operational Research, 205(1):98-105, 2010.

[99] Sujeevraja Sanjeevi and Kiavash Kianfar. A polyhedral study of triplet formulation for single row facility layout problem. Discrete Applied Mathematics, 158(16):1861-1867, 2010.

[100] Hans Schmigalla. Fabrikplanung: Begriffe und Zusammenhänge. Hanser Verlag, 1995.

[101] Leonardo D. Secchin and André R. S. Amaral. An improved mixed-integer programming model for the double row layout of facilities. Optimization Letters, 13(1):193-199, Feb 2019 .

[102] Leonardo D. Secchin and André R. S. Amaral. An improved mixed-integer programming model for the double row layout of facilities. Optimization Letters, 13(1):193-199, 2019.

[103] Hanif D. Sherali, Barbara M. P. Fraticelli, and Russell D. Meller. Enhanced model formulations for optimal facility layout. Operations Research, 51(4):629-644, 2003.

[104] James A. Tompkins, John A. White, Yavuz A. Bozer, and J. M. A. Tanchoco. Facilities planning. John Wiley \& Sons, 2010.

[105] Shengli Wang, Xingquan Zuo, Xueqing Liu, Xinchao Zhao, and Jianqiang Li. Solving dynamic double row layout problem via combining simulated annealing and mathematical programming. Applied Soft Computing, 37:303-310, 2015.

[106] Kuan Y. Wong and Komarudin. Solving facility layout problems using flexible bay structure representation and ant system algorithm. Expert Systems with Applications, 37(7):5523$5527,2010$.

[107] Taho Yang and Brett A. Peters. A spine layout design method for semiconductor fabrication facilities containing automated material-handling systems. International Journal of Operations $\&$ Production Management, 17(5):490-501, 1997. 
[108] Xuhong Yang, Wenming Cheng, Alice E. Smith, and André R. S. Amaral. An improved model for the parallel row ordering problem. Journal of the Operational Research Society, 71(3):475-490, 2020.

[109] Junfang Yu and Bhaba R. Sarker. Directional decomposition heuristic for a linear machinecell location problem. European Journal of Operational Research, 149(1):142-184, 2003.

[110] Zeqiang Zhang and Chase C. Murray. A corrected formulation for the double row layout problem. International Journal of Production Research, 50(15):4220-4223, 2012.

[111] Xingquan Zuo, Shubing Gao, Mengchu Zhou, Xin Yang, and Xinchao Zhao. A three-stage approach to a multirow parallel machine layout problem. IEEE Transactions on Automation Science and Engineering, 16(1):433-447, 2019.

[112] Xingquan Zuo, Chase C. Murray, and Alice E. Smith. Solving an extended double row layout problem using multiobjective tabu search and linear programming. IEEE Transactions on Automation Science and Engineering, 11(4):1122-1132, 2014.

[113] Xingquan Zuo, Chase C. Murray, and Alice E. Smith. Sharing clearances to improve machine layout. International Journal of Production Research, 54(14):4272-4285, 2016. 


\section{Appendix}

A. A mixed-integer linear programming approach for the T-row and the multi-bay facility layout problem

Author: Mirko Dahlbeck

resubmitted to European Journal of Operational Research, 2020. 


\title{
A mixed-integer linear programming approach for the T-row and the multi-bay facility layout problem
}

\author{
Mirko Dahlbeck $\otimes^{*}$
}

We introduce a new facility layout problem, the so-called T-Row Facility Layout Problem (TRFLP). The TRFLP consists of a set of one-dimensional departments with pairwise transport weights between them and two orthogonal rows which form a $\mathrm{T}$ such that departments in different rows cannot overlap. The aim is to find a non-overlapping assignment of the departments to the rows such that the sum of the weighted center-to-center distances measured rectilinear directions is minimized. The TRFLP is a generalization of the well-known Multi-Bay Facility Layout Problem with three rows (3-BFLP). Both problems, the TRFLP and the 3-BFLP, have wide applications, e.g., factory planning, semiconductor fabrication and arranging rooms in hospitals.

In this work we present a mixed-integer linear programming approach for the TRFLP and the 3-BFLP based on an extension of the well-known betweenness variables which now can be equal to one if the corresponding departments lie in different rows. One advantage of our formulation is the calculation of inter-row distances without big-M-type constraints. We provide cutting planes exploiting the crossroad structure in the layout, and hence T-row (3-Bay) instances with up to 18 (17) departments are solved to optimality in less than 7 hours. The best known approach for the 3-BFLP is clearly outperformed. Additionally, tight lower bounds for larger instances are calculated to evaluate our heuristically determined layouts.

Key words. Facilities planning and design; Mixed-Integer Linear Programming; Row Layout Problem

${ }^{*}$ TU Dortmund University, Faculty of Business and Economics, Vogelpothsweg 87, D-44227 Dortmund; GeorgAugust-Universität Göttingen, Institute for Numerical and Applied Mathematics, Lotzestr. 16-18, D-37083 Göttingen, mirko.dahlbeck@tu-dortmund.de 


\section{Introduction}

We introduce a new facility layout problem, the so-called T-Row Facility Layout Problem (TRFLP). We are given a set of departments $[n]:=\{1, \ldots, n\}, n \in \mathbb{N}$, with lengths $\ell_{i}, i \in[n]$, and symmetric pairwise weights $w_{i j}=w_{j i}, i, j \in[n], i<j$, and two orthogonal rows $\mathcal{R}:=\{1,2\}$ which form a $\mathrm{T}$ such that departments in different rows cannot overlap. One looks for an assignment $r:[n] \rightarrow \mathcal{R}$ of the departments to the rows $\mathcal{R}$ minimizing the weighted sum of the center-to-center distances between the departments measured in rectilinear directions such that departments in the same row do not overlap. By measuring the distances between departments in distinct rows, one has to take the width of the path $w_{\text {path }}^{T} \in \mathbb{R}_{\geq 0}$ into account.

The horizontal row is denoted by row 1 and the vertical row by row 2 . We use distance variables $d_{i j}=d_{j i}, i, j \in[n], i<j$, to measure the center-to-center distances measured in rectilinear directions between $i$ and $j$. Let $p_{M} \in \mathbb{R}$ denote the position in row 1 measured from a fixed left border which is directly opposite row 2 . If $w_{\text {path }}^{T}=0$, then $p_{M}$ is the point of intersection of row 1 and row 2. We look for a vector $r \in \mathcal{R}^{n}$ of the assignment of the departments to the rows and for a vector $p \in \mathbb{R}^{n}$ of the center positions of the departments measured from a fixed left (upper) border if $r_{i}=1\left(r_{i}=2\right), i \in[n]$, such that the following optimization problem is solved to optimality

$$
\begin{aligned}
& \min _{r \in \mathcal{R}^{n}, p \in \mathbb{R}^{n}} \sum_{\substack{i, j \in[n] \\
i<j}} w_{i j} d_{i j} \\
& \text { s.t. }\left|p_{i}-p_{j}\right| \geq \frac{\ell_{i}+\ell_{j}}{2}, \\
& d_{i j}=\left|p_{i}-p_{j}\right|, \\
& d_{i j}=\left|p_{i}-p_{M}\right|+p_{j}+w_{\text {path }}^{T}, \\
& i, j \in[n], i<j, r_{i}=r_{j}, \\
& i, j \in[n], i<j, r_{i}=r_{j}, \\
& i, j \in[n], r_{i}=1, r_{j}=2 .
\end{aligned}
$$

In this model for the TRFLP one might obtain $p_{i} \leq 0$ for some $i \in[n]$, depending on the position $p_{M}$. Setting $\mathcal{R}:=[m], m \in \mathbb{N}$, as the set of $m$ non-overlapping parallel rows and adapting the distance calculation in equations (1) with $1 \leq w_{\text {path }}^{B} \in \mathbb{R}$, such that

$$
d_{i j}=p_{i}+p_{j}+\left|r_{i}-r_{j}\right| w_{\text {path }}^{B}, \quad \quad i, j \in[n], i<j, r_{i} \neq r_{j},
$$

where $p_{i}, i \in[n]$, denotes the position of the center of $i$, measured from a fixed left border such that all departments are to the right of this border, then we obtain the Multi-Bay Facility Layout Problem (MBFLP) with $m$ rows $^{1}$, see, e.g., $[43,63,67,79]$. The distance calculation can be interpreted in the following way. There are inner-row and inter-row material handling-systems, whereby the inter-row material handling-system, e.g., an overhead bridge crane [23, 67], is fixed at the left border. The rows might be separated by equipment, some free space for maintenance or passageways [25]. The inter-row transport is more costly than the inner-row transport, because two separate material handling systems have to be coordinated, the transfer mechanism is costly (typically a larger capacity crane is used) and at this point the most delay and damages occur, see $[25,67]$. Calculating the distances between departments in row 1 (row 3 ) and row 2, we take $w_{\text {path }}^{B}$ into account. Measuring the distances between departments in row 1 and row 3 , we cross the path twice, so we take $2 \cdot w_{\text {path }}^{B}$ into account. The departments are given as one-dimensional objects, so we assume implicitly that the height of the departments equals one. Therefore, we assume $w_{\text {path }}^{B} \geq 1$ such that the height of the departments is included in the width of the path. We refer to Figure $1 \mathrm{~b}$ for an illustration. If $w_{\text {path }}^{B}>1$, then the width of the path is taken into account, and otherwise the width of the path is neglected. Therefore, we set $w_{\text {path }}^{T}+1=w_{\text {path }}^{B}$ in the following.

\footnotetext{
${ }^{1}$ In order to ensure a consistent terminology we use the term row. In the literature [67] usually the term bay is used.
} 
In Section 4 we study the relation between the TRFLP and the MBFLP with $m=3$ rows denoted by (3-BFLP). It turns out that the TRFLP is a generalization of the 3-BFLP and the optimal value of the TRFLP is less than or equal to the optimal value of the 3-BFLP if $w_{\text {path }}^{T} \leq w_{\text {path }}^{B}$.

The MBFLP and the TRFLP are of special interest in practice because these layouts are commonly used layouts in industry such as heavy manufacturing, e.g., steel production and bridge crane manufacturing, and semiconductor fabrication [63, 67, 80]. Many real-world factory layouts implicitly use these layout structures, see, e. g., [23, 63], and factory layout problems can often be decomposed, see $[36,67]$. Therefore, real factory layouts often reduce to a combination of bay layouts, T-row layouts and further layouts. In real factory layout problems often a complex path structure arises containing several crossroads. One motivation of this paper is to study a facility layout problem with a complex path structure and the path structure in the TRFLP is more complex than in the 3-BFLP, because of the more complex crossroad structure. Hence, we extend the 3-BFLP in order to include realistic aspects. A second advantage of the TRFLP in comparison to the MBFLP is that the inter-row material handling-system is not fixed at the left border, and hence the weighted transport distances can be significantly smaller. The layout of the departments highly influences the costs of the production, see, e. g., [20, 48, 77].

Additionally, the TRFLP can be applied for arranging shops in shopping malls where two levels are separated by a moving stairway or an elevator. Further, the MBFLP and the TRFLP are relevant for arranging rooms in hospitals where often only one side of a corridor has windows [72]. The task is to assign the rooms of the patients along the window side such that the sum of the traveled distances between the rooms of the patients and nurses is minimized. In the TRFLP the moving stairway or the elevator is not fixed at the border of the level (row), and hence the sum of the traveled distances can be reduced in comparison to the 3-BFLP.

The special case of the MBFLP with $m=1$ is called Single-Row Facility Layout Problem (SRFLP) and it is known to be $\mathcal{N} \mathcal{P}$-hard [3, 44, 72]. Thus, the MBFLP and the TRFLP are $\mathcal{N} \mathcal{P}$-hard as well. Besides its application in factory planning the SRFLP arises in the arrangement of rooms in hospitals $[21,40,47]$ and the arrangement of books on a shelf [6]. The SRFLP is widely studied $[1,2,55,58]$.

The MBFLP where the distance between the rows equals a constant $c \in \mathbb{R}_{+}$is called Pier-Type Material Flow Pattern (PMFP) and has application in designing the layout of cross docking warehouses, e.g., there are some dock departments for receiving incoming materials and the other departments are used for direct shipping of shipping supplies [29].

We illustrate the distance calculation of the SRFLP, the 3-BFLP and the TRFLP in the following example. We set $w_{\text {path }}^{T}+1=w_{\text {path }}^{B}$ and thus the height of the departments in the 3-BFLP is taken into account.

Example 1. We consider an instance with $n=5$ departments with lengths $\ell_{1}=\ell_{3}=\ell_{4}=4, \ell_{2}=$ $5, \ell_{5}=2$, and non-zero weights $w_{12}=w_{23}=w_{24}=w_{34}=w_{45}=1, w_{14}=3$ with $w_{\text {path }}^{T}=0$ and $w_{\text {path }}^{B}=1$. In Figure 1 optimal layouts of the SRFLP, the 3-BFLP and the TRFLP are illustrated:

a) An optimal single-row layout is illustrated in Figure 1a with an objective value of

$$
1 \cdot 4.5+3 \cdot 4+1 \cdot 4.5+1 \cdot 8.5+1 \cdot 13+1 \cdot 3=45.5
$$

b) An optimal 3-Bay layout is depicted in Figure 1 with an objective value of

$$
1 \cdot 6.5+3 \cdot 5+1 \cdot 4.5+1 \cdot 5.5+1 \cdot 10+1 \cdot 3=44.5
$$

c) An optimal T-row layout is shown in Figure 1c with an objective value of

$$
1 \cdot 6.5+3 \cdot 2+1 \cdot 4.5+1 \cdot 4.5+1 \cdot 9+1 \cdot 3=33.5 \text {. }
$$




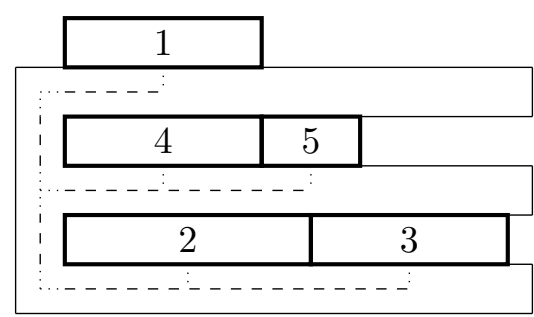

(b) An optimal 3-Bay layout with objective value 44.5.

(a) An optimal single-row layout with objective value 45.5.

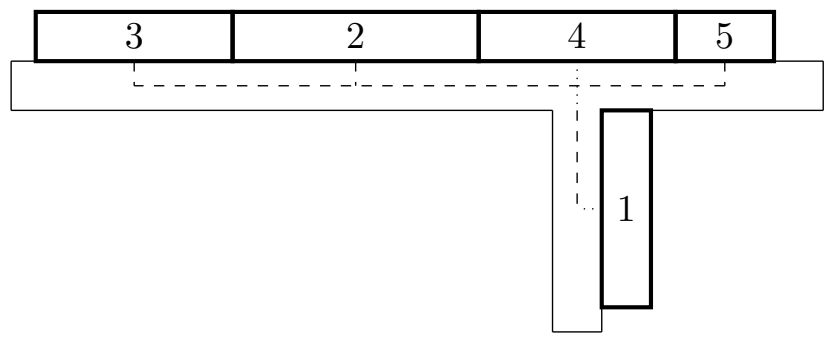

(c) An optimal T-row layout with objective value 33.5 where the center of 4 is on position $p_{M}$ in row 1.

Figure 1: We are given an instance with $n=5$ departments with lengths $\ell_{1}=\ell_{3}=\ell_{4}=4, \ell_{2}=$ $5, \ell_{5}=2$, and non-zero weights $w_{12}=w_{23}=w_{24}=w_{34}=w_{45}=1, w_{14}=3$ with $w_{\text {path }}^{T}=0, w_{\text {path }}^{B}=1$. We illustrate optimal layouts for the SRFLP, the 3-BFLP and the TRFLP where the center of 4 is on position $p_{M}$ in row 1 in the illustrated T-row layout.

In the following example we consider three optimal T-row layouts with different departments on position $p_{M}$ in row 1 . Note that, if we consider only the departments in an optimal T-row layout, then such an optimal layout might have the shape of an L.

Example 2. We are given a T-row instance with $n=5$ departments with lengths $\ell_{1}=\ell_{2}=$ $5, \ell_{3}=\ell_{5}=3, \ell_{4}=2$, and non-zero weights $w_{12}=w_{13}=3, w_{23}=w_{34}=w_{35}=2, w_{24}=w_{45}=$ $1, w_{\text {path }}^{T}=1$, and $p_{M}=15$. We obtain an optimal layout where the center of 1 (3) is on position $p_{M}$ in row 1, see Figure 2a (2c). In the optimal T-row layout illustrated in Figure $2 b$ the center positions of the departments are the following: $p_{1}=16.5, p_{2}=2.5, p_{3}=12.5, p_{4}=10, p_{5}=7.5$, i. e., $p_{i} \neq p_{M}, i \in[5]$.

a) An optimal T-row layout is illustrated in Figure 2a with an objective value of

$$
3 \cdot 3.5+3 \cdot 4+2 \cdot 7.5+1 \cdot 10+2 \cdot 2.5+2 \cdot 5+1 \cdot 2.5=65
$$

b) An optimal T-row layout is depicted in Figure $2 b$ with an objective value of

$$
3 \cdot 5+3 \cdot 4+2 \cdot 6+1 \cdot 8.5+2 \cdot 2.5+2 \cdot 5+1 \cdot 2.5=65
$$

c) An optimal T-row layout is shown in Figure 2c with an objective value of

$$
3 \cdot 7.5+3 \cdot 4+2 \cdot 3.5+1 \cdot 6+2 \cdot 2.5+2 \cdot 5+1 \cdot 2.5=65 .
$$

\subsection{Literature Review}

Almost all exact approaches for the SRFLP are either based on integer linear programming (ILP), see, e.g., $[1,2,3,11]$, or semidefinite programming (SDP), see $[15,16,18,55,56]$. The current 


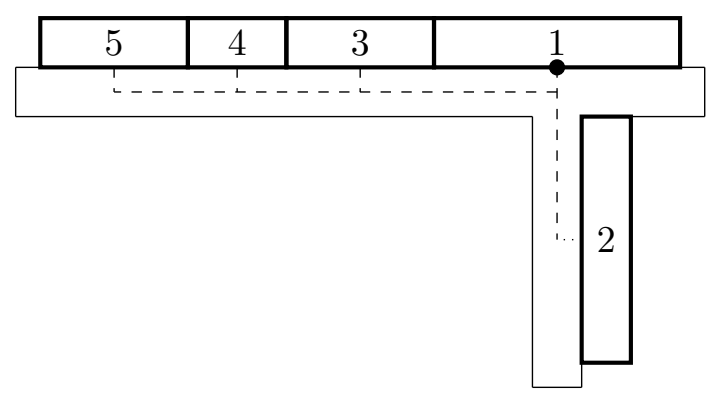

(a) An optimal T-row layout with $p_{1}=p_{M}$ and 1 is in row 1.

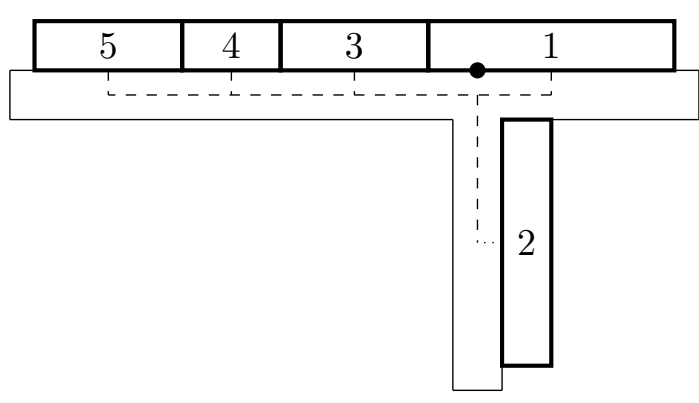

(b) An optimal T-row layout with $p_{i} \neq p_{M}, i \in[n]$.

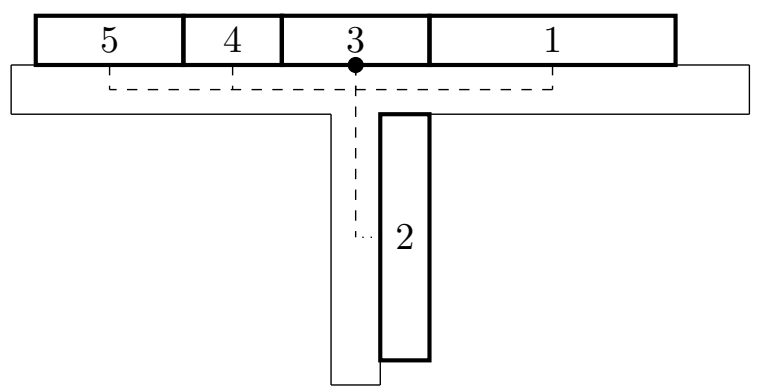

(c) An optimal T-row layout with $p_{3}=p_{M}$ and 3 is in row 1.

Figure 2: We are given a T-row instance with $n=5$ and $\ell_{1}=\ell_{2}=5, \ell_{3}=\ell_{5}=3, \ell_{4}=2$ and non-zero weights $w_{12}=w_{13}=3, w_{23}=w_{34}=w_{35}=2, w_{24}=w_{45}=1$ with $w_{\text {path }}^{T}=1$. Three optimal T-row layouts with objective value 65 are illustrated, and a black circle is displayed on position $p_{M}$ in row 1 .

best ILP approach of [3] makes use of betweenness variables and can solve instances with up to 35 departments in at most 6 hours while the current best SDP approach $[55,56]$ is able to solve instances with 36 departments in at most 20 minutes and one instance with 42 departments in less than 2 hours. Furthermore, lower and upper bounds are provided with gaps less than $2 \%$ for instances with up to 100 departments in around 200 hours. Note that the machine used in $[55,56]$ is faster than the machine used in [3]. Besides the exact approaches there have been various heuristic approaches presented in the last years [33, 37, 45, 60, 61, 70,71, 74]. We refer to [58] for a recent survey.

The MBFLP and several extensions have been extensively studied in the literature $[17,38,43$, $62,63,67]$. In [67], the MBFLP is considered where additionally the lengths of the rows are restricted. Then, a two-stage procedure is presented where in the first step the assignment of the departments is determined by solving a mixed-integer linear programming (MILP) approach neglecting inner-row distances and minimizing the weighted inter-row distances. In the second step a dummy department $n+1$ with length $\ell_{n+1}=0$ and weights $w_{i(n+1)}=w_{(n+1) i}, i \in[n]$, which are equal to the sum of the weights of $i$ to all departments which lie in a different row than $i$, is added. Then, the layout in each row is determined by setting the lengths of the departments to one and applying the dynamic programming algorithm of [72] to solve $m$ single-row instances independently. This approach is extended by [24] to instances containing departments of the same type, i. e., departments with the same length and the same transport weights to the remaining departments. Two-stage procedures are often used for the MBFLP and its extensions, see, e.g., $[24,25,67]$, but without knowledge about the quality of the obtained solution. In [43] an ILP model is presented for the MBFLP where the assignment of the departments to the rows is fixed, and instances with up to 25 departments and up to 5 rows are solved in less than one second. In order to compute an optimal solution for the MBFLP one has to enumerate over all distinguishable 
assignments of the departments to the rows. A survey for the MBFLP is given in [38].

The Flexible Multi-Bay Facility Layout Problem (FBFLP) is an extension of the MBFLP, see, e. g., $[17,25,59,63]$, where the departments are given as two-dimensional objects, i. e., the departments have a length and a height. The height of a row equals the height of a department with the highest height in that row. The vertical distance between two rows equals half of the heights of these two rows plus the sum of the heights of the rows between them. In contrast to the MBFLP, there is no upper bound for the number of rows given. The MILP approach of [59] can solve one instance with 12 departments in less than two hours but is not able to solve an instance with 11 departments within a given time limit of 24 hours. Besides the exact approach, the above described two-stage procedure is adapted to this case by [25] and several further heuristics are presented $[27,62,63,66,79]$.

In [29] a MILP model for the PMFP is presented, and an instance with 12 departments and up to five rows is considered where a gap of around $95 \%$ was obtained after a time limit of 2 hours. The authors conclude with the research question to develop a more efficient approach for the PMFP. We refer to $[14,31,51,53,64,80]$ for further extensions of the MBFLP.

Highly related to the MBFLP is the Multi-Row Facility Layout Problem (MRFLP) where distances are measured only in horizontal directions. For $m=2$ this problem is called Double-Row Facility Layout Problem (DRFLP) and several MILP approaches are available for the DRFLP $[6,8,9,26,30,76]$ (see [82] for a correction of [30]). The enumerative approach of $[42,43]$ is the current best approach for the DRFLP and the MRFLP and is able to solve double-row instances with up to 16 departments in less than 12 hours and multi-row instances with up to 13 departments and 5 rows in at most 7 hours. Heuristics for the DRFLP and the MRFLP, including extensions, are given in $[10,19,30,46,69,73,78,83,84]$. The special case where all departments have the same length is considered in $[4,12,13,54]$, and the current best approach is given in [13] where instances with up to 25 departments and up to 5 rows are solved to optimality within a time limit of 3 hours. For further literature about facility layout planning we refer to the surveys $[17,39,50]$.

\subsection{Our Contribution}

In this section, we describe our main contribution. We study the well-known betweenness variables and we show that it is sufficient to choose $(n-1)(n-2)$ of the betweenness variables as binary variables in the MILP model of [3]. Currently, all betweenness variables, i. e., $\frac{n(n-1)(n-2)}{3}$, are chosen to be binary, see, e. g. [3, 22, 43].

The TRFLP is a generalization of the SRFLP and the 3-BFLP with a more complex path structure, and hence we continue the line of research to extend facility layout problems in order to include realistic aspects. At first, we show that there always exists an optimal T-row layout where one department has its center position on position $p_{M}$ in row 1 . Then, we enumerate over each department with its center fixed on position $p_{M}$ in row 1 . We present a MILP model for this subproblem of the TRFLP based on an extension of the betweenness variables which can be in contrast to the literature equal to one if the corresponding departments lie in different rows. This extension has two advantages. At first, the distances between departments in different rows can be calculated without big- $M$-constraints. At second, we use transitivity constraints (instead of standard linearization) to ensure that we obtain the correct relation of the extended betweenness variables and the remaining variables. We provide cutting planes exploring the crossroad structure of the TRFLP to compute lower bounds for the extended betweenness variables. In order to evaluate the performance of our approach, we set up a MILP approach based on betweenness variables which can only be equal to one if the corresponding three departments are in the same row. Further, we use a variant which uses standard linearization instead of the transitivity inequalities and in another variant, we neglect some of the cutting planes. In our computational study we show that we clearly outperform these approaches.

Further, we adapt our approach and obtain a MILP model for the 3-BFLP. The current best 
known approach for the 3-BFLP can be adapted to the TRFLP, and for both problems our approach clearly outperforms the best known approach from the literature. In addition, we are able to compute tight lower bounds for even larger T-row and 3-Bay instances to evaluate the quality of our heuristically determined layouts.

\section{The Single-Row Facility Layout Problem}

In this paper we study betweenness variables and we present an extension in Section 3.2. In this section we prove that it is sufficient to choose $(n-1)(n-2)$ of the $\frac{n(n-1)(n-2)}{3}$ betweenness variables as binary variables. Betweenness variables are defined as follows in the literature, see, e. g., $[3,22,28,43]$

$$
x_{j k i}=x_{i k j}= \begin{cases}1, & \text { if } k \text { lies between } i \text { and } j \text { in the same row } \\ 0, & \text { otherwise }\end{cases}
$$

$i, j, k \in[n],|\{i, j, k\}|=3, i<j$. Based on these betweenness variables the following ILP model is introduced in [3] for the SRFLP with the constant $C:=\sum_{\substack{i, j \in[n] \\ i<j}} \frac{\ell_{i}+\ell_{j}}{2} w_{i j}$

$$
\begin{aligned}
& C+\min \sum_{\substack{i, j \in[n] \\
i<j}} w_{i j} \sum_{k \in[n] \backslash\{i, j\}} \ell_{k} x_{i k j} \\
& \text { s.t. } \quad x_{i k j}+x_{j i k}+x_{i j k}=1, \quad i, j, k \in[n], i<j<k \text {, } \\
& -x_{i h j}+x_{i h k}+x_{j h k} \geq 0, \quad i, j, k, h \in[n],|\{i, j, k, h\}|=4, \\
& x_{i h j}+x_{i h k}+x_{j h k} \leq 2, \quad i, j, k, h \in[n], i<j<k,|\{i, j, k, h\}|=4 \text {, } \\
& x_{i k j} \in\{0,1\}, \\
& i, j, k \in[n], i<j,|\{i, j, k\}|=3 \text {. }
\end{aligned}
$$

Equations (3) ensure that, given three departments, exactly one of them lies in the middle. The departments satisfy certain transitivity conditions, see inequalities (4)-(5). There always exists an optimal space-free single-row layout, i. e., a single-row layout without spaces between two neighboring departments, so the distance between $i \in[n]$ and $j \in[n], i<j$, is calculated by summing up the lengths of all departments which lie between them, see (2). By inequalities (3)-(6) one obtains a feasible ordering of the departments, see [3]. A polyhedral study is given in [75].

In [3] a lower bounding strategy is used where the LP (2)-(5) with $x_{i k j} \in[0,1], i, j, k \in[n], i<$ $j,|\{i, j, k\}|=3$, is solved and, while there exists violated cuts and the solution is not integral, the violated cuts are added and the resulting LP is solved again. All instances considered in [3] are solved to optimality by this method but in general, there is no guarantee to obtain an optimal solution. One reason for using this lower bounding approach instead of using a branch-and-cut algorithm might be that this model has $\mathcal{O}\left(n^{3}\right)$ binary variables. We provide the following result

Proposition 1. Given inequalities (3)-(5), it is sufficient to choose $(n-1)(n-2)$ betweenness variables as binary variables to obtain a correct ordering of the departments.

Proof. Let $s \in[n]$ be fixed. We set $x_{s i j}, x_{s j i}, x_{i s j} \in\{0,1\}, i, j \in[n], i<j$, and $x_{i k j} \in[0,1]$, $i, j, k \in[n] \backslash\{s\},|\{i, j, k\}|=3, i<j$. We will show that $x_{i k j}, i, j, k \in[n] \backslash\{s\},|\{i, j, k\}|=3, i<j$, is equal to one if $x_{s k i}+x_{s k j}=1$, and equal to zero otherwise, i. e., $x_{i k j} \in\{0,1\}$. Therefore, we distinguish between the following three cases

1) Let $x_{s k i}+x_{s k j}=1$. Then, we obtain $x_{i k j}=1$ by inequalities (4), as desired.

2) Let $x_{s k i}=x_{s k j}=1$. By inequalities (5) we get $x_{i k j}=0$.

3) Let $x_{s k i}=x_{s k j}=0$. It follows by inequalities (4) that $x_{i k j}=0$. 
Therefore, we obtain $x_{i k j} \in\{0,1\}, i, j, k \in[n] \backslash\{s\},|\{i, j, k\}|=3, i<j$, and according to [3] we obtain a feasible ordering of the departments. Furthermore, one can use equations (3) to reduce the number of variables as done in [3], i. e., $x_{s j i}=1-x_{s i j}-x_{i s j}, i, j \in[n] \backslash\{s\}, i<j$, and hence we obtain $(n-1)(n-2)$ binary variables.

By fixing $s \in[n]$ and choosing only $x_{s i j}$ and $x_{i s j}, i, j \in[n] \backslash\{s\}, i<j$, as binary variables, one influences the branching strategy of the MILP solver using a branch-and-cut algorithm, and hence the performance of the branch-and-cut algorithm can be improved. However, the number of branches is in general not reduced by this method, but it supports the idea to apply branch-and-cut algorithms on MILP models based on betweenness variables.

\section{The T-Row Facility Layout Problem}

Approaches for the MBFLP are often based on heuristics without knowledge about the quality of the solution, see, e.g., [24, 25, 67], and MILP models for the MBFLP and its extensions are not able to solve instances with 12 departments in reasonable time $[29,59]$. Therefore, we present a new exact approach for the TRFLP. At first, in Section 3.1, we prove that there always exists an optimal T-row layout where the center of one department in row 1 is on position $p_{M}$. In a branch-and-cut approach we enumerate over each department with its center fixed on position $p_{M}$ in row 1 and in Section 3.2 we present a MILP model for this problem based on ordering, assignment and betweenness variables. We extend the betweenness variables such that they can be equal to one if the corresponding three departments lie in distinct rows, and thus we are able to calculate the distance between departments in distinct rows without big- $M$-constraints. Transitivity constraints are used to ensure the correct relation of these betweenness variables and the remaining variables. In Section 3.3 we present various cutting planes especially designed for the TRFLP, where the center of $s_{M} \in[n]$ is fixed on position $p_{M}$ in row 1 , to strengthen our branch-and-cut algorithm and in Section 3.4 we derive diverse heuristic approaches. We describe our separation strategy in Section 3.5.

\subsection{Our Algorithm}

We start this section with the following proposition, which is essential for our algorithm

Proposition 2. Given a T-row instance, then there exists an optimal T-row layout where the center of one department lies on position $p_{M}$ in row 1.

The proof is related to a proof of [34]. Given a DRFLP instance with objective function $\min \sum_{j \in[n] \backslash\{i\}} w_{i j} d_{i j}, i \in[n]$, there exists an double-row optimal layout where $k \in[n] \backslash\{i\}$ lies directly opposite $i$.

Proof. We assume, w. l.o.g., that an optimal T-row layout contains at least one department in row 1 because if all departments would be in row 2, one can simply shift these departments to row 1 without changing the order of the departments and without increasing the objective value of the layout. So let an optimal T-row layout with at least one department in row 1 be given and we assume for all $i \in[n]$ in row 1 that $p_{i} \neq p_{M}$. Let $B_{1}\left(B_{2}\right)$ denote the set of departments in row 1 with $p_{i}<p_{M}\left(p_{i}>p_{M}\right), i \in[n]$, and let $B_{3}$ denote the set of departments in row 2 . We assume, w. l.o.g., $\sum_{\substack{k \in B_{3} \\ i \in B_{1}}} w_{i k} \geq \sum_{\substack{k \in B_{3} \\ i \in B_{2}}} w_{i k}$, and we shift all departments in row 1 to the right until the center of the first department lies on position $p_{M}$. By this method we do not increase the objective value, and the desired result is proven.

Therefore, we fix the center of $s_{M} \in[n]$ on position $p_{M}$ in row 1 and we denote the resulting problem by $\left(s_{M}\right.$-TRFLP). Our algorithm for the TRFLP works as follows. For each $s_{M} \in[n]$ we heuristically determine a $s_{M}$-T-row layout, and we sort the departments by increasing order of the 
objective values of the $s_{M}$-T-row layouts. Let $s_{M} \in[n]$ be the first not yet considered department in this sorting. Then, we solve the $s_{M}$-TRFLP with our branch-and-cut algorithm where the current best objective value is set as an upper bound, i. e., the branch-and-cut algorithm is interrupted if the best lower bound exceeds the upper bound. We repeat this until the $s_{M}$-TRFLP with the described upper bound is solved for each $s_{M} \in[n]$. Our algorithm is summarized in Algorithm 1.

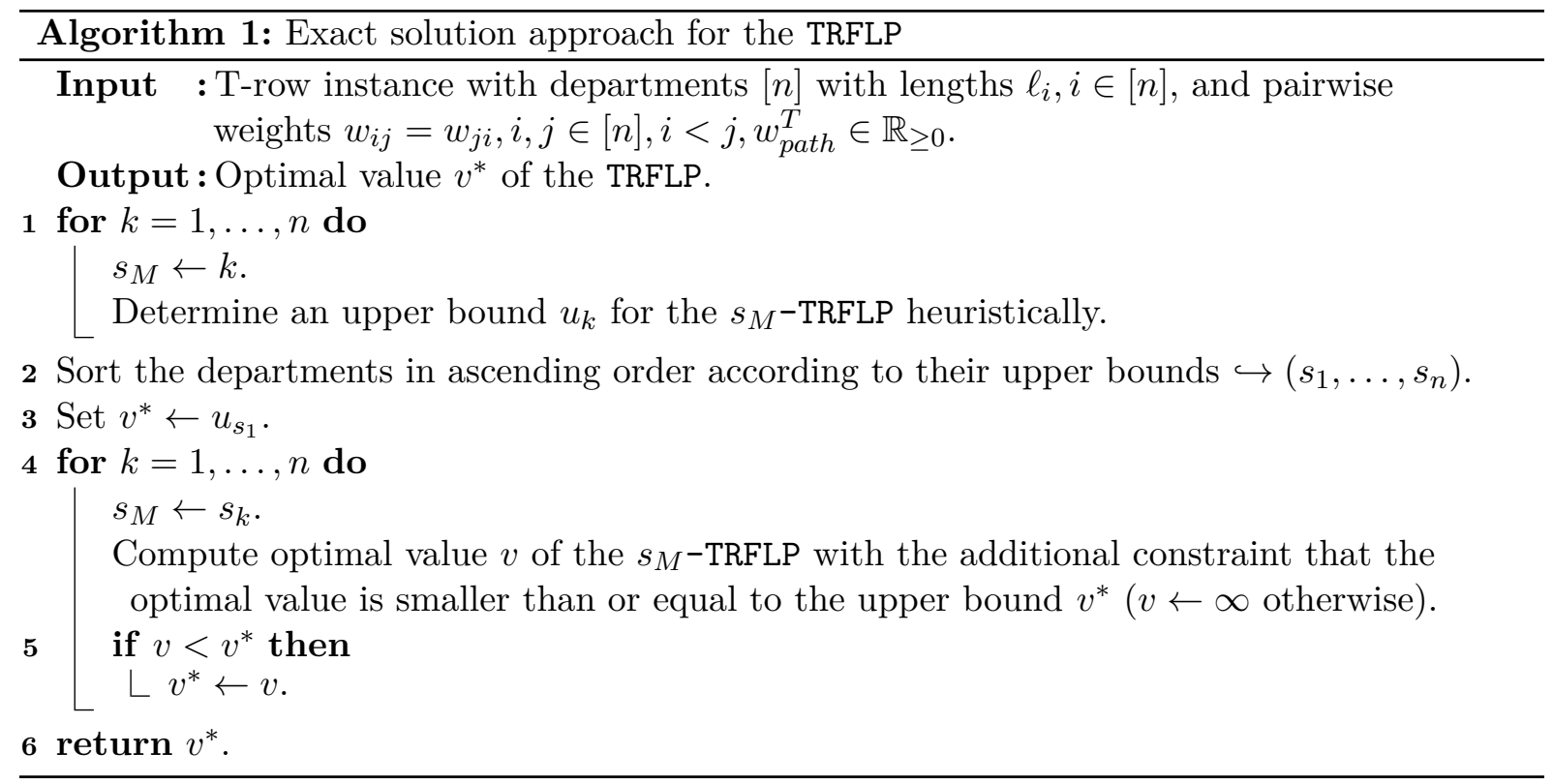

The question arises, whether the number of departments where the centers are fixed on position $p_{M}$ in row 1 can be reduced, e. g., one could try to arrange only the centers of the three departments on position $p_{M}$ in row 1 , which are at the left border of an optimal 3-Bay layout

Example 3. We consider an instance with $n=4$ departments with lengths $\ell_{1}=\ell_{3}=5, \ell_{2}=$ $\ell_{4}=7$, and non-zero weights $w_{12}=w_{13}=w_{23}=1, w_{14}=3$, with $w_{\text {path }}^{T}=0$ and $w_{\text {path }}^{B}=1$. In an optimal 3-Bay layout, 1 and 4 are neighboring without free-space in the same row and 1, 2 and 3 are on the left border of a row, see Figure 3 a for an illustration. Therefore, the optimal value of the 3-BFLP is 39. In an optimal T-row layout the center of 4 lies on position $p_{M}$ in row 1, illustrated in Figure 3b, with objective value 29.5.

However, if there are departments of the same type as recently considered in [20, 24, 46] for related layout problems, one just has to fix the center of one department of each type on position $p_{M}$ in row 1.

\subsection{A MILP model for the $s_{M}$-TRFLP}

In this section we present a MILP model for the $s_{M}$-TRFLP, $s_{M} \in[n]$. So, in the following, let $s_{M} \in[n]$ be fixed. Our MILP model makes use of the well-known ordering and assignment variables. If $i \in[n]$ and $j \in[n] \backslash\{i\}$ lie in row 2 and $i$ is above (below) $j$, we say that $i$ is left (right) to $j$. Then, we define

$$
z_{i j}= \begin{cases}1, & i \text { is left to } j \text { and } i \text { and } j \text { are in the same row } \\ 0, & \text { otherwise }\end{cases}
$$

$i, j \in[n], i \neq j$. Furthermore, we use assignment variables

$$
y_{i}= \begin{cases}1, & \text { if } i \text { lies in row } 1 \\ 0, & \text { otherwise }\end{cases}
$$




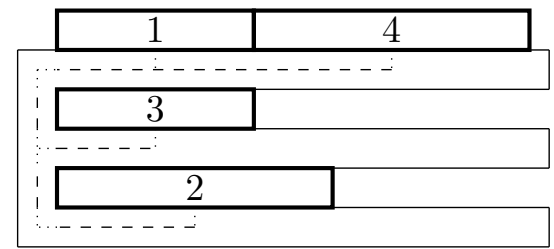

(a) optimal 3-Bay layout with 1,2 and 3 at the left border.

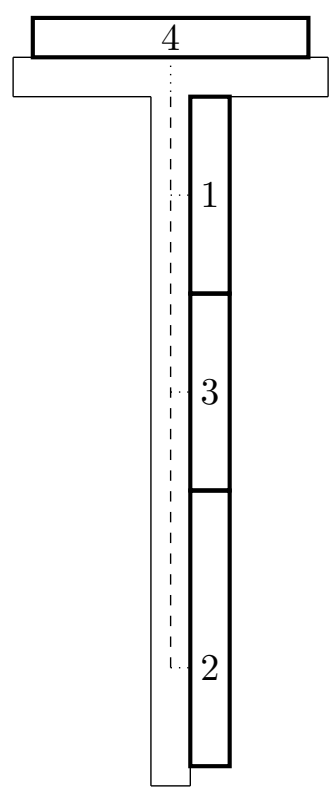

(b) optimal T-row layout where the center of 4 is on position $p_{M}$ in row 1 .

Figure 3: We are given an instance with $n=4$ departments with $\ell_{1}=\ell_{3}=5, \ell_{2}=\ell_{4}=7$, and non-zero weights $w_{12}=w_{13}=w_{23}=1, w_{14}=3$, with $w_{\text {path }}^{T}=0, w_{\text {path }}^{B}=1$. An optimal 3 -Bay layout is illustrated on the left-hand side with objective value 39 and an optimal T-row layout is illustrated on the right-hand side with objective value 29.5.

$i \in[n]$. At first, we fix $s_{M}$ to row 1 , see equation (7), and we ensure that $i \in[n] \backslash\left\{s_{M}\right\}$ is left or right to $s_{M}$ if and only if $i$ is in row 1 , see equations (8)

$$
\begin{aligned}
& y_{s_{M}}=1 \text {, } \\
& z_{i s_{M}}+z_{s_{M} i}-y_{i}=0 \text {, } \\
& i \in[n] \backslash\left\{s_{M}\right\} .
\end{aligned}
$$

We add two dummy departments $n+1$ and $n+2$ with lengths $\ell_{n+1}=\ell_{n+2}=0$ and weights $w_{i(n+1)}=w_{(n+1) i}=w_{(n+2) i}=w_{i(n+2)}=0, i \in[n] \backslash\left\{s_{M}\right\}$, to our model, and we fix $n+1$ at the left border of row 1 and $n+2$ at the left (upper) border of row 2 . Then, we define betweenness variables in the following way where at least one of the three departments is a dummy department or $s_{M}$

$$
x_{i k j}=x_{j k i}= \begin{cases}1, & \text { if } k \text { lies between } i \text { and } j \text { in the same row } \\ 0, & \text { otherwise }\end{cases}
$$

$i, j, k \in[n+2],\left|\{i, j, k\} \cap\left\{s_{M}, n+1, n+2\right\}\right| \geq 1, i<j,|\{i, j, k\}|=3$. Remark that, given three departments and one of them is equal to $s_{M}, n+1$ or $n+2$, then the corresponding betweenness variables are only equal to one if all three departments lie in the same row. In this way betweenness variables were used successfully in the literature, see, e.g., [3, 22, 43, 76]. The correct relation of the betweenness and the assignment variables are ensured by the following inequalities

$$
\begin{array}{ll}
x_{i s j}+x_{s i j}+x_{s j i}-y_{i} \leq 0, & i, j \in[n] \backslash\left\{s_{M}\right\}, i \neq j, s \in\left\{s_{M}, n+1\right\}, \\
x_{(n+2) i j}+x_{(n+2) j i}+y_{i} \leq 1, & i, j \in[n] \backslash\left\{s_{M}\right\}, i \neq j, \\
x_{i s j}+x_{s i j}+x_{s j i}-y_{i}-y_{j} \geq-1, & i, j \in[n] \backslash\left\{s_{M}\right\}, i<j, s \in\left\{s_{M}, n+1\right\}, \\
x_{(n+2) i j}+x_{(n+2) j i}+y_{i}+y_{j} \geq 1, & i, j \in[n] \backslash\left\{s_{M}\right\}, i<j .
\end{array}
$$


Inequalities (9) ensure that betweenness variables containing $i, j$ and $s_{M}$ or $n+1, i, j \in$ $[n] \backslash\left\{s_{M}\right\}, i \neq j$, are equal to zero if $i$ or $j$ (or both) are assigned to row 2. The sum of the corresponding three betweenness variables equals one if $i$ and $j$ are assigned to row 1 , see inequalities (11). Similar inequalities are used for the dummy department $n+2$, see inequalities (10) and inequalities (12).

Betweenness variables containing $s_{M}$ are equal to the sum of two products of ordering variables, e. g., $x_{s_{M} i j}=z_{s_{M} i} \cdot z_{i j}+z_{j i} \cdot z_{i s_{M}}, i, j \in[n] \backslash\left\{s_{M}\right\}, i \neq j$. Therefore, we use lower bounds related to the standard linearization, see inequalities (13)-(15). Note that the corresponding upper bounds are implied by inequalities (7)-(10)

$$
\begin{array}{ll}
x_{i s_{M} j} \geq z_{i s_{M}}+z_{s_{M} j}-1, & i, j \in[n] \backslash\left\{s_{M}\right\}, i \neq j, \\
x_{s_{M} i j} \geq z_{s_{M} i}+z_{i j}-1, & i, j \in[n] \backslash\left\{s_{M}\right\}, i \neq j, \\
x_{s_{M} i j} \geq z_{j i}+z_{i s_{M}}-1, & i, j \in[n] \backslash\left\{s_{M}\right\}, i \neq j, \\
x_{(n+1) i j}+x_{(n+2) i j}-z_{i j}=0, & i, j \in[n] \backslash\left\{s_{M}\right\}, i \neq j, \\
x_{i s_{M} j}+x_{s_{M} i j}+x_{s_{M} j i}-x_{(n+1) i j}-x_{(n+1) j i}=0, & i, j \in[n] \backslash\left\{s_{M}\right\}, i<j, \\
x_{i(n+1) j}+x_{i(n+2) j}=0, & i, j \in[n], i<j .
\end{array}
$$

Let $i, j \in[n] \backslash\left\{s_{M}\right\}, i \neq j$. Then, $i$ and $j$ are in the same row and $i$ is left to $j$ if and only if $i$ is left to $j$ in row 1 or row 2, see equations (16). Further, $i$ is left or right to $j$ in row 1 if and only if $x_{i s_{M} j}+x_{s_{M} i j}+x_{s_{M} j i}$ equals one, see equations (17). Equations (18) ensure that the dummy departments are fixed at the border. While the dummy department $n+2$ is necessary for our formulation for the $s_{M}$-TRFLP, we mainly use the dummy department $n+1$ to relate $x_{(n+2) i j}$ and $x_{(n+2) j i}, i, j \in[n] \backslash\left\{s_{M}\right\}, i<j$, with the ordering variables, see equations (16). Hence, we avoid the usage of standard linearization in this case.

In [8] a MILP model for the DRFLP is presented which consists of ordering variables $\sigma_{i j}$ which are equal to one if $i \in[n]$ is to the left of $j \in[n], i \neq j$, and $i$ and $j$ are in the same row or $i$ is in row 2 and $j$ is in row 1 . The distances between the centers of the departments are calculated via big- $M$-type constraints. Let $R_{1}\left(R_{2}\right)$ denote the set of departments assigned to row 1 (row 2) in a double-row layout. Then, we assign the departments in $R_{1}$ to row 2 such that the departments in $R_{1}$ are to the right of the departments in $R_{2}$ without changing the order of the departments in $R_{1}$ and in $R_{2}$. By this method, the value of the ordering variables is not influenced, so the ordering variables $\sigma$ are equal to ordering variables in a single-row layout. Therefore, this method differs significantly from our method described below.

The distance between $i \in[n]$ and $j \in[n] \backslash\{i\}$ in the $s_{M}$-TRFLP can be expressed by $\mid p_{i}-$ $p_{M} \mid+p_{j}+w_{\text {path }}^{T}$ if $i$ lies in row 1 and $j$ in row 2. Using this idea in a branch-and-cut algorithm coupled with big- $M$-constraints to couple the row assignment of $i$ and $j$, leads to a rather bad root relaxation and to a poorly performing algorithm as we will see in Section 5. We aim to calculate the distances between the departments by betweenness, ordering and assignment variables without big- $M$-constraints. Therefore, we extend the definition of the betweenness variables. Given a $s_{M}$-T-row layout, we define $B_{1}\left(B_{2}\right)$ as the set of departments left (right) to $s_{M}$ and $B_{3}$ as the set of departments in row 2. Let $i^{\prime}\left(j^{\prime}\right)$ be the department in $B_{a}\left(B_{b}\right)$ closest to $p_{M}, a, b \in\{1,2,3\}, a \neq b$. We arrange the departments $B_{a} \cup B_{b}$ on a straight line such that $i^{\prime}$ and $j^{\prime}$ are neighboring as well as neighboring departments in the $s_{M}$-T-row layout are neighboring. Let $i, j, k \in[n] \backslash\left\{s_{M}\right\},|\{i, j, k\}|=3, i<j$. We say that $k \in[n] \backslash\left\{s_{M}\right\}$ lies between $i$ and $j$ if there exists $a, b \in\{1,2,3\}$ such that $k$ lies between $i$ and $j$ on the with $B_{a} \cup B_{b}$ associated straight line. If $a=b$, then the departments in the set $B_{a}$ are already arranged on a straight line. In the $s_{M}$-TRFLP an automated guided vehicle has to traverse every $k$ which lies between $i$ and $j$. This motivates the following extension of the betweenness variables

$$
x_{i k j}=x_{j k i}= \begin{cases}1, & \text { if } k \text { lies between } i \text { and } j \\ 0, & \text { otherwise }\end{cases}
$$


$i, j, k \in[n] \backslash\left\{s_{M}\right\},|\{i, j, k\}|=3, i<j$. So our new betweenness variables $x_{i k j}$ might also be equal to one if $i \in[n]$ and $j \in[n], i<j$, lie in distinct rows, $k \in[n] \backslash\{i, j\}$. Since this version of betweenness variables seems to be a novelty in the layout planning literature, we illustrate their usage by an example

Example 4. We are given a T-row instance with $n=6$ departments with lengths $\ell_{1}=\ell_{5}=$ $3, \ell_{2}=4, \ell_{3}=1, \ell_{4}=\ell_{6}=2$. A $s_{M}$-T-row layout with $s_{M}=2$ is illustrated in Figure 4 including the dummy departments 7 and 8 . We summarize all betweenness variables which are equal to one. We start with $x_{i k j}, i, j, k \in[n], i<j,|\{i, k, j\}|=3$, where $i$ and $j$ lie in distinct rows: $x_{156}=x_{356}=x_{435}=x_{436}=x_{456}=1$. For betweenness variables containing $s_{M}$ or at least one of the dummy departments, we get: $x_{123}=x_{124}=x_{234}=x_{217}=x_{317}=x_{327}=x_{417}=x_{427}=$ $x_{437}=x_{856}=1$. Besides that we get $x_{134}=1$ and the remaining betweenness variables are equal to zero.

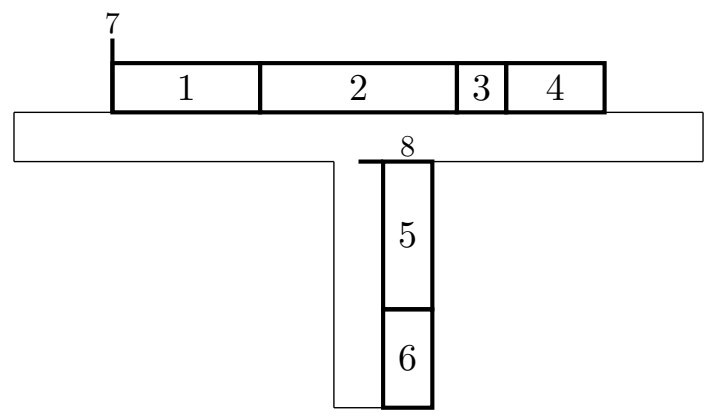

Figure 4: Illustration of a $s_{M}$-TRFLP layout with $s_{M}=2$ where the dummy departments 7 (8) is at the left (upper) border of row 1 (row 2). We summarize all extended betweenness variables which are equal to one: $x_{156}=x_{356}=x_{435}=x_{436}=x_{456}=1$.

We present the following inequalities to obtain the correct relation between our new extended betweenness variables and betweenness variables containing $s_{M}$ or a dummy department

$$
\begin{array}{ll}
x_{i k j}-x_{s_{M} k i}+x_{s_{M} k j}-x_{(n+2) k i}+x_{(n+2) k j} \geq 0, & i, j, k \in[n] \backslash\left\{s_{M}\right\},|\{i, j, k\}|=3, \\
x_{i k j}-x_{s_{M} k i}-x_{s_{M} k j}-x_{(n+2) k i}-x_{(n+2) k j} \leq 0, & i, j, k \in[n] \backslash\left\{s_{M}\right\},|\{i, j, k\}|=3, i<j, \\
x_{i k j}+x_{j i k}+x_{i j k} \leq 1, & i, j, k \in[n] \backslash\left\{s_{M}\right\}, i<j<k .
\end{array}
$$

Considering three departments, at most one of them lies in the middle, see inequalities (21). Let $i, j, k \in[n] \backslash\left\{s_{M}\right\},|\{i, j, k\}|=3$, be fixed, and we consider inequalities (19). Recall that $x_{s_{M} k i}+x_{(n+2) k i} \leq 1$ by inequalities (9)-(10). Let $k$ lie between $i$ and $s_{M}(n+2)$ and let $k$ not lie between $s_{M}(n+2)$ and $j$. Then, $k$ lies between $i$ and $j$, we refer to Figure $5 \mathrm{a}(5 \mathrm{~b})$ for an illustration. Otherwise, inequalities (19) are redundant. Now, let $i, j, k \in[n] \backslash\left\{s_{M}\right\},|\{i, j, k\}|=3, i<j$. Recall that $B_{1}\left(B_{2}\right)$ denotes the set of departments left (right) to $s_{M}$ and $B_{3}$ denotes the set of departments in row 2. If $k \in B_{a}, a \in\{1,2,3\}$, and $i, j \notin B_{a}$, then $k$ does not lie between $i$ and $j$, see inequalities (20). Now, let $i, k \in B_{a}, a \in\{1,2,3\}$, and we set $s=s_{M}$ if $a \in\{1,2\}$ and $s=n+2$ if $a=3$. Let $j \in B_{b}, b \in\{1,2,3\}, b \neq a$, and let $k$ lie between $i$ and $j$, then $k$ lies between $i$ and $s$, see inequalities (20). Let $j \in B_{a}$ and let $k$ lie between $i$ and $j$, then $k$ lies between $i$ and $s$ or $j$ and $s$ and inequalities (9) are satisfied. This leads to the following remark

Remark 1. Let $i, j$ and $k$ lie in the same row, $i, j, k \in[n] \backslash\left\{s_{M}\right\},|\{i, j, k\}|=3, i<j$. We set $s=s_{M}(s=n+2)$ if $i$ lies in row 1 (row 2) and then we obtain by inequalities (19)-(20)

$$
\begin{aligned}
& x_{i k j}-x_{s k i}+x_{s k j} \geq 0, \\
& x_{i k j}-x_{s k j}+x_{s k i} \geq 0, \\
& x_{i k j}-x_{s k i}-x_{s k j} \leq 0 .
\end{aligned}
$$

Hence, inequalities (19)-(20) are an extension of inequalities (4) with departments $[n] \backslash\left\{s_{M}\right\}$. 


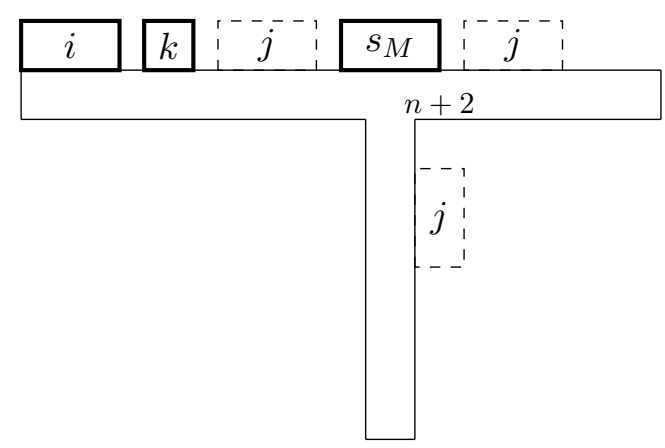

(a) Let $x_{i k s_{M}}=1$. Then, $k$ lies between $i$ and $j$ if and only if $k$ does not lie between $s_{M}$ and $j$.

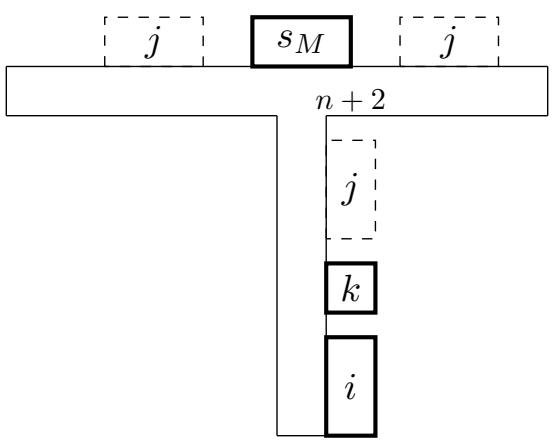

(b) Let $x_{(n+2) k i}=1$. Then, $k$ lies between $i$ and $j$ if and only if $k$ is not left to $j$ in row 2, i. e., $x_{(n+2) k j}=0$.

Figure 5: Let $i, j, k \in[n] \backslash\left\{s_{M}\right\},|\{i, j, k\}|=3$. Visualization of inequalities (19) with $x_{s_{M} k i}=1$ in Figure 5a and $x_{(n+2) k i}=1$ in Figure $5 \mathrm{~b}$. We illustrate possible positions for $j$ in dashed rectangles such that $x_{i k j}=1$. The dummy department $n+1$ is not illustrated here.

It remains to calculate the rectilinear center-to-center distances between the departments. The minimal distance between $i$ and $j, i, j \in[n] \backslash\left\{s_{M}\right\}, i<j$, in the $s_{M}$-TRFLP equals $\frac{\ell_{i}+\ell_{j}}{2}$ and, assuming that $i$ and $j$ lie in different rows, one can add $\frac{\ell_{s_{M}}}{2}+w_{\text {path }}^{T}$. Further, the minimal distance between $i \in[n] \backslash\left\{s_{M}\right\}$ and $s_{M}$ is $\frac{\ell_{i}}{2}$ and assuming that $i$ lies in row 2 we add $w_{\text {path }}^{T}$, so altogether we exclude the following constant value from our model

$$
W_{s_{M}}:=\sum_{i \in[n] \backslash\left\{s_{M}\right\}} w_{i s_{M}}\left(\frac{\ell_{i}}{2}+w_{\text {path }}^{T}\right)+\sum_{\substack{i, j \in[n] \backslash\left\{s_{M}\right\} \\ i<j}} w_{i j}\left(\frac{\ell_{i}}{2}+\frac{\ell_{j}}{2}+\frac{\ell_{s_{M}}}{2}+w_{\text {path }}^{T}\right) .
$$

For the $s_{M}$-TRFLP there always exists an optimal space-free layout, i.e., an optimal layout without free-spaces between neighboring departments in the same row. So we neglect distance variables and we compute the distances between the departments in the following way

$$
\begin{array}{ll}
\left(-w_{\text {path }}^{T}-\frac{\ell_{s_{M}}}{2}\right)\left(z_{i j}+z_{j i}\right)+\sum_{k \in[n] \backslash\{i, j\}} \ell_{k} x_{i k j}, & i, j \in[n] \backslash\left\{s_{M}\right\}, i<j, \\
\left(\frac{\ell_{s_{M}}}{2}-w_{\text {path }}^{T}\right) y_{i}+\sum_{k \in[n] \backslash\left\{s_{M}, i\right\}} \ell_{k}\left(x_{i k s_{M}}+x_{(n+2) k i}\right), & i \in[n] \backslash\left\{s_{M}\right\} .
\end{array}
$$

By (23) and (22) we measure the rectilinear center-to-center distance between $i \in[n] \backslash\left\{s_{M}\right\}$ and $j \in[n] \backslash\left\{s_{M}\right\}, i<j$. We subtract $\frac{\ell_{s_{M}}}{2}+w_{\text {path }}^{T}$ if $i$ and $j$ lie in the same row, see (22), and we add the sum of the lengths of the departments between $i$ and $j$. If $i$ and $j$ lie in row 1 and $s_{M}$ lies between them, then $x_{i s_{M} j}=1$ and we take $\ell_{s_{M}}$ into account. By (24) and (22) we calculate the rectilinear center-to-center distance between $s_{M}$ and $i \in[n] \backslash\left\{s_{M}\right\}$ by summing up the lengths of all departments which are between $i$ and $s_{M}$ and $i$ and $n+2$. Additionally, we add $\frac{\ell_{s_{M}}}{2}-w_{\text {path }}^{T}$ if $i$ lies in row 1 . This leads to the following result

Theorem 3. Let $s_{M} \in[n]$ and $W_{s_{M}}$ be calculated as described in equation (22). Then

$$
\begin{gathered}
W_{s_{M}}+\min \sum_{\substack{i, j \in[n] \backslash\left\{s_{M}\right\} \\
i<j}} w_{i j}\left(-w_{\text {path }}^{T}-\frac{\ell_{s_{M}}}{2}\right)\left(z_{i j}+z_{j i}\right) \\
+\sum_{\substack{i, j \in[n] \backslash\left\{s_{M}\right\} \\
i<j}} w_{i j} \sum_{k \in[n] \backslash\{i, j\}} \ell_{k} x_{i k j}
\end{gathered}
$$




$$
\begin{aligned}
& +\sum_{i \in[n] \backslash\left\{s_{M}\right\}} w_{i s_{M}}\left(\frac{\ell_{s_{M}}}{2}-w_{\text {path }}^{T}\right) y_{i} \\
& +\sum_{i \in[n] \backslash\left\{s_{M}\right\}} w_{i s_{M}} \sum_{k \in[n] \backslash\left\{s_{M}, i\right\}} \ell_{k}\left(x_{i k s_{M}}+x_{(n+2) k i}\right)
\end{aligned}
$$

$$
\begin{array}{ll}
\text { s.t. } & y_{i} \in\{0,1\}, \\
& z_{i j} \in\{0,1\}, \\
& z_{i j} \in[0,1], \\
& z_{i s_{M}} \in\{0,1\}, \\
& z_{s_{M} i} \in[0,1], \\
& 0 \leq x_{i k j} \leq 1,
\end{array}
$$

$$
\begin{aligned}
& i \in[n], \\
& i, j \in[n] \backslash\left\{s_{M}\right\}, i<j, \\
& i, j \in[n] \backslash\left\{s_{M}\right\}, i>j, \\
& i \in[n] \backslash\left\{s_{M}\right\}, \\
& i \in[n] \backslash\left\{s_{M}\right\}, \\
& i, j, k \in[n+2], i<j, \\
& |\{i, j, k\}|=3,
\end{aligned}
$$

subject to inequalities (7)-(21) is a MILP model for the $s_{M}-$ TRFLP.

Proof. Let $s_{M} \in[n]$ be fixed. This proof is structured as follows. At first, we show that the $z$ variables are set to binary values and we prove that the $z$ and $y$ variables satisfy inequalities of a model from the scheduling literature [32]. Hence, we obtain the correct relation of the $z$ and $y$ variables and a correct ordering of the departments in the same row. Then we show that the $x$ variables are set to binary values as well. We show the correct relation of the $x$ and $y$ and $z$ variables where we distinguish between our new extended $x$ variables and $x$ variables containing $s_{M}$ or dummy departments.

By equations (8) we obtain binary values for $z_{s_{M}}, i \in[n] \backslash\left\{s_{M}\right\}$. Let $i, j \in[n] \backslash\left\{s_{M}\right\}, i<j$. By equations (16) and inequalities (9)-(10) together with $z_{i j} \in\{0,1\}$, we obtain $x_{(n+1) i j}, x_{(n+2) i j} \in$ $\{0,1\}$. If $y_{i} \neq y_{j}$ we obtain by inequalities (9)-(10) that $0=x_{(n+1) j i}+x_{(n+2) j i}=z_{j i}$, see equations (16). Otherwise let, w.l.o.g., $y_{i}=y_{j}=1$. Then, by inequalities (9) and inequalities (11) we get $x_{(n+1) i j}+x_{(n+1) j i}=1$. Since $x_{(n+1) i j} \in\{0,1\}$, we obtain $x_{(n+1) j i} \in\{0,1\}$ as well. This implies $z_{j i} \in\{0,1\}$, see equations (16). In conclusion, the $z$ variables and the $x$ variables containing dummy departments are set to binary values and these $x$ variables are coupled correctly to the $z$ variables.

Next we show that the following inequalities are satisfied

$$
\begin{array}{ll}
z_{i j}+z_{j i}+y_{i}-y_{j} \leq 1, & i, j \in[n], i \neq j, \\
y_{i}+y_{j}-z_{i j}-z_{j i} \leq 1, & i, j \in[n], i<j, \\
y_{i}+y_{j}+z_{i j}+z_{j i} \geq 1, & i, j \in[n], i<j, \\
z_{k i}+z_{i j}+z_{j k}-z_{i k}-z_{j i}-z_{k j} \leq 1, & i, j, k \in[n],|\{i, j, k\}|=3 .
\end{array}
$$

Let $i, j \in[n] \backslash\left\{s_{M}\right\}, i \neq j$, be given. By equations (16) and inequalities (9)-(10) we obtain

$$
z_{i j}+z_{j i}+y_{i}-y_{j}=x_{(n+1) i j}+x_{(n+1) j i}+x_{(n+2) i j}+x_{(n+2) j i}+y_{i}-y_{j} \leq 1,
$$

and thus inequalities (25) are satisfied. By similar arguments, inequalities (16) together with inequalities (11)-(12) imply inequalities (26)-(27). If $i, j \in[n]$ and $\left|\{i, j\} \cap\left\{s_{M}\right\}\right|=1$, then inequalities (25)-(27) are implied by inequalities (7)-(8).

It remains to prove that inequalities (28) are satisfied if $y_{i} \in\{0,1\}, i \in[n]$, and $z_{i j} \in\{0,1\}, i, j \in$ $[n], i \neq j$. Assume, on the contrary, inequalities (28) are violated by some $i, j, k \in[n],|\{i, j, k\}|=3$. Let $z_{i j}=z_{j k}=1$ and by inequalities (25) we obtain $y_{i}=y_{j}=y_{k}$. If $z_{k i}=0$, we obtain by inequalities (26)-(27) that $z_{i k}=1$, and thus inequalities (28) are satisfied. It remains to consider the case $z_{k i}=1$.

a) Let $y_{i}=y_{j}=y_{k}=0$. It follows by equation (7) that $\left|\{i, j, k\} \cap\left\{s_{M}\right\}\right|=0$. Then, we 
obtain by inequalities (19), inequalities (9) and inequalities (16) that

$$
\begin{aligned}
& x_{i k j} \geq x_{(n+2) k i}-x_{(n+2) k j}=z_{k i}-z_{k j}=1 \\
& x_{j i k} \geq x_{(n+2) i j}-x_{(n+2) i k}=z_{i j}-z_{i k}=1,
\end{aligned}
$$

a contradiction to inequalities (21).

b) Let $y_{i}=y_{j}=y_{k}=1,\left|\{i, j, k\} \cap\left\{s_{M}\right\}\right|=1$, and we assume, w. l.o.g., $k=s_{M}$. Then, by inequalities (13)-(14) we obtain

$$
\begin{aligned}
& x_{j_{s_{M}} i} \geq z_{j s_{M}}+z_{s_{M} i}-1=1, \\
& x_{s_{M} i j} \geq z_{s_{M} i}+z_{i j}-1=1,
\end{aligned}
$$

a contradiction to inequalities (9).

c) Let $y_{i}=y_{j}=y_{k}=1$ and $\left|\{i, j, k\} \cap\left\{s_{M}\right\}\right|=0$. By symmetry, it is sufficient to consider the case that two or three departments of the set $\{i, j, k\}$ are to the right of $s_{M}$.

1) Let $z_{s_{M} k}=z_{s_{M} i}=z_{s_{M} j}=1$. Then, $x_{s_{M} i j}=x_{s_{M} k i}=x_{s_{M} j k}=1$, see inequalities (14), and thus we get by inequalities (9)-(10) and inequalities (19) that

$$
\begin{aligned}
& x_{i k j} \geq x_{s_{M} k i}-x_{s_{M} k j}=1, \\
& x_{k i j} \geq x_{s_{M} i j}-x_{s_{M} i k}=1,
\end{aligned}
$$

a contradiction to inequalities (21).

2) Let $z_{k_{s_{M}}}=1$ and $z_{s_{M} i}=z_{s_{M} j}=1$. Then, we obtain by inequalities (13)-(14) that $x_{k s_{M} j}=1$ and $x_{s_{M} k j} \geq z_{k s_{M}}+z_{j k}-1=1$, a contradiction to inequalities (9).

According to [32], we obtain by inequalities (25)-(28) together with $y_{i} \in\{0,1\}, i \in[n], z_{i j} \in$ $\{0,1\}, i, j \in[n], i \neq j$, the correct relation between the $z$ and $y$ variables and a correct ordering of the departments in the same row.

The $x$ variables containing $s_{M}$ are coupled by inequalities (13)-(15), which are highly related to the standard linearization, see, e.g., $[14,52,76]$, to the $z$ variables. Inequalities (7)-(10) imply the upper bounds from the standard linearization. Hence, these variables obtain binary values and are coupled correctly to the $z$ and $y$ variables.

It remains to consider our new extended $x$ variables. Let $i, j, k \in[n] \backslash\left\{s_{M}\right\},|\{i, j, k\}|=3$, be fixed. We use the following notation: $s \in\left\{s_{M}, n+2\right\}$ with $s=s_{M}$ if $y_{i}=1$ and $s=n+2$ if $y_{i}=0$. Note that if $y_{i}=y_{k}$, we obtain $x_{t k i}=x_{t k j}=0, t \in\left\{s_{M}, n+2\right\} \backslash\{s\}$, by inequalities (9)-(10). Similar to the proof of Proposition 1, it is sufficient to show that $x_{i k j}$ equals one if $x_{s k i}+x_{s k j}=1$ and equals zero otherwise. We distinguish between the following three cases depending on the assignment of $i, j$ and $k$ to the rows

a) Let $y_{i}=y_{j}=y_{k}$. The following three cases can be distinguished

1) Let $x_{s k i}+x_{s k j}=1$. By inequalities (19) we obtain $x_{i k j}=1$.

2) Let $x_{s k i}=x_{s k j}=1$. The $x$ variables containing $s$ have the correct relation to the $z$ variables, so they satisfy transitivity properties, i. e., $x_{i s j}=0$, and thus $x_{s i j}+x_{s j i}=1$ by inequalities (11)-(12). Further, by inequalities (21) we get $x_{s i k}+x_{s j k}=0$. As a result, we obtain by inequalities (19)

$$
x_{j i k}+x_{i j k} \geq x_{s i j}+x_{s j i}=1,
$$

and thus $x_{i k j}=0$, see inequalities (21).

3) Let $x_{s k i}=x_{s k j}=0$. By inequalities (20) it follows immediately that $x_{i k j}=0$. 
b) Let, w.l.o.g., $y_{k}=y_{i}$ and $y_{k} \neq y_{j}$. By inequalities (9)-(10) we get $x_{s k j}=0$. So by inequalities (19)-(20) we obtain $x_{i k j}=x_{s k i}$.

c) Let $y_{i}=y_{j}$ and $y_{i} \neq y_{k}$. Then, we obtain $x_{i k j}=0$ by inequalities (20).

There always exists an optimal space-free T-row layout, the $x, z$ and $y$ variables are set to binary values and are coupled correctly, so the distance calculation, see (23)-(24), is correct. This concludes the proof.

Let $S=\left(\pi_{1}, \ldots, \pi_{n-1}\right)$ denote an arbitrary sorting of the departments $[n] \backslash\left\{s_{M}\right\}$. In order to eliminate symmetrical layouts, we fix one department in row 1 to the left of $s_{M}$, see equation (29). However, if this department is assigned to row 2, we fix another department in row 1 to the left of $s_{M}$. We continue in this manner, see inequalities (30). These inequalities are related to symmetry breaking constraints for the graph coloring problem, see, [65, 68]. We set

$$
\begin{aligned}
& z_{\pi_{1} s_{M}}-y_{\pi_{1}}=0 \\
& z_{\pi_{j} s_{M}}-y_{\pi_{j}}+\sum_{k=1}^{j-1} y_{\pi_{k}} \geq 0, \quad j=2, \ldots, n-1 .
\end{aligned}
$$

If $\frac{\ell_{s_{M}}}{2}=w_{\text {path }}^{T}$, one can use stronger symmetry breaking constraints, let $S=\left(\pi_{1}, \ldots, \pi_{n-1}\right)$ denote an arbitrary sorting of the departments $[n] \backslash\left\{s_{M}\right\}$. Then,

$$
\begin{aligned}
& y_{\pi_{1}}=1, \\
& z_{\pi_{1} s_{M}}=1, \\
& y_{\pi_{2}}=1, \\
& y_{\pi_{j}}-\sum_{k=2}^{j-1} z_{\pi_{k} s_{M}} \geq 3-j,
\end{aligned}
$$

In the case $\frac{\ell_{s_{M}}}{2}=w_{\text {path }}^{T}$, we may fix $\pi_{1} \in[n] \backslash\left\{s_{M}\right\}$ in row 1 to the left of $s_{M}$, see equations (31)-(32), and in addition, we fix $\pi_{2} \in[n] \backslash\left\{s_{M}\right\}$ to row 1 , see equation (33). If $\pi_{2}$ is to the left of $s_{M}$, we fix another department to row 1 until one department is to the right of $s_{M}$, see inequalities (34). Note that inequalities (34) are redundant for $j \in[n-1], j \geq 3$, if $z_{\pi_{k} s_{M}}=0$ for some $k \in[n] \backslash\left\{s_{M}\right\}, 2 \leq k<j$.

Further, in an optimal $s_{M}$-T-row layout at least one department is contained in row 2 if $w_{\text {path }}^{T}=0$, so in this case we set

$$
\sum_{i \in[n] \backslash\left\{s_{M}\right\}} y_{i} \leq n-2
$$

\subsection{Cutting Planes}

In the following, let $s_{M} \in[n]$ be fixed. In this section we describe further inequalities to strengthen our formulation for the $s_{M}$-TRFLP. Let $i, j, k \in[n] \backslash\left\{s_{M}\right\}, i<j<k$, be given. Remark again that in the SRFLP the sum of the corresponding three betweenness variables, i.e., $x_{i k j}+x_{j i k}+x_{i j k}$, equals one, see equations (3), but might be equal to zero in the $s_{M}$-TRFLP. This is a significant difference because in the root relaxation of our branch-and-cut algorithm the value of the betweenness variables might be close to zero. Therefore, in this section, we present various lower bounds for the sum of the betweenness variables

Proposition 4. Let $s_{M} \in[n]$. The following inequalities are valid for the $s_{M}$-TRFLP

$$
\begin{array}{ll}
x_{i k j}+x_{j i k}+x_{i j k}+x_{i s_{M} j}+x_{i s_{M} k}+x_{j s_{M} k} \geq 1, & i, j, k \in[n] \backslash\left\{s_{M}\right\}, i<j<k, \\
x_{i k j}+x_{j i k}+x_{i j k}+x_{i s_{M} k}+x_{j s_{M} k}-y_{k} \geq 0, & i, j, k \in[n] \backslash\left\{s_{M}\right\},|\{i, j, k\}|=3, i<j, \\
x_{i k j}+x_{j i k}+x_{i j k}-y_{i}-y_{j}-y_{k} \geq-2, & i, j, k \in[n] \backslash\left\{s_{M}\right\}, i<j<k .
\end{array}
$$


Proof. Let $S:=\{i, j, k\} \subseteq[n] \backslash\left\{s_{M}\right\},|\{i, j, k\}|=3, i<j$, be given. Note first that inequalities (36)-(38) are satisfied if $x_{i k j}+x_{i j k}+x_{j i k}$ equals one. It turns out that $x_{i k j}+x_{i j k}+x_{j i k}$ equals zero if and only if $s_{M}$ lies between two departments of the set $S$ and the remaining department in $S$ lies in row 2. Otherwise, two or three departments are left or right to $s_{M}(n+2)$ and then by inequalities (19) one of the betweenness variables equals one. Thus, inequalities (36) are valid. Consider inequalities (37) with $y_{k}=1$ and let the sum of the corresponding betweenness variables be equal to zero, then $s_{M}$ lies between $i$ and $k$ or between $j$ and $k$. Inequalities (37) are satisfied if $y_{k}=0$. Given three departments which are assigned to row 1 , then the sum of the corresponding betweenness variables equals one, see inequalities (38).

Note that inequalities (36)-(37) are not valid for betweenness variables known in literature and in Section 5 we demonstrate that inequalities (36)-(37) significantly improve the performance of our branch-and-cut algorithm.

In the SRFLP we obtain $\sum_{i, j, k \in[n]} x_{i k j}=\left(\begin{array}{l}n \\ 3\end{array}\right)$ by equations (3). However, this equation is not $i<j$
$i \neq k \neq j$

valid for the $s_{M}$-TRFLP, and thus we present lower bounds for the sum of the betweenness variables in the $s_{M}$-TRFLP distinguishing whether $s_{M}$ or $n+2$ are contained or not.

Proposition 5. Let $s_{M} \in[n]$ and $S \subseteq[n] \backslash\left\{s_{M}\right\},|S| \geq 4$. Then, the following inequality is valid for the $s_{M}-T R F L P$

$$
\sum_{\substack{i, j \in S \\
i<j}}\left(x_{i_{s_{M}} j}+x_{s_{M} i j}+x_{s_{M} j i}+x_{(n+2) i j}+x_{(n+2) j i}\right) \geq\left(\begin{array}{c}
{\left[\frac{|S|}{2}\right\rceil} \\
2
\end{array}\right)+\left(\begin{array}{c}
\left\lfloor\frac{|S|}{2}\right\rfloor \\
2
\end{array}\right),
$$

and for $S \subseteq[n] \backslash\left\{s_{M}\right\}, n_{1}=\left\lceil\frac{|S|}{3}\right\rceil, n_{2}=\left\lfloor\frac{|S|}{3}\right\rfloor, n_{3}=|S|-n_{1}-n_{2}$, the following inequality

$$
\sum_{\substack{i, j, k \in S \\
i<j \\
i \neq k \neq j}} x_{i k j} \geq\left(\begin{array}{c}
|S| \\
3
\end{array}\right)-n_{1} \cdot n_{2} \cdot n_{3}
$$

is valid for the $s_{M}-T R F L P$.

Proof. Let $S \subseteq[n] \backslash\left\{s_{M}\right\},|S| \geq 4$. Recall that, given a $s_{M}$-T-row layout, $B_{1}\left(B_{2}\right)$ denotes the set of departments left (right) to $s_{M}$ and $B_{3}$ denotes the set of departments in row 2 . Then, we obtain

$$
\sum_{\substack{i, j \in S \\
i<j}}\left(x_{i_{s_{M}} j}+x_{s_{M} i j}+x_{s_{M} j i}+x_{(n+2) i j}+x_{(n+2) j i}\right)=\left(\begin{array}{c}
\left|B_{1}\right|+\left|B_{2}\right| \\
2
\end{array}\right)+\left(\begin{array}{c}
\left|B_{3}\right| \\
2
\end{array}\right),
$$

if $\left|B_{1}\right|+\left|B_{2}\right| \geq 2,\left|B_{3}\right| \geq 2$ (otherwise we can neglect the corresponding binomial coefficient on the right-hand side). Interpreting $B^{\prime}=B_{1} \cup B_{2}$ and computing the minimum value of the right-hand side subject to $B^{\prime} \cup B_{3}=S, B^{\prime}, B_{3} \subseteq S, B^{\prime} \cap B_{3}=\emptyset$, we obtain the desired result, see inequalities (39).

Now we consider inequalities (40), so let $S \subseteq[n] \backslash\left\{s_{M}\right\}$ be given. We are given a $s_{M}$-T-row layout, then $x_{i k j}+x_{i j k}+x_{j i k}, i, j, k \in[n], i<j<k$, equals zero if and only if $i, j$ and $k$ lie pairwise in distinct sets $B_{1}, B_{2}$ and $B_{3}$. So we obtain

$$
\sum_{\substack{i, j, k \in S \\
i<j \\
i \neq k \neq j}} x_{i k j}=\left(\begin{array}{c}
|S| \\
3
\end{array}\right)-\left|B_{1}\right| \cdot\left|B_{2}\right| \cdot\left|B_{3}\right| \cdot
$$

Computing the minimum value of the right-hand side is equivalent to maximize $\left|B_{1}\right| \cdot\left|B_{2}\right| \cdot\left|B_{3}\right|$ subject to $B_{1} \cup B_{2} \dot{\cup} B_{3}=S, B_{1}, B_{2}, B_{3} \subseteq S, B_{1} \cap B_{2} \cap B_{3}=\emptyset$, and thus we obtain the desired result. 
In addition, we are able to adapt inequalities (39) to the case with $|S|=3$ in the following way

$$
\sum_{\substack{i, j \in S \\ i<j}}\left(x_{(n+2) i j}+x_{(n+2) j i}\right)+\sum_{i \in S} y_{i} \geq 2, \quad S \in[n] \backslash\left\{s_{M}\right\},|S|=3 .
$$

Let $S \subseteq[n] \backslash\left\{s_{M}\right\},|S|=3$, and a $s_{M}$-T-row layout be given. Inequalities (41) are satisfied if at least two departments of the set $S$ are in row 1 . So let two (three) departments be in row 2 . Then, $\sum_{\substack{i, j \in S \\ i<j}} x_{(n+2) i j}+x_{(n+2) j i}=1(=3)$ and inequalities (41) are satisfied.

Next we consider the special case of inequalities (40) with $|S|=4, S \subseteq[n] \backslash\left\{s_{M}\right\}$, and we obtain $\sum_{\substack{i, j, k \in S \\ i \neq k \neq j \\ i<j}} x_{i k j} \geq 2$. This lower bound is tight if and only if each of the sets $B_{1}, B_{2}$ and $B_{3}$ contains at least one department of the set $S$, because otherwise $S \subseteq B_{a} \cup B_{b}, a, b \in\{1,2,3\}$, and hence the sum of the betweenness variables of the departments in $S$ equals 4 . So we present further lower bounds for the case with $|S|=4$.

Proposition 6. Let $s_{M} \in[n]$ and $S \subseteq[n] \backslash\left\{s_{M}\right\},|S|=4$. Then, the following inequalities are valid for the $s_{M}-T R F L P$

$$
\begin{aligned}
& \sum_{\substack{i, j, k \in S \\
i<j}} x_{i k j}+\sum_{i \in S} y_{i} \geq 4 \\
& i \neq k \neq j \\
& \sum_{\substack{i, j, k \in S \\
i<j}} x_{i k j}-\sum_{i \in S} y_{i} \geq-1, \\
& i \neq k \neq j \\
& \sum_{\substack{i, j, k \in S \\
i<j}} x_{i k j}-\sum_{s \in\left\{s_{M}, n+2\right\}} 2\left(x_{s p q}+x_{s q p}+x_{s p t}+x_{s t p}\right) \geq 0, \quad p, q, t \in S,|\{p, q, t\}|=3, q<t, \\
& i \neq k \neq j \\
& \sum_{\substack{i, j, k \in S \\
i<j}} x_{i k j}+\sum_{\substack{i, j \in S \\
i<j}} x_{i_{M} j}-\sum_{i \in S} y_{i}+x_{s_{M} q p}+x_{s_{M} p q} \geq 1, \quad p, q \in S, p<q .
\end{aligned}
$$

Proof. Let $S=\{i, j, k, h\} \subseteq[n] \backslash\left\{s_{M}\right\},|S|=4$, and let a $s_{M}$-T-row layout be given. Recall that $B_{1}\left(B_{2}\right)$ denotes the set of departments left (right) to $s_{M}$ and $B_{3}$ denotes the set of departments in row 2. If $\sum_{p \in S} y_{p} \geq 2$, then inequalities (42) are satisfied by inequalities (40) with $|S|=4$. Otherwise, at most one department is assigned to row 1 . Then, $S \subseteq B_{a} \cup B_{3}, a \in\{1,2\}$, and thus the sum of the corresponding betweenness variables equals 4 . Inequalities (43) are satisfied by inequalities (40) if at most three departments are assigned to row 1. Otherwise, all departments are in row 1 and inequalities (43) are satisfied. Inequalities (44) ensure that if three departments lie in the same set $B_{a}, a \in\{1,2,3\}$, then the sum of the corresponding betweenness variables is equal to 4 . Otherwise, inequalities (44) are satisfied by inequalities (40).

It remains to consider inequalities (45), let $p, q \in S, p<q$. If at most one department is assigned to row 1 , then inequalities (45) are satisfied by inequalities (40). So let two (three) departments be in row 1, and we assume at first, that the departments are, w. l.o.g., left to $s_{M}$. Then, $S \subseteq B_{1} \cup B_{3}$ and the sum of the corresponding betweenness variables equals 4 . Otherwise we obtain $\sum_{\substack{i, j \in S \\ i<j}} x_{i s_{M} j}=1\left(\sum_{\substack{i, j \in S \\ i<j}} x_{i s_{M} j}=2\right)$, and thus inequalities (45) are satisfied. It remains to consider the case where all departments are assigned to row 1 . Then, the sum of the corresponding betweenness variables equals 4 and in addition, we get by inequalities (11) $x_{p s_{M} q}+x_{s_{M} q q}+x_{s_{M} q p}=1$, which proves the desired result.

In the next proposition, we show that inequalities from the SRFLP can be used for the $s_{M}$-TRFLP, although an extension of the betweenness variables is used. The slightly adapted inequalities (46)-(47) and inequalities (49) were introduced in [3] for the SRFLP. 
Proposition 7. Let $s_{M} \in[n]$. The following inequalities are valid for the $s_{M}-T R F L P$

$$
\begin{array}{ll}
-x_{i h j}+x_{i h k}+x_{j h k} \geq 0, & i, j, k, h \in[n] \backslash\left\{s_{M}\right\},|\{i, j, k, h\}|=4, \\
x_{i h j}+x_{i h k}+x_{j h k} \leq 2, & i, j, k, h \in[n],|\{i, j, k, h\}|=4, i<j<k, h \neq\left\{s_{M}\right\}, \\
& \\
x_{i_{s_{M}}}+x_{i s_{M} k}-x_{s_{M} k j}-x_{s_{M} j k}-y_{i} \leq 0, & i, j, k \in[n] \backslash\left\{s_{M}\right\}, i \neq j, j<k .
\end{array}
$$

Let $\beta \geq 6$ be an even integer, $k \in S \subseteq[n] \backslash\left\{s_{M}\right\}$ and let $S_{1}, S_{2} \subseteq S \backslash\{k\}$ with $\left|S_{1}\right|=\frac{\beta}{2}$ such that $S_{1} \cup S_{2}=S \backslash\{k\}, S_{1} \cap S_{2}=\emptyset$. Then, the following inequalities

$$
\sum_{\substack{i, j \in S_{1} \\ i<j}} x_{i k j}+\sum_{\substack{i, j \in S_{2} \\ i<j}} x_{i k j}-\sum_{\substack{i \in S_{1} \\ j \in S_{2}}} x_{i k j} \leq 0,
$$

are valid for the $s_{M}-T R F L P$.

The proof of the correctness of inequalities (49) for the $s_{M}$-TRFLP is an extension of the proof of [41] for the correctness of inequalities (49) for the SRFLP.

Proof. Let $i, j, k, h \in[n] \backslash\left\{s_{M}\right\},|\{i, j, k, h\}|=4$, and a $s_{M}$-T-row layout be given. Recall that $B_{1}$ $\left(B_{2}\right)$ denotes the set of departments in row 1 left (right) to $s_{M}$ and $B_{3}$ the set of departments in row 2. If $i, j, k, h \in B_{a} \cup B_{b}, a, b \in\{1,2,3\}$, inequalities (46) are satisfied since these inequalities are valid for the SRFLP. If $x_{i h j}=0$, then inequalities (46) are redundant, so it remains to consider the case $x_{i h j}=1$. We assume, w.l.o.g., $i, h \in B_{a}, j \in B_{b}, a, b \in\{1,2,3\}, a \neq b$, and $k \in B_{c}, c \in\{1,2,3\} \backslash\{a, b\}$. It follows immediately that $x_{i h k}=1$, see inequalities (19), and thus inequalities (46) are satisfied.

Next we consider inequalities (47). Let a $s_{M}$-T-row layout and $i, j, k, h \in[n],|\{i, j, k, h\}|=$ $4, i<j<k, h \neq s_{M}$, be given. We shift the departments in the set $B_{2} \cap\{i, j, k, h\}$ to the left of $s_{M}$ without changing the order of the departments $B_{1} \cup\left(B_{2} \cap\{i, j, k, h\}\right)$. By this method we only increase the sum of the corresponding betweenness variables. Then, $i, j, k, h \in B_{1} \cup B_{3}$ and inequalities (47) are satisfied because these inequalities are valid for the SRFLP. Now we investigate inequalities (48). Let $i$ lie in row 1 , let $s_{M}$ lie between $i$ and $j$ and let $s_{M}$ lie between $i$ and $k$. Then, $k$ lies between $s_{M}$ and $j$ or $j$ lies between $k$ and $s_{M}$, see inequalities (48). Otherwise, inequalities (48) are redundant.

Next we consider inequalities (49). We are given a $s_{M}$-T-row layout and let $k \in S \subseteq[n] \backslash\left\{s_{M}\right\}$ lie in row 1, w.l.o.g., left to $s_{M}$. Let $\beta \geq 6$ be an even integer and let $S_{1}, S_{2} \subseteq S \backslash\{k\},\left|S_{1}\right|=$ $\frac{\beta}{2}, S_{1} \dot{\cup} S_{2}=S \backslash\{k\}, S_{1} \cap S_{2}=\emptyset$.

Then, we denote by $\ell^{1}\left(\ell^{2}\right)$ the number of departments in $S_{1}\left(S_{2}\right)$ left to $k$. Let $r^{1}\left(r^{2}\right)$ denote the number of departments right to $k$ in row 1 and let $u^{1}\left(u^{2}\right)$ denote the number of departments in $S_{1}\left(S_{2}\right)$ in row 2 . Then

$$
\begin{aligned}
& \sum_{\substack{i, j \in S_{1} \\
i<j}} x_{i k j}+\sum_{\substack{i, j \in S_{2} \\
i<j}} x_{i k j}-\sum_{\substack{i \in S_{1} \\
j \in S_{2}}} x_{i k j} \\
& =\ell^{1}\left(r^{1}+u^{1}\right)+\ell^{2}\left(r^{2}+u^{2}\right)-\ell^{1}\left(r^{2}+u^{2}\right)-\ell^{2}\left(r^{1}+u^{1}\right) \\
& =\left(\ell^{1}-\ell^{2}\right)\left(r^{1}+u^{1}-r^{2}-u^{2}\right) \leq 0,
\end{aligned}
$$

where the last inequality is satisfied since $\ell^{1}+r^{1}+u^{1}=1+\ell^{2}+r^{2}+u^{2}$. The proof is similar if $k$ lies in row 2.

\subsection{Heuristic approaches}

Let $s_{M} \in[n]$. In this section we describe heuristic approaches for the $s_{M}$-TRFLP which are applied in Algorithm 1. We use five heuristics to compute start layouts for the $s_{M}$-TRFLP. Afterwards we apply exchange approaches to further improve these start layouts. 
We fix the center of $s_{M} \in[n]$ on position $p_{M}$ in row 1 , and then iteratively add the remaining departments to the layout, see [30] for a related heuristic approach for the DRFLP. Let $B_{1}\left(B_{2}\right)$ denote the set of departments left (right) to $s_{M}$ and $B_{3}$ the set of departments in row 2 in the current constructed layout. We set $\bar{\ell}_{i}:=\frac{\ell_{s_{M}}}{2}+\sum_{j \in B_{i}} \ell_{j}, i=1,2, \bar{\ell}_{3}:=w_{\text {path }}^{T}+\sum_{j \in B_{3}} \ell_{j}$ and $a:=\arg \min \left\{\bar{\ell}_{1}, \bar{\ell}_{2}, \bar{\ell}_{3}\right\}$. In the first heuristic we sort the departments $[n] \backslash\left\{s_{M}\right\}$ in ascending order according to their lengths. The sorted departments are sequentially added space-free to the layout, if $a=1$ at the leftmost position in row 1 and otherwise at the rightmost position in row 1 or row 2, respectively, see Algorithm 2.

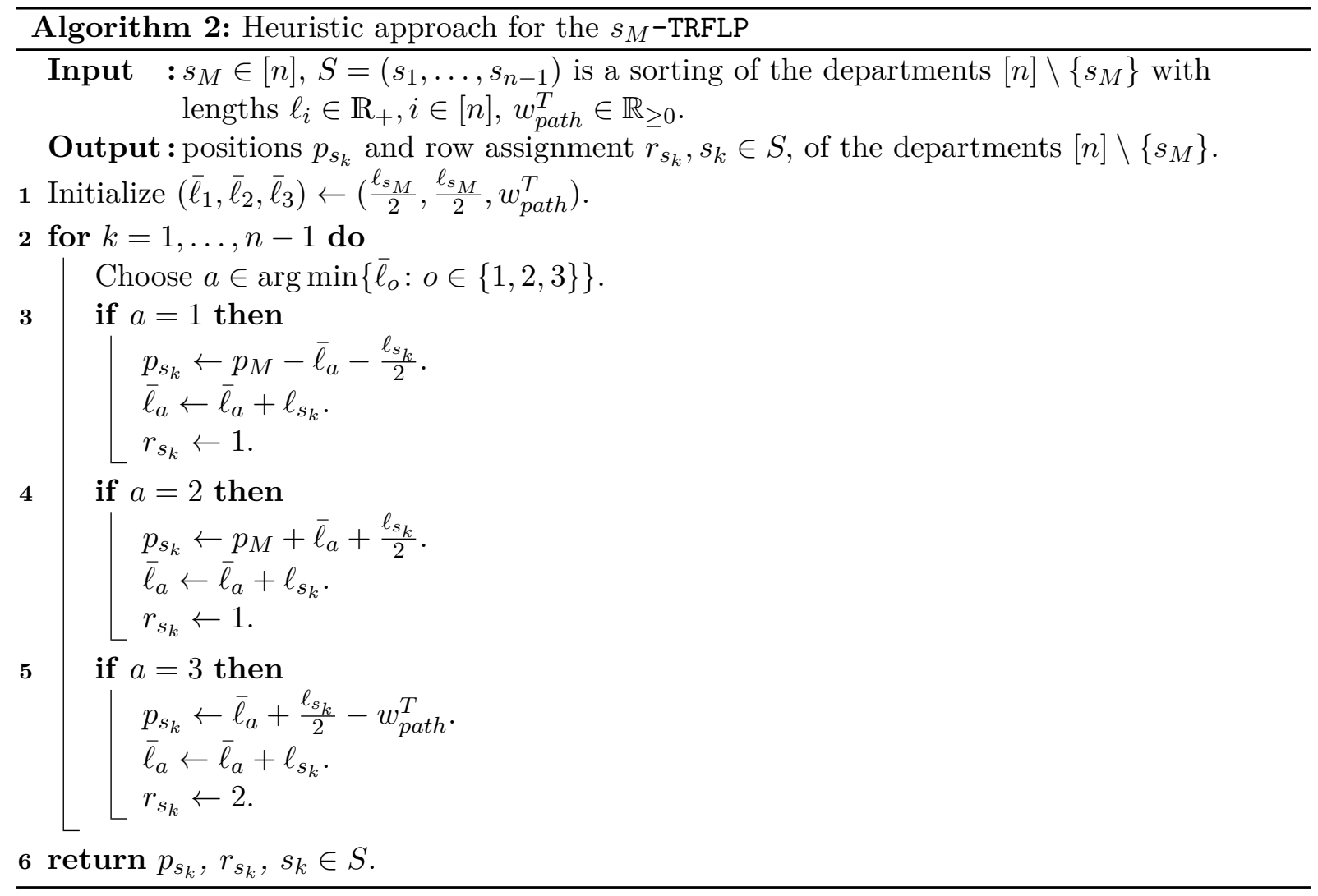

In the following heuristic approaches we assign the departments in the following way. Let, w.l.o.g., the departments $1, \ldots, j \in[n] \backslash\left\{s_{M}\right\}$ be assigned to the $s_{M}$-T-row layout and let, $R_{1}=\left(1,2, \ldots, s_{M}, \ldots, i\right), i \in[n] \backslash\left\{s_{M}\right\}, i \leq j,\left(R_{2}=(i+1, \ldots, j)\right)$ denote the set of departments assigned to row 1 (row 2), as well as the sorting of the departments in row 1 (row 2). We assign $h \in[n], h>j$, on all possible positions in $R_{1}$ and $R_{2}$ and then we choose a layout with minimal objective value, i. e., $h$ is assigned at its current best position (note that the center of $h$ is not assigned on position $p_{M}$ in row 1 ).

In our second heuristic approach we sort the departments in ascending order according to their lengths and, in contrast to the first heuristic, in each step a department is placed at its current best position. Next we divide the departments $[n] \backslash\left\{s_{M}\right\}$ into two sets depending whether $w_{i s_{M}}=0$ or $w_{i s_{M}}>0, i \in[n] \backslash\left\{s_{M}\right\}$, i. e., $S_{1}:=\left\{i \in[n] \backslash\left\{s_{M}\right\}: w_{i s_{M}}=0\right\}$ and $S_{2}:=\left\{i \in[n] \backslash\left\{s_{M}\right\}: w_{i s_{M}}>0\right\}$. Then, we sort the departments in each set in decreasing order according to their relative weights, i. e., $\sum_{\substack{j \in[n] \\ i \neq j}} \frac{w_{i j}}{\ell_{i}}, i \in[n] \backslash\left\{s_{M}\right\}$. In the third (fourth) heuristic we assign the departments in the set $S_{2}\left(S_{1}\right)$, and afterwards the departments in the set $S_{1}\left(S_{2}\right)$ in the determined order to the layout. In each step the considered department is assigned at its current best position.

In [35] a good or optimal single-row layout is given and according to different rules a double-row layout is constructed. Given an optimal or near-optimal single-row layout, in the fifth heuristic 
we assign departments in that order space-free to row 1, where the center of $s_{M}$ is fixed on position $p_{M}$ in row 1 . In the first step we arrange one department to row 2 which reduces the objective value of the current layout at most. Then, in further steps, we choose one department from row 1 which reduces the objective value at most when we assign it on its best position in row 2 , and we arrange it at that position. We apply these steps until we do not improve the objective value by rearranging one department from row 1 to row 2 .

After determining start layouts, we use a 1-opt and a 2-opt improvement heuristic. At first we apply a 1-opt heuristic where we place in each step one department on its current best position. Afterwards, in our 2-opt heuristic, we simply exchange the position of two departments. Whenever we obtain a better solution during the opt heuristics, we swap the departments and we apply the $k$-opt heuristic until the layout is $k$-optimal, $k \in\{1,2\}$.

\subsection{Separation}

In this section we describe three variants for branch-and-cut algorithms based on inequalities (7)-(21) and inequalities (29)-(49). In the first variant we include inequalities (7),(9)-(16), (18)-(19), (35) and inequalities (39) with $S=[n] \backslash\left\{s_{M}\right\}$ from the beginning. Due to equations (8) we can eliminate $n-1$ ordering variables by setting

$$
z_{s_{M} i}=y_{i}-z_{i s_{M}}, \quad i \in[n] \backslash\left\{s_{M}\right\} .
$$

The sorting of the departments in our symmetry breaking constraints $(29)-(30)$ or, if $\frac{\ell_{s_{M}}}{2}=w_{\text {path }}^{T}$, inequalities (31)-(34), is determined in the following way. We choose a heuristically determined $s_{M^{-}}$-T-row layout with minimal objective value, see Section 3.4 , and denote $B_{1}\left(B_{2}\right)$ as the set of departments left (right) to $s_{M}$ and let $B_{3}$ denote the set of departments in row 2 . Then, we compute $v(i):=\sum_{\substack{j \in B_{i} \\ k \in[n] \\ k \neq j}} \frac{w_{j k}}{\ell_{k}}, i \in\{1,2\}$, and we set $a:=\arg \min \{v(1), v(2)\}$. In inequalities (29)-(30) we assign the departments in the set $B_{a}$ first, then $B_{b}, b \in\{1,2\} \backslash\{a\}$, and at last $B_{3}$ where the departments in each set $B_{m}, m \in\{1,2,3\}$, are sorted in ascending order according of their positions, i. e., we start with the leftmost department of each set.

In the next part we describe the separation of the remaining inequalities. We only add inequalities for a set $S \subseteq[n]$ which are violated (at least) by the constant 0.4 . We separate inequalities (20)-(21) and inequalities (41) by brute-force enumeration.

Given a relaxation $\bar{x}, \bar{z}$ and $\bar{y}$. Preliminary tests indicate to separate equations (17) if $i, j \in$ $[n] \backslash\left\{s_{M}\right\}, i<j$, lie in the same row, hence, due to equations (16), we add equations (17) if $\bar{x}_{i s_{M} j}+\bar{x}_{s_{M} i j}+\bar{x}_{s_{M j i}}-\bar{x}_{(n+2) i j}-\bar{x}_{(n+2) j i}<-0.4$.

Let $i, j, k \in[n] \backslash\left\{s_{M}\right\}, i<j<k$, be given. Inequalities (36)-(38) are mainly used for computing lower bounds for $x_{i k j}+x_{i j k}+x_{j i k}$, so, given a relaxation $\bar{x}, \bar{y}$, we compute

$$
\max \left\{\begin{array}{l}
1-\bar{x}_{i s_{M} j}-\bar{x}_{i s_{M} k}-\bar{x}_{j s_{M} k}, \\
-2+\bar{y}_{i}+\bar{y}_{j}+\bar{y}_{k}, \\
\bar{y}_{k}-\bar{x}_{i s_{M} k}-\bar{x}_{j s_{M} k}, \\
\bar{y}_{j}-\bar{x}_{i s_{M} j}-\bar{x}_{k s_{M} j}, \\
\bar{y}_{i}-\bar{x}_{k_{s_{M} i}}-\bar{x}_{j_{s_{M} i} .}
\end{array}\right.
$$

Then, we add one of the five inequalities (36)-(38) with $S=\{i, j, k\}$ where the maximum value above is attained. Recall that we only add the inequality if it is violated by at least 0.4 .

In variant 2 of our separation strategy we use the same separation strategy as in variant 1 but we neglect inequalities (36)-(37) in order to verify that these inequalities improve the performance of our branch-and-cut algorithm.

In variant 3 we use the same strategy as in variant 1 , and additionally we add inequalities (40) with $S=[n] \backslash\left\{s_{M}\right\}$ from the beginning and we separate inequalities (40) by brute-force 
enumeration for $S \subseteq[n] \backslash\left\{s_{M}\right\}$ with $|S|=4,5,6,7$, where we only add inequalities which are violated by at least 1,2,4 or 8 , respectively. We separate inequalities (42)-(48) by brute-force enumeration where we only add inequalities (42)-(45) and inequalities (46)-(48) violated by at least 1 or 0.5 , respectively. We separate inequalities (49) with $\beta=6$ by complete brute-force enumeration and we only add inequalities violated by at least 2 .

\section{The Multi-Bay Facility Layout Problem with three rows}

The current known MILP models for the MBFLP and its extensions, are not able to solve instances with 12 departments in reasonable time $[29,59]$, so we describe the adaption of our MILP model for the $s_{M}$-TRFLP, $s_{M} \in[n]$, to the 3-BFLP.

Given a T-row instance, we add an additional dummy department $n+3$ with length $\ell_{n+3}=$ $2 w_{\text {path }}^{B}$ and weights $w_{i(n+3)}=w_{(n+3) i}=0, i \in[n+2]$, to our model and we fix the center of the dummy department $n+3$ on position $p_{M}$ in row 1 , the obtained problem is denoted by $((n+3)$-TRFLP $)$. We use the following connecting between the 3-BFLP and the $(n+3)$-TRFLP. Let an optimal $(n+3)$-T-row layout be given with $w_{\text {path }}^{T}=0$. We assign the departments in the T-row layout to the left (right) of $n+3$ to row 1 (row 3 ) in the 3 -Bay layout and the departments in row 2 in the T-row layout to row 2 in the 3-Bay layout without changing the order of the departments in the same row. Hence, we obtain an optimal 3-Bay layout and vice versa. An immediate consequence is the following

Proposition 8. Given a 3-BFLP instance with $w_{\text {path }}^{B} \in \mathbb{R}_{\geq 0}$ and a dummy department with length $\ell_{n+3}=2 w_{\text {path }}^{B}$ and weights $w_{i(n+3)}=w_{(n+3) i}=0, i \in[n+2]$. Then, the 3-BFLP is equivalent to the $(n+3)-T R F L P$ with $w_{\text {path }}^{T}=0$.

Now we compare the optimal values of the SRFLP, the TRFLP and the 3-BFLP. Note first that if $w_{\text {path }}^{T} \leq w_{\text {path }}^{B}$, then the optimal value of the TRFLP is less than or equal to the optimal value of the $(n+3)$-TRFLP with $w_{\text {path }}^{T}=0$, and thus smaller than or equal to the optimal value of the 3-BFLP by Proposition 8. In the following, we consider an instance which cannot be divided into two or more smaller independent instances. If $w_{\text {path }}^{T} \leq w_{\text {path }}^{B}$ and $w_{\text {path }}^{B} \geq 1$, then the optimal value of the TRFLP is equal to the optimal value of the 3-BFLP if and only the optimal value of the TRFLP is equal to the optimal value of the SRFLP. If $w_{p a t h}^{T}<\frac{\max _{i \in[n]} \ell_{i}}{2}$, then the optimal value of the TRFLP is smaller than the optimal value of the SRFLP. So for $w_{\text {path }}^{T}=0$ and $w_{\text {path }}^{B}=1$, the optimal value of the TRFLP is smaller than the optimal value of the 3-BFLP. However, one can construct instances such that the optimal value of the TRFLP is equal to the optimal value of the SRFLP if $w_{\text {path }}^{T} \geq \frac{\max _{i \in[n]} \ell_{i}}{2}$.

One main difference between solving the TRFLP and the 3-BFLP with our approach is that for the 3-BFLP only one MILP model has to be solved. We adapt our MILP model for the $s_{M}$-TRFLP, $s_{M} \in[n]$, to the $(n+3)$-TRFLP with $w_{p a t h}^{T}=0$ and we only describe the differences in the following. We use the $z, y$ and $x$ variables in the same manner as above including the dummy department $n+3$, i. e., we define $z_{i j}$ for $i, j \in[n] \cup\{n+3\}, i \neq j$, and $x_{i k j}$ with $i, j, k \in[n+3], i<j,|\{i, j, k\}|=3$. We neglect inequality (35). The distances between the $i \in[n]$ and $j \in[n], i<j$, are calculated as described in (23), see (22) for the calculation of the excluded constant. We are able to use symmetry breaking constraints (29)-(30) from the $s_{M}$-TRFLP and the departments are sorted as described in Section 3.5.

To further strengthen our branch-and-cut algorithm, we use the following inequalities for the $(n+3)$-TRFLP where inequalities $(50)$ are used in $[6,76]$ for the DRFLP

$$
\begin{array}{ll}
z_{i j}+z_{j i}+z_{i k}+z_{k i}+z_{j k}+z_{k j} \geq 1, & i, j, k \in[n], i<j<k, \\
x_{i k j}+x_{i j k}+x_{j i k}+x_{i(n+3) j}-y_{k} \leq 1, & i, j, k \in[n], i<j<k .
\end{array}
$$


Given three departments, at least two of them lie in the same row, see inequalities (50). Let $i, j, k \in[n], i<j<k$, and let $y_{k}=0$, then either the sum of the corresponding three betweenness variables equals zero or $n+3$ does not lie between $i$ and $j$, see inequalities (51). Inequalities (51) are redundant if $y_{k}=1$, see inequalities $(21)$.

In order to get upper bounds on the optimal value of the $(n+3)$-TRFLP, we use the first three heuristic approaches for the $s_{M}$-TRFLP, $s_{M} \in[n]$, presented in Section 3.4. Since $w_{i(n+3)}=$ $w_{(n+3) i}=0, i \in[n]$, the fourth heuristic equals the third heuristic so we exclude it. In the fifth heuristic, we arrange the dummy department $n+3$ to the right of the $\left\lfloor\frac{n}{2}\right\rfloor$ leftmost department in a given single-row layout. Then, we proceed as described in the fifth heuristic.

We mainly use the same separation strategy for the $(n+3)$-TRFLP as for the $s_{M}$-TRFLP, $s_{M} \in[n]$, so we only describe the differences here. We include inequalities (20) from the beginning if $w_{\text {path }}^{B}>1$, otherwise we separate inequalities (20) as described in Section 3.5. We separate inequalities (50) by brute-force enumeration. Given a relaxation $\bar{x}, \bar{y}$ and $i, j, k \in[n], i<j<k$, we separate inequalities (21) and inequalities (51) in the following way. We calculate

$$
\min \left\{\begin{array}{l}
1 \\
\bar{y}_{k}-\bar{x}_{i(n+3) j}, \\
\bar{y}_{j}-\bar{x}_{i(n+3) k}, \\
\bar{y}_{i}-\bar{x}_{j(n+3) k},
\end{array}\right.
$$

and we add one of the four corresponding inequalities where the minimum value is attained.

\section{Computational Experiments}

In this section we present our computational results. The computational experiments are implemented in $\mathrm{C}++$, and we use Cplex 12.9 as an MILP Solver [57]. All results were conducted on a $2.10 \mathrm{GHz}$ quad-core using Virtual Box 6 running on Debian GNU/Linux 8 in single processor mode. In all tests Cplex generated cuts are not added and we set $w_{\text {path }}^{T}+1=w_{\text {path }}^{B}$, as discussed in the introduction.

\subsection{Computational Results for the TRFLP and the 3-BFLP}

In this paper we focus on the TRFLP and the 3-BFLP, so applying Proposition 1 is left for future work. At first, we describe the usage of our heuristic approaches in Algorithm 1. For $s_{M} \in[n]$, we determine five start layouts with our heuristics for the $s_{M}$-TRFLP and we apply a 1-opt and 2-opt improvement heuristic on each start layout. Our heuristic approach for the TRFLP simply chooses a $s_{M}$-T-row layout with minimal objective value, $s_{M} \in[n]$, and the minimal objective value is used as an upper bound in Algorithm 1. The single-row instances in the fifth heuristic were solved to optimality using the model summarized in Section 2. We generate new instances which are larger than literature instances in the same way as described in [7]. All instances are available from the author.

In the first two columns in Table 1 the instance, whereby the number denotes the number of departments in that instance, and its source is given. In the next three columns we compare the optimal solution values of the SRFLP, the 3-BFLP and the TRFLP, and we write "TL" if the time limit of 8 hours is exceeded. If the 3 -BFLP is not solved to optimality, the obtained lower bound is displayed and marked with " ". Our heuristically determined upper bounds for the

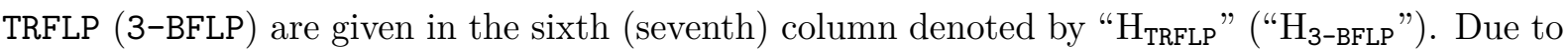
computational accuracy, some $z_{i j} \in[0,1], i, j \in[n] \backslash\left\{s_{M}\right\}, j>i$, achieve values with very small gaps to zero or one. Hence, the values in Table 1, Table 3 and Table 4 are optimal neglecting computational accuracy. The corresponding values are rounded up such that $2 \cdot O P T(\mathcal{I}) \in \mathbb{Z}$ for T-row and 3-Bay instances $\mathcal{I}$ since $w_{i j} \in \mathbb{Z}, i, j \in[n], i<j$. 


\begin{tabular}{lrrrrrr} 
Instances & Source & SRFLP & 3-BFLP & TRFLP & $\mathrm{H}_{3 \text {-BFLP }}$ & $\mathrm{H}_{\text {TRFLP }}$ \\
\hline$A m 11 a$ & {$[9]$} & 10630.5 & 8795.5 & 8407.0 & 8814.5 & 8411.5 \\
$A m 11 b$ & {$[9]$} & 7375.5 & 6021.5 & 5665.0 & 6021.5 & 5667.5 \\
$A m 12 a$ & {$[5,6]$} & 2901.0 & 2508.0 & 2354.5 & 2515.0 & 2354.5 \\
$A m 12 b$ & {$[5,6]$} & 3280.5 & 2691.5 & 2539.5 & 2697.5 & 2548.5 \\
$A m 13 a$ & {$[5]$} & 4902.5 & 4021.5 & 3836.0 & 4204.5 & 3836.0 \\
$A m 13 b$ & {$[5]$} & 5698.0 & 4529.0 & 4362.5 & 4529.0 & 4362.5 \\
$A m 14 \_1$ & {$[43]$} & 5481.5 & 4560.5 & 4350.5 & 4565.5 & 4358.0 \\
$A m 14 a$ & {$[76]$} & 5673.0 & 4687.0 & 4446.5 & 4734.0 & 4448.0 \\
$A m 14 b$ & {$[76]$} & 5595.0 & 4665.0 & 4430.5 & 4683.0 & 4433.0 \\
$A m 15$ & {$[1]$} & 6305.0 & 5291.0 & 5071.0 & 5294.0 & 5079.0 \\
$H K 15$ & {$[49]$} & 33220.0 & 26494.0 & 26124.0 & 26495.0 & 26125.0 \\
$P 16 a$ & {$[7]$} & 14829.0 & 12287.5 & 11943.0 & 12326.0 & 11943.0 \\
$P 16 b$ & {$[7]$} & 11878.5 & 9781.0 & 9469.5 & 9784.5 & 9469.5 \\
$P 17 a$ & new & 14436.5 & 11852.0 & 11524.5 & 11888.5 & 11528.0 \\
$P 17 b$ & new & 15682.0 & 12691.5 & 12317.0 & 12874.0 & 12389.0 \\
$A m 17$ & {$[2]$} & 9254.0 & 7647.0 & 7315.0 & 7690.0 & 7318.0 \\
$P 18 a$ & new & 16118.5 & 12022.0 & TL & 12863.5 & 12516.0 \\
$P 18 b$ & new & 17716.5 & 12972.5 & TL & 14616.5 & 14072.0 \\
$A m 18$ & {$[2]$} & 10650.5 & 7990.5 & 8413.5 & 8835.5 & 8413.5 \\
\hline
\end{tabular}

Table 1: Optimal values of the SRFLP, the 3-BFLP and the TRFLP as well as heuristically determined upper bounds are displayed for instances from the literature with $w_{\text {path }}^{T}=$ $0, w_{\text {path }}^{B}=1$. We write "TL" if the time limit of 8 hours is exceeded. Lower bounds for the 3-BFLP are marked with ", " if the time limit is exceeded or if we run out of memory storage. The optimal value of the TRFLP is up to $6.1 \%$ smaller than the optimal value of the 3-BFLP and up to $23.4 \%$ than the optimal value of the SRFLP.

In Table 1 we set $w_{\text {path }}^{T}=0$ and $w_{\text {path }}^{B}=1$. The optimal value of the TRFLP (3-BFLP) is between $18.8 \%$ and $23.4 \%$ (13.5\% and $20.5 \%$ ) smaller than the optimal value of the SRFLP, so the optimal values of the TRFLP and the 3-BFLP are significantly smaller than the optimal value of the SRFLP. Further, the optimal value of the TRFLP is between $1.4 \%$ and $6.1 \%$ smaller than the optimal value of the 3-BFLP, see Table 1. So for these instances the TRFLP is preferable to the 3-BFLP and the SRFLP. These reductions of the optimal values are remarkable since factories are built for a long period, and the rearrangement of the departments is expensive. For the TRFLP, the heuristic derives gaps with less than $1 \%$ to the optimal solution values and five optimal layouts. In addition, in eleven instances the department with its center arranged first on position $p_{M}$ in Algorithm 1 for the TRFLP has its center position on $p_{M}$ in the calculated optimal T-row layout. The heuristics were computed in less than one second. For the 3-BFLP the heuristic derives two optimal layouts and for the instance $A m 13 a$ the gap is around $4.4 \%$. For the remaining instances, the gaps are less than $1.5 \%$. The 3-Bay instances with 18 departments are not solved to optimality, but we obtain lower bounds with tight gaps to the heuristically determined upper bounds, i. e., the gaps are between $4.0 \%$ and $7.9 \%$.

In Table 2 we compare the running times of several approaches for the 3-BFLP and the TRFLP, given in sec, min:sec and h:min:sec. In column 2 we display the running time of the current best exact approach for the 3-BFLP by [43] where the 3-BFLP is solved with fixed row assignment and we enumerate over all distinguishable row assignments as suggested in [43]. Adapting this approach to the $s_{M}$-TRFLP and using Algorithm 1, we obtain an optimal solution for the TRFLP and the running time is displayed in column 4 . In both approaches, a heuristically determined upper bound is used. In the fifth, sixth and seventh column we display the running time of our separation variant $i \in[3]$, see Section 3.5, denoted by "MILP $i$ ". In the eights column we mainly use the same separation strategy as in variant 1 , but we use standard linearization, see inequalities (59)-(61) in the appendix, to couple our new extended betweenness variables to the ordering, 
assignment and betweenness variables containing dummy departments or $s_{M}$. This variant is denoted by variant 4 and we do not use the transitivity inequalities (19)-(20). Additionally, we set up a MILP model for the $s_{M}$-TRFLP which consists of betweenness variables which are only equal to one if all departments are in the same row (in this version, betweenness variables are known in the literature). The distances between $i \in[n] \backslash\left\{s_{M}\right\}$ and $j \in[n] \backslash\left\{s_{M}\right\}, i<j$, are calculated via big- $M$-constraints and the betweenness variables are coupled via standard linearization to the ordering variables. Note that the transitivity inequalities (19)-(20) are not valid for this MILP model. We refer to the appendix for a description and the running time of Algorithm 1 using this MILP model is summarized in the ninth column. We use a time limit of 8 hours and we write "TL" if the time limit is exceeded.

Considering the TRFLP, variant 1 clearly outperforms the other approaches because all considered T-row instances with 17 departments and even one instance with 18 departments were solved to optimality within the time limit of 8 hours. The second best approach is variant 2 where one T-row instance with 17 departments is solved to optimality. Note that inequalities (36)-(37), which are neglected in variant 2 , are not valid for betweenness variables used in the literature, i. e., betweenness variables which can only be equal to one if the three departments are in the same row. The running time of our approach using variant 2 is for almost all instances with at least 14 departments more than twice as high as the running time of our approach using variant 1. Thus, the inequalities (36)-(37) significantly improve the performance of our algorithm.

The $s_{M}$-TRFLP model summarized in the appendix is significantly weaker than variant 1 because standard linearization is used to couple the betweenness variables and the ordering variables and big- $M$-constraints are used to calculate distances between departments in distinct rows. Using this approach, only instances with up to 15 departments were solved to optimality within the time limit of 8 hours while in the approach of variant 1 instances with 15 departments were solved to optimality in less than 20 minutes. Considering variant 4 , the performance is even worse than the performance of the $s_{M}$-TRFLP model summarized in the appendix. This shows that the transitivity inequalities (19)-(20) significantly improve the performance of our approach in comparison to the standard linearization. In comparison to variant 1 , in variant 3 significantly more inequalities are added, and thus the gap at the root node is smaller. However, this increases the running time in the further branching steps, and hence variant 3 is slower than variant 1. In conclusion, our experiments show that our extension of the betweenness variables in combination with the transitivity inequalities (19)-(20) and inequalities (36)-(37) significantly improve the performance of our branch-and-cut algorithm. For all instances which were solved to optimality, our best approach for the TRFLP is faster than our approach for the 3-BFLP.

The enumerative approach of [43] is only able to solve 3-Bay instances with up to 14 departments and T-row instances with up to 13 departments within 8 hours. For larger instances, the running time is exceeded. In the corresponding TRFLP approach one enumerates over each department with its center position fixed on position $p_{M}$ in row 1 , and therefore one has to consider more MILP models than in the 3-BFLP. Thus, the running time is higher. In contrast, our approach (variant 1) is able to solve T-row instances with 13 departments in a few minutes and 3-Bay instances with 14 departments in less than 15 minutes. So for both problems we clearly outperform the approach of [43].

The inter-row transport is more costly than the inner-row transport, see, e. g., [25, 67]. Therefore, we investigate the effect of enlarging $w_{\text {path }}^{T}+1=w_{\text {path }}^{B}$ on the optimal value of the TRFLP and the 3-BFLP. In the previous results we observed that our approaches clearly outperformed the enumerative approaches, so in Table 3 and Table 4 we apply only our best fitting approaches, i.e., variant 1 for the TRFLP. The notation in Table 3 and Table 4 is similar to the notation above and we denote by "Time 3 -BFLP" and "Time 3-BFLP and the TRFLP, respectively, and by "TRFLP" and "3-BFLP" the objective value of the TRFLP and 3-BFLP.

We consider literature instances in Table 3 with $w_{\text {path }}^{T}+1=w_{\text {path }}^{B}=4$ and $w_{\text {path }}^{T}+1=w_{\text {path }}^{B}=$ 


\begin{tabular}{|c|c|c|c|c|c|c|c|c|}
\hline Instances & $\mathrm{Enu}_{3-\mathrm{BFLP}}$ & MILP $_{3-B F L P}$ & $\mathrm{Enu}_{\mathrm{TRFLP}}$ & MILP $_{1}$ & MILP $_{2}$ & MILP $_{3}$ & $\mathrm{MILP}_{4}$ & MILP $_{\text {same-row }}$ \\
\hline$A m 11 a$ & $3: 26$ & 48 & $9: 31$ & 33 & 54 & 34 & $1: 04$ & $1: 37$ \\
\hline$A m 11 b$ & $3: 35$ & $1: 19$ & $10: 11$ & 20 & 41 & 16 & 41 & $1: 27$ \\
\hline$A m 12 a$ & $13: 26$ & $1: 53$ & $38: 29$ & 51 & $1: 29$ & $1: 20$ & $2: 44$ & $6: 18$ \\
\hline$A m 12 b$ & $13: 30$ & $1: 01$ & $40: 19$ & 42 & $1: 15$ & 50 & $2: 35$ & $6: 40$ \\
\hline$A m 13 a$ & $53: 46$ & $4: 16$ & $2: 58: 52$ & $2: 17$ & 3:05 & $3: 56$ & $14: 03$ & $24: 18$ \\
\hline$A m 13 b$ & $53: 34$ & 9:31 & $3: 01: 51$ & $2: 17$ & $2: 58$ & 5:02 & 10:16 & $18: 33$ \\
\hline Am14_1 & $3: 45: 33$ & $1: 28: 24$ & TL & $9: 11$ & $16: 27$ & $24: 47$ & $1: 16: 10$ & $1: 17: 49$ \\
\hline$A m 14 \bar{a}$ & $3: 28: 30$ & $13: 23$ & TL & $6: 00$ & $15: 32$ & $12: 13$ & $52: 54$ & $58: 26$ \\
\hline$A m 14 b$ & $3: 28: 47$ & $13: 37$ & TL & $6: 51$ & $15: 59$ & $17: 34$ & $1: 22: 07$ & $1: 17: 58$ \\
\hline$A m 15$ & TL & $25: 24$ & TL & $15: 31$ & $33: 45$ & $1: 49: 33$ & $3: 20: 07$ & $3: 35: 25$ \\
\hline$H K 15$ & $\mathrm{TL}$ & $17: 51$ & TL & $13: 48$ & $20: 05$ & $58: 54$ & $3: 54: 52$ & $2: 56: 30$ \\
\hline$P 16 a$ & $\mathrm{TL}$ & 44:00 & TL & $1: 12: 31$ & $3: 27: 04$ & TL & TL & TL \\
\hline$P 16 b$ & TL & $1: 21: 35$ & $\mathrm{TL}$ & 1:09:58 & $2: 42: 40$ & $6: 04: 38$ & $\mathrm{TL}$ & $\mathrm{TL}$ \\
\hline$P 17 a$ & TL & $3: 51: 48$ & TL & $3: 32: 27$ & TL & TL & $\mathrm{TL}$ & $\mathrm{TL}$ \\
\hline$P 17 b$ & TL & $6: 31: 46$ & TL & $3: 37: 59$ & TL & TL & $\mathrm{TL}$ & $\mathrm{TL}$ \\
\hline$A m 17$ & $\mathrm{TL}$ & $4: 58: 37$ & TL & $2: 19: 20$ & $5: 53: 06$ & $\mathrm{TL}$ & $\mathrm{TL}$ & $\mathrm{TL}$ \\
\hline$P 18 a$ & $\mathrm{TL}$ & TL & TL & TL & TL & TL & $\mathrm{TL}$ & $\mathrm{TL}$ \\
\hline$P 18 b$ & $\mathrm{TL}$ & $5: 23: 22^{\dagger}$ & $\mathrm{TL}$ & $\mathrm{TL}$ & $\mathrm{TL}$ & $\mathrm{TL}$ & $\mathrm{TL}$ & $\mathrm{TL}$ \\
\hline$A m 18$ & TL & $\mathrm{TL}$ & TL & $6: 01: 58$ & TL & TL & TL & $\mathrm{TL}$ \\
\hline
\end{tabular}

Table 2: Running times are given in sec, min:sec or in h:min:sec for instances from the literature with $w_{\text {path }}^{T}=0, w_{\text {path }}^{B}=1$. We write "TL" if the time limit of 8 hours is exceeded and the running time is marked with " $\dagger$ " if we run out of memory storage. For the TRFLP, variant 1 delivers the fastest approach. We clearly outperform the enumerative approach of [43] for the TRFLP and the 3-BFLP.

11. By enlarging $w_{\text {path }}^{B}$, the running time of the 3-BFLP approach is for some instances significantly increased and for some instances significantly decreased. In contrast to the case of $w_{\text {path }}^{B}=1$, we are able to solve one 3-Bay instance with 18 departments to optimality. Note that for larger values of $w_{\text {path }}^{B}$ we run more often out of memory storage. For the TRFLP the running time is only slightly influenced (neglecting instance $P 17 b$ ) by enlarging $w_{\text {path }}^{T}$, so our approach works well with large values of $w_{\text {path }}^{T}$ and $w_{\text {path }}^{B}$. For $w_{\text {path }}^{T}=3$ and $w_{\text {path }}^{B}=4\left(w_{\text {path }}^{T}=10\right.$ and $\left.w_{\text {path }}^{B}=11\right)$, the optimal value of the TRFLP is between $2.4 \%$ and $11.4 \%(3.7 \%$ and $12.2 \%)$ smaller than the optimal value of the 3-BFLP. So by enlarging $w_{\text {path }}^{T}$ and $w_{\text {path }}^{B}$, the gap between the optimal value of the TRFLP and the optimal value of the 3-BFLP is increased in our computational results. The optimal value of the TRFLP with $w_{\text {path }}^{T}=3\left(w_{\text {path }}^{T}=10\right)$ is between $1.2 \%$ and $7.7 \%(3.9 \%$ and $18.3 \%$ ) greater than the optimal value of the TRFLP with $w_{p a t h}^{T}=0$. The optimal value of the 3-BFLP with $w_{\text {path }}^{B}=4$ is between $2.3 \%$ and $13.0 \%$ greater than the optimal value of the 3-BFLP with $w_{\text {path }}^{B}=0$ and the 3-BFLP with $w_{\text {path }}^{B}=11$ has 13 times the same optimal value as the SRFLP. So with $w_{\text {path }}^{B} \geq 11$ the departments are often arranged in one row. In contrast, the optimal value of the TRFLP with $w_{\text {path }}^{T}=10$ is up to $12.3 \%$ smaller than the optimal value of the SRFLP.

In addition, we generate star instances with $\ell_{1}=20$ and we choose the remaining integer department lengths randomly between 1 and 15 . We set integer transport weights $w_{1 i}, i \in[n], i \geq$ 2, randomly between 10 and 20 and for the remaining departments we set the transport density of $20 \%$ and we choose integer transport weights randomly between 1 and 10 . We generate 5 instances for each $n$ and we use a time limit of 4 hours for our branch-and-cut algorithm. If the 3-BFLP is not solved to optimality, the obtained lower bound is displayed and marked it with " , ".

The TRFLP was solved to optimality for all instances with up to 19 departments and we were able to solve four of the five instances with 20 departments in less than four hours, see Table 


\begin{tabular}{|c|c|c|c|c|c|c|c|c|}
\hline \multirow[b]{2}{*}{ Instances } & \multicolumn{4}{|c|}{$w_{\text {path }}^{T}=3, w_{\text {path }}^{B}=4$} & \multicolumn{4}{|c|}{$w_{\text {path }}^{T}=10, w_{\text {path }}^{B}=11$} \\
\hline & 3-BFLP & TRFLP & Time $_{3-\text { BFLP }}$ & Time $_{\text {TRFLP }}$ & 3-BFLP & TRFLP & Time $_{3-\text { BFLP }}$ & Time $_{\text {TRFLP }}$ \\
\hline$A m 11 a$ & 9774.5 & 8902.0 & 25 & 28 & 10630.5 & 9852.5 & 13 & 26 \\
\hline$A m 11 b$ & 6890.5 & 6118.5 & 10 & 16 & 7375.5 & 6930.5 & 10 & 12 \\
\hline$A m 12 a$ & 2862.0 & 2552.0 & $1: 29$ & 51 & 2901.0 & 2793.5 & 20 & 37 \\
\hline$A m 12 b$ & 3093.5 & 2740.5 & $1: 38$ & 40 & 3280.5 & 3081.5 & 40 & 38 \\
\hline$A m 13 a$ & 4489.5 & 4077.0 & 5:02 & 2:01 & 4902.5 & 4517.5 & $1: 26$ & $2: 04$ \\
\hline$A m 13 b$ & 4956.0 & 4581.5 & $1: 41$ & $1: 54$ & 5698.0 & 4999.0 & 8:07 & $1: 54$ \\
\hline Am14_1 & 5114.5 & 4642.0 & $31: 33$ & $8: 53$ & 5481.5 & 5169.5 & $3: 17$ & $10: 17$ \\
\hline$A m 14 a$ & 5296.0 & 4751.0 & $17: 11$ & $5: 35$ & 5673.0 & 5327.5 & $4: 43$ & $5: 10$ \\
\hline$A m 14 b$ & 5248.0 & 4739.5 & $8: 36$ & $6: 47$ & 5595.0 & 5323.0 & $3: 28$ & $7: 45$ \\
\hline$A m 15$ & 5869.0 & 5378.0 & $22: 30$ & $15: 53$ & 6305.0 & 5946.5 & $12: 20$ & $17: 26$ \\
\hline$H K 15$ & 27107.0 & 26446.0 & $52: 28$ & 13:04 & 28486.0 & 27180.0 & $15: 57$ & 12:04 \\
\hline$P 16 a$ & 13142.5 & 12381.0 & $2: 22: 28$ & 1:18:48 & 14828.5 & 13233.0 & $2: 38: 45$ & $1: 13: 21$ \\
\hline$P 16 b$ & 10583.5 & 9882.5 & 2:00:08 & $1: 12: 04$ & 11878.5 & 10627.5 & $2: 57: 12$ & 1:05:12 \\
\hline$P 17 a$ & 10770.0' & 11956.5 & $4: 04: 58^{\dagger}$ & $3: 57: 30$ & 13429.5 & 12871.0 & $5: 46: 33^{\dagger}$ & $3: 48: 33$ \\
\hline$P 17 b$ & 13596.5 & 12779.0 & $6: 51: 30$ & $3: 06: 40$ & 14332.5' & 13761.0 & $4: 46: 06^{\dagger}$ & 5:09:02 \\
\hline$A m 17$ & 8516.0 & 7767.5 & $3: 32: 13$ & $2: 20: 52$ & 9254.0 & 8590.0 & $2: 19: 17$ & $1: 51: 48$ \\
\hline$P 18 a$ & $11750.0^{\prime}$ & TL & TL & TL & 13496.5 & TL & $6: 22: 30^{\dagger}$ & TL \\
\hline$P 18 b$ & 13732.0' & 5289.5 & $6: 27: 55^{\dagger}$ & TL & 14250.5 & TL & $4: 19: 38^{\dagger}$ & TL \\
\hline$A m 18$ & 9744.5 & 8911.5 & $5: 51: 56$ & $5: 41: 57$ & 10650.5 & 9807.5 & 4:00:48 & $5: 49: 05$ \\
\hline
\end{tabular}

Table 3: Optimal values of the 3-BFLP and the TRFLP for instances from the literature with $w_{\text {path }}^{T}+1=w_{\text {path }}^{B}=4$ as well as $w_{\text {path }}^{T}+1=w_{\text {path }}^{B}=11$. We write "TL" if the time limit of 8 hours is exceeded and the running time is marked with " $\dagger$ " if we run out of memory storage. The running times are given in sec, min:sec or h:min:sec. If the 3-BFLP is not solved to optimality, the obtained lower bounds are marked with " ".

4. For the 3-BFLP, all instances with 18 departments and four (two) instances with 19 (20) departments were solved to optimality. For most star instances, the TRFLP is solved faster than the 3-BFLP. The optimal value of the TRFLP is between $26.2 \%$ and $40.8 \%$ smaller than the optimal value of the SRFLP and by $8.5 \%$ to $33.1 \%$ smaller than the optimal value of the 3-BFLP. So for the considered instances, the TRFLP and the 3-BFLP are preferable to the SRFLP and the TRFLP is preferable to the 3-BFLP. Again, our heuristic approaches obtain small gaps, i. e., less than $6.3 \%$ for the 3-Bay instances and for almost all T-row instances less than $2.0 \%$.

Furthermore, we use variant 3 to compute lower bounds for larger T-row instances and all additionally added inequalities in variant 3 in comparison to variant 1 are also added to the 3-BFLP using the same separation strategy. The calculation of heuristics by Cplex is disabled. We interrupt the branch-and-cut algorithm when we reach the root node, i. e., at first the LP consisting of the inequalities included in the beginning is solved with $x_{i k j} \in[0,1],|\{i, j, k\}|=$ $3, i<j, z_{i j} \in[0,1], i, j \in[n] \backslash\left\{s_{M}\right\}, i \neq j, z_{i s_{M}} \in[0,1], i \in[n] \backslash\left\{s_{M}\right\}, y_{i} \in[0,1], i \in[n]$, and then violated cutting planes are added according to our separation strategy. This LP is solved again until we obtain a binary solution, i. e., the $y, z$ and $x$ variables are binary, or until no violated cutting plane can be found. Besides this, we use Algorithm 1 as described above for the TRFLP. We set a time limit of 4 hours and if we exceed this time limit we display the current best lower bound for the 3-BFLP. The instances $P 19 a, P 19 b$ and $P 19 c$ are generated as described in [7], the instances with $n \in\{20,21,22,23\}$ are taken from [7] and the instances with $n=24$

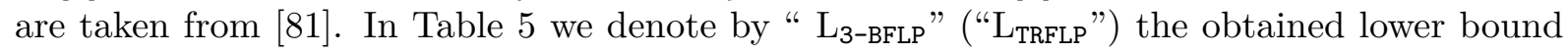
for the 3-BFLP (TRFLP) in the second and third column and we rounded the lower bounds such that $2 \cdot \mathrm{L}_{3 \text {-BFLP }} \in \mathbb{Z}\left(2 \cdot \mathrm{L}_{\text {TRFLP }} \in \mathbb{Z}\right)$. The objective values of our heuristically determined upper bounds are denoted by $\mathrm{H}_{3 \text {-BFLP }}$ and $\mathrm{H}_{\text {TRFLP }}$ for the 3-BFLP and the TRFLP, respectively. The gap 


\begin{tabular}{|c|c|c|c|c|c|c|c|}
\hline Instance & SRFLP & 3-BFLP & TRFLP & $\mathrm{H}_{3-\mathrm{BFLP}}$ & $\mathrm{H}_{\text {TRFLP }}$ & Time3-BFLP & Time \\
\hline $11 \mathrm{a}$ & 2875.0 & 2543.0 & 1702.0 & 2550.0 & 1702.0 & 5 & 0 \\
\hline $11 b$ & 4346.5 & 3482.5 & 2847.5 & 3513.5 & 2847.5 & 12 & 2 \\
\hline $11 \mathrm{c}$ & 3417.0 & 3019.0 & 2301.0 & 3112.0 & 2301.0 & 10 & 1 \\
\hline $11 d$ & 4180.0 & 3412.0 & 2878.0 & 3412.0 & 2878.0 & 5 & 2 \\
\hline $11 \mathrm{e}$ & 4334.5 & 3679.5 & 3098.5 & 3720.5 & 3122.5 & 8 & 4 \\
\hline $12 \mathrm{a}$ & 7903.0 & 6333.0 & 5540.0 & 6403.0 & 5540.0 & $2: 20$ & 13 \\
\hline $12 b$ & 5587.0 & 4598.0 & 3911.0 & 4642.0 & 3925.0 & 10 & 6 \\
\hline $12 \mathrm{c}$ & 3914.0 & 3286.0 & 2529.0 & 3338.0 & 2529.0 & 15 & 2 \\
\hline $12 \mathrm{~d}$ & 5876.5 & 4803.5 & 4027.5 & 4892.5 & 4040.5 & 21 & 8 \\
\hline $12 \mathrm{e}$ & 7809.0 & 6199.0 & 5583.0 & 6299.0 & 5583.0 & $1: 08$ & 15 \\
\hline $13 \mathrm{a}$ & 5584.5 & 4700.5 & 3823.5 & 4779.5 & 3829.5 & 40 & 11 \\
\hline $13 b$ & 5036.5 & 4247.5 & 3290.5 & 4247.5 & 3290.5 & 18 & 4 \\
\hline $13 \mathrm{c}$ & 6023.5 & 4877.5 & 4040.5 & 4877.5 & 4040.5 & 50 & 20 \\
\hline $13 d$ & 5952.0 & 4838.0 & 4036.0 & 4956.0 & 4036.0 & 55 & 27 \\
\hline $13 \mathrm{e}$ & 4944.0 & 4170.0 & 3266.0 & 4220.0 & 3281.0 & 51 & 8 \\
\hline $14 \mathrm{a}$ & 7153.5 & 5900.5 & 5276.5 & 6023.5 & 5276.5 & $1: 45$ & $1: 01$ \\
\hline $14 \mathrm{~b}$ & 7933.0 & 6524.0 & 5640.0 & 6703.0 & 5640.0 & $1: 36$ & 48 \\
\hline $14 \mathrm{c}$ & 6251.0 & 5195.0 & 4150.0 & 5259.0 & 4185.0 & $1: 32$ & 23 \\
\hline $14 \mathrm{~d}$ & 6782.0 & 5784.0 & 4884.0 & 5830.0 & 4884.0 & $1: 31$ & 26 \\
\hline $14 \mathrm{e}$ & 6913.5 & 5800.5 & 4935.5 & 5874.5 & 5034.5 & 55 & 49 \\
\hline $15 \mathrm{a}$ & 7668.0 & 6120.0 & 5312.0 & 6208.0 & 5312.0 & $2: 02$ & $1: 31$ \\
\hline $15 b$ & 7730.5 & 6317.5 & 5298.5 & 6317.5 & 5355.5 & $5: 38$ & $1: 36$ \\
\hline $15 \mathrm{c}$ & 6387.0 & 5199.0 & 4225.0 & 5199.0 & 4225.0 & $7: 02$ & $1: 27$ \\
\hline $15 \mathrm{~d}$ & 6559.5 & 5608.5 & 4609.5 & 5662.5 & 4609.5 & 3:06 & 48 \\
\hline $15 \mathrm{e}$ & 6929.0 & 5567.0 & 4643.0 & 5601.0 & 4662.0 & 8:09 & $3: 37$ \\
\hline $16 \mathrm{a}$ & 9461.0 & 7663.0 & 6564.0 & 8142.0 & 6779.0 & $7: 48$ & $3: 54$ \\
\hline $16 \mathrm{~b}$ & 11912.0 & 9376.0 & 8356.0 & 9376.0 & 8356.0 & 10:00 & $10: 15$ \\
\hline $16 \mathrm{c}$ & 11351.5 & 9122.5 & 8082.5 & 9255.5 & 8113.5 & $8: 58$ & $6: 27$ \\
\hline $16 \mathrm{~d}$ & 8073.5 & 6548.5 & 5521.5 & 6609.5 & 5535.5 & $8: 28$ & $3: 23$ \\
\hline $16 \mathrm{e}$ & 8184.0 & 6541.0 & 5561.0 & 6541.0 & 5577.0 & $3: 49$ & $4: 49$ \\
\hline $17 \mathrm{a}$ & 11063.0 & 8985.0 & 7853.0 & 9036.0 & 7853.0 & $1: 50: 14$ & $13: 47$ \\
\hline $17 \mathrm{~b}$ & 13692.5 & 10913.5 & 9876.5 & 10970.5 & 10009.5 & $36: 31$ & $34: 34$ \\
\hline $17 \mathrm{c}$ & 11101.0 & 8812.0 & 7640.0 & 8865.0 & 7640.0 & $27: 24$ & $12: 06$ \\
\hline $17 \mathrm{~d}$ & 9753.0 & 7981.0 & 6823.0 & 8213.0 & 6929.0 & $23: 10$ & 9:59 \\
\hline $17 \mathrm{e}$ & 9879.4 & 7957.5 & 6736.5 & 8027.5 & 6810.5 & $26: 19$ & $9: 37$ \\
\hline $18 \mathrm{a}$ & 15157.5 & 12288.5 & 11108.5 & 12455.5 & 11214.5 & $1: 16: 55$ & $36: 08$ \\
\hline $18 \mathrm{~b}$ & 10254.5 & 8142.5 & 7037.5 & 8315.5 & 7088.5 & $1: 01: 53$ & $25: 32$ \\
\hline $18 \mathrm{c}$ & 12847.5 & 10370.5 & 9264.5 & 10406.5 & 9285.5 & $1: 14: 52$ & $38: 36$ \\
\hline $18 \mathrm{~d}$ & 9437.0 & 7638.0 & 6464.0 & 7720.0 & 6483.0 & 1:58:08 & $21: 11$ \\
\hline $18 \mathrm{e}$ & 11769.0 & 9661.0 & 8538.0 & 9661.0 & 8538.0 & 1:00:53 & $32: 16$ \\
\hline $19 \mathrm{a}$ & 14371.5 & 11353.0 & 10046.5 & 11433.5 & 10148.5 & $1: 48: 11$ & $1: 40: 54$ \\
\hline $19 b$ & 14110.5 & 11414.5 & 10203.5 & 11693.5 & 10203.5 & $3: 53: 33$ & $1: 19: 15$ \\
\hline $19 \mathrm{c}$ & 10119.0 & 8100.0 & 6637.0 & 8337.0 & 6731.0 & 1:11:44 & $25: 56$ \\
\hline $19 d$ & 12821.0 & 8805.5 & 8848.0 & 10200.0 & 8848.0 & TL & $1: 58: 16$ \\
\hline $19 \mathrm{e}$ & 13209.0 & 10380.0 & 9495.0 & 10484.0 & 9575.0 & $1: 56: 34$ & $2: 37: 10$ \\
\hline $20 \mathrm{a}$ & 11826.0 & 9529.0 & 8257.0 & 9828.0 & 8288.0 & $2: 08: 43$ & $1: 27: 44$ \\
\hline $20 \mathrm{~b}$ & 15956.5 & 12397.0 & TL & 12446.5 & 12127.5 & $3: 12: 02$ & $\mathrm{TL}$ \\
\hline $20 \mathrm{c}$ & 14191.0 & 8471.0' & 9826.0 & 11192.0 & 9827.0 & TL & $3: 28: 52$ \\
\hline $20 d$ & 16047.5 & $11876.0^{\prime}$ & 11540.5 & 12826.5 & 11540.5 & TL & $3: 51: 36$ \\
\hline $20 \mathrm{e}$ & 11882.5 & 8384.0' & 8097.5 & 9462.5 & 8105.5 & TL & $2: 39: 25$ \\
\hline
\end{tabular}

Table 4: Optimal values for the SRFLP, the 3-BFLP and the TRFLP for randomly generated star instances are displayed. The running times are given in sec, min:sec or in h:min:sec. Note that the TRFLP was solved to optimality for four instances with $n=20$ departments. The optimal value of the TRFLP is up to $33.1 \%$ smaller than the optimal value of the 3-BFLP and up to $40.8 \%$ smaller than the optimal value of the SRFLP. 
is calculated by

$$
\operatorname{Gap}_{a}=\frac{H_{a}-L_{a}}{H_{a}} \cdot 100, \quad a \in\{3-\text { BFLP,TRFLP }\}
$$

and the gap for the 3-BFLP (TRFLP) is given in the sixth (seventh) column denoted by "Gap 3 -BFLP"

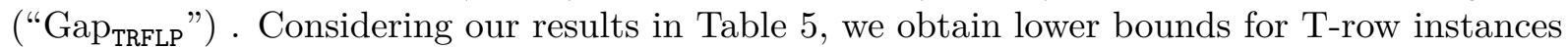
with up to 22 departments with gaps less than $9.68 \%$ to heuristically determined upper bounds. For four of the five instances with 22 departments and for all larger instances the time limit was exceeded, and hence we are not able to derive non-trivial lower bounds for the TRFLP. If the root node is reached in the time limit of 4 hours, we obtain gaps between $2.23 \%$ and $7.90 \%$ to heuristically determined upper bounds for 3-Bay instances with up to 24 departments. Considering the instances $P 24 a$ and $P 24 c$, the root node was not reached and therefore the gaps are higher, i. e., up to $10.12 \%$. In conclusion, our approach is able to calculate tight lower bounds for the TRFLP and the 3-BFLP whereby the lower bounds for the 3-BFLP are generally better.

\section{Conclusion and Future Work}

In this paper we introduced a new facility layout problem, the so-called T-Row Facility Layout Problem (TRFLP), which is a generalization of the Multi-Bay Facility Layout Problem (MBFLP) with $m=3$ rows denoted by (3-BFLP). The TRFLP and the MBFLP have several applications, e.g., in heavy manufacturing and semiconductor fabrication. The TRFLP has a more complex path structure than the 3-BFLP and we proved there always exists an optimal T-row layout where one department has its center position on $p_{M}$ in row 1 . In a branch-and-cut approach we enumerated over each department with its center fixed on position $p_{M}$ in row 1 and we presented a mixed-integer linear programming (MILP) model for this problem based on an extension of the betweenness variables which might be in contrast to the literature equal to one if the corresponding departments lie in different rows. Transitivity inequalities are used to ensure the correct relation of these extended betweenness variables to the remaining variables. To strengthen our formulation we provided cutting planes exploring the crossroad structure of the TRFLP. In addition, we adapted our MILP model to the 3-BFLP.

We were able to solve T-row and 3-bay instances from the literature with up to 18 departments within a given time limit of 8 hours and we clearly outperform the current best approach from the literature. Further, we outperform a MILP approach based on betweenness variables as known in the literature and we observed in our computational results that the transitivity inequalities (in comparison to the standard linearization) and the cutting planes significantly improve the performance of our approach. Additionally, we considered star instances and we were able to solve even larger instances of this type for the TRFLP and the 3-BFLP. According to our computational results, the TRFLP is preferable to the 3-BFLP and the SRFLP if a factory is built from the ground up, because the optimal value is significantly smaller and in factory planning the departments are arranged for a long period since the rearrangement of the departments is expensive. So even a small reduction of the yearly production costs can reduce the total production costs over a long period significantly. In addition, our approach is able to calculate tight lower bounds for even larger T-row and 3-Bay instances to evaluate the quality of heuristically determined layouts.

It remains for future work to consider facility layouts in the shape of an $\mathrm{X}$ or an $\mathrm{U}$ and the MBFLP with $m=4$ and $m=5$. Therefore, one can use our extension of the betweenness variables and one can adapt our MILP approach as well as our cutting planes. From a practical point of view, it is interesting to extend the betweenness model for the SRFLP such that it is capable of more realistic extensions such as individual in- and output positions of the departments and to use the result of Proposition 1 to improve the performance of the branch-and-cut algorithm for the SRFLP. 


\begin{tabular}{|c|c|c|c|c|c|c|c|c|}
\hline Instance & $\mathrm{L}_{3-\mathrm{BFLP}}$ & $\mathrm{L}_{\mathrm{TRFLP}}$ & $\mathrm{H}_{3 \text {-BFLP }}$ & $\mathrm{H}_{\text {TRFLP }}$ & $\mathrm{Gap}_{3-\mathrm{BFLP}}$ & $\mathrm{Gap}_{\mathrm{TRFLP}}$ & Time $_{3-\text { BFLP }}$ & Time $_{\text {TRFLP }}$ \\
\hline Am11a & 8450.0 & 7823.5 & 8814.5 & 8411.5 & $4.14 \%$ & $6.99 \%$ & 5 & 8 \\
\hline$A m 11 b$ & 5882.0 & 5431.0 & 6021.5 & 5667.5 & $2.32 \%$ & $4.17 \%$ & 5 & 4 \\
\hline$A m 12 a$ & 2382.5 & 2207.5 & 2515.0 & 2354.5 & $5.27 \%$ & $6.24 \%$ & 18 & 13 \\
\hline$A m 12 b$ & 2621.5 & 2482.0 & 2697.5 & 2548.5 & $2.82 \%$ & $2.61 \%$ & 15 & 25 \\
\hline$A m 13 a$ & 3872.5 & 3688.0 & 4204.5 & 3836.0 & $7.90 \%$ & $3.86 \%$ & 37 & 43 \\
\hline$A m 13 b$ & 4357.0 & 4215.5 & 4529.0 & 4362.5 & $3.80 \%$ & $3.37 \%$ & 38 & $1: 12$ \\
\hline Am14_1 & 4359.0 & 4079.5 & 4565.5 & 4358.0 & $4.52 \%$ & $6.39 \%$ & $1: 21$ & 2:03 \\
\hline$A m 14 a$ & 4560.0 & 4273.5 & 4734.0 & 4448.0 & $3.68 \%$ & $3.92 \%$ & 59 & $1: 43$ \\
\hline$A m 14 b$ & 4482.5 & 4200.5 & 4683.0 & 4433.0 & $4.28 \%$ & $5.24 \%$ & 1:03 & 2:03 \\
\hline$A m 15$ & 4953.0 & 4744.5 & 5294.0 & 5079.0 & $6.44 \%$ & $6.59 \%$ & 1:06 & 4:33 \\
\hline$H K 15$ & 25521.5 & 24528.5 & 26495.0 & 26125.0 & $3.67 \%$ & $6.11 \%$ & 2:04 & 4:30 \\
\hline$P 16 a$ & 11536.0 & 10911.0 & 12326.0 & 11943.0 & $6.41 \%$ & $8.64 \%$ & $1: 58$ & $12: 02$ \\
\hline$P 16 b$ & 9305.5 & 8677.5 & 9784.5 & 9469.5 & $4.90 \%$ & $8.36 \%$ & $2: 25$ & $14: 26$ \\
\hline$P 17 a$ & 11344.5 & 10428.5 & 11888.5 & 11528.0 & $4.58 \%$ & $9.54 \%$ & $3: 23$ & $21: 26$ \\
\hline$P 17 b$ & 12207.5 & 11388.0 & 12874.0 & 12389.0 & $5.18 \%$ & $8.08 \%$ & 5:06 & $33: 43$ \\
\hline Am17 & 7267.5 & 6788.5 & 7690.0 & 7318.0 & $5.49 \%$ & $7.24 \%$ & 5:02 & $18: 38$ \\
\hline$P 18 a$ & 12333.0 & 11611.0 & 12863.5 & 12516.0 & $4.12 \%$ & $7.23 \%$ & $8: 52$ & $56: 12$ \\
\hline$P 18 b$ & 13746.0 & 12578.0 & 14616.5 & 14072.0 & $6.00 \%$ & $0.62 \%$ & $6: 50$ & $25: 47$ \\
\hline Am18 & 8318.0 & 7758.0 & 8835.5 & 8413.5 & $5.86 \%$ & $7.79 \%$ & $6: 56$ & $36: 06$ \\
\hline$P 19 a$ & 14049.5 & 13140.5 & 14715.5 & 14289.5 & $4.53 \%$ & $8.04 \%$ & $6: 26$ & $53: 53$ \\
\hline$P 19 b$ & 18074.5 & 16975.5 & 19166.5 & 18660.0 & $5.70 \%$ & $9.03 \%$ & $10: 24$ & $1: 14: 46$ \\
\hline$P 19 c$ & 9865.0 & 9230.0 & 10455.0 & 9989.5 & $5.64 \%$ & $7.60 \%$ & $6: 53$ & 59:02 \\
\hline$P 20 a$ & 18485.5 & 17297.0 & 19204.5 & 18716.5 & $3.74 \%$ & $7.58 \%$ & $20: 46$ & $2: 46: 18$ \\
\hline$P 20 b$ & 19277.5 & 17897.0 & 20427.0 & 19816.0 & $5.63 \%$ & $9.68 \%$ & $18: 18$ & 1:52:04 \\
\hline$H 20$ & 11882.5 & 11138.0 & 12576.0 & 12087.0 & $5.51 \%$ & $7.85 \%$ & $38: 06$ & $2: 52: 48$ \\
\hline$P 21 a$ & 10382.5 & 9645.5 & 10862.5 & 10439.0 & $4.42 \%$ & $7.60 \%$ & $23: 50$ & $3: 10: 40$ \\
\hline$P 21 b$ & 17646.0 & 16354.5 & 18233.0 & 17806.0 & $3.22 \%$ & $8.15 \%$ & $17: 07$ & $1: 26: 56$ \\
\hline$P 21 c$ & 16811.0 & 15799.0 & 17806.0 & 17100.0 & $5.59 \%$ & $7.61 \%$ & $21: 22$ & $2: 55: 49$ \\
\hline$P 22 a$ & 12912.5 & - & 13757.0 & 13238.0 & $6.14 \%$ & - & $32: 21$ & TL \\
\hline$P 22 b$ & 22686.0 & - & 24096.0 & 23359.5 & $5.85 \%$ & - & $36: 59$ & TL \\
\hline$P 22 c$ & 21413.0 & 19922.5 & 22940.0 & 22036.0 & $6.66 \%$ & $9.59 \%$ & $35: 55$ & $3: 28: 12$ \\
\hline$P 23 a$ & 14790.5 & - & 15975.0 & 15248.5 & $7.41 \%$ & - & $3: 39: 59$ & TL \\
\hline$P 23 b$ & 22532.5 & - & 23484.5 & 22968.0 & $4.05 \%$ & - & $1: 34: 25$ & $\mathrm{TL}$ \\
\hline$P 23 c$ & 22206.0 & - & 23617.0 & 22956.5 & $5.97 \%$ & - & $1: 34: 36$ & TL \\
\hline$P 24 a$ & 16529.0 & - & 18390.0 & 17728.0 & $10.12 \%$ & - & TL & TL \\
\hline$P 24 b$ & 23478.5 & - & 25219.5 & 24578.5 & $6.90 \%$ & - & $2: 13: 54$ & TL \\
\hline$P 24 c$ & 25114.0 & - & 27020.0 & 26058.5 & $7.05 \%$ & - & TL & TL \\
\hline$P 24 d$ & 23519.0 & - & 25113.0 & 24366.0 & $6.35 \%$ & - & $3: 37: 30$ & TL \\
\hline$P 24 e$ & 27066.0 & - & 28809.0 & 28200.0 & $6.05 \%$ & & $2: 00: 17$ & TL \\
\hline
\end{tabular}

Table 5: Calculation of lower bounds for the 3-BFLP and the TRFLP with a given time limit of 4 hours and the computation of the branch-and-cut algorithm is interrupted at the root node. We use variant 3 of our separation strategy. The gaps of the lower bounds for the 3-BFLP are less than $10.12 \%$ to heuristically determined upper bounds for the 3-BFLP and even better if the root node is reached during the time limit. The computation time in the last two columns are given in sec, min:sec, h:min:sec and we write "TL " if the time limit of 4 hours is exceeded. 


\section{Acknowledgment}

This work is supported by the Simulation Science Center Clausthal-Göttingen.

\section{References}

[1] André R. S. Amaral. On the exact solution of a facility layout problem. European Journal of Operational Research, 173(2):508-518, 2006.

[2] André R. S. Amaral. An exact approach to the one-dimensional facility layout problem. Operations Research, 56(4):1026-1033, 2008.

[3] André R. S. Amaral. A new lower bound for the single row facility layout problem. Discrete Applied Mathematics, 157(1):183-190, 2009.

[4] André R. S. Amaral. On duplex arrangement of vertices. Technical report, Departamento de Informática, Universidade Federal do Espírito Santo (UFES), Brazil, 2011.

[5] André R. S. Amaral. The corridor allocation problem. Computers \& Operations Research, 39(12):3325-3330, 2012.

[6] André R. S. Amaral. Optimal solutions for the double row layout problem. Optimization Letters, 7(2):407-413, 2013.

[7] André R. S. Amaral. A parallel ordering problem in facilities layout. Computers \& Operations Research, 40(12):2930-2939, 2013.

[8] André R. S. Amaral. A mixed-integer programming formulation of the double row layout problem based on a linear extension of a partial order. Technical report, Graduate School of Computer Science (PPGI), Federal University of Espírito Santo (UFES), Brazil, 2018. Available at http://www.optimization-online.org/DB_HTML/2020/04/7739.html.

[9] André R. S. Amaral. A mixed-integer programming formulation for the double row layout of machines in manufacturing systems. International Journal of Production Research, $57(1): 34-47,2019$.

[10] André R. S. Amaral. A heuristic approach for the double row layout problem. Annals of Operations Research, pages 1-36, 2020.

[11] André R. S. Amaral and Adam N. Letchford. A polyhedral approach to the single row facility layout problem. Mathematical Programming, 141(1-2):453-477, 2013.

[12] Miguel F. Anjos, Anja Fischer, and Philipp Hungerländer. Solution approaches for the double-row equidistant facility layout problem. In Marco Lübbecke, Arie Koster, Peter Letmathe, Reinhard Madlener, Britta Peis, and Grit Walther, editors, Operations Research Proceedings 2014, pages 17-23, Cham, 2016. Springer International Publishing.

[13] Miguel F. Anjos, Anja Fischer, and Philipp Hungerländer. Improved exact approaches for row layout problems with departments of equal length. European Journal of Operational Research, 270(2):514-529, 2018.

[14] Miguel F. Anjos, Philipp Hungerländer, and Kerstin Maier. An integer linear programming approach for the combined cell layout problem. In 2018 IEEE International Conference on Industrial Engineering and Engineering Management (IEEM), pages 705-709. IEEE, 2018. 
[15] Miguel F. Anjos, Andrew Kennings, and Anthony Vannelli. A semidefinite optimization approach for the single-row layout problem with unequal dimensions. Discrete Optimization, $2(2): 113-122,2005$.

[16] Miguel F. Anjos and Anthony Vannelli. Computing globally optimal solutions for single-row layout problems using semidefinite programming and cutting planes. INFORMS Journal On Computing, 20(4):611-617, 2008.

[17] Miguel F. Anjos and Manuel V.C. Vieira. Mathematical optimization approaches for facility layout problems: The state-of-the-art and future research directions. European Journal of Operational Research, 261(1):1-16, 2017.

[18] Miguel F. Anjos and Ginger Yen. Provably near-optimal solutions for very large single-row facility layout problems. Optimization Methods and Software, 24(4):805-817, 2009.

[19] Maria M. Azevedo, José A. Crispim, and Jorge P. de Sousa. A dynamic multi-objective approach for the reconfigurable multi-facility layout problem. Journal of manufacturing systems, 42:140-152, 2017.

[20] Uwe Bracht, Mirko Dahlbeck, Anja Fischer, and Thomas Krüger. Combining simulation and optimization for extended double row facility layout problems in factory planning. In Marcus Baum, Gunther Brenner, Jens Grabowski, Thomas Hanschke, Stefan Hartmann, and Anita Schöbel, editors, Simulation Science, pages 39-59, Cham, 2018. Springer International Publishing.

[21] Timothy W. Butler, Kirk R. Karwan, James R. Sweigart, and Gary R. Reeves. An integrative model-based approach to hospital layout. IIE transactions, 24(2):144-152, 1992.

[22] Alberto Caprara, Marcus Oswald, Gerhard Reinelt, Robert Schwarz, and Emiliano Traversi. Optimal linear arrangements using betweenness variables. Mathematical Programming Computation, 3(3):261-280, 2011.

[23] Gino Cardarelli and Pacifico M. Pelagagge. Simulation tool for design and management optimization of automated interbay material handling and storage systems for large wafer fab. IEEE Transactions on Semiconductor Manufacturing, 8(1):44-49, 1995.

[24] Ignacio Castillo and Brett A. Peters. Integrating design and production planning considerations in multi-bay manufacturing facility layout. European Journal of Operational Research, 157(3):671-687, 2004.

[25] Junjae Chae and Brett A. Peters. Layout design of multi-bay facilities with limited bay flexibility. Journal of manufacturing systems, 25(1):1-11, 2006.

[26] Junjae Chae and Amelia C. Regan. A mixed integer programming model for a double row layout problem. Computers \& Industrial Engineering, 140, 2020.

[27] Mei-Shiang Chang and Hsin-Yi Lin. An immunized ant colony system algorithm to solve unequal area facility layout problems using flexible bay structure. In Proceedings of the Institute of Industrial Engineers Asian Conference 2013, pages 9-17. Springer, 2013.

[28] Thomas Christof, Marcus Oswald, and Gerhard Reinelt. Consecutive ones and a betweenness problem in computational biology. In International Conference on Integer Programming and Combinatorial Optimization, pages 213-228. Springer, 1998.

[29] Jaewoo Chung and Yunjung Suh. Analysis on the pier-type material flow pattern for facility layout applications. Journal of Applied Sciences, 14(3):237-244, 2014. 
[30] Jaewoo Chung and J.M.A. Tanchoco. The double row layout problem. International Journal of Production Research, 48(3):709-727, 2010.

[31] Jaewoo Chung and J.M.A. Tanchoco. Layout design with hexagonal floor plans and material flow patterns. International Journal of Production Research, 48(12):3407-3428, 2010.

[32] Pablo E. Coll, Celso C. Ribeiro, and Cid C. de Souza. Multiprocessor scheduling under precedence constraints: Polyhedral results. Discrete Applied Mathematics, 154(5):770-801, 2006.

[33] Gildásio L. Cravo and André R. S. Amaral. A grasp algorithm for solving large-scale single row facility layout problems. Computers $\&$ Operations Research, 106:49-61, 2019.

[34] Mirko Dahlbeck, Anja Fischer, and Frank Fischer. Decorous combinatorial lower bounds for row layout problems. European Journal of Operational Research, 286(3):929-944, 2020.

[35] Mirko Dahlbeck, Anja Fischer, and Philipp Hungerländer. A study of the relation between the single-row and the double-row facility layout problem. Working paper, 2020.

[36] Mirko Dahlbeck, Anja Fischer, Philipp Hungerländer, and Kerstin Maier. New exact approaches for the combined cell layout problem and extensions of the multi-bay facility layout problem. Working paper, 2020.

[37] Dilip Datta, André R. S. Amaral, and José R. Figueira. Single row facility layout problem using a permutation-based genetic algorithm. European Journal of Operational Research, 213(2):388-394, 2011.

[38] Hamid Davoudpour, Amir A. Jaafari, and Leila N. Farahani. Facility layout problems using bays: A survey. AIP Conference Proceedings, 1247(1):485-491, 2010.

[39] Amine Drira, Henri Pierrval, and Sonia Hajri-Gabouj. Facility layout problems: A survey. Annual Reviews in Control, 31(2):255-267, 2007.

[40] Alwalid N. Elshafei. Hospital layout as a quadratic assignment problem. Journal of the Operational Research Society, 28(1):167-179, 1977.

[41] Anja Fischer and Frank Fischer. An extended polyhedral study of the betweenness model. Working paper, 2020.

[42] Anja Fischer, Frank Fischer, and Philipp Hungerländer. A new exact approach to the space-free double row layout problem. In Karl F. Doerner, Ivana Ljubic, Georg Pflug, and Gernot Tragler, editors, Operations Research Proceedings 2015, Selected Papers of the International Conference of the German, Austrian and Swiss Operations Research Societies (GOR, ÖGOR, SVOR/ASRO), University of Vienna, Austria, September 1-4, 2015, pages 125-130. Springer, 2015.

[43] Anja Fischer, Frank Fischer, and Philipp Hungerländer. New exact approaches to row layout problems. Mathematical Programming Computation, 11(4):703-754, 2019.

[44] Michael Randolph Garey, David S. Johnson, and Larry J. Stockmeyer. Some simplified NP-complete problems. In STOC '74: Proceedings of the sixth annual ACM symposium on Theory of computing, pages 47-63. ACM, 1974.

[45] Jian Guan and Geng Lin. Hybridizing variable neighborhood search with ant colony optimization for solving the single row facility layout problem. European Journal of Operational Research, 248(3):899-909, 2016. 
[46] Mehmet Gülşen, Chase C. Murray, and Alice E. Smith. Double-row facility layout with replicate machines and split flows. Computers \& Operations Research, 108:20-32, 2019.

[47] Peter M. Hahn and Jakob Krarup. A hospital facility layout problem finally solved. Journal of Intelligent Manufacturing, 12(5-6):487-496, 2001.

[48] Mohsen M. D. Hassan. Machine layout problem in modern manufacturing facilities. International Journal of Production Research, 32(11):2559-2584, 1994.

[49] Sunderesh S. Heragu and Andrew Kusiak. Machine Layout Problem in Flexible Manufacturing Systems. Operations Research, 36(2):258-268, 1988.

[50] Hasan Hosseini-Nasab, Sepideh Fereidouni, Seyyed M. T. F. Ghomi, and Mohammad B. Fakhrzad. Classification of facility layout problems: a review study. The International Journal of Advanced Manufacturing Technology, 94(1-4):957-977, 2018.

[51] Michael H. Hu and Meei-Yuh Ku. A study on the spine layout for semiconductor manufacturing facility using simulated annealing. Journal of Statistics and Management Systems, $9(3): 591-612,2006$.

[52] Philipp Hungerländer. The checkpoint ordering problem. Optimization, 66(10):1699-1712, 2017.

[53] Philipp Hungerländer and Miguel F. Anjos. An exact approach for the combined cell layout problem. In Stefan Helber, Michael Breitner, Daniel Rösch, Cornelia Schön, Johann-Matthias Graf von der Schulenburg, Philipp Sibbertsen, Marc Steinbach, Stefan Weber, and Anja Wolter, editors, Operations Research Proceedings 2012, pages 275-281, Cham, 2014. Springer International Publishing.

[54] Philipp Hungerländer and Miguel F. Anjos. A semidefinite optimization-based approach for global optimization of multi-row facility layout. European Journal of Operational Research, 245(1):46-61, 2015.

[55] Philipp Hungerländer and Franz Rendl. A computational study and survey of methods for the single-row facility layout problem. Computational Optimization and Applications, $55(1): 1-20,2013$.

[56] Philipp Hungerländer and Franz Rendl. Semidefinite relaxations of ordering problems. Mathematical Programming, 140(1):77-97, 2013.

[57] ILOG CPLEX Optimization Studio 12.9, 2019.

[58] Birgit Keller and Udo Buscher. Single row layout models. European Journal of Operational Research, 245(3):629-644, 2015.

[59] Abdullah Konak, Sadan Kulturel-Konak, Bryan A. Norman, and Alice E. Smith. A new mixed integer programming formulation for facility layout design using flexible bays. Operations Research Letters, 34(6):660-672, 2006.

[60] Ravi Kothari and Diptesh Ghosh. Tabu search for the single row facility layout problem using exhaustive 2-opt and insertion neighborhoods. European Journal of Operational Research, 224(1):93-100, 2013.

[61] Ravi Kothari and Diptesh Ghosh. A scatter search algorithm for the single row facility layout problem. Journal of Heuristics, 20(2):125-142, 2014. 
[62] Sadan Kulturel-Konak and Abdullah Konak. A new relaxed flexible bay structure representation and particle swarm optimization for the unequal area facility layout problem. Engineering Optimization, 43(12):1263-1287, 2011.

[63] Sadan Kulturel-Konak and Abdullah Konak. Unequal area flexible bay facility layout using ant colony optimisation. International Journal of Production Research, 49(7):1877-1902, 2011.

[64] André Langevin, Benoit Montreuil, and Diane Riopel. Spine layout design. International Journal of Production Research, 32(2):429-442, 1994.

[65] Enrico Malaguti and Paolo Toth. A survey on vertex coloring problems. International transactions in operational research, 17(1):1-34, 2010.

[66] Mostafa Mazinani, Mostafa Abedzadeh, and Navid Mohebali. Dynamic facility layout problem based on flexible bay structure and solving by genetic algorithm. The International Journal of Advanced Manufacturing Technology, 65(5-8):929-943, 2013.

[67] R. D. Meller. The multi-bay manufacturing facility layout problem. International Journal of Production Research, 35(5):1229-1237, 1997.

[68] Isabel Méndez-Díaz and Paula Zabala. A cutting plane algorithm for graph coloring. Discrete Applied Mathematics, 156(2):159-179, 2008.

[69] Chase C. Murray, Alice E. Smith, and Zeqiang Zhang. An efficient local search heuristic for the double row layout problem with asymmetric material flow. International Journal of Production Research, 51(20):6129-6139, 2013.

[70] Gintaras Palubeckis. Fast local search for single row facility layout. European Journal of Operational Research, 264(3):800-814, 2015.

[71] Gintaras Palubeckis. Single row facility layout using multi-start simulated annealing. Computers 8 Industrial Engineering, 103:1-16, 2017.

[72] Jean-Claude Picard and Maurice Queyranne. On the one-dimensional space allocation problem. Operations Research, 29(2):371-391, 1981.

[73] Soroush Safarzadeh and Hamidreza Koosha. Solving an extended multi-row facility layout problem with fuzzy clearances using ga. Applied Soft Computing, 61:819-831, 2017.

[74] Hamed Samarghandi and Kourosh Eshghi. An efficient tabu algorithm for the single row facility layout problem. European Journal of Operational Research, 205(1):98-105, 2010.

[75] Sujeevraja Sanjeevi and Kiavash Kianfar. A polyhedral study of triplet formulation for single row facility layout problem. Discrete Applied Mathematics, 158(16):1861-1867, 2010.

[76] Leonardo D. Secchin and André R. S. Amaral. An improved mixed-integer programming model for the double row layout of facilities. Optimization Letters, 13(1):193-199, 2019.

[77] James A. Tompkins, John A. White, Yavuz A. Bozer, and J. M. A. Tanchoco. Facilities planning. John Wiley \& Sons, 2010.

[78] Shengli Wang, Xingquan Zuo, Xueqing Liu, Xinchao Zhao, and Jianqiang Li. Solving dynamic double row layout problem via combining simulated annealing and mathematical programming. Applied Soft Computing, 37:303-310, 2015. 
[79] Kuan Y. Wong and Komarudin. Solving facility layout problems using flexible bay structure representation and ant system algorithm. Expert Systems with Applications, 37(7):5523-5527, 2010 .

[80] Taho Yang and Brett A. Peters. A spine layout design method for semiconductor fabrication facilities containing automated material-handling systems. International Journal of Operations 8 Production Management, 17(5):490-501, 1997.

[81] Xuhong Yang, Wenming Cheng, Alice E. Smith, and André R. S. Amaral. An improved model for the parallel row ordering problem. Journal of the Operational Research Society, 71(3):475-490, 2020.

[82] Zeqiang Zhang and Chase C. Murray. A corrected formulation for the double row layout problem. International Journal of Production Research, 50(15):4220-4223, 2012.

[83] Xingquan Zuo, Chase C. Murray, and Alice E. Smith. Solving an extended double row layout problem using multiobjective tabu search and linear programming. IEEE Transactions on Automation Science and Engineering, 11(4):1122-1132, 2014.

[84] Xingquan Zuo, Chase C. Murray, and Alice E. Smith. Sharing clearances to improve machine layout. International Journal of Production Research, 54(14):4272-4285, 2016.

\section{Appendix}

We present a MILP model for the $s_{M}$-TRFLP, based on betweenness variables which can only be equal to one, if the corresponding three departments are in the same row, see, e. g., [3, 22, 28, 43]. The dummy departments $n+1$ and $n+2$ are added to the model as described in Section 3.2. Then

$$
x_{j k i}=x_{i k j}= \begin{cases}1, & \text { if } k \text { lies between } i \text { and } j \text { in the same row } \\ 0, & \text { otherwise }\end{cases}
$$

$i, j, k \in[n+2],|\{i, j, k\}|=3, i<j$. The ordering and assignment variables are used as described in Section 3.2

$$
z_{i j}= \begin{cases}1, & i \text { is left to } j \text { and } i \text { and } j \text { are in the same row } \\ 0, & \text { otherwise }\end{cases}
$$

$i, j \in[n], i \neq j$, and

$$
y_{i}= \begin{cases}1, & \text { if } i \text { lies in row } 1 \\ 0, & \text { otherwise }\end{cases}
$$

$i \in[n]$. Further, let $d_{i j}=d_{j i}$ denote the distance between $i \in[n]$ and $j \in[n], i<j$, measured in rectilinear directions. Let $s_{M} \in[n]$ be fixed. We exclude the constant $W^{T}:=$ $\sum_{i, j \in[n] \backslash\left\{s_{M}\right\}} \frac{\ell_{i}+\ell_{j}}{2} w_{i j}+\sum_{i \in[n] \backslash\left\{s_{M}\right\}} w_{i s_{M}} \frac{\ell_{i}}{2}$. Then our MILP model for the $s_{M}$-TRFLP with $M:=2 \cdot \sum_{k \in[n]} \ell_{k}$ reads as follows

$$
\sum_{\substack{i, j \in[n] \\ i<j}} w_{i j} d_{i j}
$$

s.t. $(7)-(18),(21)$, 


$$
\begin{array}{ll}
x_{i k j}-z_{i k}-z_{k j} \geq-1, & i, j, k \in[n] \backslash\left\{s_{M}\right\}, i<j,|\{i, j, k\}|=3, \\
x_{i k j}-z_{j k}-z_{k i} \geq-1, & i, j, k \in[n] \backslash\left\{s_{M}\right\}, i<j,|\{i, j, k\}|=3, \\
d_{i j}-\sum_{k \in[n] \backslash\{i, j\}} \ell_{k} x_{i k j} \geq 0, & i, j \in[n], i<j, \\
d_{i s_{M}}+y_{i}\left(w_{p a t h}^{T}-\frac{\ell_{s_{M}}}{2}\right) & \\
-\sum_{k \in[n] \backslash\left\{i, s_{M}\right\}} \ell_{k}\left(x_{i k s_{M}}+x_{(n+2) k i}\right)=w_{p a t h}^{T}, & i \in[n] \backslash\left\{s_{M}\right\}, \\
d_{i j} \geq d_{i s_{M}}+d_{j s_{M}}-M\left(1-y_{i}+y_{j}\right), & i, j \in[n] \backslash\left\{s_{M}\right\}, i<j, \\
d_{i j} \geq d_{i s_{M}}+d_{j s_{M}}-M\left(1+y_{i}-y_{j}\right), & i, j \in[n] \backslash\left\{s_{M}\right\}, i<j, \\
d_{i j} \geq 0, & i, j \in[n], i \neq j .
\end{array}
$$

Inequalities (52)-(53) are related to the standard linearization to calculate lower bounds for the betweenness variables. Note that upper bounds are obtained by inequalities (21). Previous tests, which we do not mention here, indicate that upper bounds obtained by the standard linearization do not improve this approach. The distance between $i \in[n] \backslash\left\{s_{M}\right\}$ and $j \in[n] \backslash\left\{s_{M}\right\}, i<j$, is calculated via inequalities (54) if $i$ and $j$ are in the same row, otherwise inequalities (54) are redundant. The distance between $i \in[n] \backslash\left\{s_{M}\right\}$ and $s_{M}$ is calculated by inequalities (55). If $i \in[n] \backslash\left\{s_{M}\right\}$ lies in row 1 (row 2) and $j \in[n] \backslash\left\{s_{M}\right\}, i<j$, in row 2 (row 1), their distance is calculated by inequalities (56) $((57))$. The $y, z$ and $x$ variables are chosen as described in Theorem 3. The $z$ variables are set to binary values as shown in the proof of Theorem 3. Further, $x_{i k j}, i, j, k \in[n], i<j,|\{i, j, k\}|=3$, is set to a binary value if $w_{i j}>0$ by inequalities (52)-(53), inequalities (21) and since the objective function is minimized. If $w_{i j}=0$, the objective function is not influenced by the value of $x_{i k j}$.

Inequalities (7), inequalities (9)-(18) and inequalities (52)-(57) are included from the beginning and inequalities (21) are separated as described in Section 3.3. For the usage of equations (8) we refer to Section 3.3. Additionally, we use inequalities (38), (39), (41) as described in Section 3.3 and we use the symmetry breaking constraints (29)-(30). Here, betweenness variables can only be equal to one if the corresponding departments lie in the same row, hence inequalities (19)-(20) and inequalities (36)-(37) are not valid for this MILP model.

In the following, we describe the standard linearization used in the approach denoted by variant 4 for the TRFLP in Table 2. We use standard linearization (instead of inequalities (19)-(20)) to couple the extended betweenness variables, i. e.,

$$
\begin{array}{ll}
x_{i k j}-z_{i k}-z_{k j} \geq-1, & i, j, k \in[n] \backslash\left\{s_{M}\right\},|\{i, j, k\}|=3, \\
x_{i k j}-x_{s_{M} k i}+y_{j} \geq 0, & i, j, k \in[n] \backslash\left\{s_{M}\right\},|\{i, j, k\}|=3, \\
x_{i k j}-x_{(n+2) k i}-y_{j} \geq-1, & i, j, k \in[n] \backslash\left\{s_{M}\right\},|\{i, j, k\}|=3 .
\end{array}
$$

The inequalities (59)-(61) are included from the beginning and the inequalities (19)-(20) are neglected. Besides this, we use variant 1 as described in Section 3.5. The corresponding upper bounds are obtained by inequalities (21) and previous tests, which we do not mention here, indicate that upper bounds related to the standard linearization do not improve this approach. The $y, z$ and $x$ variables are chosen as described in Theorem 3. The $z$ variables are set to binary values as shown in the proof of Theorem 3. Further, $x_{i k j}, i, j, k \in[n], i<j,|\{i, j, k\}|=3$, is set to a binary value if $w_{i j}>0$ by inequalities (59)-(61), inequalities (21) and since the objective function is minimized. If $w_{i j}=0$, the objective function is not influenced by the value of $x_{i k j}$. 
B. New exact approaches for the combined cell layout problem and extensions of the multi-bay facility layout problem

Authors: Mirko Dahlbeck, Anja Fischer, Kerstin Maier, Philipp Hungerländer submitted to European Journal of Operational Research, 2020. 


\title{
New exact approaches for the combined cell layout problem and extensions of the multi-bay facility layout problem
}

\author{
Mirko Dahlbeck, ${ }^{*}$ Anja Fischer ${ }^{\dagger}$ Philipp Hungerländer ${ }^{\ddagger}$ Kerstin Maier ${ }^{\S}$
}

October 21, 2020

In this paper we consider the Combined Cell Layout Problem (CCLP), the Multi-Bay Facility Layout Problem (MBFLP) and several generalizations of the MBFLP, which have wide applications, e.g., in factory planning, heavy manufacturing, semiconductor fabrication and arranging rooms in hospitals. Given a set of cells of type single-row or directed-circular and a set of one-dimensional departments with pairwise transport weights between them, the CCLP asks for an assignment of the departments to the cells such that departments in the same cell do not overlap and such that the sum of the weighted center-to-center distances is minimized. Distances between departments in the same cell are measured according to the layout type of the cell and otherwise their distance equals the sum of the distances to the associated (un-) loading stations of the cells plus possible space between the cells. We solve the CCLP exactly by enumerating over all assignments of the departments to the cells and solving several CCLP with fixed-cell assignment. We show how to reduce the number of distinguishable cell assignments significantly by merging two cells of type single-row. This leads to new well-performing exact approaches for the CCLP, the MBFLP and its generalizations where arising subproblems are solved via (new) mixed-integer linear programming models. In a computational study we compare the computation times and the optimal values of various facility layout problems in order to support the decision maker to choose a layout.

Key words. Facility Planning and Design, Combined Cell Layout Problem, Multi-Bay Layout Problem, Pier-Type Layouts, Exact Approaches

\footnotetext{
${ }^{*}$ TU Dortmund University, Faculty of Business and Economics; Georg-August-Universität Göttingen, Institute for Numerical and Applied Mathematics, mirko.dahlbeck@tu-dortmund.de

${ }^{\dagger}$ TU Dortmund University, Faculty of Business and Economics, anja2.fischer@tu-dortmund.de

${ }^{\ddagger}$ Institute for Mathematics, Alpen-Adria-Universität Klagenfurt, Austria, philipp.hungerlaender@aau.at

${ }^{\S}$ MANSIO Karl Popper Kolleg, Alpen-Adria-Universität Klagenfurt, Austria, kerstin.maier@aau.at
} 


\section{Introduction}

The aim of facility layout problems is to find an optimal non-overlapping arrangement of departments inside a plant according to a given objective function, e.g., minimizing materialhandling costs. It constitutes an important problem for manufacturing industries as up to $50 \%$ of manufacturing costs are due to moving parts between different facilities, and thus a good arrangement of facilities might reduce up to $30 \%$ of material-handling costs [53]. In contrast, a poor layout can add up to $36 \%$ of material-handling costs [16]. Especially in terms of Industry 4.0 and Smart Manufacturing minimizing material-handling costs, and hence finding an optimal layout of the departments within the facilities, plays an enormous role $[2,46]$.

In this work we consider various facility layout problems and propose new well-performing exact approaches for solving them. We are given the following setting for all layout problems considered: A set of $n \in \mathbb{N}$ one-dimensional departments $[n]:=\{1, \ldots, n\}$, with lengths $\ell_{i}, i \in[n]$, and pairwise weights $w_{i j}, i, j \in[n], i \neq j$, and a set of cells $\mathcal{C}$. The task is to minimize the sum of the weighted center-to-center distances of the departments such that departments in the same cell do not overlap. The considered problems differ in two aspects - the structure of the layout, i. e., the number of cells and their (un-) loading positions - and the distance calculation. We describe the differences in the following.

At first, we consider problems consisting of one cell and we deal with the Single-Row Facility Layout Problem (SRFLP) and the Directed Circular Facility Layout Problem (DCFLP). In the SRFLP one looks for an arrangement of the departments in one row such that the weighted sum of the horizontal center-to-center distances between the departments is minimized. Like for all other layout problems considered in this work, the arrangement of machines within flexible manufacturing systems is a perfect application example [29]. Further, applications can be found in the alignment of departments in office buildings, hospitals or in supermarkets [52], the assignment of files to disk cylinders in computer storage, and the design of warehouse layouts [42, 48]. In the DCFLP the task is to find an arrangement of the departments along a circle such that the weighted sum of the center-to-center distances measured in clockwise direction is minimized. According to [1, 44], the DCFLP has several practical advantages over the SRFLP, e. g., relative low initial investment costs because of their space-saving design and high material handling flexibility. The DCFLP arises by, e.g., determining a space-free alignment around a cyclic conveyor system or the cyclic motion path of an industrial robot. Both, the SRFLP and the DCFLP are widely studied, see, e. g., $[5,15,36,37,38,40]$.

The focus of this work lies on facility layout problems consisting of several cells where we concentrate on problems with up to four cells in the computational experiments. At first, we consider the Combined Cell Layout Problem (CCLP), which is a generalization of the SRFLP and the DCFLP. We are given a set of cells $\mathcal{C}:=\{1, \ldots, m\}, m \in[n], m \geq 2$, each with an (un-) loading station whose position is denoted by $p_{E_{k}}, k \in \mathcal{C}$ (in this paper we always assume that loading and unloading station of a cell are the same). The function $t: \mathcal{C} \rightarrow\{$ SRFLP, DCFLP $\}$ specifies the associated layout type of each cell. For $t(k)=\operatorname{SRFLP}, k \in \mathcal{C}$, the position $p_{E_{k}}$ of the loading station is fixed at the left or right border of cell $k$ and for $t(k)=$ DCFLP, $k \in \mathcal{C}$, the loading station can be placed on an arbitrary position along the circle. The inner-cell distances depend on the type of the cell. The inter-cell distance between cell $k \in \mathcal{C}$ and cell $o \in \mathcal{C}, k<o$, is denoted by $u_{k o}$ and the distance between departments in different cells equals the sum of the distances of the departments to the respective loading station in the same cell plus the corresponding inter-cell distance $u_{k o}$. We also write $\operatorname{CCLP}\left(m_{1}, m_{2}\right), m=m_{1}+m_{2}$, where $m_{1} \in \mathbb{Z}_{\geq 0}$ denotes the number of cells of type SRFLP and $m_{2} \in \mathbb{Z}_{\geq 0}$ denotes the number of cells of type DCFLP.

Several variants of the CCLP have been studied in the literature in more detail. If the assignment of the departments to the cells is fixed, the problem is called Fixed-Cell Combined Cell Layout Problem $(\mathrm{FC}-\mathrm{CCLP})^{1}$. If $t(k)=\operatorname{SRFLP}$ for all $k \in \mathcal{C}$, then this problem is denoted as Multi-Bay

\footnotetext{
${ }^{1}$ In $[15,32]$ this problem is denoted by Combined Cell Layout Problem instead of Fixed-Cell Combined Cell
} 
Facility Layout Problem (MBFLP), see, e. g., [26, 41, 47]. The cells are arranged in a non-overlapping and in a parallel way, and hence the inter-cell distance is set to $u_{k o}=w_{p a t h}(o-k), k, o \in \mathcal{C}, k<$ $o, w_{\text {path }} \in \mathbb{R}_{\geq 0}$. The MBFLP is in particular interesting in practice in heavy manufacturing, e. g., steel production and bridge crane manufacturing, and semiconductor fabrication [45, 47, 54], as well as arranging the rooms of the patients in hospitals where often only one side of a corridor has windows [48]. Many real-world factory layouts implicitly use these layout structures, see, e.g., $[18,45]$. The layout problem similar to the MBFLP but with $u_{k o}=w_{\text {path }}$ for some $w_{\text {path }} \in \mathbb{R}_{\geq 0}$ is denoted by Pier-Type Material Flow Pattern (PMFP), see [21]. The PMFP has applications in the design of cross docking warehouses [21]. The size of the factory, and thus the costs of the initial investment increases with a large number of cells. Hence, we focus on the MBFLP and the PMFP with $m=3$ and $m=4$ which we call (3-BFLP), (4-BFLP), (3-PMFP) and (4-PMFP), respectively. For instance, in a hospital each floor corresponds to a cell and the initial investment costs for building a hospital usually increase with the number of floors. The departments are given as one-dimensional objects. Hence, we assume implicitly that the departments have the same height and we assume, w.l.o.g., that the height equals one. In the MBFLP with $m \geq 3$ the vertical distance between a department in cell $i$ and a department in cell $j, i, j \in[m], i<j$, is at least the height of row $i$ plus the height of the cells between cell $i$ and cell $j$. The vertical distances can be included by enlarging $w_{\text {path }}$, thus, in the following, we assume that the vertical distance is included in $w_{\text {path }}$ if $w_{\text {path }}>0$.

The T-Row Facility Layout Problem (TRFLP) was introduced in [23] and consists of two cells of type SRFLP where the position of the loading station in cell 2 is fixed at the border and in cell 1 the position $p_{E_{1}}$ can be chosen arbitrarily. It is shown in [23] that the TRFLP is an extension of the 3-BFLP, and hence has the same applications. For further applications we refer to [23].

We continue this line of research and introduce the X-Row Facility Layout Problem (XRFLP). Recall that we assume that the height of the departments equals one. Given four non-overlapping cells of type SRFLP which form an X and let $d_{i p_{E_{k}}}$ denote the distance of the center of $i \in[n]$ to $p_{E_{k}}$ if $i$ is assigned to cell $k \in[4]$. Let $C_{2}\left(C_{3}\right)$ denote the set of departments assigned to cell 2 (cell 3), then one has to ensure that the departments in cell 2 and in cell 3 do not overlap, i. e., either $d_{i p_{E_{2}}} \geq 1+\frac{\ell_{i}}{2}, i \in C_{2}$, or $d_{i p_{E_{3}}} \geq 1+\frac{\ell_{i}}{2}, i \in C_{3}$, has to be satisfied. For an illustration we refer to Figure 1d. The XRFLP is an extension of the 4-BFLP because one has to ensure additionally that departments in cell 2 and cell 3 do not overlap. In factory planning it is realistic to take the width of the path between cells into account because the products have to be transported between the departments by a forklift or an automatic guided vehicle and the transportation systems usually travel in a rectangular manner. Considering the XRFLP, let $w_{\text {path }}^{1}$ $\left(w_{\text {path }}^{2}\right)$ denote the width of the path between cell 1 and cell 3 (cell 2 and cell 4 ). Measuring the distances between cell 2 and cell 3 we do not cross a path, and hence we set the inter-cell distance to zero. In contrast, going from cell 1 to cell 4 we cross both paths, i. e., $u_{14}=w_{\text {path }}^{1}+w_{\text {path }}^{2}$. For the remaining inter-cell distances we obtain $u_{12}=u_{13}=w_{\text {path }}^{1}$ and $u_{24}=u_{34}=w_{\text {path }}^{2}$.

We illustrate the distance calculation of the problems considered above by the following example.

Example 1. We are given an instance with $n=5$ departments with lengths $\ell_{2}=1, \ell_{1}=\ell_{3}=$ $\ell_{4}=2, \ell_{5}=3$ and non-zero weights $w_{15}=w_{24}=w_{31}=1, w_{21}=w_{23}=w_{52}=2, w_{42}=3$. The inter-cell distances $w_{\text {path }}^{1}$, $w_{\text {path }}^{2}$ for the XRFLP are set to zero as well as $u_{12}=0$ for the CCLP $(1,1)$ and the CCLP $(0,2)$. Optimal layouts of different facility layout problems are depicted in Figure 1. Note that in the X-row layout department 2 is in cell 3.

a) An optimal single-row layout is illustrated in Figure 1a with objective value

$$
2 \cdot 1.5+1 \cdot 5+1 \cdot 2.5+2 \cdot 3.5+(3+1) \cdot 1.5+2 \cdot 4=31.5
$$

Layout Problem and additionally one department in each cell is fixed opposite the (un-) loading station. 
b) an optimal directed-circular layout is illustrated in Figure $1 b$ with objective value

$$
1 \cdot 2.5+2 \cdot 3.5+2 \cdot 1.5+1 \cdot 8.5+1 \cdot 2+3 \cdot 1.5+2 \cdot 4=35.5
$$

c) an optimal T-row layout is illustrated in Figure 1c with objective value

$$
2 \cdot 0.5+1 \cdot 2+1 \cdot 2.5+2 \cdot 2.5+(3+1) \cdot 1.5+2 \cdot 3=22.5
$$

d) an optimal X-row layout is illustrated in Figure $1 d$ with objective value

$$
2 \cdot 1.5+1 \cdot 3+1 \cdot 2.5+2 \cdot 2.5+(3+1) \cdot 1.5+2 \cdot 2=23.5
$$

e) an optimal combined cell layout with two circular layout cells is illustrated in Figure 1e with objective value

$$
2 \cdot 1.5+1 \cdot(2+1.5)+1 \cdot 2.5+2 \cdot(0+2)+(3+1) \cdot 0+2 \cdot 2=17
$$

f) an optimal combined cell layout with one single-row and one circular layout cell is illustrated in Figure 1f with objective value

$$
2 \cdot 1.5+1 \cdot(3+1.5)+1 \cdot 2.5+2 \cdot(0+3)+(3+1) \cdot(0+1)+2 \cdot 2=24 .
$$

In the following example we compare the distance calculation of the 3-BFLP and the 4-BFLP with the distance calculation of the 3-PMFP and the 4-PMFP.

Example 2. We are given an instance with $n=5$ departments with lengths $\ell_{1}=\ell_{5}=6, \ell_{3}=$ $\ell_{4}=5, \ell_{2}=2$ and non-zero weights $w_{12}=2, w_{13}=w_{14}=w_{15}=w_{34}=w_{35}=1, w_{\text {path }}=1$. Optimal 3-Bay, 4-Bay, 3-Pier-Type and 4-Pier-Type layouts are illustrated in Figure 2.

a) An optimal 3-Bay layout is illustrated in Figure 2a with an objective value of

$$
2 \cdot 4+6.5+11.5+7+5+7.5=45.5
$$

b) An optimal 4-Bay layout is illustrated in Figure $2 b$ with an objective value of

$$
2 \cdot 4+6.5+7.5+7+6+7.5=42.5
$$

c) An optimal 3-Pier-Type layout is illustrated in Figure 2c with an objective value of

$$
2 \cdot 4+6.5+11.5+7+5+6.5=44.5
$$

d) An optimal 4-Pier-Type layout is illustrated in Figure 2d with an objective value of

$$
2 \cdot 4+6.5+6.5+7+6+6.5=40.5 \text {. }
$$




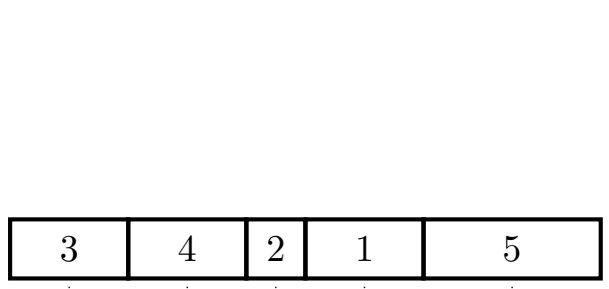

( $\ldots \ldots \ldots \ldots$

(a) Optimal single-row layout with objective value 31.5 .

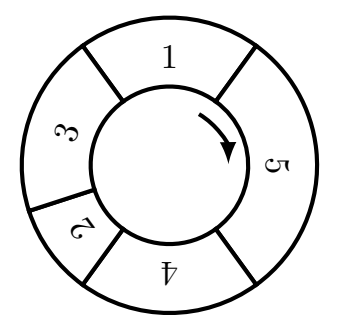

(b) Optimal directed-circular layout with objective value 35.5 .

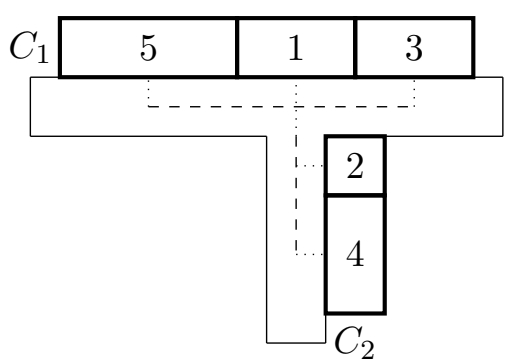

(c) Optimal T-row layout with objective value 22.5 .

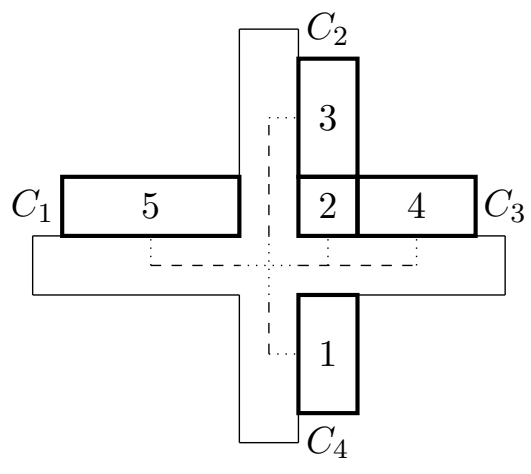

(d) Optimal X-row layout with objective value 23.5.
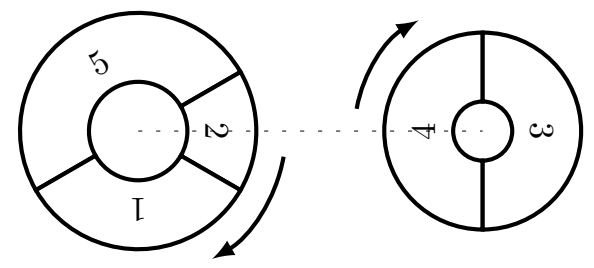

(e) Optimal combined cell layout for two circular layout cells $\left(p_{E_{1}}\right.$ and $p_{E_{2}}$ lie above departments 2 and 4 , respectively) with objective value 17 .

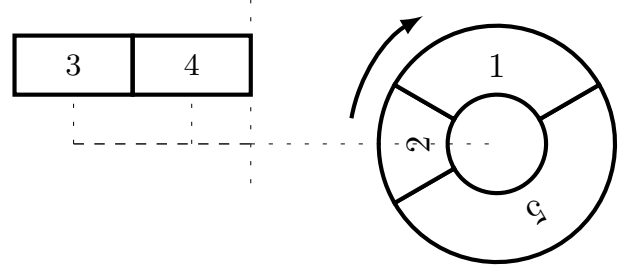

(f) Optimal combined cell layout for one single-row and one circular layout cell $\left(p_{E_{1}}\right.$ lies on the right border and $p_{E_{2}}$ lies above department 2$)$ with objective value 24 .

Figure 1: We are given an instance with $n=5$ departments with lengths $\ell_{2}=1, \ell_{1}=\ell_{3}=\ell_{4}=$ $2, \ell_{5}=3$ and non-zero weights $w_{15}=w_{24}=w_{31}=1, w_{21}=w_{23}=w_{52}=2, w_{42}=3$. The inter-cell distances are set to zero. Illustration of optimal layouts and the associated distance calculations for the SRFLP, the DCFLP, the TRFLP, the XRFLP, the CCLP $(0,2)$ and the CCLP $(1,1)$. Detailed calculations of the objective values are given in Example 1. Dotted lines are neglected in the distance calculations.

\subsection{Literature review}

There are several facility layout problems studied in the literature, see, e.g., [12, 30] for two recent surveys. In the following we give an overview of existing solution approaches for facility layout problems considered in this work as well as related ones:

- The SRFLP is one of a few layout types for which strong lower and upper bounds for even large-sized instances exist which are based on Semidefinite Programming (SDP) and Integer Linear Programming (ILP) formulations. The strongest SDP approach $[35,36]$ is able to solve one instance with 42 departments to optimality while the current best ILP approach [5], based on betweenness variables, is able to solve instances with up to 35 departments. Several heuristic approaches were presented, see, e. g., [25, 43, 49], (see [24] 


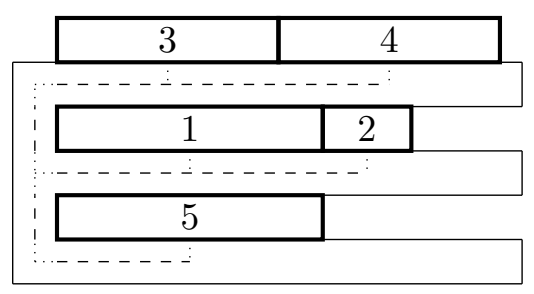

(a) Optimal 3-Bay layout with objective value 45.5 .

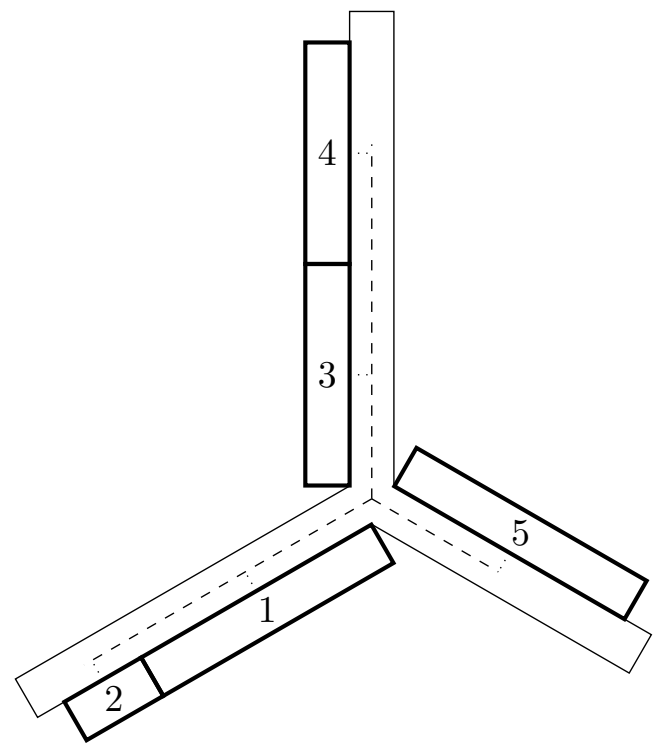

(c) Optimal 3-Pier-Type layout with objective value 44.5 .

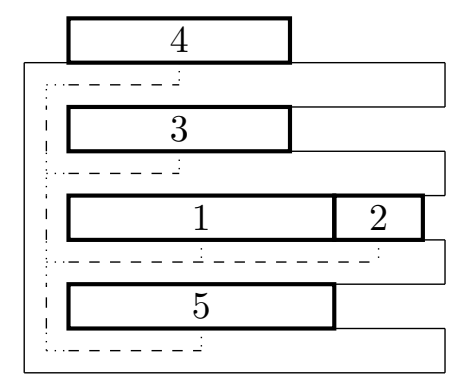

(b) Optimal 4-Bay layout with objective value 42.5 .

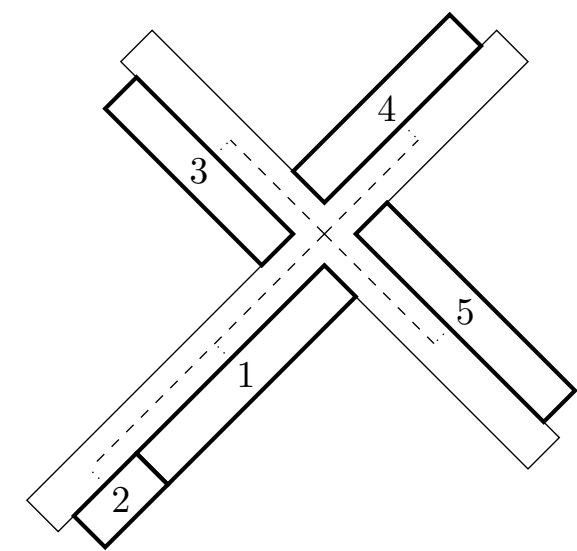

(d) Optimal 4-Pier-Type layout with objective value 40.5

Figure 2: We are given an instance with $n=5$ departments with lengths $\ell_{1}=\ell_{5}=6, \ell_{3}=\ell_{4}=5$, $\ell_{2}=2$ and non-zero weights $w_{12}=2, w_{13}=w_{14}=w_{15}=w_{34}=w_{35}=1, w_{\text {path }}=1$. We illustrate optimal layouts and the associated distance calculations for the 3-BFLP, the 4-BFLP, the 3-PMFP and the 4-PMFP. Dotted lines are neglected in these calculations. For details on the distances we refer to Example 2. 
for a correction of the proof for the main result the heuristic of [49] is based on). A recent survey is given in [40].

- The Checkpoint Ordering Problem (COP) asks for a space-free non-overlapping arrangement of the departments in one cell such that the sum of the weighted distances of the centers of the departments to a checkpoint whose position is given in advance is minimized. In [31] a dynamic programming algorithm and an ILP approach is suggested for solving the COP. Further, [34] proposed the Multiple Checkpoint Ordering Problem, which generalizes the COP to an arbitrary but fixed number of checkpoints.

- Let a set of nodes $V$ with $|V|=n$ and weights $w_{i j}$ and $w_{j i}, i, j \in V, i<j$, be given. The Linear Ordering Problem (LOP) looks for a bijective mapping $\sigma:[n] \rightarrow[n]$ such that $\sum_{\substack{i, j \in V \\ \sigma^{-1}(i)<\sigma^{-1}(j)}} w_{i j}$ is maximized, see, e.g., [27, 28]. The DCFLP can be modeled as an LOP, and hence the DCFLP can be solved faster in practice than the SRFLP, see [37, 38], which was so far considered as the simplest available layout type. An SDP and an ILP approach are given in [38] as well as heuristic approaches such that tight lower and upper bounds for instances with up to 100 departments are provided. We refer to [38] for an overview of further circular layout problems.

- For the FC-CCLP where additionally in each cell one department is fixed as the loading station, the ILP model of [15] outperformed the SDP approach of [32]. To the best of our knowledge, our paper is the first that considers the CCLP without fixing one department as loading station and without pre-assigning departments to given cells.

- There are several two-stage procedure heuristics for the MBFLP, see, e. g., [19, 20, 47], where at first, the departments are assigned to the cells and second, the order of the departments within each cell is determined. In [26] an ILP model for the MBFLP with fixed cell assignment was proposed and optimal solutions for instances with up to 25 departments and up to 5 rows are obtained within one second. Further, a Mixed-Integer Linear Programming (MILP) model for solving the PMFP can be found in [21]. The current best approach for the 3-BFLP as well as for the TRFLP is given in [23] where instances with up to 18 departments are solved to optimality and tight lower bounds for the 3-BFLP with up to 24 departments are provided.

- The Multi-Row Facility Layout Problem (MRFLP) consists of a set of $m$ non-overlapping parallel cells of type SRFLP where free-space between neighboring departments in the same cell may arise and where the distance between the departments equals their horizontal distance. The special case with two cells is denoted by Double-Row Facility Layout Problem (DRFLP). There are several MILP approaches for the DRFLP, see, [6, 9, 22, 51, 55], and an SDP approach for the MRFLP [33]. The current best approach is an enumerative approach by [26], which is able to solve double-row instances with up to 16 departments and multi-row instances with up to 5 cells and 13 departments in reasonable time. Recently a two-stage approach for the MRFLP was presented in [11] which allows to derive good solutions quickly for $m \geq 3$.

\subsection{Our contribution}

The main contributions of this paper are the following:

- We present a new exact approach for the CCLP where we enumerate over all cell assignments of the departments and then solve several FC-CCLP. We show how to reduce the number of cell assignments that have to be considered significantly. Indeed, given a CCLP instance where all cells of type SRFLP have the same inter-cell distances and let an assignment of 
the departments to the cells be given, then we can merge two cells of type SRFLP. If the number of cells of type SRFLP is even, this result allows us to halve the number of cells of type SRFLP.

- We extend the previous mentioned results to the 3-BFLP and to the 4-BFLP with positive inter-cell distances. We introduce the XRFLP, which is a realistic extension of the 4-BFLP with a more complex path structure, and we show that we can use our main result for this problem as well even with positive inter-cell distances.

- In $[15,32]$ a fixed department deals as (un-) loading station. We omit this assumption by adding a dummy department with appropriate length and weights to each cell which deals as (un-) loading station. Considering cells of type DCFLP, one department may overlap with the dummy department and for the arising optimization problem we present a new MILP model which outperforms an associated enumerative approach.

- At first, we present a theoretical study between the relationship of the optimal values of several facility layout problems, see Section 3. Then, in a computational study, see Section 6 , we compare the optimal values of these layout problems on instances from the literature in order to support the decision maker to choose the layout of a factory which is built from the ground up. We also display the running time for solving these problems as this might influence the decision.

- Our approach outperforms the current best approach for the 3-BFLP as well as the 3-PMFP and 4-PMFP, and hence we partially answer a research question of [21] to derive a more efficient exact solution approach for the PMFP.

\section{Summary of exact approaches for the SRFLP and the DCFLP}

In this section we summarize MILP models for the SRFLP and the DCFLP. Let $\mathcal{D}$ denote a set of departments. For single-row instances with up to 20 departments the MILP model of [5] based on betweenness variables allows to calculate optimal layouts faster than other approaches from the literature, see, e.g. [35, 36]. For solving the DCFLP, we choose the MILP formulation suggested in $[15,37,38]$.

\subsection{The Single-Row Facility Layout Problem}

In the following we recall the respective MILP formulation of [5]. First, we make use of betweenness variables $x_{i k j}, i, j, k \in \mathcal{D},|\{i, j, k\}|=3, i<j$, where $\mathcal{D}$ represents a set of departments (a subset of $[n]$ plus partially some added dummy departments in the following where we assume that $w, \ell$ are known), with the interpretation

$$
x_{i k j}= \begin{cases}1, & \text { if } k \text { lies between } i \text { and } j, \\ 0, & \text { otherwise. }\end{cases}
$$

Then the MILP model reads as follows, where we neglect the constant weights

$$
\begin{aligned}
& W^{S}=\sum_{\substack{i, j \in \mathcal{D} \\
i<j}}\left(w_{i j}+w_{j i}\right) \frac{\ell_{i}+\ell_{j}}{2} \text {. } \\
& \min \sum_{\substack{i, j \in \mathcal{D} \\
i<j}}\left(w_{i j}+w_{j i}\right) \sum_{k \in \mathcal{D} \backslash\{i, j\}} \ell_{k} x_{i k j} \\
& \text { s.t. } x_{i k j}+x_{j i k}+x_{i j k}=1 \text {, } \\
& -x_{i h j}+x_{i h k}+x_{j h k} \geq 0, \\
& x_{i h j}-x_{i h k}+x_{j h k} \geq 0 \text {, } \\
& i, j, k \in \mathcal{D}, i<j<k, \\
& i, j, k, h \in \mathcal{D}, i<j<k,|\{i, j, k, h\}|=4 \text {, } \\
& i, j, k, h \in \mathcal{D}, i<j<k,|\{i, j, k, h\}|=4 \text {, }
\end{aligned}
$$




$$
\begin{array}{ll}
x_{i h j}+x_{i h k}-x_{j h k} \geq 0, & i, j, k, h \in \mathcal{D}, i<j<k,|\{i, j, k, h\}|=4, \\
x_{i h j}+x_{i h k}+x_{j h k} \leq 2, & i, j, k, h \in \mathcal{D}, i<j<k,|\{i, j, k, h\}|=4, \\
x_{i k j} \in\{0,1\}, & i, j, k \in \mathcal{D}, i<j,|\{i, j, k\}|=3 .
\end{array}
$$

Note that for the SRFLP there always exists an optimal layout without free-spaces between neighboring departments, hence, neglecting $W^{S}$ the distance between two departments equals the lengths of the departments between them, see (1). Equations (2) ensure that for three departments $i, j, k \in \mathcal{D}, i<j<k$, exactly one of the departments lies between the other two. In a feasible arrangement of the departments $\mathcal{D}$ for each subset $\{i, j, k, h\} \subseteq \mathcal{D},|\{i, j, k, h\}|=4$, the following holds: if department $h$ lies between departments $i$ and $j$, then department $h$ additionally lies between a) departments $i$ and $k$ or b) departments $j$ and $k$, see Inequalities (3), but not both a) and b), see Inequalities (6). Hence, Inequalities (3)-(6) ensure transitivity and according to $[5,50]$ together with the binarity of the $x$-variables $(7)$ we obtain a feasible ordering of the departments.

\subsection{The Directed Circular Facility Layout Problem}

The approach of $[15,37,38]$ makes use of distance variables $d_{i j}, i, j \in \mathcal{D}, i \neq j$, measuring the center-to-center distances between $i$ and $j$ in clockwise direction. Given a directed-circular layout, we interpret this layout as a single-row layout with a different distance calculation by splitting the circle at one department and unwinding it. Indeed, in order to obtain an ordering of the departments with respect to the splitting department, we choose an arbitrary department $f \in \mathcal{D}$ to be the leftmost department and then we say that $i \in \mathcal{D} \backslash\{f\}$ is to the left of $j \in \mathcal{D} \backslash\{f\}, i<j$, if $d_{f i}<d_{f j}$. Therefore we use ordering variables

$$
z_{i j}= \begin{cases}1, & \text { if } i \text { lies to the left of } j \\ 0, & \text { otherwise }\end{cases}
$$

$i, j \in \mathcal{D} \backslash\{f\}, i<j$. Note that $f$ is the leftmost department, and hence we neglect ordering variables containing $f$. Further, we can exclude some constants, let $L:=\sum_{k \in \mathcal{D}} \ell_{k}$. If both $w_{i j}$ and $w_{j i}, i, j \in \mathcal{D}, i<j$, are greater than zero, then we set $\tilde{w}_{i j}=w_{i j}-\min \left\{w_{i j}, w_{j i}\right\}$ and $\tilde{w}_{j i}=w_{j i}-\min \left\{w_{i j}, w_{j i}\right\}$ and add the constant $W:=\sum_{\substack{i, j \in \mathcal{D} \\ i<j}} \min \left\{w_{i j}, w_{j i}\right\} L$ to the objective value, see [37]. In total, we neglect the constant weights $W^{D}=\sum_{\substack{i, j \in \mathcal{D} \\ i \neq j}} \tilde{w}_{i j} \frac{\ell_{i}+\ell_{j}}{2}+W$. Let $D_{i j}, i, j \in \mathcal{D} \backslash\{f\}, i<j$, denote the sum of the lengths of the departments $k \in \mathcal{D} \backslash\{f, i\}$ which are left of $j$ minus the sum of the lengths of the departments $k \in \mathcal{D} \backslash\{f, j\}$ which are left of $i$. Note that $D_{i j}, i, j \in \mathcal{D}, i<j$, is negative if $j$ is left to $i$. Then the MILP can be written as follows where we set $L_{i j}=L_{j i}=L-\ell_{i}-\ell_{j}, i, j \in \mathcal{D}, i<j$.

$$
\begin{array}{lll}
\min & \sum_{\substack{i, j \in \mathcal{D} \\
i \neq j}} \tilde{w}_{i j} d_{i j} & \\
\text { s.t. } 0 \leq z_{i j}+z_{j k}-z_{i k} \leq 1, & i, j, k \in \mathcal{D} \backslash\{f\}, i<j< \\
& d_{f i}-\sum_{\substack{k \in \mathcal{D} \backslash\{f\} \\
k<i}} \ell_{k} z_{k i}+\sum_{\substack{k \in \mathcal{D} \backslash\{f\} \\
k>i}} \ell_{k} z_{i k}=\sum_{\substack{k \in \mathcal{D} \backslash\{f\} \\
k>i}} \ell_{k}, \quad & i \in \mathcal{D} \backslash\{f\}, \\
& d_{i f}+d_{f i}=L_{f i}, & i \in \mathcal{D} \backslash\{f\}, \\
& D_{i j}-\sum_{\substack{k \in \mathcal{D} \backslash\{f, i\} \\
k<j}} \ell_{k} z_{k j}+\sum_{\substack{k \in \mathcal{D} \backslash\{f, i\} \\
k>j}} \ell_{k} z_{j k} & \\
& +\sum_{\substack{k \in \mathcal{D} \backslash\{f, j\} \\
k<i}} \ell_{k} z_{k i}-\sum_{\substack{k \in \mathcal{D} \backslash\{f, j\} \\
k>i}} \ell_{k} z_{i k}=-\sum_{\substack{k \in \mathcal{D} \backslash\{f\} \\
i<k<j}} \ell_{k}, & \\
&
\end{array}
$$




$$
\begin{aligned}
& d_{i j}+L_{i j} z_{i j}-D_{i j}=L_{i j}, \\
& d_{j i}-L_{i j} z_{i j}+D_{i j}=0, \\
& d_{i j} \geq 0, \\
& z_{i j} \in\{0,1\},
\end{aligned}
$$

$$
\begin{aligned}
& i, j \in \mathcal{D} \backslash\{f\}, i<j, \\
& i, j \in \mathcal{D} \backslash\{f\}, i<j, \\
& i, j \in \mathcal{D}, i \neq j, \\
& i, j \in \mathcal{D} \backslash\{f\}, i<j .
\end{aligned}
$$

Inequalities (8) are the well-known three-cycle-inequalities, which ensure transitivity and suffice to induce a feasible ordering of the departments, see, e.g., [15, 37, 38]. Via Equations (9)-(10) we determine for each department $i \in \mathcal{D} \backslash\{f\}$ the sum of the lengths of the departments which lie between $f$ and $i$ and between $i$ and $f$, respectively (calculated in clockwise direction). Equations (11) compute for each pair of departments $i$ and $j$ with $i, j \in \mathcal{D} \backslash\{f\}$, the sum of the lengths of the departments $k \in \mathcal{D} \backslash\{f, i\}$ which are left of $j$ minus the sum of the lengths of the departments $k \in \mathcal{D} \backslash\{f, j\}$ which are left of $i$. By Equations (12) and (13) we obtain the correct distance between $i$ and $j$ with $i, j \in \mathcal{D} \backslash\{f\}, i<j$, i. e., if $i$ lies left to $j$ we get $d_{i j}=D_{i j}$ and $d_{j i}=L_{i j}-D_{i j}$ and otherwise $d_{i j}=L_{i j}+D_{i j}$ and $d_{j i}=-D_{i j}$ with negative values of $D_{i j}$ in the latter case.

\section{Relation between the optimal values of several facility layout problems}

After repeating and introducing several facility layout problems we want to study in this section the relation of the optimal objective values of the considered layout problems. We start with a comparison of the SRFLP and the DCFLP. Let $d_{i j}^{\text {DCFLP }}, i, j \in[n], i \neq j$, denote the center-to-center distances between $i$ and $j$ measured in clockwise direction in the DCFLP and let $d_{i j}^{\mathrm{SRFLP}}=d_{j i}^{\mathrm{SRFLP}}, i, j \in[n], i<j$, denote the horizontal center-to-center distance between $i$ and $j$ in the SRFLP. It holds that $d_{i j}^{\text {SRFLP }}<\sum_{k \in[n]} \ell_{k}, i, j \in[n], i<j$, in the SRFLP and $d_{i j}^{\text {DCFLP }}+d_{j i}^{\text {DCFLP }}=\sum_{k \in[n]} \ell_{k}$ in the DCFLP. Hence, for an instance with symmetric weights, i. e., if $w_{i j}=w_{j i}$ for all $i, j \in[n], i<j$, the optimal value of the SRFLP is less than the optimal value of the DCFLP if $n \geq 3$ (if not all weights are equal to zero). In contrast to this, consider an instance with lengths $\ell_{i}=1, i \in[n]$, and non-zero (asymmetric) weights $w_{i(i+1)}=1, i \in[n-1]$, and $w_{n 1}=1$. Then, the optimal value of the DCFLP equals $C:=\sum_{i, j \in[n], i \neq j} w_{i j} \frac{\ell_{i}+\ell_{j}}{2}=n$ and for the SRFLP one obtains $C+n-2$. We illustrate two optimal single-row layouts for $n=7$ in Figure 3 . This shows that it is not possible to provide a general statement comparing the optimal values of the SRFLP and the DCFLP.

Next we extend our study to layout problems with more than one cell. In the following consideration let $w_{\text {path }}=w_{\text {path }}^{1}=w_{\text {path }}^{2}$ and for the CCLP $(2,0)$, the CCLP $(1,1)$ and the CCLP $(0,2)$ let $u_{12}=w_{\text {path }}$. The digraph $D=(O, A(O))$ illustrated in Figure 4 shows the relations of the optimal objective values of the considered layout problems. An $\operatorname{arc}(i, j) \in A(O)$ from $i \in O:=\{\operatorname{SRFLP}$, DCFLP, CCLP $(2,0), \operatorname{CCLP}(1,1)$, CCLP $(0,2)$, TRFLP, 3-BFLP, 4-BFLP, 3-PMFP, 4-PMFP, XRFLP $\}$ to $j \in O, i \neq j$, is added if the optimal value of the layout problem $i$ is at least as high as the optimal value of layout problem $j$. Note that transitive arcs are not illustrated. The displayed digraph is correct. This can be shown by considering the associated optimal layouts. Let $(i, j) \in A(O)$, then usually the optimal layout for problem $i \in O$ is a feasible layout for problem $j \in O$ and comparing bay and pier-type layouts just the distance calculation varies. It was mentioned in [23] that the optimal value of the TRFLP is not higher than the one for the 3-BFLP under the described conditions. One can construct instances such that each of the problems TRFLP, XRFLP and 4-PMFP has the smallest optimal value of these three layout problems. Additionally, one can construct instances such that the optimal value of the 4-BFLP is less than or equal to the optimal value of the XRFLP and vice versa. In Section 6 we will compare the objective values of the various layout problems for instances from the literature. 


\begin{tabular}{|l|l|l|l|l|l|l|}
\hline 1 & 2 & 3 & 4 & 5 & 6 & 7 \\
\hline
\end{tabular}

(a) Optimal single-row layout with objective value 12 and distances, e.g., $d_{71}=6$ and $d_{i(i+1)}=$ $1, i \in[6]$.

\begin{tabular}{|l|l|l|l|l|l|l|}
\hline 5 & 4 & 6 & 7 & 1 & 3 & 2 \\
\hline
\end{tabular}

(b) Optimal single-row layout with objective value 12 and distances, e.g., $d_{12}=d_{56}=2, d_{71}=$ $1, d_{34}=4$, and $d_{i(i+1)}=1, i \in\{2,4,6\}$.

Figure 3: We are given an SRFLP instance with $n=7$, lengths $\ell_{i}=1, i \in[7]$, and non-zero weights $w_{i(i+1)}=1, i \in[6], w_{71}=1$. We illustrated two optimal singe-row layouts.

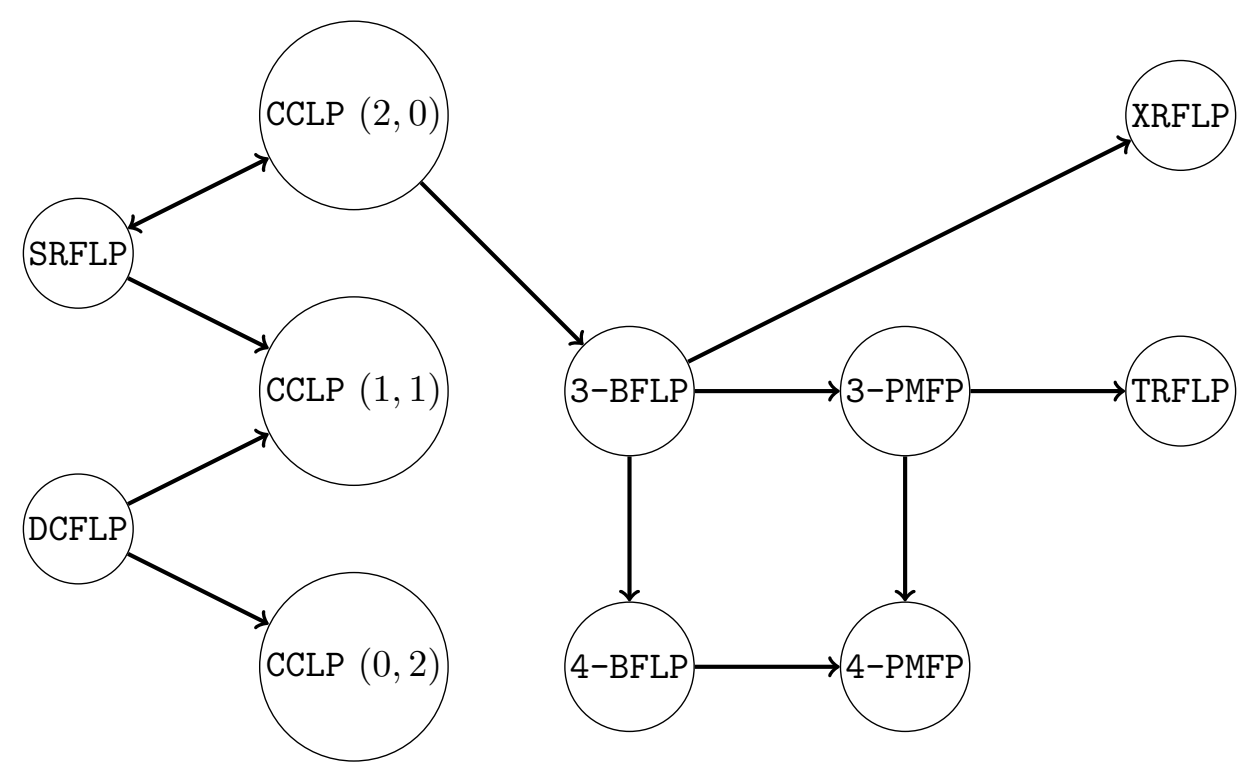

Figure 4: A comparison of the optimal values of several facility layout problems where an arc between $i \in O=\{\operatorname{SRFLP}$, DCFLP, CCLP $(0,2)$, CCLP $(1,1)$, CCLP $(2,0)$, TRFLP, 3-BFLP, 4-BFLP, 3-PMFP, 4-PMFP, XRFLP $\}$ and $j \in O$ indicates that the optimal value of the layout problem $i$ is at least as high as the optimal value of the layout problem $j$. Transitive arcs are not illustrated.

\section{The Combined Cell Layout Problem}

Our goal is to solve the CCLP exactly by enumerating over all (distinguishable) cell assignments and then solving several FC-CCLP. In [47] it is stated that if the cell assignment is given, one can solve the cells in the MBFLP independently by adding appropriate dummy departments. We extend this result to the CCLP. In Section 4.1 we provide a significant reduction on the number of cell assignments of type SRFLP that have to be considered if the inter-cell distances $u_{k o}$ are the same for all $k, o \in \mathcal{C}, k<o$, with $\{t(k), t(o)\} \cap\{$ SRFLP $\} \neq \emptyset$, by merging two cells of type SRFLP, and hence solving larger single-row instances including an additional dummy department.

In Section 4.2 we show how to solve the problems associated with circular cells. Indeed, in the DCFLP one department may overlap with the dummy department and we prove that there always exists an optimal directed-circular layout where one department lies opposite the dummy department. Based on this result we present an MILP model for determining an optimal layout for the departments contained in a cell of type DCFLP including the additional dummy department. Alternatively one can use an enumerative approach instead of the MILP model. The running times of both variants are compared in Section 6 .

\subsection{Our Algorithm}

Our goal is to solve the optimization problems in each cell of the FC-CCLP independently as done in [47]. Therefore we define the following problems where the weights of exactly one department 
are adjusted appropriately.

Definition 3. Given an $F C-C C L P$ instance and let $\mathcal{D} \subseteq\left[n+m^{\prime}\right], m^{\prime} \in \mathbb{N}_{\geq 0}$, be a set of departments where $n+1, n+2, \ldots, n+m^{\prime}$ are dummy departments with lengths $\ell_{n+1}, \ell_{n+2}, \ldots, \ell_{n+m^{\prime}} \in \mathbb{R}_{\geq 0}$ and weights $w_{i z}=w_{z i}=0, i \in\left[n+m^{\prime}\right], z \in\left\{n+1, \ldots, n+m^{\prime}\right\}, i \neq z$. For the department $s_{M} \in \mathcal{D}$ we set

$$
\begin{array}{ll}
w_{i s_{M}} \leftarrow w_{i s_{M}}+\sum_{j \in[n] \backslash \mathcal{D}} w_{i j}, & i \in \mathcal{D} \backslash\left\{s_{M}\right\}, \\
w_{s_{M} i} \leftarrow w_{s_{M}}+\sum_{j \in[n] \backslash \mathcal{D}} w_{j i}, & i \in \mathcal{D} \backslash\left\{s_{M}\right\},
\end{array}
$$

all other weights remain the same. Then the aim of the optimization problem $W_{\left(s_{M}, p_{z}\right)}^{A}(\mathcal{D})$ for the updated weights is to find an optimal layout of the departments $\mathcal{D}$ respective the structure of the cell $A \in\{S R F L P, D C F L P\}$. Additionally, $p_{z}, z \in\{a, b\}$, specifies the position of $s_{M}$, where $p_{a}$ denotes that the position of $s_{M}$ can be chosen arbitrarily and $p_{b}$ expresses that $s_{M}$ has to lie at the leftmost position of the layout. Let $A=D C F L P$ and $s_{M} \in\left\{n+1, \ldots, n+m^{\prime}\right\}$, then one department of the set $\mathcal{D} \backslash\left\{s_{M}\right\}$ may overlap with $s_{M}$.

Given an FC-CCLP instance, we add a dummy department to each cell with length zero and weights as described above, and hence we obtain the following result.

Lemma 4. Given a fixed-cell combined cell layout instance where $C_{k}, k \in \mathcal{C}$, denotes the set of departments assigned to cell $k$ and let the dummy department $n+k$ be added to cell $k$ with length $\ell_{n+k}=0$ and adapted weights for dummy department $n+k$ as described in (14)-(15) for $\mathcal{D}=C_{k} \cup\{n+k\}$ and $s_{M}=n+k$. Then, the FC-CCLP is equivalent to solving the problems

$$
\begin{array}{ll}
W_{\left(n+k, p_{b}\right)}^{S}\left(C_{k} \cup\{n+k\}\right), & k \in \mathcal{C} \text { with } t(k)=S R F L P, \\
W_{\left(n+k, p_{a}\right)}^{D}\left(C_{k} \cup\{n+k\}\right), & k \in \mathcal{C} \text { with } t(k)=D C F L P,
\end{array}
$$

and the sum of the optimal values (plus constant inter-cell weights) is equal to the optimal value of the FC-CCLP.

The result of Lemma 4 is stated in [47] for the MBFLP without a proof and this result was implicitly used in $[15,32]$. For the convenience of the reader we present a proof.

Proof. Remark that inter-cell distances lead to constant weights $C:=\sum_{\substack{k, o \in \mathcal{C} \\ k<o}} \sum_{\substack{i \in C_{k} \\ j \in C_{o}}} u_{k o}\left(w_{i j}+w_{j i}\right)$ in the FC-CCLP, and thus we may exclude them. As we interpret the dummy departments as the (un-) loading stations of the cells, we can express the inter-cell distances by summing up inner-cell distances, i. e., $d_{i j}=d_{i(n+k)}+d_{(n+o) j}, k, o \in \mathcal{C}, k \neq o, i \in C_{k}, j \in C_{o}$. This proves the desired result.

Hence, the FC-CCLP can be divided into $m$ sub-problems. In this paper we concentrate on cells of type SRFLP and DCFLP, but note that the result of Lemma 4 is independent of the layout type of the cells, and thus our approach can be extended to cells of other types such as the DRFLP.

We provide the following result which breaks some symmetries and which allows us to reduce the number of cell assignments of type SRFLP that have to be considered significantly if $u_{k o}=c$ for some constant $c \in \mathbb{R}_{\geq 0}$ for all $k, o \in \mathcal{C}, k<o$, with $\{t(k), t(o)\} \cap\{\operatorname{SRFLP}\} \neq \emptyset$.

Theorem 5. The CCLP $\left(m_{1}, m_{2}\right)$ with $u_{k o}=c \in \mathbb{R}_{\geq 0}$ for all $k, o \in \mathcal{C}, k<o$, with $\{t(k), t(o)\} \cap$ $\{S R F L P\} \neq \emptyset$ is equivalent to enumerate over $\frac{\left(\left\lceil\frac{m_{1}}{2}\right\rceil+m_{2}\right)^{n}}{\left[\frac{m_{1}}{2}\right] ! m_{2} !}$ cell assignments and solve the following optimization problems for a fixed cell assignment exactly

$$
\begin{array}{ll}
W_{\left(n+k, p_{a}\right)}^{S}\left(C_{k} \cup C_{k+1} \cup\{n+k\}\right), & k=1,3, \ldots, h, \\
W_{\left(n+m_{1}, p_{b}\right)}^{S}\left(C_{m_{1}} \cup\left\{n+m_{1}\right\}\right), & \text { if } m_{1} \text { is odd, } \\
W_{\left(n+k, p_{a}\right)}^{D}\left(C_{k} \cup\{n+k\}\right), & k=m_{1}+1, \ldots, m,
\end{array}
$$


with $h=m_{1}-1$ if $m_{1}$ is even and $h=m_{1}-2$ if $m_{1}$ is odd and the departments $C_{k}$ are assigned to cell $k \in[m]$. Apart from this the dummy department $n+k$ is added to cell $k$ for $k=1,3, \ldots, h$, $k=m_{1}$ if $m_{1}$ odd and $k=m_{1}+1, \ldots, m$. Additionally, we have to compute some constants such that inter-cell distances are calculated correctly.

Further, the SRFLP is equivalent to the CCLP (2,0).

In [23] it is shown that the TRFLP is a generalization of the 3-BFLP. Therefore, an additional dummy department $n+1$ with lengths $\ell_{n+1}=2 w_{\text {path }}$ and weights $w_{i(n+1)}=0, i \in[n]$, is added to the TRFLP and its center position is fixed on position $p_{E_{1}}$ in row 1. In such an optimal T-row layout, the departments left (right) to $n+1$ are assigned to cell 3 (cell 1 ) in the 3-BFLP and the departments in cell 2 are assigned to cell 2 in the 3-BFLP without changing the order of the departments in the same row. We use this idea in the following proof.

Proof. Let $C_{k}$ denote the set of departments assigned to cell $k \in[m]$ in the FC-CCLP. By Lemma 4 the CCLP is equivalent to enumerate over $\frac{\left(m_{1}+m_{2}\right)^{n}}{m_{1} !}$ distinguishable cell assignments and solve the problems

$$
\begin{array}{ll}
W_{\left(n+k, p_{b}\right)}^{S}\left(C_{k} \cup\{n+k\}\right), & k=1,2, \ldots, m_{1}, \\
W_{\left(n+k, p_{a}\right)}^{D}\left(C_{k} \cup\{n+k\}\right), & k=m_{1}+1, \ldots, m,
\end{array}
$$

where the dummy department $n+k$ is assigned to cell $k$. Note that by our assumptions on $u_{k o}$ with $k, o \in \mathcal{C}, k<o$, such that $\{t(k), t(o)\} \cap\{\operatorname{SRFLP}\} \neq \emptyset$ it is sufficient to determine which departments lie in a common cell of type SRFLP. Hence, we can divide $\left(m_{1}+m_{2}\right)^{n}$ by $m_{1}$ !.

For the improved formula, let us first consider cells of type DCFLP. If we know which departments should be together in one cell of type DCFLP, it remains to determine the exact cell of each of the departments. For this note that the associated inter-cell weights do not have to be the same. So, given $C_{m_{1}+1}, \ldots, C_{m}$, we calculate $m_{2}$ ! constants and determine a best bijection $\pi:[m] \backslash\left[m_{1}\right] \rightarrow$ $[m] \backslash\left[m_{1}\right]$ minimizing the associated inter-cell distances $\sum_{k, o \in[m] \backslash\left[m_{1}\right], k<o} u_{k o} \sum_{i \in C_{\pi(k)}, j \in C_{\pi(o)}} w_{i j}$. Thus, we can break the symmetries with respect to the cells of type DCFLP and divide the number of cell assignments that have to be checked by $m_{2}$ !.

It remains to consider cells of type SRFLP. If $m_{1}=1$, we are done. So, let $m_{1} \geq 2$. We exclude the constant $\left(w_{i j}+w_{j i}\right) \frac{c}{2}$ if $i \in[n]$ lies in a cell of type SRFLP and $j \in[n]$ lies in a cell of type DCFLP. Let $k, k+1 \in\left[2\left\lfloor\frac{m_{1}}{2}\right\rfloor\right]$ and we consider the departments $C_{k} \cup C_{k+1}$ without changing the cell assignments of the remaining departments. We merge the cells $k$ and $k+1$ and the resulting cell is called merged cell in the following. We add a dummy department $n+k$ to the merged cell with length $\ell_{n+k}=c$ and weights $w_{i(n+k)}=\sum_{j \in[n] \backslash\left(C_{k} \cup C_{k+1}\right)} w_{i j}, w_{(n+k) i}=\sum_{j \in[n] \backslash\left(C_{k} \cup C_{k+1}\right)} w_{j i}$ (see (14)-(15)). Then, enumerating over all possible cell assignments of the departments $C_{k} \cup C_{k+1}$ to two cells of type SRFLP is equivalent to solve the $W_{\left(n+k, p_{a}\right)}^{S}\left(C_{k} \cup C_{k+1} \cup\{n+k\}\right)$ because in an optimal layout of the $W_{\left(n+k, p_{a}\right)}^{S}\left(C_{k} \cup C_{k+1} \cup\{n+k\}\right)$ one can assign all departments left (right) to $n+k$ to cell $k($ cell $k+1)$ and vice versa. Therefore, we solve the $W_{\left(n+k, p_{a}\right)}^{S}\left(C_{k} \cup C_{k+1} \cup\{n+k\}\right)$ and we obtain a new cell assignment $\tilde{C}_{k}\left(\tilde{C}_{k+1}\right)$, which contains all departments to the left (right) of $n+k$. Note that the inter-cell distances are taken into account by the length of the dummy department $n+k$ as well as the excluded constant.

So, if $m_{1}$ is even, it is sufficient to consider $\frac{m_{1}}{2}$ cells of type SRFLP. If $m_{1}$ is odd, we have to take one additional cell into account and solve the ${\underset{W}{\left(n+m_{1}, p_{b}\right)}}_{\left({ }^{\prime}\right.}^{S}\left(C_{m_{1}} \cup\left\{n+m_{1}\right\}\right)$ with dummy department $\left(n+m_{1}\right)$ with $\ell_{n+m_{1}}=0$ and weights $w_{i\left(n+m_{1}\right)}=\sum_{j \in[n] \backslash C_{m_{1}}} w_{i j}, w_{\left(n+m_{1}\right) i}=\sum_{j \in[n] \backslash C_{m_{1}}} w_{j i}$ (see (14)-(15)) and we additionally exclude the constant

$$
\sum_{\substack{i \in C_{m_{1}} \\ j \in[n] \backslash C_{m_{1}}}}\left(w_{i j}+w_{j i}\right) \frac{c}{2} .
$$


Breaking again the symmetries concerning the merged SRFLP cells we have to consider $\frac{\left(\left\lceil\frac{m_{1}}{2}\right\rceil+m_{2}\right)^{n}}{\left[\frac{m_{1}}{2}\right] ! m_{2} !}$ cell assignments.

One immediate consequence of these considerations is that the SRFLP is equivalent to the CCLP $(2,0)$ with $u_{12}=0$. If $u_{12}>0$, then there exists an optimal solution for the CCLP $(2,0)$ where all departments are arranged in one cell, and hence the SRFLP is equivalent to the CCLP $(2,0)$.

The last result leads to the following definition which allows us to specify the (generalized) cell assignments which have to be considered more precisely.

Definition 6. Let a CCLP $\left(m_{1}, m_{2}\right)$ instance with $u_{k o}=c \in \mathbb{R}_{\geq 0}$ for all $k, o \in \mathcal{C}, k<o$, with $\{t(k), t(o)\} \cap\{S R F L P\} \neq \emptyset$ be given. We denote by $\tilde{m}:=\left\lceil\frac{m_{1}}{2}\right\rceil+m_{2}$ the number of generalized cells. Then, a generalized cell assignment $\tilde{c}:[n] \rightarrow[\tilde{m}]$ is called proper if the following conditions are satisfied where $\tilde{C}_{k}=\{i \in[n]: \tilde{c}(i)=k\}, k \in[\tilde{m}]$.

$$
\begin{aligned}
& \inf \left\{i \in \tilde{C}_{k}\right\} \leq \inf \left\{i \in \tilde{C}_{l}\right\}, \\
& k, l \in\left[\left\lfloor\frac{m_{1}}{2}\right\rfloor\right], k<l, \\
& \inf \left\{i \in \tilde{C}_{k}\right\} \leq \inf \left\{i \in \tilde{C}_{l}\right\} \text {, } \\
& k, l \in[\tilde{m}] \backslash\left[\left\lceil\frac{m_{1}}{2}\right\rceil\right], k<l .
\end{aligned}
$$

Indeed, it suffices to consider proper generalized cell assignments where the concrete cell of departments in cells of type DCFLP might has to be determined in our algorithm.

Our approach for solving the CCLP is summarized in Algorithm 1. If the upper bound $v^{*}$ is exceeded, we neglect the current generalized cell assignment and go to the next one. We solve cells of type DCFLP first since the DCFLP is in practice easier to solve than the SRFLP, see [37, 38], and the results of $[15,32]$ indicate that in general the optimal values of cells of type DCFLP are higher than the optimal values of cells of type SRFLP, and hence we hope to exceed the upper bound $u$ earlier such that we can neglect the current cell assignment.

We describe in Section 5 how to include inter-cell distances for the 3-BFLP and the 4-BFLP. Considering cells of type SRFLP, it remains to present our approach for solving $W_{\left(n+\tilde{m}_{1}, p_{b}\right)}^{S}\left(\tilde{C}_{\tilde{m}_{1}} \cup\right.$ $\left.\left\{n+\tilde{m}_{1}\right\}\right)$ if $m_{1}$ is odd with $\tilde{m}_{1}=\left\lceil\frac{m_{1}}{2}\right\rceil$. We can simply fix the dummy department $n+\tilde{m}_{1}$ at the border, i. e.,

$$
x_{i\left(n+\tilde{m}_{1}\right) j}=0, \quad i, j \in C_{\tilde{m}_{1}}, i<j,
$$

and then solve a single-row instance with departments $C_{\tilde{m}_{1}} \cup\left\{n+\tilde{m}_{1}\right\}$ and these additional equations.

\subsection{Circular Cells in the Fixed-Cell Combined Cell Layout Problem}

In this section we focus on the subproblems of the FC-CCLP concerning circular cells. Let $C_{k}$ denote the set of departments assigned to some cell $k$ with $t(k)=$ DCFLP. By the following proposition we can use techniques from the directed-circular literature, see Section 2.2, for solving the $W_{\left(n+k, p_{a}\right)}^{D}\left(C_{k} \cup\{n+k\}\right)$ to optimality.

Proposition 7. Let an FC-CCLP instance be given where $C_{k}, k \in \mathcal{C}$, denotes the set of departments assigned to the cell $k$ with $t(k)=D C F L P$. Let dummy department $n+k$ be assigned to cell $k$ with $\ell_{n+k}=0$ and $w_{i(n+k)}=\sum_{j \in[n] \backslash C_{k}} w_{i j}, w_{(n+k) i}=\sum_{j \in[n] \backslash C_{k}} w_{j i}$ (see (14), (15)) and let $V_{k}:=\left\{i \in C_{k}: w_{i(n+k)}+w_{(n+k) i}>0\right\} \neq \emptyset$. Further, let $v_{n+k}^{*}$ denote the optimal value of the $W_{\left(n+k, p_{a}\right)}^{D}\left(C_{k} \cup\{n+k\}\right)$ and let $v_{s_{M}}^{*}$ denote the optimal value of the $W_{\left(s_{M}, p_{a}\right)}^{D}\left(C_{k}\right), s_{M} \in V_{k}$, where in $W_{\left(s_{M}, p_{a}\right)}^{D}\left(C_{k}\right)$ the weights of $s_{M}$ are adjusted according to (14), (15). Then

$$
v_{n+k}^{*}=\min \left\{v_{s_{M}}^{*}: s_{M} \in V_{k}\right\}
$$

The proof is related to a proof in [24]. Given a DRFLP instance with objective function $\min \sum_{j \in S} w_{i j} d_{i j}, i \in[n], S \subseteq[n] \backslash\{i\}$, there exists an optimal layout where some $j \in S$ lies directly opposite $i$. 


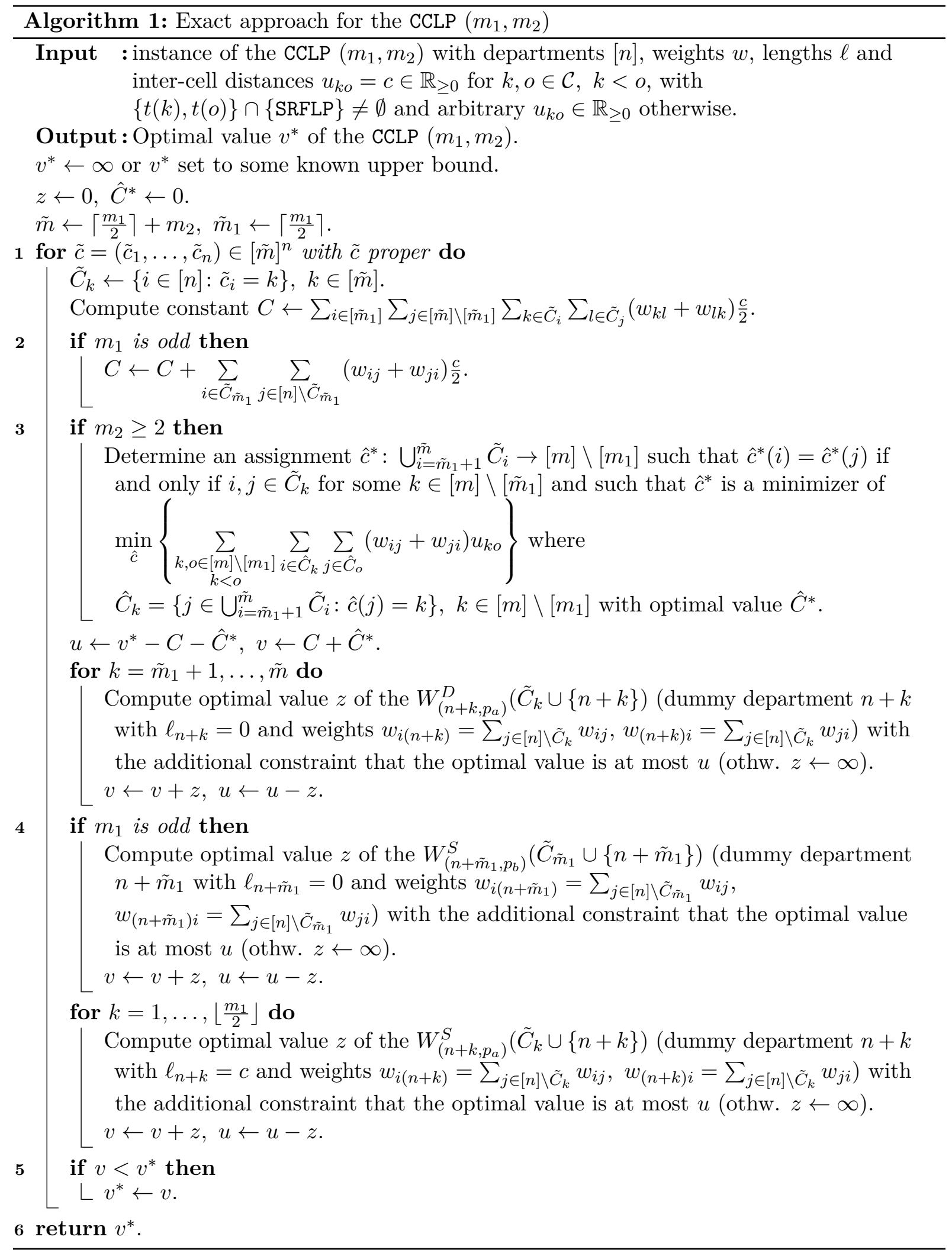


Proof. Let $V_{k} \neq \emptyset, k \in \mathcal{C}$, with $t(k)=$ DCFLP, and assume that an optimal layout of the $W_{\left(n+k, p_{a}\right)}^{D}\left(C_{k} \cup\{n+k\}\right)$ is given where no department of the set $V_{k}$ lies directly opposite $n+k$. Then, shifting all departments to the, w.l.o.g., right by some small $\varepsilon$ does not change the distances between departments $i, j \in C_{k}, i \neq j$, and hence influences the objective value by

$$
\sum_{j \in V_{k}} \varepsilon\left(w_{(n+k) j}-w_{j(n+k)}\right) .
$$

By the optimality of the layout we do not change the objective value by shifting all departments, w. l. o. g., to the right until the first department contained in $V_{k}$ lies directly opposite $n+k$. Thus, there always exists an optimal solution of $W_{\left(n+k, p_{a}\right)}^{D}\left(C_{k} \cup\{n+k\}\right)$ where an $s_{M} \in V_{k}$ has the same position as $n+k$ and (16) follows for the described weight adjustment.

If $V_{k}=\emptyset$ for some $k \in \mathcal{C}$ with $t(k)=$ DCFLP (hence, the departments in this cell do not have relations to departments in other cells), we simply neglect the dummy department $n+k$ and then the $W_{\left(n+k, p_{a}\right)}^{D}\left(C_{k} \cup\{n+k\}\right)$ is equivalent to the DCFLP with departments $C_{k}$. If $V_{k} \neq \emptyset$, one can fix one department $i \in V_{k}$ opposite $n+k$ and enumerate over each department fixed opposite $n+k$, see Proposition 7. In summary, we obtain an optimal layout of the $W_{\left(n+k, p_{a}\right)}^{D}\left(C_{k} \cup\{n+k\}\right)$ by solving $\max \left\{1,\left|V_{k}\right|\right\}$ directed-circular instances.

However, our goal is to reduce the number of directed-circular instances that have to be solved, and therefore we set up an MILP model for the $W_{\left(n+k, p_{a}\right)}^{D}\left(C_{k} \cup\{n+k\}\right), C_{k} \subseteq[n], k \in \mathcal{C}$, with dummy department $n+k$ with $\ell_{n+k}=0, w_{i(n+k)}=\sum_{j \in[n] \backslash C_{k}} w_{i j}, w_{(n+k) i}=\sum_{j \in[n] \backslash C_{k}} w_{j i}$. An advantage of an MILP model is to obtain good lower bounds quickly, and hence to exclude unbalanced cell assignments earlier in Algorithm 1. Therefore, we use the following binary variables

$$
y_{i}= \begin{cases}1, & \text { if } i \text { lies opposite } n+k, \\ 0, & \text { otherwise }\end{cases}
$$

for $i \in C_{k}$. Let $L=\sum_{i \in C_{k}} \ell_{i}$ and $\hat{w}_{i j}=w_{i j}-\min \left\{w_{i j}, w_{j i}\right\}, i, j \in C_{k}, i \neq j, \hat{w}_{i(n+k)}=$ $w_{i(n+k)}, \hat{w}_{(n+k) i}=w_{(n+k) i}, i \in C_{k}$. We exclude the constant

$$
W_{k}^{D}=\sum_{\substack{i, j \in C_{k} \cup\{n+k\} \\ i \neq j}} \hat{w}_{i j} \frac{\ell_{i}+\ell_{j}}{2}+\sum_{\substack{i, j \in C_{k} \\ i<j}} \min \left\{w_{i j}, w_{j i}\right\} L .
$$

We define $L_{i}=\sum_{j \in C_{k} \backslash\{i\}} \ell_{j}$ and then our MILP with $V_{k} \neq \emptyset$ reads as follows

$$
\begin{aligned}
& \min \sum_{\substack{i, j \in C_{k} \cup\{n+k\} \\
i \neq j}} \hat{w}_{i j} d_{i j} \\
& \text { s.t. (8), (11)-(13), } \\
& \sum_{i \in C_{k}} y_{i}=1 \text {, } \\
& \mathcal{D}:=C_{k} \cup\{n+k\}, f:=n+k, \\
& z_{i j}-y_{i} \geq 0 \\
& i, j \in C_{k}, i<j, \\
& z_{i j}+y_{j} \leq 1, \\
& i, j \in C_{k}, i<j \text {, } \\
& y_{i}=0 \text {, } \\
& i \in C_{k} \backslash V_{k} \text {, } \\
& d_{(n+k) i}-\sum_{\substack{j \in C_{k} \\
j<i}} \ell_{j} z_{j i}+\sum_{\substack{j \in C_{k} \\
j>i}} \ell_{j} z_{i j}+\sum_{j \in C_{k}} \frac{\ell_{j}}{2} y_{j}=\sum_{\substack{j \in C_{k} \\
j>i}} \ell_{j}, \quad i \in C_{k}, \\
& d_{i(n+k)}+d_{(n+k) i}+\left(L_{i}+\ell_{i}\right) y_{i}=L_{i}, \quad i \in C_{k}, \\
& d_{i j} \geq 0 \text {, } \\
& i, j \in C_{k}, i \neq j \text {, }
\end{aligned}
$$




$$
\begin{aligned}
& d_{i j} \geq-\frac{\ell_{i}}{2}, \\
& z_{i j} \in\{0,1\}, \\
& y_{i} \in\{0,1\},
\end{aligned}
$$

$$
\begin{aligned}
& i, j \in C_{k} \cup\{n+k\}, i \neq j, \\
& |\{i, j\} \cap\{n+k\}|=1, \\
& i, j \in C_{k}, i<j, \\
& i \in C_{k} .
\end{aligned}
$$

Let $k \in \mathcal{C}$. Equations (18) ensure that exactly one department lies opposite $n+k$, see Proposition 7. According to the $z$-variables we obtain an ordering of the departments, see Section 2.2, and we ensure via Inequalities (19)-(20) that the department $i^{\prime} \in C_{k}$ with $y_{i^{\prime}}=1$ is the leftmost department. By Proposition 7 and our assumption $V_{k} \neq \emptyset$, we set $y_{i}, i \in C_{k} \backslash V_{k}$, equal to zero, see Equations (21). The distance calculation in Equations (22) is similar to the distance calculation in Equations (9) where we additionally subtract half the length of the department that is fixed opposite $n+k$ because we excluded the constant $W_{k}^{D}$. By Equations (23) and Inequalities (25) we obtain $d_{i(n+k)}=d_{(n+k) i}=-\frac{\ell_{i}}{2}, i \in C_{k}$, if $i$ lies opposite $n+k$. In this case the distances $d_{i(n+k)}$ as well as $d_{(n+k) i}, i \in C_{k}$, are negative since we excluded the constant $\sum_{i \in C_{k}}\left(w_{i(n+k)}+w_{(n+k) i}\right) \frac{\ell_{i}}{2}$. If $i \in C_{k}$ does not lie opposite $n+k$, then we obtain $d_{i(n+k)}+d_{(n+k) i}=L_{i}$, see Equations (23).

Proposition 8. Let $C_{k}$ denote the set of departments assigned to cell $k \in \mathcal{C}$ with $t(k)=D C F L P$. Then

$$
\begin{aligned}
& \min \sum_{\substack{i, j \in C_{k} \cup\{n+k\} \\
i<j}} \hat{w}_{i j} d_{i j} \\
& \text { s.t. (8), (11)-(13), (18) }
\end{aligned}
$$

is a MILP model for the $W_{\left(n+k, p_{a}\right)}^{D}\left(C_{k} \cup\{n+k\}\right)$.

Proof. Let $i^{\prime} \in C_{k}$ such that $y_{i^{\prime}}=1$, see Equation (18). By Inequalities (8) together with the binary constraints we obtain a feasible ordering of the departments and the distances $d_{i j}, i, j \in C_{k} \backslash\left\{i^{\prime}\right\}$, are calculated correctly by Equations (11)-(13), see [15, 37, 38]. According to the $z$-variables we obtain an ordering of the departments and by Inequalities (19)-(20) $i^{\prime}$ is the leftmost department. We neglect the constant $\sum_{i \in C_{k}}\left(\hat{w}_{i(n+k)}+\hat{w}_{(n+k) i}\right) \frac{\ell_{i}}{2}$ and by Equations (22) and Inequalities (25) we obtain $d_{i^{\prime}(n+k)}=d_{(n+k) i^{\prime}}=-\frac{\ell_{i}^{\prime}}{2}$ if $y_{i}^{\prime}=1, i^{\prime} \in C_{k}$, and $d_{i(n+k)}+d_{(n+k) i}=L_{i}$ in the case $y_{i}=0, i \in C_{k}$. Hence, the distance calculation between $i \in C_{k}$ and $n+k$ is correct as well, see Equations (22)-(23).

We conclude this section by pointing out how further realistic extensions can be included in our approach.

Remark 9. 1) Our approach presented above can be combined with further aspects relevant in practice. Let the size of the cells be equal to $F \in \mathbb{R}_{\geq 0}$ (restriction on the sum of the lengths of all departments in one cell). We can neglect all cell assignments where the sum of the lengths of the departments in one cell exceeds F, see [17, 26]. However, a solution of the $W_{\left(n+k, p_{a}\right)}^{D}\left(C_{k} \cup\{n+k\}\right)$ contains free-spaces if the sum of the lengths of the departments assigned to cell $k$ is smaller than $F$. By the same shifting argument as used in the proof of Proposition 7 there exists an optimal layout where the free-space is interruption-free, i. e., it is sufficient to add one additional dummy department $n+k^{\prime}$ with $\ell_{n+k^{\prime}}=F-\sum_{i \in C_{k}} \ell_{i}, w_{i\left(n+k^{\prime}\right)}=w_{\left(n+k^{\prime}\right) i}=0, i \in C_{k} \cup\{n+k\}$, as done in [14] for the MREFLP and solve the $W_{\left(n+k, p_{a}\right)}^{D}\left(C_{k} \cup\left\{n+k, n+k^{\prime}\right\}\right)$ without spaces. Thus, one can use the methods presented above. In cells of type SRFLP, there always exists an optimal layout where the possible free-space arises only at the borders of the layout. Hence, it suffices to restrict the horizontal center-to-center distances of all departments to the loading stations, i. e., $d_{i(n+k)} \leq F-\frac{\ell_{i}}{2}, i \in C_{k}, k \in[m], t(k)=S R F L P$. Clearly, the length of the dummy department $n+k$ can be neglected. 
2) In the facility layout planning literature it is a standard assumption that the (un-) loading points of the departments are fixed at their centers, see, e.g., [5, 7, 12]. However, if the input and output positions are fixed on the left (or right) border of the departments, one can treat $n+k$ as an ordinary department, i. e., no department may overlap with $n+k$, and we just need to solve one directed-circular instance with departments $C_{k} \cup\{n+k\}$ in order to solve the $W_{\left(n+k, p_{a}\right)}^{D}\left(C_{k} \cup\{n+k\}\right)$.

\section{Extensions of the Multi-Bay Facility Layout Problem}

In this section we describe the adaption of our approach presented in Section 4 to the 3-BFLP, the XRFLP and the 4-BFLP. Adding appropriate dummy departments we are able to use the main ideas of Algorithm 1. Even for the problems with four cells we have to consider at most $2^{n}$ distinguishable generalized cell assignments of the departments. Considering the 3-BFLP and the 4-BFLP, we are able to further reduce the number of cell assignments that have to be considered.

\subsection{The 3-Bay Facility Layout Problem}

In [23] it is shown that the 3-BFLP is equivalent to the TRFLP where a dummy department $n+1$ is fixed on the (un-) loading station with length $\ell_{n+1}=2 w_{\text {path }}$ and weights $w_{i(n+1)}=$ $w_{(n+1) i}=0, i \in[n]$. In order to reduce the number of distinguishable cell assignments that have to be considered, we present a different approach. We merge cell 1 and cell 2 as a new cell and interpret cell 3 as new cell 2. Let $C_{1}, C_{2} \subseteq[n], C_{1} \cap C_{2}=\emptyset$, such that $C_{1} \cup C_{2}=[n]$ and let $C_{1}\left(C_{2}\right)$ denote the set of departments assigned to the merged cell 1 (cell 2 ). The dummy department $n+1(n+2)$ is added to the merged cell 1 (cell 2) with $\ell_{n+1}=0$ $\left(\ell_{n+2}=0\right)$ and weights $w_{i(n+1)}=\sum_{j \in[n] \backslash C_{1}} w_{i j}, w_{(n+1) i}=\sum_{j \in[n] \backslash C_{1}} w_{j i}\left(w_{i(n+2)}=\sum_{j \in[n] \backslash C_{2}} w_{i j}\right.$, $\left.w_{(n+2) i}=\sum_{j \in[n] \backslash C_{2}} w_{j i}\right)$ (see (14)-(15)). We exclude the constant $\sum_{i \in C_{1}, j \in C_{2}}\left(w_{i j}+w_{j i}\right) \cdot w_{\text {path }}$ and we fix an additional dummy department $n+3$ to the merged cell 1 neighboring $n+1$ with lengths $\ell_{n+3}=w_{\text {path }}$ and weights $w_{i(n+3)}=w_{(n+3) i}=0, i \in C_{1} \cup\{n+1\}$. Then, we obtain an optimal solution for the 3-BFLP by solving the $W_{\left(n+1, p_{a}\right)}^{S}\left(C_{1} \cup\{n+1, n+3\}\right)$ and the $W_{\left(n+2, p_{b}\right)}^{S}\left(C_{2} \cup\{n+2\}\right)$. Hence, we can solve the 3-BFLP similar to the approach summarized in Algorithm 1.

As done in [23] one can use symmetry breaking for the 3-BFLP. If $w_{\text {path }}=0$, we can fix two departments to $C_{1}$ and one has to consider $2^{n-2}$ generalized cell assignments. If $w_{\text {path }}>0$, one department can be fixed to $C_{1}$, and hence we have to consider $2^{n-1}$ generalized cell assignments. In the 3-PMFP and the 4-PMFP one can fix two departments to the merged cell $C_{1}$, and hence it is sufficient to consider $2^{n-2}$ generalized cell assignments for $w_{\text {path }} \geq 0$.

In contrast to the previous presented approaches, the computation time of our approach for the TRFLP is slightly higher than the approach of [23]. Thus, we summarize our approach for the TRFLP in the appendix.

\subsection{The X-Row and the 4-Bay Facility Layout Problem}

Next we consider layout problems with four original cells in more detail. Recall that we assume that the height of each department equals one. The following proposition is essential to solve the cells independently, and thus we derive a result similar to Theorem 5

Proposition 10. Given an XRFLP instance. There always exists an optimal X-row layout where some $i \in[n]$ is contained in cell 3 and $d_{i p_{E_{3}}}=\frac{\ell_{i}}{2}$.

Proof. Let an optimal X-row layout be given. We denote by $C_{a}$ the set of departments assigned to cell $a \in[4]$ and we assume that $d_{i p_{E_{3}}}>\frac{\ell_{i}}{2}$ for all $i \in C_{3}$. If all departments are contained in $C_{a} \cup C_{b}, a, b \in[4], a<b$, then we arrange all departments space-free in cell 3 respecting the 
order of the departments in cell $a$ and cell $b$ such that the department in cell $a$ which is closest to $p_{E_{a}}$ is neighboring the department in cell $b$ which is closest to $p_{E_{b}}$. Then we can shift the departments such that afterwards one department $i \in[n]$ satisfies $d_{i p_{E_{3}}}=\frac{\ell_{i}}{2}$ and clearly, we do not increase the objective value by this method.

Next, let $\left|C_{a}\right|,\left|C_{b}\right|,\left|C_{c}\right| \geq 1, a, b, c \in[4],|\{a, b, c\}|=3$. If there exists $i \in C_{3}$ with $d_{i p_{E_{3}}}<$ $\frac{\ell_{i}}{2}+1, i \in C_{3}$, then we shift all departments in cell 3 to the left until the left border of the leftmost department reaches the position $p_{E_{3}}$ and the resulting layout is feasible since the previous layout was feasible. The objective value of the layout is not increased by this method. Further, we assume there exists $i \in C_{2}$ with $d_{i p_{E_{2}}}<\frac{\ell_{i}}{2}+1$ or $d_{i p_{E_{2}}} \geq \frac{\ell_{i}}{2}+1$ for all $i \in C_{2}$ and $C_{3}=\emptyset$. Then, we shift all departments in cell 2 to the left in the direction to $p_{E_{2}}$ until the leftmost department $j \in C_{2}$ satisfies $d_{j p_{E_{2}}}=\frac{\ell_{j}}{2}$. Then we arrange the departments in cell 3 (cell 2) to cell 2 (cell 3 ) space-free respecting the order of the departments. We do not increase the objective value by this method since $u_{12}=u_{13}$ and $u_{24}=u_{34}$. It remains to consider the case $d_{i p_{E_{3}}} \geq \frac{\ell_{i}}{2}+1, i \in C_{3} \neq \emptyset$, and $d_{i p_{E_{2}}} \geq \frac{\ell_{i}}{2}+1, i \in C_{2}$. Then we shift all departments in cell 3 to the left until the first department has its left border on position $p_{E_{3}}$. Clearly, we do not increase the objective value by this method.

Given an X-row instance, we want to ensure that departments in cell 2 and cell 3 do not overlap. Therefore, we fix an additional dummy department $n+3$ at the border of cell 2 with lengths $\ell_{n+3}=1$ and weights $w_{i(n+3)}=w_{(n+3) i}=0, i \in[n+2]$, see Proposition 10. If $w_{\text {path }}^{1}=w_{\text {path }}^{2}=0$, then one can solve the cells independently, see Lemma 4, and hence we can apply Theorem 5 with $u_{k o}=0, k, o \in[4], k<o$, and interpret cell 1 and cell 3 as well as cell 2 and cell 4 as a new (larger) cell. The dummy department $n+1(n+2)$ is added to the merged cell 1 (cell 2) with $\ell_{n+1}=0\left(\ell_{n+2}=0\right)$ and weights as described in (14)-(15) and the merged cell 2 additionally contains the dummy department $n+3$ which is neighboring $n+2$.

It remains to include inter-cell distances in our approach for the XRFLP. Let $C_{1}, C_{2} \subseteq[n], C_{1} \cap$ $C_{2}=\emptyset$, such that $C_{1} \dot{\cup} C_{2}=[n]$ and let $C_{1}\left(C_{2}\right)$ denote the set of departments assigned to the merged cell 1 (cell 2). We add the additional dummy department $n+4$ to the merged cell 1 with lengths $\ell_{(n+4)}=w_{\text {path }}^{1}$ and weights $w_{i(n+4)}=w_{(n+4) i}=0, i \in[n+3]$, and then we solve the $W_{\left(n+1, p_{a}\right)}^{S}\left(C_{1} \cup\{n+1, n+4\}\right)$ where $n+4$ is neighboring $n+1$. In the merged cell 2 we avoid adding another dummy department by adapting the lengths of the dummy departments $n+2$ and $n+3$ such that $\ell_{n+2}=2 \cdot \min \left\{1, w_{\text {path }}^{2}\right\}$ and $\ell_{n+3}=\left|w_{\text {path }}^{2}-1\right|$. In both cases, the distance of $n+2$ and the departments to the, w.l.o.g., left of $n+2$ is at least $w_{\text {path }}^{2}$ and to the departments to the right of $n+2$ is at least 1, and thus the distances are calculated correctly. We refer to Figure 5 for an illustration. This leads to the following result.

Corollary 11. Given an X-row instance with $w_{\text {path }}^{1}, w_{\text {path }}^{2} \geq 1$, and we set $\ell_{n+1}=0, \ell_{n+2}=$ $2 \cdot \min \left\{w_{\text {path }}^{2}, 1\right\}, \ell_{n+3}=\left|w_{\text {path }}^{2}-1\right|, \ell_{n+4}=w_{\text {path }}^{1}$, and $n+1(n+2)$ is neighboring $n+4(n+3)$. We obtain an optimal $X$-row layout by enumerating over all assignments of the departments to the cells $\mathcal{C}=\{1,2\}$ and solving the $W_{\left(n+1, p_{a}\right)}^{S}\left(C_{1} \cup\{n+1, n+4\}\right)$ and the $W_{\left(n+2, p_{a}\right)}^{S}\left(C_{2} \cup\{n+2, n+3\}\right)$ where $C_{k}, k \in[2]$, denotes the set of departments assigned to (merged) cell $k$ and the weights of the additional dummy departments are set to $w_{i(n+3)}=w_{(n+3) i}=w_{i(n+4)}=w_{(n+4) i}=0, i \in[n+2]$.

Proof. By adding dummy departments $n+3$ and $n+4$ with length $\ell_{n+4}=w_{\text {path }}^{1}, \ell_{n+3}=\left|w_{\text {path }}^{2}-1\right|$ as well as $\ell_{n+2}=2 \cdot \min \left\{w_{\text {path }}^{2}, 1\right\}$, we obtain the correct distance calculation. Thus, similar to Theorem 5 it is sufficient to consider two cells.

In Algorithm 1 one has to consider $2^{n}$ cell assignments to obtain an optimal X-row layout since we use symmetry breaking in the proof of Proposition 10. In our algorithm we solve the $W_{\left(n+2, p_{a}\right)}^{S}\left(C_{2} \cup\{n+2, n+3\}\right)$ first if $\left|C_{2}\right| \leq\left|C_{1}\right|+1$ and otherwise we solve the $W_{\left(n+1, p_{a}\right)}^{S}\left(C_{1} \cup\right.$ $\{n+1, n+4\})$ first with the idea to exclude unbalanced cell assignments earlier. For the whole 


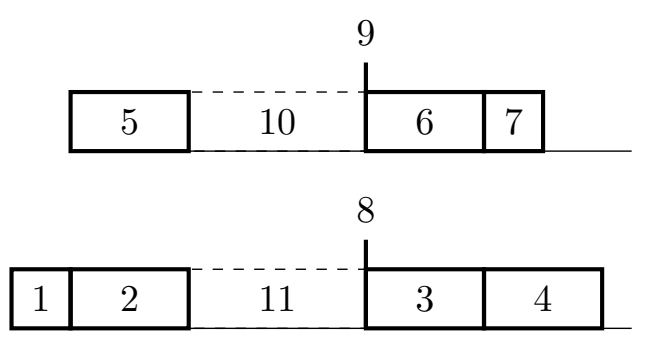

(a) Feasible layouts for the $W_{\left(8, p_{a}\right)}^{S}(\{1,2,3,4\} \cup$ $\{8,11\})$ and the $W_{\left(9, p_{a}\right)}^{S}(\{5,6,7\} \cup\{9,10\})$ with $\ell_{10}=\ell_{11}=w_{\text {path }}=3$.

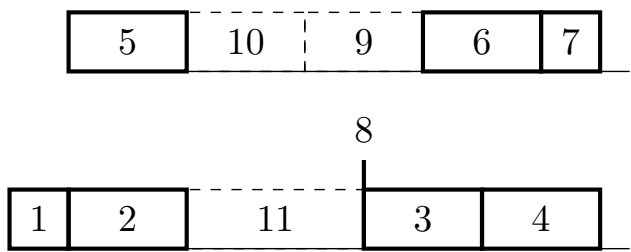

(c) Feasible layouts for the $W_{\left(8, p_{a}\right)}^{S}(\{1,2,3,4\} \cup$ $\{8,11\})$ and the $W_{\left(9, p_{a}\right)}^{S}(\{5,6,7\} \cup\{9,10\})$ with $w_{\text {path }}^{1}=w_{\text {path }}^{2}=3$ and $\ell_{9}=\ell_{10}=2, \ell_{11}=3$.

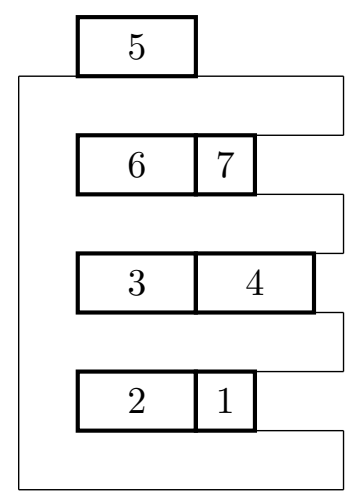

(b) 4-Bay layout with $\ell_{10}=\ell_{11}=w_{\text {path }}$ deduced from the single-row layouts illustrated in Figure 5 a.

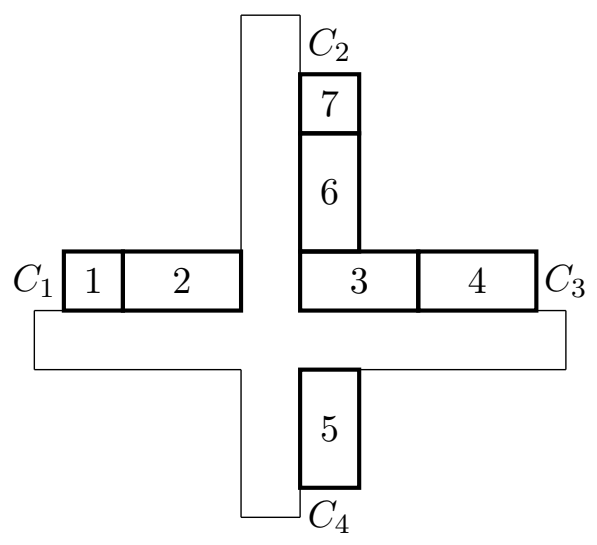

(d) X-row layout with $\ell_{9}=\ell_{10}=2, \ell_{11}=3$, deduced from the single-row layouts illustrated in Figure 5c.

Figure 5: Visualization of obtained 4-Bay and X-row layouts with positive inter-cell distances by solving appropriate single-row instances. In order to construct, e.g., a 4-Bay layout, with $n=7$ departments, the departments left (right) to the dummy department 8 are assigned to cell 1 (cell 2) in reversed (the same) order and the departments left (right) to the dummy department 9 are assigned to cell 4 (cell 3) in reversed (the same) order. The distances between each pair of departments in the layouts illustrated in Figure 5a and in Figure 5b as well as in Figure 5c and in Figure 5d are the same.

problem we exclude the constant

$$
W:=\sum_{\substack{i, j \in[n] \\ i<j}}\left(w_{i j}+w_{j i}\right) \frac{\ell_{i}+\ell_{j}}{2}+\sum_{i \in C_{2}}\left(w_{i(n+2)}+w_{(n+2) i}\right) \frac{\ell_{n+2}}{2} .
$$

We neglect the dummy department $n+4$ if $w_{\text {path }}^{1}=0$. However, we additionally add the following constraints

$$
\begin{array}{ll}
x_{j i(n+2)}-x_{j i(n+3)}=0, & i, j \in[n], i \neq j, \\
x_{j i(n+1)}-x_{j i(n+4)}=0, & i, j \in[n], i \neq j,
\end{array}
$$

where inequalities (28) are only added if $w_{\text {path }}^{1}, w_{\text {path }}^{2}>0$.

Next we describe how to include inter-cell distances to the 4-BFLP in Algorithm 1. At first, we merge cell 1 and cell 2 as well as cell 3 and cell 4 . Hence, let $C_{1}\left(C_{2}\right)$ denote the set of departments 
assigned to the merged cell 1 (cell 2). We exclude the constant $\sum_{i \in C_{1}, j \in C_{2}}\left(w_{i j}+w_{j i}\right) \cdot w_{p a t h}$ and solve the $W_{\left(n+k, p_{a}\right)}^{S}\left(C_{k} \cup\{n+k, n+k+2\}\right), k \in\{1,2\}$, with an additional dummy department $n+k+2$ with length $\ell_{n+k+2}=w_{\text {path }}$ and weights $w_{i(n+k+2)}=w_{(n+k+2) i}=0, i \in C_{k} \cup\{n+k\}$, where $n+k+2$ is a neighboring department of $n+k$. By Corollary 11 we obtain an optimal solution for the 4-BFLP by this method. For an illustration we refer to Figure 5 . In our algorithm we solve the $W_{\left(n+2, p_{a}\right)}^{S}\left(C_{2} \cup\{n+2, n+3\}\right)$ first if $\left|C_{2}\right| \leq\left|C_{1}\right|$ and otherwise we solve the $\left.W_{\left(n+1, p_{a}\right)}^{S}\left(C_{1} \cup\{n+1, n+4\}\right)\right)$ first. Considering the 4-BFLP with $w_{\text {path }}=0$, we can fix two departments (randomly chosen) to cell 1 , and hence it is sufficient to consider $2^{n-2}$ cell assignments. If $w_{\text {path }}>0$, one can fix one department to cell 1 , and hence it is sufficient to consider $2^{n-1}$ cell assignments.

\section{Computational results}

In this section we present our computational results. The computational experiments are implemented in C++ and we use Cplex 12.10 as an MILP Solver [39]. All results were conducted on a 2.10GHz quad-core using Virtual Box 6 and running on Debian GNU/Linux 8 in single processor mode.

\subsection{Computational experiments}

In our computational results we focus on optimization problems which consist of at most 2 merged cells in view of our results derived in Theorem 5. The instances from the literature are symmetric, see e. g., $[10,13]$. Hence, let $w_{i j}, i, j \in[n], i<j$, be given, we choose uniformly at random $\tilde{w}_{i j} \in\left\{0,1, \ldots, w_{i j}\right\}, i, j \in[n], i<j$, and set $\tilde{w}_{j i}=w_{i j}-\tilde{w}_{i j}, i, j \in[n], j<i$, to obtain asymmetric instances. All instances can be downloaded from https://tinyurl.com/ instances-DaFiHuMa20. At first, we present results for the FC-CCLP, see Table 1. We assign the departments $1, \ldots,\left\lfloor\frac{n}{2}\right\rfloor$ to the first cell and the remaining departments to the second cell. For the FC-CCLP $(1,1)$ we assume that the cell of type SRFLP is the first cell. The inter-cell distance is constant, and hence set to zero. In the first column in Table 1 the instance names are given, and the first number in the names indicates the number of the departments. Instances with second number equal to 1 are equidistant instances, and hence we omit them here. In the next three columns we display the optimal values and in the last five columns the corresponding running times are given where "enu" describes the enumerative approach and "MILP" describes our MILP model for cells of type DCFLP. We write $<1$ if the running time is less than one second. For all instances tested here the optimal value of the FC-CCLP $(2,0)$ is smaller than the optimal value of the FC-CCLP $(1,1)$ which is smaller than the optimal value of the FC-CCLP $(0,2)$. Hence, it follows that in our tests a cell of type SRFLP has a smaller optimal value than a cell of type DCFLP with the same departments. One can observe that even large-sized instances were solved quickly and it seems that a cell of type SRFLP is easier to solve than a single-row instance with the same number of departments. The reason is the additionally dummy department which has usually high weights to all departments. However, cells of type DCFLP could be solved to optimality in a few seconds while cells of type SRFLP partially need a few minutes.

In order to compare our approach for the TRFLP, the 3-BFLP and the 3-PMFP with the approach of [23], we make use of the heuristically determined upper bounds in [23]. In Table 2 we compare the optimal values of several facility layout problems where the inter-cell distances are set to zero and in Table 3 the inter-cell distances are set to three. In the column "Source" we display the source of the symmetric instances. We observe that the optimal value of the TRFLP is smaller than the optimal value of the 3-BFLP and the 3-PMFP. The optimal value of the 4-BFLP is slightly smaller than the optimal value of the XRFLP if the inter-cell distances are set to zero, and if $w_{\text {path }}=w_{\text {path }}^{1}=w_{\text {path }}^{2}=3$, then the optimal value of XRFLP is smaller than the optimal value of the 4-BFLP and greater than the optimal value of the 4-PMFP, see Table 3 . For all instances the 


\begin{tabular}{|c|c|c|c|c|c|c|c|c|c|}
\hline Instance & Source & FC-CCLP $(2,0)$ & FC-CCLP $(1,1)$ & FC-CCLP $(0,2)$ & FC-CCLP $(2,0)$ & \multicolumn{2}{|c|}{ FC-CCLP $(1,1)$} & \multicolumn{2}{|c|}{ FC-CCLP $(0,2)$} \\
\hline & & opt value & opt value & opt value & time & enu & MILP & enu & MILP \\
\hline AV25-2 & [10] & 42745.5 & 47933.5 & 51169.5 & $<1$ & $<1$ & $<1$ & $<1$ & $<1$ \\
\hline AV25-3 & [10] & 27602.0 & 29890.0 & 31001.0 & $<1$ & $<1$ & $<1$ & $<1$ & $<1$ \\
\hline AV25-4 & [10] & 55549.5 & 62776.5 & 67306.5 & $<1$ & $<1$ & $<1$ & $<1$ & $<1$ \\
\hline AV25-5 & [10] & 18044.0 & 19692.0 & 20937.0 & $<1$ & $<1$ & $<1$ & $<1$ & $<1$ \\
\hline AV30-2 & [10] & 24397.5 & 25505.5 & 27447.5 & $<1$ & $<1$ & $<1$ & $<1$ & $<1$ \\
\hline AV30-3 & [10] & 52018.0 & 56298.0 & 61122.0 & 1 & $<1$ & $<1$ & $<1$ & $<1$ \\
\hline AV30-4 & {$[10]$} & 65516.5 & 71443.5 & 78201.5 & 1 & $<1$ & $<1$ & $<1$ & $<1$ \\
\hline AV30-5 & [10] & 134026.0 & 141672.0 & 162364.0 & 1 & 1 & $<1$ & $<1$ & $<1$ \\
\hline ste36-2 & [13] & 181508.0 & 223338.0 & 307066.0 & 5 & 4 & 4 & 1 & $<1$ \\
\hline ste36-3 & [13] & 101644.0 & 128985.0 & 206897.0 & 8 & 7 & 7 & 1 & $<1$ \\
\hline ste36-4 & [13] & 95805.5 & 111559.0 & 186153.0 & 7 & 6 & 5 & 1 & $<1$ \\
\hline ste36-5 & [13] & 91651.5 & 114715.0 & 175358.0 & 4 & 4 & 3 & 1 & $<1$ \\
\hline sko42-2 & [13] & 249986.0 & 278212.0 & 308533.0 & 43 & 32 & 23 & 4 & $<1$ \\
\hline sko42-3 & [13] & 198270.0 & 220596.0 & 242858.0 & 5 & 4 & 2 & 3 & $<1$ \\
\hline sko42-4 & [13] & 154057.0 & 172482.0 & 190150.0 & 22 & 5 & 4 & 2 & $<1$ \\
\hline sko42-5 & [13] & 287194.0 & 318344.0 & 351982.0 & 20 & 12 & 10 & 3 & $<1$ \\
\hline sko49-2 & [13] & 459140.0 & 519684.0 & 558166.0 & $3: 32$ & $2: 26$ & $2: 51$ & 7 & $<1$ \\
\hline sko49-3 & [13] & 357705.0 & 410297.0 & 441037.0 & $2: 25$ & $1: 29$ & $1: 54$ & 13 & $<1$ \\
\hline sko49-4 & [13] & 262364.0 & 292652.0 & 315012.0 & $2: 51$ & 45 & 52 & 9 & $<1$ \\
\hline sko49-5 & [13] & 737087.0 & 785207.0 & 860911.0 & $15: 00$ & 59 & 56 & 14 & 4 \\
\hline
\end{tabular}

Table 1: Optimal values ("opt value") and running times (in sec or min:sec) for different variants of the FC-CCLP, where $\left\lfloor\frac{n}{2}\right\rfloor$ departments are assigned to the first cell and the remaining departments to the second cell.

optimal value of the XRFLP is smaller than the optimal value of the TRFLP and for all instances the 4-PMFP (the 4-BFLP if $w_{\text {path }}=0$ ) has the smallest optimal value. Recall that the SRFLP is equivalent to the CCLP $(2,0)$, see Theorem 5 . The optimal value of the SRFLP is smaller than the optimal value of the CCLP $(0,2)$ here but the CCLP $(1,1)$ has the smallest optimal value of these three problems in our tests. The optimal values of the CCLP are greater than the ones of the 3-BFLP. The CCLP $(0,2)$ with up to 18 departments could be solved in less than 30 minutes, see Table 4, by using our MILP approach. Our MILP approach clearly outperforms the enumerative approach on the instances considered. Therefore, our approach outperforms the approach of $[15,32]$ as well because their models do not contain dummy departments, and hence one would have to use the enumerative approach.

The approach of [23] can easily be extended to the 3-PMFP. The optimal values derived by the approach of [23] are optimal neglecting computational accuracy, we refer to [23] for details. Considering the 3-BFLP with positive inter-cell distance as well as with inter-cell distance of zero we outperform the approach of [23], see Table 4 and Table 5. With our approach the TRFLP and the 4-BFLP could be solved with up to 17 departments within a time limit of 8 hours and the XRFLP with up to 16 departments. Our MILP model for the optimization problem in cell 1 in the TRFLP leads to a smaller running time than the corresponding enumerative approach, see Table 4 and Table 5. However, the approach of [23] can even solve one instance with 18 departments and is for most instances faster than our approach. The 3-PMFP (4-PMFP) is solved faster than the 3-BFLP and the TRFLP (the 4-BFLP and the XRFLP) since the number of distinguishable cell assignments is significantly smaller, see Table 5 .

\section{Conclusion and Future work}

In this paper we presented a new exact approach for the Combined Cell Layout Problem (CCLP) and we focus on the special cases of the CCLP, i. e., the Multi-Bay Facility Layout Problem (MBFLP) and the Pier-Type Material Flow Pattern (PMFP) with $m=3$ and $m=4$ denoted by (3-BFLP), (3-PMFP), (4-BFLP), (4-PMFP). Further, we considered the T-Row Facility Layout Problem (TRFLP) and we introduced a new layout problem, the so called X-Row Facility Layout Problem (XRFLP), which is a generalization of the 4-BFLP with a more complex path structure. 


\begin{tabular}{lcrrrrrrr} 
Instances & Source & \multicolumn{1}{c}{ SRFLP } & CCLP $(1,1)$ & CCLP $(0,2)$ & 3-BFLP & TRFLP & 4-BFLP & XRFLP \\
\hline$A m 11 a$ & {$[9]$} & 10630.5 & 9840.0 & 11178.5 & 8466.5 & 8407.0 & 6899.5 & 7038.5 \\
$A m 11 b$ & {$[9]$} & 7375.5 & 6802.5 & 7262.0 & 5694.5 & 5665.0 & 4864.5 & 4990.5 \\
$A m 12 a$ & {$[6,7]$} & 2901.0 & 2702.5 & 3266.5 & 2382.0 & 2354.5 & 1994.0 & 2047.0 \\
$A m 12 b$ & {$[6,7]$} & 3280.5 & 3042.5 & 3389.5 & 2557.5 & 2539.5 & 2172.5 & 2234.5 \\
$A m 13 a$ & {$[6]$} & 4902.5 & 4404.5 & 5283.5 & 3863.5 & 3836.0 & 3258.5 & 3327.5 \\
$A m 13 b$ & {$[6]$} & 5698.0 & 5046.0 & 6029.0 & 4376.0 & 4362.5 & 3642.0 & 3702.0 \\
$A m 14 \_1$ & {$[26]$} & 5481.5 & 5132.0 & 5766.5 & 4370.5 & 4350.5 & 3557.5 & 3634.5 \\
$A m 14 a$ & {$[51]$} & 5673.0 & 5263.0 & 6619.0 & 4475.0 & 4446.5 & 3773.0 & 3872.0 \\
$A m 14 b$ & {$[51]$} & 5595.0 & 5166.0 & 5725.0 & 4451.0 & 4430.5 & 3749.0 & 3838.0 \\
$A m 15$ & {$[3]$} & 6305.0 & 5961.5 & 6899.0 & 5093.0 & 5071.0 & 4237.0 & 4319.0 \\
$H K 15$ & {$[29]$} & 33220.0 & 30880.0 & 37440.0 & 26290.0 & 26125.0 & 21810.0 & 21891.0 \\
$P 16 a$ & {$[8]$} & 14829.0 & 14087.0 & 15125.0 & 11999.0 & 11943.0 & 10076.0 & 10194.0 \\
$P 16 b$ & {$[8]$} & 11878.5 & 11360.0 & 12768.5 & 9499.5 & 9469.5 & 7805.5 & 7921.5 \\
$P 17 a$ & {$[23]$} & 14436.5 & 14066.0 & 15930.0 & 11551.5 & 11524.5 & 9574.5 & - \\
$P 17 b$ & {$[23]$} & 15682.0 & 14870.5 & 16034.0 & 12389.0 & 12317.0 & 10174.0 & - \\
$A m 17$ & {$[4]$} & 9254.0 & 8604.0 & 10896.0 & 7345.0 & 7315.0 & 6044.0 & - \\
$P 18 a$ & {$[23]$} & 16118.5 & 15043.5 & 17904.0 & 12528.5 & - & 10266.5 & - \\
$P 18 b$ & {$[23]$} & 17716.5 & 16733.0 & 18022.5 & 14138.5 & - & - & - \\
$A m 18$ & {$[23]$} & 10650.5 & 10050.5 & 12274.5 & 8446.5 & 8413.5 & 6914.5 & -
\end{tabular}

Table 2: We illustrated the optimal values of several facility layout problems for instances from the literature where the inter-cell distances are set to zero. Instances marked with "_" could not be solved to optimality within the time limit of 8 hours.

\begin{tabular}{|c|c|c|c|c|c|c|c|c|c|}
\hline Instances & SRFLP & $\operatorname{CCLP}(1,1)$ & $\operatorname{CCLP}(0,2)$ & 3-BFLP & 3-PMFP & TRFLP & 4-BFLP & 4-PMFP & XRFLP \\
\hline$A m 11 a$ & 10630.5 & 10075.5 & 11727.5 & 9453.5 & 9237.5 & 8902.0 & 8261.5 & 7763.5 & 7882.5 \\
\hline$A m 11 b$ & 7375.5 & 7240.5 & 7739.0 & 6619.5 & 6444.5 & 6118.5 & 6152.5 & 5723.5 & 5793.5 \\
\hline$A m 12 a$ & 2901.0 & 2782.0 & 3468.0 & 2748.0 & 2672.0 & 2552.0 & 2519.0 & 2333.0 & 2374.0 \\
\hline$A m 12 b$ & 3280.5 & 3213.0 & 3584.5 & 2959.5 & 2872.5 & 2740.5 & 2733.5 & 2565.5 & 2593.5 \\
\hline$A m 13 a$ & 4902.5 & 4573.5 & 5532.5 & 4337.5 & 4232.5 & 4077.0 & 3926.5 & 3706.5 & 3741.5 \\
\hline$A m 13 b$ & 5698.0 & 5262.0 & 6260.0 & 4820.0 & 4727.0 & 4581.5 & 4293.0 & 4065.0 & 4102.0 \\
\hline Am14_1 & 5481.5 & 5278.5 & 6117.5 & 4940.5 & 4802.5 & 4642.0 & 4313.5 & 4049.5 & 4106.5 \\
\hline$A m 14 a$ & 5673.0 & 5386.0 & 6964.0 & 5093.0 & 4967.0 & 4751.0 & 4641.0 & 4365.0 & 4408.0 \\
\hline$A m 14 b$ & 5595.0 & 5400.0 & 6094.0 & 5059.0 & 4939.0 & 4739.5 & 4623.0 & 4325.0 & 4379.0 \\
\hline$A m 15$ & 6305.0 & 6102.0 & 7298.0 & 5687.0 & 5546.0 & 5378.0 & 5047.0 & 4768.0 & 4822.0 \\
\hline$H K 15$ & 33220.0 & 31174.0 & 37773.0 & 26905.0 & 26755.0 & 26446.0 & 22680.0 & 22368.0 & 22429.0 \\
\hline$P 16 a$ & 14829.0 & 14447.0 & 15551.0 & 12866.0 & 12668.0 & 12381.0 & 11276.0 & 10844.0 & 10940.0 \\
\hline$P 16 b$ & 11878.5 & 11638.5 & 13194.5 & 10324.5 & 10147.5 & 9882.5 & 8974.5 & 8561.5 & 8645.5 \\
\hline$P 17 a$ & 14436.5 & 14237.0 & 16443.0 & 12429.5 & 12232.5 & 11956.5 & 10811.5 & 10361.5 & - \\
\hline$P 17 b$ & 15682.0 & 15341.5 & 16526.0 & 13298.0 & 13094.0 & 12779.0 & 11446.0 & 10969.0 & - \\
\hline$A m 17$ & 9254.0 & 8814.0 & 11328.0 & 8245.0 & 8046.0 & 7767.5 & 7292.0 & 6836.0 & - \\
\hline$P 18 a$ & 16118.5 & 15529.5 & 18480.0 & 13506.5 & 13272.5 & - & - & 11155.5 & - \\
\hline$P 18 b$ & 17716.5 & 17300.0 & 18544.5 & 15110.5 & 14885.5 & - & - & 12531.5 & - \\
\hline$A m 18$ & 10650.5 & 10272.5 & 12823.5 & 9430.5 & 9202.5 & 8911.5 & - & 7791.5 & - \\
\hline
\end{tabular}

Table 3: Optimal values of facility layout problems for instances from the literature with inter-cell distances $w_{\text {path }}=w_{\text {path }}^{1}=w_{\text {path }}^{2}=3$ and for the CCLP $(1,1)$ and CCLP $(0,2)$ we set $u_{12}=3$. Instances marked with "-" could not be solved to optimality within the time limit of 8 hours. 


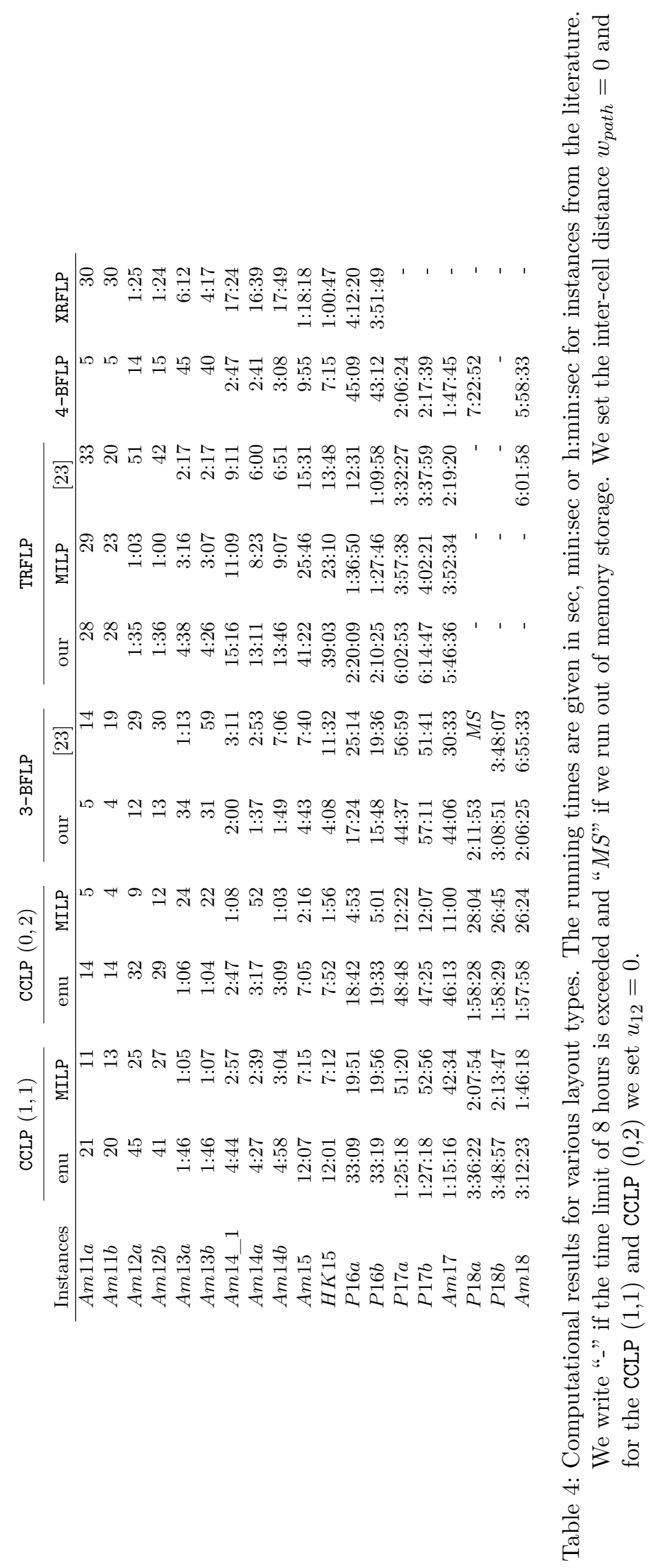




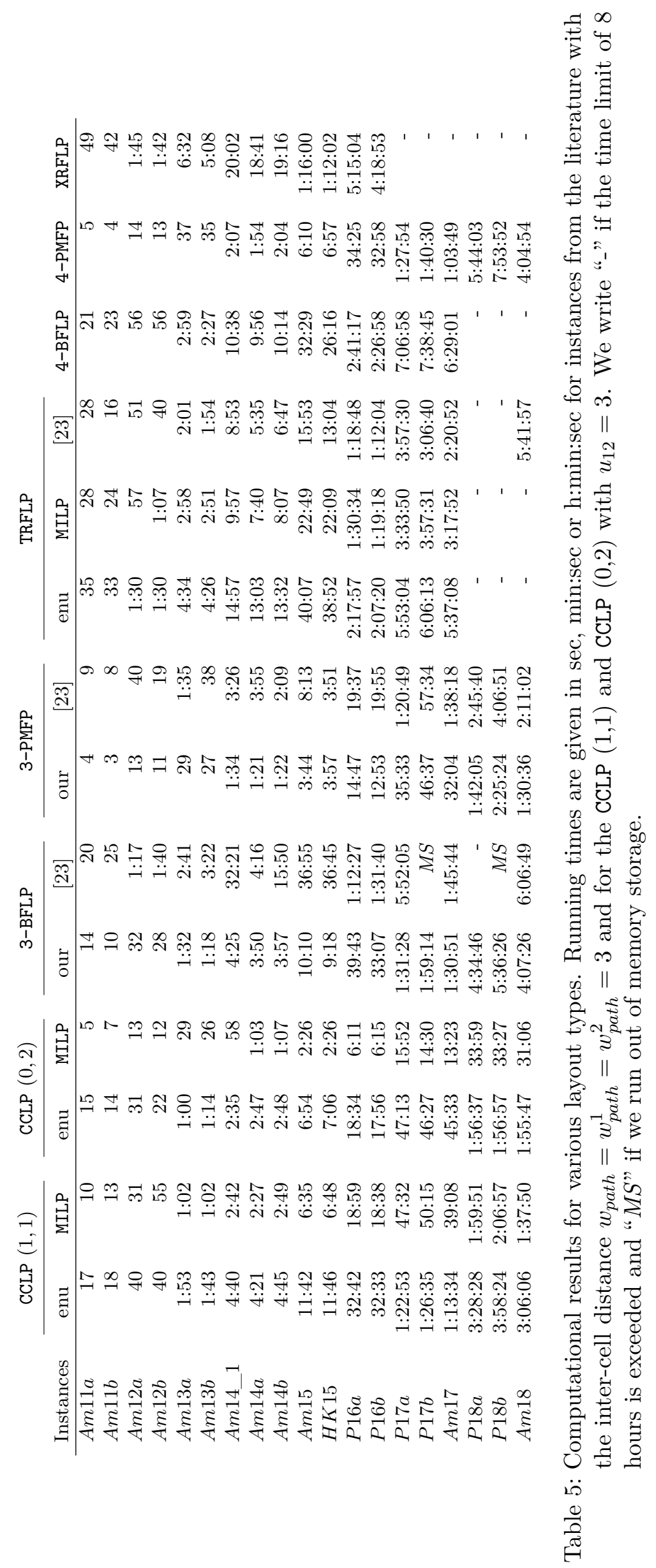


The CCLP, the TRFLP and the XRFLP have several applications such as in heavy manufacturing and semiconductor fabrication.

We extend the approaches known from the literature in various ways. Given a CCLP instance where all cells of type SRFLP have the same inter-cell distances, we proved that by enumerating over all cell assignments and solving the CCLP with fixed-cell assignment, one can merge two cells of type SRFLP and therefore reduce the number of cell assignments that have to be considered significantly. Further, we omit fixing one department on the loading station by adding a dummy department with appropriate lengths and weights to each cell dealing as loading and unloading station. Considering cells of type DCFLP, one department overlaps with the dummy department and for the arising optimization problem a Mixed-Integer Linear Programming (MILP) model is presented. The main result is adapted to the 3-BFLP, 4-BFLP, TRFLP and the XRFLP with positive inter-cell distances which are leveled by dummy departments of appropriate lengths. The optimal values of the optimization problems are studied from a theoretical point of view and compared in our computational study to support the decision maker to choose the layout of a factory which is built from the ground up. The CCLP consisting of two cells can be solved fast and our MILP model for cells of type DCFLP works well and clearly outperforms the enumerative approach. Hence, we can solve instances with up to 18 departments in at most 30 minutes if both cells are of type DCFLP. For the 3-BFLP as well as the 3-PMFP we outperform the current best approach from the literature.

It remains for future work to apply our approach on facility layout problems consisting of a higher amount of cells as well as to consider cells of other types, e. g., cells of type Double-Row Facility Layout Problem. A further realistic extension is to consider two-dimensional departments with varying widths. Then it might be harder to ensure in the XRFLP that departments in cell 2 and cell 3 do not overlap.

\section{Acknowledgment}

This work is partially supported by the Simulation Science Center Clausthal-Göttingen.

\section{References}

[1] Panos Afentakis. A loop layout design problem for flexible manufacturing systems. International Journal of Flexible Manufacturing Systems, 1(2):175-196, 1989.

[2] Luis O. Alpala, Maria del Mar Eva Alemany, Diego H. Peluffo-Ordoñez, Fabio Bolaños, Aura M. Rosero, and Juan C. Torres. Methodology for the design and simulation of industrial facilities and production systems based on a modular approach in an "industry 4.0" context. Dyna, 85(207): $243-252,2018$.

[3] André R. S. Amaral. On the exact solution of a facility layout problem. European Journal of Operational Research, 173(2):508-518, 2006.

[4] André R. S. Amaral. An exact approach to the one-dimensional facility layout problem. Operations Research, 56(4):1026-1033, 2008.

[5] André R. S. Amaral. A new lower bound for the single row facility layout problem. Discrete Applied Mathematics, 157(1):183-190, 2009.

[6] André R. S. Amaral. The corridor allocation problem. Computers \& Operations Research, 39(12): $3325-3330,2012$.

[7] André R. S. Amaral. Optimal solutions for the double row layout problem. Optimization Letters, 7 (2):407-413, 2013.

[8] André R. S. Amaral. A parallel ordering problem in facilities layout. Computers E Operations Research, 40(12):2930-2939, 2013. 
[9] André R. S. Amaral. A mixed-integer programming formulation for the double row layout of machines in manufacturing systems. International Journal of Production Research, 57(1):34-47, 2019.

[10] Miguel F. Anjos and Anthony Vannelli. Computing Globally Optimal Solutions for Single-Row Layout Problems Using Semidefinite Programming and Cutting Planes. INFORMS Journal On Computing, 20(4):611-617, 2008.

[11] Miguel F. Anjos and Manuel V. C. Vieira. Mathematical optimization approach for facility layout on several rows. Optimization Letters, pages 1-15, 2020.

[12] Miguel F. Anjos and Manuel V.C. Vieira. Mathematical optimization approaches for facility layout problems: The state-of-the-art and future research directions. European Journal of Operational Research, 261(1):1-16, 2017.

[13] Miguel F. Anjos and Ginger Yen. Provably near-optimal solutions for very large single-row facility layout problems. Optimization Methods and Software, 24(4):805-817, 2009.

[14] Miguel F. Anjos, Anja Fischer, and Philipp Hungerländer. Improved exact approaches for row layout problems with departments of equal length. European Journal of Operational Research, 270(2):514-529, 2018. ISSN 0377-2217. doi: https://doi.org/10.1016/j.ejor.2018.04.008. URL http://www.sciencedirect.com/science/article/pii/S0377221718303114.

[15] Miguel F. Anjos, Philipp Hungerländer, and Kerstin Maier. An integer linear programming approach for the combined cell layout problem. In 2018 IEEE International Conference on Industrial Engineering and Engineering Management (IEEM), pages 705-709. IEEE, 2018.

[16] Jaydeep Balakrishnan and Chun H. Cheng. Multi-period planning and uncertainty issues in cellular manufacturing: A review and future directions. European Journal of Operational Research, 177(1): 281-309, February 2007. ISSN 03772217. doi: 10.1016/j.ejor.2005.08.027. URL https://linkinghub. elsevier.com/retrieve/pii/S0377221705009045.

[17] Uwe Bracht, Mirko Dahlbeck, Anja Fischer, and Thomas Krüger. Combining simulation and optimization for extended double row facility layout problems in factory planning. In Marcus Baum, Gunther Brenner, Jens Grabowski, Thomas Hanschke, Stefan Hartmann, and Anita Schöbel, editors, Simulation Science, pages 39-59, Cham, 2018. Springer International Publishing. ISBN 978-3-319-96271-9.

[18] Gino Cardarelli and Pacifico M. Pelagagge. Simulation tool for design and management optimization of automated interbay material handling and storage systems for large wafer fab. IEEE Transactions on Semiconductor Manufacturing, 8(1):44-49, 1995.

[19] Ignacio Castillo and Brett A. Peters. Integrating design and production planning considerations in multi-bay manufacturing facility layout. European Journal of Operational Research, 157(3):671-687, 2004 .

[20] Junjae Chae and Brett A Peters. Layout design of multi-bay facilities with limited bay flexibility. Journal of Manufacturing Systems, 25(1):1-11, 2006. ISSN 0278-6125. doi: https://doi.org/ 10.1016/S0278-6125(07)00004-0. URL http://www.sciencedirect.com/science/article/pii/ S0278612507000040.

[21] Jaewoo Chung and Yunjung Suh. Analysis on the pier-type material flow pattern for facility layout applications. Journal of Applied Sciences, 14(3):237-244, 2014.

[22] Jaewoo Chung and J.M.A. Tanchoco. The double row layout problem. International Journal of Production Research, 48(3):709-727, 2010.

[23] Mirko Dahlbeck. A mixed-integer linear programming approach for the T-row and the multi-bay facility layout problem. Submitted; preprint available at http://www.optimization-online.org/ DB_HTML/2020/03/7670.html, 2020.

[24] Mirko Dahlbeck, Anja Fischer, and Frank Fischer. Decorous combinatorial lower bounds for row layout problems. European Journal of Operational Research, 286(3):929-944, 2020. ISSN 0377-2217. doi: https://doi.org/10.1016/j.ejor.2020.04.010. URL http://www.sciencedirect.com/science/ article/pii/S037722172030343X. 
[25] Dilip Datta, André R. S. Amaral, and José Rui Figueira. Single row facility layout problem using a permutation-based genetic algorithm. European Journal of Operational Research, 213(2):388-394, 2011.

[26] Anja Fischer, Frank Fischer, and Philipp Hungerländer. New exact approaches to row layout problems. Mathematical Programming Computation, 11(4):703-754, 2019.

[27] Martin Grötschel, Michael Jünger, and Gerhard Reinelt. A cutting plane algorithm for the linear ordering problem. Operations Reasearch, 32(6):1195-1220, 1984.

[28] Martin Grötschel, Michael Jünger, and Gerhard Reinelt. Facets of the linear ordering polytope. Mathematical Programming, 33(1):43-60, 1985.

[29] Sunderesh S. Heragu and Andrew Kusiak. Machine Layout Problem in Flexible Manufacturing Systems. Operations Research, 36(2):258-268, 1988.

[30] Hasan Hosseini-Nasab, Sepideh Fereidouni, Seyyed M. T. F. Ghomi, and Mohammad B. Fakhrzad. Classification of facility layout problems: a review study. The International Journal of Advanced Manufacturing Technology, 94(1-4):957-977, 2018.

[31] Philipp Hungerländer. The checkpoint ordering problem. Optimization, 66(10):1699-1712, 2017.

[32] Philipp Hungerländer and Miguel F. Anjos. An exact approach for the combined cell layout problem. In Stefan Helber, Michael Breitner, Daniel Rösch, Cornelia Schön, Johann-Matthias Graf von der Schulenburg, Philipp Sibbertsen, Marc Steinbach, Stefan Weber, and Anja Wolter, editors, Operations Research Proceedings 2012, pages 275-281, Cham, 2014. Springer International Publishing. ISBN 978-3-319-00795-3.

[33] Philipp Hungerländer and Miguel F. Anjos. A semidefinite optimization-based approach for global optimization of multi-row facility layout. European Journal of Operational Research, 245(1):46-61, 2015 .

[34] Philipp Hungerländer and Kerstin Maier. The multiple checkpoint ordering problem. In Natalia Kliewer, Jan Fabian Ehmke, and Ralf Borndörfer, editors, Operations Research Proceedings 2017, pages 171-177, Cham, 2018. Springer International Publishing. ISBN 978-3-319-89920-6.

[35] Philipp Hungerländer and Franz Rendl. Semidefinite relaxations of ordering problems. Mathematical Programming, 140(1):77-97, 2013.

[36] Philipp Hungerländer and Franz Rendl. A computational study and survey of methods for the single-row facility layout problem. Computational Optimization and Applications, 55(1):1-20, 2013.

[37] Philipp Hungerländer, Kerstin Maier, Jörg Pöcher, and Christian Truden. On a new modelling approach for circular layouts and its practical advantages. In 2017 IEEE International Conference on Industrial Engineering and Engineering Management (IEEM), pages 1581-1585, Dec 2017. doi: 10.1109/IEEM.2017.8290159.

[38] Philipp Hungerländer, Kerstin Maier, Veronika Pachatz, and Christian Truden. Exact and heuristic approaches for a new circular layout problem. SN Applied Sciences, 2(6):1035, May 2020. ISSN 2523-3971. doi: 10.1007/s42452-020-2734-z. URL https ://doi .org/10.1007/s42452-020-2734-z.

[39] IBM. ILOG CPLEX Optimization Studio 12.10, 2019.

[40] Birgit Keller and Udo Buscher. Single row layout models. European Journal of Operational Research, 245(3):629-644, 2015.

[41] Abdullah Konak, Sadan Kulturel-Konak, Bryan A. Norman, and Alice E. Smith. A new mixed integer programming formulation for facility layout design using flexible bays. Operations Research Letters, 34(6):660-672, 2006.

[42] Ravi Kothari and Diptesh Ghosh. The single row facility layout problem: state of the art. Opsearch, 49(4):442-462, 2012. 
[43] Ravi Kothari and Diptesh Ghosh. Tabu search for the single row facility layout problem using exhaustive 2-opt and insertion neighborhoods. European Journal of Operational Research, 224(1): 93-100, 2013.

[44] Panagiotis Kouvelis, Wen-Chyuan Chiang, and Ali S. Kiran. A survey of layout issues in flexible manufacturing systems. Omega, 20(3):375-390, 1992.

[45] Sadan Kulturel-Konak and Abdullah Konak. Unequal area flexible bay facility layout using ant colony optimisation. International Journal of Production Research, 49(7):1877-1902, 2011.

[46] Andrew Kusiak. Smart manufacturing. International Journal of Production Research, 56(1-2): $508-517,2018$.

[47] R. D. Meller. The multi-bay manufacturing facility layout problem. International Journal of Production Research, 35(5):1229-1237, 1997.

[48] Jean-Claude Picard and Maurice Queyranne. On the one-dimensional space allocation problem. Operations Research, 29(2):371-391, 1981.

[49] Hamed Samarghandi and Kourosh Eshghi. An efficient tabu algorithm for the single row facility layout problem. European Journal of Operational Research, 205(1):98-105, 2010.

[50] Sujeevraja Sanjeevi and Kiavash Kianfar. A polyhedral study of triplet formulation for single row facility layout problem. Discrete Applied Mathematics, 158(16):1861-1867, 2010.

[51] Leonardo D. Secchin and André R. S. Amaral. An improved mixed-integer programming model for the double row layout of facilities. Optimization Letters, 13(1):193-199, 2019.

[52] Donald M. Simmons. One-Dimensional Space Allocation: An Ordering Algorithm. Operations Research, 17(5):812-826, 1969.

[53] James A. Tompkins, John A. White, Yavuz A. Bozer, and J. M. A. Tanchoco. Facilities planning. John Wiley \& Sons, 2010.

[54] Taho Yang and Brett A. Peters. A spine layout design method for semiconductor fabrication facilities containing automated material-handling systems. International Journal of Operations $\&$ Production Management, 17(5):490-501, 1997.

[55] Zeqiang Zhang and Chase C. Murray. A corrected formulation for the double row layout problem. International Journal of Production Research, 50(15):4220-4223, 2012.

\section{Appendix}

In the appendix we summarize our approach for the TRFLP. We denote by $C_{1}\left(C_{2}\right)$ the set of departments assigned to cell 1 (cell 2) and if the cell assignment of the departments is known, we denote the resulting problem as Fixed-Row T-Row Facility Layout Problem (FR-TRFLP). Given an FR-TRFLP instance, by Lemma 4 we obtain the problems $W_{\left(n+2, p_{b}\right)}^{S}\left(C_{2} \cup\{n+2\}\right)$ and the $W_{\left(n+1, p_{a}\right)}^{S}\left(C_{1} \cup\{n+1\}\right)$ with the dummy department $n+2$ or $n+1$, respectively, where additionally one department in cell 1 may overlap with the dummy department $n+1$. We denote the obtained problem by $W_{\left(n+1, p_{a}, o\right)}^{S}\left(C_{1} \cup\{n+1\}\right)$. For solving the $W_{\left(n+2, p_{b}\right)}^{S}\left(C_{2} \cup\{n+2\}\right)$ we refer to Section 4.2. Thus, it remains to study the $W_{\left(n+1, p_{a}, o\right)}^{S}\left(C_{1} \cup\{n+1\}\right)$.

We study new exact methods for solving the $W_{\left(n+1, p_{a}, o\right)}^{S}\left(C_{1} \cup\{n+1\}\right)$. In [23] the following result is proven for the TRFLP and this result is valid for the FR-TRFLP as well.

Proposition 12. Given an FR-TRFLP instance where $C_{1} \neq \emptyset\left(C_{2}\right)$ denotes the set of departments assigned to cell 1 (cell 2) and let $V_{1}:=\left\{i \in C_{1}: \sum_{j \in C_{2}}\left(w_{i j}+w_{j i}\right)>0\right\} \neq \emptyset$. Let the dummy department $n+1$ be assigned to cell 1 with $\ell_{n+1}=0$ and $w_{i(n+1)}=\sum_{j \in[n] \backslash C_{1}} w_{i j}$, $w_{(n+1) i}=\sum_{j \in[n] \backslash C_{1}} w_{j i}$ (see (14), (15)) and let the optimal value of the $W_{\left(n+1, p_{a}, o\right)}^{S}\left(C_{1} \cup\{n+1\}\right)$ 
be denoted by $v_{n+1}^{*}$. Further, we denote by $v_{s_{M}}^{*}$ the optimal value of the $W_{\left(s_{M}, p_{a}\right)}^{S}\left(C_{1}\right)$ where the weights of $s_{M} \in C_{1}$ are adjusted according to (14), (15). Then,

$$
v_{n+1}^{*}=\min \left\{v_{s_{M}}^{*}: s_{M} \in V_{1}\right\}
$$

Further, there always exists an optimal T-row layout with $C_{1} \neq \emptyset$. If $V_{1}=\emptyset$, we neglect the dummy department $n+1$ and then the $W_{\left(n+1, p_{a}, o\right)}^{S}\left(C_{1} \cup\{n+1\}\right.$ is equivalent to the SRFLP with the set of departments $C_{1}$. Given an FR-TRFLP instance, we fix $s_{M} \in C_{1}$ directly opposite the (un-) loading station, and hence we have to solve the $W_{\left(s_{M}, p_{a}\right)}^{S}\left(C_{1} \cup\{n+1\}\right)$. By this method one has to consider $2^{n}-1$ cell assignments for the TRFLP and solve the $W_{\left(n+2, p_{b}\right)}^{S}\left(C_{2} \cup\{n+2\}\right)$ once and the $W_{\left(s_{M}, p_{a}\right)}^{S}\left(C_{1} \cup\{n+1\}\right), s_{M} \in C_{1}$, has to be solved $\max \left\{1,\left|V_{1}\right|\right\}$ times for every cell assignment.

Hence, we present an MILP model for solving the $W_{\left(n+1, p_{a}, o\right)}^{S}\left(C_{1}\right)$ with $V_{1} \neq \emptyset$ in order to solve this problem faster as well as to obtain good lower bounds and neglect unbalanced cell assignments early in our algorithm. The idea is to split the dummy department $n+1$ into two dummy departments $n+3$ and $n+4$ with lengths $\ell_{n+3}=\ell_{n+4}=0$ and weights $w_{i(n+3)}=w_{i(n+4)}=\frac{w_{i(n+1)}}{2}, w_{(n+3) i}=w_{(n+4) i}=\frac{w_{(n+1) i}}{2}, i \in C_{1}$. We ensure that exactly one department lies between $n+3$ and $n+4$ and this department lies on position $p_{E_{1}}$. Neglecting the constant weights $W=\sum_{\substack{i, j \in[n] \\ i<j}}\left(w_{i j}+w_{j i}\right) \frac{\ell_{i}+\ell_{j}}{2}$ for the whole problem our MILP model for the subproblem in cell 1 reads as follows

$$
\begin{array}{ll}
\min \sum_{\substack{i, j \in C_{1} \\
i<j}}\left(w_{i j}+w_{j i}\right) \sum_{k \in C_{1} \backslash\{i, j\}} \ell_{k} x_{i k j} & \\
\quad+\sum_{\substack{i \in C_{1} \\
j \in\{n+3, n+4\}}}\left(w_{i j}+w_{j i}\right)\left(\sum_{k \in C_{1} \backslash\{i\}} \ell_{k} x_{i k j}-\frac{\ell_{i}}{2} x_{(n+3) i(n+4)}\right) \\
\quad(2)-(6), & \mathcal{D}=C_{1} \cup\{n+3, n+4\}, \\
\sum_{i \in C_{1}} x_{(n+3) i(n+4)}=1, & \\
\quad x_{(n+3) i(n+4)}=0, & i \notin V_{1}, \\
\quad x_{i k j} \in\{0,1\}, & i, j, k \in C_{1} \cup\{n+3, n+4\},
\end{array}
$$

By Equation (30) exactly one department is arranged between the dummy departments $n+3$ and $n+4$ and if $i \in C_{1} \backslash V_{1}$, then $i$ does not lie between the dummy departments, see Equations (31). In the objective function (29) the distances between $i \in C_{1}$ and $j \in C_{1} \cup\{n+3, n+4\}, i<j$, are measured similar as in the SRFLP if $x_{(n+3) i(n+4)}=0$. Otherwise, if $x_{(n+3) i(n+4)}=1$, then the distance between $i$ and $j \in\{n+3, n+4\}$ equals $-\frac{\ell_{i}}{2}$ because we excluded the value $\left(w_{i j}+w_{j i}\right) \frac{\ell_{i}}{2}$ in the calculation of the constant $W$. Measuring the distance between $j \in C_{1} \backslash\{i\}$ and the dummy departments we subtract $\frac{\ell_{i}}{2}$, and thus we do not take the length $\ell_{i}$ into account, as desired by the calculation of the constant $W$. Hence, our distance calculation and the MILP model are correct. In our algorithm we solve the $W_{\left(n+2, p_{b}\right)}^{S}\left(C_{2} \cup\{n+2\}\right)$ first and then we solve the $W_{\left(n+1, p_{a}\right)}^{S}\left(C_{1} \cup\{n+1\}\right)$ because the $W_{\left(n+2, p_{b}\right)}^{S}\left(C_{2} \cup\{n+2\}\right)$ can often be solved faster, see Table 1 and the corresponding conclusions. Note that in the FR-TRFLP the inter-cell distance $u_{12}$ leads only to constant weights, and thus we can exclude them. 


\section{Combining simulation and optimization for extended double row facility layout problems in factory planning}

Authors: Uwe Bracht, Mirko Dahlbeck, Anja Fischer, Thomas Krüger published in Simulation Science, 2018.

Reprinted by permission from Springer Nature Customer Service Centre GmbH: Springer Nature, Simulation Science, Combining Simulation and Optimization for Extended Double Row Facility Layout Problems in Factory Planning, Uwe Bracht, Mirko Dahlbeck, Anja Fischer, Thomas Krüger, 08.08.2018, https://doi.org/10.1007/978-3-319-96271-9_3 


\title{
Combining Simulation and Optimization for Extended Double Row Facility Layout Problems in Factory Planning
}

\author{
Uwe Bracht $^{1}$, Mirko Dahlbeck ${ }^{2,3(\bowtie)}$, Anja Fischer ${ }^{3}$, and Thomas Krüger ${ }^{1}$ \\ 1 Technische Universität Clausthal, Clausthal-Zellerfeld, Germany \\ \{uwe.bracht, thomas.krueger\}@imab.tu-clausthal.de \\ ${ }^{2}$ Georg-August-Universität Göttingen, Göttingen, Germany \\ m.dahlbeck@math.uni-goettingen.de \\ 3 Technische Universität Dortmund, Dortmund, Germany \\ anja2.fischer@tu-dortmund.de
}

\begin{abstract}
We investigate the so called Double Row Facility Layout Problem (DRFLP). Given a set of departments with given lengths and pairwise transport weights between them, the aim is to assign the departments to two rows such that the weighted sum of the distances between them is minimized and such that the departments do not overlap. The DRFLP is known to be rather challenging. Even with the best approach known in literature, which is based on an enumeration over all row assignments of the departments and where only the center-to-center distances are measured, the largest instance solved to optimality contains only 16 departments. In this paper we show how the existing models can be extended in various directions in order to handle more aspects that are important in real-world applications such as vertical distances between the departments and restricting the size of the layout area. We also show how the structure of real-world instances, which often contain several departments of the same type, can be exploited in mathematical optimization. This allows us to solve a realistic instance with 21 departments in reasonable time. Furthermore, we propose a new approach which combines optimization and simulation. Here simulation allows the evaluation of the optimized solutions with respect to several performance indicators which play an important role for a smooth production apart from the weighted transport distances. If problems are detected, this information is included in the mathematical models by extending these.
\end{abstract}

Keywords: Facility layout problem $\cdot$ Exact solution $\cdot$ Simulation

\section{Introduction}

Globalization, the growing dynamics of the markets, the increase in customized products, decreasing product life cycles and technological innovations are only some of the challenges manufacturing enterprises have to cope with. As a result,

(C) Springer Nature Switzerland AG 2018

M. Baum et al. (Eds.): SimScience 2017, CCIS 889, pp. 39-59, 2018.

https://doi.org/10.1007/978-3-319-96271-9_3 
manufacturing enterprises are forced to implement a cost efficient production in order to remain competitive. The layout of the production areas and operating equipments (assets and departments) is one of the main influencing factors and provides a basis to uphold the long-term productivity and competitiveness [19]. In this work we present a combined optimization-simulation approach for determining a good start solution for the layout of the departments along both sides of a single path. For this we extend the mathematical optimization approach in [7]. The start solution obtained via mathematical optimization is then the basis for the following steps of the factory planners on a much finer level of detail. In order to handle many of the requirements posed on the layout in real-world production, the existing mathematical optimization models have to be extended.

From a mathematical point of view, the described factory planning problem leads to so called facility layout problems, which are widely studied [4]. Several methods have been developed in this area ranging from graphical methods, heuristics, which allow deriving solutions rather fast but without some knowledge of the quality of the solutions, and exact optimization methods. Unfortunately, solving even small instances exactly without additional restrictions on the path structure is extremely challenging. So deriving an exact solution or even a good solution with appropriate solution guarantees for small to medium-sized instances is often rather time-consuming. For this reason, one often concentrates on special cases where one restricts the structure of the layout and the paths. We investigate the so called Double Row Facility Layout Problem (DRFLP). Given $n$ departments with positive lengths $\ell_{i}, i \in\{1, \ldots, n\}=:[n]$, and symmetric pairwise transport weights $c_{i j}, i, j \in[n], i<j$, between them, the classic DRFLP asks for an assignment of $n$ departments to two rows (the two sides of a path) and horizontal positions of the departments such that the weighted sum of the center-to-center distances, measured in horizontal direction, is minimized. Moreover, two departments in the same row may not overlap. So we look for a vector $p \in \mathbb{R}^{n}$ of positions and a vector $r \in\{1,2\}^{n}$ of the assignment of the departments to the two rows such that

$$
\begin{aligned}
& \min \sum_{\substack{i, j \in[n] \\
i<j}} c_{i j}\left|p_{i}-p_{j}\right| \\
& \text { subject to }\left|p_{i}-p_{j}\right| \geq \frac{\ell_{i}+\ell_{j}}{2}, i, j \in[n], i<j, \text { if } r_{i}=r_{j} .
\end{aligned}
$$

In [9], Chung and Tanchoco present a model for the DRFLP (see also [24]) which can solve instances with up to 8 departments in about 10 min. Amaral suggests a mixed-integer program that can solve instances with up to 12 departments in less than one hour [3]. The current best known approach for solving the DRFLP is presented in [12], where Fischer et al. solve instances with up to 16 departments in less than $12 \mathrm{~h}$. Beside the exact methods, there are several heuristic approaches for solving the DRFLP and extensions of it, see, e.g., [17,25]. Highly related to the DRFLP is the Single Row Facility Layout Problem (SRFLP), introduced in [22]. 
In contrast to the DRFLP, in the SRFLP the departments are arranged in only one row. With the best known approach, presented in [13,14], Hungerländer and Rendl are able to solve instances with up to 42 departments to optimality and they receive very small gaps for instances with up to 110 departments. For an overview of layout problems in general we refer to $[4,11]$.

Our paper is structured as follows. In Sect.2, we summarize the current best approach for the DRFLP [12]. There, one combines a strong model for the DRFLP with fixed row assignment, i. e., the row assignment of each department is known in advance, with a branching scheme enumerating over all possible row assignments.

In Sect. 3, we extend this approach in various directions. We consider departments as 2-dimensional objects which have a length and a width. In real-world applications the size of a factory is limited, so in Sect. 3.1 we show how to restrict the area used for the DRFLP layout. In Sect. 3.2, we allow the consideration of vertical distances between the departments. Afterwards, in Sect. 3.3 we consider instances that contain departments of the same type, i. e., these departments have the same length and the same transport weights to all other departments. We exploit this structural property of departments of the same type by reducing the number of relevant row assignments significantly.

Unfortunately, in the classic mathematical DRFLP models only the transport weights and so the weighted transport loads are taken into account. But there are several further indicators that are important for guaranteeing a smooth production, e. g., the throughput of the factory, the cycle times of the products or the used storage and buffer capacities. Therefore, we combine in Sect. 4 the mathematical DRFLP model with a simulation of the production that allows determining various key performance indicators. Thus, we can detect potential problems and conflicts.

In Sect. 5, we solve a realistic instance with 21 departments in less than $14 \mathrm{~h}$ by exploiting that there are several departments of the same type. We compare our solutions obtained via mathematical optimization to solutions derived by applying classic methods used in factory planning. The results obtained via simulation are then the starting point in Sect. 5.2 for extending the mathematical models such that the transport distances are considered not only in an aggregated form, but for each single product. We summarize our results and give suggestions for future work in Sect. 6 .

\section{Basic Model for the DRFLP}

In the classic models for the DRFLP, see, e. g., [3, 9,12], the following three assumptions are made

1. the total size of the area needed for the arrangement is not limited,

2. vertical distances between the departments are neglected,

3. each department can be assigned to any of the two rows. 
In contrast to the SRFLP, there might occur free spaces between departments in the same row in optimal DRFLP solutions.

The current best solution approach for the DRFLP is described in [12]. The main idea is to enumerate over all possible row assignments and solve the DRFLP with fixed row assignment (FR-DRFLP). In the following, we summarize the model of [12] for solving the FR-DRFLP. First we add two dummy departments $n+1$ and $n+2$ representing the left and right border of the layout. The lengths and transport weights of the dummy departments are set to zero, i. e., $\ell_{n+1}=\ell_{n+2}=$ $0, c_{i(n+1)}=c_{i(n+2)}=0$ for $i \in[n]$ and $c_{(n+1)(n+2)}=0$.

In order to consider a fixed row assignment, let $R=\{1,2\}$ be the set of rows and $r_{i} \in R, i \in[n]$, be an assignment of the departments to the two rows. For $h \in R$ we will write: $j \in R_{h} \Leftrightarrow r_{j}=h$. The dummy departments $n+1$ and $n+2$ are assigned to both rows and we define $\tilde{R}_{h}=R_{h} \cup\{n+1, n+2\}$. We use betweenness variables

$$
x_{i k j}=x_{j k i}= \begin{cases}1, & k \text { lies between } i \text { and } j \text { in the same row } \\ 0, & \text { otherwise, }\end{cases}
$$

for $l \in R, i, j, k \in \tilde{R}_{l}, i \neq k \neq j, i<j$. The betweenness variables induce an order of the departments in each row, because $x_{(n+1) i j}$ is equal to 1 if and only if department $i$ is left to department $j$ in the same row. We consider the following integer linear programming model

$$
\begin{array}{ll}
x_{i j k}+x_{i k j}+x_{j i k}=1, & l \in R, i, j, k \in \tilde{R}_{l}, i<j<k, \\
x_{(n+1) i(n+2)}=1, & i \in[n], \\
x_{i k j}=0, & l \in R, i, j \in R_{l}, i<j, k \in\{n+1, n+2\}, \\
x_{(n+1) i j}=x_{i j(n+2)}, & l \in R, i, j \in R_{l}, i \neq j, \\
x_{i h j}+x_{i h k}+x_{j h k} \leq 2, & l \in R, i, j, k, h \in \tilde{R}_{l},|\{i, j, k, h\}|=4, i<j<k, \\
-x_{i h j}+x_{i h k}+x_{j h k} \geq 0, & l \in R, i, j, k, h \in \tilde{R}_{l},|\{i, j, k, h\}|=4, \\
x_{i j k} \in\{0,1\}, & l \in R, i, j, k \in \tilde{R}_{l},|\{i, j, k\}|=3, i<k .
\end{array}
$$

If three departments lie in the same row, by (1) exactly one of them lies in the middle. The constraints (2)-(4) ensure that every department lies between the dummy departments $n+1$ and $n+2$, i. e., the dummy departments are the left and right border of the layout. Inequalities (5)-(6) imply that the departments satisfy certain transitivity properties. According to [2], (1) and (5)-(7) induce a correct ordering of the departments in each row.

Next we need to calculate the distance between two distinct departments. The horizontal position $p_{i}$ of the center of department $i, i \in[n]$, is given by $d_{i(n+1)}=p_{i}$. The value $d_{i(n+2)}$ is defined as the distance between the right border of the layout, i. e., department $n+2$, and department $i$ for $i \in[n]$. The distance between the left and the right border of a layout is given by $d_{(n+1)(n+2)}$. The distance is calculated according to (see [12])

$$
d_{j i}=d_{i j} \geq\left|p_{i}-p_{j}\right|=\left|d_{(n+1) i}-d_{(n+1) j}\right|,
$$


for $i, j \in[n] \cup\{n+2\}, i<j$. We set $M:=\sum_{i=1}^{n} \ell_{i}$ and we obtain in the following a model for the FR-DRFLP, which we call IP FR-DRFLP. This model is given in its basic form in [12] and we add the inequalities (9) and (10), because we later want to extend this model.

$$
\min \sum_{\substack{i, j \in[n] \\ i<j}} c_{i j} d_{i j}
$$

s.t. (1)-(7),

$$
\begin{array}{ll}
d_{j(n+1)}-d_{i(n+1)} \geq M\left(x_{(n+1) i j}-1\right)+\frac{\ell_{i}+\ell_{j}}{2}, & l \in R, i, j \in R_{l}, i \neq j, \\
d_{j(n+2)}-d_{i(n+2)} \geq M\left(x_{j i(n+2)}-1\right)+\frac{\ell_{i}+\ell_{j}}{2}, & l \in R, i, j \in R_{l}, i \neq j, \\
d_{i(n+1)}+d_{i(n+2)}=d_{(n+1)(n+2)}, & i \in[n], \\
d_{i(n+1)} \geq \frac{\ell_{i}}{2}, d_{i(n+2)} \geq \frac{\ell_{i}}{2}, & i \in[n], \\
d_{i k}+d_{k j} \geq d_{i j}, & i, j, k \in[n+2], i<j, \\
d_{i j} \geq 0, & |\{i, j, k\}|=3, \\
& i, j \in[n+2], i<j .
\end{array}
$$

By inequalities (8), (9) and (11) there is a minimal distance of $\frac{\ell_{i}+\ell_{j}}{2}$ between the centers of the departments $i$ and $j$ if they lie in the same row and with respect to the dummy departments. Inequalities (12) are triangle inequalities that also connect departments lying in different rows. We argued above that the betweenness inequalities (1) and (5)-(7) induce a correct ordering of the departments in each row. Combining this with (8)-(13) we get the following theorem:

Theorem 1. The model (1)-(13) is correct for the FR-DRFLP.

This result follows immediately from [12] which we adapted only slightly such that also the distance between some department $i, i \in[n]$, and department $n+2$ is calculated correctly.

Furthermore, we can add a lower bound on the distance between the centers of two departments $i$ and $j$ in the same row by summing up the lengths of all departments between $i$ and $j$

$$
d_{i j} \geq \frac{\ell_{i}+\ell_{j}}{2}+\sum_{k \in R_{l} \backslash\{i, j\}} \ell_{k} x_{i k j}, \quad l \in R, i, j \in \tilde{R}_{l}, i<j .
$$

We want to point out that the distance between two departments might be greater than this bound, because in an optimal solution of the FR-DRFLP there might occur free space between two neighboring departments.

In order to solve the DRFLP using the model above, we have to test exponentially many row assignments. We can reduce the number of distinguishable row 
assignments by reducing $M$, the big- $M$-value in inequalities (8) and (9), which is also an upper bound on the sum of the lengths of the departments in each single row. Certainly we can assume that in an optimal solution the sum of the lengths of the departments in row one is the same as or larger than the sum in row two. Let the leftmost department $i$ of the considered layout start at position $p_{n+1}=0$ with its center $p_{i}=\frac{\ell_{i}}{2}$ and let the rightmost department $j \in[n]$ apart from $n+2$ of this layout finish at $t$ with its center $p_{j}=t-\frac{\ell_{j}}{2}$.

Lemma 1 ([12]). Given a DRFLP instance that satisfies, w.l.o.g., $\ell_{i} \leq \ell_{i+1}$ for $i \in[n-1]$, there always exists an optimal DRFLP layout on the interval $[0, t]$ with

$$
t \leq \sum_{i=\left\lfloor\frac{n+1}{3}\right\rfloor+1}^{n} \ell_{i}
$$

Moreover, this bound is tight.

Due to [12], we can neglect all row assignments where the sum of the lengths of the departments in one of the rows exceeds $t$.

Usually, in factory planning the incoming warehouse and the shipping warehouse of a factory are arranged at the left and at the right border, respectively. If this is the case, the dummy departments $(n+1, n+2)$ can be interpreted as these warehouses. For an illustration we refer to Fig. 1. Of course, we might obtain a better overall solution value if we drop the restriction on the position of both warehouses. In this case they are treated as ordinary departments that have transport connections to other departments and need a certain space. Later in Sect. 5 we compare the quality of the solutions with and without this restriction on the positions.

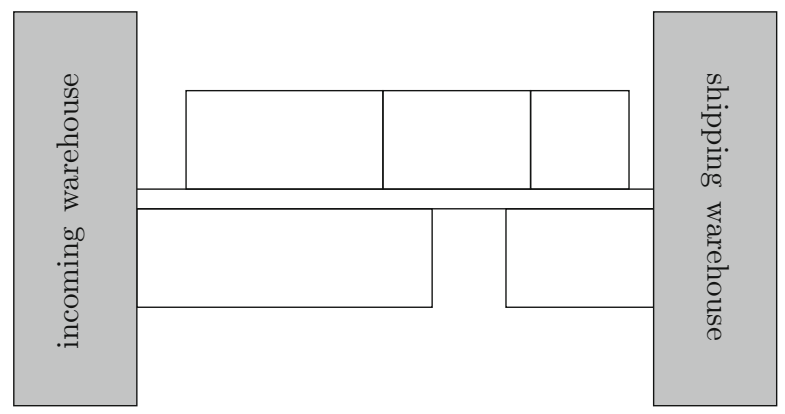

Fig. 1. Visualization of an extended DRFLP layout where we fixed the incoming and the shipping warehouse to the border of the layout. One motivation for this arrangement is that one hopes to receive rather linear transport flows between the departments. 


\section{Extensions of the Basic DRFLP Model}

In this section we show how the DRFLP and the FR-DRFLP can be extended such that further aspects, which are relevant in practice, can be handled in optimization.

\subsection{Restricted Area of the Whole Layout and Blocked Areas}

We consider the case that the departments not only have a length but also a width, i. e., they are given as 2-dimensional objects. Our aim is to place the departments in a restricted area. In [21], a restricted area is taken into account by a penalty function. However, we will restrict the area by additional constraints. In factory planning the layout area is usually defined as follows:

Definition 1. The area of a given layout is defined as the area of the minimum boundary rectangle containing all departments.

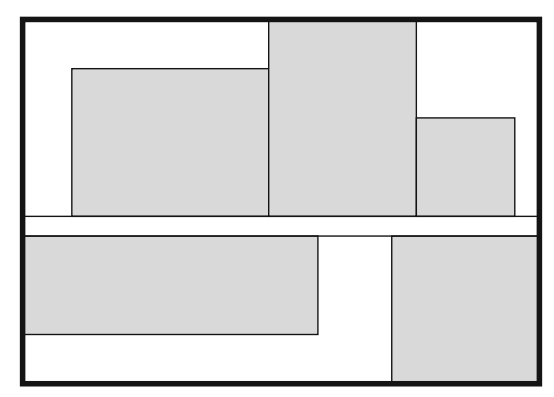

Fig. 2. Minimum boundary rectangle of a layout (marked black) enclosing five 2dimensional departments. The area of the layout corresponds to the size of this rectangle.

By definition the area of a layout is equal to $d_{(n+1)(n+2)} \cdot w$, where $w$ is the width of the layout (in our FR-DRFLP model). An example is illustrated in Fig. 2. Let $w_{i}$ denote the width of department $i \in[n]$. In the FR-DRFLP the row assignment is fixed, so we compute the width of the layout by summing up the width of the department with the largest width in each row plus the width of the path $w_{\text {path }}$ between the two rows, i. e., $w=\max _{i \in R_{1}} w_{i}+\max _{i \in R_{2}} w_{i}+w_{\text {path }}$. In particular, for a fixed row assignment the width of a layout is constant. Thus, we only need to restrict the distance $d_{(n+1)(n+2)}$ in an appropriate way to restrict the used area. Assume the area of the layout may be at most $F \in \mathbb{R}_{\geq 0}$. Then the linear inequality $d_{(n+1)(n+2)} \leq \frac{F}{w}$ ensures that the area of the layout is bounded by $F$.

So, given a row assignment, all departments lie in the interval $\left[0, \frac{F}{w}\right]$. Thus, we can neglect all row assignments where the sum of the lengths of the departments 
in the same row exceeds $\frac{F}{w}$. Moreover, we can improve the big- $M$-value used in inequalities (8) and (9) to

$$
M=\min \left\{\frac{F}{w}, \sum_{i=1}^{n} \ell_{i}\right\} .
$$

Hence, for every row assignment we have to compute a new big- $M$-value. Note that the upper bound $t$, as computed in (14), is not valid anymore.

Apart from a restriction of the used area, there might appear so called blocked areas in real-world factory planning problems. It is not allowed to place departments in these areas. This might be due to already existing departments or due to safety restrictions. Let $B_{1}=\left\{\left[b_{1}, b_{1}+g_{1}\right], \ldots,\left[b_{u}, b_{u}+g_{u}\right]\right\}$ be the blocked areas in row 1 and $B_{2}=\left\{\left[b_{u+1}, b_{u+1}+g_{u+1}\right], \ldots,\left[b_{v}, b_{v}+g_{v}\right]\right\}$ be the blocked areas in row 2 for given $b_{k}, g_{k} \geq 0, k \in[v], b_{k}+g_{k} \leq b_{k+1}, k \in[v] \backslash\{u\}$. For each blocked area we introduce a new dummy department, which we will call blocked department, with length equal to the length of the blocked area. We place the center of the blocked department in the middle of the blocked area. So we get the blocked departments $n+3, n+4, \ldots,\left(n+2+\left|B_{1}\right|+\left|B_{2}\right|\right)$ with length $\ell_{n+2+k}=g_{k}$ for $k \in[v]$. The row assignment of the blocked departments is fixed, namely $R^{1}=\left\{(n+3), \ldots,\left(n+2+\left|B_{1}\right|\right)\right\}$ are assigned to row 1 and $R^{2}=\left\{\left(n+2+\left|B_{1}\right|+1\right), \ldots,\left(n+2+\left|B_{1}\right|+\left|B_{2}\right|\right)\right\}$ to row 2 . To ensure that the blocked department $n+2+k$ lies exactly on the interval $\left[b_{k}, b_{k}+g_{k}\right]$, we set the distance variable to

$$
d_{(n+1)(n+2+k)}=b_{k}+\frac{g_{k}}{2}, k \in[v] .
$$

Additionally, we extend the inequalities (8) such that they are satisfied for all departments $i, j \in R_{l} \cup R^{l}$ for $l \in\{1,2\}$. Apart from this we can fix the betweenness variables that belong to each three departments with index at least $n+1$. For correctness of the model an update of the big- $M$-value to $\tilde{M}=\max \left\{b_{u}+g_{u}, b_{v}+g_{v}\right\}+t$, where $t$ is defined as in (14), is needed, since it might happen that in an optimal solution the $n$ departments are all arranged right to the blocked departments (possible if the area of the layout is not additionally bounded; this is possible by restricting $\left.d_{(n+1)(n+2)}\right)$. Note that $M$ has to be further enlarged if the blocked areas have non-zero transport weights to departments in $[n]$. This can happen if we extend an existing factory and do not want to move some of the old departments.

\subsection{Vertical and Inter-row Distances}

We want to overcome the second assumption of the basic model as stated in Sect. 2-vertical distances between the departments are neglected - by adding inter-row distances between departments in distinct rows as well as vertical distances between departments in the same row. For this, note again that we assume that the departments are 2-dimensional objects. The center-to-center distance 
between two departments $i$ and $j$ is computed as follows: First we add the distance between the center of $i$ to the path, then, as in the 1-dimensional case, we compute the distance $\left|p_{i}-p_{j}\right|$ and afterwards we add the distance from the path to the center of $j$. Furthermore, if $i$ and $j$ are in distinct rows, we add the width of the path $w_{\text {path }}$. An example is illustrated in Fig. 3.

In order to solve this extended DRFLP, we use again our fixed-assignment model IP $\mathrm{IR}_{F-D R F L P}$. For the FR-DRFLP, the inter-row distances and associated transport weights are constant. The inter-row weights are calculated by

$$
\sum_{\substack{j \in R_{1} \\ k \in R_{2}}}\left(\frac{w_{j}+w_{k}}{2}+w_{\text {path }}\right) c_{j k}
$$

and for departments in the same row we get

$$
\sum_{\substack{j, k \in R_{1} \\ j<k}} \frac{w_{j}+w_{k}}{2} c_{j k}+\sum_{\substack{j, k \in R_{2} \\ j<k}} \frac{w_{j}+w_{k}}{2} c_{j k} .
$$

All in all, in our setting we only need to add a constant to the objective value of some FR-DRFLP to include inter-row distances and compare the total objective values in the enumeration scheme. We want to note again, that the reduction of $M$ according to (14) is not possible because of this constant.

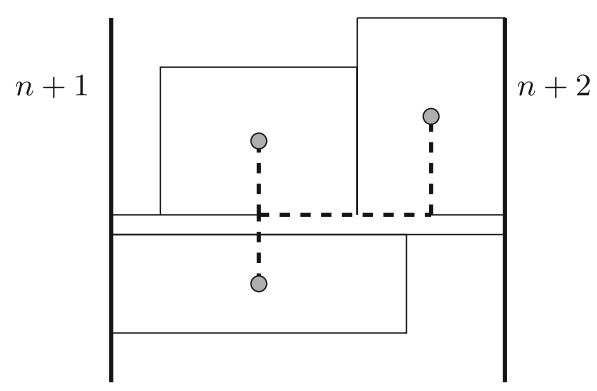

Fig. 3. Calculation of the vertical and horizontal distances between three departments

Naturally, the third assumption mentioned at the beginning of Sect. 2 - each department can be assigned to any of the two rows - can easily be dropped. If the row assignment of some departments is fixed in advance, this only helps us because the number of possible row assignments decreases.

\subsection{Departments of the Same Type}

In order to compute an optimal DRFLP layout we enumerate over all row assignments of the departments and solve some IP ${ }_{\mathrm{FR} \text {-DRFLP }}$ in each step. In principle, 
we have to solve the IP $\mathrm{FR}_{\text {-DRFLP }}$ for all distinguishable row assignments (some layouts might be neglected due to further considerations). So restricting the number of distinguishable row assignments is essential. Let $n$ denote the number of departments. In general, there are $\frac{1}{2} \cdot 2^{n}$ distinguishable row assignments because by assumption we can place every department in row 1 or in row 2 and we can fix the row assignment of exactly one department. In realistic instances there appear departments of the same type, see also our test case in Sect. 5.1, i. e., the departments have the same length and the same transport weight $c$ to all other departments. We use this additional information to reduce the number of distinguishable row assignments significantly.

Theorem 2. Let $m$ denote the number of different department types and let $a_{i}$ be the number of departments of type $i \in[m]$. Then there are at most

$$
\left\lceil\frac{1}{2} \prod_{i \in[m]}\left(a_{i}+1\right)\right\rceil
$$

distinguishable row assignments.

Proof. We will prove this result by induction on $m$. Let $m=1$. We only take row assignments into account that contain at least as many departments in row 1 as in row 2. By symmetry, these are all distinguishable row assignments. So we assign $a_{1}, \ldots,\left\lceil\frac{a_{1}}{2}\right\rceil$ departments to row 1 and we obtain $a_{1}-\left\lceil\frac{a_{1}}{2}\right\rceil+1=\left\lceil\frac{a_{1}+1}{2}\right\rceil$ distinguishable row assignments. Let us now assume that the result is true for $m$ and we consider $m+1$ department types.

Case 1: $a_{m+1}$ is odd. The idea of the proof is to assign more departments of type $m+1$ to row 1 than to row 2 . By this method, we take all distinguishable row assignments into account. For $a_{m+1}, \ldots,\left\lceil\frac{a_{m+1}}{2}\right\rceil$ departments of type $m+1$ in row 1 we obtain $\prod_{i=1}^{m}\left(a_{i}+1\right)$ distinguishable row assignments in each subcase. Altogether we obtain

$$
\begin{aligned}
\left(a_{m+1}-\left\lceil\frac{a_{m+1}}{2}\right\rceil+1\right) \prod_{i=1}^{m}\left(a_{i}+1\right) & =\left\lceil\frac{a_{m+1}+1}{2}\right\rceil \prod_{i=1}^{m}\left(a_{i}+1\right) \\
& =\left\lceil\frac{1}{2} \prod_{i=1}^{m+1}\left(a_{i}+1\right)\right\rceil
\end{aligned}
$$

distinguishable row assignments.

Case 2: $a_{m+1}$ is even. We assume that $a_{i}$ is even for all $i \in[m]$, otherwise the proof is analogous to Case 1 . Similar to Case 1 we assign $a_{m+1}, \ldots, \frac{a_{m+1}}{2}+1$ departments of type $m+1$ to row 1 and obtain

$$
\frac{a_{m+1}}{2} \prod_{i=1}^{m}\left(a_{i}+1\right)
$$


distinguishable row assignments. It remains to consider the case with $\frac{a_{m+1}}{2}$ departments of type $m+1$ in row 1 . Then, there are also $\frac{a_{m+1}}{2}$ departments of type $m+1$ in row 2 . Now we use our induction hypothesis to create distinguishable row assignments. Altogether we get

$$
\begin{aligned}
\frac{a_{m+1}}{2} \prod_{i=1}^{m}\left(a_{i}+1\right)+\left\lceil\frac{1}{2} \prod_{i=1}^{m}\left(a_{i}+1\right)\right\rceil & =\left(a_{m+1}+1\right) \frac{1}{2} \prod_{i=1}^{m}\left(a_{i}+1\right)+\frac{1}{2} \\
& =\left\lceil\frac{1}{2} \prod_{i=1}^{m+1}\left(a_{i}+1\right)\right\rceil
\end{aligned}
$$

distinguishable row assignments.

This formula is also correct if all departments have a different type, because then $a_{i}=1$ for all $i \in[m]$ and $m=n$. We illustrate the advantages of Theorem 2 by a realistic example, see [18] and Sect. 5 .

Example 1. We are given $n=21$ departments, where two departments appear four times, three departments twice and seven departments just once. Without reduction, we have to test $2^{20}=1048576$ row assignments. By Theorem 2 we obtain at most $\frac{1}{2} \cdot 5 \cdot 5 \cdot 3 \cdot 3 \cdot 3 \cdot 2^{7}=43200$ distinguishable row assignments.

Apart from reducing the number of row assignments if there are several departments of the same type, we additionally can strengthen our model. Indeed, we can break some symmetries of the arrangement by fixing the order of departments of the same type in the same row. This symmetry breaking is done in such a way that at least one optimal solution is preserved. Let $a_{i_{1}}$ departments of the same type $i, i \in[m]$, be in row 1 . We denote these departments, w.l.o.g., by $1, \ldots, a_{i_{1}}$. Then, we fix the order of these departments by additional constraints, w.l.o.g., we use an ascending order. Since these departments are of the same type, they have the same length and we can add

$$
d_{(n+1) 1} \leq d_{(n+1) 2}+\ell_{1} \leq \ldots \leq d_{(n+1)\left(a_{i_{1}}\right)}+\left(a_{i_{1}}-1\right) \cdot \ell_{1}
$$

to our model. It follows immediately that we can set the ordering variables to

$$
x_{(n+1) k l}= \begin{cases}1, & k, l \in\left[a_{i_{1}}\right], k<l \\ 0, & k, l \in\left[a_{i_{1}}\right], k>l .\end{cases}
$$

Similar equations can be added for department $n+2$. Furthermore, we fix the associated betweenness variables

$$
x_{k u v}= \begin{cases}1, & k, u, v \in\left[a_{i_{1}}\right], k<u<v \\ 0, & k, u, v \in\left[a_{i_{1}}\right], k<v \text { and }(u<k \text { or } u>v) .\end{cases}
$$




\section{Iterative Combination of Optimization and Simulation}

Using an extended version of the algorithm of [12] we are now able to solve the DRFLP. For further details on the software we used as well as on our test environment we refer to Sect. 5. As already mentioned in the introduction, in the mathematical models usually only the transport loads are taken into account. But for a successful production system, which highly depends on the decisions made during the factory planning process, several further key performance indicators play a significant role. To determine these we use simulation. If problems are detected, the optimization model is extended.

To verify the quality of the extended DRFLP model we apply discrete event simulation, see also [7]. As a software tool we use Tecnomatix Plant Simulation [23]. Starting point is the development of a basic simulation model that includes different controls and import functions. These controls are necessary for

- the management of processing sequences and times,

- the implementation of imported processing parameters or production programs and

- an automated generation of the layout specific simulation model after the import of the DRFLP solution.

In addition, the controls allow

- the consideration of different distribution strategies for the material flow,

- adding different products,

- adjusting the processing sequences, i. e., in which order the products have to be processed, and

- adjusting a production program which includes a production schedule and the number of products.

By running the simulation of the processes on and between the departments or machines, which are arranged according to some layout, we generate dynamic and realistic information about the transport processes.

Additionally, we implemented some statistical tools for the evaluation of the respective layouts. Related to the input data for the DRFLP we analyze the total product distances [1], specific product distances and the transport momentum. Furthermore, a first benefit of the simulation is that we can consider additional key performance indicators of the production systems, among them output, throughput times, inventory, capacities, utilization of resources. Apart from this a second benefit is the visualization of processes that simplifies the understanding of complex relations [8].

An analysis of the results including the key performance indicators is then the basis to see needs for improvement. So, if the current layout has to be improved, the DRFLP models are customized by extending or adapting the mathematical models and the interplay between optimization and simulation continues as illustrated in Fig. 4. One big advantage of our iterative layout creation 


\section{Layout Planning Issue}

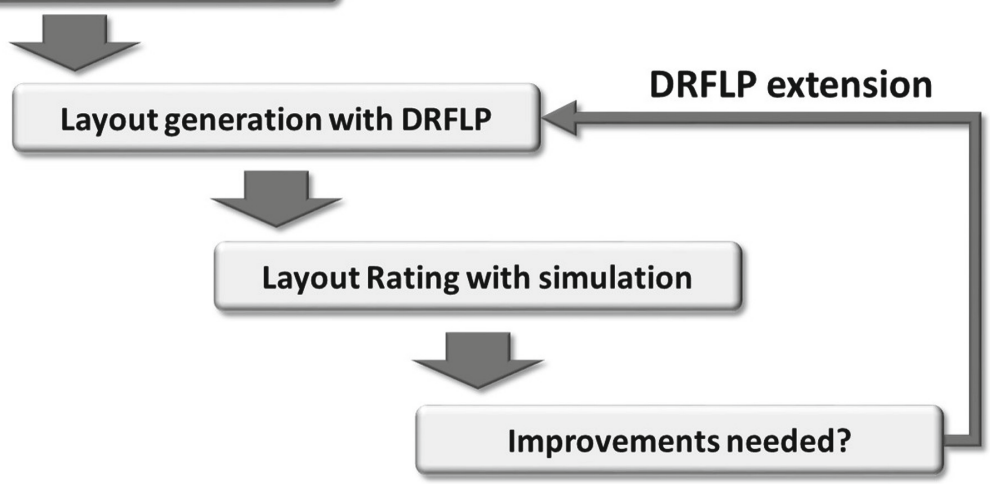

Fig. 4. Extended and evaluated DRFLP-method: The quality of a layout determined via mathematical optimization for some layout planning issue is measured using simulation. If improvements are needed, the mathematical models are extended.

is that afterwards we can nicely compare the found solutions with respect to several indicators. So, the effect of certain decisions becomes clear.

The simulation might show that the distances between certain departments are too large for a smooth production. Then, we can restrict these distances in the model. Furthermore, the simulation gives some information about the size of the storage and buffer areas needed during production. If more or less space is needed, the sizes of the departments have to be adapted in the next step. Additionally, in the mathematical model we always assume that the transport weights between each pair of departments is known in advance. If there are several departments of the same type we assume that the intermediate products are evenly distributed among the departments of the same type. With the help of simulation we can check whether this is a good distribution strategy by testing several ones and if necessary we can adapt our model.

Many simulation models are generated in 2D. This kind of department representation is quite abstract and impedes the intuitive understanding of the layout and the production process. Especially for layouts with an increased number of departments, the transparency of a DRFLP solution with the 2D simulation model is limited. The integration of 3D models provides a better overview for the planner as illustrated in Fig. 5. Especially the product flow can be demonstrated very quickly. Using a 3D simulation model on basis of the optimized layout can simplify the virtual validation of the planned production area and the detection of bottlenecks. All in all, simulation allows to control whether it will be possible to achieve the desired output of the production system afterwards in real production. 


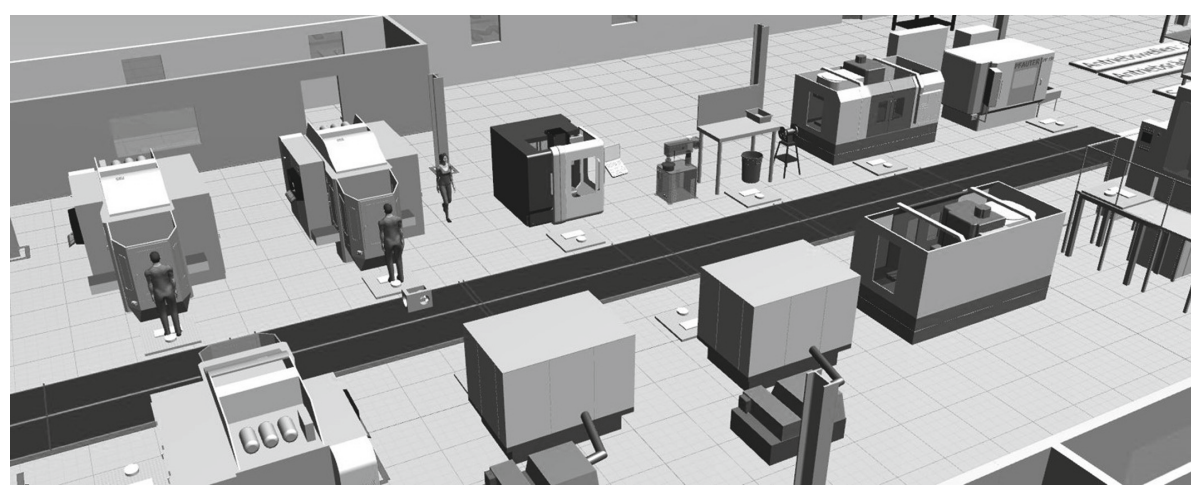

Fig. 5. Illustration of a 3-dimensional simulation including the workers where the departments are arranged on both sides of a common path

\section{Computational Experiments}

In this section, we present our computational results. All experiments were conducted on an INTEL-Core-I7-4770 $(4 \times 3400 \mathrm{MHz}, 8 \mathrm{MB}$ Cache $)$ with 32 GB RAM in single processor mode using openSUSE Linux 42.1. We used CPLEX 12.7.0 [15]. As mentioned above all simulations were done with Tecnomatrix Plant Simulation [23].

\subsection{Test Case and Computational Results}

For testing our new solutions of the extended DRFLP models in the simulation we use a well-known application example [18]. It represents a real gearbox production and includes 21 departments (with 12 types) and eight different products which are combined in an assembly department to an end product. This example provides a solid data basis for the layout planning problem and the simulation model. All necessary information like the transport matrix, processing sequences, processing times, set up times and production rates are given in [18]. For the convenience of the reader we present them here, where we only specify the transport amount between the single types. We have $m=12$ types with multiplicities $a_{1}=a_{2}=a_{4}=a_{8}=a_{10}=a_{11}=a_{12}=1, a_{5}=a_{6}=a_{9}=2$, $a_{3}=a_{7}=4$ and so $n=21$ departments. The lengths (given in meter) are $\ell_{a_{1}}=4, \ell_{a_{2}}=3.4, \ell_{a_{3}}=4.6, \ell_{a_{4}}=4, \ell_{a_{5}}=4.7, \ell_{a_{6}}=3.3, \ell_{a_{7}}=4.5, \ell_{a_{8}}=$ $2.3, \ell_{a_{9}}=3.8, \ell_{a_{10}}=5.2, \ell_{a_{11}}=4, \ell_{a_{12}}=4$ and the transport weights $c_{i j}=c_{j i}$ between types $i, j$ are given via 


$$
C=\left(\begin{array}{cccccccccccc}
0 & 240 & 204 & 0 & 0 & 570 & 0 & 0 & 120 & 0 & 0 & 0 \\
240 & 0 & 240 & 0 & 0 & 0 & 0 & 0 & 0 & 0 & 0 & 0 \\
204 & 240 & 0 & 60 & 60 & 144 & 0 & 0 & 180 & 0 & 0 & 0 \\
0 & 0 & 60 & 0 & 60 & 0 & 0 & 0 & 0 & 0 & 0 & 0 \\
0 & 0 & 60 & 60 & 0 & 0 & 0 & 120 & 0 & 0 & 0 & 0 \\
570 & 0 & 144 & 0 & 0 & 0 & 570 & 0 & 0 & 0 & 144 & 0 \\
0 & 0 & 0 & 0 & 0 & 570 & 0 & 570 & 0 & 0 & 0 & 0 \\
0 & 0 & 0 & 0 & 120 & 0 & 570 & 0 & 0 & 0 & 690 & 0 \\
120 & 0 & 180 & 0 & 0 & 0 & 0 & 0 & 0 & 60 & 240 & 0 \\
0 & 0 & 0 & 0 & 0 & 0 & 0 & 0 & 60 & 0 & 60 & 0 \\
0 & 0 & 0 & 0 & 0 & 144 & 0 & 690 & 240 & 60 & 0 & 720 \\
0 & 0 & 0 & 0 & 0 & 0 & 0 & 0 & 0 & 0 & 720 & 0
\end{array}\right) .
$$

In optimization, we assume that the transports are equally divided among the departments of the same type. In simulation also other strategies can be tested, but we only implemented a division of the transports according to a discrete uniform distribution. A 3D illustration of some layout for this instance, where the incoming and the shipping warehouse are arranged at the borders, is given in Fig. 6.

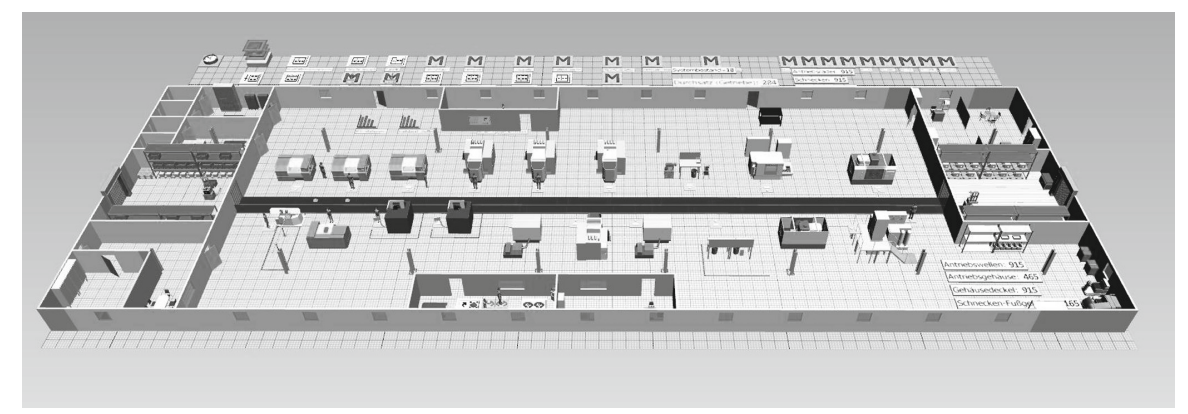

Fig. 6. 3D illustration of a DRFLP layout with 21 departments [18]. This simulation was derived using mathematical optimization. The incoming and the shipping warehouse are arranged at the left and the right border, respectively.

This example has already been taken into account in Example 1. Indeed, it contains several departments of the same type. In our computational tests we used Theorem 2 and the additional symmetry breaking constraints for departments of the same type in the same row. In Fig. 7 we show the development of the running times of our optimization approach if we enlarge the number of the departments. The instance of [18] contains departments of 12 different types. In the tests we start with 12 departments and successively add one department in each step. So, department type 3 appears twice when we consider 13 departments and three times when we consider 14 departments and so on. Figure 7 shows that the original instance with all departments could be solved in less 
than $14 \mathrm{~h}$, although it contains 21 departments and so five departments more than the largest DRFLP instance solved to optimality in the literature before.

In the simulation we tested the following five different solutions:

1. A solution determined according to criteria usually used in factory planning where apart from the transport weights one had a special look at the linearity of the flows. The heuristic of Schmigalla $[16,20]$ was applied and afterwards the solution was improved by hand. Incoming and shipping warehouse were arranged at the border.

2. A solution determined according to criteria usually used in factory planning where all departments of a type were interpreted as one big block and then these blocks were arranged. The number of blocks that had to be arranged is smaller than the total number of departments. So it was easier to build this layout by hand. Incoming and shipping warehouse were arranged at the border.

3. We used our mathematical DRFLP model for deriving a solution but as it is often done in practice incoming and shipping warehouse were arranged at the border (see end of Sect. 2). A 3D visualization of this warehouse can be found in Fig. 6.

4. We used our mathematical DRFLP model for deriving a solution and the incoming and shipping warehouse were arranged at the border. Additionally, all departments of the same type were interpreted as one big block and then these 12 blocks were arranged.

5. Solution derived using our mathematical DRFLP model with arbitrary position of all departments as well as of the incoming and the shipping warehouse (in our model these are departments, too). This approach was also used for deriving the results in Fig. 7.

In all five simulations we manufactured 36000 end products and determined afterwards the average distance of each single product and the total distance traveled. The results can be found in Table 1 . The end product, which is obtained by combining all eight products in an assembly department, is denoted as product 9. The second column in Table 1 shows the number of transports ("Trans") needed for each product $1, \ldots, 9$, and the next ten columns show the distances for the five simulation variants where the left column ("Single") for each type contains the information on the average transport distance of each single product and the right column the total distance ("Total") traveled for all products of the same type.

The results show that the use of our optimization model allows to improve the solution significantly in comparison to the solution determined by hand, especially if we do not restrict the position of the warehouses (Layout 5). Comparing Layout 1 and Layout 3, where the two warehouses have been fixed to the border, the solution obtained by optimization is better than the solution obtained by applying the heuristic of Schmigalla followed by some improvement steps by the factory planners. But even the optimized solution with blocks and fixed border, illustrated in Layout 4, is better than the solutions determined 


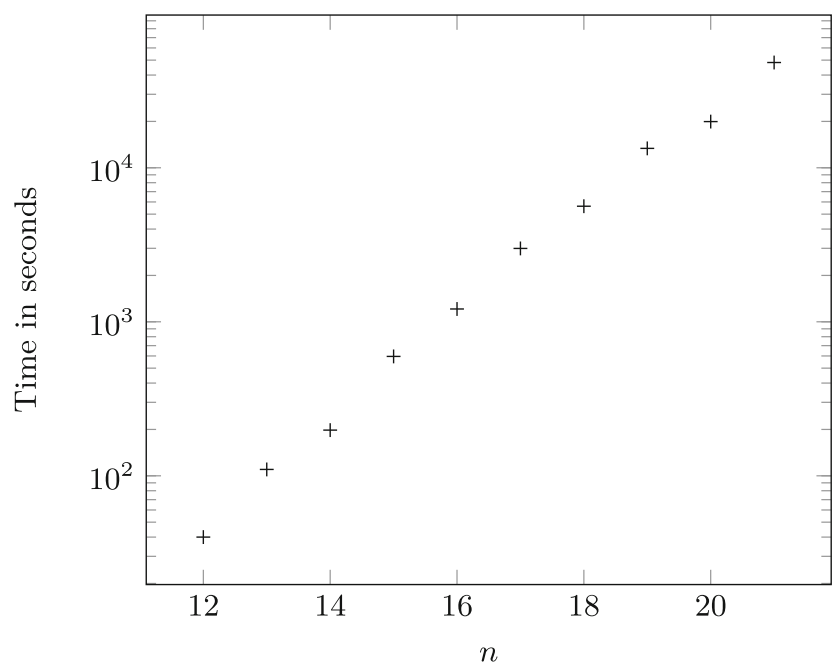

Fig. 7. Running times in seconds for variations of some realistic instance from [18]. We start with 12 different department types and 12 departments and we add departments successively according to the following order of the number of departments of each of the 12 types: 11412241211 . The largest instance contains 21 departments.

Table 1. Visualization of the results of the five simulations for our test case. In this production all eight products are combined to an end product, denoted as product 9, see column "P". The entry "Total" in the first column refers to the total distance traveled in each of the five layouts. Note that using our approach from mathematical optimization with arbitrary department positions reduces the total distances significantly in comparison with the other four variants.

\begin{tabular}{|c|c|c|c|c|c|c|c|c|c|c|c|}
\hline \multirow[t]{2}{*}{$\mathrm{P}$} & \multirow[t]{2}{*}{ Trans } & \multicolumn{2}{|c|}{ Layout 1} & \multicolumn{2}{|c|}{ Layout 2} & \multicolumn{2}{|c|}{ Layout 3} & \multicolumn{2}{|c|}{ Layout 4} & \multicolumn{2}{|c|}{ Layout 5} \\
\hline & & Single & Total & Single & Total & Single & Total & Single & Total & Single & Total \\
\hline 1 & 480 & 37.90 & 18192.0 & 38.70 & 18576.0 & 28.70 & 13776.00 & 29.15 & 13992.00 & 16.31 & 7828.8 \\
\hline 2 & 90 & 37.90 & 3411.0 & 38.70 & 3483.0 & 28.70 & 2583.00 & 29.15 & 2623.50 & 16.31 & 1467.9 \\
\hline 3 & 144 & 38.50 & 5544.0 & 44.85 & 6458.4 & 29.32 & 4222.08 & 35.91 & 5171.04 & 30.75 & 4428.0 \\
\hline 4 & 60 & 37.90 & 2274.0 & 37.60 & 2256.0 & 28.70 & 1722.00 & 29.15 & 1749.00 & 39.60 & 2376.0 \\
\hline 5 & 30 & 65.60 & 1968.0 & 71.90 & 2157.0 & 69.20 & 2076.00 & 70.40 & 2112.00 & 66.51 & 1995.3 \\
\hline 6 & 60 & 55.96 & 3357.6 & 49.03 & 2941.8 & 55.66 & 3339.60 & 54.18 & 3250.80 & 54.27 & 3256.2 \\
\hline 7 & 30 & 66.12 & 1983.6 & 71.89 & 2156.7 & 66.42 & 1992.60 & 70.90 & 2127.00 & 66.51 & 1995.3 \\
\hline 8 & 120 & 37.90 & 4548.0 & 37.60 & 4512.0 & 28.70 & 3444.00 & 29.15 & 3498.00 & 39.00 & 4680.0 \\
\hline 9 & 720 & 2.00 & 1440.0 & 2.00 & 1440.0 & 10.80 & 7776.00 & 11.20 & 8064.00 & 0.00 & 0.0 \\
\hline Total & & & 42718.2 & & 43980.9 & & 40931.28 & & 42587.34 & & 28027.5 \\
\hline
\end{tabular}

by hand, illustrated in Layout 2. A visualization of all layouts can be found in Fig. 8, where blocks consisting of departments of the same type are highlighted with lines in bold type. 
1)

\begin{tabular}{|c|c|c|c|c|c|c|c|c|c|c|}
\hline$M_{1}$ & $M_{3}^{1}$ & $M_{3}^{2}$ & $M_{3}^{3}$ & $M_{7}^{1}$ & $M_{7}^{2}$ & $M_{8}$ & $M_{10}$ & $M_{5}^{1}$ & $M_{4}$ \\
\hline \hline$M_{2}$ & $M_{3}^{4}$ & $M_{6}^{1}$ & $M_{6}^{2}$ & $M_{7}^{3}$ & $M_{7}^{4}$ & $M_{9}^{1}$ & $M_{9}^{2}$ & $M_{5}^{2}$ & $M_{11}$ \\
\hline
\end{tabular}

2)

\begin{tabular}{|c|c|c|c|c|c|c|c|c|c|c|}
\hline \multirow{2}{*}{$M_{1}$} & $M_{3}^{1}$ & \multicolumn{2}{|c|}{$M_{3}^{2}$} & $M_{3}^{3}$ & $M_{3}^{4}$ & $M_{4}$ & $M_{5}^{1}$ & $M_{5}^{2}$ & $M_{9}^{1}$ & $M_{9}^{2}$ \\
\cline { 2 - 10 } & $M_{2}$ & $M_{6}^{1}$ & $M_{6}^{2}$ & $M_{7}^{1}$ & $M_{7}^{2}$ & $M_{7}^{3}$ & $M_{7}^{4}$ & $M_{8}$ & $M_{10}$ & $M_{11}$ \\
\hline
\end{tabular}

3)

\begin{tabular}{|c|c|c|c|c|c|c|c|c|c|c|}
\hline \multirow{2}{*}{$M_{1}$} & $M_{3}^{1}$ & $M_{3}^{2}$ & \multicolumn{2}{|c|}{$M_{3}^{3}$} & $M_{7}^{1}$ & $M_{7}^{2}$ & $M_{7}^{3}$ & $M_{8}$ & $M_{10}$ & $M_{5}^{1}$ \\
\cline { 2 - 9 } & $M_{2}$ & $M_{3}^{4}$ & $M_{6}^{1}$ & $M_{6}^{2}$ & $M_{9}^{1}$ & $M_{7}^{4}$ & $M_{9}^{2}$ & $M_{11}$ & $M_{5}^{2}$ & $M_{4}$ \\
\hline
\end{tabular}

4)

\begin{tabular}{|c|c|c|c|c|c|c|c|c|c|c|}
\hline$M_{1}$ & $M_{3}^{1}$ & $M_{3}^{2}$ & $M_{3}^{3}$ & $M_{3}^{4}$ & $M_{9}^{1}$ & $M_{9}^{2}$ \\
\cline { 2 - 8 } & $M_{2}$ & $M_{6}^{1}$ & $M_{6}^{2}$ & $M_{7}^{1}$ & $M_{7}^{2}$ & $M_{7}^{3}$ & $M_{7}^{4}$ & $M_{8}$ & $M_{5}^{1}$ & $M_{5}^{2}$ \\
\hline
\end{tabular}

5)

\begin{tabular}{|c|c|c|c|c|c|c|c|c|c|c|c|}
\hline$M_{10}$ & $M_{9}^{1}$ & $M_{12}$ & $M_{8}$ & $M_{7}^{1}$ & $M_{7}^{2}$ & $M_{6}^{1}$ & $M_{3}^{1}$ & $M_{3}^{2}$ & $M_{3}^{3}$ & $M_{5}^{2}$ \\
\hline & $M_{9}^{2}$ & $M_{11}$ & $M_{7}^{3}$ & $M_{7}^{4}$ & $M_{6}^{2}$ & $M_{1}$ & $M_{2}$ & $M_{3}^{4}$ & $M_{5}^{2}$ & $M_{4}$ \\
\hline
\end{tabular}

Fig. 8. Visualization of the five layouts tested in the simulations. These five layouts were constructed by the five variants stated above, i. e., Layout 1 and Layout 2 were derived according to general approaches used in factory planning and Layout 3, Layout 4 and Layout 5 were derived using the optimization model. The last layout shows an optimal solution if the position of none of the departments is restricted.

In the simulation the different products that are produced are considered separate, but in the mathematical models the transport weights are based on aggregated information for the transports of all products. Studying the results and indicators provided by the simulation of the five layout variants we realized that the time needed for the transport processes of different products can vary significantly. Next we show how to overcome this situation by adapting our optimization model.

\subsection{Manufacturing Different Products}

Let us assume that we manufacture an amount of different products. Let $P$ denote the set of products and $h_{p}$ be the desired number of product $p \in P$. Every product has its own transport matrix $C^{p}$. The $i j$-th entry of the matrix $C^{p}$ denotes the transport weights between department $i$ and $j$ for producing product $p$. The transport weight matrix $C$ that we used before is built on the sum of the transport matrices times the associated desired number of products, i. e., $C=\sum_{p \in P} h_{p} \cdot C^{p}$. Our aim is now to investigate the influence of single products to the whole production.

Definition 2. For a product $p \in P$ we define the transport distance as

$$
\sum_{\substack{i, j \in[n] \\ i<j}} c_{i j}^{p} d_{i j}
$$

where $c_{i j}^{p}$ is the ij-th entry of the matrix $C^{p}$. 
The simulation showed that these transport distances might be rather high in an optimal layout if the number of products of this type is small in comparison to the others. But high transport distances can increase the cycle time. So, for a smooth production we want to bound the transport distances associated to single products. Therefore, we present two possibilities: At first we can restrict this by an upper bound on the transport distance.

A second way is to set up a desired distance $d_{p} \in \mathbb{R}_{+}$for the transport distance for each single product $p \in P$. Of course, the desired transport distance depends on the amount of products $h_{p}$ for $p \in P$. If this value is exceeded, we want to penalize this with a quadratic function $\tilde{f}$ which is later approximated by some piecewise linear function. Let $a \geq d_{p}$ be the highest possible transport distance of product $p \in P$. Then we set $\tilde{f}:[0, a] \rightarrow[0, \tilde{f}(a)]$ such that

$$
\tilde{f}(x)= \begin{cases}d_{p}-x, & x<d_{p} \\ 0, & x=d_{p} \\ \left(x-d_{p}\right)^{2}, & x>d_{p} .\end{cases}
$$

To avoid a non-linear objective function, we approximate $\tilde{f}$ on the interval $\left[d_{p}, a\right]$ with a piecewise linear, continuous, convex function $f$. Therefore, we use linear interpolation [6]. Let a product $p \in P$ and points $h_{1}, \ldots, h_{m}$ be given with $m \geq 1$ and $h_{i} \geq d_{p}$ sorted in ascending order for $i \in[m]$. Then we compute a linear approximation of $\tilde{f}$ between the points $\left(h_{i},\left(h_{i}-d_{p}\right)^{2}\right)$ and $\left(h_{i+1},\left(h_{i+1}-d_{p}\right)^{2}\right)$ for $i=1, \ldots, m-1$. The resulting function $f$ is piecewise linear and can be written as $f(x)=\max _{i=1, \ldots, m}\left(a_{i}\right)^{T} x+b_{i}$ for $a_{i}, b_{i} \in \mathbb{R}$ and $i \in[m]$. We add the following term to the objective function of our model (1)-(13)

$$
h_{p} \cdot f\left(\sum_{i, j \in[n], i<j} c_{i j}^{p} d_{i j}\right) .
$$

This term can be linearized by replacing (15) with

$$
h_{p} \cdot t
$$

in the objective function and adding the constraints

$$
a_{i}^{T} x+b_{i} \leq t, \quad i \in[m] .
$$

We may set up such a penalty function for every product $p \in P$.

\section{Conclusion and Future Work}

In this paper we presented a new approach that allows combining mathematical optimization and simulation in facility layout planning. We concentrated on the Double Row Facility Layout Problem. In contrast to the literature we showed 
how the existing models can be extended in order to cover several aspects important in practice. To evaluate the facility layout we used simulation to determine further key performance indicators. If problems occur, the mathematical models have to be adapted appropriately. For the first time we were able to solve an instance with 21 departments to optimality in reasonable time. We compared our mathematical model with classic methods from factory planning and we could reduce the total transport distance significantly, especially by using arbitrary positions for the warehouses.

It remains for future work to include more aspects in the mathematical models. One important topic is the treatment of asymmetric transport weights in combination with input and output positions of the departments that might not lie in the center of the department. Furthermore, due to safety restrictions or quality requirements certain clearance conditions between departments have to be satisfied. From the mathematical point of view it is interesting to further study the polyhedral structure of the associated models as well as to develop new (mixed-) integer programming models that combine the assignment of the department to the rows as well as the positioning of the departments in each row. The hope would be that intelligent branching orders can reduce the overall running time. Apart from the Double Row Facility Layout Problem it seems worth to consider more complex path structures in the shape of a $\mathrm{T}$ or an $\mathrm{X}$ or along some closed path.

A further important goal is the inclusion of robustness aspects because the facility layout decision has an impact for several years, but the production program, which is the basis for the transport weights, might change. Apart from an extension of the mathematical models, simulation allows testing different scenarios for future production programs [5] easily. Using simulation, different layout variants can be evaluated with regard to changing production requirements [10]. So, a flexible and adaptable production layout can be identified.

Acknowledgement. This work was supported by the Simulation Science Center Clausthal-Göttingen.

\section{References}

1. Altinkilinc, M.: Simulation-based layout planning of a production plant. In: Proceedings of the 36th Conference on Winter simulation, pp. 1079-1084 (2004)

2. Amaral, A.R.: A new lower bound for the single row facility layout problem. Discrete Appl. Math. 157(1), 183-190 (2009)

3. Amaral, A.R.: Optimal solutions for the double row layout problem. Optim. Lett. 7(2), 407-413 (2013)

4. Anjos, M.F., Vieira, M.V.: Mathematical optimization approaches for facility layout problems: the state-of-the-art and future research directions. Eur. J. Oper. Res. 261(1), 1-16 (2017)

5. Arnhold, D.: Digitale Produktionsprozessplanung variantenreicher Produkte unter Berücksichtigung von intervallbasierten Eingangsdaten. Shaker (2013)

6. Blu, T., Thévenaz, P., Unser, M.: Linear interpolation revitalized. IEEE Trans. Image Process. 13(5), 710-719 (2004) 
7. Bracht, U., Fischer, A., Krüger, T.: Mathematische Anordnungsoptimierung und Simulation - ein kombinierter Ansatz zur Fabriklayoutplanung. Werkstattstechnik online 107(4), 200-207 (2017)

8. Bracht, U., Geckler, D., Wenzel, S.: Digitale Fabrik: Methoden und Praxisbeispiele. Springer, Deutschland (2011). https://doi.org/10.1007/978-3-662-55783-9

9. Chung, J., Tanchoco, J.: The double row layout problem. Int. J. Prod. Res. 48(3), 709-727 (2010)

10. Dombrowski, U., Ernst, S.: Scenario-based simulation approach for layout planning. Procedia CIRP 12, 354-359 (2013)

11. Drira, A., Pierreval, H., Hajri-Gabouj, S.: Facility layout problems: a survey. Ann. Rev. Control 31(2), 255-267 (2007)

12. Fischer, A., Fischer, F., Hungerländer, P.: New exact approaches to row layout problems. Technical report 2015-11, Preprint-Reihe, Institut für Numerische und Angewandte Mathematik, Georg-August Universität Göttingen (2015)

13. Hungerländer, P., Rendl, F.: A computational study and survey of methods for the single-row facility layout problem. Comput. Optim. Appl. 55(1), 1-20 (2013)

14. Hungerländer, P., Rendl, F.: Semidefinite relaxations of ordering problems. Math. Program. 140(1), 77-97 (2013)

15. IBM ILOG CPLEX Optimization Studio 12.7 (2017)

16. Kettner, H., Schmidt, J., Greim, H.-R.: Leitfaden der systematischen Fabrikplanung. Hanser München (1984)

17. Murray, C.C., Smith, A.E., Zhang, Z.: An efficient local search heuristic for the double row layout problem with asymmetric material flow. Int. J. Prod. Res. 51(20), 6129-6139 (2013)

18. Prêt, U.: Komplexes Fallbeispiel Teilefertigungs- und Montageprojekt "Schneckengetriebeproduktion" (2017). http://www.uwe-pret.de/getriebe.pdf. Accessed 06 Apr 2018

19. Rooks, T.: Rechnergestützte Simulationsmodellgenerierung zur dynamischen Absicherung der Montagelogistikplanung bei der Fahrzeugneutypplanung im Rahmen der digitalen Fabrik. Shaker (2009)

20. Schmigalla, H.: Fabrikplanung: Begriffe und Zusammenhänge. Hanser Verlag (1995)

21. Scholz, D., Petrick, A., Domschke, W.: STaTS: a slicing tree and tabu search based heuristic for the unequal area facility layout problem. Eur. J. Oper. Res. 197(1), 166-178 (2009)

22. Simmons, D.M.: One-dimensional space allocation: an ordering algorithm. Oper. Res. 17(5), 812-826 (1969)

23. Tecnomatrix Plant Simulation. Siemens PLM Software (2017)

24. Zhang, Z., Murray, C.C.: A corrected formulation for the double row layout problem. Int. J. Prod. Res. 50(15), 4220-4223 (2012)

25. Zuo, X., Murray, C.C., Smith, A.E.: Solving an extended double row layout problem using multiobjective tabu search and linear programming. IEEE Trans. Autom. Sci. Eng. 11(4), 1122-1132 (2014) 


\section{Decorous combinatorial lower bounds for row layout problems}

Authors: Mirko Dahlbeck, Anja Fischer, Frank Fischer

published in European Journal of Operational Research, 2020, available at https://doi.org/ $10.1016 /$ j.ejor.2020.04.010. 
Production, Manufacturing, Transportation and Logistics

\title{
Decorous combinatorial lower bounds for row layout problems
}

\author{
Mirko Dahlbeck $^{\mathrm{a}, \mathrm{b}}$, Anja Fischer ${ }^{\mathrm{a}, *}$, Frank Fischer ${ }^{\mathrm{c}}$ \\ ${ }^{a}$ Faculty of Business and Economics, TU Dortmund University, Vogelpothsweg 87, Dortmund D-44227, Germany \\ ${ }^{\mathrm{b}}$ Institute for Numerical and Applied Mathematics, Georg-August-Universität Göttingen, Lotzestr. 16-18, D-37083 Göttingen, Germany \\ ${ }^{\mathrm{C}}$ Institute of Computer Science, Johannes Gutenberg University Mainz, Staudingerweg 9, Mainz D-55128, Germany
}

\section{A R T I C L E I N F O}

\section{Article history:}

Received 4 June 2019

Accepted 4 April 2020

Available online 18 April 2020

\section{Keywords:}

Facilities planning and design

Integer programming

Row layout problem

Lower bounds

\begin{abstract}
A B S T R A C T
In this paper we consider the Double-Row Facility Layout Problem (DRFLP). Given a set of departments and pairwise transport weights between them the DRFLP asks for a non-overlapping arrangement of the departments along both sides of a common path such that the weighted sum of the center-to-center distances between the departments is minimized. Despite its broad applicability in factory planning, only small instances can be solved to optimality in reasonable time. Apart from this even deriving good lower bounds using existing integer programming formulations and branch-and-cut methods is a challenging problem. We focus here on deriving combinatorial lower bounds which can be computed very fast. These bounds generalize the star inequalities of the Minimum Linear Arrangement Problem. Furthermore we exploit a connection of the DRFLP to some parallel identical machine scheduling problem. Our lower bounds can be further improved by combining them with a new distance-based mixed-integer linear programming model, which is not a formulation for the DRFLP, but can be solved close to optimality quickly. We compare the new lower bounds to some heuristically determined upper bounds on mediumsized and large DRFLP instances. Special consideration is given to the case when all departments have the same length. Furthermore we show that the lower bounds that we derive using adapted variants of our approaches for the Parallel Row Ordering Problem, a DRFLP variant where the row assignment of the departments is given in advance and spaces between neighboring departments are not allowed, are even better with respect to the gaps.
\end{abstract}

(c) 2020 Elsevier B.V. All rights reserved.

\section{Introduction}

In this paper we consider special facility layout problems which have several applications, in particular in factory planning. For recent surveys on facility layout problems in general we refer, e. g., to Anjos and Vieira (2017), Drira, Pierrval, and Hajri-Gabouj (2007), Ahmadi, Pishvaee, and Jokar (2017), Hosseini-Nasab, Fereidouni, Fatemi Ghomi, and Fakhrzad (2018), Keller and Buscher (2015), Anjos and Liers (2012). An instance of the Multi-Row Facility Layout Problem (MRFLP) consists of $n$ one-dimensional departments $[n]:=\{1, \ldots, n\}$ with given positive lengths $\ell_{i}, i \in[n]$, pairwise non-negative weights $w_{i j}=w_{j i} \in \mathbb{R}_{+}, \quad i, j \in[n], \quad i<j$, which usually correspond to the amount of transport between the departments, and a set $\mathcal{R}:=[m], m \in \mathbb{N}$, of rows. The objective is to find an assignment $r:[n] \rightarrow \mathcal{R}$ of departments to rows and horizontal positions for the centers of the departments such that departments in the same row do not overlap and such that the total

\footnotetext{
* Corresponding author.

E-mail addresses: m.dahlbeck@math.uni-goettingen.de (M. Dahlbeck), anja2.fischer@tu-dortmund.de (A. Fischer), frank.fischer@uni-mainz.de (F. Fischer).
}

weighted sum of the center-to-center distances, measured in horizontal direction, between all pairs of departments is minimized. So we look for a vector $q \in \mathbb{R}^{n}$ of positions and a vector $r \in \mathcal{R}^{n}$ of the assignment of the departments to the $m$ rows solving the following optimization problem

$$
\begin{aligned}
\min _{r \in \mathcal{R}^{n}, q \in \mathbb{R}^{n}} & \sum_{\substack{i, j \in[n] \\
i<j}} w_{i j}\left|q_{i}-q_{j}\right| \\
\text { s.t. } & \left|q_{i}-q_{j}\right| \geq \frac{\ell_{i}+\ell_{j}}{2}, \quad i, j \in[n], i<j, \text { if } r_{i}=r_{j} .
\end{aligned}
$$

The special case of the MRFLP with $m=2$ is called Double-Row Facility Layout Problem (DRFLP), see, e. g., Amaral (2019), Fischer, Fischer, and Hungerländer (2019). The DRFLP is in particular relevant for real-world applications because material handling and thus real factory layouts most often reduce to double-row layouts and variants thereof. Indeed, it was noted by several authors that in factory planning the costs of the production are highly influenced by the layout of the departments, see, e. g., Tompkins, White, Bozer, and Tanchoco (2010), Hassan (1994), Bracht, Dahlbeck, Fischer, and Krüger (2018). Besides its applications in factory planning, the DRFLP can be used to find an arrangement of rooms in 


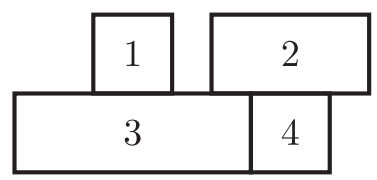

Fig. 1. An optimal double-row layout for an instance with $\ell_{i}=i, i=1,2,3, \ell_{4}=1$, and non-zero weights $w_{13}=w_{23}=1, w_{24}=2$. Note that there is some free space between the neighboring departments 1 and 2 in row 1 .

hospitals (Butler, Karwan, Sweigart, \& Reeves, 1992; Elshafei, 1977; Hahn \& Krarup, 2001), office centers or schools (Amaral, 2012). Further applications include setting books on a shelf (Amaral, 2013a), balancing hydraulic turbine runners and optimal data memory layout generation for digital signal processors (Anjos \& Vieira, 2017). We refer to Hungerländer and Anjos (2015) for further applications.

In the following, we denote the center-to-center distance between two departments $i, j \in[n], i<j$, by $d_{i j}=d_{j i}$. To illustrate the structure of double-row layouts and the corresponding distance calculation we give an example. Note that in an optimal doublerow layout there might be free space between two neighboring departments in the same row.

Example 1. We consider four departments with lengths $\ell_{i}=i, i=$ $1,2,3, \ell_{4}=1$ and pairwise non-zero weights $w_{13}=w_{23}=1, w_{24}=$ 2. Fig 1 illustrates an optimal double-row layout with solution value $1 \cdot 2=2$.

\subsection{Literature Review}

A well-studied special case of the MRFLP is the Single-Row Facility Layout Problem (SRFLP) with $m=1$, i. e., all departments are assigned to the same row. Exact optimization approaches for the SRFLP are based on relaxations of integer linear programming (ILP) and semidefinite programming (SDP) formulations, see, e. g., Amaral (2006), Amaral (2008), Amaral (2009), Amaral and Letchford (2013) as well as Anjos, Kennings, and Vannelli (2005), Anjos and Vannelli (2008), Anjos and Yen (2009), Hungerländer and Rendl (2013b), Hungerländer and Rendl (2013a). The strongest ILP approach is a linear programming based cutting plane algorithm using betweenness variables that can solve instances with up to 35 departments within a few hours (Amaral, 2009). The strongest SDP approach to date, using products of ordering variables, is even stronger and allows to solve instances with up to 40 departments in less than 140 minutes, to solve one instance with 42 departments in about one hour and to obtain small gaps for instances with up to 81 departments within 60 hours (Hungerländer \& Rendl, 2013a; 2013b). Additionally, several heuristic algorithms have been suggested that are able to obtain good layouts (Cravo \& Amaral, 2019; Datta, Amaral, \& Figueira, 2011; Kothari \& Ghosh, 2013; 2014; Palubeckis, 2015a; 2017). One of the leading heuristics was presented in Palubeckis (2017), where a multi-start simulated annealing heuristic obtains the best known solutions or small gaps for instances from the literature with 60 to 80 departments. Furthermore this heuristic is tested on instances with up to 1000 departments. A recent heuristic in Cravo and Amaral (2019) could improve results of Palubeckis (2017) for instances with $200 \leq n \leq 500$ departments and obtained solutions with objective value close to the best known values presented in Palubeckis (2017) for instances with up to 1000 departments. A recent survey on the SRFLP is given in Keller and Buscher (2015).

In contrast to the SRFLP, the DRFLP has received much less attention in the literature. From a practical point of view the DRFLP seems much harder than the SRFLP. The ILP-based approach in Chung and Tanchoco (2010) (see also the corresponding corrections by Zhang \& Murray, 2012) can handle instances with up to 10 departments whereas the exact ILP approach of Amaral (2013a) can solve instances with up to 12 departments to optimality. The latter model was improved in Secchin and Amaral (2019) such that one is able to solve a DRFLP instance with 15 departments in at most 11 hours. Recently, Fischer et al. (2019) presented an algorithm which can solve DRFLP instances with up to 16 departments in less than 12 hours.

To the best of our knowledge there has not been research on computing non-trivial lower bounds for the DRFLP. The enumeration scheme of Fischer et al. (2019) cannot be used to obtain lower bounds for larger instances because one would have to calculate a lower bound for each of the exponentially many row assignments, which is out of scope for $n$ large. The mixed-integer programming models, see, e. g., Amaral (2013a), Secchin and Amaral (2019), are based on big- $M$-type constraints to couple continuous position variables with binary ordering variables. Thus, their linear relaxations are rather weak. So using them in a branch-andcut approach leads to weak lower bounds and so to large gaps for medium-sized and large DRFLP instances, even after a longer time limit because the root node gaps are hardly improved. For detailed computational results we refer to Section 4.

For the DRFLP only a limited number of problem-specific heuristics were presented in the literature (Chung \& Tanchoco, 2010; Guan, Lin, Feng, \& Ruan, 2020; Gülșen, Murray, \& Smith, 2019; Murray, Smith, \& Zhang, 2013; Wang, Zuo, Liu, Zhao, \& Li, 2015; Zuo, Gao, Zhou, Yang, \& Zhao, 2019; Zuo, Murray, \& Smith, 2014; 2016), partially handling some extended versions that include, e. g., certain clearance conditions between departments in the same row or dynamic aspects. But without the knowledge of good lower bounds it is hard to evaluate the quality of these heuristics.

Because the MRFLP and the DRFLP are very challenging problems in practice, several special cases have been studied in the literature. There are two main classes of simplifications. First one reduces the freedom in the arrangement of the departments. In the Space-Free MRFLP and DRFLP (SF-MRFLP and SF-DRFLP) one restricts to a common left border of the rows and spaces between neighboring departments in the same row are not allowed. For the SF-DRFLP, which is also known as Corridor Allocation Problem, heuristics and exact approaches were presented in Kothari and Ghosh (2012), Ahonen, de Alvarenga, and Amaral (2014) and Amaral (2012), Hungerländer and Anjos (2012), Fischer et al. (2019), Fischer, Fischer, and Hungerländer (2015). Similar to the general DRFLP the enumeration approach of Fischer et al. (2019) can solve space-free double-row instances with up to 16 departments in less than 12 hours. If one additionally fixes the row assignment of each of the departments we derive the $k$-Parallel Row Ordering Problem (kPROP) (in our notation $k$ equals the number of rows $m$ ) and the Parallel Row Ordering Problem (PROP) for $m=2$ (Amaral, 2013b; Hungerländer, 2014a; Maadi, Javidnia, \& Jamshidi, 2017; Yang, Cheng, Smith, \& Amaral, 2020). The best approach for these problems in Fischer et al. (2019) is the basis for the enumerative approach for the DRFLP. Instances with up to 25 departments are solved to optimality. For larger $n$ one can derive lower bounds via the SDP approach in Hungerländer (2014a).

The second area of simplifications for the DRFLP considers the departments and not their arrangement. The Multi-Row Equidistant Facility Layout Problem (MREFLP) is a special case of the MRFLP with all departments equal in shape Amaral (2011) and the DRFLP with departments of equal length is called (DREFLP). Recently, in Anjos, Fischer, and Hungerländer (2018), see also Anjos, Fischer, and Hungerländer (2016), it is shown that in the MREFLP the departments can be arranged on an integer grid and an ILP and an SDP model are presented. As a result, equidistant double-row and equidistant multi-row instances with up to 25 departments were solved to optimality for $2 \leq m \leq 5$ and gaps with less than $4 \%$ were obtained for instances with up to 50 departments and $2 \leq m \leq 5$. 
Due to the grid structure of optimal solutions the MREFLP can be seen as a special case of the Quadratic Assignment Problem (QAP), see, e. g., Loiola, de Abreu, Boaventura-Netto, Hahn, and Querido (2007). In Hungerländer (2014b) it is shown that the best method for the SRFLP is better than methods especially tailored to the equidistant SRFLP, see, e. g., Palubeckis (2012, 2015b).

If we restrict the SRFLP with departments of equal length to binary weights $w_{i j} \in\{0,1\}, i, j \in[n], i<j$, we obtain the wellstudied Minimum Linear Arrangement Problem (LA), see, e. g., Harper (1964, 1966). Given a graph $G=(V, E)$ with, w. l. o. g., $V=\{1, \ldots, n\}$, the LA looks for a bijection $q: V \rightarrow V$ such that

$\sum_{i j \in E}\left|q_{i}-q_{j}\right|$

is minimized. The LA is already an $\mathcal{N} \mathcal{P}$-hard problem (Garey, Johnson, \& Stockmeyer, 1974) and hence all other row layout problems mentioned above are also $\mathcal{N} \mathcal{P}$-hard. Several authors determined lower bounds for the LA, see, e. g., Liu and Vannelli (1995), Caprara, Letchford, and Salazar-González (2011), Amaral, Caprara, Letchford, and Salazar-Gonzalez (2008). The combinatorial lower bounds for the LA presented in Caprara et al. (2011) were the starting point for our investigations together with a research question in Hungerländer (2017). In Hungerländer (2017) the so called Checkpoint Ordering Problem (CPOP) was introduced. Given a set of $n$ departments with lengths $\ell_{i}$ and weights $w_{i}, i \in[n]$, the CPOP asks for a space-free non-overlapping arrangement of the departments in one row such that the sum of the weighted distances of the centers of the departments to a checkpoint whose position is given in advance is minimized. The CPOP is closely related to the SRFLP and it was asked in Hungerländer (2017) whether some partial relation of the SRFLP and certain scheduling problems can be exploited further in the row layout setting.

\subsection{Our Contribution}

The main contributions of this paper are the following:

- We indicate a relation between some special DRFLP, where we only explicitly measure the (weighted) distance of some specific department to the others, to the parallel identical machine scheduling problem with minimum (weighted) completion time.

- We develop the first non-trivial combinatorial lower bounds for the DRFLP, the DREFLP and the PROP. These bounds can also be extended to the multi-row case, i. e., to the MRFLP, the MREFLP and the KPROP.

- We show how to combine these lower bounds with a new mixed-integer linear programming model to compute even stronger lower bounds for the DRFLP, the DREFLP and the PROP via some branch-and-cut algorithm within a given time limit of a few minutes.

- We present a corrected and short proof of a result of Samarghandi and Eshghi (2010) which states that the SRFLP with weights $w_{i j}=1, i, j \in[n], i<j$, can be solved to optimality in polynomial time by using some specific order of the departments. We use this result to further strengthen our lower bounding model for the PROP.

- In a computational study we compare our lower bounds for DRFLP instances from the literature as well as for mediumsized and large randomly generated instances with lower bounds received via some branch-and-cut algorithm within a time limit of one hour for a well-known DRFLP formulation (Amaral, 2013a). Furthermore we compare them to some heuristically determined upper bounds. Apart from this we investigate the strength of our DREFLP and PROP lower bounds.
This paper is structured as follows. In Section 2 we present combinatorial lower bounds for the DRFLP and prove their correctness. In Section 3 we introduce a distance-based ILP model to further improve these bounds. Furthermore we shortly explain in both sections which adaptations are needed for deriving lower bounds for the PROP (and partially the kPROP). In Section 4 we computationally investigate the strength of our newly derived lower bounds for medium-sized and large DRFLP, DREFLP as well as PROP instances by comparing them to some bounds from the literature and heuristically determined upper bounds. We conclude this paper in Section 5 and present directions for future work.

\section{Combinatorial lower bounds}

To the best of our knowledge combinatorial lower bounds specialized to the DRFLP are not known in the literature and lower bounds received via some branch-and-cut algorithm within a given time limit of one hour for some DRFLP formulation from the literature (Amaral, 2013a) are rather weak as we will see in Section 4. In this section we present three possibilities to compute combinatorial lower bounds for the DRFLP. To simplify the presentation we concentrate on lower bounds for the DRFLP and show at the end of this section how to extend these lower bounds to the MRFLP and to the $\mathrm{kPROP}$ and the PROP as well. Apart from this we will have a closer look at the equidistant case of the DRFLP.

In the following we generalize the so called star inequalities of the LA, see, e. g., Caprara et al. (2011), and we indicate a connection of a special DRFLP to the parallel identical machine scheduling problem with minimum weighted completion time with four machines (an exact definition is given below). With these results we partially answer a research question in Hungerländer (2017) whether one can use ideas from the scheduling literature for row layout problems.

\subsection{Weighted Star Lower Bound}

We start with a description of the star inequalities, which are used for determining lower bounds for the optimal solution value of the LA in Caprara et al. (2011) given some graph $G=(V, E)$. Let $q$ be a solution of the LA. Then the star inequalities for a fixed node $i \in V$ and a set $S \subseteq V \backslash\{i\}$ read as follows

$\sum_{j \in S}\left|q_{i}-q_{j}\right| \geq\left\lfloor\frac{(|S|+1)^{2}}{4}\right\rfloor$,

see, e. g., Caprara et al. (2011) and Amaral et al. (2008). One can derive this formula by arranging all nodes in $S$ as close as possible to node $i$. With $S_{i}=\{j \in V: i j \in E\}$ a lower bound for the optimal solution value of the LA is given by

$\frac{1}{2} \sum_{i \in V}\left\lfloor\frac{\left(\left|S_{i}\right|+1\right)^{2}}{4}\right\rfloor$,

because we count the minimal contribution of each node (each pairwise absolute difference is counted twice and so we have to divide the sum by two).

In the following we present three different ways to measure the contribution of each department to the sum of the weighted distances in the DRFLP. These three approaches are related to the Parallel Identical Machine Scheduling Problem with minimum weighted completion time, see, e. g., Kawaguchi and Kyan (1986), Smith (1956), Hall, Schulz, Shmoys, and Wein (1997), Lenstra, Kan, and Brucker (1977), often called $P \| \sum w_{k} C_{k}$ where $C_{k}$ denotes the completion time of some job $k$.

Definition 2. Given a set of jobs $J$ with processing times $p_{k} \in \mathbb{R}_{+}$ and weights $w_{k} \in \mathbb{R}_{+}, k \in J$, one looks for an assignment of start times $t_{k} \in \mathbb{R}_{+}$of the jobs $J$ to $u \in \mathbb{N}$ parallel identical machines such 
that no two jobs overlap on one machine and such that the sum of the weighted completion times $\sum_{k \in J} w_{k} C_{k}$ with $C_{k}=t_{k}+p_{k}$ is minimized. For constant $u$ we denote this problem by $P_{u} \| \sum w_{k} C_{k}$ and for $u$ part of the input by $P \| \sum w_{k} C_{k}$.

The scheduling problem $P_{u} \| \sum w_{k} C_{k}$ is weakly $\mathcal{N} \mathcal{P}$-hard, see, e. g., Lee and Uzsoy (1992), and $P \| \sum w_{k} C_{k}$ is $\mathcal{N} \mathcal{P}$-hard in the strong sense (Lee \& Uzsoy, 1992). For $u=1$, this problem is a single machine scheduling problem and can be solved in polynomial time by the so called Smith rule (Smith, 1956). The Smith rule states that in an optimal solution the jobs are ordered nonincreasingly by their relative weights $\frac{w_{k}}{p_{k}}$ for $k \in J$. In the literature the Smith rule has also been extended to the parallel machine case, i. e., the jobs are ordered non-increasingly by their relative weights $\frac{w_{k}}{p_{k}}$ for $k \in J$ and we assign each of the jobs using this order to the next machine that gets idle. As we will see below, in general optimality might be lost for a schedule determined like this. Further, it is well known that the unweighted case, i. e., $P \| \Sigma C_{k}$ with $w_{k}=1$ for $k \in J$, can be solved to optimality in polynomial time by the Shortest Processing Time rule (SPT), where one processes the jobs in increasing order of their processing time. We will show next how to use these rules for deriving combinatorial lower bounds for the optimal value of some DRFLP instance. For this we will frequently use the following notation.

Definition 3. Let $(n, w, \ell)$ be a DRFLP instance. We denote by

$\mathcal{Q}(n, w, \ell)=\{(r, q): r, q$ is a feasible solution for the DRFLP instance $(n, w, \ell)\}$

the set of feasible solutions. For a fixed department $i \in[n]$, a set $S \subseteq[n] \backslash\{i\}$ and some $(r, q) \in \mathcal{Q}(n, w, \ell)$ we denote the sum of the weighted distances from all departments in $S$ to $i$ by

$W_{i}(q, S):=\sum_{j \in S} w_{i j}\left|q_{i}-q_{j}\right|$.

The best possible value of $W_{i}(\cdot, S)$ for some fixed set $S$ over all feasible solutions is denoted by

$\widehat{W}_{i}(S):=\min _{(r, q) \in \mathcal{Q}(n, w, \ell)} W_{i}(q, S)$,

and the optimal value of the DRFLP is then

$\widehat{W}:=\frac{1}{2} \min _{(r, q) \in \mathcal{Q}(n, w, \ell)} \sum_{i \in[n]} W_{i}(q,[n] \backslash\{i\})$.

Note that in the calculation of $\widehat{W}$ we have to divide the sum of the $W_{i}(.$, .) by two because each pairwise distance is counted twice.

The common idea for our combinatorial bounding procedure is to find lower bounds for $\widehat{W}_{i}([n] \backslash\{i\})$, which will give rise to lower bounds for $\widehat{W}$ : for each feasible solution $(r, q) \in \mathcal{Q}(n, w, \ell)$ we have

$$
\begin{aligned}
\frac{1}{2} \sum_{i \in[n]} \widehat{W}_{i}([n] \backslash\{i\})= & \frac{1}{2} \sum_{i \in[n]} \min _{(r, q) \in \mathcal{Q}(n, w, \ell)} W_{i}(q,[n] \backslash\{i\}) \\
& \leq \frac{1}{2} \min _{(r, q) \in \mathcal{Q}(n, w, \ell)} \sum_{i \in[n]} W_{i}(q,[n] \backslash\{i\})=\widehat{W} .
\end{aligned}
$$

The following proposition is essential for our considerations. It reduces the set of possibly optimal solutions.

Proposition 4. Let $(n, w, \ell)$ be a DRFLP instance and let $i \in[n]$, $S \subseteq[n] \backslash\{i\}$. Then there exists a solution $(r, q) \in \mathcal{Q}(n, w, \ell)$ for which $\widehat{W}_{i}(S)$ is attained such that there is some $j \in S$ with $q_{i}=q_{j}, i$. e., $j$ lies directly opposite $i$.

Proof. Let $i \in[n], S \subseteq[n] \backslash\{i\}$ and let $(r, q) \in \mathcal{Q}(n, w, \ell)$ be a solution minimizing $W_{i}(\cdot, S)$. Assume, w. l. o. g., that $r_{i}=1$ and that $\left\{j \in S: r_{j}=2\right\} \neq \emptyset$, otherwise we can easily place one department $\hat{j}$ opposite to $i$ and reduce the distance of $i$ and $\hat{j}$. We get

$$
\begin{aligned}
W_{i}(q, S) & =\sum_{\substack{j \in S \\
r_{j}=1}} w_{i j}\left|q_{i}-q_{j}\right|+\sum_{\substack{j \in S \\
q_{j}<q_{i} \\
r_{j}=2}} w_{i j}\left(q_{i}-q_{j}\right) \\
& +\sum_{\substack{j \in S \\
q_{i}<q_{j} \\
r_{j}=2}} w_{i j}\left(q_{j}-q_{i}\right)+\underbrace{\sum_{\substack{j \in S \\
q_{i}=q_{j}}} w_{i j}\left(q_{j}-q_{i}\right) .}_{=0} .
\end{aligned}
$$

If there does not exist a $j \in S$ with $q_{i}=q_{j}$, then by the optimality of $(r, q)$ shifting all departments $j \in S$ with $r_{j}=2$ to the left or to the right by some small $\varepsilon>0$ does not change the objective value. So we can shift all departments in row 2, w. 1. o. g., to the left until one department lies opposite $i$.

This result shows that in order to determine a lower bound for $\widehat{W}_{i}(S)$ for $i \in[n], S \subseteq[n] \backslash\{i\}$ it suffices to determine a lower bound for varying $j \in S$ opposite $i$. This motivates the following definition.

Definition 5. Let $(n, w, \ell)$ be a DRFLP instance, and let $i \in[n]$, $S \subseteq[n] \backslash\{i\}$ and $j \in S$. Denote

$\widehat{W}_{(i, j)}(S):=\min \left\{W_{i}(q, S):(r, q) \in \mathcal{Q}(n, w, \ell), q_{i}=q_{j}\right\}$.

An immediate consequence of Proposition 4 is the following corollary.

Corollary 6. Let $(n, w, \ell)$ be a DRFLP instance and let $i \in[n]$, $S \subseteq[n] \backslash\{i\}$. Then $\widehat{W}_{i}(S)=\min _{j \in S} \widehat{W}_{(i, j)}(S)$.

Thus, in order to compute lower bounds for $\widehat{W}$, it suffices to determine lower bounds for $\widehat{W}_{(i, j)}(S)$ for all valid choices of $i, j$ and $S$. In the following we determine three different lower bounds for $\widehat{W}_{(i, j)}(S)$ given some DRFLP instance. In all three variants we interpret the optimization problem (2) for computing $\widehat{W}_{(i, j)}(S)$ as a scheduling problem $P_{4} \| \sum w_{k} C_{k}$ with weights $w_{k}=w_{i k}$. The departments correspond to the jobs in the $P_{4} \| \sum w_{k} C_{k}$ and the lengths of the departments to the processing times, i. e., $p_{k}=$ $\ell_{k}, k \in S \backslash\{j\}$. Given a feasible solution of the optimization problem (2), then, as illustrated in Fig. 2, machine 1 and machine 2 of the scheduling problem correspond to row 1 in this solution and machine 3 and machine 4 to row 2 .

Thus we are able to use methods from the scheduling literature to compute lower bounds for the DRFLP. All lower bound calculations have in common that we sort the jobs in $S \backslash\{j\}$ by some given order. Respecting some machine-dependent non-availability times from zero to $a=\left(a_{1}, \ldots, a_{4}\right) \in \mathbb{R}_{+}^{4} \cup\{\infty\}$ (i. e., no job on machine $k$ may start before $\left.a_{k}, k=1, \ldots, 4\right)$, the jobs are assigned in a greedy manner. Whenever a machine becomes idle and is available one assigns the next unscheduled job in the list non-preemptively. Our basic algorithm is summarized in Algorithm 1.

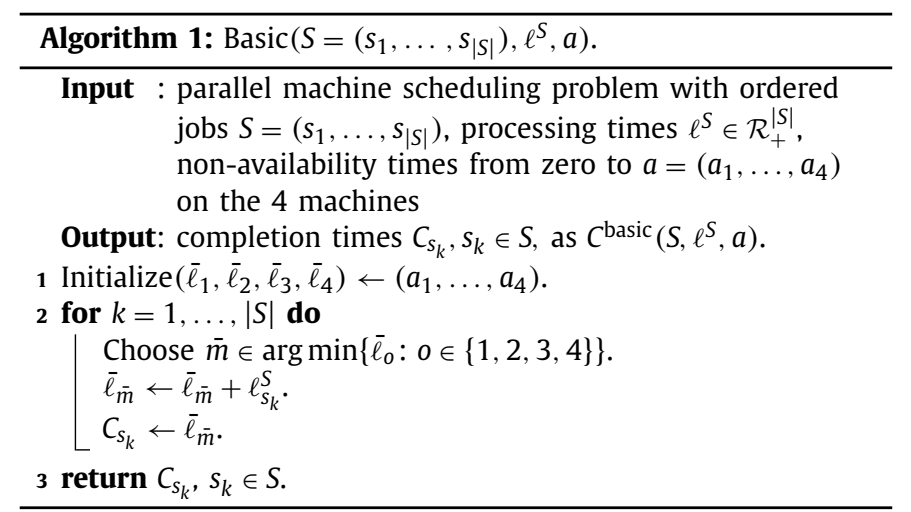




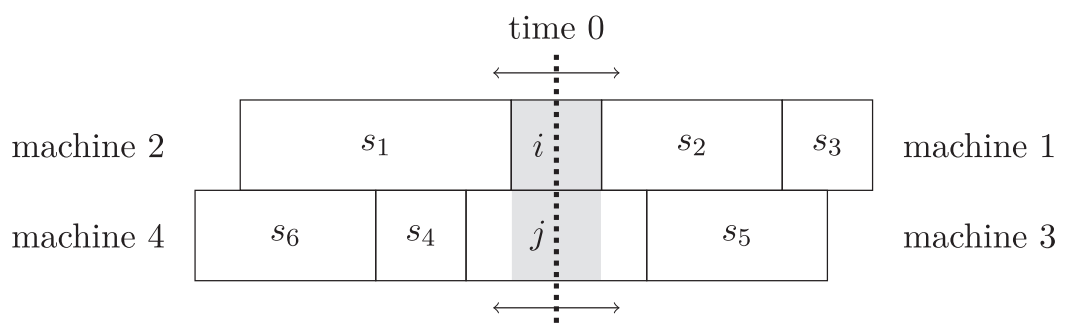

Fig. 2. Visualization of the connection of the DRFLP and parallel machine scheduling on four machines. Here departments $i$ and $j$ lie opposite and we have to arrange departments $\left\{s_{1}, \ldots, s_{6}\right\}$. In the lower bound calculations we will partially adjust the start of the jobs (departments) at a machine by half the length of $i$ (see gray area) or half the length of $j$. Furthermore we have to keep in mind that in scheduling one considers the completion times of the jobs but in the DRFLP we measure the distances between the centers of the departments.

Definition 7. Let $S=\left(s_{1}, \ldots, s_{|S|}\right)$ be an ordered sequence of jobs (departments) with processing times (lengths) $\ell^{S} \in \mathbb{R}_{+}^{|S|}$ and let $a \in$ $\mathbb{R}_{+}^{4}$ denote four non-availability times. Then we denote by $C^{\text {basic }}(S$, $\left.\ell^{S}, a\right)$ the greedy solution returned by Algorithm 1 when scheduling the jobs in this order.

For the first lower bound we use the SPT rule, i. e., we order the jobs (departments) by increasing length. Furthermore, machine 1 and machine 2 are non-available from 0 to $\frac{\ell_{i}}{2}$ and machine 3 and machine 4 from 0 to $\frac{\ell_{j}}{2}$.

Definition 8. Let $(n, w, \ell)$ be a DRFLP instance. Let $i \in[n]$, $S \subseteq[n] \backslash\{i\}, j \in S$ with $S_{j}^{\mathrm{spt}}=\left(s_{1}, \ldots, S_{|S|-1}\right)$ a sequence of departments in $S \backslash\{j\}$ with length $\ell^{S_{j}^{\text {spt }}}=\left(\ell_{s_{1}}, \ldots, \ell_{s_{|S|-1}}\right)$ ordered by increasing lengths and let

$C^{\text {spt, }(i, j)}(S, \ell):=C^{\text {basic }}\left(S_{j}^{\text {spt }}, \ell^{S_{j}^{\text {spt }}},\left(\frac{\ell_{i}}{2}, \frac{\ell_{i}}{2}, \frac{\ell_{j}}{2}, \frac{\ell_{j}}{2}\right)\right)$.

Furthermore, let $w_{i \bullet}^{\prime}=\left(w_{i 1}^{\prime}, \ldots, w_{i(|S|-1)}^{\prime}\right)$ be the weights $w_{i k}$ of $k \in S \backslash\{j\}$, ordered decreasingly. Then the $S P T$-lower-bound is

$W_{(i, j)}^{\mathrm{spt}}(S):=\sum_{k=1}^{|S|-1} w_{i k}^{\prime}\left(C_{S_{k}}^{\mathrm{spt},(i, j)}(S, \ell)-\frac{\ell_{S_{k}}}{2}\right)$

In the special case of all weights being equal to one the $S P T$ distance-bound is

$W_{(i, j)}^{\mathrm{dst}}(S):=\sum_{k=1}^{|S|-1}\left(C_{S_{k}}^{\mathrm{spt},(i, j)}(S, \ell)-\frac{\ell_{s_{k}}}{2}\right)$.

The SPT-distance-bound cannot be used to derive bounds for the optimal value of the DRFLP. However, it can be used to derive lower bounds for the (geometric) distances between the departments themselves without regarding the amount of transports. We will make use of them later in the lower bound ILP model presented in Section 3. In $W_{(i, j)}^{\mathrm{spt}}(S)$ we assign the highest weights to the earliest jobs (the departments closest to department $i$ ) in order to get a lower bound for $\widehat{W}_{(i, j)}(S)$. For an illustration we refer to Fig. 3.

Proposition 9. Let $(n, w, \ell)$ be a DRFLP instance, and let $i \in[n]$, $S \subseteq[n] \backslash\{i\}, j \in S$ and $W_{(i, j)}^{\mathrm{dst}}(S)$ and $W_{(i, j)}^{\mathrm{spt}}(S)$ as defined above. Then

$W_{(i, j)}^{\mathrm{dst}}(S)=\min \left\{\sum_{k=1}^{|S|-1}\left|q_{i}-q_{s_{k}}\right|:(r, q) \in \mathcal{Q}(n, w, \ell), q_{i}=q_{j}\right\}$,

$W_{(i, j)}^{\mathrm{spt}}(S) \leq \widehat{W}_{(i, j)}(S)$.

Proof. Let $i \in[n], S \subseteq[n] \backslash\{i\}, j \in S$. The correctness of (3) follows directly by the correctness of the SPT rule for the problem $P_{4} \| \Sigma C_{k}$. Note that in comparison to the scheduling problem in the DRFLP the distances are measured between the centers of the departments, i. e., we obtain $d_{i k}=C_{k}-\frac{\ell_{k}}{2}, k \in S \backslash\{j\}$, and $d_{i j}=0$.

The only difference in (4) is that we additionally assign the highest weights to the departments closest to $i$ which maintains correctness.

Example 10. Consider a double-row instance with $n=8, \ell_{k}=k$, $k \in[6], \ell_{7}=4, \ell_{8}=1$, and non-zero weights $w_{18}=\frac{1}{2}, w_{28}=1$, $w_{38}=3, w_{48}=3, w_{58}=1, w_{68}=7, w_{78}=5$. Our aim is to compute $W_{(8,7)}^{\mathrm{dst}}([7])$ and $W_{(8,7)}^{\mathrm{spt}}([7])$. Therefore we consider the problem $P_{4} \| \sum_{k \in[6]} w_{k 8} C_{k}$ where the non-availability times range from zero to $\ell_{8} / 2=0.5$ on machines 1 and 2 and to $\ell_{7} / 2=2$ on machines 3 and 4 . We apply the SPT rule for the jobs (departments) [6] and obtain the schedule illustrated in Fig. 3(a). Then we arrange the departments on machine 1 and machine 2 space-free to row 1 in the double-row layout by respecting their order and the departments in machine 3 and machine 4 space-free to row 2 such that department 8 lies directly opposite 7 as illustrated in Fig. 3(b). So we get $W_{(8,7)}^{\text {dst }}([7])=1+1.5+3+4+4.5+5.5=19.5$. Next, we assign the highest weights to departments closest to department 8 . For instance, $w_{68}$ is assigned to department 1 and $w_{38}$ to department 2. In total, we obtain

$W_{(8,7)}^{\mathrm{spt}}([7])=7 \cdot 1+3 \cdot 1.5+3 \cdot 3+1 \cdot 4+1 \cdot 4.5+0.5 \cdot 5.5=31.75$.

Note that in general the value $\min _{j \in S} W_{(i, j)}^{\mathrm{dst}}(S)$ (and thus the value $\left.\min _{j \in S} W_{(i, j)}^{\mathrm{spt}}(S)\right)$ is not obtained by arranging $i \in[n]$ directly opposite a shortest department of $S \subseteq[n] \backslash\{i\}$.

Example 11. Consider a DRFLP instance with $\ell_{1}=\ldots=\ell_{4}=$ $1, \ell_{5}=5$ and non-zero weights $w_{i j}=1, i, j \in[5], i<j$. Then $W_{(1, j)}^{\mathrm{dst}}(S)=5$ for $j=2,3,4$ and $S=\{2, \ldots, 5\}$, but $W_{(1,5)}^{\mathrm{dst}}(S)=$ $W_{(1,5)}^{\mathrm{spt}}(S)=1+2+1=4$. So it is the best to assign the largest department directly opposite department $i$ in this example. The corresponding layout is illustrated in Fig. 4.

\subsection{Scheduling Lower Bound}

In this section we suggest two further possibilities to bound $\widehat{W}_{(i, j)}(S)$ with $i \in[n], S \subseteq[n] \backslash\{i\}, j \in S$ from below. Our main tool is the approximation algorithm in Kawaguchi and Kyan (1986) for the $P \| \sum w_{k} C_{k}$. The associated algorithm determines a schedule by applying the Smith rule in the parallel machine case. The jobs are ordered non-increasingly by $\frac{w_{k}}{p_{k}}$ and the corresponding schedule is determined by Algorithm 1.

Theorem 12. (Kawaguchi E Kyan (1986)) We consider the problem $P \| \sum w_{k} C_{k}$ with jobs J. Then using the Smith rule for sorting the jobs leads to a $\frac{1+\sqrt{2}}{2}$-approximation algorithm for the $P \| \sum w_{k} C_{k}$ with the running time $\mathcal{O}(|J| \cdot \log (|J|))$. Moreover, in the case with at least two machines, the bound $\frac{1+\sqrt{2}}{2}$ is tight. 


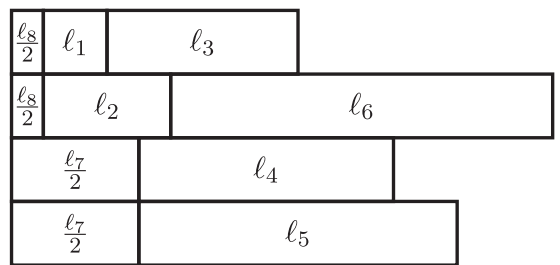

(a) A schedule obtained by the SPT rule for set of jobs [6] where $\frac{\ell_{8}}{2}$ and $\frac{\ell_{7}}{2}$ are fixed.

\begin{tabular}{|c|c|c|c|c|c|c|}
\hline \multicolumn{2}{|c|}{6} & 2 & 8 & 1 & 3 \\
\hline \multirow{2}{*}{5} & \multicolumn{3}{|c|}{7} & 4 \\
\hline
\end{tabular}

(b) Double-row layout obtained by arranging the departments from Figure 3(a) to row 1 and row 2 such that department 8 lies directly opposite department 7 .

Fig. 3. Consider an instance with $n=8, \ell_{k}=k, k \in[6], \ell_{7}=4, \ell_{8}=1$, and non-zero weights $w_{18}=\frac{1}{2}, w_{28}=1, w_{38}=3, w_{48}=3, w_{58}=1, w_{68}=7$, $w_{78}=5$. Then we obtain $W_{(8.7)}^{\text {dst }}([7])=19.5$ and $W_{(8.7)}^{\text {spt }}([7])=31.75$.

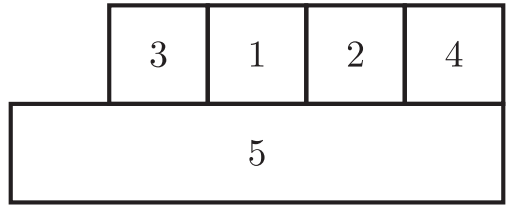

Fig. 4. Consider a double-row instance with $\ell_{1}=\ldots=\ell_{4}=1, \ell_{5}=5$ and weights $w_{i j}=1, i, j \in[5], i<j$. The sum of the (weighted) distances of department 1 to the remaining departments is minimized by arranging it directly opposite department 5 , which is the largest department in this instance.

We will write $\alpha^{K K}:=\frac{1+\sqrt{2}}{2} \approx 1.207$. In order to determine a lower bound for $\widehat{W}_{(i, j)}(S)$ for $i \in[n], S \subseteq[n] \backslash\{i\}, j \in S$, we again interpret the departments $S \backslash\{j\}$ as jobs of $P_{4} \| \sum_{k \in S \backslash\{j\}} w_{i k} C_{k}$ and apply the approximation algorithm of Kawaguchi and Kyan (1986). However, in the lower bound calculation we have to take care of two facts. First, the distance calculations for the DRFLP are center-tocenter whereas the algorithm by Kawaguchi and Kyan is based on completion times. Second, because the $P_{4} \| \sum w_{k} C_{k}$ solution is not exact but only approximate, we must respect the approximation factor $\alpha^{K K}$.

Definition 13. Let $(n, w, \ell)$ be a DRFLP instance, and let $i \in[n]$, $S \subseteq[n] \backslash\{i\}$ and $j \in S$. We denote by $S_{j}^{s c}=\left(s_{1}, \ldots, s_{|S|-1}\right)$ a sequence of departments $S \backslash\{j\}$ with length vector $\ell^{S_{j}^{s c}}$ ordered according to the Smith rule, i. e., non-increasingly by $\frac{w_{i k}}{\ell_{k}}$. Denote by

$C^{\mathrm{sc},(i, j)}(S, \ell):=C^{\text {basic }}\left(S_{j}^{\mathrm{sc}}, \ell^{S_{j}^{\mathrm{sc}}},(0,0,0,0)\right)$

the completion times returned by Algorithm 1 for this ordering. Then the SCHED1-lower-bound is

$$
\begin{aligned}
W_{(i, j)}^{\mathrm{sc}}(S) & :=\frac{1}{\alpha^{K R}} \sum_{k=1}^{|S|-1} w_{i s_{k}} \cdot C_{S_{k}}^{\mathrm{sc},(i, j)}(S, \ell) \\
& +\sum_{k=1}^{|S|-1} w_{i s_{k}} \cdot\left(\frac{1}{2} \min \left\{\ell_{i}, \ell_{j}\right\}-\frac{\ell_{s_{k}}}{2}\right) .
\end{aligned}
$$

Proposition 14. Let $(n, w, \ell)$ be a DRFLP instance and $i \in[n]$, $j \in S \subseteq[n] \backslash\{i\}$. Then $W_{(i, j)}^{\mathrm{sc}}(S) \leq \widehat{W}_{(i, j)}(S)$.

Proof. Let $i \in[n], j \in S \subseteq[n] \backslash\{i\}$ be given. Here $i$ lies opposite $j$ and we want to bound the sum of the weighted distances of $i$ to all other departments. We want to interpret this as a variant of $P_{4} \| \sum_{k \in S \backslash\{j\}} w_{i k} C_{k}$. Let $(r, q) \in \mathcal{Q}(n, w, \ell)$ be an optimal solution of (2). A corresponding solution of $P_{4} \| \sum_{k \in S \backslash\{j\}} w_{i k} C_{k}$ is then $C_{k}=$ $\left|q_{k}-q_{i}\right|+\frac{\ell_{k}}{2}-\frac{\ell_{h_{k}}}{2}, k \in S \backslash\{j\}$, where $h_{k}=i$ if $k$ is in the same row as $i$ and $h_{k}=j$ otherwise. The completion times of the scheduling problem are formed by taking into account that the DRFLP measures center-to-center distances. Let $v^{*}$ denote the optimal value of the scheduling problem $P_{4} \| \sum_{k \in S \backslash\{j\}} w_{i k} C_{k}$, then

$$
\begin{aligned}
\widehat{W}_{(i, j)}(S)= & \sum_{k \in S \backslash\{j\}} w_{i k}\left|q_{k}-q_{i}\right| \geq \sum_{k \in S \backslash\{j\}} w_{i k} C_{k} \\
& +\frac{1}{2} \sum_{k \in S \backslash\{j\}} w_{i k}\left(\min \left\{\ell_{i}, \ell_{j}\right\}-\ell_{k}\right) \\
\geq & v^{*}+\frac{1}{2} \sum_{k \in S \backslash\{j\}} w_{i k}\left(\min \left\{\ell_{i}, \ell_{j}\right\}-\ell_{k}\right) \\
\geq & \frac{1}{\alpha^{K K}} \sum_{k=1}^{|S|-1} w_{i s_{k}} C_{S_{k}}^{\mathrm{sc},(i, j)}(S, \ell) \\
& +\frac{1}{2} \sum_{k=1}^{|S|-1} w_{i s_{k}}\left(\min \left\{\ell_{i}, \ell_{j}\right\}-\ell_{s_{k}}\right)=W_{(i, j)}^{\mathrm{sc}}(S),
\end{aligned}
$$

where the last inequality follows by Theorem 12 .

The following Example 15 illustrates the differences to the calculation of the star lower bounds.

Example 15. We consider again Example 10 and our aim is to compute $W_{(8,7)}^{s c}([7])$. We sort the departments by decreasing relative weights, i. e., $S_{j}^{s c}=(6,3,4,2,1,5)$, and compute $C^{s c,(i, j)}(S, \ell)$. The obtained schedule is illustrated in Fig. 5(a) and the corresponding double-row layout is illustrated in Fig. 5(b), where we do not show department 7 because it overlaps with departments 2 and 4 while the distance calculation is done as illustrated here. Then we obtain

$$
\begin{aligned}
W_{(8,7)}^{\mathrm{sc}}([7])= & \frac{1}{\alpha^{K K}}\left(w_{18}\left(\ell_{1}+\ell_{2}\right)+w_{28} \ell_{2}+w_{38} \ell_{3}+w_{48} \ell_{4}\right. \\
& \left.+w_{58}\left(\ell_{5}+\ell_{3}\right)+w_{68} \ell_{6}\right) \\
+ & \sum_{k \in[6]} \frac{w_{i k}}{2}\left(\min \left\{\ell_{7}, \ell_{8}\right\}-\ell_{k}\right) \approx 34.2>31.75=W_{(8,7)}^{\mathrm{spt}}([7]) .
\end{aligned}
$$

By Proposition 14 we obtain a lower bound for $\widehat{W}_{(i, j)}(S), i \in$ $[n], S \subseteq[n] \backslash\{i\}, j \in S$. But, as shown in Example 15 and Fig. 5(b), we do not receive a valid double-row layout. The reason for this is that we only use $\frac{\min \left\{\ell_{i}, \ell_{j}\right\}}{2}$ and neglect that one of the two departments might be longer. For calculating the next bound we introduce two artificial jobs (departments) with length $\frac{1}{2}$. 


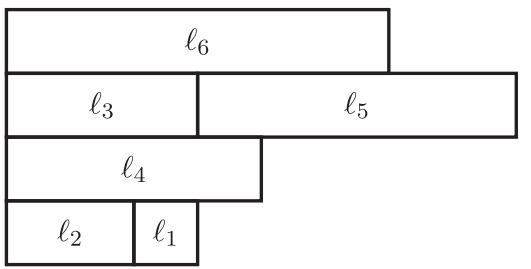

(a) Schedule on four machines obtained using the Smith rule.

\begin{tabular}{|l|l|l|l|l|l|}
\hline 5 & \multicolumn{2}{|c|}{3} & 8 & \multicolumn{2}{|c|}{6} \\
\hline & 1 & 2 & & \multicolumn{2}{|c|}{4} \\
\cline { 3 - 5 } & & & &
\end{tabular}

(b) Double-row layout deduced from the schedule above where 8 lies directly opposite 7 . Note that department 7 is not drawn because it is larger than department 8 and would overlap with departments 2 and 4 .

Fig. 5. Consider an instance with $n=8, \ell_{k}=k, k \in[6], \ell_{7}=4, \ell_{8}=1$, and non-zero weights $w_{18}=\frac{1}{2}, w_{28}=1, w_{38}=3, w_{48}=3, w_{58}=1, w_{68}=7, w_{78}=5$. We get $W_{(8,7)}^{\mathrm{sc}}([7]) \approx 34.2$.

$\left(\max \left\{\ell_{i}, \ell_{j}\right\}-\min \left\{\ell_{i}, \ell_{j}\right\}\right)=\frac{\left|\ell_{i}-\ell_{j}\right|}{2}$ and weights such that they are chosen first by the Smith rule. In order to get a best possible lower bound afterwards the weight is chosen minimal with respect to the desired property.

Definition 16. Let $(n, w, \ell)$ be a DRFLP instance, and let $i \in[n]$, $S \subseteq[n] \backslash\{i\}$ and $j \in S$. Define $\beta:=\max \left\{\frac{w_{i k}}{\ell_{k}}: k \in S \backslash\{j\}\right\}$. We add two dummy departments $n+1$ and $n+2$ with lengths $\ell_{n+1}=$ $\ell_{n+2}=\frac{\left|\ell_{i}-\ell_{j}\right|}{2}$ and weights $w_{i(n+1)}=w_{i(n+2)}=\beta \frac{\left|\ell_{i}-\ell_{j}\right|}{2}$. Let $S_{j}^{\mathrm{sc} 2}=$ $\left(s_{1}, \ldots, s_{|S|+1}\right)$ be a sequence of departments $S \cup\{n+1, n+2\} \backslash\{j\}$ ordered according to the Smith rule with $s_{1}=n+1, s_{2}=n+2$ and with length vector $\ell_{j}^{S_{j}^{c 2}}$ and denote by

$C^{s c 2,(i, j)}(S, \ell):=C^{\text {basic }}\left(S_{j}^{s c 2}, \ell^{S_{j}^{s c 2}},(0,0,0,0)\right)$

the completion times returned by Algorithm 1 for this ordering. Then the SCHED2-lower-bound is

$$
\begin{aligned}
W_{(i, j)}^{\mathrm{sc} 2}(S) & :=\frac{1}{\alpha^{K K}} \sum_{k=1}^{|S|+1} w_{i s_{k}} \cdot C_{s_{k}}^{\mathrm{sc} 2,(i, j)}(S, \ell) \\
& +\sum_{k=3}^{|S|+1} w_{i s_{k}} \cdot\left(\frac{1}{2} \min \left\{\ell_{i}, \ell_{j}\right\}-\frac{\ell_{s_{k}}}{2}\right)-\beta \frac{\left(\ell_{i}-\ell_{j}\right)^{2}}{2} .
\end{aligned}
$$

Proposition 17. Let $(n, w, \ell)$ be a DRFLP instance and $i \in[n]$, $j \in S \subseteq[n] \backslash\{i\}$. Then $W_{(i, j)}^{s c 2}(S) \leq \widehat{W}_{(i, j)}(S)$.

Proof. Let $i \in[n], j \in S \subseteq[n] \backslash\{i\}$ be given. The proof of this result is similar to the proof of Proposition 14. In contrast to this proof we introduce two dummy departments $n+1, n+2$ here to level different lengths of $i$ and $j$. In the associated scheduling problem we then also count the completion times of the dummy departments. So we have to subtract this value afterwards. Let all objects be as defined in Definition 16 and let $(r, q) \in \mathcal{Q}(n, w, \ell)$ be an optimal solution of (2). The corresponding scheduling solution is $C_{s_{k}}=\left|q_{s_{k}}-q_{i}\right|+\frac{1}{2}\left(\ell_{s_{k}}-\min \left\{\ell_{i}, \ell_{j}\right\}\right)$ for $k=3, \ldots,\left|S_{j}^{\text {sc2 }}\right|$. Let $v^{*}$ be the optimal solution value of $P_{4} \| \sum_{k \in S_{j}^{s c 2}} w_{i k} C_{k}$, then

$$
\begin{aligned}
\widehat{W}_{(i, j)}(S)= & \sum_{k \in S \backslash\{j\}} w_{i k}\left|q_{k}-q_{i}\right| \\
\geq & \sum_{k=1}^{|S|+1} w_{i s_{k}} C_{s_{k}}+\frac{1}{2} \sum_{k=3}^{|S|+1} w_{i s_{k}}\left(\min \left\{\ell_{i}, \ell_{j}\right\}-\ell_{S_{k}}\right) \\
& -w_{i(n+1)} \ell_{n+1}-w_{i(n+2)} \ell_{(n+2)}
\end{aligned}
$$

$$
\begin{aligned}
\geq & v^{*}+\frac{1}{2} \sum_{k=3}^{|S|+1} w_{i s_{k}}\left(\min \left\{\ell_{i}, \ell_{j}\right\}-\ell_{s_{k}}\right)-\beta \frac{\left(\ell_{i}-\ell_{j}\right)^{2}}{2} \\
\geq & \frac{1}{\alpha^{K K}} \sum_{k=1}^{|S|+1} w_{i s_{k}} C_{S_{k}}^{\mathrm{sc} 2,(i, j)}(S, \ell) \\
& +\frac{1}{2} \sum_{k=3}^{|S|+1} w_{i s_{k}}\left(\min \left\{\ell_{i}, \ell_{j}\right\}-\ell_{S_{k}}\right)-\beta \frac{\left(\ell_{i}-\ell_{j}\right)^{2}}{2} \\
= & W_{(i, j)}^{\mathrm{sc} 2}(S),
\end{aligned}
$$

where the last inequality follows by Theorem 12 . Furthermore note that by the choice of $\beta, \ell_{n+1}=\ell_{n+2}, w_{i(n+1)}=w_{i(n+2)}$ using the Smith rule it is possible to set $s_{1}=n+1, s_{2}=n+2$.

The combination of the previous results leads to one of our main results - a first combinatorial lower bound for the optimal value of the DRFLP.

Theorem 18. Let $(n, w, \ell)$ be a DRFLP instance. Let $V_{i}:=\{k \in[n] \backslash$ $\left.\{i\}: w_{i k}>0\right\}$ for $i \in[n]$. Then

$\frac{1}{2} \sum_{i \in[n]} \min _{j \in V_{i}} \max \left\{W_{(i, j)}^{\mathrm{spt}}\left(V_{i}\right), W_{(i, j)}^{\mathrm{sc}}\left(V_{i}\right), W_{(i, j)}^{\mathrm{sc} 2}\left(V_{i}\right)\right\}$

is a lower bound for the optimal value $\widehat{W}$ of the DRFLP. The bound (7) can be computed in $\mathcal{O}\left(n^{3} \cdot \log (n)\right)$.

Proof. The correctness follows from Propositions 9, 14, 17 and the definition of $\widehat{W}$. The running time for fixed $i \in[n], j \in V_{i}$, is $\mathcal{O}(n \cdot \log (n))$ because one has to sort the jobs (departments) in order to apply Algorithm 1 . Since there are $\mathcal{O}\left(n^{2}\right)$ such summands the total running time is $\mathcal{O}\left(n^{3} \cdot \log (n)\right)$.

So we mainly need Algorithm 1 and a sorting algorithm to calculate our combinatorial lower bound (7). The different sorting criteria for the three lower bounds $W_{(i, j)}^{\mathrm{spt}}\left(V_{i}\right), W_{(i, j)}^{\mathrm{sc}}\left(V_{i}\right), W_{(i, j)}^{\mathrm{sc} 2}\left(V_{i}\right)$ for fixed $i, j \in[n], j \in V_{i}$, (see Propositions 9, 14, 17) are described in Definitions 8, 13, 16.

\subsection{Extensions}

In this section we discuss extensions of the combinatorial lower bounds to the general MRFLP, the DREFLP and the PROP.

\subsubsection{MRFLP}

In general, all the lower bounds presented above for the DRFLP can be extended to lower bounds for the MRFLP. Indeed, for the 
bounds in Proposition 9 we can use the same approach but we have to check all $\left(\begin{array}{c}|S| \\ m-1\end{array}\right)$ (with $S \subseteq[n] \backslash\{i\}$ ) choices for departments directly opposite the fixed department $i \in[n]$. The same is true for the scheduling bounds SCHED1 and SCHED2. For the MRFLP we have to slightly extend Algorithm 1 to handle scheduling problems on $2 \mathrm{~m}$ parallel machines. However, the running time for the calculation of (7) is increased significantly in comparison to the doublerow case, but remains polynomial if $m$ is fixed.

\subsubsection{DREFLP}

In the calculation of the SPT-lower-bound we assign high weights to small departments. So the question arises if we can simplify the calculation of the combinatorial lower bounds in the equidistant case, because there we do not need Algorithm 1 to determine an optimal arrangement of the departments depending on their lengths. We start with the special case of all weights being equal to one. Note that we assume as done in the literature, see, e. g., Anjos et al. (2018) and Amaral (2011), that the department lengths are equal to one.

Proposition 19. Let $(n, w, \mathbf{1})$ be a DREFLP instance. Let $i \in[n]$ and $S \subseteq[n] \backslash\{i\}$, then for all $j \in S \backslash\{i\}$ and all solutions $(r, q) \in \mathcal{Q}(n, w, \mathbf{1})$ we get

$W_{(i, j)}^{\mathrm{dst}}(S)=\left\lfloor\frac{\left(\left\lceil\frac{|S|-1}{2}\right\rceil+1\right)^{2}}{4}\right\rfloor+\left\lfloor\frac{\left(\left\lfloor\frac{|S|-1}{2}\right\rfloor+1\right)^{2}}{4}\right\rfloor \leq \sum_{k \in S}\left|q_{i}-q_{k}\right|$.

Proof. For $i \in[n]$ we arrange one department of $S \subseteq[n] \backslash\{i\}$ directly opposite $i$ and we assign the remaining $\left\lceil\frac{|S|-1}{2}\right\rceil$ departments to row 1 and $\left\lfloor\frac{|S|-1}{2}\right\rfloor$ departments to row 2 . The result follows from the star inequalities (1) for the LA.

Consequently, we can also simplify the calculation of the SPTlower-bound. For $i \in[n]$ we sort the departments in $S \subseteq[n] \backslash\{i\}$ by decreasing weights $w_{i k}, k \in S$, and assign the departments in that order as close as possible to $i$, i. e., a department with highest weight $w_{i k}, k \in S$, lies directly opposite $i$. We denote this lower bound by $W_{i}^{\text {sort }}(S)$.

If we know that two departments $i, j \in[n], i<j$, overlap and so lie exactly opposite due to the grid structure (Anjos et al., 2018), we can determine a lower bound for the weighted distances of $i$ and $j$ to the departments $S \subseteq[n] \backslash\{i, j\}$. For this we order the departments in $S$ by decreasing weight $w_{i k}+w_{j k}, k \in S$, and get a sequence $S_{i, j}^{\mathrm{E}-\mathrm{spt}}=\left(s_{1}, \ldots, s_{|S|}\right)$. With

$C^{\mathrm{E}-\mathrm{spt},(i, j)}(S)=C^{\text {basic }}\left(S_{i, j}^{\mathrm{E}-\mathrm{spt}},(1, \ldots, 1),(0,0,0,0)\right)$

we get

$W_{(i, j)}^{\mathrm{E}-\mathrm{spt}}(S):=\sum_{k=1}^{|S|}\left(w_{i s_{k}}+w_{j s_{k}}\right)\left(C_{S_{k}}^{\mathrm{E}-\mathrm{spt},(i, j)}(S)\right)$.

Proposition 20. Let $(n, w, \mathbf{1})$ be a DREFLP instance. Let $i, j \in[n]$, $i<j$, and $S \subseteq[n] \backslash\{i, j\}$, then for all DREFLP solutions $(r, q) \in$ $\mathcal{Q}(n, w, \mathbf{1})$ with $q_{i}=q_{j}$ we have

$W_{(i, j)}^{\mathrm{E}-\mathrm{spt}}(S) \leq \sum_{k \in S}\left(w_{i k}+w_{j k}\right)\left|q_{i}-q_{k}\right|$.

Proof. The result follows directly by Proposition 9 and its proof.

\subsection{3. $P R O P$}

Finally we have a look at row layout problems where the assignment of the departments to the rows is already known like the SRFLP, the PROP and the kPROP. We concentrate on the PROP in the description, but the other cases follow analogously.
For the PROP the lower bound calculation of $W_{(i, j)}^{\mathrm{dst}}$ and $W_{(i, j)}^{\mathrm{spt}}, i$, $j \in[n], i \neq j$, can be adapted as follows. Because the row assignment is fixed, we can split the calculation of the distances in inner-row and inter-row distances. Let $i \in[n]$ and $S \subseteq[n] \backslash\{i\}$. We first order the departments $S_{1}:=\left\{j \in S: r_{i}=r_{j}\right\} \subseteq S$ which are in the same row as $i$ increasingly by their lengths and get $S^{\text {inn }}=\left(s_{1}, \ldots, s_{\left|S_{1}\right|}\right)$ with length vector $\ell^{S^{\text {inn }}}$. Applying Algorithm 1 we get the completion times

$C^{\text {inn }, i}(S, \ell)=C^{\text {basic }}\left(S^{\text {inn }}, \ell^{S^{\text {inn }}},\left(\frac{\ell_{i}}{2}, \frac{\ell_{i}}{2}, \infty, \infty\right)\right)$

and with $\left(w_{i 1}^{\prime}, \ldots, w_{i\left|S_{1}\right|}^{\prime}\right)$ being a decreasingly sorted list of the weights $w_{i k}, k \in S_{1}$, the bounds

$$
\begin{aligned}
& W_{i}^{\mathrm{dst}-\mathrm{inn}}(S):=\sum_{k=1}^{\left|S_{1}\right|}\left(C_{S_{k}}^{\mathrm{inn}, i}(S, \ell)-\frac{\ell_{s_{k}}}{2}\right), \\
& W_{i}^{\mathrm{spt}-\mathrm{inn}}(S):=\sum_{k=1}^{\left|S_{1}\right|} w_{i k}^{\prime}\left(C_{S_{k}}^{\mathrm{inn}, i}(S, \ell)-\frac{\ell_{s_{k}}}{2}\right),
\end{aligned}
$$

For the inter-row distances we have to consider all possible departments lying opposite $i$. So let $j \in S$ with $r_{j} \neq r_{i}$ be fixed. Now order the remaining departments in the other row, i. e. $S_{2, j}:=\{k \in S$ : $\left.j \neq k, r_{i} \neq r_{k}\right\} \subseteq S$, by increasing length and get $S_{j}^{\text {int }}=\left(s_{1}^{\prime}, \ldots, s_{\left|S_{2, j}\right|}^{\prime}\right)$ with length vector $\ell^{S_{j}^{\text {int }}}$. Applying Algorithm 1 we get the completion times

$C^{\text {int },(i, j)}(S, \ell)=C^{\text {basic }}\left(S_{j}^{\text {int }}, \ell^{S_{j}^{\text {int }}},\left(\infty, \infty, \frac{\ell_{j}}{2}, \frac{\ell_{j}}{2}\right)\right)$.

As before, with $\left(w_{i 1}^{\prime \prime}, \ldots, w_{i\left|S_{2}\right|}^{\prime \prime}\right)$ being a decreasingly sorted list of the weights $w_{i k}, k \in S_{2 j}$, we get the bounds

$$
\begin{aligned}
W_{(i, j)}^{\mathrm{dst}-\mathrm{int}}(S) & :=\sum_{k=1}^{\left|S_{2, j}\right|}\left(C_{s_{k}^{\prime}}^{\mathrm{int},(i, j)}(S, \ell)-\frac{\ell_{s_{k}^{\prime}}}{2}\right), \\
W_{(i, j)}^{\mathrm{spt}-\mathrm{int}}(S): & : \sum_{k=1}^{\left|S_{2, j}\right|} w_{i k}^{\prime \prime}\left(C_{S_{k}^{\prime}}^{\mathrm{int},(i, j)}(S, \ell)-\frac{\ell_{s_{k}^{\prime}}}{2}\right) .
\end{aligned}
$$

Combining the inner-row and inter-row bounds leads to $W_{(i, j)}^{\text {P-dst }}(S)$ and $W_{(i, j)}^{\mathrm{P} \text {-spt }}(S)$. Similar adaptions are possible for improving $W_{(i, j)}^{\mathrm{sc}}(S)\left(W_{(i, j)}^{\mathrm{sc} 2}(S)\right.$ for the PROP is then the same as $\left.W_{(i, j)}^{\mathrm{sc}}(S)\right)$ in the case of fixed row assignments. We denote the improved PROP bounds by prepending "P-" to the name.

In order to compute a lower bound for the PROP we can sum up the lower bounds for inner-row distances of each of the departments to the others and divide this sum by two. To obtain a global lower bound for the inter-row distances in the PROP we sum up the weighted distances of each department in row 1 to row 2 , i. e., for each $i \in[n]$ with $r_{i}=1$ we compute $\min _{j \in[n], r_{j}=2} W_{(i, j)}^{\text {spt-int }}(S)$ and vice versa and we take the maximum value of these. By this method we do not have to divide the obtained value by two.

\section{A distance-based lower bounding ILP model}

For the SRFLP a distance-based model was introduced in Amaral and Letchford (2013) to compute a lower bound for the optimal solution value. The lower bound calculation was combined with some branch-and-cut algorithm. These results were based on investigations of the LA in Caprara et al. (2011) where the authors combined a distance model with combinatorial bounds. In Section 3.1 we introduce an ILP model consisting of distance variables and so called overlap variables, which is not a formulation for the DRFLP. The optimal solution value of that model is a lower bound for the optimal value of the DRFLP. Our model is based on 
our combinatorial lower bounds presented in the previous section. In the equidistant case of the DRFLP the model can be strengthened and we will also mention which adaptions are possible in the case of PROP or kPROP. We want to use the newly derived cutting planes in a branch-and-cut algorithm. So we describe in Section 3.2 appropriate separators.

\subsection{The Lower Bounding Model}

In the description of our ILP model we start with the variables. We use distance variables $d_{i j}=d_{j i} \geq 0, i, j \in[n], i<j$. In contrast to the literature, see, e. g., Fischer et al. (2019), Secchin and Amaral (2019), where left-right ordering variables were used, we use binary overlap variables $x_{i j}=x_{j i} \in\{0,1\}, i, j \in[n], i<j$. Two departments $i$ and $j$ overlap if their positions satisfy $\left|q_{i}-q_{j}\right|<\frac{\ell_{i}+\ell_{j}}{2}$. The associated variables have the following interpretation

$x_{i j}=\left\{\begin{array}{l}1, \text { departments } i \text { and } j \text { lie in different rows and overlap, } \\ 0, \text { otherwise. }\end{array}\right.$

We want to note that the model does not contain position variables for the departments.

It was proven in Fischer et al. (2019) that there always exists an optimal double-row layout where the distance from the left border of the leftmost department to the right border of the rightmost department is at most $M:=\sum_{i=\left\lfloor\frac{n+1}{3}\right\rfloor+1}^{n} \ell_{i}$ where the departments are sorted in ascending order according to their length. Apart from this we define a parameter $\kappa \in\{0,1\}$ which is one if and only if all department lengths are integral. This is the case in almost all test instances in the literature. Our lower bounding model for the DRFLP reads as follows.

$$
\begin{aligned}
& \min \sum_{\substack{i, j \in[n] \\
i<j}} w_{i j} d_{i j} \\
& \sum_{\substack{i, j \in S \\
i<j}} x_{i j} \leq|S|-1, \quad S \subseteq[n],|S| \geq 2,
\end{aligned}
$$$$
\sum_{j \in S \cup T} x_{i j} \leq|S|+1, \quad i \in[n], S \subset[n] \backslash\{i\} \text { with } \sum_{j \in S} \ell_{j} \geq \ell_{i},
$$$$
T:=\left\{j \in[n] \backslash(S \cup\{i\}): \ell_{j} \geq \max _{k \in S} \ell_{k}\right\},
$$

$d_{i j}+\left(\frac{\ell_{i}+\ell_{j}}{2}\right) x_{i j} \geq \frac{\ell_{i}+\ell_{j}}{2}$

$i, j \in[n], i<j$,

$d_{i j}+\left(M-\ell_{i}-\ell_{j}+\frac{1}{2} \kappa\right) x_{i j} \leq M-\frac{\ell_{i}+\ell_{j}}{2}, \quad i, j \in[n], i<j$,

$d_{i j}+d_{j k}-d_{i k} \geq 0, \quad i, j, k \in[n],|\{i, j, k\}|=3, i<k$,

$$
\sum_{j \in S} d_{i j} \geq \min _{j \in S} W_{(i, j)}^{\mathrm{dst}}(S), \quad i \in[n], S \subseteq[n] \backslash\{i\},
$$

$$
\sum_{j \in S} w_{i j} d_{i j} \geq \min _{j \in S} \max \left\{\begin{array}{l}
W_{(i, j)}^{\mathrm{spt}}(S), \\
W_{(i, j)}^{\mathrm{sc}}(S), \\
W_{(i, j)}^{\mathrm{sc} 2}(S)
\end{array}\right\}, \quad i \in[n], S \subseteq V_{i},
$$

$\sum_{\substack{i, j \in S \\ i<j}} d_{i j}+o_{S, 3} \sum_{\substack{i, j \in S \\ i<j}} x_{i j} \geq o_{S, 3}, \quad S \subset[n],|S|=3, o_{S, 3}=\sum_{i \in S} \ell_{i}+\min _{i \in S} \ell_{i}$,

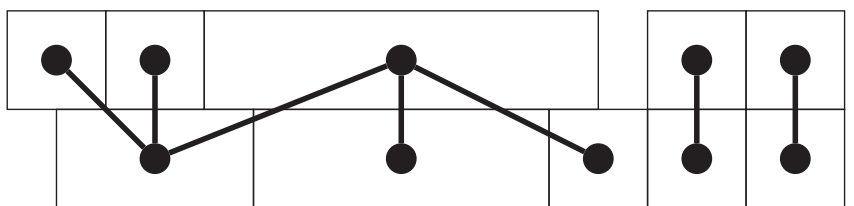

Fig. 6. Visualization of the forest associated to the overlap variables of a doublerow layout. Each node represents a department and there exists an edge between two different departments if and only if both overlap.

$$
\begin{gathered}
\sum_{\substack{i, j \in S \\
i<j}} d_{i j}+o_{S, 4} \sum_{\substack{i, j \in S \\
i<j}} x_{i j} \geq 0_{S, 4}, \\
o_{S, 4}=\frac{3}{2} \sum_{i \in S} \ell_{i}+2 \cdot \min _{\substack{i_{1}, i_{2} \in S \\
i_{1} \neq i_{2}}}\left(\ell_{i_{1}}+\ell_{i_{2}}\right) \\
\sum_{\substack{i, j \in S \\
i<j}} \ell_{i} \ell_{j} d_{i j}+o_{S} \sum_{\substack{i, j \in S \\
i<j}} x_{i j} \geq o_{S}, \quad S \subseteq[n],|S| \geq 3 \\
x_{i j}=x_{j i} \in\{0,1\}, \quad o_{S}=\frac{1}{6}\left(\left(\sum_{i \in S} \ell_{i}\right)^{3}-\sum_{i \in S} \ell_{i}^{3}\right) \\
d_{i j}=d_{j i} \geq 0, \quad \begin{array}{l}
i, j \in[n], i<j \\
i, j \in[n], i<j .
\end{array}
\end{gathered}
$$

The overlap variables in a double-row layout have to build a forest if we interpret them as edges in a graph where each department represents a single node of the associated graph and two nodes (departments) are connected by an edge if both overlap in the layout, see Fig. 6. We ensure this by the well-known subtour elimination constraints (9), see, e. g., Dantzig, Fulkerson, and Johnson (1954), Edmonds (1971), for the complete description of the forest polytope. But the forest also has to satisfy further properties concerning the degree of certain nodes. Let $i \in[n]$ be fixed and let $S \subset[n] \backslash\{i\}$ with $\Sigma_{j \in S} \ell_{j} \geq \ell_{i}$, i. e., the departments in $S$ are in total at least as long as department $i$, then at most $|S|+1$ departments of the set $S \cup\left\{k \in[n] \backslash(S \cup\{i\}): \ell_{k} \geq \max _{j \in S} \ell_{j}\right\}$ can overlap with $i$. This results in (10). So, for instance, a department $i$ can overlap with at most two departments that are at least as long as $i$ itself.

The distance and the overlap variables are coupled via (11) and (12). On the one hand, if two departments do not overlap, then the distance between the centers of both is at least the sum of half the lengths of the departments. On the other hand the distance of two departments that overlap cannot be larger than the sum of half the lengths of both departments. Assuming integral department length we can even enforce that this value is $\frac{1}{2}$ less because the overlap is then at least one half (the departments are arranged on the half grid according to Hungerländer \& Anjos, 2015). As used in previous layout models, see, e. g., Amaral and Letchford (2013), the distance variables have to satisfy the triangle inequalities (13). Furthermore, we use our combinatorial bounds to bound the sum of the (weighted) distances between all departments of some set $S \subset[n]$, see $(14)-(15)$.

If we know that certain departments do not overlap pairwise, then we can treat them as departments in an SRFLP instance and use constraints known to be valid for the SRFLP, see (16) and (17). For the validity of these inequalities and especially for the calculation of the right-hand side $o$ one compares all different orderings of the associated departments and counts how often the length of each single department appears. Inequalities (18) are an adapted version of the so called clique inequalities presented in Amaral and Letchford (2013) for the SRFLP. Note, inequalities (16)-(18) are 
trivially satisfied if one of the associated $x$-variables equals one. Finally, we have the integrality of the overlap variables (19).

\subsubsection{Adaptations of Our Lower Bounding Model for the DREFLP}

For the DREFLP there always exists an optimal solution on the grid (Anjos et al., 2018). Therefore we can restrict to solutions where two departments overlap if and only if they lie directly opposite each other. So the interpretation of our overlap variables changes to

$x_{i j}^{e}=x_{j i}^{e}= \begin{cases}1, & \text { if } i \text { and } j \text { lie directly opposite each other, } \\ 0, & \text { otherwise, }\end{cases}$

$i, j \in[n], i<j$. Our model specialized to the DREFLP reads as follows.

$\min \sum_{\substack{i, j \in[n] \\ i<j}} w_{i j} d_{i j}$

(13),

$$
\sum_{\substack{j \in[n] \\ j \neq i}} x_{i j}^{e} \leq 1, \quad i \in[n]
$$

$\sum_{\substack{i, j \in[n] \\ i<j}} x_{i j}^{e} \leq\left\lfloor\frac{n}{2}\right\rfloor$

$\sum_{\substack{i, j \in[n] \\ i<j}} x_{i j}^{e} \geq n-\left\lceil\frac{2 n}{3}\right\rceil+1, \quad n \geq 9$,

$d_{i j}+x_{i j}^{e} \geq 1$

$i, j \in[n], i<j$,

$d_{i j}+M x_{i j}^{e} \leq M$,

$i, j \in[n], i<j$,

$\sum_{\substack{i, j \in[n] \\ i<j}} d_{i j} \geq \begin{cases}\frac{(n+1) n(n-1)}{12}, & n \text { odd } \\ \frac{(n+2) n(n-2)}{12}, & n \text { even }\end{cases}$

$\sum_{j \in[n] \backslash\{i\}} d_{i j} \geq\left\lfloor\frac{\left(\left\lceil\frac{n-2}{2}\right\rceil+1\right)^{2}}{4}\right\rfloor+\left\lfloor\frac{\left(\left\lfloor\frac{n-2}{2}\right\rfloor+1\right)^{2}}{4}\right\rfloor, \quad i \in[n]$,

$\sum_{j \in S} w_{i j} d_{i j} \geq W_{i}^{\text {sort }}(S), \quad i \in[n], S \subseteq V_{i}$,

$\sum_{k \in S}\left(w_{i k} d_{i k}+w_{j k} d_{j k}\right)-x_{i j}^{e}\left(W_{(i, j)}^{\mathrm{E}-\mathrm{spt}}(S)\right) \geq 0$,

$i, j \in[n], i<j, S \subseteq[n] \backslash\{i, j\}$,

$\sum_{\substack{i, j \in S \\ i<j}} d_{i j}+o_{S} \sum_{\substack{i, j \in S \\ i<j}} x_{i j}^{e} \geq o_{S}, \quad S \subseteq[n],|S| \geq 3, o_{S}=\frac{1}{6}\left(|S|^{3}-|S|\right)$,

$x_{i j}^{e}=x_{j i}^{e} \in\{0,1\}$,

$i, j \in[n], i<j$.

There always exists an optimal solution to the DREFLP on the grid (Anjos et al., 2018). So each department may overlap with at most one department, see (20). Additionally, we can bound the total sum of the overlap variables by $\left\lfloor\frac{n}{2}\right\rfloor$. We can also generalize (21) to $\sum_{i, j \in S, i<j} x_{i j}^{e} \leq\left\lfloor\frac{|S|}{2}\right\rfloor$ for sets $S \subseteq[n]$. Constraints (20) and (21) can be seen as a strengthened version of inequalities (9) and (10). For $n \geq 9$ there always exists an optimal equidistant double-row layout which uses at most $\left\lceil\frac{2 n}{3}\right\rceil-1$ columns of the grid (Anjos et al., 2018). It follows that at least $n-\left\lceil\frac{2 n}{3}\right\rceil+1$ columns contain two departments, see (22). If two departments overlap, then their distance is zero, see (24), and at least one otherwise, see (23). In the unweighted case of the DREFLP, i. e., if all weights are equal to one, an optimal solution for this problem can be derived by arranging $\left\lfloor\frac{n+1}{2}\right\rfloor$ departments in row 1 and $\left\lceil\frac{n-1}{2}\right\rceil$ departments in row 2. So the sum of the weighted distances can be calculated. This value is a lower bound for the sum of the distances in the DREFLP, see (25) (for details we refer to the upcoming paper Dahlbeck, Fischer, \& Hungerländer, 2020). Note that we are not aware of a similar result for the DRFLP. So we take advantage of the DREFLP structure here. Apart from this we can bound the sum of the distances of some $i \in[n]$ to all departments $S \subseteq V_{i}$ from below using our combinatorial bounds, see (27). If two departments $i, j \in[n]$, $i<j$, overlap, we can use $W_{(i, j)}^{\mathrm{E}-\mathrm{spt}}(S)$ defined in (8) as a lower bound for the weighted distances of $i$ and $j$ to the departments $S \subseteq[n] \backslash\{i$, $j$ \}, see (28). If $i$ and $j$ do not overlap, inequality (28) is redundant. Inequalities (29) are the clique inequalities (18) used before with $\ell_{i}=1, i \in[n]$.

\subsubsection{Adaptations of Our Model for the PROP}

For the PROP further improvements of our lower bounding model (9)-(18) are possible because the row assignment of the departments is given. So one can hope to achieve smaller gaps for the PROP in comparison to the DRFLP, especially if the rows are balanced, i. e., the sum of the lengths of the departments in row 1 is close to the corresponding sum in row 2. Balanced layouts are of special interest in practice because in factory planning the size of the factory influences the production costs (Langevin, Montreuil, \& Riopel, 1994).

For the departments in some row $r \in \mathcal{R}$ we can use results from the SRFLP literature because the departments are arranged without spaces. For bounding the sum of the distances of the departments we use the fact that the SRFLP with $w_{i j}=1, i, j \in[n], i<j$, can easily be solved in polynomial time.

Theorem 21 (Samarghandi \& Eshghi (2010)). Let $(n, w, \ell)$ with $w_{i j}=1, i, j \in[n], i<j$, be an SRFLP instance. Let a single-row layout with associated permutation $\pi^{*}$ be derived by sorting the departments in an ascending order according to their lengths and placing the first department in the middle and the remaining ones right and left in an alternating manner to the ones already assigned, see Fig. 7. Then this layout is optimal with objective value $\frac{n-1}{2} \sum_{i=1}^{n} \ell_{i}+$ $\sum_{k=2}^{n-1}(k-1)(n-k) \ell_{\pi^{*}(k)}$.

This result was stated in Samarghandi and Eshghi (2010) but not proven correctly. So we present a new proof here.

Proof. Let an arbitrary permutation $\pi:[n] \rightarrow[n]$ of the departments be given where $\pi(k)$ denotes the $k$ th department from the left border. The distance $d_{\pi(i) \pi(j)}$ of two departments $\pi(i), \pi(j), i$, $j \in[n], \pi(i)<\pi(j)$, equals $d_{\pi(i) \pi(j)}=\frac{\ell_{\pi(i)}+\ell_{\pi(j)}}{2}+\sum_{k=i+1}^{j-1} \ell_{\pi(k)}$. Then with $C:=\sum_{i, j=1, i<j}^{n} \frac{\ell_{i}+\ell_{j}}{2}=\frac{n-1}{2} \sum_{i=1}^{n} \ell_{i}$ we get

$$
\begin{aligned}
\sum_{\substack{i, j=1 \\
i<j}}^{n} w_{i j} d_{i j} & =\sum_{\substack{i, j=1 \\
i<j}}^{n} d_{i j}=\sum_{\substack{i, j=1 \\
i<j}}^{n} d_{\pi(i) \pi(j)}=C+\sum_{\substack{i, j=1 \\
i<j}}^{n} \sum_{k=i+1}^{j-1} \ell_{\pi(k)}=C \\
& +\sum_{k=2}^{n-1}(k-1)(n-k) \ell_{\pi(k)} .
\end{aligned}
$$




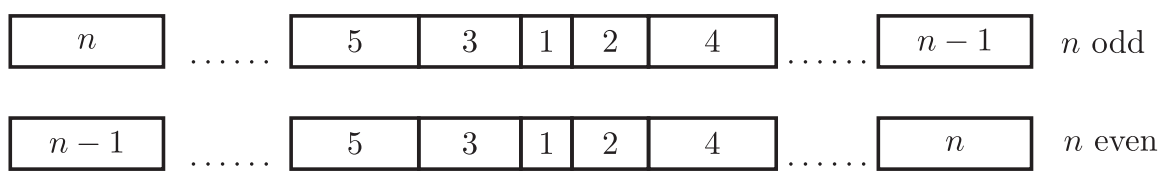

Fig. 7. Visualization of optimal solutions (and permutations $\pi^{*}$ of $[n]$ ) for SRFLP instances with weights $w_{i j}=1, i, j \in[n], i<j$, and $\ell_{i} \leq \ell_{i+1}$ for $i \in[n-1]$ for the different parities of $n$.

For proving the optimality of $\pi^{*}$ we consider the quadratic function $f:[2, n-1] \rightarrow \mathbb{R}$ with $f(k)=(k-1)(n-k)$. The function $f$ is strongly concave with its unique maximum point at $k^{*}=\frac{n+1}{2} \in$ $[2, n-1]$ and is symmetric to $k^{*}$. Then $(31)$ is minimized by $\pi^{*}$ and the result follows.

Let $R_{k}, k \in \mathcal{R}$, denote the indices of the departments which are assigned to row $k$. Then we can use the following constraints for PROP.

$$
\begin{aligned}
& \sum_{\substack{i, j \in R_{k} \\
i<j}} \ell_{i} \ell_{j} d_{i j}=\frac{1}{6}\left(\left(\sum_{i \in R_{k}} \ell_{i}\right)^{3}-\sum_{i \in R_{k}} \ell_{i}^{3}\right), \quad k \in \mathcal{R}, \\
& \sum_{\substack{i, j \in R_{k} \\
i<j}} d_{i j} \geq \sum_{\substack{i, j \in R_{k} \\
i<j}} \frac{\ell_{i}+\ell_{j}}{2}+\sum_{z=2}^{\left|R_{k}\right|-1}(z-1)\left(\left|R_{k}\right|-z\right) \ell_{\pi^{*, k}(z)}, \quad k \in \mathcal{R},
\end{aligned}
$$

$$
d_{i j} \geq \frac{\ell_{i}+\ell_{j}}{2}, \quad k \in \mathcal{R}, i, j \in R_{k}, i<j
$$

$$
d_{i j} \leq\left(\sum_{z \in R_{k}} \ell_{z}\right)-\frac{\ell_{i}+\ell_{j}}{2}, \quad k \in \mathcal{R}, i, j \in R_{k}, i<j,
$$

$d_{i j} \leq \max \left\{\sum_{z \in R_{1}} \ell_{z}, \sum_{z \in R_{2}} \ell_{z}\right\}-\frac{\ell_{i}+\ell_{j}}{2}, \quad i \in R_{1}, j \in R_{2}$.

Treating the departments in the same row as a SRFLP we can use the clique Eq. (32) as shown to be valid for the SRFLP in (Amaral \& Letchford, 2013) (we can still use (18)). As already mentioned the sum of the distances between departments in the same row can be bounded using Theorem 21 where in (33) an optimal layout of departments $R_{k}$ of the unweighted SRFLP according to Theorem 21 is denoted by $\pi^{*, k}$.

Of course, two departments in the same row satisfy a minimal distance condition, see (34). So we do not need (11) for departments in the same row. Note that in the PROP one can bound distances between two departments in the same row (35) and also in different rows (36) because the departments are arranged without spaces and with a fixed left border. So we do not need (12) for departments lying in the same row. Apart from this we can use the improved combinatorial lower bounds in (14) and (15). For a summary of the complete PROP model we refer to the appendix.

\subsection{Separation}

In this section we describe a branch-and-cut algorithm which is based on the inequalities (9)-(18) and we explain for which subsets $S \subseteq[n]$ the constraints are indeed used in the calculation of the lower bounds. We include inequalities (11) from the beginning as well as inequalities (9) for $S=[n]$ and (15) for $S=V_{i}$. Inequalities (14) are included for $S=V_{i}$ and $S=[n]$. Furthermore, we add all triangle inequalities (13).
The remaining constraints are separated in the following way. We separate inequalities (12) by complete enumeration. It is wellknown that the problem to decide whether the vector of the $x$ variables is contained in the forest polytope, i. e., the convex hull over all incidence vectors of forests of a complete undirected graph with $n$ nodes, can be solved in polynomial time, see, e. g., Schrijver (2003) pp. 880-881. Indeed, assuming non-negativity of the $x$ variables, one can determine a maximally violated subtour elimination constraint (9) solving special minimum cut problems on an associated directed graph.

There are potentially exponentially many inequalities of type (10), so we use the following heuristic approach. First we sort the departments according to their lengths in ascending order, i. e., $\ell_{1} \leq \ell_{2} \leq \ldots \leq \ell_{i} \leq \ldots \leq \ell_{n}$. For each $i \in[n]$ we determine the set $\bar{S}_{i}:=\left\{j \in[n] \backslash\{i\}: \ell_{j} \geq \ell_{i}\right\}$ and separate constraints

$\sum_{j \in \bar{S}_{i}} x_{i j} \leq 2$

explicitly. These inequalities imply all inequalities of (10) with $|S|=1$. For $2 \leq|S| \leq 3$ we add an inequality if $S$ satisfies $\Sigma_{j \in S} \ell_{k} \geq \ell_{i}$ and $\Sigma_{j \in S \backslash\{k\}} \ell_{j}<\ell_{i}$ for all $k \in S$, because the inequality is redundant otherwise.

We separate inequalities (16) and (17) by brute-force enumeration. In Amaral and Letchford (2013) it is conjectured that the separation problem of the general clique inequalities of the SRFLP is $\mathcal{N P}$-hard. For this reason we restrict to sets $S$ of size three and four in (18) and check all these inequalities by complete enumeration.

It remains the usage of our combinatorial lower bounds in (14) and (15). Given a relaxation $\bar{x}, \bar{d}$, we construct two sets for fixed $i \in[n]$. At first, we consider all departments which are according to the distance variables $\bar{d}$ close to $i$, i. e., $S_{1}^{i}=\{j \in[n] \backslash\{i\}$ : $\left.\bar{d}_{i j} \leq \frac{\ell_{i}+\ell_{j}}{2}\right\}$ and previous tests, which are not included in this paper, show that it is worth to check $S_{2}^{i}=\left\{j \in[n] \backslash\{i\}: \bar{d}_{i j} \leq \ell_{i}+\ell_{j}\right\}$ as well.

For the PROP similar separation strategies were used. Testing our DREFLP model with a branch-and-cut algorithm we include inequalities (13), (20)-(23), (25) and (26) from the beginning as well as inequalities (27) for $S=V_{i}$. We separate inequalities (24), (28) by brute force enumeration and as done in our DRFLP lower bounding model we restrict inequalities (29) to sets $|S|=3$ and $|S|=4$ and check all these inequalities by complete enumeration.

\section{Computational results}

In this section we present our computational experiments implemented in C++. We used Cplex 12.8 (IBM, 2018) as an ILP solver. All results were conducted on a 2.30 gigahertz dual-core computer running on Debian GNU/Linux 8 in single processor mode.

We compare our combinatorial lower bounds (7) as well as our branch-and-cut algorithm, described in Section 3.2, interrupted after a time limit of a few minutes on instances from the literature as well as on randomly generated medium-sized and large doublerow instances. All instances are available from the authors.

In this paper we present the first non-trivial lower bounds for the DRFLP. In order to show the strength of our lower bound approaches we want to compare to lower bounds derived using 
Table 1

Results for instances from the literature where the optimal solution values are known (Fischer et al., 2019). The gaps are given in percent.

\begin{tabular}{|c|c|c|c|c|c|c|c|c|c|}
\hline Instance & Source & Amaral & (7) & $I L P_{3 \min }$ & optimal & heuristic & Gap $_{\text {Ama. }}$ & $\operatorname{Gap}_{(7)}$ & $\operatorname{Gap}_{I L P}$ \\
\hline$s 9$ & Amaral $(2012,2013 a)$ & 1179.0 & 778.0 & 1093.0 & 1179.0 & 1183.5 & 0 & 34.01 & 7.29 \\
\hline s9h & Amaral $(2012,2013 a)$ & 2293.0 & 1636.5 & 1713.5 & 2293.0 & 2309.5 & 0 & 28.63 & 25.27 \\
\hline s10 & Amaral $(2012,2013 a)$ & 1351.0 & 822.0 & 1213.5 & 1351.0 & 1392.0 & 0 & 39.16 & 10.18 \\
\hline s11 & Amaral $(2012,2013 a)$ & 3424.5 & 1998.5 & 2658.5 & 3424.5 & 3427.5 & 0 & 41.64 & 22.37 \\
\hline Am12a & Amaral $(2012,2013 a)$ & 1493.0 & 795.0 & 1136.5 & 1493.0 & 1556.5 & 0 & 46.75 & 23.88 \\
\hline Am12b & Amaral $(2012,2013 a)$ & 1606.5 & 854.0 & 1223.0 & 1606.5 & 1610.5 & 0 & 46.84 & 23.87 \\
\hline Am13a & Amaral (2012) & 2175.5 & 1213.0 & 1682.5 & 2456.5 & 2624.5 & 11.44 & 50.62 & 31.51 \\
\hline Am13b & Amaral (2012) & 2486.0 & 1294.5 & 1980.0 & 2864.0 & 2886.5 & 13.20 & 54.80 & 30.87 \\
\hline Am14a & Secchin and Amaral (2019) & 1757.0 & 1483.5 & 2082.0 & 2904.0 & 2920.0 & 39.50 & 48.92 & 28.31 \\
\hline Am14b & Secchin and Amaral (2019) & 1451.5 & 1386.0 & 1893.5 & 2736.0 & 2736.0 & 46.95 & 49.34 & 30.79 \\
\hline Am15_1 & Amaral (2006) & 1554.5 & 1543.0 & 2209.0 & 3195.0 & 3272.0 & 51.35 & 51.71 & 30.86 \\
\hline HK15 & Heragu and Kusiak (1991) & 9059.0 & 7900.0 & 11777.0 & 16570.0 & 16740.0 & 45.33 & 52.32 & 28.93 \\
\hline P16_a & Amaral (2013b) & 2247.5 & 3676.5 & 4937.0 & 7365.5 & 7466.5 & 69.49 & 50.08 & 32.97 \\
\hline P16_b & Amaral (2013b) & 1598.5 & 2968.0 & 4053.0 & 5870.5 & 6306.0 & 72.77 & 49.44 & 30.96 \\
\hline
\end{tabular}

Table 2

Results for randomly generated double-row instances with integral department lengths between 1 and 15 . We display the average values over ten instances each. The average gaps are given in percent. Note that for six instances with $n=50$ and density $10 \%$ we had to enlarge the time limit to five minutes for ILPure.

\begin{tabular}{|c|c|c|c|c|c|c|c|c|c|c|c|}
\hline Instance & Amaral & (7) & $I L P_{3 \min }^{\text {pure }}$ & $I L P_{3 \min }$ & $I L P_{10 \min }$ & heuristic & Gap $_{\text {Ama. }}$ & $\operatorname{Gap}_{(7)}$ & Gap ILPp & $\operatorname{Gap}_{I L P}$ & time heur. \\
\hline $20_{10}$ & 338.79 & 289.86 & 468.14 & 500.60 & 505.45 & 617.35 & 38.13 & 51.29 & 19.11 & 14.21 & 3.15 \\
\hline $20_{50}$ & 1527.12 & 3202.19 & 3626.43 & 5469.71 & 5495.22 & 8506.05 & 81.89 & 62.45 & 57.23 & 35.39 & 5.21 \\
\hline $20_{100}$ & 3310.24 & 10431.77 & 8529.66 & 13986.62 & 14058.16 & 20943.10 & 83.95 & 50.29 & 59.15 & 32.84 & 7.09 \\
\hline $30_{10}$ & 331.84 & 802.17 & 1018.18 & 1514.81 & 1520.63 & 2544.90 & 86.45 & 68.37 & 58.93 & 39.48 & 15.30 \\
\hline $30_{50}$ & 2101.71 & 9839.95 & 8233.34 & 17484.33 & 17498.97 & 30996.05 & 93.22 & 68.33 & 73.41 & 43.56 & 30.81 \\
\hline $30_{100}$ & 4291.18 & 34059.95 & 18287.52 & 43916.72 & 43931.98 & 69736.45 & 93.85 & 51.20 & 73.75 & 37.03 & 58.69 \\
\hline $40_{10}$ & 432.03 & 1796.76 & 1904.74 & 3801.49 & 3973.69 & 8007.10 & 94.42 & 77.08 & 75.90 & 49.43 & 55.09 \\
\hline $40_{50}$ & 3043.79 & 22713.90 & 13841.45 & 42319.98 & 42332.02 & 76055.40 & 95.99 & 70.16 & 81.78 & 44.39 & 142.88 \\
\hline $40_{100}$ & 6090.44 & 80325.81 & 29964.70 & 102936.41 & 102974.79 & 167635.30 & 96.36 & 52.06 & 82.11 & 38.56 & 280.10 \\
\hline $50_{10}$ & 17.82 & 3184.06 & 3053.28 & 6667.47 & 8725.34 & 18006.80 & 99.89 & 82.23 & 82.89 & 51.39 & 159.35 \\
\hline $50_{50}$ & 52.36 & 42486.84 & 22313.42 & 79545.26 & 79550.13 & 149788.90 & 99.97 & 71.61 & 85.08 & 46.84 & 427.47 \\
\hline $50_{100}$ & 63.18 & 156449.80 & 48166.27 & 199949.20 & 199949.20 & 328566.70 & 99.98 & 52.37 & 85.32 & 39.13 & 1009.80 \\
\hline
\end{tabular}

Table 3

Results for randomly generated double-row instances with integral department lengths between 5 and 10 . We display the average values over ten instances each. The average gaps are given in percent.

\begin{tabular}{|c|c|c|c|c|c|c|c|c|c|c|c|}
\hline Instance & Amaral & (7) & $I L P_{3 \min }^{\text {pure }}$ & $I L P_{3 \min }$ & $I L P_{10 \min }$ & heuristic & $\operatorname{Gap}_{\text {Ama. }}$ & $\operatorname{Gap}_{(7)}$ & Gap $_{\text {ILPpure }}$ & $\operatorname{Gap}_{I L P}$ & time heur. \\
\hline $20_{10}$ & 251.69 & 334.18 & 402.79 & 502.13 & 502.13 & 674.85 & 54.01 & 47.03 & 33.92 & 21.12 & 2.80 \\
\hline $20_{50}$ & 1276.73 & 4015.68 & 3428.96 & 6047.42 & 6054.10 & 8832.65 & 85.52 & 54.61 & 61.15 & 31.52 & 5.08 \\
\hline $20_{100}$ & 2424.67 & 13225.15 & 8506.21 & 15833.20 & 15842.39 & 22573.75 & 89.24 & 41.45 & 62.31 & 29.85 & 7.20 \\
\hline $30_{10}$ & 310.58 & 950.50 & 890.62 & 1723.57 & 1729.66 & 2905.65 & 88.49 & 65.80 & 67.78 & 39.01 & 15.73 \\
\hline $30_{50}$ & 1838.96 & 12560.22 & 7744.96 & 21068.41 & 21070.50 & 32132.35 & 94.27 & 60.95 & 75.88 & 34.42 & 31.74 \\
\hline $30_{100}$ & 3663.20 & 43408.32 & 18239.38 & 53278.66 & 53278.66 & 78339.90 & 95.32 & 44.57 & 76.71 & 31.98 & 50.78 \\
\hline $40_{10}$ & 446.25 & 2072.82 & 1628.15 & 4171.48 & 4176.62 & 8168.50 & 94.32 & 74.07 & 79.66 & 50.86 & 54.25 \\
\hline $40_{50}$ & 3039.25 & 28568.01 & 13403.33 & 50194.18 & 50194.18 & 79917.90 & 96.20 & 64.26 & 83.22 & 37.15 & 135.46 \\
\hline $40_{100}$ & 5760.78 & 101189.12 & 29338.09 & 125597.20 & 125597.20 & 187431.80 & 96.93 & 46.00 & 84.35 & 32.98 & 242.43 \\
\hline $50_{10}$ & 11.56 & 3652.35 & 2802.76 & 7695.32 & 7943.25 & 17620.70 & 99.94 & 79.24 & 84.04 & 54.58 & 176.94 \\
\hline $50_{50}$ & 21.36 & 53907.31 & 21815.19 & 96871.04 & 96871.04 & 160570.10 & 99.99 & 66.42 & 86.41 & 39.64 & 383.10 \\
\hline $50_{100}$ & 51.45 & 194844.10 & 47001.25 & 243675.60 & 243675.60 & 368747.80 & 99.99 & 47.15 & 87.25 & 33.90 & 886.15 \\
\hline
\end{tabular}

mixed-integer linear programming techniques. Because the use of the enumerative approach of Fischer et al. (2019), the best exact DRFLP approach, for computing lower bounds is out of scope due to the exponential number of subproblems that have to be solved (approximately), we compare our lower bounds to the lower bounds that can be achieved via branch-and-cut on the model presented in Amaral (2013a) within a given time limit of one hour on our computer. We decided to use the weaker model presented in Amaral (2013a) and not to use the model in Secchin and Amaral (2019) because this contains a huge number of variables and constraints for larger $n$ and so even the calculation of the root node value was rather time-consuming in our tests. Further note that we do not test instances from Chung and Tanchoco (2010) and Murray et al. (2013) because there only double-row instances with clearance conditions were considered.

Since only smaller instances were solved to optimality in the literature (see Table 1), we generate random instances with $n \in\{20$,
$30,40,50\}$. To obtain a wide set of random instances we set the transport density to $10 \%, 50 \%$ and $100 \%$ and we choose integer transport weights randomly between 1 and 10 . The integral lengths of the departments are chosen randomly between 1 and 15 (see Table 2) as well as between 5 and 10 (see Table 3). For each type we created ten instances. We denote these instances by $n_{k}$ where $n$ is the number of departments and $k$ is the transport density. The first column "Instance" of the tables displays the instance name. For the instances from the literature the second column contains its source. The lower bound value obtained by applying Cplex with a given time limit of one hour for the DRFLP formulation in Amaral (2013a) is given in column "Amaral". Apart from this the tables contain our combinatorial bound (7) as well as the lower bounds derived using branch-and-cut for our lower bounding model within a given time limit of three resp. ten minutes, see columns "ILP $P_{3 \min }$ " and "ILP 10 min". In order to show that our combinatorial lower bounds significantly strengthen our lower 
Table 4

Results for equidistant instances from the literature (Anjos et al., 2018; Yu \& Sarker, 2003). The upper bounds "best ub" are taken from Anjos et al. (2018). We compared our lower bounding model with the ILP and the SDP from Anjos et al. (2018) with a given time limit of three hours. The values of (7) and $I L P_{3 \min }$ are rounded to integers.

\begin{tabular}{llllllll}
\hline Instances & (7) for DREFLP & $I_{3 \min }$ & best ub & $\operatorname{Gap}_{(7)}$ & $\operatorname{Gap}_{I L P}$ & Gap $_{\text {Anjos ILP } 3 h}$ & Gap $_{S D P 3 h}$ \\
\hline$Y_{20}$ & 4301 & 5821 & 6046 & 28.86 & 3.72 & 0.00 & 0.00 \\
$Y_{25}$ & 7032 & 9887 & 10170 & 30.86 & 2.78 & 1.22 & 0.36 \\
$Y_{30}$ & 9237 & 13315 & 13790 & 33.02 & 3.44 & 2.78 & 0.14 \\
$Y_{35}$ & 12607 & 18595 & 19087 & 33.95 & 2.58 & 21.27 & 0.26 \\
$Y_{40}$ & 15332 & 22809 & 23739 & 35.41 & 3.92 & 23.88 & 0.37 \\
$Y_{45}$ & 19952 & 29639 & 31442 & 36.54 & 5.73 & 26.35 & 0.65 \\
$Y_{50}$ & 25839 & 39450 & 41517 & 37.76 & 4.98 & 28.35 & 0.62 \\
\hline
\end{tabular}

Table 5

Results for equidistant instances from the literature (Anjos \& Yen, 2009). We compare to the best upper bounds, gaps and SDP lower bounds that are derived using the approach presented in Anjos et al. (2018) with a time limit of three hours.

\begin{tabular}{lllllll}
\hline Instances & (7) for DREFLP & $I_{1 L P_{15 \min }}$ & best ub & $\operatorname{Gap}_{(7)}$ & $\operatorname{Gap}_{\text {ILP }}$ & $\operatorname{Gap}_{\text {SDP } 3 h}$ \\
\hline sko42-1 & 5957 & 11717 & 12731 & 53.21 & 7.96 & 0.72 \\
sko49-1 & 9142 & 18736 & 20512 & 55.43 & 8.66 & 1.91 \\
sko56-1 & 13942 & 29201 & 31988 & 56.41 & 8.71 & 1.95 \\
sko64-1 & 20705 & 43408 & 48574 & 57.37 & 10.64 & 4.27 \\
\hline
\end{tabular}

\section{Table 6}

Results for equidistant instances from the literature (Anjos \& Yen, 2009). We compare to the best upper bounds, gaps and SDP lower bounds that are derived using the approach presented in Anjos et al. (2018) with a time limit of three hours. Because of the large $n$ we use a time limit of one hour for our ILP lower bounding approach.

\begin{tabular}{lllllll}
\hline Instances & (7) for DREFLP & $I_{L P}$ & best ub & $\operatorname{Gap}_{(7)}$ & $\operatorname{Gap}_{I L P}$ & $\operatorname{Gap}_{S D P 3 h}$ \\
\hline sko72-1 & 29912 & 61905 & 69621 & 57.04 & 11.08 & 4.87 \\
sko81-1 & 43114 & 89288 & 102793 & 58.06 & 13.14 & 8.78 \\
\hline
\end{tabular}

bounding model we tested our ILP without using the combinatorial results from Section 2. These results can be found in column $I L P_{3 \mathrm{~min}}^{\text {pure }}$. On order to obtain upper bounds we applied our heuristic to all instances. The value of the best solution determined via our heuristic is given in column "heuristic" and the time spent for the heuristic in seconds is given in column "time heur.". The optimal solution values are known for the instances from the literature considered in Table 1. They are given in column "optimal". For the convenience of the reader we also calculate the average gaps. The gaps are calculated via

$G a p=\frac{\text { upper bound }- \text { lower bound }}{\text { upper bound }} \cdot 100$,

and are given in percent. "Gap $A$ Ama." refers to the average gaps using Cplex for the model in Amaral (2013a) after a time limit of one

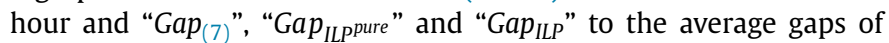
our combinatorial lower bound and of our lower bounding model without and with the use of the combinatorial lower bounds, respectively.

In order to obtain upper bounds we use a heuristic approach similar to the one in Chung and Tanchoco (2010). Note that, given the row assignment and the order of the departments in each row, we only need to solve a small linear program to obtain the exact position of the departments and hence possible spaces between neighboring departments, see, e. g., Murray et al. (2013). In Chung and Tanchoco (2010) five ways for determining a DRFLP start solution were presented. For each instance we test all five variants and afterwards apply a 1-opt and a 2-opt heuristic to a best start layout, combined with determining the best positions via the associated linear program. In the 1-opt improvement heuristic, considering department $i \in[n]$ we fix the row assignment and the ordering of all departments $[n] \backslash\{i\}$ and determine a best position for $i$. For each possible position (usually there are $n+1$ choices) we calcu- late the associated spaces with the mentioned linear program. In the 2-opt we exchange two departments and apply our linear program. We only accept changes if the objective value is improved.

\subsection{Results for the DRFLP}

In Table 1 we show results for some DRFLP instances from the literature, see, e. g., Fischer et al. (2019), where optimal solutions are known. Since the instances are rather small, the heuristic needs less than 20 seconds for each instance. The gap of our combinatorial lower bounds (7) are close to $50 \%$ and by our branch-and-cut algorithm we reduce the gap to between $28 \%$ and $33 \%$. The heuristically determined solutions are rather good, but even for these small instances the heuristic could only determine an optimal solution in one case.

Tables 2 and 3 show that our combinatorial lower bounds, which were computed in less than one second, clearly outperform the lower bounds obtained via using Cplex within a time limit of one hour for the DRFLP formulation in Amaral (2013a) on the randomly generated instances. These lower bounds are rather weak because of big-M type constraints and a quadratic number of binary decision variables and so the gaps are close to $100 \%$ for large $n$. Using branch-and-cut to improve our bounds allows a significant strengthening to final gaps between $14 \%$ and 55\%. For the ILP variant that does not use the combinatorial bounds the gaps are much higher and grow by increasing the number of departments. For instances with at least 40 departments the average gaps are higher than $70 \%$. Regarding (7) and our ILP the gaps are smaller for dense instances. Enlarging the time limit for our ILP approach from 3 to 10 minutes usually has only a very small effect on the bound. So three minutes seem to be a good value (for the larger instances this is even faster than our heuristic). Comparing the gaps in Tables 2 and 3 the instance type does not seem to have a large 
Table 7

Results for PROP instances from the literature (Amaral, 2013b; Fischer et al., 2019): The instance name includes the number of departments $n$. The first $\left\lfloor\frac{n}{t}\right\rfloor$ departments of an instance are assigned to row 1 . The table shows the average values of our lower bounds using the variant of (7) adapted for the PROP and using our lower bounding approach $\left(I L P_{10 \mathrm{sec}}\right)$, average optimal solution values ("optimal") and average gaps ("Gap ILP") over five instances each (and over two instances for $n=16$ and $n=20$ ). The gaps are given in percent.

\begin{tabular}{llllll}
\hline Instance & $i$ & (7) for PROP & ILP $P_{10 \mathrm{sec}}$ & optimal & GaP $_{\text {ILP }}$ \\
\hline P16 & 2 & 3756.80 & 6554.99 & 6934.75 & 5.46 \\
P16 & 3 & 4598.28 & 7298.76 & 9452.25 & 22.99 \\
P16 & 4 & 5028.68 & 8103.06 & 10522.75 & 23.33 \\
P16 & 5 & 5350.14 & 8797.24 & 11767.75 & 25.44 \\
P20 & 2 & 6587.88 & 11322.10 & 12772.75 & 11.36 \\
P20 & 3 & 8455.90 & 12799.60 & 17520.75 & 26.69 \\
P20 & 4 & 9184.90 & 14052.45 & 20493.25 & 31.12 \\
P20 & 5 & 9815.33 & 15375.70 & 22558.75 & 31.76 \\
P21 & 2 & 5722.76 & 9870.59 & 11109.40 & 10.98 \\
P21 & 3 & 6792.91 & 11066.58 & 13297.20 & 16.64 \\
P21 & 4 & 7887.36 & 13195.82 & 17310.80 & 23.63 \\
P21 & 5 & 8203.20 & 13665.52 & 19159.40 & 28.33 \\
P22 & 2 & 7203.15 & 12479.41 & 14090.30 & 11.48 \\
P22 & 3 & 8729.44 & 14212.50 & 18295.50 & 22.24 \\
P22 & 4 & 9941.98 & 16823.44 & 22567.10 & 25.48 \\
P22 & 5 & 10232.11 & 17523.40 & 24132.10 & 27.25 \\
P23 & 2 & 7590.92 & 13483.86 & 15048.40 & 10.52 \\
P23 & 3 & 9604.89 & 15550.08 & 20248.40 & 23.19 \\
P23 & 4 & 10777.65 & 18001.96 & 25070.60 & 28.11 \\
P23 & 5 & 11171.86 & 19012.92 & 27313.80 & 30.13 \\
P24 & 2 & 8604.50 & 15281.90 & 17563.20 & 13.03 \\
P24 & 3 & 9808.99 & 16746.94 & 20632.00 & 18.59 \\
P24 & 4 & 11445.04 & 19266.70 & 25632.40 & 24.58 \\
P24 & 5 & 12543.23 & 21904.52 & 30316.80 & 27.45 \\
P25 & 2 & 9507.26 & 17015.60 & 19393.30 & 12.40 \\
P25 & 3 & 11746.93 & 19965.92 & 25477.90 & 21.32 \\
P25 & 4 & 13478.91 & 23248.80 & 31095.10 & 25.13 \\
P25 & 5 & 14043.55 & 24771.06 & 33228.50 & 25.34 \\
AV25 & 2 & 6227.73 & 11166.12 & 13394.00 & 15.44 \\
AV25 & 3 & 7635.86 & 13241.86 & 17278.20 & 22.94 \\
AV25 & 4 & 8223.55 & 14455.58 & 19577.80 & 25.60 \\
AV25 & 5 & 8868.04 & 15681.86 & 21808.00 & 27.26 \\
\hline & & & & &
\end{tabular}

impact on the quality of our lower bounding approach, especially for the non-sparse instances. Furthermore the tables show that the quality of our lower bounds hardly deteriorates if one increases the number of departments.

\subsection{Results for the DREFLP}

We consider the results of our lower bounding model (13) and (20)-(30) specialized to the DREFLP. In Table 4 we compare our lower bounding model with a time limit of three minutes with

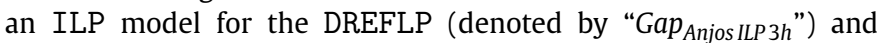
an SDP approach for the DREFLP (Anjos et al., 2018) (denoted by "Gap ${ }_{S D P 3 h}$ ") with a time limit of three hours. The upper bounds ("best ub") in Table 4 are taken from Anjos et al. (2018). For benchmark instances from the literature, see, e. g., Yu and Sarker (2003) and Hungerländer (2014b), with 20 to 50 departments the gaps of our combinatorial bounds are around 35\% and the gaps of our lower bounding model are between $2.58 \%$ and $5.73 \%$. While our lower bounding model outperforms the ILP approach of Anjos et al. (2018) for $n \geq 35$, the SDP approach provides the best lower bounds, but with a higher running time. One reason for the better behavior of the SDP approach is that in the equidistant case of the DRFLP the structure of optimal solutions is much simpler than for arbitrary department lengths. This property can nicely be exploited in appropriate SDP models.

Additionally we test the equidistant so called "sko" benchmark instances of Anjos and Yen (2009) with up to 81 departments. As $n$ is rather large we increase the time limit of our lower bound- ing model to 15 minutes and 60 minutes, respectively. In order to simplify a comparison with the results in Table 4 we used the program and the computer of Anjos et al. (2018) for the SDP and ILP lower bounds of Anjos et al. (2018). The results are shown in Tables 5 and 6. One can see that even for such large instances all gaps of our lower bounding model are less than $13.14 \%$ and usually smaller. Our combinatorial bounds (7), which lie between $53.21 \%$ and $58.06 \%$, are significantly improved by our ILP. Similarly to the Y-instances the SDP bounds are better.

Comparing the results of the standard DRFLP and the DREFLP, one can see that the gaps of our lower bounding model are much better in the equidistant case. One reason for this behavior is that in the equidistant case we have a lower bound for the sum of the distances (25).

\subsection{Results for the PROP}

For the PROP we test the same instances as in Fischer et al. (2019). The optimal solution values of all the instances are given in Fischer et al. (2019). Looking at the strength of our lower bounding approach adapted to the PROP one sees in Table 7 that the average gaps are even better than for the DRFLP. Most often they are less than $30 \%$ and for the balanced instances with (approximately) half of the departments in each row the average gaps are at most $16 \%$. So the approach seems to work well for balanced instances. One reason for the worse performance on unbalanced instances is that the lower bound calculation cannot take into account that some of the departments in a longer row will not overlap with some of the departments in the other row at all. For this remember that the left border of the layout is fixed and free spaces are not allowed between departments in the same row.

\section{Conclusion}

The Double-Row Facility Layout Problem (DRFLP) is a very challenging problem with various application areas, including factory planning. Despite its broad applicability it can only be solved to optimality in reasonable time for rather small instances. Apart from this, using the integer-programming based solution approaches from the literature one derives even with high running times very large gaps for large instances. So heuristics are currently the only way to determine solutions for larger instances. In order to evaluate the quality of heuristically determined solutions, we developed in this paper combinatorial lower bounds for the optimal solution value of the DRFLP. Indeed, interpreting some subproblem of the DRFLP as a parallel identical machine scheduling problem we computed the first known non-trivial combinatorial lower bounds for the DRFLP. Furthermore we combined these bounds with a new mixed-integer linear programming model, which is indeed not a formulation for the DRFLP, to obtain even better lower bounds. Only few heuristics are present in the literature for the standard DRFLP. We compare our lower bounds to upper bounds derived by some construction heuristic presented in Chung and Tanchoco (2010) which is combined with a 1-opt and a 2-opt improvement heuristic. Our computational results show that we were able to obtain non-trivial lower bounds for large double-row instances. We received average gaps of $32 \%$ to $46 \%$ for large dense instances and of about 50 to $55 \%$ for large sparse instances using our lower bounding approach. Note that the pure combinatorial bounds, which can be determined very fast, could usually be strengthened significantly, but with average gaps from $40 \%$ to $80 \%$ they are still much better than the model (Amaral, 2013a) from the literature after a time limit of one hour for larger instances. Additionally, we showed how our bounds can be specialized to the equidistant DRFLP and can be extended to the $(k-)$ 
parallel row ordering problem. In both cases the gaps are better than for the DRFLP.

As already mentioned, only few heuristic approaches are known for the DRFLP in the literature. Now, with this new possibility for evaluation, it remains for future work to construct new heuristic approaches. Another interesting research question is to exploit which is the best way to extend our lower bounding model to a DRFLP formulation. For good solution times the development of sophisticated branching strategies in a branch-and-cut algorithm might be important.

From a practical point of view, it is important to extend the Single-Row Facility Layout Problem and the DRFLP in order to handle more characteristics important in practice. For instance, the standard models do not allow for individual input and output positions of the departments and certain clearance conditions. Additionally, it remains for future work to investigate more complex path structures in the shape of a $U$, a $T$ or an X. Here the lowerbounding approaches developed in the current paper might help in determining non-trivial lower bounds as well.

\section{Acknowledgment}

This work is partially supported by the Simulation Science Center Clausthal-Göttingen. We thank the anonymous referees for their helpful suggestions on this paper.

\section{Appendix A}

For the PROP with given row assignment $r$ and sets $R_{i}=\{j \in$ $\left.[n]: r_{j}=i\right\}, i=1,2$, we use the following lower bounding model with $M=\max \left\{\sum_{z \in R_{1}} \ell_{z}, \sum_{z \in R_{2}} \ell_{z}\right\}$.

$$
\begin{aligned}
& \min \sum_{\substack{i, j \in[n] \\
i<j}} w_{i j} d_{i j} \\
& \sum_{\substack{i \in S \in R_{1} \\
j \in S \cap R_{2}}} x_{i j} \leq|S|-1 \\
& \sum_{j \in S \cup T} x_{i j} \leq|S|+1 \\
& S \subseteq[n],|S| \geq 2, \\
& d_{i j}+\left(\frac{\ell_{i}+\ell_{j}}{2}\right) x_{i j} \geq \frac{\ell_{i}+\ell_{j}}{2} \\
& d_{i j}+\left(M-\ell_{i}-\ell_{j}+\frac{1}{2} \kappa\right) x_{i j} \leq M-\frac{\ell_{i}+\ell_{j}}{2}, \\
& d_{i j}+d_{j k}-d_{i k} \geq 0 \text {, } \\
& \sum_{j \in S} d_{i j} \geq \min _{j \in\left\{k \in[n]: r_{k} \neq r_{i}\right\}} W_{(i, j)}^{\text {P-dst }}(S), \\
& \sum_{j \in S} w_{i j} d_{i j} \geq \min _{j \in\left\{k \in[n]: r_{k} \neq r_{i}\right\}} \max \left\{\begin{array}{c}
W_{(i, j)}^{\text {P.spt }}(S), \\
W_{(i, j)}^{\text {p.sc }}(S)
\end{array}\right\}, \\
& \sum_{\substack{i, j \in S \\
i<j}} d_{i j}+o_{S, 3} \sum_{\substack{i \in S S R_{1} \\
j \in S \cap R_{2}}} x_{i j} \geq o_{S, 3} \\
& \sum_{\substack{i, j \in S \\
i<j}} d_{i j}+o_{S, 4} \sum_{\substack{i \in S \cap R_{1} \\
j \in S \cap R_{2}}} x_{i j} \geq o_{S, 4} \\
& i \in[n], S \subset\left\{j \in[n]: r_{j} \neq r_{i}\right\} \text { with } \sum_{j \in S} \ell_{j} \geq \ell_{i}, \\
& T:=\left\{j \in\left\{k \in[n]: r_{k} \neq r_{i}\right\}: \ell_{j} \geq \max _{k \in S} \ell_{k}\right\}, \\
& i \in R_{1}, j \in R_{2} \text {, } \\
& i \in R_{1}, j \in R_{2} \text {, } \\
& i, j, k \in[n],|\{i, j, k\}|=3, i<k, \\
& i \in[n], S \subseteq[n] \backslash\{i\}, \\
& i \in[n], S \subseteq V_{i}, \\
& S \subset[n],|S|=3, o_{S, 3}=\sum_{i \in S} \ell_{i}+\min _{i \in S} \ell_{i}, \\
& S \subset[n],|S|=4, \\
& o_{S, 4}=\frac{3}{2} \sum_{i \in S} \ell_{i}+2 \cdot \min _{\substack{i_{1}, i_{\in} \in S \\
i_{1} \neq i_{2}}}\left(\ell_{i_{1}}+\ell_{i_{2}}\right), \\
& \sum_{\substack{i, j \in S \\
i<j}} \ell_{i} \ell_{j} d_{i j}+o_{S} \sum_{\substack{i \in S \cap R_{1} R_{1} \\
j \in S \cap R_{2}}} x_{i j} \geq o_{S}, \\
& S \subseteq[n],|S| \geq 3, \\
& o_{S}=\frac{1}{6}\left(\left(\sum_{i \in S} \ell_{i}\right)^{3}-\sum_{i \in S} \ell_{i}^{3}\right) \\
& \sum_{\substack{i, j \in R_{k} \\
i<j}} \ell_{i} \ell_{j} d_{i j}=\frac{1}{6}\left(\left(\sum_{i \in R_{k}} \ell_{i}\right)^{3}-\sum_{i \in R_{k}} \ell_{i}^{3}\right), \quad k \in \mathcal{R}, \\
& \sum_{\substack{i, j \in \mathcal{R}_{k} \\
i<j}} d_{i j} \geq \sum_{\substack{i, j \in R_{k} \\
i<j}} \frac{\ell_{i}+\ell_{j}}{2}+\sum_{z=2}^{\left|R_{k}\right|-1}(z-1)\left(\left|R_{k}\right|-z\right) \ell_{\pi^{* k, k}(z)}, \quad k \in \mathcal{R}, \\
& d_{i j} \geq \frac{\ell_{i}+\ell_{j}}{2}, \\
& k \in \mathcal{R}, i, j \in R_{k}, i<j,
\end{aligned}
$$

$$
\begin{array}{ll}
d_{i j} \leq\left(\sum_{z \in R_{k}} \ell_{z}\right)-\frac{\ell_{i}+\ell_{j}}{2}, & \\
d_{i j} \leq M-\frac{\ell_{i}+\ell_{j}}{2}, & i \in R_{1}, j \in R_{2}, \\
x_{i j}=x_{j i} \in\{0,1\}, & i \in R_{1}, j \in R_{2}, \\
d_{i j}=d_{j i} \geq 0, & i, j \in[n], i<j .
\end{array}
$$

\section{References}

Ahmadi, A., Pishvaee, M. S., \& Jokar, M. R. A. (2017). A survey on multi-floor facility layout problems. Computers E Industrial Engineering, 107, 158-170. doi:10.1016/ j.cie.2017.03.015.

Ahonen, H., de Alvarenga, A. G., \& Amaral, A. R. S. (2014). Simulated annealing and tabu search approaches for the corridor allocation problem. European Journal of Operational Research, 232(1), 221-233.

Amaral, A. R. S. (2019). A mixed-integer programming formulation for the double row layout of machines in manufacturing systems. International Journal of Production Research, 57(1), 34-47. doi:10.1080/00207543.2018.1457811.

Amaral, A. R. S., Caprara, A., Letchford, A. N., \& Salazar-Gonzalez, J.-J. (2008). A new lower bound for the minimum linear arrangement of a graph. Electronic Notes in Discrete Mathematics, 30, 87-92. doi:10.1016/j.endm.2008.01.016. The IV LatinAmerican Algorithms, Graphs, and Optimization Symposium

Amaral, A. R. S. (2006). On the exact solution of a facility layout problem. European Journal of Operational Research, 173(2), 508-518.

Amaral, A. R. S. (2008). An exact approach to the one-dimensional facility layout problem. Operations Research, 56(4), 1026-1033.

Amaral, A. R. S. (2009). A new lower bound for the single row facility layout problem. Discrete Applied Mathematics, 157(1), 183-190.

Amaral, A. R. S. (2011). On duplex arrangement of vertices. Technical Report. Departamento de Informática, Universidade Federal do Espírito Santo (UFES), Brazil.

Amaral, A. R. S. (2012). The corridor allocation problem. Computers \& Operations Research, 39(12), 3325-3330.

Amaral, A. R. S. (2013a). Optimal solutions for the double row layout problem. Optimization Letters, 7(2), 407-413.

Amaral, A. R. S. (2013b). A parallel ordering problem in facilities layout. Computers E' Operations Research, 40(12), 2930-2939.

Amaral, A. R. S., \& Letchford, A. N. (2013). A polyhedral approach to the single row facility layout problem. Mathematical Programming, 141(1-2), 453-477.

Anjos, M. F., Fischer, A., \& Hungerländer, P. (2016). Solution approaches for the double-row equidistant facility layout problem. In M. Lübbecke, A. Koster, P. Letmathe, R. Madlener, B. Peis, \& G. Walther (Eds.), Operations research proceedings 2014 (pp. 17-23). Cham: Springer International Publishing.

Anjos, M. F., Fischer, A., \& Hungerländer, P. (2018). Improved exact approaches for row layout problems with departments of equal length. European Journal of Operational Research, 270(2), 514-529. doi:10.1016/j.ejor.2018.04.008.

Anjos, M. F., Kennings, A., \& Vannelli, A. (2005). A semidefinite optimization approach for the single-row layout problem with unequal dimensions. Discrete Optimization, 2(2), 113-122.

Anjos, M. F., \& Liers, F. (2012). Global approaches for facility layout and VLSI floorplanning. In M. F. Anjos, \& J. B. Lasserre (Eds.), Handbook on semidefinite, conic and polynomial optimization. In International Series in Operations Research E Management Science: 166 (pp. 849-877). Springer US.

Anjos, M. F., \& Vannelli, A. (2008). Computing globally optimal solutions for single-row layout problems using semidefinite programming and cutting planes. INFORMS Journal On Computing, 20(4), 611-617.

Anjos, M. F., \& Vieira, M. V. C. (2017). Mathematical optimization approaches for facility layout problems: The state-of-the-art and future research directions. European Journal of Operational Research, 261, 1-16.

Anjos, M. F., \& Yen, G. (2009). Provably near-optimal solutions for very large single-row facility layout problems. Optimization Methods and Software, 24(4), 805-817.

Bracht, U., Dahlbeck, M., Fischer, A., \& Krüger, T. (2018). Combining simulation and optimization for extended double row facility layout problems in factory planning. In M. Baum, G. Brenner, J. Grabowski, T. Hanschke, S. Hartmann, \& A. Schöbel (Eds.), Simulation science (pp. 39-59). Cham: Springer International Publishing.

Butler, T. W., Karwan, K. R., Sweigart, J. R., \& Reeves, G. R. (1992). An integrative model-based approach to hospital layout. IIE Transactions, 24(2), 144-152.

Caprara, A., Letchford, A. N., \& Salazar-González, J.-J. (2011). Decorous lower bounds for minimum linear arrangement. INFORMS Journal on Computing, 23(1), 26-40.

Chung, J., \& Tanchoco, J. M. A. (2010). The double row layout problem. International Journal of Production Research, 48(3), 709-727.

Cravo, G. L., \& Amaral, A. R. S. (2019). A grasp algorithm for solving large-scale single row facility layout problems. Computers \& Operations Research, 106, 49-61.

Dahlbeck, M., Fischer, A., \& Hungerländer, P. (2020). Heuristics for the double row facility layout problem based on solutions of the single row facility layout problem. Working paper.

Dantzig, G. B., Fulkerson, D. R., \& Johnson, S. (1954). Solution of a large scale traveling salesman problem. Journal of the Operations Research Society of America, 2, 393-410

Datta, D., Amaral, A. R. S., \& Figueira, J. R. (2011). Single row facility layout problem using a permutation-based genetic algorithm. European Journal of Operational Research, 213(2), 388-394. 
Drira, A., Pierrval, H., \& Hajri-Gabouj, S. (2007). Facility layout problems: A survey. Annual Reviews in Control, 31, 255-267.

Edmonds, J. (1971). Matroids and the greedy algorithm. Mathematical Programming, 1(1), 127-136. doi:10.1007/BF01584082.

Elshafei, A. N. (1977). Hospital layout as a quadratic assignment problem.. Journal of the Operational Research Society, 28(1), 167-179.

Fischer, A., Fischer, F., \& Hungerländer, P. (2015). A new exact approach to the space-free double row layout problem. In K. F. Doerner, I. Ljubic, G. Pflug, \& G. Tragler (Eds.), Operations research proceedings 2015, selected papers of the international conference of the German, Austrian and Swiss operations research societies (GOR, ÖGOR, SVOR/ASRO), University of Vienna, austria, September 1-4 2015.. In Operations Research Proceedings (pp. 125-130). Springer. doi:10.1007/ 978-3-319-42902-1_17.

Fischer, A., Fischer, F., \& Hungerländer, P. (2019). New exact approaches to row layout problems. Mathematical Programming Computation. doi:10.1007/ s12532-019-00162-6.

Garey, M. R., Johnson, D. S., \& Stockmeyer, L. J. (1974). Some simplified NP-complete problems. In Proceedings of the sixth annual ACM symposium on theory of computing (pp. 47-63). ACM.

Guan, J., Lin, G., Feng, H.-B., \& Ruan, Z.-Q. (2020). A decomposition-based algorithm for the double row layout problem. Applied Mathematical Modelling, 77, 963 979. doi:10.1016/j.apm.2019.08.015.

Gülsen, M., Murray, C. C., \& Smith, A. E. (2019). Double-row facility layout with replicate machines and split flows. Computers E' Operations Research, 108, 20-32.

Hahn, P. M., \& Krarup, J. (2001). A hospital facility layout problem finally solved. Journal of Intelligent Manufacturing, 12(5-6), 487-496.

Hall, L. A., Schulz, A. S., Shmoys, D. B., \& Wein, J. (1997). Scheduling to minimize average completion time: Off-line and on-line approximation algorithms. Mathematics of Operations Research, 22(3), 513-544. doi:10.1287/moor.22.3.513.

Harper, L. H. (1964). Optimal assignments of numbers to vertices. SIAM Journal on Applied Mathematics, 12(1), 131-135.

Harper, L. H. (1966). Optimal numberings and isoperimetric problems on graphs. Journal of Combinatorial Theory, 1(3), 385-393.

Hassan, M. M. D. (1994). Machine layout problem in modern manufacturing facilities. International Journal of Production Research, 32(11), 2559-2584.

Heragu, S. S., \& Kusiak, A. (1991). Efficient models for the facility layout problem. European Journal of Operational Research, 53(1), 1-13.

Hosseini-Nasab, H., Fereidouni, S., Fatemi Ghomi, S. M. T., \& Fakhrzad, M. B. (2018). Classification of facility layout problems: a review study. The International Journal of Advanced Manufacturing Technology, 94(1), 957-977. doi:10.1007| s00170-017-0895-8.

Hungerländer, P. (2014a). A Semidefinite Optimization Approach to the Parallel Row Ordering Problem. Technical Report. Alpen-Adria Universität Klagenfurt, Mathematics, Optimization Group, TR-ARUK-M-O-14-05.

Hungerländer, P. (2014b). Single-row equidistant facility layout as a special case of single-row facility layout. International Journal of Production Research, 52(5) 1257-1268.

Hungerländer, P. (2017). The checkpoint ordering problem. Optimization, 66(10), 1699-1712.

Hungerländer, P., \& Anjos, M. F. (2012). A Semidefinite Optimization Approach to Space-Free Multi-Row Facility Layout. Cahiers du GERAD. Montreal, QC, Canada: GERAD

Hungerländer, P., \& Anjos, M. F. (2015). A semidefinite optimization-based approach for global optimization of multi-row facility layout. European Journal of Operational Research, 245(1), 46-61.

Hungerländer, P., \& Rendl, F. (2013a). A computational study and survey of methods for the single-row facility layout problem. Computational Optimization and Applications, 55(1), 1-20.

Hungerländer, P., \& Rendl, F. (2013b). Semidefinite relaxations of ordering problems. Mathematical Programming, 140(1), 77-97.

IBM (2018). ILOG CPLEX Optimization Studio 12.8

Kawaguchi, T., \& Kyan, S. (1986). Worst case bound of an lrf schedule for the mean weighted flow-time problem. SIAM Journal on Computing, 15(4), 11191129. doi: $10.1137 / 0215081$

Keller, B., \& Buscher, U. (2015). Single row layout models. European Journal of Operational Research, 245(3), 629-644.

Kothari, R., \& Ghosh, D. (2012). Population heuristics for the corridor allocation problem. Technical Report. IIMA Working Papers WP2012-09-02, Indian Institute of Management Ahmedabad, Research and Publication Department.
Kothari, R., \& Ghosh, D. (2013). Tabu search for the single row facility layout problem using exhaustive 2-opt and insertion neighborhoods. European Journal of Operational Research, 224(1), 93-100.

Kothari, R., \& Ghosh, D. (2014). A scatter search algorithm for the single row facility layout problem. Journal of Heuristics, 20(2), 125-142.

Langevin, A., Montreuil, B., \& Riopel, D. (1994). Spine layout design. The International Journal of Production Research, 32(2), 429-442.

Lee, C.-Y., \& Uzsoy, R. (1992). A new dynamic programming algorithm for the paralel machines total weighted completion time problem. Operations Research Letters, 11(2), 73-75.

Lenstra, J. K., Rinnooy Kan, A. H. G., \& Brucker, P. (1977). Complexity of machine scheduling problems. In P. Hammer, E. Johnson, B. Korte, \& G. Nemhauser (Eds.), Studies in integer programming. In Annals of Discrete Mathematics: 1 (pp. 343 362). Elsevier. doi:10.1016/S0167-5060(08)70743-X. http://www.sciencedirect. com/science/article/pii/S016750600870743X

Liu, W., \& Vannelli, A. (1995). Generating lower bounds for the linear arrangement problem. Discrete Applied Mathematics, 59(2), 137-151. doi:10.1016 0166-218X(93)E0168-X.

Loiola, E. M., de Abreu, N. M. M., Boaventura-Netto, P. O., Hahn, P., \& Querido, T. (2007). A survey for the quadratic assignment problem. European Journal of Operational Research, 176(2), 657-690.

Maadi, M., Javidnia, M., \& Jamshidi, R. (2017). Two strategies based on metaheuristic algorithms for parallel row ordering problem (PROP). Iranian Journal of Management Studies, 10(2), 467-498. doi:10.22059/ijms.2017.216663.672285.

Murray, C. C., Smith, A. E., \& Zhang, Z. (2013). An efficient local search heuristic for the double row layout problem with asymmetric material flow. International Journal of Production Research, 51(20), 6129-6139.

Palubeckis, G. (2012). A branch-and-bound algorithm for the single-row equidistant facility layout problem. OR Spectrum, 34(1), 1-21.

Palubeckis, G. (2015a). Fast local search for single row facility layout. European Journal of Operational Research, 264(3), 800-814.

Palubeckis, G. (2015b). Fast simulated annealing for single-row equidistant facility layout. Applied Mathematics and Computation, 263, 287-301.

Palubeckis, G. (2017). Single row facility layout using multi-start simulated annealing. Computers \& Industrial Engineering, 103, 1-16.

Samarghandi, H., \& Eshghi, K. (2010). An efficient tabu algorithm for the single row facility layout problem. European Journal of Operational Research, 205(1), 98-105.

Schrijver, A. (2003). Combinatorial optimization. Polyhedra and efficiency. Vol. B. Algorithms and Combinatorics: 24. Berlin: Springer-Verlag.

Secchin, L. D., \& Amaral, A. R. S. (2019). An improved mixed-integer programming model for the double row layout of facilities. Optimization Letters, 13(1), 193199. doi:10.1007/s11590-018-1263-9.

Smith, W. E. (1956). Various optimizers for single-stage production. Naval Research Logistics Quarterly, 3(1-2), 59-66. doi:10.1002/nav.3800030106.

Tompkins, J. A., White, J. A., Bozer, Y. A., \& Tanchoco, J. M. A. (2010). Facilities planning. John Wiley \& Sons.

Wang, S., Zuo, X., Liu, X., Zhao, X., \& Li, J. (2015). Solving dynamic double row layout problem via combining simulated annealing and mathematical programming. Applied Soft Computing, 37, 303-310. doi:10.1016/j.asoc.2015.08.023.

Yang, X., Cheng, W., Smith, A. E., \& Amaral, A. R. S. (2020). An improved model for the parallel row ordering problem. Journal of the Operational Research Society, 71(3), 475-490.

Yu, J., \& Sarker, B. R. (2003). Directional decomposition heuristic for a linear machine-cell location problem. European Journal of Operational Research, 149(1), $142-184$.

Zhang, Z., \& Murray, C. C. (2012). A corrected formulation for the double row layout problem. International Journal of Production Research, 50(15), 4220-4223.

Zuo, X., Gao, S., Zhou, M., Yang, X., \& Zhao, X. (2019). A three-stage approach to a multirow parallel machine layout problem. IEEE Transactions on Automation Science and Engineering, 16(1), 433-447. doi:10.1109/TASE.2018.2866377.

Zuo, X., Murray, C. C. \& Smith, A. E. (2014). Solving an extended double row layout problem using multiobjective tabu search and linear programming. IEEE Transactions on Automation Science and Engineering, 11(4), 1122-1132.

Zuo, X. Q., Murray, C. C., \& Smith, A. E. (2016). Sharing clearances to improve machine layout. International Journal of Production Research, 54(14), 4272-4285. doi: $10.1080 / 00207543.2016 .1142134$. 
E. A study of the relation between the single-row and the double-row facility layout problem

Authors: Mirko Dahlbeck, Anja Fischer, Philipp Hungerländer

Working Paper, 2020, available at http://www.optimization-online.org/DB_HTML/2020/11/ 8110.html. 


\title{
A study of the relation between the single-row and the double-row facility layout problem
}

\author{
Mirko Dahlbeck* Anja Fischer ${ }^{\dagger}$ Philipp Hungerländer ${ }^{\ddagger}$
}

November 13, 2020

\begin{abstract}
The NP-hard Multi-Row Facility Layout Problem (MRFLP) consists of a set of one-dimensional departments and pairwise transport weights between them. It asks for a non-overlapping arrangement of the departments along a given number of rows such that the weighted sum of the horizontal center-to-center distances between the departments is minimized. We mainly focus on the MRFLP with exactly two rows, the so called Double-Row Facility Layout Problem (DRFLP), and on the case with exactly one row, the so called Single-Row Facility Layout Problem (SRFLP). Although the MRFLP has wide applications in factory planning, only small instances can be solved to optimality in reasonable time for the MRFLP with at least two rows while provably good or optimal solutions for the SRFLP can be derived very fast. In the equidistant case, where all departments have the same size, we prove that the optimal value of the MRFLP is less than or equal to the optimal value of the SRFLP divided by the number of rows of the MRFLP. We derive equidistant double-row layouts satisfying this property in a very short time and we improve some of the best known upper bounds for the equidistant DRFLP. Given a double-row instance with arbitrary department lengths we provide a formula for the relation of the optimal value of the DRFLP and the SRFLP and provide an example which shows that this bound is tight. In addition, we present heuristic approaches for the DRFLP based on good or optimal single-row layouts. For instances with up to 40 departments we obtain small gaps to the best known upper bounds and for even larger instances we improve the best known upper bounds. Our approaches are significantly faster than the ones in the literature.
\end{abstract}

Key words. Facilities planning and design; Row Layout Problem; Heuristic

\section{Introduction}

Given a set of departments $\{1, \ldots, n\}=:[n], n \in \mathbb{N}$, with length $\ell_{i}, i \in[n]$, and pairwise nonnegative weights $w_{i j}=w_{j i}, i, j \in[n], i<j$, the Multi-Row Facility Layout Problem (MRFLP) asks for an assignment of the departments to the rows $\mathcal{R}:=[m] \in \mathbb{N}, m \geq 1$, such that departments in the same row do not overlap and such that the weighted sum of the horizontal center-to-center distances between the departments is minimized. So we look for an assignment $r:[n] \rightarrow \mathcal{R}$ of the

\footnotetext{
${ }^{*}$ TU Dortmund University, Faculty of Business and Economics; Georg-August-Universität Göttingen, Institute for Numerical and Applied Mathematics, mirko.dahlbeck@tu-dortmund.de

${ }^{\dagger}$ TU Dortmund University, anja2.fischer@tu-dortmund.de

${ }^{\ddagger}$ Alpen-Adria Universität Klagenfurt, philipp.hungerlaender@aau.at
} 
departments to the $m$ rows and for a vector $p \in \mathbb{R}^{n}$ of the center positions of the departments such that

$$
\begin{aligned}
\min _{r \in \mathcal{R}^{n}, p \in \mathbb{R}^{n}} & \sum_{\substack{i, j \in[n] \\
i<j}} w_{i j}\left|p_{i}-p_{j}\right| \\
\text { s. t. }\left|p_{i}-p_{j}\right| \geq \frac{\ell_{i}+\ell_{j}}{2}, & i, j \in[n], i<j, \text { if } r_{i}=r_{j} .
\end{aligned}
$$

The special case of the MRFLP with $m=1$ is called Single-Row Facility Layout Problem (SRFLP) and is well-known to be $\mathcal{N} \mathcal{P}$-hard $[3,29,47]$. The SRFLP arises in factory planning, in the arrangement of departments in office buildings, hospitals or supermarkets [50] as well as of books on a shelf [4]. Further applications are the assignment of files to disk cylinders in computer storage and the design of warehouse layouts [38, 47]. There always exists an optimal single-row layout without spaces between neighboring departments, so the SRFLP is equivalent to finding a permutation of the departments that minimizes the weighted sum of the horizontal centerto-center distances. Most often exact approaches for the SRFLP are based on integer linear programming (ILP) models, see, e.g., [1, 2, 3, 7], or semidefinite optimization (SDP) formulations, see, e. g., $[10,11,14,34,35]$. The current fastest approach of $[34,35]$ is able to solve one instance with 42 departments to optimality in less than 2 hours and obtains gaps of less than $2 \%$ for instances with up to 110 departments in 400 hours. Additionally, several heuristic algorithms have been suggested that are able to obtain high quality solutions $[20,24,40,41,44,46]$. The heuristic in [24] is applied on instances from the literature with 60 to 80 departments. Almost half of their solutions were improved by [40,41] and their heuristic is applied on instances with up to 110 departments. Afterwards, [44] presented a heuristic which derived layouts of the same quality but the running time was reduced and hence instances with up to 300 departments were considered. One of the leading heuristics is given in [46], where a multi-start simulated annealing heuristic obtains the best known solutions or small gaps for instances from the literature with 60 to 80 departments. This heuristic is applied on instances with up to 1000 departments. A recent survey on the SRFLP is given in [37].

The SRFLP with departments of equal length is denoted by Single-Row Equidistant Facility Layout Problem (SREFLP) and is a special case of the Quadratic Assignment Problem (QAP). In [32] it is shown that the best method for the SRFLP is better than methods especially designed for the SREFLP, see, e. g., [43], and methods for the QAP. For a heuristic approach we refer to [45].

For $m=2$ the MRFLP is called Double-Row Facility Layout Problem (DRFLP). Problems in factory layout planning can often be decomposed, see [23], and hence most often real factory layout problems reduce to a combination of single-row and double-row layouts. Determining good solutions is important since the costs of the production are highly influenced by the layout of the departments, see, e.g., $[16,31,51]$. In contrast to the SRFLP, the DRFLP is very challenging even for instances with a small number of departments. Several mixed-integer linear programming (MILP) models have been developed for the DRFLP and the MRFLP, see, e. g. [4, 5, 13, 18, 19, 49] (see [53] for a correction of [19]). However, the current fastest exact approach for the DRFLP and the MRFLP in $[27,28]$ is able to solve double-row instances with up to 16 departments in less than 12 hours and multi-row instances with up to 13 departments and 5 rows in at most 7 hours. Heuristics for the MRFLP and the DRFLP, partially handling some extended versions, are given in $[6,13,15,19,30,42,48,52,54,55]$. A large disadvantage of the heuristic presented in [6], which performs well with respect to the obtained solution values, is its large computational effort and so the long running times. The question arises whether one can get good solutions quickly. The quality of heuristically determined solution can be judged to some extend using a method for calculating lower bounds on the optimal value of the DRFLP [22].

The special case of the MRFLP where all departments have the same length is called Multi-Row Equidistant Facility Layout Problem (MREFLP) and Double-Row Equidistant Facility Layout Problem (DREFLP) if $m=2$, considered in [8, 9, 33]. In the current fastest approach [9] instances 
with up to 25 departments and up to 5 rows are solved to optimality within a time limit of 3 hours. For further papers on facility layout planning we refer to the recent surveys [12, 25].

In this paper we mainly focus on the SRFLP and the DRFLP, so we present an example in order to compare the distance calculation. Note that in an optimal double-row layout one might obtain free-spaces between neighboring departments.

Example 1. Given an instance with 5 departments and lengths $\ell_{1}=\ell_{3}=\ell_{4}=2, \ell_{2}=\ell_{5}=1$ and non-zero weights $w_{12}=w_{45}=3, w_{23}=w_{34}=1$.

- An optimal single-row layout is depicted in Figure 1a with objective value

$$
3 \cdot 1.5+1 \cdot 1.5+1 \cdot 2+3 \cdot 1.5=12.5 .
$$

- An optimal double-row layout is illustrated in Figure $1 b$ with objective value

$$
3 \cdot 0+1 \cdot 1.5+1 \cdot 1.5+3 \cdot 0=3 .
$$

\begin{tabular}{|l|l|l|l|l|}
\hline 1 & 2 & 3 & 4 & 5 \\
\hline
\end{tabular}

(a) Illustration of an optimal single-row layout.

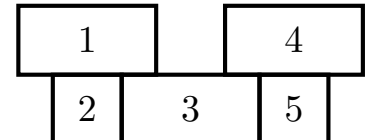

(b) Illustration of an optimal double-row layout.

Figure 1: We consider an instance with 5 departments, lengths $\ell_{1}=\ell_{3}=\ell_{4}=2, \ell_{2}=\ell_{5}=1$ and non-zero weights $w_{12}=w_{45}=3, w_{23}=w_{34}=1$.

\subsection{Main contribution}

Our main contribution in this paper is the following. We provide a theoretical study of the relationship between optimal values of the MREFLP and the SREFLP as well as between the optimal values of the DRFLP and the SRFLP. Therefore, we present the following results.

- Given an equidistant instance, we prove that $v_{m}^{*} \leq \frac{v_{1}^{*}}{m}$ where $v_{m}^{*}$ denotes the optimal value of the MREFLP with $m$ rows, see Section 2.1.

- Given a (general) double-row instance with $n$ departments, we prove that $v_{2}^{*} \leq \frac{n-2}{n-1} v_{1}^{*}$ where $v_{1}^{*}\left(v_{2}^{*}\right)$ denotes the optimal value of the SRFLP (DRFLP). Further we present an example which shows that this bound is tight, see Section 3.1.

In addition, we present new heuristic approaches for the MREFLP and the DRFLP based on a good or optimal single-row layout $\pi$ with objective value $v_{1}$, see Sections 2.2 and 3.2.

- Given an equidistant instance, we derive a multi-row layout with objective value less than or equal to $\frac{v_{1}}{m}$. The constructed layout contains free-spaces only at the left or right border of the layout. We set up an MILP model which simplifies to a linear programming (LP) model to include free-spaces, and thus to further improve the quality of the determined layouts. We improve some of the best known upper bounds, in particular, for large-sized instances. Additionally, given a good or optimal single-row layout and combining our approach with some improvement heuristics, good double-row layouts can be derived in a few minutes while the SDP lower bounding approach, which includes a construction heuristic, in [9] had a time limit of three hours.

- We present two heuristics for the DRFLP which are extensions of the heuristic in the equidistant case. For instances with more than 40 departments we outperform the approach of [19] in combination with a shorter running time. Considering instances with 30 or 40 departments we obtain tight gaps, i. e., less than $1 \%$, to the upper bounds derived in [6] while our approaches are significantly faster. 


\section{The multi-row equidistant facility layout problem}

In Section 2.1 we study the relation of the optimal solution values of the MREFLP and the SREFLP. Therefore, let $v_{m}^{*}$ denote the optimal value of the MREFLP with $m$ rows. One of our main results in this paper is that $v_{m}^{*} \leq \frac{v_{1}^{*}}{m}$. Further, we show that this bound is tight. Several heuristics are able to compute good or optimal single-row layouts for large-sized instances, see, e. g., [20, 34, 35, 46]. We show how to construct (at least) one equidistant multi-row layout with objective value $v_{m}$ based on a single-row layout with objective value $v_{1}$ such that $v_{m} \leq \frac{v_{1}}{m}$. All constructed layouts contain free space only at the border of the layout. We further can improve the layouts by allowing free spaces. In Section 2.2 we present an ILP, which simplifies to some LP, to determine the exact positions of the departments and include possible free space. Further improvement heuristics from the literature can be applied.

\subsection{Relation between equidistant single- and multi-row layouts}

In this paper we study the relation between the optimal solution values of single-row and doublerow layouts, but in the equidistant case all result can be extended to the MREFLP, so we present our results for the MREFLP in this part. First, we repeat a combinatorial property of the MREFLP given in [33].

Theorem 2. Given an MREFLP instance, there always exists an optimal multi-row layout where the departments are arranged on the grid.

We say that $i \in[n]$ lies in column $j \in[n]$ if the center of $i$ is located at the $j^{\text {th }}$ grid point. In order to study the relation of the equidistant single-row and double-row layouts, we consider unweighted distances first. Let $d_{i j}=d_{j i}, i, j \in[n], i<j$, denote the horizontal center-to-center distance between $i$ and $j$.

Proposition 3. The following properties hold independent of the order of the departments:

1. The sum of the distances between all pairs of departments in (space-free) equidistant single-row layouts satisfies $\sum_{\substack{i, j \in[n] \\ i<j}} d_{i j}=\frac{(n+1) n(n-1)}{6}=: f(n)$.

2. The sum of the distances between all pairs of departments in a space-free equidistant double-row layouts satisfies

$$
\sum_{\substack{i, j \in[n] \\ i<j}} d_{i j}= \begin{cases}\frac{(n+1) n(n-1)}{12}, & \text { for } n \text { odd }, \\ \frac{(n+2) n(n-2)}{12}, & \text { for } n \text { even } .\end{cases}
$$

Proof. 1. For equidistant single-row layouts the result follows directly from the clique equations in [7] using the fact that all department lengths are equal to one, i. e., $\frac{1}{6}\left(\left(\sum_{i=1}^{n} 1\right)^{3}-\sum_{i=1}^{n} 1^{3}\right)=$ $\frac{1}{6}\left(n^{3}-n\right)=\frac{(n+1) n(n-1)}{6}=f(n)$.

2. For space-free equidistant double-row layouts with $n$ even we have

$$
\sum_{\substack{i, j \in[n] \\ i<j}} d_{i j}=4 f\left(\frac{n}{2}\right)=\frac{(n+2) n(n-2)}{12}
$$

because each row contains $\frac{n}{2}$ departments and we count the inner- as well as the inter-row horizontal distances. For space-free equidistant double-row layouts with $n$ odd we have

$$
\sum_{\substack{i, j \in[n] \\ i<j}} d_{i j}=4 f\left(\frac{n-1}{2}\right)+2 \cdot \frac{\left(\frac{n-1}{2}\right)\left(\frac{n-1}{2}+1\right)}{2}=\frac{(n+1) n(n-1)}{12} .
$$


In comparison to the even case we first arrange $n-1$ departments without spaces in two rows and then add the distance of the remaining department to all others.

Note that in the equidistant case we cannot hope to reduce the sum of the (unweighted) distances by going from single- to double-row layouts by more than one half because for $n$ odd the sum of the pairwise distances is reduced exactly by one half.

Definition 4. We are given an equidistant multi-row instance and an equidistant single-row layout $\pi$. Then, the equidistant multi-row layout $L_{k}(\pi), k \in[m]$, is constructed by assigning the first $k$ departments in the order of $\pi$ to the first column and totally filling up all other columns with the remaining departments in the order of $\pi$. The objective value of layout $L_{k}(\pi), k \in[m]$, is denoted by $v_{L_{k}(\pi)}$.

Let $\pi$ be an equidistant single-row layout. Note that the layouts $L_{k}(\pi), k \in[m]$, contain possible spaces only in the first and last column. We refer to Figure 2 for an illustration of the special case $m=2$. In the following theorem we provide a relation between the objective value of an equidistant single-row layout $\pi$ and the objective value of the associated multi-row layouts $L_{1}(\pi), \ldots, L_{m}(\pi)$.
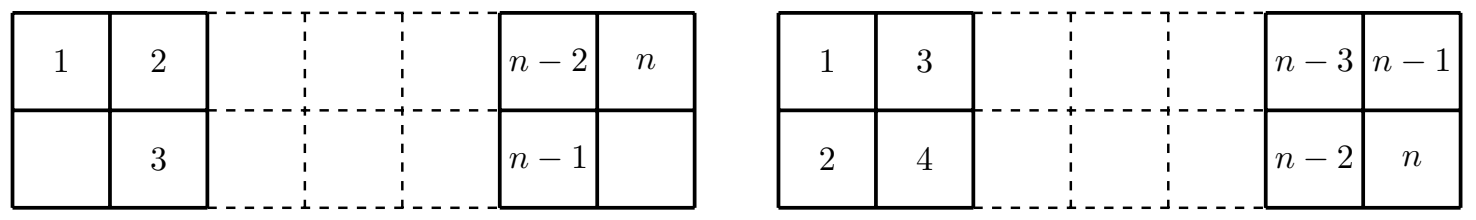

Figure 2: Illustration of the equidistant double-row layouts $L_{1}(\pi)$ on the left-hand side and $L_{2}(\pi)$ on the right-hand side deduced from the single-row layout $\pi=(1, \ldots, n)$. Note that in this drawing we assume, w. l.o.g., that $n$ is even.

Theorem 5. Given an equidistant multi-row instance and an equidistant single-row layout $\pi$ with objective value $v_{1}$ and let $v_{L_{k}(\pi)}$ denote the objective value of layout $L_{k}(\pi), k \in[m]$. Then

$$
\sum_{k \in[m]} v_{L_{k}(\pi)}=v_{1}
$$

Proof. Given an equidistant single-row layout $\pi$ with, w. l. o.g., $\pi=(1, \ldots, n)$, and the layouts $L_{1}(\pi), \ldots, L_{m}(\pi)$. Let $i, j \in[n], i<j$, and let $d_{i j}^{k}$ and $d_{i j}^{\pi}$ denote the horizontal center-to-center distance between $i$ and $j$ in layout $L_{k}(\pi), k \in[m]$, and layout $\pi$, respectively. We get $d_{i j}^{\pi}=j-i$ and our aim is to show that

$$
\sum_{k \in[m]} d_{i j}^{k}=d_{i j}^{\pi}, \quad i, j \in[n], i<j .
$$

So let $i, j \in[n], i<j$. For calculating the distances $d_{i j}^{k}, k \in[m]$, we start with the special case $j-i=1(=2)$. Then, in $m-1(m-2)$ of the layouts $L_{1}(\pi), \ldots, L_{m}(\pi)$ the departments $i$ and $j$ lie in the same column and in one (two) layout (layouts) they lie in neighboring columns. So we obtain:

a) Let $j-i \leq m$. Then, $i$ and $j$ are in $m-j+i$ of the layouts $L_{1}(\pi), \ldots, L_{m}(\pi)$ in the same column, and hence their distance is zero. In the remaining $j-i$ layouts, $i$ and $j$ are in neighboring columns and hence we obtain $\sum_{k \in[m]} d_{i j}^{k}=j-i$.

b) Let $j-i>m$. Then, we choose $j^{\prime}=j-z m, z \in \mathbb{N}_{\geq 1}$, such that $j^{\prime}>i$ and $j^{\prime}-i \leq m$. By the result in a) it follows that

$$
\sum_{k \in[m]} d_{i j}^{k}=\sum_{k \in[m]}\left(d_{i j^{\prime}}^{k}+z\right)=z m+j^{\prime}-i=j-i .
$$


This proves equations (1). The desired result follows immediately: Let $v_{L_{k}(\pi)}, k \in[m]$, and $v_{1}$, denote the objective value of layout $L_{k}(\pi)$ and layout $\pi$, respectively. Then

$$
\sum_{k \in[m]} v_{L_{k}(\pi)}=\sum_{\substack{i, j \in[n] \\ i<j}} w_{i j} \sum_{\substack{k \in[m] \\ i j}} d_{i j}^{k}=\sum_{\substack{i, j \in[n] \\ i<j}} w_{i j} d_{i j}^{\pi}=v_{1}
$$

Note that the result derived by equations (1) is even stronger than the result stated in Theorem 5. In view of Proposition 3 and Theorem 5 we present the relation between $v_{1}^{*}$ and $v_{m}^{*}$ in the equidistant case.

Corollary 6. Given an equidistant multi-row instance and let $v_{m}^{*}\left(v_{1}^{*}\right)$ denote the optimal value of the MREFLP (SREFLP). Then

$$
v_{m}^{*} \leq \frac{v_{1}^{*}}{m}
$$

Given an equidistant multi-row instance and a single-row layout $\pi$ with objective value $v_{1}$, then Corollary 6 and Theorem 5 provide an easy way to construct an equidistant multi-row layout with objective value $v_{m}$ based on $\pi$ that satisfies $v_{m} \leq \frac{v_{1}}{m}$ by computing the layouts $L_{1}(\pi), \ldots, L_{m}(\pi)$ and choosing one layout with minimal objective value.

\subsection{Heuristics for the MREFLP building on combinatorial properties}

Let an equidistant single-row layout $\pi$ be given with objective value $v_{1}$ and we assume, w. l.o.g., $\pi=(1, \ldots, n)$. One can determine the layouts $L_{i}(\pi), i \in[m]$, easily, see Corollary 6 , but the question arises whether one can improve the layouts $L_{i}(\pi)$ by including free-space not only in the first and last column. For determining such a layout, we use an ILP approach based on the following variables

$$
x_{i}= \begin{cases}1, & \text { the } i \text {-th department in the single-row layout } \pi \text { opens a new column } \\ 0, & \text { otherwise }\end{cases}
$$

$i \in[n]$. Then, the ILP approach reads as follows

$$
\begin{array}{ll}
\min \sum_{\substack{i, j \in[n] \\
i<j}} w_{i j} \sum_{k=i+1}^{j} x_{k} & \\
\sum_{j=i}^{i+m-1} x_{j} \geq 1, & i \in[n-m+1], \\
& \\
x_{1}=1, & \\
x_{i} \in\{0,1\}, & i \in[n] .
\end{array}
$$

The distance of $i$ and $j, i, j \in[n], i<j$, equals the number of columns between $i$ and $j$ plus one if $i$ and $j$ lie in distinct columns (3). Inequalities (4) ensure that at most $m$ departments are assigned to each column. Of course, the first department opens a new column, see (5). The matrix corresponding to inequalities (4) satisfies the consecutive ones property, i.e., the ones in each column appear consecutively, and so the integrality conditions (6) can be replaced by $x_{i} \in[0,1], i \in[n]$, see, e.g., [17], and our ILP simplifies to some LP. We illustrate the usage of this LP by the following example. 
Example 7. Given an instance with $\ell_{i}=1, i \in[8]$, with non-zero weights $w_{12}=w_{34}=w_{56}=$ $w_{67}=1$ and $w_{23}=w_{45}=w_{78}=5$. Then, an optimal single-row layout is $\pi^{*}=(1,2,3,4,5,6,7,8)$ with $v_{1}^{*}=19$. The layouts $L_{1}\left(\pi^{*}\right)$ and $L_{2}\left(\pi^{*}\right)$ have objective value 8 and 11 , respectively. Applying the $L P(3)-(5)$ with $x_{i} \in[0,1], i \in[n]$, with $m=2$, we obtain the double-row layout illustrated in Figure 3 with objective value 4 and this layout has free-space in columns 1 and 4.

\begin{tabular}{|l|l|l|l|l|}
\hline 1 & 2 & 4 & 6 & 8 \\
\hline$s$ & 3 & 5 & 7 & $s$ \\
\hline
\end{tabular}

\begin{tabular}{|l|l|l|l|}
\hline 1 & 3 & 5 & 7 \\
\hline 2 & 4 & 6 & 8 \\
\hline
\end{tabular}

\begin{tabular}{|l|l|l|l|l|}
\hline 1 & 2 & 4 & 6 & 7 \\
\hline$s$ & 3 & 5 & $s$ & 8 \\
\hline
\end{tabular}

Figure 3: We consider an instance with $\ell_{i}=1, i \in[8]$, non-zero weights $w_{12}=w_{34}=w_{56}=w_{67}=$ 1 and $w_{23}=w_{45}=w_{78}=5$ and an optimal single-row layout $\pi^{*}=(1,2,3,4,5,6,7,8)$. Then, layout $L_{1}\left(\pi^{*}\right)$ illustrated on the left-hand side has objective value 8 , layout $L_{2}\left(\pi^{*}\right)$ illustrated in the middle has objective value 11 and a double-row layout obtained by solving the LP (3)-(5) with $x_{i} \in[0,1], i \in[n]$, has objective value 4 , where free space between two neighboring departments in the same row is illustrated by some dashed rectangle denoted by $s$.

A layout obtained by solving the LP (3)-(5) with $x_{i} \in[0,1], i \in[n]$, is our initial start layout for further heuristic approaches. At first, we insert $n-2$ dummy departments with length $\ell_{k}=1, k=n+1, \ldots, 2 n-2$, and weights $w_{i k}=0, i \in[2 n-2], k=n+1, \ldots, 2 n-2, i \neq k$, and we fix them on the free spaces in the initial layout. Remaining dummy departments, if they exist at all, are assigned uniformly to the left and to the right border of the layout. It is sufficient to add $n-2$ dummy departments such that the resulting equidistant double-row layout is space-free.

Then, we try to improve the layout by using exchange heuristics. The exchange heuristics 2-opt, 3-opt, 1-column-opt, 2-column-opt and 3-column-opt were used in a related version in [9]. Given a double-row layout with $n-1$ columns, we say that $i \in[2 n-2]$ which lies in column $k \in[n-1]$ is on position $q=k$ if $i$ is in row 1 and on position $q=k+n-1$ if $i$ is in row 2. At first, we apply a 1-opt heuristic, where we place $i \in[n]$ on every possible position $q=1, \ldots, 2 n-2$ on the grid. If there is a dummy department on position $q$, we place the dummy department on the previous position of $i$. Otherwise, let $j \in[n]$ be on position $q$. We shift $j$ to the right or to the left, depending on the case whether $i$ was to the right of to the left of position $q$ before. We assume, w. l.o.g., we shift $j$ to the right. If there is a dummy department on position $q+1$, we are done. Otherwise we shift the department which was previously on position $q+1$ to the right. We continue in this manner until we reach a dummy department or until every department to the right of $j$ (which is in the same row as $j$ ) was shifted.

We say that column $i \in[n-1]$ is on column position $q^{\prime}=i$. Similar to the 1 -opt, we use a 1-column-opt, where we arrange the departments in column $i, i=1, \ldots, n-1$, on every possible position $q^{\prime}, q^{\prime}=1, \ldots, n-1$, and shift the departments in the column on position $q^{\prime}$ to the right or left, depending on the previous position of column $i$. Furthermore, we apply a 2-opt and a 3 -opt algorithm, where we simply change the position of 2 or 3 departments respectively, and a 2 -column-opt as well as a 3-column-opt, where we swap the position of 2 or 3 columns. In the 3-opt algorithm at most one of the considered three departments may be a dummy department while we neglect dummy departments in the 2-opt algorithm. During our improvement algorithm, we compute the objective value of the space-free layouts, since we added dummy departments. Whenever we obtain a better solution, we swap the departments and we apply each opt-algorithm until the solution is $k$-optimal for $k=1,2,3$. The opt-algorithms are applied in increasing order of $k, k=1,2,3$, i. e., in the order 1-column-opt, 1-opt, 2-column-opt, 2-opt, 3-column-opt, 3-opt. 


\section{The double-row facility layout problem}

In Section 3.1 we study the relation between the optimal values of the SRFLP and the DRFLP. So let $v_{1}^{*}\left(v_{2}^{*}\right)$ denote the optimal value of the SRFLP (DRFLP). At first, we prove that $\frac{v_{2}^{*}}{v_{1}^{*}} \leq \frac{n-2}{n-1}$. Let $n \geq 3$. One of our main results in this paper is that there exists a constant $C>0$ such that for every $0<\varepsilon \leq \frac{1}{10}$ there exists a $\delta$ with $0<\delta \leq C \varepsilon$ such that there exists an instance with $\frac{v_{2}^{*}}{v_{1}^{*}}>\frac{n-2}{n-1}-\delta$. This shows that our bound is tight.

However, there are three reasons for constructing double-row layouts based on single-row layouts. First, good or optimal single-row layouts can be obtained very fast, see, e. g., [20, 34, 44, 46]. Second, our computational results, see Section 4, indicate that by going from single-row layouts to double-row layouts the objective value is approximately halved. A third reason is that these double-row layouts can be calculated very fast, in particular, for heuristically determined singlerow layouts. So in Section 3.2.1 we present a generalization of the equidistant layouts $L_{1}(\pi)$ and $L_{2}(\pi)$ with the aim to construct a balanced double-row layout, i. e., a layout where the sum of the lengths of the departments in row 1 is almost equal to the sum of the lengths of the departments in row 2. In Section 3.2.2 we present a new heuristic based on the idea that the ordering of departments in the same row is given by the single-row layout and it remains to determine the row assignment of the departments as well as their exact positions.

\subsection{Relation between optimal single-row and double-row layouts}

We construct double-row layouts based on given single-row layouts in the following way.

Definition 8. Let a single-row layout $\pi$ be given, then we construct layouts $\tilde{L}_{i}(\pi), i \in[n-1]$, in the following way: We assign $i$ to row 1 if $\ell_{i}>\ell_{i+1}$ and otherwise we assign $i$ to row 2. Then, we fix $i+1$ directly opposite $i$. The remaining departments are assigned space-free to row 2 respecting the order of $\pi$.

We refer to Figure 4 for an illustration. The optimal value of the DRFLP is less than or equal to the optimal value of the SRFLP since every single-row layout is also a valid double-row layout. Using the layouts $\tilde{L}_{i}, i \in[n-1]$, we provide the following result:

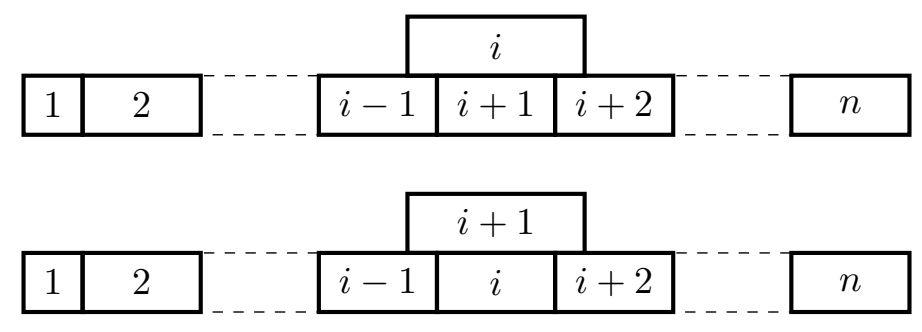

Figure 4: Let a single-row layout $\pi=(1, \ldots, n)$ be given. The layout $\tilde{L}_{i}(\pi), i \in[n-1]$, is illustrated in the two cases $\ell_{i}>\ell_{i+1}$ and $\ell_{i} \leq \ell_{i+1}$.

Proposition 9. Let $v_{1}^{*}\left(v_{2}^{*}\right)$ denote the optimal value of the SRFLP (DRFLP). Then we get

$$
(n-1) v_{2}^{*} \leq(n-2) v_{1}^{*} .
$$

Proof. This result is clear for $n=1$, so let $n \geq 2$. Let, w. l.o.g., $\pi^{*}=(1, \ldots, n)$ be an optimal single-row layout, and hence the distance between $i \in[n]$ and $j \in[n], i<j$, with respect to $\pi^{*}$ simplifies to

$$
d_{i j}^{\pi^{*}}:=\frac{\ell_{i}+\ell_{j}}{2}+\sum_{\substack{h \in[n] \\ i<h<j}} \ell_{h}
$$


Further, we define $d_{i j}^{k}, i, j \in[n], i<j, k \in[n-1]$, as the distance between $i$ and $j$ in layout $\tilde{L}_{k}\left(\pi^{*}\right)$. At first, we will show that

$$
\sum_{k \in[n-1]} d_{i j}^{k} \leq(n-2) d_{i j}^{\pi^{*}}, \quad i, j \in[n], i<j .
$$

Let two departments $i, j \in[n], i<j$, be fixed. If $j=i+1$, then we obtain $d_{i(i+1)}^{i}=0$ as well as $d_{i(i+1)}^{k} \leq d_{i(i+1)}^{\pi^{*}}, k \in[n-1], k \neq i$, and hence inequalities (9) are satisfied in this case. So let $j>i+1$. We distinguish between the following four cases to calculate an upper bound for $d_{i j}^{k}, k \in[n-1]$.

a) $k<i$ or $k \geq j$ : $k$ or $k+1$ is assigned to row 1 . For $k<i-1$ and $k>j$ we get $d_{i j}^{k}=d_{i j}^{\pi^{*}}$ and if $k=i-1$ or $k=j$, we obtain $d_{i j}^{k} \leq d_{i j}^{\pi^{*}}$.

b) $k=i$ : $i$ or $i+1$ is assigned to row 1 in layout $\tilde{L}_{i}\left(\pi^{*}\right)$. Then we get $d_{i j}^{i}=d_{i j}^{\pi^{*}}-\ell_{i+1}-\frac{\ell_{i}}{2}+$ $\frac{\min \left\{\ell_{i}, \ell_{i+1}\right\}}{2}$. We refer to Figure 5a and Figure $5 \mathrm{~b}$ for an illustration.

c) $k=j-1$ : Similar to b) we get $d_{i j}^{j-1}=d_{i j}^{\pi^{*}}-\frac{\ell_{j}}{2}-\ell_{j-1}+\frac{\min \left\{\ell_{j-1}, \ell_{j}\right\}}{2}$.

d) $i<k<j-1: k$ or $k+1$ is assigned to row 1 . Then it follows $d_{i j}^{k}=d_{i j}^{\pi^{*}}-\max \left\{\ell_{k}, \ell_{k+1}\right\}$. An illustration is given in Figure 5c.

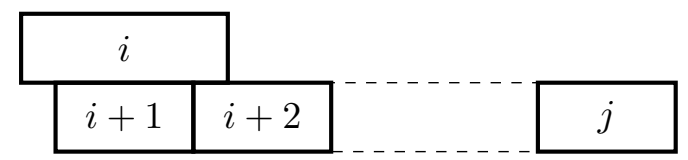

(a) Illustrated layout $\tilde{L}_{i}\left(\pi^{*}\right)$ with $\ell_{i}>\ell_{i+1}$ and $d_{i j}^{i}=d_{i j}^{\pi^{*}}-\frac{\ell_{i}+\ell_{i+1}}{2}$

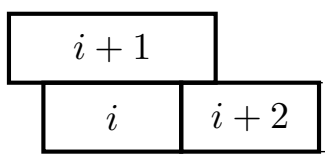

(b) Illustrated layout $\tilde{L}_{i}\left(\pi^{*}\right)$ with $\ell_{i+1} \geq \ell_{i}$ and $d_{i j}^{i}=d_{i j}^{\pi^{*}}-\ell_{i+1}$

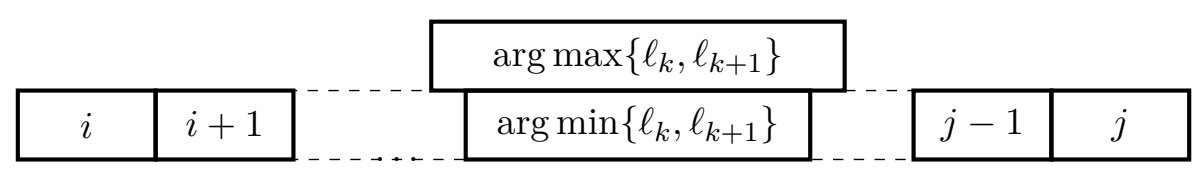

(c) Layout $\tilde{L}_{k}\left(\pi^{*}\right)$ is illustrated and if $i<k<j-1$, we obtain $d_{i j}^{k}=d_{i j}^{\pi^{*}}-\max \left\{\ell_{k}, \ell_{k+1}\right\}$

Figure 5: Illustration of layouts $\tilde{L}_{k}\left(\pi^{*}\right), k \in[n-1]$, with $\pi^{*}=(1, \ldots, n)$ where we only illustrate departments $i, \ldots, j, i, j \in[n], i+1<j$.

Since $\frac{\min \left\{\ell_{i}, \ell_{i+1}\right\}+\min \left\{\ell_{j-1}, \ell_{j}\right\}}{2} \leq \max \left\{\ell_{i+1}, \ell_{j-1}\right\}$ we obtain the desired inequalities (9) by summing up the distances in all layouts $\tilde{L}_{1}\left(\pi^{*}\right), \ldots, \widetilde{L}_{n-1}\left(\pi^{*}\right)$ and using (8)

$$
\begin{aligned}
\sum_{k \in[n-1]} d_{i j}^{k} & \leq \sum_{k \in[n-1]} d_{i j}^{\pi^{*}}-\ell_{i+1}-\ell_{j-1}-\frac{\ell_{i}+\ell_{j}}{2}+\frac{\min \left\{\ell_{i}, \ell_{i+1}\right\}+\min \left\{\ell_{j-1}, \ell_{j}\right\}}{2} \\
& -\sum_{k=i+1}^{j-2} \max \left\{\ell_{k}, \ell_{k+1}\right\} \\
& \leq \sum_{k \in[n-1]} d_{i j}^{\pi^{*}}-\left(\sum_{k=i+1}^{j-2} \max \left\{\ell_{k}, \ell_{k+1}\right\}\right)-\min \left\{\ell_{i+1}, \ell_{j-1}\right\}-\frac{\ell_{i}+\ell_{j}}{2} \\
& \leq(n-1) d_{i j}^{\pi^{*}}-d_{i j}^{\pi^{*}}=(n-2) d_{i j}^{\pi^{*}} .
\end{aligned}
$$

Let $v_{\tilde{L}_{k}\left(\pi^{*}\right)}, k \in[n-1]$, denote the objective value of layout $\tilde{L}_{k}\left(\pi^{*}\right)$, and let $v_{1}^{*}\left(v_{2}^{*}\right)$ denote the optimal value of the SRFLP (DRFLP). By inequalities (9) we obtain

$$
(n-1) v_{2}^{*} \leq \sum_{k \in[n-1]} v_{\tilde{L}_{k}\left(\pi^{*}\right)}=\sum_{\substack{i, j \in[n] \\ i<j}} w_{i j} \sum_{k \in[n-1]} d_{i j}^{k} \leq \sum_{\substack{i, j \in[n] \\ i<j}} w_{i j}(n-2) d_{i j}^{\pi^{*}}=(n-2) v_{1}^{*} .
$$


The optimality of the single-row layout $\pi^{*}$ is not used in the proof of Proposition 9. Therefore, the result can be extended such that $v_{2} \leq \frac{n-2}{n-1} v_{1}$ where $v_{1}$ denotes the objective value of a given single-row layout $\pi$ and $v_{2}$ denotes the minimum value of the objective values of the layouts $\tilde{L}_{1}(\pi), \ldots, \tilde{L}_{n-1}(\pi)$. This result can be used to derive a double-row layout with a slightly smaller objective value than the corresponding single-row layout $\pi$.

However, we show that inequality (7) is tight. We start with instances consisting of $n=3,4,5$ departments and afterwards we enlarge the instances recursively.

Example 10. a) We are given 3 departments with length $\ell_{1}=\ell_{2}=\ell_{3}=\varepsilon>0$ and non-zero weights $w_{12}=w_{23}=1$. Then, we get $v_{1}^{*}=2 \varepsilon$ and $v_{2}^{*}=\varepsilon$, and hence inequality $\frac{v_{2}^{*}}{v_{1}^{*}}>\frac{1}{2}-\delta$ is satisfied for $\delta>0$.

b) Now we add a fourth department with length $\ell_{4}=2-\varepsilon$ and non-zero weight $w_{34}=\varepsilon$. Clearly, $v_{1}^{*}=3 \varepsilon$ and an optimal double-row layout for $\varepsilon \leq \frac{1}{10}$ is depicted in Figure 6 and has objective value $v_{2}^{*}=\varepsilon+(1-\varepsilon) \varepsilon$. Hence we have $\frac{v_{2}^{*}}{v_{1}^{*}}=\frac{\varepsilon(2-\varepsilon)}{3 \varepsilon}=\frac{2-\varepsilon}{3}>\frac{2}{3}-\delta$ if $\varepsilon<3 \delta$.

c) Next we add a fifth department and we choose $0<\varepsilon \leq \frac{1}{10}$ such that $\ell_{5}=\frac{2}{\varepsilon}-\ell_{4}=\frac{2}{\varepsilon}-2+\varepsilon$ and non-zero weight $w_{45}=\varepsilon^{2}$. Then $v_{1}^{*}=4 \varepsilon$. An optimal double-row layout can be obtained by arranging the first 4 departments as good as possible as illustrated in Figure 6 and then additionally arrange 5 such that the centers of departments 4 and 5 are as close as possible, see Figure 6. Then $d_{45} w_{45}=\left(p_{5}-p_{4}\right) w_{45}=\left(\frac{\ell_{5}}{2}+\ell_{3}-\frac{\ell_{4}}{2}\right) \varepsilon^{2}=\varepsilon-2 \varepsilon^{2}+2 \varepsilon^{3}$ and thus $v_{2}^{*}=\varepsilon+(1-\varepsilon) \varepsilon+\varepsilon\left(1-2 \varepsilon+2 \varepsilon^{2}\right)$ and the desired inequality $\frac{v_{2}^{*}}{v_{1}^{*}}>\frac{3}{4}-\delta$ is satisfied for $\delta>\frac{3 \varepsilon-2 \varepsilon^{2}}{4}$.

\begin{tabular}{|l|l|l|}
\hline 1 & 3 & \\
\hline 2 & 4 \\
\hline
\end{tabular}

Figure 6: We are given an instance with $n=4$ departments with lengths $\ell_{1}=\ell_{2}=\ell_{3}=\varepsilon>$ $0, \ell_{4}=2-\varepsilon$, and non-zero weights $w_{12}=w_{23}=1, w_{34}=\varepsilon$. An optimal single-row layout is $\pi^{*}=(1,2,3,4)$ and has objective value $v_{1}^{*}=3 \varepsilon$. The structure of an optimal double-row layout for $\varepsilon \leq \frac{1}{10}$ is depicted above and has objective value $v_{2}^{*}=\varepsilon+(1-\varepsilon) \varepsilon$. One can add a fifth department with $\ell_{5}=\frac{2}{\varepsilon}-\ell_{4}$ and the non-zero weight $w_{45}=\varepsilon^{2}$. Then, we obtain $v_{1}^{*}=4 \varepsilon$ and $v_{2}^{*}=\varepsilon+(1-\varepsilon) \varepsilon+\varepsilon\left(1-2 \varepsilon+2 \varepsilon^{2}\right)$ and thus $\frac{v_{2}^{*}}{v_{1}^{*}}>\frac{3}{4}-\delta$ for $\delta>\frac{3 \varepsilon-2 \varepsilon^{2}}{4}$.

In the following we prove that the double-row layouts constructed in Example 10 are optimal and we show how recursively enlarged double-row instances can be solved to optimality.

Lemma 11. Let an instance with $n$ departments with lengths $\ell_{1}=\ell_{2}=\ell_{3}=\varepsilon$ and $\ell_{k}=$ $\frac{2}{\varepsilon^{k-4}}-\ell_{k-1}, k \in[n], k \geq 4$, and non-zero weights $w_{12}=1$ and $w_{(k-1) k}=\varepsilon^{k-3}, k \in[n], k \geq 3$ be given with $0<\varepsilon \leq \frac{1}{10}$. Then an optimal double-row layout is obtained by arranging the departments in the order $1, \ldots, n$ in an alternating manner to the rows. In each step, one department is assigned at the rightmost possible position such that the layout is space-free and such that 1 lies directly opposite 2.

Proof. Let $d_{i j}\left(d_{i j}^{\prime}, d_{i j}^{\prime \prime}\right.$ and $\left.\tilde{d}_{i j}\right), i, j \in[n], i<j$, denote the horizontal center-to-center distance between $i$ and $j$ in layout $L$ which is constructed as described in the statement of Lemma 11 (in layout $L^{\prime}$, layout $L^{\prime \prime}$ and layout $\tilde{L}$ with its construction described below) and let $p_{i}, i \in[n]$, $\left(p_{i}^{\prime}, p_{i}^{\prime \prime}\right.$ and $\tilde{p}_{i}$ ) denote the center position of department $i$ in layout $L$ (layout $L^{\prime}$, layout $L^{\prime \prime}$ and 
layout $\tilde{L})$. Further, let $r_{i} \in\{1,2\}\left(r_{i}^{\prime}, r_{i}^{\prime \prime}, \tilde{r}_{i} \in\{1,2\}\right)$ denote the row assignment of $i \in[n]$ in layout $L\left(L^{\prime}, L^{\prime \prime}\right.$ and $\left.\tilde{L}\right)$. We divide the proof into three parts. In the first two parts we assume that 1 lies opposite 2 with $p_{1}=p_{2}=\frac{1}{2}$ and then we show in part 1) that the departments to the, w.l.o.g., right of 1 or 2 are assigned in an alternating manner with respect to the rows, at the rightmost possible position such that the resulting layout is space-free in an increasing order. In this step there might be departments right and left to 1 and 2. In part 2) we show that in an optimal layout all the departments $3, \ldots, n$ are to the, w. l. o. g., right of 1 or 2 and we complete the proof by showing in part 3 ) that in an optimal layout 1 lies opposite 2 .

1) Let a double-row layout be given where 1 lies directly opposite 2 and let $S \subseteq\{3, \ldots, n\}$ denote the set of departments to the right of 1 or 2 . We show that in an optimal layout the departments in $S$ are arranged in an alternating manner to the rows in increasing order. In each step, one department is assigned at the rightmost possible position such that the layout is space-free. The layout is denoted by $\tilde{L}$ and we assume, w.l.o.g., $\tilde{p}_{1}=\tilde{p}_{2}=\frac{1}{2}$. An illustration is given in Figure 6 for $n=5$ and $S=\{3,4,5\}$. Assume, on the contrary, there exists an optimal layout $L^{\prime}$ where 1 lies directly opposite 2 (with $p_{1}^{\prime}=p_{2}^{\prime}=\frac{1}{2}$ ) where exactly the departments in $S$ lie right of 1 or 2 and the others lie left of 1 or 2 and at least one department in $S$ is not arranged as in $\tilde{L}$. Let $k \in S$ be the department with its left border closest to 1 in layout $L^{\prime}$ with $\tilde{d}_{1 k} \neq d_{1 k}^{\prime}$ (if two departments satisfy this property, we choose the department with the smaller index). Let $k$ be to the right of $h \in S \cup\{1,2\}$ in the same row with possible free-space between $h$ and $k$ such that no department lies between $h$ and $k$.

At first, we show that in layout $L^{\prime}$ the departments $\{z \in S: z \leq h\}$ have the same position as in layout $\tilde{L}$. This in particular shows that in an optimal layout $L^{\prime}$ it holds that $h<k$. Let $i:=\min \left\{z \in S: p_{z}^{\prime} \neq \tilde{p}_{z}\right\}$ and we assume, on the contrary, $i \leq h$. Then, by the definition of $k, h, i$ there is free-space around $\tilde{p}_{i}$ in $L^{\prime}$ such that one can arrange $i$ on position $\tilde{p}_{i}$ in layout $L^{\prime}$ without overlapping other departments (the positions of all other departments remain the same). We denote the resulting layout by $L^{\prime \prime}$. Note that either $i-1 \in S \cup\{1,2\}$ and $p_{i-1}^{\prime}=\tilde{p}_{i-1}$ or $i-1$ is to the left of 1 or 2 in layouts $L^{\prime}$ and $L^{\prime \prime}$. So it follows that $p_{i}^{\prime \prime}>p_{i-1}^{\prime \prime}$. Let $\delta=p_{i}^{\prime}-p_{i}^{\prime \prime}>0$. Then, we obtain $d_{(i-1) i}^{\prime \prime}+\delta=d_{(i-1) i}^{\prime}$ and $d_{i(i+1)}^{\prime \prime}-\delta \leq d_{i(i+1)}^{\prime}$. The remaining weighted distances in layouts $L^{\prime}$ and $L^{\prime \prime}$ are equal. Since $w_{(i-1) i}>w_{i(i+1)}$, the objective value of layout $L^{\prime \prime}$ is smaller than the objective value of layout $L^{\prime}$, a contradiction. Thus, we have $p_{z}^{\prime}=\tilde{p}_{z}, z \in S, z \leq h$.

We distinguish now between the following two cases where the first case has two subcases in the description below depending on $h$. For the first case, let $k=\min \{z \in S: z \geq h+1\}$ if $h \geq 2$, and $k=\min \{z \in S: z \geq 3\}$ if $h=1$. If $h \geq 2(h=1)$, we obtain either $k-1=h$ $(k=3)$ or $k-1>h(k>3)$ and $k-1$ is to the left of 1 or 2 . By the construction of the layout and the definition of $k$, at most one department $j \in S \cup\{1,2\}$ overlaps with $h$. We obtain $j<h$ if $h \neq 1$ and $j=2$ if $h=1$. Therefore, department $j$ satisfies $\tilde{p}_{j}=p_{j}^{\prime}$ and $p_{j}^{\prime}+\frac{\ell_{j}}{2}<p_{k}^{\prime}-\frac{\ell_{k}}{2}$. Note that if $j \in\{1,2\}$, then there is free-space between $h$ and $k$. Therefore, we obtain a feasible layout by changing the row assignment of the departments $z \in S$ with $p_{z}^{\prime}>p_{h}^{\prime}$ without changing their positions. Then, we shift $k$ without spaces to the right of $j$, if $j$ exists, and to the right of 1 or 2 otherwise. We denote the resulting layout by $L^{\prime \prime}$ and we obtain $p_{h}^{\prime \prime}<p_{k}^{\prime \prime}$ because we have $\tilde{p}_{z}=p_{z}^{\prime \prime}, z \in S, z \leq h$ and $\ell_{h}<\ell_{k}$ or $k=3$. Let $\delta:=p_{k}^{\prime}-p_{k}^{\prime \prime}>0$. Therefore, we obtain $d_{(k-1) k}^{\prime \prime}+\delta=d_{(k-1) k}^{\prime}$ and, if $k+1 \in[n]$, $d_{k(k+1)}^{\prime \prime}-\delta \leq d_{k(k+1)}^{\prime}$. The remaining weighted distances in layout $L^{\prime \prime}$ are the same as in layout $L^{\prime}$. Since $w_{(k-1) k}>w_{k(k+1)}$ if $k+1 \in[n]$, the objective value of layout $L^{\prime \prime}$ is smaller than the objective value of layout $L^{\prime}$, a contradiction. 
Now we consider the case of $k>\min \{z \in S: z \geq h+1\}$ and we set

$$
j:= \begin{cases}2, & h=1, \\ 1, & h=2, \\ \min \{z \in S: z \geq h+1\}, & h \geq 3 .\end{cases}
$$

We aim to show that $p_{j}^{\prime}=\tilde{p}_{j}$. This result is clear if $j \in\{1,2\}$, so let $h \geq 3$. Then, either $j-1=h$ or $j-1$ is left to 1 or 2 . By the definition of $k$ we can arrange $j$ on position $\tilde{p}_{j}$ either in row 1 or row 2 such that the resulting layout $L^{\prime \prime}$ is feasible. Let $\delta:=\left|p_{j}^{\prime}-\tilde{p}_{j}\right|>0$. If $\delta \leq \ell_{j}$, then $j$ is in layout $L^{\prime}$ and $L^{\prime \prime}$ in the same row (since $\ell_{k}>\ell_{j}$ ) and layout $L^{\prime \prime}$ is feasible since $p_{z}^{\prime \prime}=\tilde{p}_{z}$ for all $z \in S, z \leq h$. We obtain $d_{(j-1) j}^{\prime \prime}+\delta=d_{(j-1) j}^{\prime}$ and $d_{j(j+1)}^{\prime \prime}-\delta \leq d_{(j-1) j}^{\prime}$. By $w_{(j-1) j}>w_{j(j+1)}$ it follows that the objective value of layout $L^{\prime \prime}$ is smaller than the objective value of layout $L^{\prime}$, a contradiction. Now, let $\delta>\ell_{j}$. Then, to avoid the overlapping of departments, we shift all departments which are in layout $L^{\prime \prime}$ to the right of $j$ in the same row to the right by the smallest possible value such that a feasible layout is obtained. The departments are shifted at most by the value $\ell_{j}$, so we obtain $d_{(j-1) j}^{\prime \prime}+\delta=d_{(j-1) j}^{\prime}$ and $d_{z(z+1)}^{\prime \prime} \leq d_{z(z+1)}^{\prime}+\delta, z \in[n-1], z \geq j$. Note that for $0<\varepsilon \leq \frac{1}{10}$ and $o \in[n-1], o \geq 3$, we obtain

$$
w_{(o-1) o} \quad>2 w_{o(o+1)}+\sum_{z=o+1}^{n-1} w_{z(z+1)} .
$$

Hence, the objective value of layout $L^{\prime \prime}$ is smaller than the objective value of layout $L^{\prime}$, a contradiction. Therefore, we have $p_{j}^{\prime}=\tilde{p}_{j}$.

Now we consider $o:=\min \{q \in S: q \geq \max \{h, j\}+1\}$. We obtain either $\max \{h, j\}=o-1$ or $\max \{h, j\}<o-1$ and $o-1$ is to the left of 1 or 2 . Let $k=o$, then there is free-space between $h$ and $k$ because of the choice of $k$. We simply shift $k$ to the left such that $k$ is to the right of $h$ without free-space and the resulting layout is denoted by $L^{\prime \prime}$. By construction of the layout we obtain $p_{k}^{\prime \prime}>p_{k-1}^{\prime \prime}$ and we set $\delta=p_{k}^{\prime}-p_{k}^{\prime \prime}>0$. We obtain $d_{(k-1) k}^{\prime \prime}+\delta=d_{(k-1) k}^{\prime}$ and, if $k+1 \in[n], d_{k(k+1)}^{\prime \prime}-\delta \leq d_{k(k+1)}^{\prime}$. The remaining weighted distances are equal in layout $L^{\prime}$ and layout $L^{\prime \prime}$. Since $w_{(k-1) k}>w_{k(k+1)}$ if $k+1 \in[n]$, the objective value of layout $L^{\prime \prime}$ is smaller than the objective value of layout $L^{\prime}$, a contradiction. So let $k>o$ and let $\delta>0$ denote the horizontal distance between the right border of $h$ and the left border of $o$. We distinguish between the following two cases.

a) Let $\delta \geq \ell_{o}$, then we arrange $o$ space-free to the right of $h$ and, if necessary, we shift the departments which are to the right of $h$ or $j$ to the right by the smallest possible value such that $o$ and $k$ do not overlap. Note that these departments are shifted at most by $\ell_{o}$, we refer to Figure $7 \mathrm{a}$ and Figure $7 \mathrm{~b}$ for an illustration. The resulting layout is denoted by $L^{\prime \prime}$ and we obtain $p_{o}^{\prime \prime}>p_{o-1}^{\prime \prime}$. Comparing the distances in layout $L^{\prime}$ and $L^{\prime \prime}$, we obtain $d_{(o-1) o}^{\prime \prime}+\delta=d_{(o-1) o}^{\prime}$ and $d_{o(o+1)}^{\prime \prime} \leq d_{o(o+1)}^{\prime}+\delta+\ell_{o}$. Additionally, we obtain $d_{z(z+1)}^{\prime \prime} \leq d_{z(z+1)}^{\prime}+\ell_{o}, z \in[n-1], z \geq o+1$. The remaining weighted distances in layout $L^{\prime \prime}$ and $L^{\prime}$ are equal. By (10) the following inequality is satisfied

$$
\delta w_{(o-1) o} \quad>\left(\delta+\ell_{o}\right) w_{o(o+1)}+\ell_{o} \sum_{z=o+1}^{n-1} w_{z(z+1)} .
$$

So the objective value of layout $L^{\prime \prime}$ is smaller than the objective value of layout $L^{\prime}$, a contradiction.

b) It remains to consider the case $\delta<\ell_{o}$. Since $k>o$ and $\ell_{k}>\ell_{o}$ it follows that $o$ and $j$ are neighboring (with possible free-space), see Figure 7c. Let $\delta^{\prime}$ denote the 
horizontal distance of the left border of $k$ and the right border of $j$ if $k$ and $j$ overlap and otherwise we set $\delta^{\prime}=0$. Then we change the row assignment of all departments to the right of $h$ or $j$ (without changing the order of these departments), and we shift these departments (without $o$ ) by $\delta^{\prime}>0$ to the right such that $j$ and $k$ do not overlap. Then we shift $o$ to the left by the value $\delta$ such that there is no free-space between $h$ and $o$, see Figure $7 \mathrm{~d}$. By construction of the layout, we obtain $\delta^{\prime} \leq \delta$. The resulting layout is denoted by $L^{\prime \prime}$. We compare the weighted distances in layout $L^{\prime \prime}$ and $L^{\prime}$, similar as done above, and it turns out that the objective value of layout $L^{\prime \prime}$ is smaller than the objective value of layout $L^{\prime}$ if

$$
\delta w_{(o-1) o}>\left(\delta+\delta^{\prime}\right) w_{o(o+1)}+\delta^{\prime} \sum_{z=o+1}^{n-1} w_{z(z+1)} .
$$

This inequality is satisfied for $0<\varepsilon \leq \frac{1}{10}$, see inequalities (10), a contradiction.

2) Let 1 lie directly opposite 2 . We prove that the departments $3, \ldots, n$ are to the, w. l. o. g., right of 1 or 2 . Assume, on the contrary, there exists an optimal double-row layout $L^{\prime}$ where 1 lies directly opposite 2 with $p_{1}^{\prime}=p_{2}^{\prime}=\frac{1}{2}$ and the departments $S \subset[n], S \neq \emptyset$, are to the right of 1 or 2 and the departments $T \subset[n], T \neq \emptyset$, are to the left of 1 or 2 such that $S \dot{\cup} T=\{3, \ldots, n\}, S \cap T=\emptyset$. Since $L^{\prime}$ is an optimal double-row layout, the departments in $S$ and $T$ are arranged as described in 1$)$. Let $i \in S, i+1, \ldots, j \in T$ and $j+1 \notin T$. Note that, if $i \in T$ and $i+1, \ldots, j \in S, j+1 \notin S$, we simply arrange the departments left (right) to 1 or 2 to the right (left) of 1 or 2 without changing the row assignment and the order of the departments in $S(T)$, and hence we obtain $i \in S$ and $i+1, \ldots, j \in T, j+1 \notin T$. Our goal is to show that $\sum_{z=i}^{j-1} w_{z(z+1)} d_{z(z+1)}<\sum_{z=i}^{j-1} w_{z(z+1)} d_{z(z+1)}^{\prime}$. At first, we obtain

$$
d_{i(i+1)}^{\prime}>\frac{\ell_{i}+\ell_{i+1}}{2}>d_{i(i+1)} .
$$

If $j=i+1$, the desired inequality is satisfied. Otherwise, we obtain

$$
d_{i(i+1)}^{\prime}+d_{(i+1)(i+2)}^{\prime}>d_{i(i+1)}+d_{(i+1)(i+2)}=\frac{\ell_{i}+\ell_{i+2}}{2}
$$

where the last equation follows from $p_{i}<p_{i+1}<p_{i+2}$. We refer to Figure 8 for an illustration. We continue in this manner and we obtain

$$
d_{z(z+1)}^{\prime}+d_{(z+1)(z+2)}^{\prime}=d_{z(z+1)}+d_{(z+1)(z+2)},
$$

$z, z+1, z+2 \in T, z \geq i+1$. For $i$ even we obtain, $d_{(i+1)(i+2)}=\sum_{\substack{z \geq 4 \\ z<\leq e n \\ z \leq i}} \ell_{z}-\sum_{\substack{z \geq 3 \\ z \text { odd } \\ z \leq i}} \ell_{z}+$ $\frac{\ell_{i+2}-\ell_{i+1}}{2}$. Note that, if $i+1, i+2 \in T$, then $i+1$ and $i+2$ lie in distinct rows. We obtain

$$
d_{(i+1)(i+2)}^{\prime}-d_{(i+1)(i+2)}=\sum_{\substack{z \in T \\ z \leq i-1 \\ r_{z}^{\prime}=r_{i+2}^{\prime}}} \ell_{z}-\sum_{\substack{z \in T \\ z \leq i-1 \\ r_{z}^{\prime}=r_{i+1}^{\prime}}} \ell_{z}+\sum_{\substack{z \geq 3 \\ z \text { odd } \\ z \leq i}} \ell_{z}-\sum_{\substack{z \geq 4 \\ z \text { even } \\ z \leq i}} \ell_{z}<0
$$

since for $0<\varepsilon \leq \frac{1}{10}$ we have $\ell_{i}>2 \sum_{z \in[i-1]} \ell_{z}$. Now, let $i$ be odd. We get $d_{(i+1)(i+2)}=$ $\sum_{\substack{z \geq 3 \\ z \leq i d}} \ell_{z}-\sum_{\substack{z \leq 4 \\ z \leq i \\ z \leq i}} \ell_{z}+\frac{\ell_{i+2}-\ell_{i+1}}{2}$, and thus we get

$$
d_{(i+1)(i+2)}^{\prime}-d_{(i+1)(i+2)}=\sum_{\substack{z \in T \\ z \leq i-1 \\ r_{z}^{\prime}=r_{i+2}^{\prime}}} \ell_{z}-\sum_{\substack{z \in T \\ z \leq i-1 \\ r_{z}^{\prime}=r_{i+1}^{\prime}}} \ell_{z}-\sum_{\substack{z \geq 3 \\ z \text { odd } \\ z \leq i}} \ell_{z}+\sum_{\substack{z \geq 4 \\ z \text { even } \\ z \leq i}} \ell_{z}<0
$$




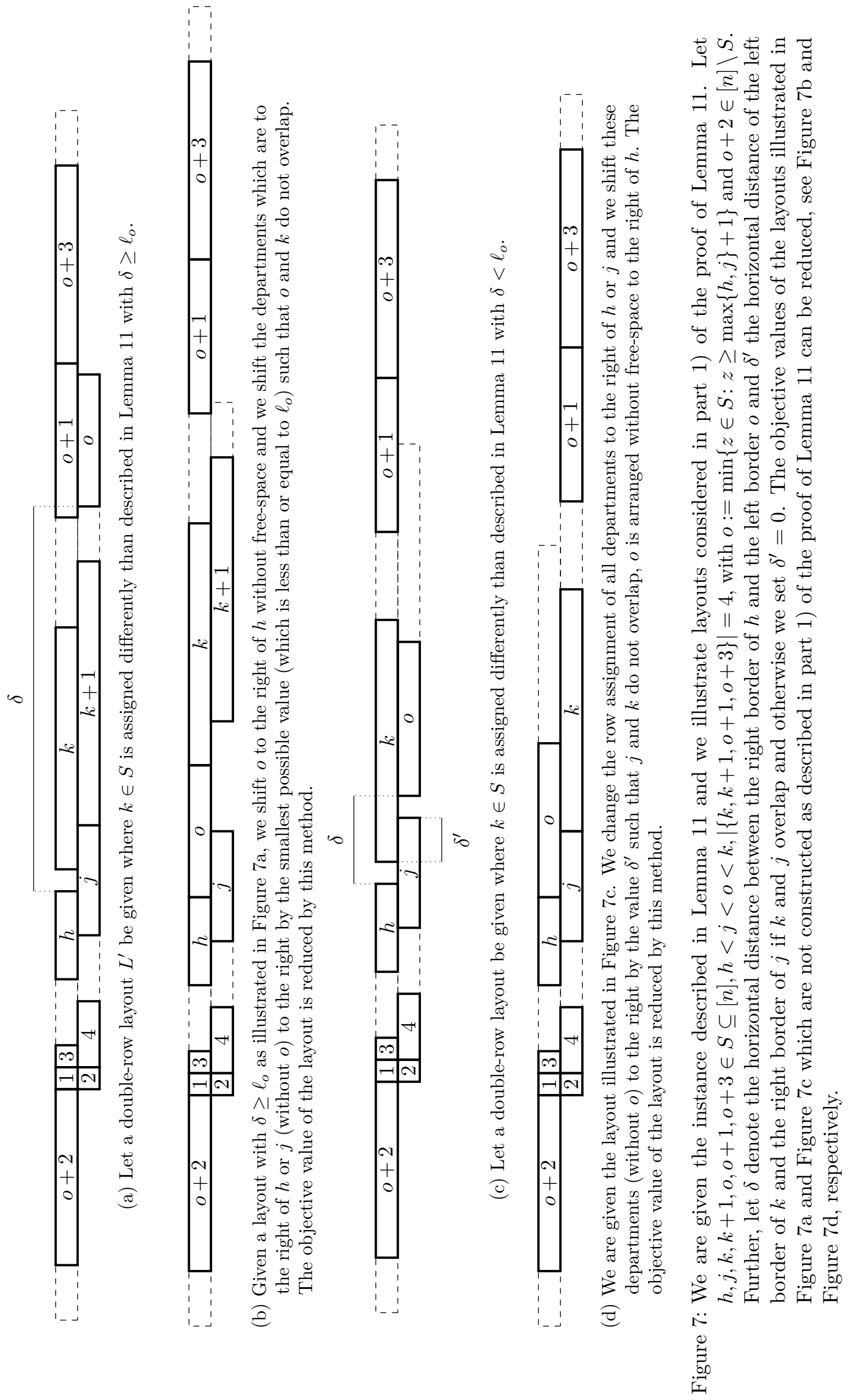




\begin{tabular}{|c|c|c|c|c|}
\hline 1 & 3 & $i-1$ & & \\
\hline 2 & 4 & & $i$ & $i+2$ \\
\hline
\end{tabular}

(a) Given a double-row layout $L$ constructed as described in Lemma 11 we obtain $d_{i(i+1)}+$ $d_{(i+1)(i+2)}=\frac{\ell_{i}+\ell_{i+2}}{2}$.

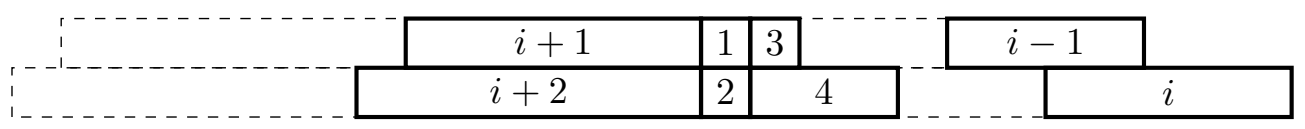

(b) Given a double-row layout $L^{\prime}$ where 1 lies opposite 2 and the departments $1, \ldots, i, i \in$ $[n], i \geq 3$, are to the right of 1 or 2 and the departments $i+1, \ldots, j \in[n]$, are to the left of 1 or 2 (with $i+1 \neq j$ here) we obtain $d_{i(i+1)}^{\prime}+d_{(i+1)(i+2)}^{\prime}>d_{i(i+1)}+d_{(i+1)(i+2)}$.

Figure 8: We are given the instance described in Lemma 11 and we compare the distances in layout $L$ and $L^{\prime}$. We obtain $d_{i(i+1)} \leq d_{i(i+1)}^{\prime}-\delta$, and $d_{(i+1)(i+2)}=d_{(i+1)(i+2)}^{\prime}+\delta$, for some $\delta>0$. We continue in this manner and since $w_{z(z+1)}>w_{(z+1)(z+2)}, z \in[n-2], z \geq 2$, the objective value of layout $L$ is smaller than the objective value of layout $L^{\prime}$.

and for $0<\varepsilon \leq \frac{1}{10}$ this inequality is satisfied since $\ell_{i}>2 \sum_{z \in[i-1]} \ell_{z}$. So we obtain $d_{(i+1)(i+2)}^{\prime}=d_{(i+1)(i+2)}-\delta$ for some $\delta>0$ and by inequalities (11) we obtain $d_{i(i+1)}^{\prime}>$ $d_{i(i+1)}+\delta$. Further, by equations $(12) d_{(i+z)(i+z+1)}=d_{(i+z)(i+z+1)}^{\prime}-\delta, \frac{z}{2} \in \mathbb{Z}, z \geq 2, i+$ $z+1 \leq j$, and $d_{(i+z+1)(i+z+2)}=d_{(i+z+1)(i+z+2)}^{\prime}+\delta, \frac{z}{2} \in \mathbb{Z}, z \geq 2, i+z+2 \leq j$. Recall that $w_{z(z+1)}>w_{(z+1)(z+2)}, z+2 \in[n], z \geq 2$, so the desired inequality is satisfied. One can continue in this manner if further departments $k$ and $k+1$ are in distinct sets $S$ and $T, k \in[n-1], k \geq j$. So the objective value of layout $L$ is smaller than the objective value of layout $L^{\prime}$, a contradiction.

3) It remains to show that 1 lies directly opposite 2 in an optimal double-row layout. Assume, on the contrary, there exists an optimal double-row layout $L^{\prime}$ where 1 does not lie directly opposite 2. We divide this proof into three cases.

a) At first, let $d_{23}^{\prime}<\varepsilon$, and we assume, w.l.o.g., $p_{2}^{\prime} \leq p_{3}^{\prime}$. We calculate a lower bound for such a layout by allowing the departments $4, \ldots, n$ to overlap with 1 in the same row (correct sublayout with respect to $1,2,3$ ). Then, the departments $4, \ldots, n$ are arranged in an alternating manner to the rows such that 4 is arranged in the same row as 2 and space-free at the rightmost position (since $p_{2}^{\prime} \leq p_{3}^{\prime}$ ) in increasing order, the proof of this result is similar to the proof of 1) and 2). We denote the resulting (in general not feasible) layout with departments $1, \ldots, n$ by $L^{\prime \prime}$ and clearly, the objective value of layout $L^{\prime \prime}$ is less than or equal to the objective value of layout $L^{\prime}$. We obtain $d_{12}+d_{23} \leq d_{12}^{\prime \prime}+d_{23}^{\prime \prime}$. Further, let $\delta:=\varepsilon-d_{23}^{\prime \prime}>0$ be the length of the line segment at which 2 and 3 overlap, then we get $d_{(2+i)(2+i+1)}=d_{(2+i)(2+i+1)}^{\prime \prime}-\delta, i \in[n-3], i$ odd, and $d_{(2+i+1)(2+i+2)}=d_{(2+i+1)(2+i+2)}^{\prime \prime}+\delta, i \in[n-4], i$ odd. Similar to 2$)$ it follows that the objective value of layout $L^{\prime \prime}$ is greater than the objective value of layout $L$, a contradiction.

b) Let $d_{13}^{\prime}<\varepsilon$ and $d_{23}^{\prime} \geq \varepsilon$, then one can swap the positions of 1 and 2 and the objective value of the layout is reduced, a contradiction.

c) It remains to consider an optimal double-row layout $L^{\prime}$ with $d_{13}^{\prime} \geq \varepsilon$ and $d_{23}^{\prime} \geq \varepsilon$. At first, let $0<d_{12}^{\prime}<\varepsilon$ and we assume, w.l.o.g., $p_{1}^{\prime}<p_{2}^{\prime}$. If $p_{3}^{\prime}<p_{1}^{\prime}$, we shift all departments in the same row as 1 to the right until 1 and 2 lie directly opposite. The resulting layout is denoted by $L^{\prime \prime}$ and we set $d_{12}^{\prime}:=\delta>0$. Then, we obtain $d_{12}^{\prime \prime}=0$, $d_{23}^{\prime \prime} \leq d_{23}^{\prime}$ and $d_{z(z+1)}^{\prime \prime} \leq d_{z(z+1)}^{\prime}+\delta, z \in[n-1], z \geq 3$. Since $w_{(z-1) z}>w_{z(z+1)}, z \in$ $[n-1], z \geq 3, w_{12}=w_{23}$, the objective value of layout $L^{\prime \prime}$ is smaller than the objective 
value of layout $L^{\prime}$, a contradiction. Now, let $p_{3}^{\prime}>p_{2}^{\prime}$. Then we shift 3 to the right of 1 without free-space and, if necessary, we shift all departments now lying to the right of 3 or 2 in the same row to the right to avoid overlapping. The resulting layout has a smaller objective value than layout $L^{\prime}$, the proof is similar to 1a) and 1b) with $o=3$, a contradiction.

So, let $d_{12}^{\prime} \geq \varepsilon$. We calculate a lower bound on a layout containing the departments $3, \ldots, n$ and then we add $w_{12} d_{12}^{\prime}+w_{23} d_{23}^{\prime} \geq 2 \varepsilon$ to this lower bound. To calculate a lower bound for the layout of the departments $3, \ldots, n$, we distinguish between two cases. If 3 and 4 overlap in layout $L^{\prime}$, then 3 and 4 lie directly opposite as described above for 1 and 2 . So let 3 and 4 lie directly opposite. Then the departments $5, \ldots, n$ are arranged in an alternating manner space-free at the rightmost position in increasing order to the rows (this result is similar to the proof of 1) and 2)). The resulting layout is denoted by $L^{\prime \prime}$ and the objective value of layout $L^{\prime \prime}$ is less than or equal to the objective value of layout $L^{\prime}$. We obtain $w_{12} d_{12}+w_{23} d_{23}+w_{34} d_{34}<2 \varepsilon$, see Example 10. Furthermore, we obtain $d_{z(z+1)}^{\prime \prime}=d_{z(z+1)}+\delta, z \in[n-1], z \geq 4, \frac{z}{2} \in \mathbb{Z}$, and $d_{z(z+1)}^{\prime \prime}=d_{z(z+1)}-\delta, z \in[n-1], z \geq 5, \frac{z+1}{2} \in \mathbb{Z}$, for some $\delta>0$. So the objective value of layout $L$ is smaller than the objective value of layout $L^{\prime \prime}$.

Now we assume that 3 and 4 do not overlap in layout $L^{\prime}$. Then, we calculate a lower bound for the double-row instance consisting of the departments $4, \ldots, n$ and add $\sum_{i \in[3]} w_{i(i+1)} d_{i(i+1)}^{\prime} \geq 3 \varepsilon$ to this lower bound. We distinguish between the two cases whether 4 and 5 overlap or not. We continue as described above and this lower bound exceeds the objective value of layout $L$.

So for these instances we obtain an optimal double-row layout as described above. Therefore, we obtain our desired result.

Theorem 12. Let $n \geq 3$. Then, there exists a constant $C>0$ such that for every $0<\varepsilon \leq \frac{1}{10}$ there exists a $\delta$ with $0<\delta \leq C \varepsilon$ such that

$$
\frac{v_{2}^{*}}{v_{1}^{*}}>\frac{n-2}{n-1}-\delta,
$$

where $v_{1}^{*}\left(v_{2}^{*}\right)$ denotes the optimal value of the SRFLP (DRFLP) with $n$ departments and lengths $\ell_{1}=\ell_{2}=\ell_{3}=\varepsilon$ and $\ell_{k}=\frac{2}{\varepsilon^{k-4}}-\ell_{k-1}, k \in[n], k \geq 4$, and non-zero weights $w_{12}=w_{23}=1$ and $w_{(k-1) k}=\varepsilon^{k-3}, k \in[n], k \geq 3$.

Proof. We prove this result by induction. For $n=3,4$ and $n=5$ we refer to Example 10. So let $n \in \mathbb{N}, n \geq 6$, and we consider instances as described in the statement of the theorem and considered in Lemma 11. We obtain $\frac{\ell_{k-1}+\ell_{k}}{2} w_{(k-1) k}=\varepsilon, k \in[n], k \geq 2$, and thus the single-row layout $\pi^{*}=(1, \ldots, n)$ has objective value $v_{1, n}^{*}:=(n-1) \varepsilon$ which proves that $\pi^{*}$ is optimal because its objective value equals the constant $\tilde{C}:=\sum_{\substack{i, j \in[n] \\ i<j}} w_{i j} \frac{\ell_{i}+\ell_{j}}{2}$ which is a lower bound on the optimal value of the SRFLP.

An optimal double-row layout can be obtained as described in Lemma 11 and it remains to compare the optimal value of the double-row instance with $v_{1, n}^{*}$. Therefore, let $p_{n}$ and $p_{n-1}$ denote the center position of $n$ and $n-1$ measured from the left border of the double-row layout. We assume, w.l.o.g., that $n$ is odd and hence $n$ is in the same row as 1 .

$$
\begin{aligned}
\left(p_{n}-p_{n-1}\right) w_{(n-1) n} & =\left(\left(\frac{\ell_{n}}{2}+\ell_{n-2}+\ldots+\ell_{3}+\ell_{1}\right)-\left(\frac{\ell_{n-1}}{2}+\ell_{n-3}+\ldots+\ell_{4}+\ell_{2}\right)\right) \varepsilon^{n-3} \\
& =\left(\frac{1}{\varepsilon^{n-4}}-\ell_{n-1}+\ell_{n-2}-\ell_{n-3}+\ell_{n-4}-\ldots-\ell_{4}+\ell_{3}\right) \varepsilon^{n-3}
\end{aligned}
$$




$$
\begin{aligned}
& =\left(\frac{1}{\varepsilon^{n-4}}-\frac{2}{\varepsilon^{n-5}}+2 \ell_{n-2}-\ell_{n-3}+\ell_{n-4}-\ldots-\ell_{4}+\ell_{3}\right) \varepsilon^{n-3} \\
& =\left(\frac{1}{\varepsilon^{n-4}}+\sum_{z=1}^{n-4}\left((-1)^{z} \frac{2 z}{\varepsilon^{n-4-z}}\right)+(n-3) \ell_{3}\right) \cdot \varepsilon^{n-3} \\
& =\varepsilon+\sum_{z=1}^{n-4}\left((-1)^{z} 2 z \varepsilon^{1+z}\right)+(n-3) \varepsilon^{n-2} .
\end{aligned}
$$

We set $\delta^{\prime \prime}=(-1) \frac{\sum_{z=1}^{n-4}\left((-1)^{z} 2 z \varepsilon^{1+z}\right)+(n-3) \varepsilon^{n-2}}{(n-1) \varepsilon}$ and for small $\varepsilon>0$ we obtain $0<\delta^{\prime \prime} \leq \varepsilon$. Let $v_{2, n}^{*}$ and $v_{2, n-1}^{*}$ denote the optimal value of an optimal double-row layout with $n$ and $n-1$ departments, respectively. By assumption there exists a constant $C^{\prime}>0$ such that there exists a $\delta^{\prime}$ with $0<\delta^{\prime} \leq C^{\prime} \varepsilon$, such that $\frac{v_{2, n-1}^{*}}{(n-2) \varepsilon}>\frac{n-3}{n-2}-\delta^{\prime}$. Then, we obtain

$$
\begin{aligned}
\frac{v_{2, n}^{*}}{v_{1, n}^{*}} & =\frac{v_{2,(n-1)}^{*}+\left(p_{n}-p_{n-1}\right) w_{n(n-1)}}{(n-1) \varepsilon} \\
& >\frac{n-3}{n-1}-\delta^{\prime} \frac{n-2}{n-1}+\frac{1}{n-1}-\delta^{\prime \prime} \\
& =\frac{n-2}{n-1}-\delta^{\prime} \frac{n-2}{n-1}-\delta^{\prime \prime} .
\end{aligned}
$$

We set $C=C^{\prime} \frac{n-2}{n-1}+1, \delta=\delta^{\prime} \frac{n-2}{n-1}+\delta^{\prime \prime}$ and we obtain $\delta \leq C \varepsilon$.

\subsection{Heuristic approaches for the DRFLP}

In Sections 3.2.1 and 3.2.2 we describe two heuristics for the DRFLP based on good or optimal single-row layouts. Considering the instances described in Lemma 11, both heuristics determine an optimal double-row layout given the optimal single-row layout $\pi=(1, \ldots, n)$. Further, both heuristics are extensions of the heuristics presented in Section 2.2. Similar as before both variants use a given single-row layout, especially the order of the departments, to construct a double-row layout. Because of the arbitrary department lengths in the DRFLP adaptations are needed. In Section 3.2.3 we describe exchange heuristics to further improve given double-row layouts. In particular, we set up an MILP model for deriving a 1-optimal solution.

\subsubsection{Balanced rows}

Given a single-row layout $\pi=\left(\pi_{1}, \ldots, \pi_{n}\right)$, we first determine the row assignment of the departments. In the order of $\pi$ we assign the current department to a row where the sum of the lengths of the departments already assigned to that row is minimal. The order in $\pi$ is then used as the order of the departments in each of the two rows. Given the assignments of the departments to the rows and the order of the departments in each row, we determine the exact positions of the departments by solving an LP, see, e.g., [4]. This approach is motivated by the construction of $L_{1}(\pi), L_{2}(\pi)$ in the equidistant case.

\subsubsection{Mincut heuristic}

Let a single-row layout $\pi$ be given and we assume, w.l.o.g., $\pi=(1, \ldots, n)$. We present a new heuristic based on the idea that the sorting of the departments in the same row is given via $\pi$, so it remains to determine the row assignment of the departments as well as their exact positions. We are given $n_{\min }, n_{\max } \in \mathbb{N}, n_{\min } \leq n_{\max }$, and in each step we add a set $S$ of departments which contains, if possible, at least $n_{\min }$ departments and at most $n_{\max }$ departments.

Let the set of departments $[h], 0 \leq h \leq n, h \in \mathbb{N}_{0}$, be already added to the double-row layout (we start with $h=0$ and we stop if $h=n$ ). If $h+n_{\max } \geq n$, all remaining departments are added 
and we set $S=\{h+1, \ldots, n\}$. Otherwise, we interpret the departments $H=\{h+1, \ldots, n\}$ as nodes in a complete graph with weights $w_{i j}, i, j \in H, i<j$. Our goal is to determine some $k^{\prime}$ and an associated set $S:=\left\{h+1, h+2, \ldots, k^{\prime}\right\}$ such that the sum of the total transport weights between $S$ and $[n] \backslash\left[k^{\prime}\right]$ is small. So we detect which departments should be considered together in the next step. We set

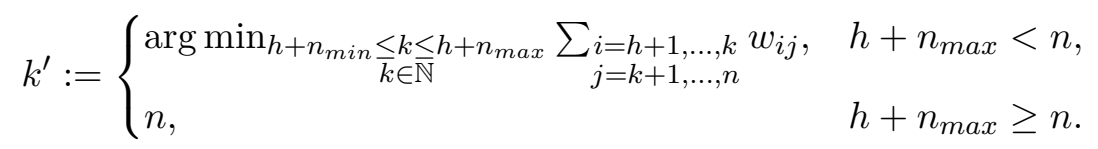

So $|S| \leq n_{\max }$, and, if $h+n_{\min } \leq n$, then $|S| \geq n_{\min }$. The calculation of $k^{\prime}$ is related to the calculation of a constrained minimum cut in the graph described above.

Then, we add the dummy department $n+1(n+2)$ to row 1 (row 2) with length $\ell_{n+1}=\ell_{n+2}=0$ and weights $w_{i(n+1)}=w_{(n+1) i}=w_{i(n+2)}=w_{(n+2) i}=\frac{1}{2} \sum_{\substack{i=h+1, \ldots, k^{\prime} \\ j=k^{\prime}+1, \ldots, n}} w_{i j}$ such that $n+1(n+2)$ is the rightmost department in row 1 (row 2). Knowing $k^{\prime}$ and so $S$, our goal is to determine a row assignment of the departments $S$ such that departments in the same row are sorted according to $\pi$ and such that $\sum_{i, j \in\left[k^{\prime}\right] \cup\{n+1, n+2\}} w_{i j} d_{i j}$ is minimized, i. e., we have to solve a (small) double-row instance where the order of the departments in the same row is known. For solving this problem we apply the approach of [28] and enumerate over all distinguishable assignments of the departments $S$ to the rows. Knowing the order of the departments in the rows, each subproblem reduces to some LP with $k^{\prime}+2$ departments. We choose one of the row assignments for $S$ where the layout has minimal objective value. In the last step, when $n \in S$, the solution of the LP corresponds to a double-row layout including possible free-spaces. The algorithm stops after returning this layout. We denote this heuristic by $\mathrm{mc}\left(n_{\min }, n_{\max }\right)$.

Considering the DREFLP and choosing $n_{\min }=n_{\max }=n$ in the approach here the resulting objective value is less than or equal to the objective value obtained by solving the LP (3)-(5) with $x_{i} \in[0,1], i \in[n]$. However, a huge number of row assignments would have to be checked.

\subsubsection{Exchange algorithms}

The layouts derived in Section 3.2.1 and Section 3.2.2 are our initial start solutions. We denote a given double-row layout by $\sigma=\left(\sigma_{1}, \ldots, \sigma_{k}, f, \sigma_{k+1}, \ldots, \sigma_{n}\right)$, where $f$ indicates that the departments which arise in the order of $\sigma$ before $f$ are assigned to row 1 and the remaining departments are assigned to row 2 (in the order of $\sigma$ ). In [22] a 1-opt algorithm is used where in each step one department is arranged at every possible position in $\sigma$, and then an LP is solved to determine the exact position of the departments. The department is arranged on a position which leads to a minimal objective value.

Instead of using this enumerative approach, we set up an MILP model for the 1-opt algorithm. Recall that $d_{i j}=d_{j i}, i, j \in[n], i<j$, denotes the horizontal center-to-center distance between $i$ and $j$ and $p_{i}, i \in[n]$, denotes the position of the center of $i$. Given a double-row layout, we remove $t \in[n]$, so let, w. l. o. g., the departments $1, \ldots, k, k \in[n-1]$, be in row 1 and $k+1, \ldots, n-1$ be in row 2 in this sorting and $t=n$. Then, we use the following variables

$$
z_{i}= \begin{cases}1, & t \text { is arranged on position } i \text { in } \sigma \\ 0, & \text { otherwise }\end{cases}
$$

$i=1, \ldots, n+1$, where $z_{1}=1$ corresponds to $t$ left to $1, z_{k+1}=1$ corresponds to $t$ right to $k$, $z_{k+2}=1$ corresponds to $t$ left to $k+1$. Then our MILP model reads as follows with $M=\sum_{i \in[n]} \ell_{i}$

$$
\min \sum_{\substack{i, j \in[n] \\ i<j}} w_{i j} d_{i j}
$$




$$
\begin{array}{ll}
p_{i+1}-p_{i} \geq \frac{\ell_{i+1}+\ell_{i}}{2}, & i \in[n-2] \backslash\{k\}, \\
\sum_{i \in[n+1]} z_{i}=1, & \\
p_{i}-p_{t}-M z_{i} \geq \frac{\ell_{i}+\ell_{t}}{2}-M, & i \in[k], \\
p_{t}-p_{i}-M z_{i+1} \geq \frac{\ell_{i}+\ell_{t}}{2}-M, & i \in[k], \\
p_{i}-p_{t}-M z_{i+1} \geq \frac{\ell_{i}+\ell_{t}}{2}-M, & i \in[n-1] \backslash[k], \\
p_{t}-p_{i}-M z_{i+2} \geq \frac{\ell_{i}+\ell_{t}}{2}-M, & i \in[n-1] \backslash[k], \\
d_{i j}-p_{j}+p_{i} \geq 0, & i, j \in[k], i<j, \\
d_{i j}-p_{j}+p_{i} \geq 0, & i, j \in[n-1] \backslash[k], i<j, \\
d_{i j}-p_{j}+p_{i} \geq 0, & i \in[k] \cup\{t\}, j \in([n-1] \backslash[k]) \cup\{t\}, i \neq j, \\
d_{i j}-p_{i}+p_{j} \geq 0, & i \in[k] \cup\{t\}, j \in([n-1] \backslash[k]) \cup\{t\}, i \neq j, \\
p_{i} \geq \frac{\ell_{i}}{2}, & i \in[n], \\
d_{i j}=d_{j i} \geq 0, & i, j \in[n], i<j, \\
z_{i} \in\{0,1\}, & i \in[n+1] .
\end{array}
$$

By inequalities (16) we ensure that departments in the same row do not overlap, recall that the sorting of the departments is known. By equation (17) we ensure that $t$ is assigned to exactly one position in $\sigma$. If $t$ is assigned to row 1 , then we ensure by inequalities (18) that $t$ and the department to the right of $t$ do not overlap and by inequalities (19) we ensure that $t$ does not overlap with the department to the left of $t$ (if $t$ is assigned to row 2 , see inequalities (20)-(21)). The distance between departments $i, j \in[n-1], i<j$, in the same row is calculated by inequalities $(22)-(23)$ and distances between departments in different rows as well as distances between $i \in[n-1]$ and $t$ are calculated by inequalities (24)-(25).

Afterwards we use a 2-opt algorithm where we swap the position of two departments in $\sigma$. We only accept changes if the objective value is reduced and the 1-opt (2-opt) algorithm is applied until the double-row layout cannot be improved by a 1-opt (2-opt) step.

\section{Computational results}

In this section we present our computational results. The computational experiments are based on a $\mathrm{C}++$ implementation which uses Cplex 12.10 as an MILP solver [36]. All results were conducted on a 2.10GHz quad-core using Virtual Box 6 and running on Debian GNU/Linux 8 in single processor mode. As often done in the literature, in our computational experiments we focus on the DREFLP and the DRFLP. We apply our heuristics based on optimal or best known single-row layouts, see, e.g., [39, 40, 41], and we use a heuristic for the SRFLP with a short running time and which is easy to implement, i. e., we start with a random single-row layout and apply a 1-opt algorithm and a 2-opt algorithm until the single-row layout cannot be improved by a 1-opt or 2-opt step, respectively. The single-row layouts and all considered instances are available from the authors.

\subsection{Results for the DREFLP}

We start our computational study with the equidistant case. In Table 1 we display in column two (column three) the objective value of an optimal or best known (heuristically determined) single-row layout denoted by "Best known" ("Heuristic"). The objective value is marked with a 


\begin{tabular}{|c|c|c|c|c|c|c|c|c|c|}
\hline \multirow[b]{2}{*}{ Instance } & \multicolumn{2}{|c|}{ SREFLP } & \multicolumn{2}{|c|}{ Start layout } & \multicolumn{2}{|c|}{ Exchange } & \multirow[b]{2}{*}[9]{} & \multicolumn{2}{|c|}{ Time } \\
\hline & Best known & Heuristic & $H_{\text {Best }}$ & $H_{\text {Heur }}$ & $H_{\text {Best }}$ & $H_{\text {Heur }}$ & & $H_{\text {Best }}$ & $H_{\text {Heur }}$ \\
\hline Y20 & $12185^{*}$ & $12185^{*}$ & 6047 & 6047 & $6046^{*}$ & $6046^{*}$ & $6046^{*}$ & $<1$ & $<1$ \\
\hline Y 25 & $20357^{*}$ & 20434 & 10170 & 10206 & 10170 & 10206 & 10170 & $<1$ & $<1$ \\
\hline Y30 & $27673^{*}$ & 27704 & 13801 & 13801 & 13800 & 13801 & 13790 & $<1$ & $<1$ \\
\hline Y35 & $38194^{*}$ & 38290 & 19093 & 19141 & 19087 & 19141 & 19087 & 1 & 1 \\
\hline Y40 & $47561^{*}$ & 47604 & 23737 & 23762 & 23732 & 23759 & 23739 & 3 & 2 \\
\hline Y45 & 62904 & 63357 & 31447 & 31671 & 31442 & 31671 & 31442 & 4 & 4 \\
\hline Y50 & 83127 & 83179 & 41523 & 41538 & 41523 & 41538 & 41517 & 7 & 7 \\
\hline Y60 & 112126 & 112735 & 56017 & 56330 & 56010 & 56328 & 55986 & 32 & 17 \\
\hline sko42-1 & 25525 & 25525 & 12749 & 12749 & 12743 & 12743 & 12731 & 2 & 2 \\
\hline sko49-1 & 40967 & 42469 & 20477 & 21226 & 20470 & 21224 & 20512 & 6 & 6 \\
\hline sko56-1 & 64024 & 66083 & 31975 & 33011 & 31972 & 32932 & 31988 & 11 & 24 \\
\hline sko64-1 & 96883 & 98122 & 48418 & 49052 & 48409 & 49004 & 48574 & 23 & 45 \\
\hline sko72-1 & 139150 & 143317 & 69535 & 71607 & 69531 & 71603 & 69621 & 41 & 42 \\
\hline sko81-1 & 205106 & 208554 & 102549 & 104263 & 102549 & 104067 & 102793 & $1: 10$ & $4: 36$ \\
\hline sko100-1 & 378234 & 384049 & 189062 & 191982 & 189056 & 191964 & - & $3: 31$ & $3: 56$ \\
\hline
\end{tabular}

Table 1: Heuristically determined upper bounds for equidistant double-row instances from the literature [9]. Our heuristics are based on optimal or best known single-row layouts as well as heuristically determined single-row layouts where known optimal solution values are marked with a "*". The running times of our heuristics are given in sec or min:sec.

"** if the associated single-row layout is known to be an optimal layout. The objective value of the start layout and of the final layout after applying our exchange algorithm is denoted by $H_{\text {Best }}$ $\left(H_{\text {Heur }}\right)$ which is based on the best known single-row layout (heuristically determined single-row layout). The current best upper bounds for these instances are derived by the semidefinite optimization approach of [9] and are given in column eight. This approach mainly focuses on determining strong lower bounds and the time limit is set to 3 hours. In the last two columns we summarize the running times of our heuristics, see Section 2.2, given in sec or min:sec. The best known or optimal single-row layouts are available at https://www.philipphungerlaender. com/benchmark-libraries/layout-lib/row-layout-instances/.

We observe that for all instances in Table 1 our heuristic based on optimal or best known single-row layouts is better than the one based on heuristically determined single-row layouts. Note that only for the instance Y20 our single-row heuristic derived an optimal solution, but the obtained gaps for the remaining instances are rather small and the running time is at most one minute, even for $n=100$. For the instance Y20 our heuristic derives an optimal double-row layout based on an optimal (heuristically determined) single-row layout. For larger instances the optimal solutions are not known. Considering the Y-instances with $25 \leq n \leq 60$ and given a best known single-row layout, we obtain three times the same objective value as the approach of [9], once we can even improve the best known upper bound and three times we obtain small gaps to the best known upper bounds. For all large sko-instances with $n \geq 49$ and given some best known single-row layout, we improve the previously known best upper bounds in [9] with a significantly smaller running time. Using our approach based on heuristically determined single-row layouts, we obtain small gaps to the approach of [9], however, these layouts can be calculated in a few minutes, including the calculation of the corresponding single-row layout. Comparing the best solution values of the SREFLP and the DREFLP one can see that the value of the DREFLP is strictly less than halve the value of the SREFLP, but rather close to this value in our tests.

\subsection{Results for the DRFLP}

In the following, we denote the balanced row heuristic by br. Given a single-row layout $\pi=\left(\pi_{1}, \ldots, \pi_{n}\right)$, we calculate a start layout via our heuristics br and mc and afterwards we calculate again a start layout based on the inverted order $\left(\pi_{n}, \ldots, \pi_{1}\right)$. In Tables $2-5$ we display the minimum value of these two layouts and we apply our improvement algorithm only on one double-row layout with minimal objective value. In our test we used $n_{\min }=8$ and $n_{\max }=14$ in 


\begin{tabular}{lrrrrrrr}
\hline & & \multicolumn{2}{c}{ Start layout } & & \multicolumn{2}{c}{ Exchange } & \\
Instance & \multicolumn{1}{c}{ SRFLP } & \multicolumn{1}{c}{$\mathrm{br}$} & $\mathrm{mc}(8,14)$ & & \multicolumn{1}{c}{ br } & $\mathrm{mc}(8,14)$ & \multicolumn{1}{c}{ DRFLP } \\
\hline Am14_1 & $5481.5^{*}$ & 2873.5 & 2743.5 & & 2738.5 & 2738.5 & $2738.5^{*}$ \\
Am14a & $5673.0^{*}$ & 2981.0 & 2907.0 & & 2907.0 & 2907.0 & $2904.0^{*}$ \\
Am14b & $5595.0^{*}$ & 2773.0 & 2736.0 & & 2736.0 & 2736.0 & $2736.0^{*}$ \\
Am15_1 & $6305.0^{*}$ & 3236.0 & 3211.0 & & 3223.0 & 3211.0 & $3195.0^{*}$ \\
HK15 & $33220.0^{*}$ & 17040.0 & 16895.0 & & 16740.0 & 16600.0 & $16570.0^{*}$ \\
$P 16 a$ & $14829.0^{*}$ & 7427.0 & 7365.5 & & 7416.0 & 7365.5 & $7365.5^{*}$ \\
$P 16 b$ & $11878.5^{*}$ & 5944.5 & 5870.5 & & 5928.5 & 5870.5 & $5870.5^{*}$ \\
\hline
\end{tabular}

Table 2: Results for instances from the literature where optimal DRFLP solution values are known [28]. Given an optimal single-row layout, the mc heuristic derived four times an optimal double-row layout and the br heuristic two times. The start layouts were only slightly improved by the exchange algorithm.

the mc heuristic.

In Table 2 we consider double-row instances with known optimal solution values. Optimal single-row layouts can be derived very fast for these small instances via, e. g., the approach of [3], so we apply our heuristics only on optimal single-row layouts in Table 2. The mc heuristic determined four optimal double-row layouts and the br heuristic calculated two optimal doublerow layouts. If an optimal single-row layout was given, the running time of the br heuristic was less than one second and the mc heuristic needed less than one minute (including the improvement algorithm). Considering the values of the start layouts derived with the mc and the br heuristics, the mc heuristic was always better. The start layouts were only slightly improved by the exchange algorithm.

In Table 3-5 we display in column two (three) the objective value of an optimal or best known (heuristically determined) single-row layout. The objective value is marked with a "*" if the single-row layout is optimal. The objective value of the start layout and the final layout is denoted by $\mathrm{br}_{\text {opt }}$ and $\mathrm{mc}(8,14)_{\text {opt }}$ or $\mathrm{br}_{\text {Best }}$ and $\mathrm{mc}(8,14)_{\text {Best }}\left(\mathrm{br}_{\text {Heur }}\right.$ and $\left.\mathrm{mc}(8,14)_{\text {Heur }}\right)$ if the heuristics are based on an optimal or best known (heuristically determined) single-row layout. The optimal single-row layouts in Table $3-4$ are obtained by the approach of [26] and are available from the authors.

In [6] four heuristics are presented and each of them is applied ten times. The minimum value is displayed in Table 3 in the twelfth column. Each single run for $n=40$ needs about an hour, which leads to a high total running time of this approach. The equidistant instance N40-1 is neglected in Table 3. Let a single-row layout be given, then our heuristics run in a few minutes. For all instances in Table 3, the mc heuristic based on an optimal single-row layout obtains gaps of less than $0.5 \%$ to the results of [6] and for one instance our heuristic obtains a layout with the same objective value. This shows that our approach is able to derive high-quality double-row layouts even for larger instances and our approach is much faster than the methods in the literature. For these instances the running time of the br and the mc heuristic hardly depends of the start layout, so we only displayed the running times based on optimal single-row layouts. Note that the start layouts are only slightly improved by the exchange algorithm and considering the mc heuristic, the final layout often equals the start layout. The quality of our start layouts depends on the quality of the single-row layouts. Given an optimal (heuristically determined) single-row layout, then the mc heuristic is better than the br heuristic. However, the br heuristic based on optimal single-row layouts is often better than the mc heuristic based on heuristically determined single-row layouts.

In Table 4-5 we compare our approach with the heuristic presented in [19] where five start layouts are computed and the minimal objective value is displayed. Then, our exchange algorithm is applied on one layout with minimal objective value. We consider randomly generated instances from the literature [22] with $n \in\{20,30,40,50\}$. The transport density is set to $10 \%, 50 \%$ and $100 \%$ and integer transport weights are chosen randomly between 1 and 10 . The integral lengths 


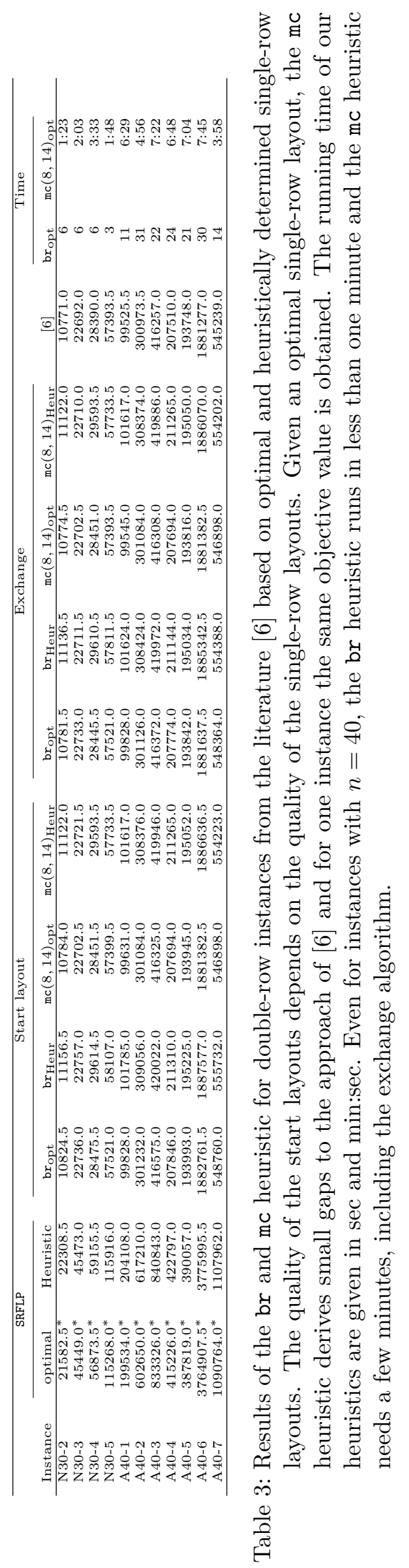


of the departments are chosen randomly between 1 and 15 as well as between 5 and 10. For each type ten instances are tested and the average values are displayed in Table 4 . We denote the instances by $n_{k, \ell_{\min }-\ell_{\max }}$ where $n$ is the number of departments, $k$ is the transport density and $\ell_{\min }$ and $\ell_{\max }$ describe the upper and the lower bound of the integral department lengths.

Considering sparse instances in Table 4, the heuristically determined layouts have large gaps to optimal single-row layouts and the corresponding double-row layouts of the br and mc heuristic have large gaps to the layouts based on optimal single-row layouts. So the quality of the start layout and of the final layout of the br and mc heuristic depend highly on the quality of the single-row layout. Note that it would be possible to improve the SRFLP solutions by using the fact that the sparse instances with $n=20$ and $n=30$ could often be divided into two or more independent smaller instances. Given an optimal single-row layout, then the objective values of the start layout and of the final layout of the br and mc heuristic are smaller than the corresponding objective values of the approach of [19]. The exchange algorithms only slightly improve the start layouts of the br and mc heuristic, however, the start layout of [19] is improved significantly by the exchange algorithms. Note again that the mc heuristic is better than the br heuristic if both heuristics are based on optimal or heuristically determined single-row layouts.

In Table 5 we only consider sko-instances where good heuristically determined single-row layouts are available at https://www.philipphungerlaender.com/benchmark-libraries/layout-lib/ row-layout-instances/. The instance sko56-5 marked with a "o" is not the best known singlerow layout but the best known single-row layout with objective value 592294.5 is not available online, so we decided to choose this single-row layout. Looking at the results for the sko-instances in Table 5 all solutions derived using the br and the mc heuristic based on best known single-row layouts are better than the results of [19]. If we use the two heuristics in combination with our simple single-row heuristic, we could improve 5 out of 9 upper bounds in comparison to the approach in [19]. As seen in Tables 3-4, for most instances the mc heuristic is slightly better than the br heuristic based on best known (heuristically determined) single-row layouts. For the mc heuristic based on best known single-row layouts, the exchange algorithm only slightly improves the start layout.

In Table 6 we compare the running times of the heuristics applied on the sko-instances. The br heuristic is the fastest heuristic for best known and heuristically determined single-row layouts. In column three we display the running time of the br heuristic based on a best known single-row layout, where the 1-opt algorithm is calculated by an enumerative approach instead of using our MILP approach. For 8 out of 9 instances the running time is at least halved by using our MILP approach. Altogether, the running times of the br and the mc heuristics are not highly influenced by choosing a best known or heuristically determined single-row layout. The mc heuristic is for both, best known and heuristically determined single-row layouts, a bit slower than the heuristic approach of [19].

\section{Conclusion and future work}

In this paper we studied the relationship of the MREFLP and the SREFLP and we proved that $v_{m}^{*} \leq \frac{v_{1}^{*}}{m}$ where $v_{m}^{*}$ denotes the optimal value of the MREFLP with $m \in \mathbb{N}$ rows. Given an equidistant single-row layout with value $v_{1}$, we presented an easy way to derive an equidistant multi-row layout whose value $v_{m}$ is at most $\frac{v_{1}}{m}$. We can further improve such a layout by the inclusion of free spaces via a new ILP model which simplifies to some LP model. For the DREFLP we improved some of the best known upper bounds and we significantly reduced the running time for calculating these layouts.

We proved that the optimal solution value of the SRFLP and the DRFLP for the same instance might be close. In particular, we showed that the following inequality holds $(n-1) v_{2}^{*} \leq(n-2) v_{1}^{*}$, where $v_{1}^{*}, v_{2}^{*}$ denote the optimal solution values of the two problems. Additionally, we presented an example where this bound is tight. Nonetheless, good or optimal single-row layouts can be a 


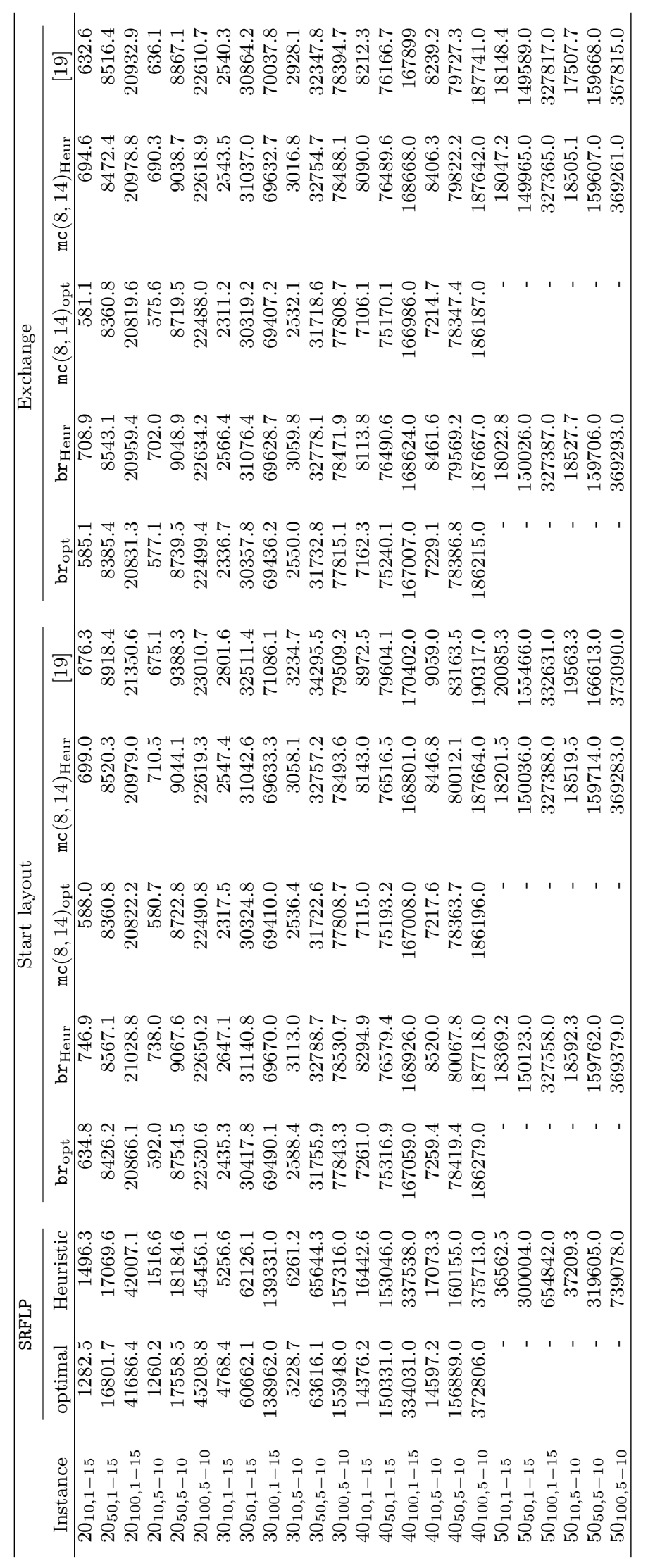

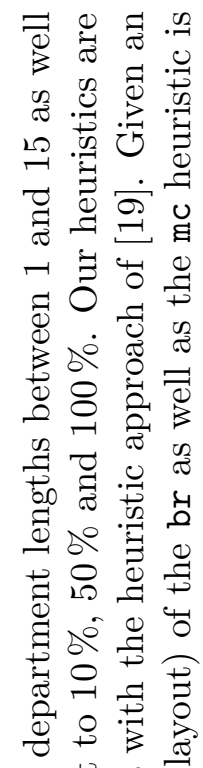

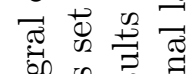

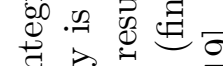

苟高表泀索

萨导

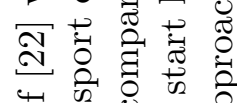

प

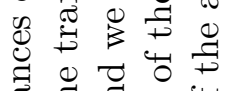

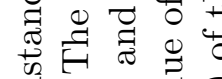

.

త

矛过

क्ष

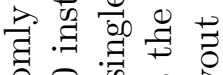

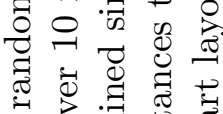

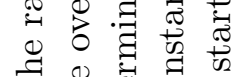

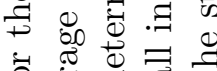

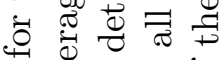

. 충

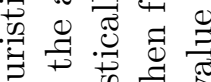

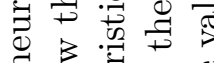

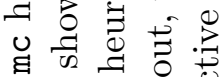

उ

ปै

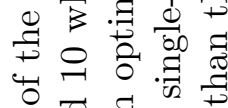

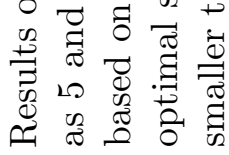

$\ddot{\forall}$

$\frac{0}{\frac{\pi}{4}}$ 


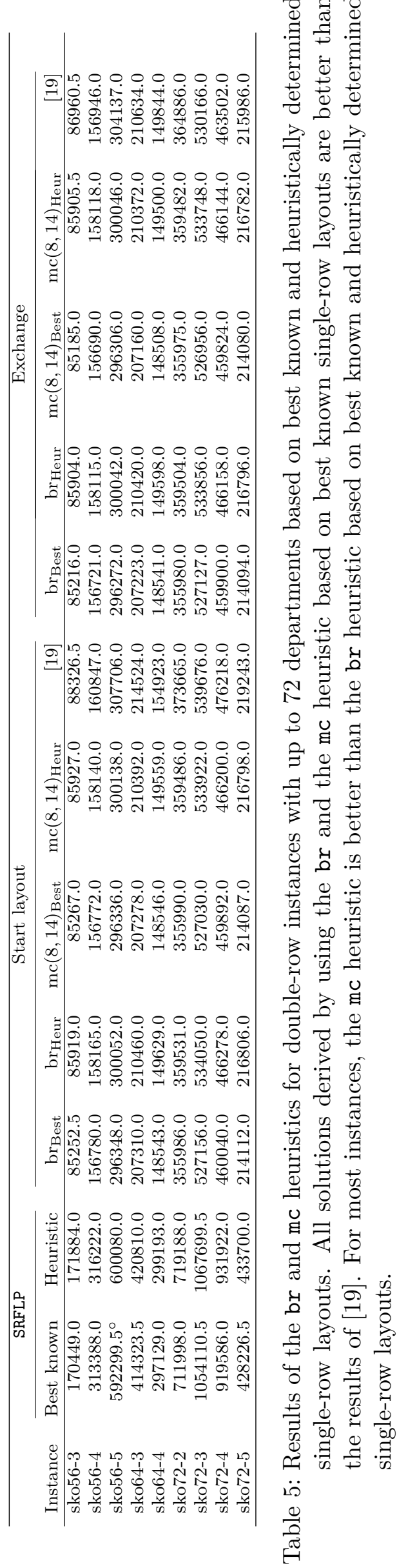




\begin{tabular}{rrrrrrr}
\hline Instance & br $_{\text {Best }}$ & br $_{\text {Best }}^{\text {nu }}$ & br $_{\text {Heur }}$ & $\mathrm{mc}(8,14)_{\text {Best }}$ & $\mathrm{mc}(8,14)_{\text {Heur }}$ & {$[19]$} \\
\hline sko 56-3 & $2: 13$ & $7: 49$ & $2: 20$ & $19: 02$ & $26: 38$ & $9: 44$ \\
sko 56-4 & $3: 10$ & $6: 37$ & $4: 50$ & $20: 36$ & $25: 10$ & $9: 50$ \\
sko 56-5 & $3: 16$ & $7: 07$ & $1: 33$ & $21: 02$ & $23: 52$ & $9: 02$ \\
sko 64-3 & $6: 59$ & $18: 15$ & $2: 43$ & $39: 36$ & $39: 29$ & $22: 50$ \\
sko 64-4 & $2: 35$ & $9: 51$ & $4: 35$ & $39: 28$ & $36: 17$ & $24: 24$ \\
sko 72-2 & $8: 46$ & $23: 20$ & $4: 54$ & $1: 03: 33$ & $54: 57$ & $39: 32$ \\
sko 72-3 & $10: 36$ & $20: 13$ & $11: 38$ & $1: 06: 41$ & $1: 22: 41$ & $53: 26$ \\
sko 72-4 & $18: 54$ & $33: 34$ & $11: 20$ & $1: 12: 32$ & $1: 17: 09$ & $1: 03: 50$ \\
sko 72-5 & $8: 44$ & $23: 17$ & $9: 03$ & $56: 28$ & $1: 02: 30$ & $40: 46$ \\
\hline
\end{tabular}

Table 6: In this table we compare the running times of the heuristic approaches considered in Table 5. The running times are given in sec, min:sec and h:min:sec, respectively. In the third column the running time of the br heuristic is displayed with an enumerative 1-opt algorithm as used in [22]. One can see that the running time is significantly reduced by using our MILP approach for the 1-opt algorithm, displayed in the second column.

good starting point for deriving good double-row layouts. Indeed, we presented two heuristics for the DRFLP, which rely on the ideas used for the MREFLP and which can be calculated very fast. We obtained very small gaps to the best known upper bounds for instances with 30 and 40 departments, but derive these solutions much faster and for instances with more departments we outperform the heuristic of [19].

It remains for future work to set up heuristics for layout problems with more complicated path structures like the T-Row Facility Layout Problem [21]. Further it is interesting from a practical point of view to extend the current exact models and heuristics for the Single-Row and Double-Row Facility Layout Problem such that more aspects like individual input and output positions of the departments or certain clearance conditions between the departments can be taken into account.

\section{References}

[1] André R. S. Amaral. On the exact solution of a facility layout problem. European Journal of Operational Research, 173(2):508-518, 2006.

[2] André R. S. Amaral. An exact approach to the one-dimensional facility layout problem. Operations Research, 56(4):1026-1033, 2008.

[3] André R. S. Amaral. A new lower bound for the single row facility layout problem. Discrete Applied Mathematics, 157(1):183-190, 2009.

[4] André R. S. Amaral. Optimal solutions for the double row layout problem. Optimization Letters, 7(2):407-413, 2013.

[5] André R. S. Amaral. A mixed-integer programming formulation for the double row layout of machines in manufacturing systems. International Journal of Production Research, $57(1): 34-47,2019$.

[6] André R. S. Amaral. A heuristic approach for the double row layout problem. Annals of Operations Research, pages 1-36, 2020.

[7] André R. S. Amaral and Adam N. Letchford. A polyhedral approach to the single row facility layout problem. Mathematical Programming, 141(1-2):453-477, 2013.

[8] Miguel F. Anjos, Anja Fischer, and Philipp Hungerländer. Solution approaches for the double-row equidistant facility layout problem. In Marco Lübbecke, Arie Koster, Peter 
Letmathe, Reinhard Madlener, Britta Peis, and Grit Walther, editors, Operations Research Proceedings 2014, pages 17-23, Cham, 2016. Springer International Publishing.

[9] Miguel F. Anjos, Anja Fischer, and Philipp Hungerländer. Improved exact approaches for row layout problems with departments of equal length. European Journal of Operational Research, 270(2):514-529, 2018.

[10] Miguel F. Anjos, Andrew Kennings, and Anthony Vannelli. A semidefinite optimization approach for the single-row layout problem with unequal dimensions. Discrete Optimization, 2(2):113-122, 2005.

[11] Miguel F. Anjos and Anthony Vannelli. Computing globally optimal solutions for single-row layout problems using semidefinite programming and cutting planes. INFORMS Journal On Computing, 20(4):611-617, 2008.

[12] Miguel F. Anjos and Manuel V. C. Vieira. Mathematical optimization approaches for facility layout problems: The state-of-the-art and future research directions. European Journal of Operational Research, 261(1):1-16, 2017.

[13] Miguel F. Anjos and Manuel V. C. Vieira. Mathematical optimization approach for facility layout on several rows. Optimization Letters, pages 1-15, 2020.

[14] Miguel F. Anjos and Ginger Yen. Provably near-optimal solutions for very large single-row facility layout problems. Optimization Methods and Software, 24(4):805-817, 2009.

[15] Maria M. Azevedo, José A. Crispim, and Jorge P. de Sousa. A dynamic multi-objective approach for the reconfigurable multi-facility layout problem. Journal of manufacturing systems, 42:140-152, 2017.

[16] Uwe Bracht, Mirko Dahlbeck, Anja Fischer, and Thomas Krüger. Combining simulation and optimization for extended double row facility layout problems in factory planning. In Marcus Baum, Gunther Brenner, Jens Grabowski, Thomas Hanschke, Stefan Hartmann, and Anita Schöbel, editors, Simulation Science, pages 39-59, Cham, 2018. Springer International Publishing.

[17] Henning Bruhn and Dieter Rautenbach. Maximal determinants of combinatorial matrices. Linear Algebra and its Applications, 553:37-57, 2018.

[18] Junjae Chae and Amelia C. Regan. A mixed integer programming model for a double row layout problem. Computers \& Industrial Engineering, 140:106244, 2020.

[19] Jaewoo Chung and J. M. A. Tanchoco. The double row layout problem. International Journal of Production Research, 48(3):709-727, 2010.

[20] Gildásio L. Cravo and André R. S. Amaral. A grasp algorithm for solving large-scale single row facility layout problems. Computers $\& 3$ Operations Research, 106:49-61, 2019.

[21] Mirko Dahlbeck. A mixed-integer linear programming approach for the T-row and the multi-bay facility layout problem. Submitted; preprint available at http://www . optimization-online.org/DB_HTML/2020/03/7670.html, 2020.

[22] Mirko Dahlbeck, Anja Fischer, and Frank Fischer. Decorous combinatorial lower bounds for row layout problems. European Journal of Operational Research, 286(3):929-944, 2020.

[23] Mirko Dahlbeck, Anja Fischer, Philipp Hungerländer, and Kerstin Maier. New exact approaches for the combined cell layout problem and extensions of the multi-bay facility layout problem. Submitted; preprint available at http://www.optimization-online.org/ DB_HTML/2020/10/8068.html, 2020. 
[24] Dilip Datta, André R. S. Amaral, and José Rui Figueira. Single row facility layout problem using a permutation-based genetic algorithm. European Journal of Operational Research, 213(2):388-394, 2011.

[25] Amine Drira, Henri Pierrval, and Sonia Hajri-Gabouj. Facility layout problems: A survey. Annual Reviews in Control, 31:255-267, 2007.

[26] Anja Fischer and Frank Fischer. An extended polyhedral study of the betweenness model. Working paper, 2020.

[27] Anja Fischer, Frank Fischer, and Philipp Hungerländer. A new exact approach to the space-free double row layout problem. In Karl F. Doerner, Ivana Ljubic, Georg Pflug, and Gernot Tragler, editors, Operations Research Proceedings 2015, Selected Papers of the International Conference of the German, Austrian and Swiss Operations Research Societies (GOR, ÖGOR, SVOR/ASRO), University of Vienna, Austria, September 1-4, 2015, pages 125-130. Springer, 2015.

[28] Anja Fischer, Frank Fischer, and Philipp Hungerländer. New exact approaches to row layout problems. Mathematical Programming Computation, 11(4):703-754, 2019.

[29] Michael Randolph Garey, David S. Johnson, and Larry J. Stockmeyer. Some simplified NP-complete problems. In STOC 'r4: Proceedings of the sixth annual ACM symposium on Theory of computing, pages 47-63. ACM, 1974.

[30] Mehmet Gülşen, Chase C. Murray, and Alice E. Smith. Double-row facility layout with replicate machines and split flows. Computers \& Operations Research, 108:20-32, 2019.

[31] M.M.D. Hassan. Machine layout problem in modern manufacturing facilities. International Journal of Production Research, 32(11):2559-2584, 1994.

[32] Philipp Hungerländer. Single-row equidistant facility layout as a special case of single-row facility layout. International Journal of Production Research, 52(5):1257-1268, 2014.

[33] Philipp Hungerländer and Miguel F. Anjos. A semidefinite optimization-based approach for global optimization of multi-row facility layout. European Journal of Operational Research, 245(1):46-61, 2015.

[34] Philipp Hungerländer and Franz Rendl. A computational study and survey of methods for the single-row facility layout problem. Computational Optimization and Applications, $55(1): 1-20,2013$.

[35] Philipp Hungerländer and Franz Rendl. Semidefinite relaxations of ordering problems. Mathematical Programming, 140(1):77-97, 2013.

[36] ILOG CPLEX Optimization Studio 12.10, 2019.

[37] Birgit Keller and Udo Buscher. Single row layout models. European Journal of Operational Research, 245(3):629-644, 2015.

[38] Ravi Kothari and Diptesh Ghosh. The single row facility layout problem: state of the art. OPSEARCH, 49(4):442-462, 2012.

[39] Ravi Kothari and Diptesh Ghosh. Insertion based Lin-Kernighan heuristic for single row facility layout. Computers \& Operations Research, 40(1):129-136, 2013.

[40] Ravi Kothari and Diptesh Ghosh. Tabu search for the single row facility layout problem using exhaustive 2-opt and insertion neighborhoods. European Journal of Operational Research, 224(1):93-100, 2013. 
[41] Ravi Kothari and Diptesh Ghosh. A scatter search algorithm for the single row facility layout problem. Journal of Heuristics, 20(2):125-142, 2014.

[42] Chase C. Murray, Alice E. Smith, and Zeqiang Zhang. An efficient local search heuristic for the double row layout problem with asymmetric material flow. International Journal of Production Research, 51(20):6129-6139, 2013.

[43] Gintaras Palubeckis. A branch-and-bound algorithm for the single-row equidistant facility layout problem. OR Spectrum, 34(1):1-21, 2012.

[44] Gintaras Palubeckis. Fast local search for single row facility layout. European Journal of Operational Research, 264(3):800-814, 2015.

[45] Gintaras Palubeckis. Fast simulated annealing for single-row equidistant facility layout. Applied Mathematics and Computation, 263:287-301, 2015.

[46] Gintaras Palubeckis. Single row facility layout using multi-start simulated annealing. Computers \& Industrial Engineering, 103:1-16, 2017.

[47] Jean-Claude Picard and Maurice Queyranne. On the one-dimensional space allocation problem. Operations Research, 29(2):371-391, 1981.

[48] Soroush Safarzadeh and Hamidreza Koosha. Solving an extended multi-row facility layout problem with fuzzy clearances using ga. Applied Soft Computing, 61:819-831, 2017.

[49] Leonardo D. Secchin and André R. S. Amaral. An improved mixed-integer programming model for the double row layout of facilities. Optimization Letters, 13(1):193-199, 2019.

[50] Donald M. Simmons. One-dimensional space allocation: An ordering algorithm. Operations Research, 17(5):812-826, 1969.

[51] James A. Tompkins, John A. White, Yavuz A. Bozer, and J. M. A. Tanchoco. Facilities planning. John Wiley \& Sons, 2010.

[52] Shengli Wang, Xingquan Zuo, Xueqing Liu, Xinchao Zhao, and Jianqiang Li. Solving dynamic double row layout problem via combining simulated annealing and mathematical programming. Applied Soft Computing, 37:303-310, 2015.

[53] Zeqiang Zhang and Chase C. Murray. A corrected formulation for the double row layout problem. International Journal of Production Research, 50(15):4220-4223, 2012.

[54] Xingquan Zuo, Chase C. Murray, and Alice E. Smith. Solving an extended double row layout problem using multiobjective tabu search and linear programming. IEEE Transactions on Automation Science and Engineering, 11(4):1122-1132, 2014.

[55] Xingquan Zuo, Chase C. Murray, and Alice E. Smith. Sharing clearances to improve machine layout. International Journal of Production Research, 54(14):4272-4285, 2016. 